\title{
Pharmacological, psychological, and non-invasive brain stimulation interventions for treating depression after stroke (Review)
}

Allida S, Cox KL, Hsieh CF, Lang H, House A, Hackett ML

Allida S, Cox KL, Hsieh CF, Lang H, House A, Hackett ML.

Pharmacological, psychological, and non-invasive brain stimulation interventions for treating depression after stroke.

Cochrane Database of Systematic Reviews 2020, Issue 1. Art. No.: CD003437.

DOI: 10.1002/14651858.CD003437.pub4.

www.cochranelibrary.com

Pharmacological, psychological, and non-invasive brain stimulation interventions for treating depression after stroke (Review) 
TABLE OF CONTENTS

HEADER

ABSTRACT

PLAIN LANGUAGE SUMMARY

SUMMARY OF FINDINGS

BACKGROUND

OBJECTIVES

METHODS

Figure 1.

RESULTS

Figure 2.

Figure 3.

DISCUSSION

Figure 4.

AUTHORS' CONCLUSIONS

ACKNOWLEDGEMENTS

REFERENCES

CHARACTERISTICS OF STUDIES

DATA AND ANALYSES

Analysis 1.1. Comparison 1 Pharmacological interventions versus placebo, Outcome 1 Depression: meeting study criteria for depression at end of treatment.

Analysis 1.2. Comparison 1 Pharmacological interventions versus placebo, Outcome 2 Depression: $<50 \%$ reduction in scale scores at end of treatment.

Analysis 1.3. Comparison 1 Pharmacological interventions versus placebo, Outcome 3 Depression: average change in scores between baseline and end of treatment.

Analysis 1.4. Comparison 1 Pharmacological interventions versus placebo, Outcome 4 Depression: mean scores at end of treatment.

Analysis 1.5. Comparison 1 Pharmacological interventions versus placebo, Outcome 5 Anxiety: meeting study criteria for anxiety at end of treatment.

Analysis 1.6. Comparison 1 Pharmacological interventions versus placebo, Outcome 6 Cognitive function: average change in scores between baseline and end of treatment.

Analysis 1.7. Comparison 1 Pharmacological interventions versus placebo, Outcome 7 Cognitive function: mean scores at end of treatment.

Analysis 1.8. Comparison 1 Pharmacological interventions versus placebo, Outcome 8 Activities of daily living: average change in scores between baseline and end of treatment.

Analysis 1.9. Comparison 1 Pharmacological interventions versus placebo, Outcome 9 Activities of daily living: mean scores at end of treatment.

Analysis 1.10. Comparison 1 Pharmacological interventions versus placebo, Outcome 10 Disability: average change in scores between baseline and end of treatment.

Analysis 1.11. Comparison 1 Pharmacological interventions versus placebo, Outcome 11 Disability: mean scores at end of treatment.

Analysis 1.12. Comparison 1 Pharmacological interventions versus placebo, Outcome 12 Neurological function: average change in scores between baseline and end of treatment.

Analysis 1.13. Comparison 1 Pharmacological interventions versus placebo, Outcome 13 Neurological function: mean scores at end of treatment.

Analysis 1.14. Comparison 1 Pharmacological interventions versus placebo, Outcome 14 Adverse events: death.

Analysis 1.15. Comparison 1 Pharmacological interventions versus placebo, Outcome 15 Adverse events: all.

Analysis 1.16. Comparison 1 Pharmacological interventions versus placebo, Outcome 16 Adverse events: leaving the study early (including death).

Analysis 2.2. Comparison 2 Non-invasive brain stimulation versus sham non-invasive brain stimulation and/or usual care, Outcome 2 Depression: $<50 \%$ reduction in scale scores at end of treatment.

Analysis 2.3. Comparison 2 Non-invasive brain stimulation versus sham non-invasive brain stimulation and/or usual care, Outcome 3 Depression: mean scores at end of treatment.

Analysis 2.4. Comparison 2 Non-invasive brain stimulation versus sham non-invasive brain stimulation and/or usual care, Outcome 4 Depression: mean scores at end of follow-up. 
Analysis 2.5. Comparison 2 Non-invasive brain stimulation versus sham non-invasive brain stimulation and/or usual care, Outcome 5 Activities of daily living: mean scores at end of treatment.

Analysis 2.6. Comparison 2 Non-invasive brain stimulation versus sham non-invasive brain stimulation and/or usual care, Outcome 6 Neurological function: average change in scores between baseline and end of treatment.

Analysis 2.7. Comparison 2 Non-invasive brain stimulation versus sham non-invasive brain stimulation and/or usual care, Outcome 7 Neurological function: mean scores at end of treatment.

Analysis 2.8. Comparison 2 Non-invasive brain stimulation versus sham non-invasive brain stimulation and/or usual care, Outcome 8 Adverse events: death.

Analysis 2.9. Comparison 2 Non-invasive brain stimulation versus sham non-invasive brain stimulation and/or usual care, Outcome 9 Adverse events: all.

Analysis 2.10. Comparison 2 Non-invasive brain stimulation versus sham non-invasive brain stimulation and/or usual care, Outcome 10 Adverse events: leaving the study early (including death).

Analysis 3.1. Comparison 3 Psychological therapy versus usual care and/or attention control, Outcome 1 Depression: meeting study criteria for depression at end of treatment.

Analysis 3.3. Comparison 3 Psychological therapy versus usual care and/or attention control, Outcome 3 Depression: average change in scores between baseline and end of treatment.

Analysis 3.4. Comparison 3 Psychological therapy versus usual care and/or attention control, Outcome 4 Depression: mean scores at end of treatment.

Analysis 3.5. Comparison 3 Psychological therapy versus usual care and/or attention control, Outcome 5 Depression: meeting study criteria for depression at end of follow-up.

Analysis 3.6. Comparison 3 Psychological therapy versus usual care and/or attention control, Outcome 6 Depression: average change in scores between baseline and end of follow-up.

Analysis 3.7. Comparison 3 Psychological therapy versus usual care and/or attention control, Outcome 7 Depression: mean scores at end of follow-up.

Analysis 3.8. Comparison 3 Psychological therapy versus usual care and/or attention control, Outcome 8 Psychological distress: average change in scores between baseline and end of treatment.

Analysis 3.9. Comparison 3 Psychological therapy versus usual care and/or attention control, Outcome 9 Psychological distress: mean scores at end of treatment.

Analysis 3.10. Comparison 3 Psychological therapy versus usual care and/or attention control, Outcome 10 Anxiety: meeting study criteria for anxiety at end of treatment.

Analysis 3.11. Comparison 3 Psychological therapy versus usual care and/or attention control, Outcome 11 Anxiety: mean scores at end of treatment.

Analysis 3.12. Comparison 3 Psychological therapy versus usual care and/or attention control, Outcome 12 Anxiety: mean scores at end of follow-up.

Analysis 3.13. Comparison 3 Psychological therapy versus usual care and/or attention control, Outcome 13 Activities of daily living: average change in scores from baseline to end of treatment.

Analysis 3.14. Comparison 3 Psychological therapy versus usual care and/or attention control, Outcome 14 Activities of daily living: mean scores at end of treatment.

Analysis 3.15. Comparison 3 Psychological therapy versus usual care and/or attention control, Outcome 15 Activities of daily living: mean scores at end of follow-up.

Analysis 3.16. Comparison 3 Psychological therapy versus usual care and/or attention control, Outcome 16 Disability: mean scores at end of treatment.

Analysis 3.17. Comparison 3 Psychological therapy versus usual care and/or attention control, Outcome 17 Adverse events: death.

Analysis 3.18. Comparison 3 Psychological therapy versus usual care and/or attention control, Outcome 18 Adverse events: all.

Analysis 3.19. Comparison 3 Psychological therapy versus usual care and/or attention control, Outcome 19 Adverse events: leaving the study early (including death).

Analysis 4.3. Comparison 4 Pharmacological intervention and psychotherapy (combination) versus a pharmacological intervention and usual care or attention control (single), Outcome 3 Depression: mean scores at end of treatment.

Analysis 4.4. Comparison 4 Pharmacological intervention and psychotherapy (combination) versus a pharmacological intervention and usual care or attention control (single), Outcome 4 Anxiety: mean scores at end of treatment.

Analysis 4.5. Comparison 4 Pharmacological intervention and psychotherapy (combination) versus a pharmacological intervention and usual care or attention control (single), Outcome 5 Activities of daily living: mean scores at end of treatment.

Analysis 4.6. Comparison 4 Pharmacological intervention and psychotherapy (combination) versus a pharmacological intervention and usual care or attention control (single), Outcome 6 Neurological function: mean scores at end of treatment. .

178 
Analysis 4.7. Comparison 4 Pharmacological intervention and psychotherapy (combination) versus a pharmacological intervention and usual care or attention control (single), Outcome 7 Adverse events: death.

Analysis 4.8. Comparison 4 Pharmacological intervention and psychotherapy (combination) versus a pharmacological intervention and usual care or attention control (single), Outcome 8 Adverse events: all.

Analysis 4.9. Comparison 4 Pharmacological intervention and psychotherapy (combination) versus a pharmacological intervention and usual care or attention control (single), Outcome 9 Adverse events: leaving the study early (including death).

Analysis 5.1. Comparison 5 Non-invasive brain stimulation and a pharmacological intervention (combination) versus a pharmacological intervention and sham stimulation or usual care (single), Outcome 1 Depression: meeting the criteria for depression at end of treatment.

Analysis 5.2. Comparison 5 Non-invasive brain stimulation and a pharmacological intervention (combination) versus a pharmacological intervention and sham stimulation or usual care (single), Outcome 2 Depression: $<50 \%$ reduction in scale scores at end of treatment.

Analysis 5.3. Comparison 5 Non-invasive brain stimulation and a pharmacological intervention (combination) versus a pharmacological intervention and sham stimulation or usual care (single), Outcome 3 Depression: mean scores at end of treatment.

Analysis 5.4. Comparison 5 Non-invasive brain stimulation and a pharmacological intervention (combination) versus a pharmacological intervention and sham stimulation or usual care (single), Outcome 4 Depression: mean scores at end of followup.

Analysis 5.5. Comparison 5 Non-invasive brain stimulation and a pharmacological intervention (combination) versus a pharmacological intervention and sham stimulation or usual care (single), Outcome 5 Cognitive function: mean scores at end of treatment.

Analysis 5.6. Comparison 5 Non-invasive brain stimulation and a pharmacological intervention (combination) versus a pharmacological intervention and sham stimulation or usual care (single), Outcome 6 Activities of daily living: mean scores at end of treatment.

Analysis 5.7. Comparison 5 Non-invasive brain stimulation and a pharmacological intervention (combination) versus a pharmacological intervention and sham stimulation or usual care (single), Outcome 7 Disability: mean scores at end of treatment.

Analysis 5.8. Comparison 5 Non-invasive brain stimulation and a pharmacological intervention (combination) versus a pharmacological intervention and sham stimulation or usual care (single), Outcome 8 Neurological function: mean scores at end of treatment.

Analysis 5.9. Comparison 5 Non-invasive brain stimulation and a pharmacological intervention (combination) versus a pharmacological intervention and sham stimulation or usual care (single), Outcome 9 Adverse events: death.

Analysis 5.10. Comparison 5 Non-invasive brain stimulation and a pharmacological intervention (combination) versus a pharmacological intervention and sham stimulation or usual care (single), Outcome 10 Adverse events: all.

Analysis 5.11. Comparison 5 Non-invasive brain stimulation and a pharmacological intervention (combination) versus a pharmacological intervention and sham stimulation or usual care (single), Outcome 11 Adverse events: leaving the study early (including death).

ADDITIONAL TABLES

APPENDICES

WHAT'S NEW

HISTORY

CONTRIBUTIONS OF AUTHORS 
[Intervention Review]

\section{Pharmacological, psychological, and non-invasive brain stimulation interventions for treating depression after stroke}

Sabine Allida ${ }^{1}$, Katherine Laura Cox ${ }^{1}$, Cheng-Fang Hsieh² ${ }^{2}$ Helen Lang ${ }^{3}$, Allan House ${ }^{4}$, Maree L Hackett1,5

1Mental Health, The George Institute for Global Health, Faculty of Medicine, University of New South Wales, Sydney, Australia. 2Division of Geriatrics and Gerontology, Department of Internal Medicine and Department of Neurology, Kaohsiung Medical University Hospital, Kaohsiung Medical University, Kaohsiung, Taiwan. ${ }^{3}$ Toronto, Canada. ${ }^{4}$ Division of Psychological and Social Medicine, Leeds Institute of Health Sciences, University of Leeds, Leeds, UK. 5 Faculty of Health and Wellbeing, University of Central Lancashire, Preston, Lancashire, UK

Contact address: Maree L Hackett, Professor, Program Head, Mental Health, The George Institute for Global Health, Faculty of Medicine, University of New South Wales, PO Box M201, Missenden Road, Sydney, NSW, 2050, Australia. mhackett@georgeinstitute.org.au.

Editorial group: Cochrane Stroke Group.

Publication status and date: New search for studies and content updated (conclusions changed), published in Issue 1, 2020.

Citation: Allida S, Cox KL, Hsieh CF, Lang H, House A, Hackett ML. Pharmacological, psychological, and non-invasive brain stimulation interventions for treating depression after stroke. Cochrane Database of Systematic Reviews 2020, Issue 1. Art. No.: CD003437. DOI: 10.1002/14651858.CD003437.pub4.

Copyright @ 2020 The Cochrane Collaboration. Published by John Wiley \& Sons, Ltd.

\section{A B S T R A C T}

\section{Background}

Depression is an important morbidity associated with stroke that impacts on recovery yet often undetected or inadequately treated. This is an update and expansion of a Cochrane Review first published in 2004 and updated in 2008.

\section{Objectives}

Primary objective

- To determine whether pharmacological therapy, non-invasive brain stimulation, psychological therapy, or combinations of these interventions reduce the prevalence of diagnosable depression after stroke

Secondary objectives

- To determine whether pharmacological therapy, non-invasive brain stimulation, psychological therapy, or combinations of these interventions reduce levels of depressive symptoms, improve physical and neurological function and health-related quality of life, and reduce dependency after stroke

- To assess the safety of and adherence to such treatments

\section{Search methods}

We searched the Specialised Registers of Cochrane Stroke and Cochrane Depression Anxiety and Neurosis (last searched August 2018), the Cochrane Central Register of Controlled Trials (CENTRAL; 2018, Issue 1), in the Cochrane Library, MEDLINE (1966 to August 2018), Embase (1980 to August 2018), the Cumulative Index to Nursing and Allied Health Literature (CINAHL) (1982 to August 2018), PsycINFO (1967 to August 2018), and Web of Science (2002 to August 2018). We also searched reference lists, clinical trial registers (World Health Organization International Clinical Trials Registry Platform (WHO ICTRP) to August 2018; ClinicalTrials.gov to August 2018), and conference proceedings, and we contacted study authors. 


\section{Selection criteria}

Randomised controlled trials comparing (1) pharmacological interventions with placebo; (2) one of various forms of non-invasive brain stimulation with sham stimulation or usual care; (3) one of various forms of psychological therapy with usual care and/or attention control; (4) pharmacological intervention and various forms of psychological therapy with pharmacological intervention and usual care and/or attention control; (5) non-invasive brain stimulation and pharmacological intervention with pharmacological intervention and sham stimulation or usual care; (6) pharmacological intervention and one of various forms of psychological therapy with placebo and psychological therapy; (7) pharmacological intervention and non-invasive brain stimulation with placebo plus non-invasive brain stimulation; (8) non-invasive brain stimulation and one of various forms of psychological therapy versus non-invasive brain stimulation plus usual care and/or attention control; and (9) non-invasive brain stimulation and one of various forms of psychological therapy versus sham brain stimulation or usual care plus psychological therapy, with the intention of treating depression after stroke.

\section{Data collection and analysis}

Two review authors independently selected studies, assessed risk of bias, and extracted data from all included studies. We calculated mean difference (MD) or standardised mean difference (SMD) for continuous data, and risk ratio (RR) for dichotomous data, with 95\% confidence intervals (Cls). We assessed heterogeneity using the $\mathrm{I}^{2}$ statistic and certainty of the evidence according to GRADE.

\section{Main results}

We included 49 trials (56 comparisons) with 3342 participants. Data were available for: (1) pharmacological interventions with placebo (with 20 pharmacological comparisons); (2) one of various forms of non-invasive brain stimulation with sham stimulation or usual care (with eight non-invasive brain stimulation comparisons); (3) one of various forms of psychological therapy with usual care and/or attention control (with 16 psychological therapy comparisons); (4) pharmacological intervention and various forms of psychological therapy with pharmacological intervention and usual care and/or attention control (with two comparisons); and (5) non-invasive brain stimulation and pharmacological intervention with pharmacological intervention and sham stimulation or usual care (with 10 comparisons). We found no trials for the following comparisons: (6) pharmacological intervention and various forms of psychological therapy interventions versus placebo and psychological therapy; (7) pharmacological intervention and non-invasive brain stimulation versus placebo plus noninvasive brain stimulation; (8) non-invasive brain stimulation and one of various forms of psychological therapy versus non-invasive brain stimulation plus usual care and/or attention control; and (9) non-invasive brain stimulation and one of various forms of psychological therapy versus sham brain stimulation or usual care plus psychological therapy.

Treatment effects observed: very low-certainty evidence from eight trials suggests that pharmacological interventions decreased the number of people meeting study criteria for depression (RR $0.70,95 \% \mathrm{Cl} 0.55$ to $0.88 ; 1025$ participants) at end of treatment, and very low-certainty evidence from six trials suggests that pharmacological interventions decreased the number of people with less than $50 \%$ reduction in depression scale scores at end of treatment (RR $0.47,95 \% \mathrm{Cl} 0.32$ to $0.69 ; 511$ participants) compared to placebo. No trials of non-invasive brain stimulation reported on meeting study criteria for depression at end of treatment. Only one trial of non-invasive brain stimulation reported on the outcome $<50 \%$ reduction in depression scale scores; thus, we were unable to perform a meta-analysis for this outcome. Very low-certainty evidence from six trials suggests that psychological therapy decreased the number of people meeting the study criteria for depression at end of treatment (RR $0.77,95 \% \mathrm{Cl} 0.62$ to $0.95 ; 521$ participants) compared to usual care/attention control. No trials of combination therapies reported on the number of people meeting the study criteria for depression at end of treatment. Only one trial of combination (non-invasive brain stimulation and pharmacological intervention) therapy reported $<50 \%$ reduction in depression scale scores at end of treatment. Thus, we were unable to perform a meta-analysis for this outcome.

Five trials reported adverse events related to the central nervous system (CNS) and noted significant harm in the pharmacological interventions group (RR 1.55, 95\% Cl 1.12 to 2.15; 488 participants; very low-certainty evidence). Four trials found significant gastrointestinal adverse events in the pharmacological interventions group (RR $1.62,95 \% \mathrm{Cl} 1.19$ to 2.19 ; 473 participants; very lowcertainty evidence) compared to the placebo group. No significant deaths or adverse events were found in the psychological therapy group compared to the usual care/attention control group. Non-invasive brain stimulation interventions and combination therapies resulted in no deaths.

\section{Authors' conclusions}

Very low-certainty evidence suggests that pharmacological or psychological therapies can reduce the prevalence of depression. This very low-certainty evidence suggests that pharmacological therapy, psychological therapy, non-invasive brain stimulation, and combined interventions can reduce depressive symptoms. Pharmacological intervention was associated with adverse events related to the CNS and the gastrointestinal tract. More research is required before recommendations can be made about the routine use of such treatments.

\section{PLAIN LANGUAGE SUMMARY}

Pharmacological, psychological, and brain stimulation treatments for depression after stroke

\section{Review question}


Do pharmacological treatments, non-invasive brain stimulation, psychological treatments, or combination treatments reduce the proportion of people with depression or the extent of depressive symptoms after stroke?

\section{Background}

Depression is common after stroke yet often is not detected or inadequately treated.

\section{Search date}

We identified studies by searches conducted on 13 August 2018.

\section{Study characteristics}

We included trials that reported on the use of pharmacological, non-invasive brain stimulation, psychological, and combination therapy interventions to treat depression after stroke. Mean age of participants ranged from 54 to 78 years. Studies were from Asia (30), Europe (11), North America (6), and Australia (2).

\section{Key results}

We included 49 trials (56 treatments) involving 3342 participants. Pharmacological treatments resulted in fewer people meeting the study criteria for depression and less than 50\% reduction in depression scale scores at end of treatment. Psychological therapy reduced the number of people meeting the study criteria for depression at end of treatment. More people in the pharmacological treatment group reported central nervous system (in five trials) and gastrointestinal side effects (in four trials) than in the placebo groups. Information on side effects of other treatments was not provided.

\section{Certainty of the evidence}

Estimates of treatment effects were imprecise due to small numbers in most studies and recruitment of people with very different baseline characteristics. We rated the certainty of evidence as very low due to these and other limitations in study design.

\section{Conclusion}

Antidepressant drugs may benefit people with persistent depressive symptoms after stroke, but care is required in their use, as little is known about their side effects. Psychological therapy may offer a treatment option. Future research should include a broader group of people with stroke. 


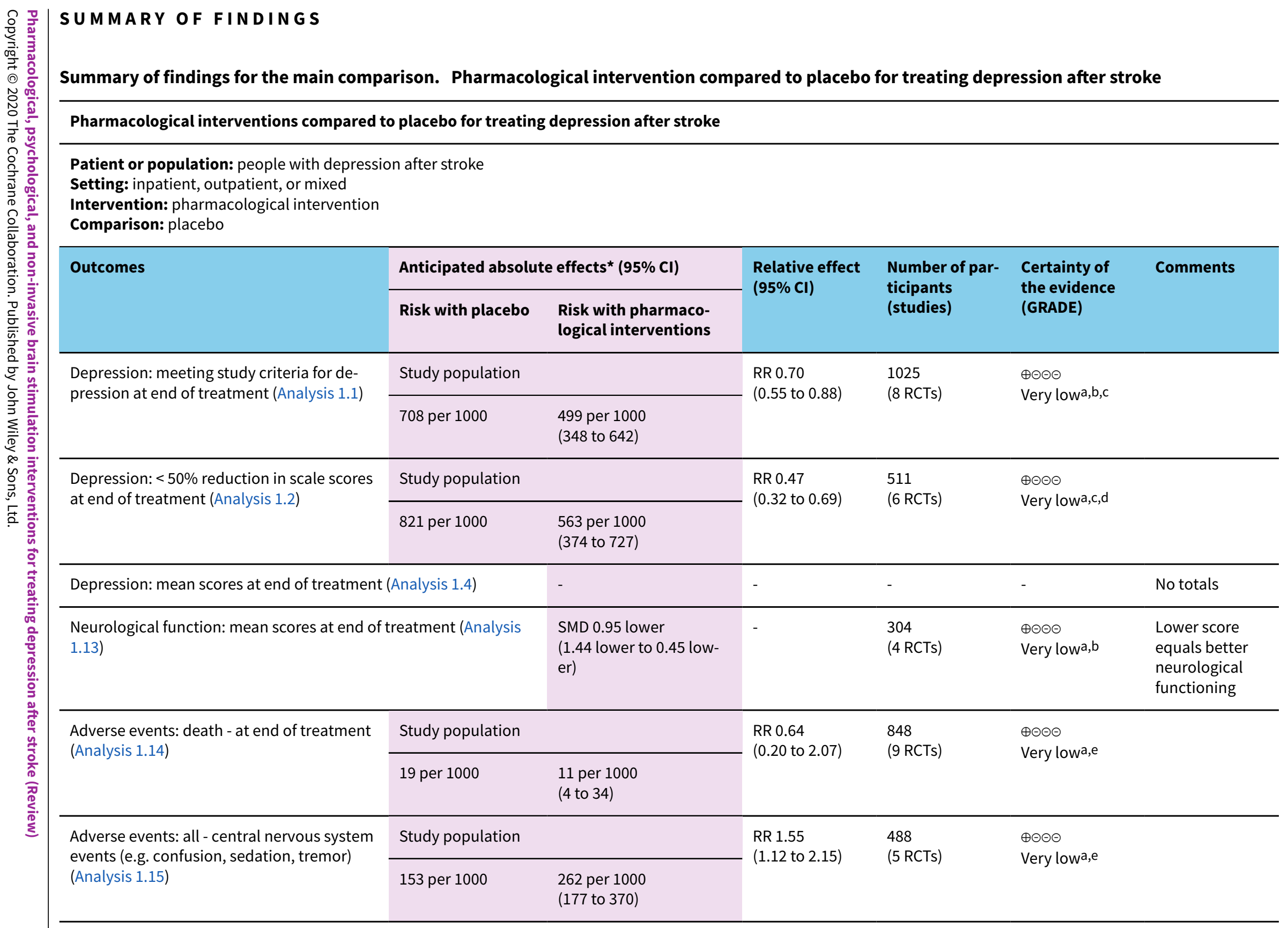




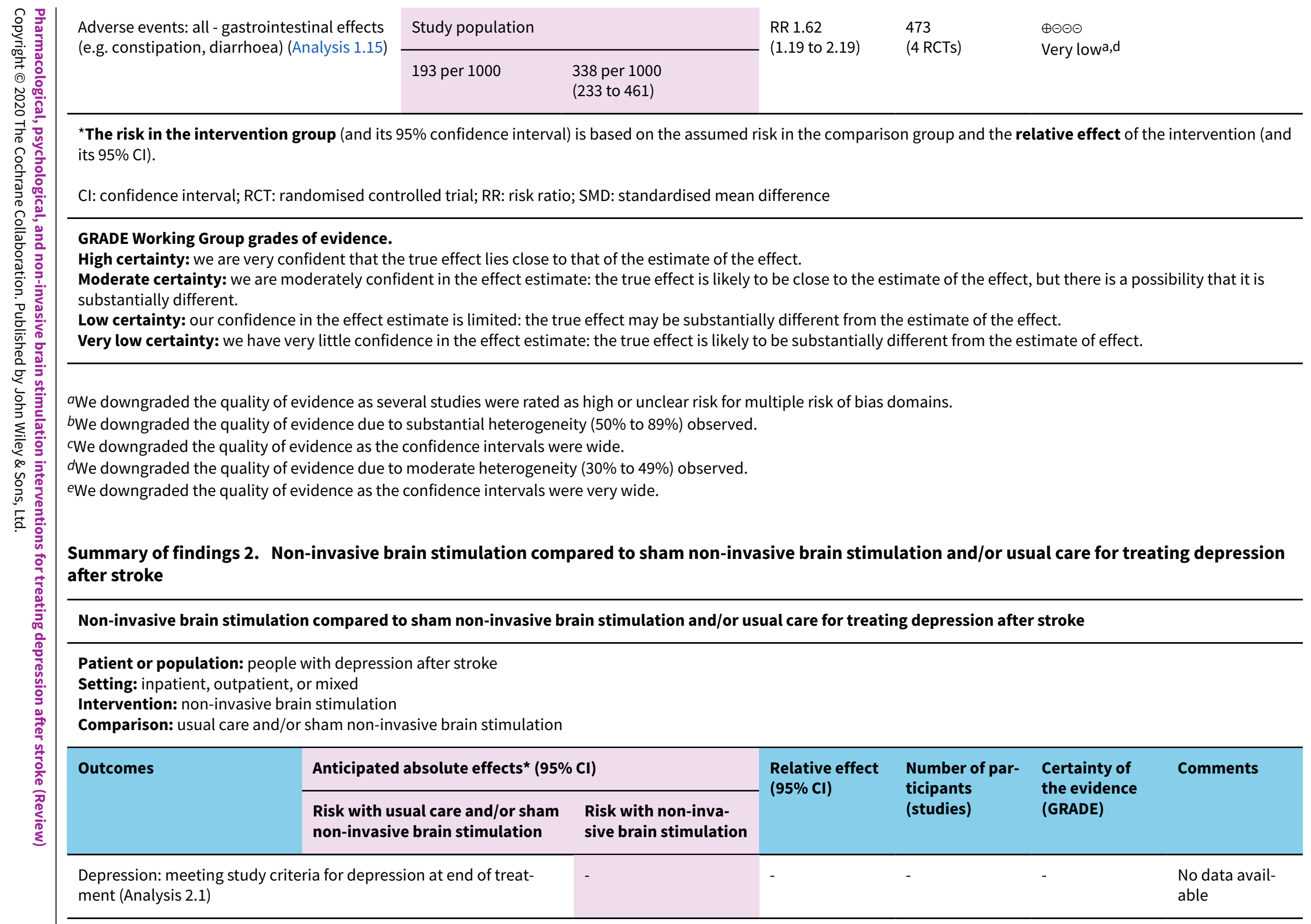




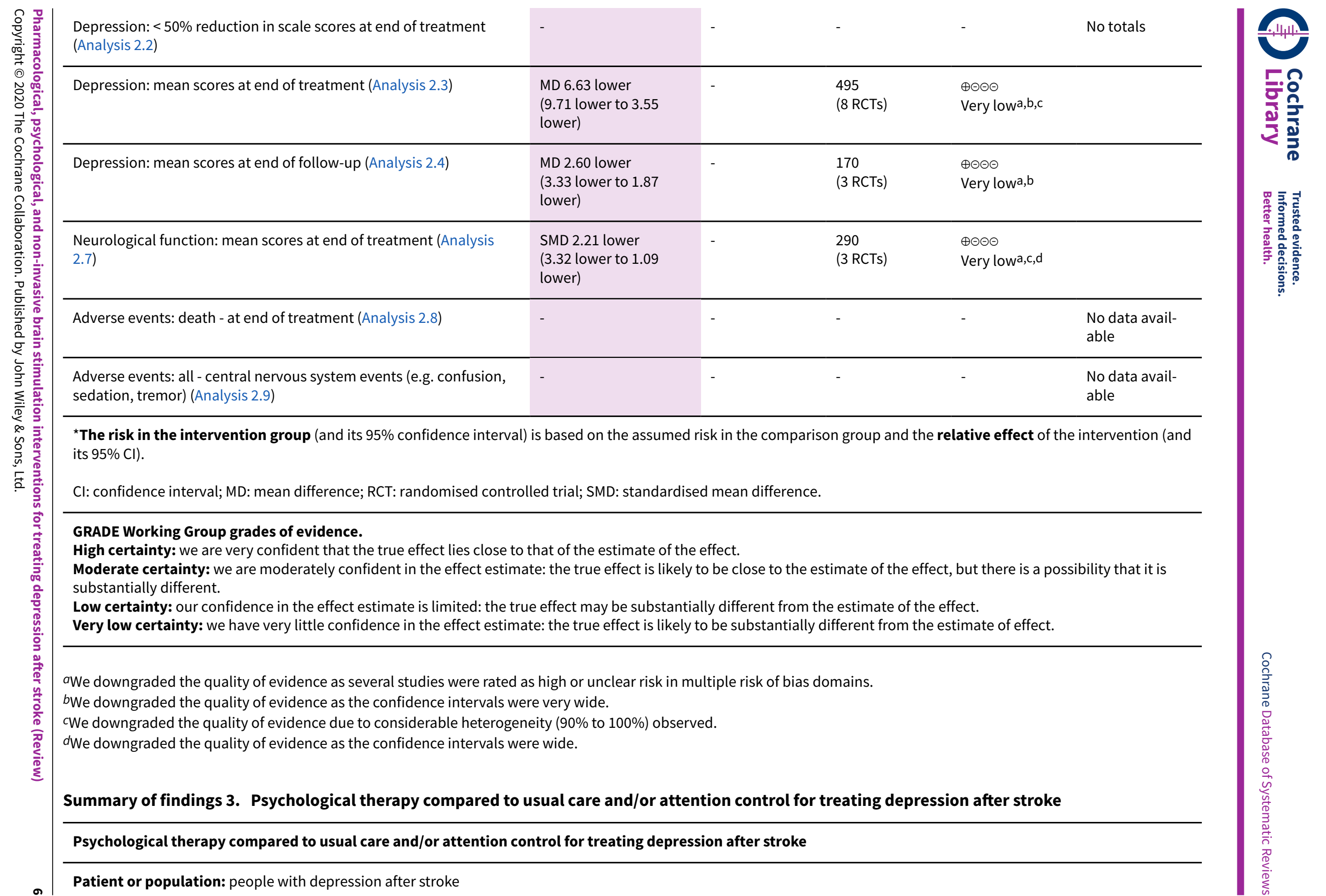




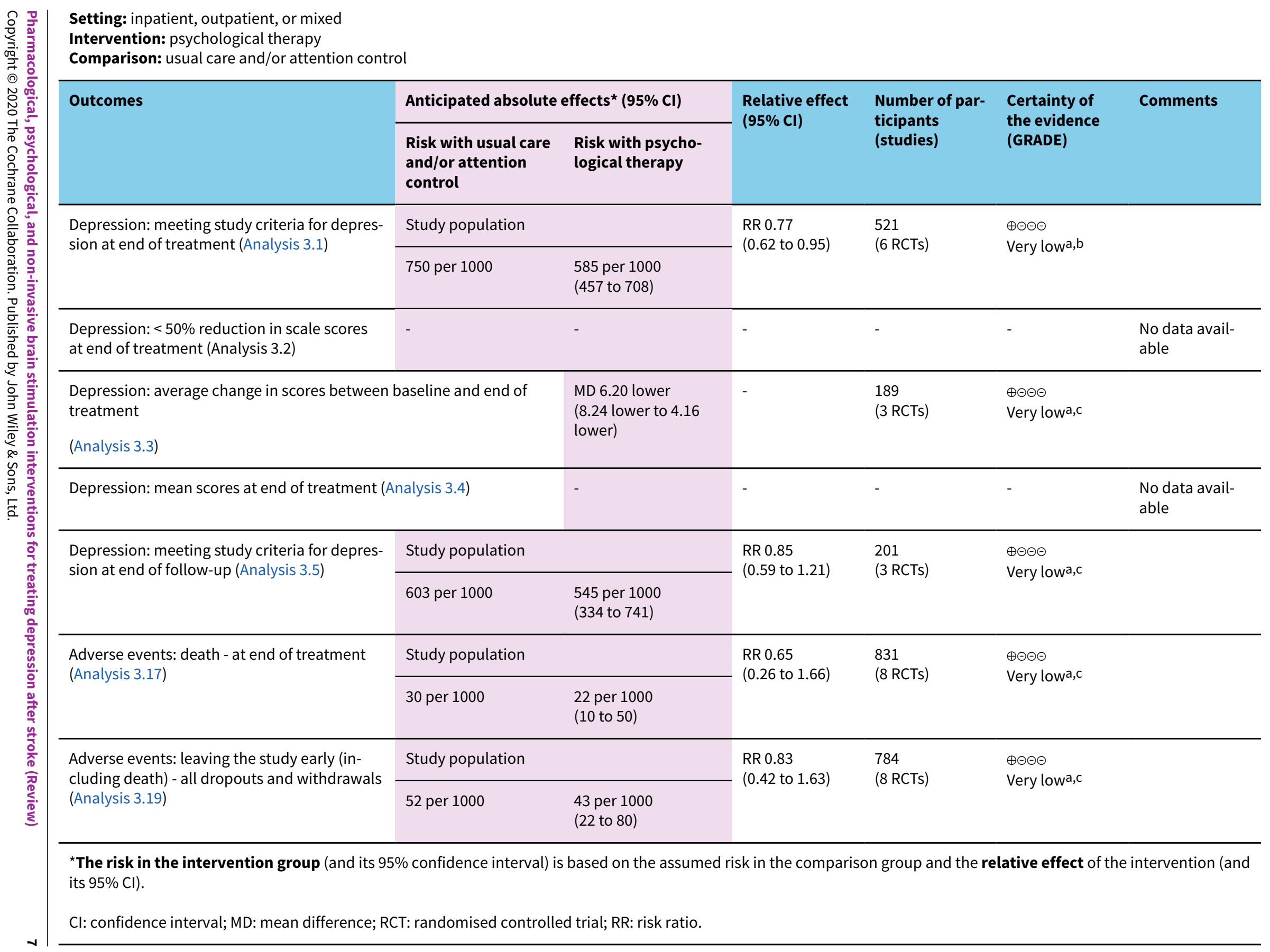


GRADE Working Group grades of evidence.

High certainty: we are very confident that the true effect lies close to that of the estimate of the effect.

Moderate certainty: we are moderately confident in the effect estimate: the true effect is likely to be close to the estimate of the effect, but there is a possibility that it is substantially different.

Low certainty: our confidence in the effect estimate is limited: the true effect may be substantially different from the estimate of the effect.

Very low certainty: we have very little confidence in the effect estimate: the true effect is likely to be substantially different from the estimate of effect.

aWe downgraded the quality of evidence as several studies were rated as unclear or high risk in multiple risk of bias domains.

bWe downgraded the quality of evidence as confidence intervals were wide.

cWe downgraded the quality of evidence as confidence intervals were very wide.

\section{Summary of findings 4. Pharmacological intervention and a form of psychotherapy (combination) compared to pharmacological intervention and} usual care or attention control (single) for treating depression after stroke

Pharmacological intervention and a form of psychotherapy (combination) compared to pharmacological intervention and usual care or attention control (single) for treating depression after stroke

Patient or population: people with depression after stroke

Setting: inpatient, outpatient, or mixed

Intervention: pharmacological intervention and a form of psychotherapy (combination)

Comparison: pharmacological intervention and usual care or attention control (single)

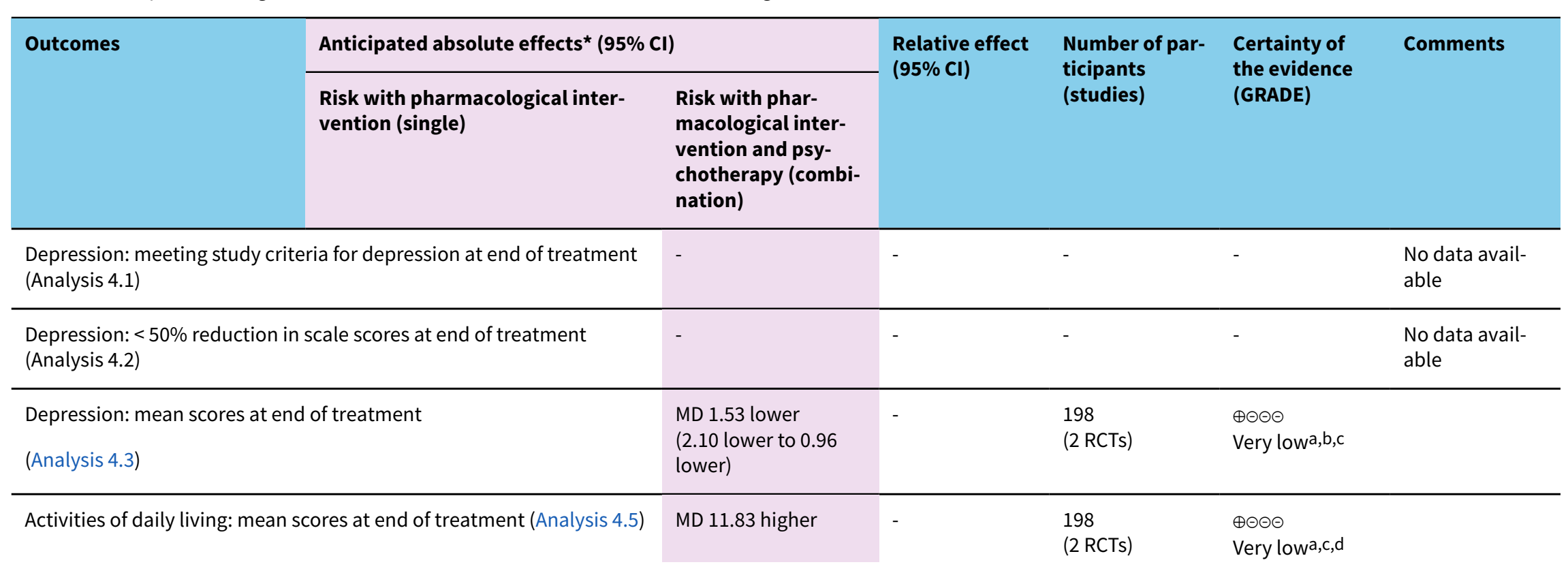




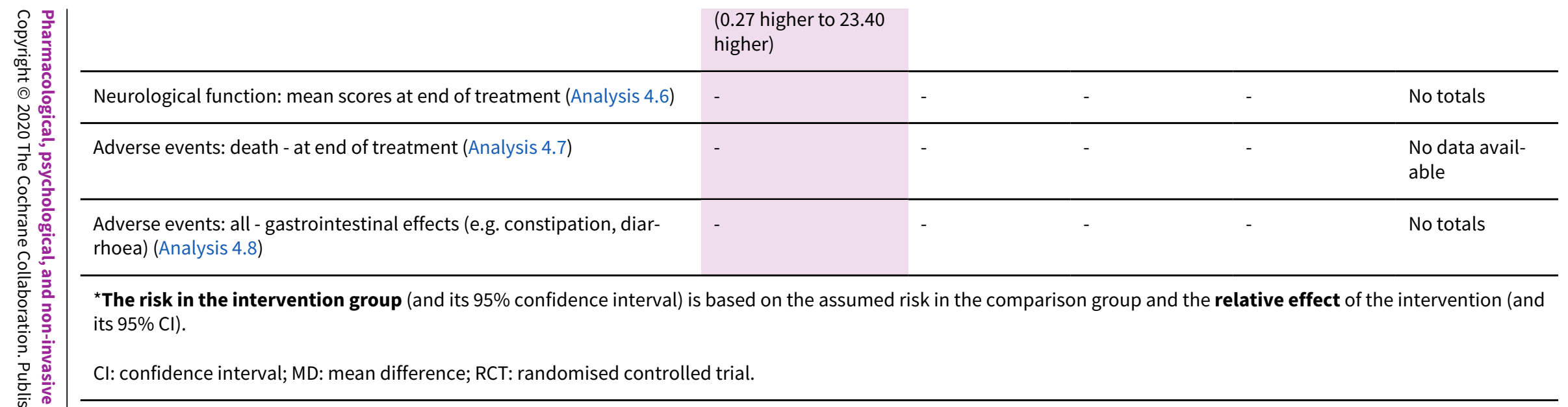

\section{GRADE Working Group grades of evidence.}

High certainty: we are very confident that the true effect lies close to that of the estimate of the effect.

Moderate certainty: we are moderately confident in the effect estimate: the true effect is likely to be close to the estimate of the effect, but there is a possibility that it is substantially different.

Low certainty: our confidence in the effect estimate is limited: the true effect may be substantially different from the estimate of the effect.

Very low certainty: we have very little confidence in the effect estimate: the true effect is likely to be substantially different from the estimate of effect.

aWe downgraded the quality of evidence as both studies were rated as unclear risk in multiple risk of bias domains.

$b$ We downgraded the quality of evidence as substantial heterogeneity $(50 \%$ to $89 \%)$ was observed.

cWe downgraded the quality of evidence as the confidence intervals were very wide.

$d$ We downgraded the quality of evidence as considerable heterogeneity (90\% to $100 \%)$ was observed.

\section{Summary of findings 5. Non-invasive brain stimulation and pharmacological intervention (combination) compared to pharmacological intervention} and sham stimulation or usual care (single) for treating depression after stroke

Non-invasive brain stimulation and pharmacological intervention (combination) compared to pharmacological intervention and sham stimulation or usual care (single) for treating depression after stroke

Patient or population: people with depression after stroke

Setting: inpatient, outpatient, or mixed

Intervention: non-invasive brain stimulation and pharmacological intervention (combination)

Comparison: pharmacological intervention and sham stimulation or usual care (single)

\begin{tabular}{|c|c|c|c|c|c|c|}
\hline \multirow[t]{2}{*}{ Outcomes } & \multicolumn{2}{|c|}{ Anticipated absolute effects ${ }^{\star}(95 \% \mathrm{Cl})$} & \multirow{2}{*}{$\begin{array}{l}\text { Relative effect } \\
(95 \% \mathrm{Cl})\end{array}$} & \multirow{2}{*}{$\begin{array}{l}\text { Number of par- } \\
\text { ticipants } \\
\text { (studies) }\end{array}$} & \multirow{2}{*}{$\begin{array}{l}\text { Certainty of } \\
\text { the evidence } \\
\text { (GRADE) }\end{array}$} & \multirow[t]{2}{*}{ Comments } \\
\hline & $\begin{array}{l}\text { Risk with pharmacological } \\
\text { intervention (single) }\end{array}$ & $\begin{array}{l}\text { Risk with non-invasive brain } \\
\text { stimulation and pharma- }\end{array}$ & & & & \\
\hline
\end{tabular}




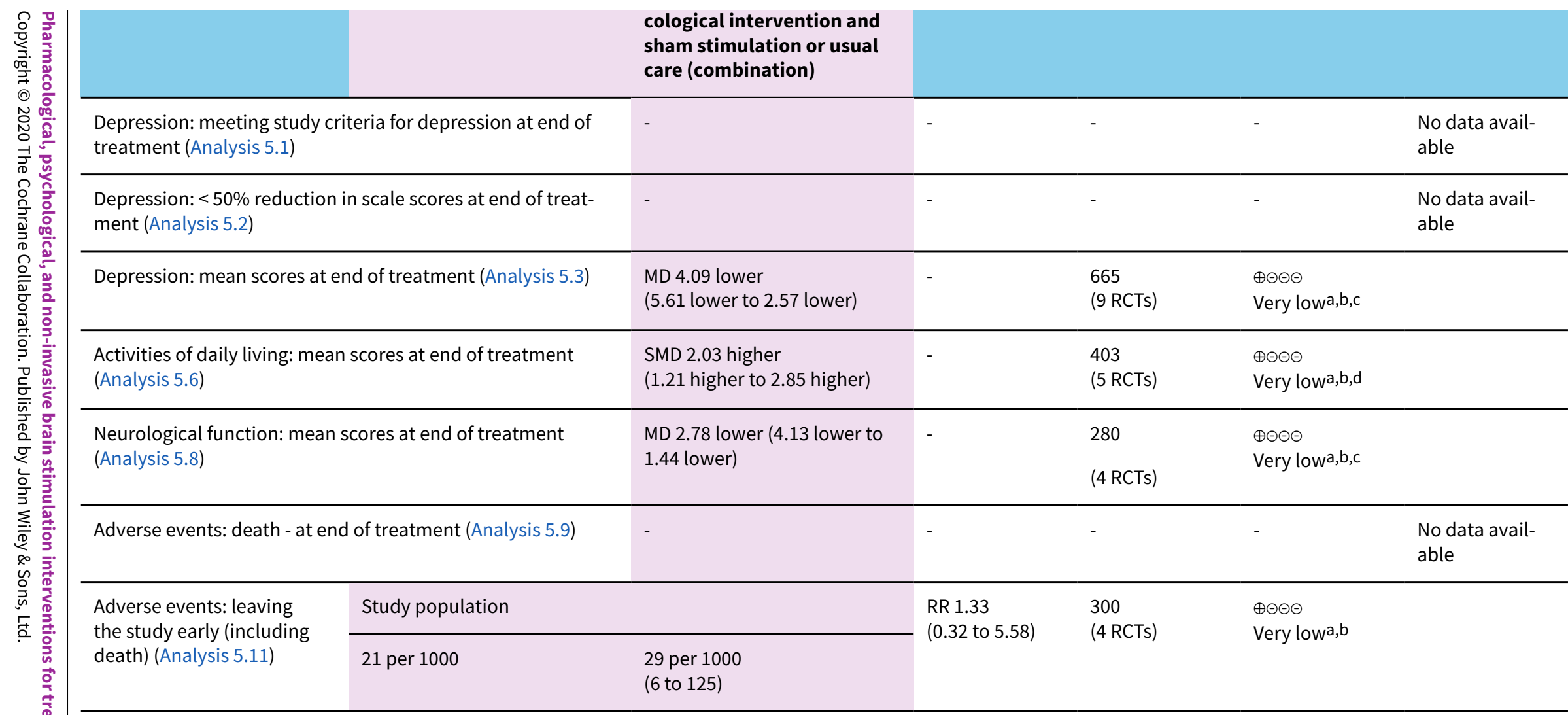

${ }^{\star}$ The risk in the intervention group (and its $95 \%$ confidence interval) is based on the assumed risk in the comparison group and the relative effect of the intervention (and its $95 \% \mathrm{Cl})$.

$\mathrm{Cl}$ : confidence interval; MD: mean difference; RCT: randomised controlled trial; RR: risk ratio; SMD: standardised mean difference.

\section{GRADE Working Group grades of evidence.}

High certainty: we are very confident that the true effect lies close to that of the estimate of the effect.

Moderate certainty: we are moderately confident in the effect estimate: the true effect is likely to be close to the estimate of the effect, but there is a possibility that it is substantially different.

Low certainty: our confidence in the effect estimate is limited: the true effect may be substantially different from the estimate of the effect.

Very low certainty: we have very little confidence in the effect estimate: the true effect is likely to be substantially different from the estimate of effect.

aWe downgraded the quality of evidence as the confidence intervals were very wide.

$b$ We downgraded the quality of evidence as several studies were rated as unclear or high risk in multiple risk of bias domains.

cWe downgraded the quality of evidence as substantial heterogeneity ( $50 \%$ to $89 \%$ ) was observed.

เ

$d$ We downgraded the quality of evidence as considerable heterogeneity ( $90 \%$ to $100 \%$ ) was observed. 


\section{B A C K G R O U N D}

\section{Description of the condition}

Depression and anxiety disorders are important sequelae of stroke. These mood disorders occur in up to half of people during the first year after onset of stroke, although estimates differ between studies due to varying definitions, populations, exclusion criteria, and timing of assessments (Ayerbe 2013; Hackett 2014). Inconsistent research findings are also due to the complexity of recognition, assessment, and diagnosis of an underlying mood disorder associated with acute stroke and cognitive, language, and other impairments. In addition, people with stroke may experience a variety of behavioural syndromes that are more specific to brain injury, including indifference, emotional lability, disinhibition, unawareness of illness (anosognosia), and difficulties with verbal emotional expression (aprosody). In particular, much of the controversy surrounding 'stroke-associated depression' as a specific type of depressive syndrome hinges on concern about whether the tools normally used for diagnosis of major depression and other depressive illnesses may mis-attribute features of ischaemic brain injury to depression (House 1987; Johnson 1991). Although several depression screening tools have been validated (against a structured clinical interview) for use in people with stroke (Turner 2012), in practice, researchers use a range of methods to diagnose depression - a psychiatric interview to apply standard diagnostic criteria such as those provided in the Diagnostic and Statistical Manual of Mental Disorders (e.g. DSM-IIIR, DSM-IV, DSM V) (APA 1987; APA 1994; APA 2013), or psychiatric rating scales such as the Montgomery Åsberg Depression Rating Scale (MADRS) (Montgomery 1979), or a rating scale of mood based on selfassessment.

Although controversy continues about whether depression after stroke is predominantly caused by physical factors (such as stroke lesion location) (Carson 2000), or by a person's psychological response to stroke, evidence suggests that clinically diagnosed stroke-associated depression is similar in frequency and nature to depression among older people with other chronic illnesses (Burvill 1996; Burvill 1997; Sharpe 1990). Although it was previously thought that the period of greatest risk appeared to be within the first few months of stroke onset (Burvill 1995a; Herrmann 1998; House 1991), this was not apparent in systematic reviews of high-quality observational studies (Hackett 2014). Although some people recover spontaneously, apparently undergoing a grieflike depressive adjustment reaction, up to one-third of people have depression that persists during the first year or longer after stroke onset (Astrom 1996; Herrmann 1998). Those with 'anxious depression' and those with more severe symptoms at presentation appear less responsive to treatment and have a worse long-term prognosis (Astrom 1996).

Evidence of a causal relationship between stroke-associated depression and adverse outcomes is complicated by potential confounding factors such as age, gender, social class, physical disability, and comorbid conditions. However, evidence provided by Parikh 1990 and Sinyor 1986 suggests that abnormal mood may impede rehabilitation by impairing physical function (Ayerbe 2013), as well as cognitive function (Robinson 1986), and by contributing to stress on carers (Anderson 1995a). Furthermore, stroke-associated depression may be associated with increased risk of death (House 2001; Morris 1993b), including death by suicide (Stenager 1998). Depressive illness among older people, in general, is associated with greater morbidity and dependency, higher use of drugs and alcohol, increased use of healthcare resources, and poor compliance with treatment of comorbid conditions (Katona 1995).

\section{Description of the intervention}

We considered three broad interventions.

- Pharmacological interventions designed to treat depression: several classes of relevant pharmacological agents include selective serotonin reuptake inhibitors (SSRIs) (e.g. fluvoxamine, fluoxetine, sertraline, citalopram, paroxetine), serotoninnorepinephrine reuptake inhibitors (SNRIs) (e.g. venlafaxine, milnacipran, sibutramine), monoamine oxidase inhibitors (MAOIs) (e.g. moclobemide), tricyclic antidepressants (TCAs) (e.g. nortriptyline, imipramine, clomipramine), and other antidepressant medications including psychostimulants (e.g. methylphenidate), mood stabilisers (e.g. lithium), or benzodiazepines.

- Non-invasive brain stimulation: electroconvulsive therapy (ECT) involves the brief passage of an electrical current through the brain via electrodes applied to the scalp to induce a generalised seizure (i.e. a fit or convulsion). The seizure comprises two components: a central element - the ictus involving depolarisation (i.e. discharge of neurotransmitter chemicals) of brain cells - and a peripheral element consisting of convulsive, jerking movements of the body, although this is now modified due to use of a short-acting anaesthetic and muscle relaxant, as part of what is called modified ECT. Modified ECT replaced the crude equipment and techniques of unmodified ECT used in the mid-1950s. The seizure is detected by electrodes placed on the scalp to monitor brain electrical activity (i.e. EEG). The ECT electrodes can be placed on both sides of the head (bilateral placement), or on one side - usually the right side of the head (unilateral placement). Passage of an electrical current through the skull to the brain is necessary to trigger a seizure. In this update, we broadened the review to include other non-invasive brain stimulation techniques such as (1) transcranial magnetic stimulation or repetitive transcranial magnetic stimulation (TMS or rTMS, where a magnetic 'coil' is placed near the head of the person receiving the treatment without making physical contact); (2) transcranial direct current stimulation (tDCS, where a constant, low current is delivered directly to the brain area of interest via small electrodes); (3) cranial electrotherapy stimulation (CES, where a small, pulsed electrical current is applied across a patient's head); and (4) magnetic seizure therapy (MST), a type of convulsive therapy that involves replacing the electrical stimulation used in ECT with a rapidly alternating strong magnetic stimulation.

- One of various forms of psychological therapy (talking therapy) designed to treat depression: as many therapies are available, we included any psychological therapy that involved direct patient-professional interaction. The content of the interaction could vary from counselling to specific psychotherapy, provided it was directed at helping people develop their social problemsolving skills and adjust to the emotional impact of stroke. All interventions had to have a psychological component - talking, listening, support, advice; they had to be based on a theory of talking therapy; had to be structured and time-tabled as a talking therapy; and had to be delivered by somebody with some explicitly stated training in and supervision of therapies. The person-professional interaction could take place in person, 
via telephone, or through other media. We did not include web-based interventions even if mediated by a healthcare professional. We did not include interventions based upon selfmanagement or supported self-management.

We further considered these combinations of three broad interventions.

- Pharmacological intervention and one of various forms of psychological therapy versus pharmacological intervention plus usual care and/or attention control.

- Non-invasive brain stimulation and pharmacological intervention versus pharmacological intervention plus sham stimulation or usual care.

- Pharmacological intervention and one of various forms of psychological therapy versus placebo plus psychological therapy.

- Pharmacological intervention and non-invasive brain stimulation versus placebo plus non-invasive brain stimulation.

- Non-invasive brain stimulation and one of various forms of psychological therapy versus non-invasive brain stimulation plus usual care and/or attention control.

- Non-invasive brain stimulation and one of various forms of psychological therapy versus sham brain stimulation or usual care plus psychological therapy.

\section{How the intervention might work}

Pharmacological interventions are thought to alter the synaptic transmission process within the brain to increase neurotransmission, for example, SSRIs are intended to block the resorption of serotonin, SNRIs are designed to increase the levels of serotonin and norepinephrine, and TCAs are designed to block the reuptake of norepinephrine.

During modified ECT, a small amount of electrical current is passed briefly across the brain to cause an artificial epileptic fit that affects the entire brain. Repeated ECT is believed to alter chemical pathways in the brain that are responsible for depression. The exact mechanism of action of rTMS, tDCS, and CES remains unclear. They are thought to induce intracerebral current flow and increase or decrease neuronal excitability and/or activate nerve cells in the specific area being stimulated. rTMS involves replacing the electrical stimulation used in ECT with a magnetic stimulus, which is purported to produce similar clinical effects but without the cognitive side effects.

Psychological therapy focuses on changing thinking, emotional, behavioural, and relationship patterns. During psychological therapies, trained therapists work with individuals to help them see patterns in their thoughts, emotions, behaviours, or relationships that may be problematic. The therapist's role is to help a person understand these patterns while assist that person in developing ways to overcome them.

\section{Why it is important to do this review}

Although depression may influence recovery and outcomes following stroke, many (perhaps most) people with stroke do not receive effective treatment because their mood disorder is undiagnosed or is inadequately treated. The UK National Sentinel Audit found that $25 \%$ of patients were not screened for depression, and only $60 \%$ of those identified as needing support received it. Ebrahim 1987a found that few people with stroke-associated depression had been given antidepressants following discharge from hospital, and House 1989 reported that general practitioners and hospital doctors had a passive attitude toward therapy. On the other hand, some more recent studies have found antidepressant prescribing persisting long term but with little attempt to match prescribing to need (Paul 2006). Although this variability may reflect problems with the diagnosis of a 'significant' mood state among older people with disability, it may also reflect uncertainty among clinicians as to the balance of benefits and risks (including side effects) of therapies in this setting. For example, it is not clear that in other settings, antidepressants are of benefit for mild or moderate depression of the sort that is common after stroke (Fournier 2010).

Indirect evidence of the effectiveness of pharmacological and psychological treatments for depression (and anxiety) for older people in general, and for those with associated physical illness, is available in several published reviews (Gill 2000; Kirsch 2008; Lima 2001; McCusker 1998; Mittmann 1997; Wilkinson 1997). However, because of the possibility that depression after stroke may differ in important ways, it may be inappropriate to extrapolate these data to people with stroke. Use of rTMS, tDCS, and CES in people with stroke is relatively new, and few data are available to guide clinical decision-making.

We undertook and updated a systematic review of all randomised controlled trials (RCTs) (published and unpublished) of pharmacological agents, non-invasive brain stimulation, psychological therapies, or their combination for treatment of depression after stroke. This is an update of a Cochrane Review first published in 2004 and last updated in 2008.

\section{O B JECT IVES}

\section{Primary objective}

- To determine whether pharmacological therapy, non-invasive brain stimulation, psychological therapy, or combinations of these interventions reduce the prevalence of diagnosable depression after stroke

\section{Secondary objectives}

- To determine whether pharmacological therapy, non-invasive brain stimulation, psychological therapy, or combinations of these interventions reduce levels of depressive symptoms, improve physical and neurological function and health-related quality of life, and reduce dependency after stroke

- To assess the safety of and adherence to such treatments

\section{METHODS}

\section{Criteria for considering studies for this review}

\section{Types of studies}

We restricted the review to all relevant randomised controlled trials (RCTs) only. There was no restriction on eligibility of RCTs on the basis of language, sample size, duration of follow-up, or publication status. Trials that met all inclusion criteria, but from which no outcome data were available (neither from the report of the trial nor from the study authors), could not contribute meaningfully to a pooled estimate of effect. These trials were regarded as 'dropouts' rather than as ineligible. 


\section{Types of participants}

We defined stroke according to clinical criteria, including cerebral infarction, intracerebral haemorrhage, and 'uncertain' pathological subtypes. We excluded trials of people with subarachnoid haemorrhage $(\mathrm{SAH})$ only, as this entity has a different natural history and management strategy from other stroke subtypes. However, we did include trials with mixed stroke subtypes, including small numbers of people with $\mathrm{SAH}$. There were no restrictions on the basis of age, sex, or other characteristics. Participants were required to have depression (diagnosed by psychiatric interview, mood scale, or treating clinician) on recruitment. We excluded trials with participants who were not depressed at recruitment, but that measured depression as the primary outcome at follow-up. These trials were included in a review of interventions for preventing depression after stroke (Hackett 2008a).

The diagnostic categories of depression considered were:

- depressive disorder, as defined by symptom scores on a standard screening instrument - scoring above a pre-defined scoring threshold;

- major depression, as defined by the American Psychiatric Association Diagnostic and Statistical Manual of Mental Disorders (DSM-IIIR, DSM-IV, DSM-V; APA 1987; APA 1994; APA 2013), or similar diagnostic criteria; and

- dysthymia or minor depression, as defined by DSM or other standard diagnostic criteria.

Trials that included mixed populations (such as those with stroke and head injury or other central nervous system (CNS) disorders) were excluded unless separate results for people with stroke could be identified. Trials were excluded if participants were being treated primarily for a stroke-associated pain syndrome, even if depression was measured as a secondary outcome.

\section{Types of interventions}

We included the following interventions.

- Comparison between a pharmacological intervention and placebo for treatment of depression after stroke. Specific pharmacological agents included tricyclic antidepressants (e.g. nortriptyline, imipramine, clomipramine), selective serotonin reuptake inhibitors (SSRIs) (e.g. fluvoxamine, fluoxetine, sertraline, citalopram, paroxetine), monoamine oxidase inhibitors (MAOIs) (e.g. moclobemide), and other antidepressant medications. Trials of mood stabilisers (e.g. lithium) or of benzodiazepines and psychostimulants (e.g. methylphenidate) were analysed separately.

- Comparison between non-invasive brain stimulation and sham stimulation or usual care for treatment of depression associated with stroke.

- Comparison between psychological therapy and usual care and/ or attention control for treatment of depression after stroke. We included any psychological therapy that involved direct person-professional interaction. The content of the interaction could vary from counselling to specific psychological therapy, provided it was directed at helping people develop their social problem-solving skills and adjust to the emotional impact of stroke. All interventions had to have a psychological component - talking, listening, support, advice - and had to be based on a theory of talking therapy; had to be structured and time-tabled as a talking therapy; and had to be delivered by somebody with some explicitly stated training in and supervision of therapies.

Alternatively, we included their combinations.

- Pharmacological intervention and one of various forms of psychological therapy versus pharmacological intervention plus usual care and/or attention control.

- Non-invasive brain stimulation and pharmacological intervention versus pharmacological intervention plus sham stimulation or usual care.

- Pharmacological intervention and one of various forms of psychological therapy versus placebo plus psychological therapy.

- Pharmacological intervention and non-invasive brain stimulation versus placebo plus non-invasive brain stimulation.

- Non-invasive brain stimulation and one of various forms of psychological therapy versus non-invasive brain stimulation plus usual care and/or attention control.

- Non-invasive brain stimulation and one of various forms of psychological therapy versus sham brain stimulation or usual care plus psychological therapy.

Exclusions included the following.

- Interventions with an agent or therapy that was being evaluated primarily for other reasons (e.g. to improve physical function, to provide neuroprotection, to facilitate neuroregeneration), even if the intervention was a recognised treatment for depression, and even if a standardised depression scale was administered at baseline and at outcome assessment (these trials are included in a separate systematic review, with depression as a secondary endpoint (Mead 2012)).

- Interventions provided with the sole purpose of educating or providing information.

- Occupational therapy (including leisure therapy and other rehabilitation services).

- Acupuncture or electro-acupuncture.

- Herbal medicines.

- Interventions that involved visits from stroke support workers, unless there was a clearly defined psychological component. Attention control in psychological therapy trials can include non-specific interventions such as relaxation classes or followup with a clinician who has no psychological training.

\section{Types of outcome measures}

\section{Primary outcomes}

Primary analyses focused on remission and included the following.

- Meeting the criteria for depression at end of treatment, as defined by DSM or similar standard diagnostic criteria.

- Less than $50 \%$ reduction in depression scale scores at end of treatment.

\section{Secondary outcomes}

- Depression scores as measured on scales such as the Hamilton Depression Rating Scale (HDRS; Hamilton 1960), the Montgomery Åsberg Depression Rating Scale (MADRS; Montgomery 1979), the Geriatric Depression Scale (GDS; 
Gompertz 1993), the Beck Depression Inventory (BDI; Beck 1961), and the Hospital Anxiety and Depression Scale (HADS Depression subscale; Zigmond 1983) at end of treatment and at follow-up

- Meeting the criteria for depression at end of follow-up, as defined by DSM or similar standard diagnostic criteria

- Less than $50 \%$ reduction in depression scale scores at end of follow-up

- Psychological distress scores, as measured on composite scales such as the General Health Questionnaire (GHQ; Goldberg 1972) at end of treatment

- Anxiety scores, as measured on scales such as the Hamilton Anxiety Scale, the Beck Anxiety Inventory, and the Hospital Anxiety and Depression Scale (HADS Anxiety subscale; Zigmond 1983) at end of treatment

- Cognitive function scores, as measured on scales such as the Mini-Mental State Examination (MMSE; Folstein 1975) at end of treatment

- Activities of daily living scores, as measured on scales such as the Barthel Index (Bl; Mahoney 1965) at end of treatment

- Disability scores, as measured on scales such as the Functional Independence Measure (FIM; Deutsch 1997)

- Neurological function scores, as measured on scales such as the National Institutes of Health Stroke Scale (NIHSS; Lyden 2001)

- Disadvantages of treatment recorded as adverse events, grouped by death, all, and leaving the study early (including death)

Participants' reasons for withdrawal from trials were examined as a marker of acceptance.

\section{Search methods for identification of studies}

This review is an update of a previously published Cochrane Review update (2008) (Hackett 2008; Appendix 1). The first review was published in 2004 (Hackett 2004). For this update, we searched all databases from inception until August 2018. We searched for relevant trials in all languages and arranged for translation of trial reports when necessary.

\section{Specialised Register of Cochrane Stroke}

See the methods for the Cochrane Stroke Group Specialised register; the Cochrane Stroke Group Information Specialist searched the Specialised Register of Cochrane Stroke on 13 August 2018.

\section{Electronic searches}

We searched the following bibliographic databases.

- Cochrane Depression Anxiety and Neurosis Trials Register (last searched August 2018).

- Cochrane Central Register of Controlled Trials (CENTRAL; 2018, Issue 1), in the Cochrane Library (Appendix 2).

- MEDLINE (OVID): 1966 to August 2018 (Appendix 3).

- Embase (OVID): 1980 to August 2018 (Appendix 4).

- PsycINFO (OVID): 1967 to August 2018 (Appendix 5).

- Cumulative Index to Nursing and Allied Health Literature (CINAHL) (EBSCO): 1982 to August 2018 (Appendix 6).
- Science Citation Index - Expanded (SCI-EXPANDED), Social Sciences Citation Index (SSCI), and Arts \& Humanities Citation Index (A\&HCl) within Web of Science: 2002 to August 2018 (Appendix 7).

Biological Abstracts has now been superseded by ISI Web of Science, which includes the Arts and Humanities Index. Several databases/citation indexes (Applied Science and Technology Plus; Biological Abstracts; BIOSIS Previews; General Science Plus; Dissertations and Theses) listed in Appendix 1 were not used for this update.

\section{Searching other resources}

We searched the following resources using "stroke" or "brain infarction" or "depression" or "low mood" from inception to August 2018.

- US National Institutes of Health Ongoing Trials Register ClinicalTrials.gov (www.clinicaltrials.gov).

- World Health Organization International Clinical Trials Registry Platform (WHO ICTRP) (www.who.int/ictrp/en/).

We also searched abstracts and conference proceedings from the following international conferences for relevant studies.

- European Stroke Organisation Conference (2015 to 2018).

- Stroke Society of Australasia Annual Scientific Meetings (2008 to 2018).

- World Stroke Congress (2000 to 2016).

- Asia Pacific Stroke Conference (2011 to 2017).

The full search strategies for other resources are provided in Appendix 8.

\section{Personal communications}

We contacted the study authors to ask for information on ongoing studies or to request additional study data and, in some instances, additional analyses.

\section{Reference lists}

We searched the reference lists of relevant trials, systematic reviews, and reviewed chapters in books on the prevention and treatment of depression and management of stroke, including but not limited to, reviews of the management of stroke, books specifically directed at treatment or prevention of depression, and writings on stroke and old age.

\section{Data collection and analysis}

\section{Selection of studies}

Two review authors (SA, KC) reviewed all new citations and discarded those that were irrelevant based on the title of the publication and its abstract. When any suggestion was made that an article was possibly relevant, we retrieved the full-length article for further assessment. Two review authors (SA, KC) independently selected the new trials for inclusion in the review from the culled citation list. Potentially relevant Chinese articles were translated by another study author (C-FH). We resolved disagreements by discussion, and $\mathrm{MH}$ and $\mathrm{AH}$ confirmed the final list and adjudicated any persisting differences of opinion. The selection process is presented in a PRISMA flow diagram (Figure 1). Due to changes in 
the inclusion criteria for this update, records were re-screened from the point of inception. Although we have tried our best to reflect this in the PRISMA flow diagram, it is likely that the numbers will not add up. We listed the included studies under Characteristics of included studies and studies that we ultimately excluded under Characteristics of excluded studies, and we provided the primary reasons for exclusion. 
Figure 1. Study flow diagram. Eligibility criteria were changed to include non-invasive brain stimulation interventions in this update. The 2015 search was rerun from the point of inception to screen for non-invasive brain stimulation and combination interventions.

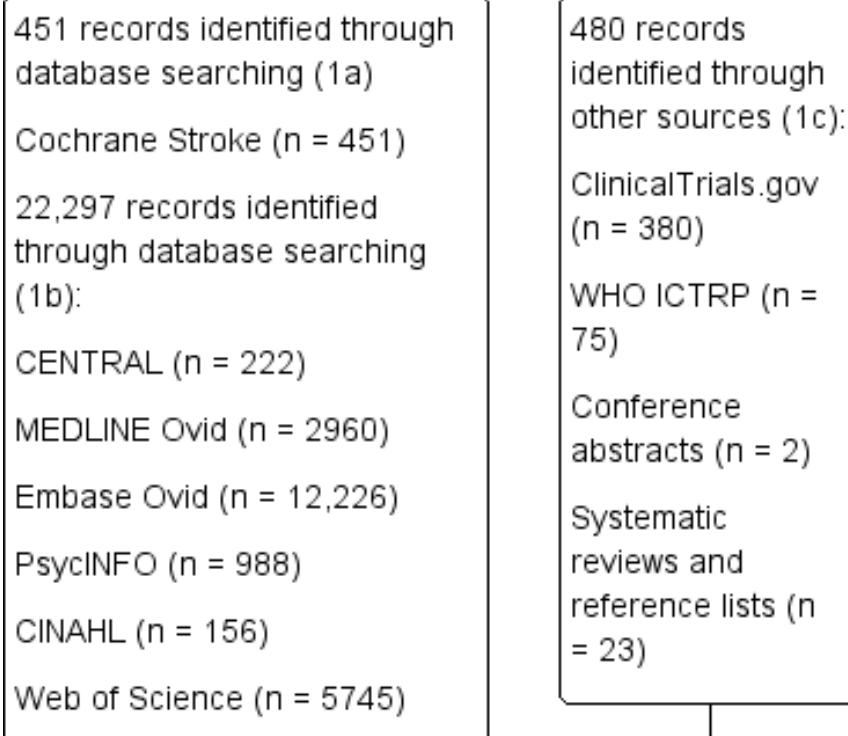


Figure 1. (Continued)

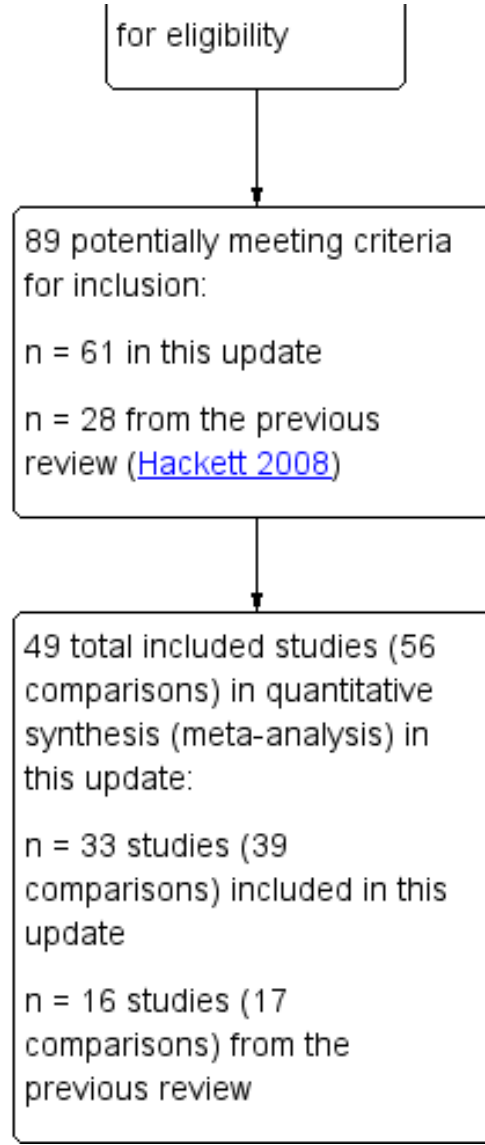

\section{Data extraction and management}

Five review authors (SA, KC, C-FH, HL, MH) independently extracted study characteristics and outcome data from included studies and entered them on specially designed forms. We cross-checked and entered the data into Review Manager 5 (Review Manager 2014). We resolved disagreements by discussion or through consultation with two other review authors ( $\mathrm{AH}$ or $\mathrm{MH}$ ). We obtained missing information from the study authors when possible. Information on funding sources is mentioned in the notes sections of the Characteristics of included studies table.

We collected data on:

- the report: author, year, and source of publication;

- the study: sample characteristics, social demography, and definition and criteria used for depression;

- the participants: stroke sequence (first ever vs recurrent), social situation, time elapsed since stroke onset, history of psychiatric illness, current neurological status, current treatment for depression, and history of coronary artery disease;

- the research design and features: sampling mechanism, treatment assignment mechanism, adherence, non-response, and length of follow up;

- the intervention: type, duration, dose, timing, and mode of delivery; and
2)

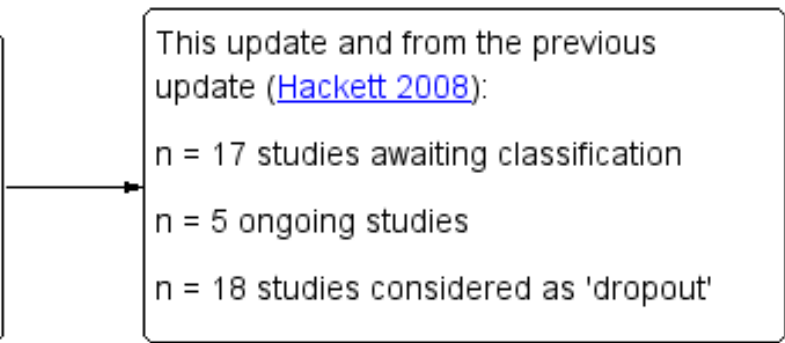

- the effect size: sample size, nature of outcome, estimate, and standard error on $\mathrm{x} d \mathrm{y}=\mathrm{SD}$.

To allow for intention-to-treat (ITT) analysis, we sought the data irrespective of adherence and fidelity of the intervention, and regardless of whether participants were subsequently deemed ineligible or were otherwise excluded from treatment or follow-up. When study authors used multiple measures to assess depression, we extracted data from the measure the study authors stated was used to assess the primary outcome. For measures assessing secondary outcomes, we extracted data from the most commonly used measure. When data for the same trial endpoint were conflicting across multiple publications, we extracted data from the first publication reporting data for that outcome.

We checked all extracted data for agreement between review authors. We obtained missing information from the primary investigators whenever possible. To avoid introducing bias, we obtained this unpublished information in writing, on forms designed for the purpose, and entered it into RevMan.

\section{Assessment of risk of bias in included studies}

Three review authors (SA, KC, C-FH) independently assessed risk of bias for each study using the criteria outlined in the Cochrane Handbook for Systematic Reviews of Interventions (Higgins 2011). We resolved any disagreements by discussion or by consultation 
with another review author (MH). Although a number of scales have been devised to assess the quality of RCTs, no convincing evidence shows that complex and time-consuming scales are more effective than simple scales (Verhagen 2001). We assessed risk of bias according to the following domains.

- Random sequence generation.

- Allocation concealment: if allocation was performed using opaque envelopes, we also categorised this as 'high risk' as it is not tamper-proof.

- Blinding of participants and personnel: for psychological interventions, we recognise that participants are unlikely to remain blinded; however we also categorised this as 'high risk'.

- Blinding of outcome assessment.

- Incomplete outcome data.

- Selective outcome reporting: if a published trial had no corresponding published or registered protocol, this was assessed as unclear risk.

- Other bias.

We also provided a quote from the study to justify our judgement in the Risk of bias in included studies table. When considering treatment effects, we have taken into account the risk of bias for studies that contributed to that outcome.

\section{Measures of treatment effect}

\section{Dichotomous dato}

For all dichotomous outcomes, we calculated risk ratios (RRs) with 95\% confidence intervals (Cls) when appropriate, using randomeffects analyses.

\section{Continuous data}

For continuous data, if ordinal scale data appeared to be normally distributed, or if the analysis suggested that parametric tests were appropriate, we treated outcome measures as continuous. If at least two studies reported the same outcomes, then we calculated a mean difference (MD) with $95 \% \mathrm{Cl}$ across trials. When different outcome measures were used, we calculated a standardised mean difference (SMD) with $95 \% \mathrm{Cl}$.

\section{Unit of analysis issues}

We predicted that randomisation would occur at the level of the individual participant in most, if not all, trials. Outcomes are reported at end of treatment and at end of follow-up when data are available. When trials included two or more active intervention arms and only one control arm (placebo, attention control, or usual care), we compared data from each treatment arm with data from the total number of participants in the control arm divided by the number of active intervention arms. Comparisons are presented as separate trials.

\section{Dealing with missing data}

We wrote to the authors of all included, ongoing, and dropout trials to request data that were unavailable or ambiguous in published articles. We also wrote to all pharmaceutical companies known to produce, or having a licence to produce, antidepressants in 2004. We received nine replies identifying no new trials, so we did not repeat this in the 2008 update nor in the current update.

\section{Assessment of heterogeneity}

Clinical and methodological heterogeneity were assessed by examining the study characteristics. We used the $I^{2}$ statistic to measure heterogeneity among the trials in each analysis (Deeks 2011). If at least two trials reported the same outcomes, we reviewed the data for appropriateness of pooling. We interpreted the amount of heterogeneity as low ( $0 \%$ to $29 \%$ ), moderate ( $30 \%$ to $49 \%$ ), substantial $(50 \%$ to $89 \%)$, and considerable $(90 \%$ to $100 \%)$ using $I^{2}$ values. We reported similarities between interventions, participants, design, and outcomes in the Included studies subsection.

\section{Assessment of reporting biases}

We assessed publication bias by using a funnel plot only if 10 or more trials were included (Higgins 2011). We attempted to avoid language bias by including trials irrespective of language of publication, and we provided translation when needed by native speakers of that language.

In some cases, similarities between trial reports indicated the possibility of multiple publications from the same trial. We contacted study authors to check whether these publications were duplicates. In the absence of a response and explicit crossreferencing, we judged articles to be from the same trial if they met the following criteria: (1) evidence suggested overlapping recruitment sites, trial dates, and grant funding numbers, and (2) similar or identical patient characteristics were reported by trial authors.

\section{Data synthesis}

We analysed data using Review Manager software and pooled data for meta-analysis when studies assessed similar treatments and had similar outcomes (Review Manager 2014). We conducted a meta-analysis using available or calculated MD or SMD for continuous outcomes, and RR for dichotomous outcomes. We included measures of uncertainty in the results, such as $95 \% \mathrm{Cls}$ and estimates of $I^{2}$.

\section{'Summary of findings' and certainty of the evidence}

We assessed the certainty of evidence according to GRADE by constructing a 'Summary of findings' table for the outcomes below, per comparison, using the GRADEPro tool (GRADEproGDT 2015) (Atkins 2004; Schunemann 2011).

These data were available for comparison: (1) pharmacological interventions versus placebo; (2) one of various forms of non-invasive brain stimulation versus sham stimulation or usual care; (3) one of various forms of psychological therapy versus usual care and/or attention control; (4) pharmacological intervention and various forms of psychological therapy versus pharmacological intervention and usual care and/or attention control (with two comparisons); and (5) non-invasive brain stimulation and pharmacological intervention versus pharmacological intervention and sham stimulation or usual care (with 10 comparisons).

For comparison 1, 'pharmacological intervention versus placebo', we reported certainty of evidence for the following outcomes: meeting study criteria for depression at end of treatment, $<50 \%$ reduction in depression scale scores at end of treatment, mean neurological function scores at end of treatment, adverse events 
related to CNS and gastrointestinal tract and death at end of treatment.

For comparison 2, 'non-invasive brain stimulation versus sham or usual care', we reported certainty of evidence for the following outcomes: mean depression scores at end of treatment, mean depression scores at end of follow-up, mean neurological function scores at end of treatment, and death at end of treatment.

For comparison 3, 'psychological intervention versus usual care or attention control', we reported certainty of evidence for the following outcomes: meeting the study criteria for depression at end of treatment, average change in depression scores between baseline and end of treatment, meeting the study criteria for depression at end of follow-up, death, and leaving the study early (including death) at end of treatment.

For comparison 4, 'pharmacological intervention and a form of psychological therapy (combination) versus pharmacological intervention and usual care or attention control (single)', we reported certainty of evidence for mean depression scores at end of treatment, mean activities of daily living at end of treatment, and death at end of treatment.

For comparison 5, 'non-invasive brain stimulation and pharmacological intervention (combination) versus pharmacological intervention and sham stimulation or usual care (single)', we reported certainty of evidence for the following outcomes: mean depression scores at end of treatment, mean activities of daily living scores at end of treatment, mean neurological function scores at end of treatment, death, and leaving the study early (including death) at end of treatment.

We found no trials for these comparisons: (6) pharmacological intervention and various forms of psychological therapy interventions versus placebo and psychological therapy; (7) pharmacological intervention and non-invasive brain stimulation versus placebo plus non-invasive brain stimulation; (8) noninvasive brain stimulation and one of various forms of psychological therapy versus non-invasive brain stimulation plus usual care and/or attention control; and (9) non-invasive brain stimulation and one of various forms of psychological therapy versus sham brain stimulation or usual care plus psychological therapy.

\section{Subgroup analysis and investigation of heterogeneity}

We planned to undertake subgroup analyses for all outcomes when feasible to explore the influence of date of publication, sample size, duration of follow-up, treatment type, high (over $20 \%$ ) number of dropouts, and blinded versus unblinded outcome assessors. If at least two trials reported the same outcomes, we reviewed the data for appropriateness of pooling. If we found definitive evidence of heterogeneity $\left(\mathrm{I}^{2}>50 \%\right)$, we explored potential reasons for differences by performing subgroup analyses and meta-regression (Normand 1999). If heterogeneity could not be explained, we combined trials using random-effects analyses with cautious interpretation, or we did not combine them at all. When possible, we performed subgroup analyses to examine the impact of treatment type and duration, and of stroke severity.

\section{Sensitivity analysis}

We explored the sensitivity of the combined estimate of individual trials for all outcomes when feasible by leaving one study out if we noted high risk of bias and methodological differences. We then calculated the combined effect of the remaining trials and compared these results with the combined effect based on all trials.

\section{RESULTS}

\section{Description of studies}

\section{Results of the search}

In total, we identified 23,228 records; of these, we retrieved 22,748 through database searching. We found 480 additional references by searching other resources. After 14,128 duplicates were removed, we screened 9100 titles and abstracts and excluded 8967 irrelevant records. We retrieved full-text reports for the remaining 133 studies. After reading the full texts, we excluded 44 trials as they did not meet the review eligibility criteria. We have provided the primary reasons for exclusions in the Characteristics of excluded studies table and in Figure 1. We identified 10 trials that met the inclusion criteria (Chang 2011; Hadidi 2014; Jorge 2004; Jorge 2008; Kim 2017; Kim 2017a; Kootker 2012; Raffaele 1996; Robinson 2000; Valiengo 2017). However, data were not available for depressed participants only (Chang 2011; Hadidi 2014; Jorge 2004; Jorge 2008; Kim 2017; Kim 2017a; Raffaele 1996; Robinson 2000; Valiengo 2017), or were not in a format suitable for meta-analysis (Kootker 2012). These trials are considered 'dropouts' (Table 1). In the previously published version of this review (Hackett 2008), eight trials met the inclusion criteria but were considered 'dropouts' (Bramanti 1989; Choi-Kwon 2006; Delbari 2011; Downes 1995; Mauri 1988; Meara 1998; Ohtomo 1985; Sun 2000), as outcome data were not available at all (Downes 1995), or outcome data were not available for depressed participants only (Choi-Kwon 2006; Delbari 2011; Ohtomo 1985; Sun 2000), or outcome data were not presented in a format suitable for meta-analysis (Bramanti 1989; Meara 1998; Mauri 1988). See Table 1 for more detailed information on these studies.

We contacted the study authors to ask for information on ongoing studies or to request additional study data and, in some instances, additional analyses. We received responses with additional data regarding seven trials (Andersen 1994; Downes 1995; Fruehwald 2003; Lincoln 2003; Murray 2002; Reding 1986, Towle 1989). In 2008, we received responses with additional data from the authors of two trials (Lai 2006a; Watkins 2007). We received responses with additional data or information from the authors of seven new trials (Cullen 2018; Fang 2017; Hoffmann 2015; Kerr 2018; Kirkness 2017a; Mitchell 2002; Robinson 2008a).

\section{Included studies}

From the first published version of this review, a total of nine included trials included 671 participants (Andersen 1994; Fruehwald 2003; Lincoln 2003; Lipsey 1984; Murray 2002; Ohtomo 1991; Reding 1986; Towle 1989; Wiart 2000). Seven additional trials (eight comparisons) with 864 participants were included in the 2008 update of this review (Jiang 2001a/Jiang 2001b; Lai 2006a; Ponzio 2001; Rampello 2005; Watkins 2007; Yang 2002; Zhao 2004). Lincoln 2003 compared an active treatment with an attention control (time spent by participants in the treatment group with a trained therapist was controlled in the attention control group 
by participants spending an equal amount of time in focused conversation), as well as another control (standard care). We combined data from the attention control and control groups, and we compared these with data from the treatment group. Jiang 2001a compared two active treatment arms versus a placebo arm. We compared data from both treatment arms against data from half the number of participants in the placebo arm and presented the results as two separate comparisons (Jiang 2001a; Jiang 2001b). More detailed information is provided in Characteristics of included studies.

This present review includes 33 trials (39 comparisons) with 2807 participants. Cao 2009a and Jiang 2014a were parallel RCTs with four arms. We compared data from both treatment arms with their respective control arms and presented the results as separate comparisons (Cao 2009a; Cao 2009b; Jiang 2014a; Jiang 2014b). Gao 2017a and Kirkness 2017a compared two active treatment arms versus a usual care or attention control arm. We compared data from both treatment arms with data from half the number of participants in the usual care or attention control arm and presented the results as separate comparisons (Gao 2017a; Gao 2017b; Kirkness 2017a; Kirkness 2017b). Similarly, Robinson 2008a compared two active treatment arms against a placebo arm. Data from both treatment arms were compared with data from half the number of participants in the placebo arm (Robinson 2008a; Robinson 2008b). Yang 2014a compared two active treatment arms versus a sham non-invasive brain stimulation arm. We compared data from both treatment arms with data from half the number of participants in the sham non-invasive brain stimulation arm (Yang 2014a; Yang 2014b).

\section{Participants}

All trials in this review included men and women. The mean age of participants ranged from 55 to 77.5 years. Most trial authors reported the time since stroke and randomisation into the trial, with the range covering 'within a few days' to 36 months post stroke. Most trials included participants with ischaemic stroke, diagnosed via a combination of standard clinical and computed tomography (CT) criteria. For more detailed information on each included trial, please refer to the Characteristics of included studies table.

\section{Interventions and comparators}

We reported results from the following comparisons: (1) pharmacological intervention versus placebo; (2) non-invasive brain stimulation versus sham non-invasive brain stimulation; (3) one of various forms of psychological therapy versus usual care and/or attention control; (4) pharmacological intervention and one of various forms of psychological therapy versus pharmacological intervention and usual care and/or attention control; and (5) non-invasive brain stimulation and pharmacological intervention versus pharmacological intervention and sham stimulation or usual care. In 18 trials, 20 pharmacological comparisons were assessed against placebo (Andersen 1994; Fruehwald 2003; Gao 2017a; Huang 2002; Jiang 2001a/Jiang 2001b; Kong 2007; Lai 2006a; Li 2008; Lipsey 1984; Murray 2002; Ohtomo 1991; Ponzio 2001; Rampello 2005; Reding 1986; Robinson 2008a/Robinson 2008b; Wang 2005; Wiart 2000; Yang 2002). Seven trials (eight comparisons) reported on non-invasive brain stimulation comparisons versus sham or usual care (Chen 2005a; Gu 2016; Jiang 2014a; Meng 2015; Yang 2013; Yang 2014a;/Yang 2014b; Zheng 2016), and the authors of 15 trials (16 comparisons) assessed various forms of psychological therapy compared to usual care or attention control (Alexopoulos 2012; Cao 2009b; Cullen 2018; Fang 2017; Gao 2017b; Hoffmann 2015; Kerr 2018; Kirkness 2017a/Kirkness 2017b; Lincoln 2003; Mitchell 2002; Thomas 2007; Towle 1989; Wang 2004a; Watkins 2007; Zhao 2004). In two trials (two comparisons), a combination of pharmacological interventions and psychological therapy was assessed against pharmacological intervention and usual care and/or attention control (Cao 2009a; Wang 2005a). In 10 trials (10 comparisons), a combination of non-invasive brain stimulation and pharmacological intervention was compared to pharmacological intervention and sham stimulation or usual care (Du 2005; Fan 2014; Jiang 2014b; Jin 2013; Li 2013; Li 2014; Liu 2015; Lu 2016; Sun 2013; Zhang 2013).

We found no trials for the following comparisons: (6) pharmacological intervention and one of various forms of psychological therapy compared to placebo and psychological therapy; (7) pharmacological intervention and non-invasive brain stimulation versus placebo plus non-invasive brain stimulation; (8) non-invasive brain stimulation and one of various forms of psychological therapy versus non-invasive brain stimulation plus usual care and/or attention control; and (9) non-invasive brain stimulation and one of various forms of psychological therapy versus sham brain stimulation or usual care plus psychological therapy.

\section{Pharmacological interventions}

Among the trials of pharmacological interventions, 12 compared an SSRI against placebo (citalopram: Andersen 1994; Gao 2017a; fluoxetine: Fruehwald 2003; Huang 2002; Kong 2007; Li 2008; Wang 2005; Wiart 2000; paroxetine: Lai 2006a; Ponzio 2001; Yang 2002; sertraline: Murray 2002); two trials compared a tricyclic antidepressant against placebo (amitriptyline: Jiang 2001a; nortriptyline: Lipsey 1984); and six trials compared other treatments with antidepressant effects (Deanxit: Jiang 2001b; Aniracetam: Ohtomo 1991; reboxetine: Rampello 2005; trazodone: Reding 1986; nefiracetam: Robinson 2008a; Robinson 2008b). We found no trials of mood stabilisers (e.g. lithium) or benzodiazepines. We found one trial of psychostimulants (e.g. methylphenidate), which was considered a 'dropout' as outcome data for those with depression at entry could not be separated from data for those without (Delbari 2011).

\section{Non-invasive brain stimulation}

Among trials reporting on non-invasive brain stimulation interventions, seven compared rTMS versus sham rTMS or usual care (no changes to antidepressant dosage and medication) (Chen 2005a; Gu 2016; Meng 2015; Yang 2013; Yang 2014a; Yang 2014b; Zheng 2016). In only one trial, TMS was compared with usual care (Jiang 2014a). Two trials compared high-frequency rTMS versus sham or usual care (Yang 2013; Yang 2014a), and one trial compared low-frequency rTMS versus sham stimulation or usual care (Yang 2014b). We found no trials of ECT. Any future trials will be included but analysed separately.

\section{Psychological therapy}

Forms of psychological therapy included structured cognitivebehavioural therapy delivered by trained psychologists or nurses (Gao 2017b; Hoffmann 2015; Lincoln 2003; Mitchell 2002; Thomas 2007); motivational interviewing (MI) delivered by nurses or non- 
clinical psychologists (Kerr 2018; Watkins 2007); psychosocial therapy delivered by psychosocial nurse practitioner therapists in person or via telephone (Fang 2017; Kirkness 2017a; Kirkness 2017b); group psychotherapy (Cao 2009b); and psychotherapy with an ecosystem aspect (Alexopoulos 2012); treatments focused on psychological support (Wang 2004a), problem-solving therapy with counselling delivered by social workers (Towle 1989), and a supportive psychological intervention including education delivered by special personnel (Cullen 2018; Zhao 2004).

\section{Combination therapy}

In two trials, a combination of psychotherapy and an SSRI was compared with an SSRI alone (fluoxetine: Cao 2009a; paroxetine: Wang 2005a). In six trials, rTMS and an SSRI were compared with an SSRI (fluoxetine: Du 2005; Li 2014; Zhang 2013; citalopram: Liu 2015; sertraline: Jiang 2014b; Jin 2013). In two trials, rTMS and an SNRI were compared with an SNRI alone (duloxetine: Fan 2014; Lu 2016). In one trial, rTMS and another antidepressant medication were compared with an antidepressant alone (mirtazapine: Li 2013). Only one trial compared rTMS and a combination of antipsychoactive agents and tricyclic antidepressants (flupenthixol and melitracen: named Deanxit) versus Deanxit alone (Sun 2013).

\section{Outcomes}

\section{Primary outcome - depression}

In 14 trials (15 comparisons), outcome data for meeting the study criteria for depression at end of treatment were assessed and reported (Alexopoulos 2012; Andersen 1994; Fang 2017; Fruehwald 2003; Kirkness 2017a/Kirkness 2017b; Lincoln 2003; Lipsey 1984; Mitchell 2002; Murray 2002; Ohtomo 1991; Ponzio 2001; Watkins 2007; Yang 2002; Zhao 2004). For the outcome less than 50\% reduction in depression scale scores at end of treatment, six trials contributed data (Andersen 1994; Lai 2006a; Li 2008; Murray 2002; Wiart 2000; Yang 2002).

\section{Secondary outcomes}

A variety of additional outcomes were assessed in each trial. Several trials assessed and reported outcome data for depression scores (Alexopoulos 2012; Andersen 1994; Cao 2009b; Chen 2005a; Cullen 2018; Fruehwald 2003; Gu 2016; Hoffmann 2015; Huang 2002; Jiang 2001a; Jiang 2001b; Kerr 2018; Kong 2007; Lai 2006a; Li 2008; Lincoln 2003; Lipsey 1984; Mitchell 2002; Murray 2002; Rampello 2005; Robinson 2008a; Robinson 2008b; Thomas 2007; Wang 2004a; Wiart 2000; Yang 2013; Yang 2014a; Yang 2014b; Zhao 2004), psychological distress scores (Lincoln 2003; Watkins 2007), anxiety scores (Cullen 2018; Fang 2017; Hoffmann 2015; Kerr 2018; Wang 2005a), cognitive function scores (Du 2005; Gao 2017a; Gao 2017b; Wang 2005; Wiart 2000), activities of daily living scores (Cao 2009a; Cao 2009b; Du 2005; Fan 2014; Gao 2017a; Gao 2017b; Hoffmann 2015; Kerr 2018; Kirkness 2017a; Kirkness 2017b; Kong 2007; Li 2008; Li 2014; Lincoln 2003; Meng 2015; Mitchell 2002; Yang 2013), disability scores (Alexopoulos 2012; Chen 2005a; Fruehwald 2003; Lu 2016; Sun 2013; Wang 2004a; Watkins 2007; Wiart 2000), and neurological function scores (Huang 2002; Jiang 2001a; Jiang 2001b; Jiang 2014a; Jiang 2014b; Jin 2013; Kong 2007; Liu 2015; Meng 2015; Zheng 2016). In 24 trials (28 comparisons), study authors reported having systematically measured and reported adverse events (Alexopoulos 2012; Andersen 1994; Du 2005; Fang 2017; Fruehwald 2003; Gao 2017a/Gao 2017b; Gu 2016; Huang 2002; Jiang 2001a/Jiang 2001b; Jiang 2014a/Jiang 2014b; Li 2008; Lincoln 2003; Lipsey 1984; Liu 2015; Meng 2015; Mitchell 2002; Murray
2002; Ponzio 2001; Robinson 2008a/Robinson 2008b; Thomas 2007; Towle 1989; Wang 2005a; Watkins 2007; Wiart 2000). Adverse event data often were not collected, were not reported, or were reported poorly.

\section{Excluded studies}

We excluded a total of 44 trials at the full-text review stage for a variety of reasons, including (1) depression not the primary outcome of the study ( $n=22)$; (2) data not available for depressed participants only $(n=20)$; or (3) data not available in a suitable format for meta-analysis $(n=2)$. See Characteristics of excluded studies.

\section{Ongoing studies}

Five trials are ongoing (Kirkevold 2018: psychological therapy; NCT03056287: non-invasive brain stimulation; Tang 2017: noninvasive brain stimulation; Thomas 2016: psychological therapy; Xu 2016: pharmacological intervention).

\section{Studies awaiting classification}

From the previously published version of this review, four trials are listed as awaiting classification (Evans 1985; Hanspal 2007; Katz 1998; Pearson 2005). We were unable to obtain more information or outcome data from these trials despite multiple attempts to contact the study authors. In the present review, 13 trials (17 comparisons) are awaiting classification (Chen 2002a/Chen 2002b; Ding 2005; Finkenzeller 2009; He 2003; He 2005; Huang 2005; Latow 1983; Lee 2005; Liu 2010; Razazian 2016; Tang 2002; Wang 2015; Yan 2010a/Yan 2010b/Yan 2010c/Yan 2010d). We were unable to obtain more information or outcome data for three of these despite multiple attempts to contact the study authors (He 2003; Latow 1983; Lee 2005). For two trials (three comparisons), we were unsure if depression was the primary outcome (Chen 2002a/Chen 2002b; Razazian 2016). In eight trials (11 comparisons), no information was provided for the psychotherapy component of the intervention to help us determine if it meets our review criteria (Ding 2005; Finkenzeller 2009; He 2005; Huang 2005; Liu 2010; Tang 2002; Wang 2015; Yan 2010a/Yan 2010b/Yan 2010c/Yan 2010d).

\section{Dropout studies}

From the previously published review, eight trials met the inclusion criteria for this review (Bramanti 1989; Choi-Kwon 2006; Delbari 2011; Downes 1995; Mauri 1988; Meara 1998; Ohtomo 1985; Sun 2000). However, no outcome data were available in one trial (unpublished data only: Downes 1995); in others, data were not presented on depressed participants at baseline (Choi-Kwon 2006; Delbari 2011; Ohtomo 1985; Sun 2000), or data were not presented in a suitable format for inclusion in the meta-analysis (Bramanti 1989; Mauri 1988; Meara 1998).

In this review, 10 additional trials met the inclusion criteria (Chang 2011; Hadidi 2014; Jorge 2004; Jorge 2008; Kim 2017; Kim 2017a; Kootker 2012; Raffaele 1996; Robinson 2000; Valiengo 2017). However, data were not presented on depressed participants only at baseline (Chang 2011; Hadidi 2014; Jorge 2004; Kim 2017; Kim 2017a; Raffaele 1996; Robinson 2000), or data were not presented in a suitable format (Kootker 2012; Valiengo 2017), or we were unable to verify if any participants had a diagnosis of stroke or if there were duplicate data from another trial (Jorge 2008). We considered these trials as 'dropouts' and have provided more detailed information in Table 1. 


\section{Risk of bias in included studies}

We present a graphical summary of risk of bias assessments performed by review authors for the included trials in Figure 2, based on the seven risk of bias domains. Figure 3 provides a summary of risk of bias for each included trial. We have provided the reasons for judgements in the Risk of bias in included studies tables.

Figure 2. Risk of bias graph: review authors' judgements about each risk of bias item presented as percentages across all included studies.

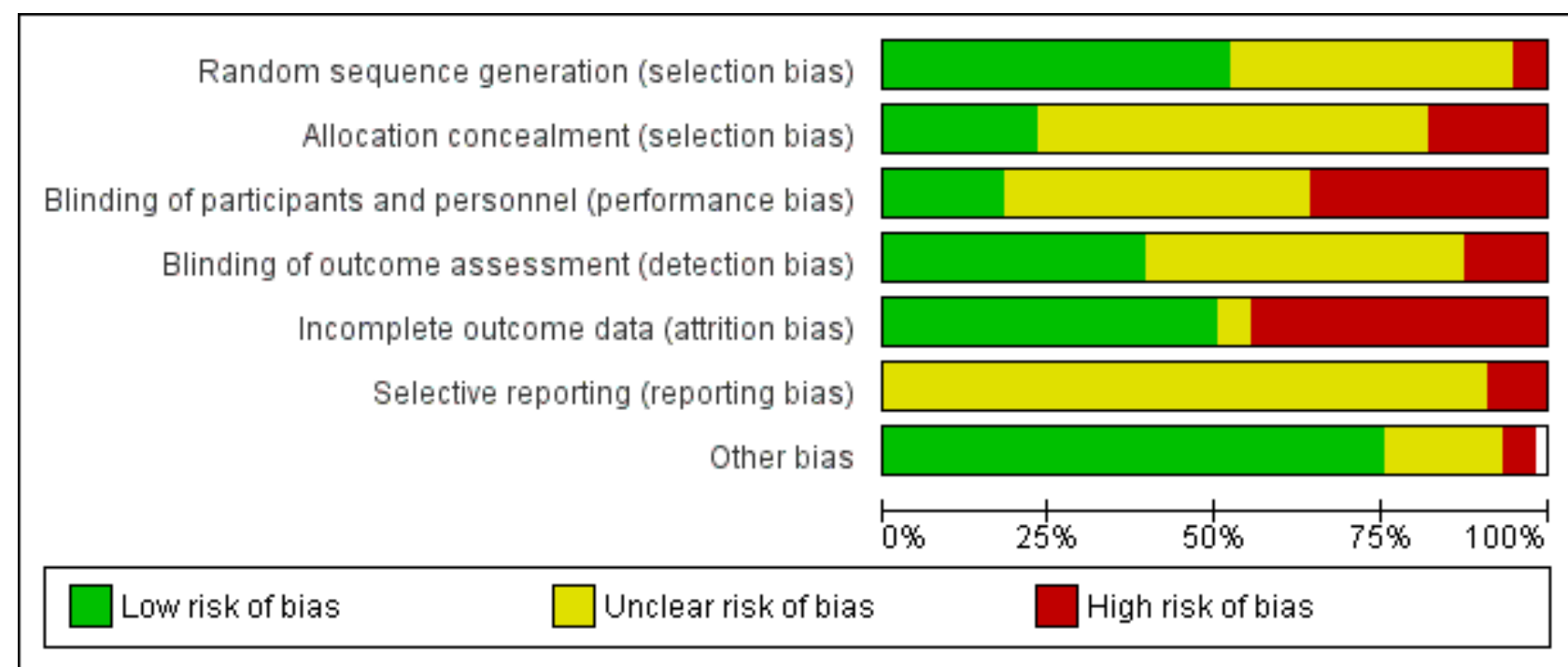


Figure 3. Risk of bias summary: review authors' judgements about each risk of bias item for each included study.

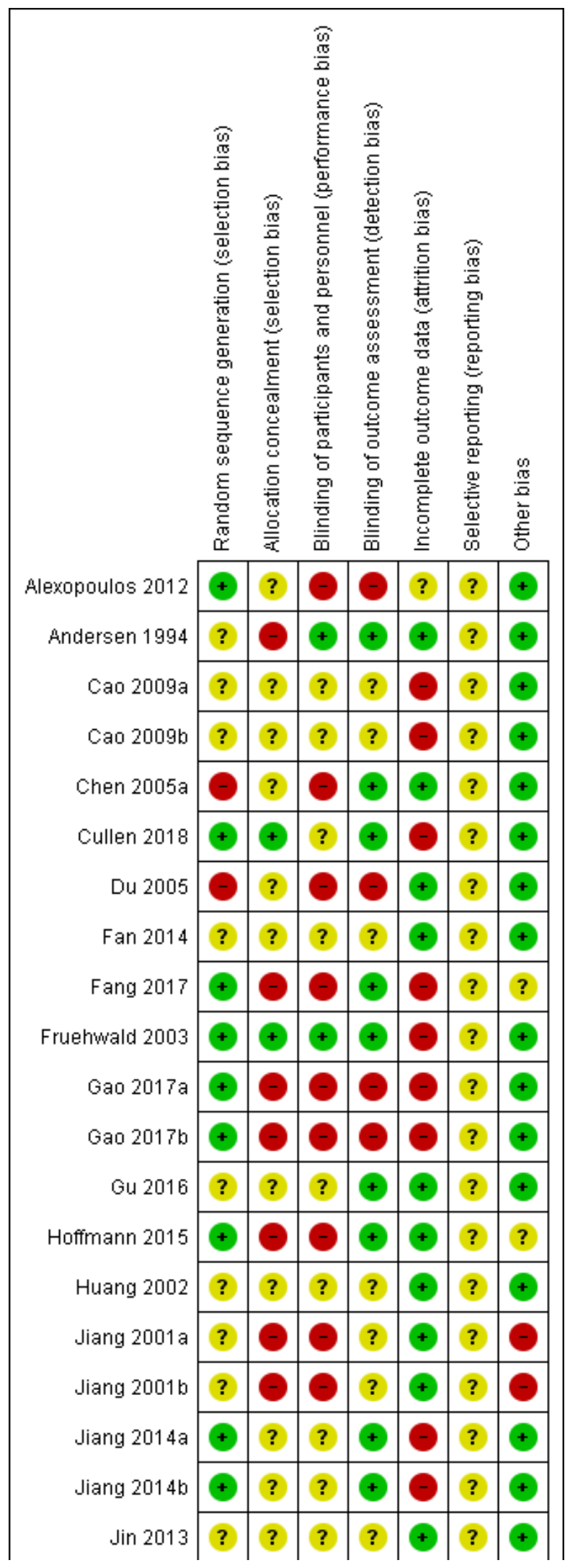


Figure 3. (Continued)

\begin{tabular}{|c|c|c|c|c|c|c|c|}
\hline Jin 2013 & $?$ & $?$ & $?$ & $?$ & + & $?$ & + \\
\hline Kerr 2018 & + & + & $\odot$ & $\odot$ & $?$ & $\odot$ & $\oplus$ \\
\hline Kirkness 2017a & + & $?$ & $\odot$ & + & $\odot$ & $?$ & + \\
\hline Kirkness $2017 \mathrm{~b}$ & + & $?$ & $\odot$ & + & $\odot$ & $?$ & + \\
\hline Kong 2007 & + & 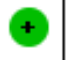 & + & $?$ & $\odot$ & $?$ & $\oplus$ \\
\hline Lai 2006a & $?$ & $?$ & $?$ & $?$ & $\odot$ & $?$ & $?$ \\
\hline Li 2008 & + & $\odot$ & $\odot$ & + & $\odot$ & $?$ & $\oplus$ \\
\hline Li 2013 & $?$ & $?$ & $?$ & $?$ & + & $?$ & $\oplus$ \\
\hline Li 2014 & + & $?$ & $?$ & $?$ & + & $?$ & + \\
\hline Lincoln 2003 & + & $\odot$ & $\Theta$ & $\odot$ & $\odot$ & $?$ & $\Theta$ \\
\hline Lipsey 1984 & + & $\odot$ & + & + & $\odot$ & $?$ & + \\
\hline Liu 2015 & $?$ & $?$ & $?$ & $?$ & + & $?$ & + \\
\hline Lu 2016 & 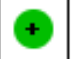 & $?$ & $?$ & $?$ & $\odot$ & $?$ & + \\
\hline Meng 2015 & + & $?$ & $?$ & $?$ & 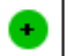 & $?$ & $?$ \\
\hline Mitchell 2002 & + & $?$ & $\Theta$ & + & $\odot$ & $\odot$ & + \\
\hline Murray 2002 & + & $\odot$ & + & + & $\odot$ & $?$ & $?$ \\
\hline Ohtomo 1991 & $O$ & $?$ & + & + & $\odot$ & $?$ & $?$ \\
\hline Ponzio 2001 & $?$ & $?$ & $?$ & $?$ & + & $?$ & + \\
\hline Rampello 2005 & + & $\odot$ & $\odot$ & + & + & $?$ & + \\
\hline Reding 1986 & + & $\odot$ & + & + & + & $?$ & $?$ \\
\hline Robinson 2008a & + & + & + & + & $\odot$ & $\odot$ & $?$ \\
\hline Robinson 2008b & + & $\odot$ & + & + & $\odot$ & $\odot$ & $?$ \\
\hline Sun 2013 & $?$ & $?$ & $?$ & $?$ & 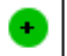 & $?$ & $\oplus$ \\
\hline Thomas 2007 & 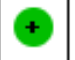 & + & - & $?$ & $\odot$ & $\odot$ & $\oplus$ \\
\hline Towle 1989 & + & $\odot$ & $\odot$ & $?$ & $\odot$ & $?$ & + \\
\hline Wang 2004a & $?$ & $?$ & $?$ & $?$ & + & $?$ & + \\
\hline Wang 2005 & $?$ & $?$ & $?$ & $?$ & $\odot$ & $?$ & $?$ \\
\hline Wang 2005a & $?$ & $?$ & $?$ & $?$ & $?$ & $?$ & + \\
\hline Watkins 2007 & + & $\odot$ & $\odot$ & $\odot$ & + & $?$ & + \\
\hline Wiart 2000 & $?$ & $\odot$ & $\odot$ & + & + & $?$ & + \\
\hline Yang 2002 & $?$ & $?$ & $?$ & $?$ & - & $?$ & \\
\hline
\end{tabular}


Figure 3. (Continued)

\begin{tabular}{|c|c|c|c|c|c|c|c|}
\hline Yang 2002 & $?$ & $?$ & $?$ & $?$ & - & $?$ & \\
\hline Yang 2013 & $?$ & $?$ & $?$ & $?$ & + & $?$ & + \\
\hline Yang 2014a & $?$ & $?$ & $?$ & $?$ & + & $?$ & + \\
\hline Yang 2014b & $?$ & $?$ & $?$ & $?$ & + & $?$ & + \\
\hline Zhang 2013 & + & $?$ & $?$ & $?$ & + & $?$ & $\oplus$ \\
\hline Zhao 2004 & $?$ & $?$ & - & + & + & $?$ & + \\
\hline Zheng 2016 & ? & ? & ? & ? & + & ? & + \\
\hline
\end{tabular}

\section{Allocation}

The randomisation sequence was appropriately generated in 25 trials (29 comparisons); thus we rated then as low risk (Alexopoulos 2012; Cullen 2018; Fang 2017; Fruehwald 2003; Gao 2017a/Gao 2017b; Hoffmann 2015; Jiang 2014a/Jiang 2014b; Kerr 2018; Kirkness 2017a/Kirkness 2017b; Kong 2007; Li 2008; Li 2014; Lincoln 2003; Lipsey 1984; Lu 2016; Meng 2015; Mitchell 2002; Murray 2002; Rampello 2005; Reding 1986; Robinson 2008a/Robinson 2008b; Thomas 2007; Towle 1989; Watkins 2007; Zhang 2013). However, 21 trials (24 comparisons) did not describe their method of sequence generation, and so we rated them as unclear risk (Andersen 1994; Cao 2009a/Cao 2009b; Fan 2014; Gu 2016; Huang 2002; Jiang 2001a/Jiang 2001b; Jin 2013; Lai 2006a; Li 2013; Liu 2015; Ponzio 2001; Sun 2013; Wang 2004a; Wang 2005; Wang 2005a; Wiart 2000; Yang 2002; Yang 2013; Yang 2014a/Yang 2014b; Zhao 2004; Zheng 2016). We rated three trials as high risk, as generation of sequence was controlled by the investigators (Ohtomo 1991), or the method was drawing lots (Chen 2005a; Du 2005), which could be manipulated.

We rated 12 trials ( 13 comparisons) as low risk, as an appropriately generated and clearly concealed allocation procedure was used in the study (Cullen 2018; Fruehwald 2003; Kerr 2018; Kong 2007; Li 2008; Lipsey 1984; Murray 2002; Rampello 2005; Reding 1986; Robinson 2008a/Robinson 2008b; Thomas 2007; Wiart 2000). Twenty-nine trials (33 comparisons) did not describe adequate concealment allocation, and we rated them as unclear risk (Alexopoulos 2012; Cao 2009a/Cao 2009b; Chen 2005a; Du 2005; Fan 2014; Gu 2016; Huang 2002; Jiang 2014a/Jiang 2014b; Jin 2013; Kirkness 2017a/Kirkness 2017b; Lai 2006a; Li 2013; Li 2014; Liu 2015; Lu 2016; Meng 2015; Mitchell 2002; Ohtomo 1991; Ponzio 2001; Sun 2013; Wang 2004a; Wang 2005; Wang 2005a; Yang 2002; Yang 2013; Yang 2014a/Yang 2014b; Zhang 2013; Zhao 2004; Zheng 2016). We rated eight trials (10 comparisons) as high risk for allocation concealment, as they used sealed opaque envelopes, which could be tampered with (Andersen 1994; Fang 2017; Gao 2017a/Gao 2017b; Hoffmann 2015; Jiang 2001a/Jiang 2001b; Lincoln 2003; Towle 1989; Watkins 2007) .

\section{Blinding}

The authors of nine trials (10 comparisons) reported that participants and personnel were blinded to the treatment allocation, and so we rated these trials as low risk for performance bias (Andersen 1994; Fruehwald 2003; Kong 2007;
Lipsey 1984; Murray 2002; Ohtomo 1991; Reding 1986; Robinson 2008a/Robinson 2008b; Wiart 2000). We rated 22 trials (25 comparisons) as unclear risk, as they did not provide information about blinding of participants and personnel (Cao 2009a/Cao 2009b; Cullen 2018; Fan 2014; Gu 2016; Huang 2002; Jiang 2014a/Jiang 2014b; Jin 2013; Lai 2006a; Li 2013; Li 2014; Liu 2015; Lu 2016; Ponzio 2001; Sun 2013; Wang 2004a; Wang 2005; Wang 2005a; Yang 2002; Yang 2013; Yang 2014a/Yang 2014b; Zhang 2013; Zheng 2016). We rated 15 trials (18 comparisons) as high risk for performance bias, as participants or personnel were not blinded to treatment allocation (Alexopoulos 2012; Chen 2005a; Du 2005; Fang 2017; Gao 2017a/Gao 2017b; Hoffmann 2015; Jiang 2001a/Jiang 2001b; Kerr 2018; Kirkness 2017a/Kirkness 2017b; Li 2008; Lincoln 2003; Rampello 2005; Towle 1989; Watkins 2007; Zhao 2004).

We rated 19 trials (24 comparisons) as low risk for detection bias, as outcome assessors were blinded to treatment allocation (Andersen 1994; Chen 2005a; Cullen 2018; Fang 2017; Fruehwald 2003; Gu 2016; Hoffmann 2015; Jiang 2014a/Jiang 2014b; Kirkness 2017a/Kirkness 2017b; Li 2008; Lipsey 1984; Mitchell 2002; Murray 2002; Ohtomo 1991; Rampello 2005; Reding 1986; Robinson 2008a/Robinson 2008b; Wiart 2000; Zhao 2004). Twenty-four trials (27 comparisons) did not provide information about blinding of outcome assessors, and we rated them as unclear risk of detection bias (Cao 2009a/Cao 2009b; Fan 2014; Huang 2002; Jin 2013; Kong 2007; Jiang 2001a/Jiang 2001b; Lai 2006a; Li 2013; Li 2014; Liu 2015; Lu 2016; Meng 2015; Ponzio 2001; Sun 2013; Thomas 2007; Towle 1989; Wang 2004a; Wang 2005; Wang 2005a; Yang 2002; Yang 2013; Yang 2014a/Yang 2014b; Zhang 2013; Zheng 2016). We rated six trials (seven comparisons) as high risk because they did not use blinded outcome assessment (Alexopoulos 2012; Du 2005; Gao 2017a/Gao 2017b; Kerr 2018; Lincoln 2003; Watkins 2007).

\section{Incomplete outcome data}

We rated 26 trials ( 29 comparisons) as low risk, as they provided ITT analyses (Andersen 1994; Chen 2005a; Du 2005; Fan 2014; Hoffmann 2015; Huang 2002; Jiang 2001a/Jiang 2001b; Jin 2013; Lai 2006a; Li 2013; Li 2014; Liu 2015; Meng 2015; Ponzio 2001; Rampello 2005; Reding 1986; Sun 2013; Wang 2004a; Wang 2005; Watkins 2007; Wiart 2000; Yang 2013; Yang 2014a/Yang 2014b; Zhang 2013; Zhao 2004; Zheng 2016). We rated 18 trials (22 comparisons) as high risk for attrition bias, as they reported per-protocol analyses only (Cullen 2018; Fang 2017; Fruehwald 2003; Gao 2017a/Gao 2017b; Jiang 2014a/Jiang 2014b; Kirkness 2017a/Kirkness 2017b; Kong 2007; Li 2008; Lincoln 2003; Lipsey 1984; Lu 2016; Mitchell 
2002; Murray 2002; Ohtomo 1991; Robinson 2008a/Robinson 2008b; Thomas 2007; Towle 1989; Yang 2002). The method of analysis was unclear in four trials (five comparisons) (Alexopoulos 2012; Cao 2009a/Cao 2009b; Kerr 2018; Wang 2005a).

\section{Selective reporting}

We rated 45 trials (51 comparisons) as unclear risk for reporting bias, as no trial protocol was available to compare a priori outcomes versus those reported in publications (Alexopoulos 2012; Andersen 1994; Cao 2009a/Cao 2009b; Chen 2005a; Cullen 2018; Du 2005; Fan 2014; Fang 2017; Fruehwald 2003; Gao 2017a/Gao 2017b; Gu 2016; Hoffmann 2015; Huang 2002; Jiang 2001a/Jiang 2001b; Jiang 2014a/Jiang 2014b; Jin 2013; Kirkness 2017a/Kirkness 2017b; Kong 2007; Lai 2006a; Li 2008; Li 2013; Li 2014; Lincoln 2003; Lipsey 1984; Liu 2015; Lu 2016; Meng 2015; Murray 2002; Ohtomo 1991; Ponzio 2001; Rampello 2005; Reding 1986; Sun 2013; Towle 1989; Wang 2004a; Wang 2005; Wang 2005a; Watkins 2007; Wiart 2000; Yang 2002; Yang 2013; Yang 2014a/Yang 2014b; Zhang 2013; Zhao 2004; Zheng 2016). We rated four trials (five comparisons) as high risk, as one or two outcomes mentioned in the study protocol or trial registry information page were not reported in the primary results publication (Kerr 2018; Mitchell 2002; Robinson 2008a/Robinson 2008b; Thomas 2007).

\section{Other potential sources of bias}

We rated 38 trials (44 comparisons) as low risk for other bias, as baseline demographics and depression scores were balanced between groups (Alexopoulos 2012; Andersen 1994; Cao 2009a/Cao 2009b; Chen 2005a; Cullen 2018; Du 2005; Fan 2014; Fruehwald 2003; Gao 2017a/Gao 2017b; Gu 2016; Huang 2002; Jiang 2001a/Jiang 2001b; Jiang 2014a/Jiang 2014b; Jin 2013; Kerr 2018; Kirkness 2017a/Kirkness 2017b; Kong 2007; Li 2008; Li 2013; Li 2014; Lipsey 1984; Liu 2015; Lu 2016; Mitchell 2002; Ponzio 2001; Rampello 2005; Sun 2013; Thomas 2007; Towle 1989; Wang 2004a; Wang 2005a; Watkins 2007; Wiart 2000; Yang 2013; Yang 2014a/Yang 2014b; Zhang 2013; Zhao 2004; Zheng 2016). We rated 10 trials (11 comparisons) as unclear, as no information about baseline demographics and depression scores between groups was provided (Fang 2017; Hoffmann 2015; Lai 2006a; Meng 2015; Murray 2002; Ohtomo 1991; Reding 1986; Robinson 2008a/Robinson 2008b; Wang 2005; Yang 2002). We rated two trials (three comparisons) as high risk, as baseline demographic or depression scores were uneven between groups (Jiang 2001a/Jiang 2001b; Lincoln 2003).

\section{Effects of interventions}

See: Summary of findings for the main comparison Pharmacological intervention compared to placebo for treating depression after stroke; Summary of findings 2 Non-invasive brain stimulation compared to sham non-invasive brain stimulation and/or usual care for treating depression after stroke; Summary of findings 3 Psychological therapy compared to usual care and/or attention control for treating depression after stroke; Summary of findings 4 Pharmacological intervention and a form of psychotherapy (combination) compared to pharmacological intervention and usual care or attention control (single) for treating depression after stroke; Summary of findings $\mathbf{5}$ Non-invasive brain stimulation and pharmacological intervention (combination) compared to pharmacological intervention and sham stimulation or usual care (single) for treating depression after stroke
Overall, we included 3342 participants in this review. In view of the large number and heterogeneous nature of the outcome measures (multiple measures often used for the same endpoint with no primary measure stated) and the reporting of results, we considered it inappropriate to pool outcome data for many endpoints. For details of all comparisons made for the trials with outcome data, refer to the Data and analyses section.

See Summary of findings for the main comparison; Summary of findings 2; Summary of findings 3; Summary of findings 4; and Summary of findings 5 for comparisons.

\section{Primary outcomes \\ Depression (remission)}

\section{Meeting study criteria for depression at end of treatment}

Eight trials (eight comparisons) on pharmacological interventions reported on the outcome meeting study criteria for depression at end of treatment (Andersen 1994; Fruehwald 2003; Lipsey 1984; Murray 2002; Ohtomo 1991; Ponzio 2001; Wang 2005; Yang 2002). We observed treatment effects favouring pharmacological interventions compared to placebo (risk ratio (RR) 0.70, $95 \%$ confidence interval (Cl) 0.55 to $0.88,1025$ participants, very low-certainty evidence; Analysis 1.1). However, substantial heterogeneity $\left(I^{2}=68 \%\right)$ and wide confidence intervals were evident across individual trials.

No trials of non-invasive brain stimulation alone assessed this outcome (Analysis 2.1).

Five trials (six comparisons) of psychological therapy reported on the outcome meeting study criteria for depression at end of treatment and demonstrated an effect favouring psychological therapy over usual care and/or attention control (RR $0.77,95 \% \mathrm{Cl}$ 0.62 to 0.95 ; 6 RCTs; 521 participants; very low-certainty evidence) (Alexopoulos 2012; Fang 2017; Kirkness 2017a/Kirkness 2017b; Mitchell 2002; Watkins 2007). We observed low heterogeneity $\left(I^{2}=\right.$ $36 \%$ ) and wide confidence intervals (Analysis 3.1).

No trials of combination therapies assessed this outcome (Analysis 4.1; Analysis 5.1).

\section{Less than $\mathbf{5 0} \%$ reduction in depression scale scores}

Six trials (six comparisons) of pharmacological interventions reported on this outcome (Andersen 1994; Lai 2006a; Li 2008; Murray 2002; Wiart 2000; Yang 2002). We observed treatment effects favouring pharmacological therapy among those who received a pharmacological intervention compared with placebo (RR 0.47, $95 \% \mathrm{Cl} 0.32$ to $0.69 ; 6 \mathrm{RCTs} ; 511$ participants; very low-certainty evidence). We observed substantial heterogeneity $\left(I^{2}=66 \%\right)$ and wide confidence intervals (Analysis 1.2).

We did not perform a meta-analysis for the comparison non-invasive brain stimulation versus sham non-invasive brain stimulation or usual care (Analysis 2.2).

No trials of psychological interventions versus usual care and/ or attention control and pharmacological intervention and psychological therapy (combination) versus pharmacological intervention and usual care or attention control (single) assessed this outcome (Analysis 3.2; Analysis 4.2). 
We did not perform a meta-analysis for the comparison noninvasive brain stimulation and pharmacological intervention (combination) versus pharmacological intervention and sham stimulation or usual care (single), as only one trial reported data on this outcome for each comparison (Analysis 5.2).

\section{Secondary outcomes}

\section{Depression scores}

\section{Average change in scores between baseline and end of treatment}

We did not perform a meta-analysis on this outcome for the comparison pharmacological interventions versus placebo (Analysis 1.3), due to the heterogenous nature of the outcome measures and single trials using multiple measures for this outcome without specifying a primary outcome measure. Two trials (three comparisons) found an effect favouring psychological therapy over usual care and/or attention control (mean difference (MD) $-6.20,95 \% \mathrm{Cl}-8.24$ to -4.16 ; 3 RCTs; 189 participants; very lowcertainty evidence; Analysis 3.3) (Kirkness 2017a/Kirkness 2017b; Mitchell 2002).

\section{Mean scores at end of treatment}

We did not perform a meta-analysis on this outcome for the comparison pharmacological interventions versus placebo due to the heterogenous nature of the outcome measures (Analysis 1.4).

Seven trials (eight comparisons) demonstrated an effect favouring non-invasive brain stimulation over sham or usual care at end of treatment (MD $-6.63,95 \% \mathrm{Cl}-9.71$ to -3.55 ; 8 RCTs; 495 participants; very low-certainty evidence) (Chen 2005a; Gu 2016; Jiang 2014a; Meng 2015; Yang 2013; Yang 2014a/Yang 2014b; Zheng 2016). However, considerable heterogeneity $\left(I^{2}=99 \%\right)$ and very wide confidence intervals (Analysis 2.3 subgroup 2.3.1) are evident.

We did not perform a meta-analysis on the outcome for comparison of psychological therapy versus usual care and/or attention control (Analysis 3.4) due to the heterogenous nature of the outcome measures.

We also observed a beneficial effect for combination therapy (pharmaceutical intervention and psychological therapy) compared to pharmaceutical intervention alone at end of treatment (MD $-1.53,95 \% \mathrm{Cl}-2.10$ to $-0.96 ; 2$ RCTs; 198 participants; very low-certainty evidence; Analysis 4.3 subgroup 4.3.1). Heterogeneity was substantial $\left(I^{2}=87 \%\right)$ and confidence intervals were very wide. We also observed this effect among those who received a combination of non-invasive brain stimulation and pharmacological intervention in comparison to those who received pharmacological intervention alone at end of treatment (MD -4.09, 95\% Cl -5.61 to -2.57; 9 RCTs; 685 participants; very lowcertainty evidence; Analysis 5.3 subgroup 5.3.1). Heterogeneity was substantial $\left(I^{2}=88 \%\right)$ and confidence intervals were very wide.

\section{Mean scores at end of follow-up}

Three trials (three comparisons) of non-invasive brain stimulation addressed the outcome of mean depression scores at end of followup and revealed an effect favouring non-invasive brain stimulation over sham or usual care (MD $-2.60,95 \% \mathrm{Cl}-3.3$ to -1.87 ; 3 RCTs; 170 participants; very low-certainty evidence; Analysis 2.4 subgroup 2.4.1) (Gu 2016; Meng 2015; Yang 2013). However, confidence intervals were very wide.
We did not perform a meta-analysis for the comparison noninvasive brain stimulation and pharmacological intervention versus pharmacological intervention alone, as only one trial reported data on this outcome (Analysis 5.4 subgroup 5.4.1).

\section{Meeting study criteria for depression at end of follow-up}

Two trials (three comparisons) of psychological therapy assessed this outcome and showed no statistically significant effects for those who received psychological therapy compared to usual care and/or attention control (RR $0.85,95 \% \mathrm{Cl} 0.59$ to 1.21 ; 3 RCTs; 201 participants; very low-certainty evidence; $\mathrm{I}^{2}=11 \%$; Analysis 3.5 , subgroup 3.5.1) (Kirkness 2017a/Kirkness 2017b; Mitchell 2002).

\section{Psychological distress scores}

No significant effect was observed in those who received psychological therapy compared to usual care and/or attention control on the outcome average change in scores between baseline and end of treatment (MD $-0.21,95 \% \mathrm{Cl}-1.89$ to $1.48 ; 2$ RCTs; 377 participants; very low-certainty evidence) (Lincoln 2003; Watkins 2007). Nor did we observe a significant effect on mean psychological distress scores at end of treatment (MD $-0.43,95 \% \mathrm{Cl}$ -2.17 to 1.31 ; 2 RCTs; 377 participants; very low-certainty evidence). See Analysis 3.8 subgroup 3.8.1 and Analysis 3.9 subgroup 3.9.1.

\section{Anxiety scores}

\section{Mean scores at end of treatment}

We did not perform a meta-analysis on this outcome for comparison of psychological therapy versus usual care and/or attention control due to the heterogenous nature of the outcome measures and single trials using multiple measures for this outcome without specifying a primary outcome measure (Analysis 3.11).

\section{Mean scores at end of follow-up}

We did not perform a meta-analysis on this outcome for comparison: psychological therapy versus usual care and/or attention control due to the heterogenous nature of the outcome measures and single trials using multiple measures for this outcome without specifying a primary outcome measure (Analysis 3.12).

\section{Cognitive function scores}

We did not perform a meta-analysis, as only one trial reported data for this outcome (Analysis 1.6; Analysis 1.7; Analysis 5.5).

\section{Activities of daily living (ADL) scores}

\section{Average change in scores between baseline and end of treatment}

Two trials (two comparisons) revealed that pharmacological intervention compared to placebo had no significant effect on the average change in scores between baseline and end of treatment (MD -8.00, 95\% Cl-24.18 to 8.18; 2 RCTs; 256 participants; very low-certainty evidence) (Ponzio 2001; Reding 1986) (Analysis 1.8 subgroup 1.8.1). Similarly, two trials (two comparisons) also showed that psychological therapy compared to usual care and/or attention control had no significant effect on the average change in scores between baseline and end of treatment (SMD $-0.03,95 \% \mathrm{Cl}$ -0.24 to 0.18 ; 2 RCTs; 377 participants; very low-certainty evidence; Analysis 3.13) (Lincoln 2003; Watkins 2007). 


\section{Mean scores at end of treatment}

Three trials of pharmacological interventions (three comparisons) found no significant effect on mean ADL scores at end of treatment compared with placebo (MD 3.14, 95\% Cl -0.97 to 7.26; 3 RCTs; 316 participants; very low-certainty evidence; Analysis 1.9 subgroup 1.9.1) (Gao 2017a; Kong 2007; Li 2008). Two trials (two comparisons) demonstrated no effect among those who received non-invasive brain stimulation compared to sham or usual care (SMD 1.84, $95 \% \mathrm{Cl}-1.40$ to $5.08 ; 2 \mathrm{RCTs} ; 208$ participants; very low-certainty evidence; Analysis 2.5) (Jiang 2014a; Meng 2015). However, we observed considerable heterogeneity $\left(I^{2}=99 \%\right)$ and very wide confidence intervals.

We did not perform a meta-analysis on this outcome for comparison: psychological therapy versus usual care and/or attention control (Analysis 3.14), due to the heterogenous nature of the outcome measures and single trials using multiple measures for this outcome without specifying a primary outcome measure.

Two trials (two comparisons) found that a combination of pharmacological intervention and psychological therapy had no effect on mean ADL scores compared to a single pharmacological intervention at end of treatment (MD $11.83,95 \% \mathrm{Cl} 0.27$ to 23.40 ; 2 RCTs; 198 participants; very low-certainty evidence; Analysis 4.5 subgroup 4.5.1) (Cao 2009a; Wang 2005a). Similarly, five trials (five comparisons) showed that combination therapy (non-invasive brain stimulation and pharmacological intervention) had no effect compared to pharmacological intervention alone (single) (SMD 2.03, $95 \% \mathrm{Cl} 1.21$ to 2.85; $5 \mathrm{RCTs} ; 003$ participants; very lowcertainty evidence; Analysis 5.6) (Du 2005; Fan 2014; Jiang 2014b; Li 2013; Li 2014). However, the two comparisons showed considerable heterogeneity $\left(\mathrm{I}^{2}=94 \%\right.$ and $\left.\mathrm{I}^{2}=91 \%\right)$ and very wide confidence intervals.

\section{Mean scores at end of follow-up}

We did not perform a meta-analysis, as only one trial reported data on this outcome (Analysis 3.15).

\section{Disability scores}

Two trials (two comparisons) found that psychological therapy had no effect on mean disability scores at end of treatment compared to usual care and/or attention control (SMD $-0.16,95 \% \mathrm{Cl}-0.48$ to 0.17 ; 2 RCTs; 162 participants; very low-certainty evidence; Analysis 3.16) (Alexopoulos 2012; Gao 2017b). Although two trials (two comparisons) reported that non-invasive brain stimulation and pharmacological intervention (combination) had an effect on mean disability scores at end of treatment compared to pharmacological intervention alone (MD -10.02, 95\% Cl -20.14 to 0.11; 2 RCTs; 180 participants; very low-certainty evidence; Analysis 5.7 subgroup 5.7.1) (Lu 2016; Sun 2013).

\section{Neurological function scores}

\section{Mean scores at end of treatment}

Four trials (four comparisons) showed that pharmacological interventions had an effect on mean scores at end of treatment compared to placebo (SMD $-0.95,95 \% \mathrm{Cl}-1.44$ to $-0.45 ; 4 \mathrm{RCTs}$; 304 participants; very low-certainty evidence; Analysis 1.13) (Huang 2002; Jiang 2001a; Kong 2007; Wang 2005). Heterogeneity was substantial $\left(I^{2}=75 \%\right)$ and confidence intervals were very wide. Similarly, we observed an effect among those who received non- invasive brain stimulation compared to sham or usual care (SMD $-2.21,95 \% \mathrm{Cl}-3.32$ to -1.09 ; $3 \mathrm{RCTs} ; 290$ participants; very lowcertainty evidence; Analysis 2.7) (Meng 2015; Jiang 2014a; Zheng 2016). However, we noted considerable heterogeneity $\left(I^{2}=93 \%\right)$ and wide confidence intervals.

We did not perform a meta-analysis for this comparison: pharmacological intervention and psychological therapy versus pharmacological intervention alone (Analysis 4.6 subgroup 4.6.1), as only one trial reported data on this outcome.

In contrast, four trials (four comparisons) found that a combination of non-invasive brain stimulation and pharmacological intervention had an effect on mean scores at end of treatment compared to pharmacological intervention alone (MD $-2.78,95 \% \mathrm{Cl}$ -4.13 to -1.44 ; 4 RCTs; 280 participants; very low-certainty evidence; Analysis 5.8 subgroup 5.8.1) (Jiang 2014b; Jin 2013; Li 2013; Liu 2015). Heterogeneity was substantial $\left(I^{2}=82 \%\right)$ and confidence intervals were very wide.

\section{Adverse events: death}

Nine trials (nine comparisons) found that pharmacological intervention had no effect on adverse events compared to placebo: death (RR 0.64, 95\% $\mathrm{Cl} 0.20$ to 2.07; 9 RCTs; 848 participants; very low-certainty evidence; Analysis 1.14 subgroup 1.14.1) (Andersen 1994; Fruehwald 2003; Gao 2017a; Huang 2002; Li 2008; Lipsey 1984; Murray 2002; Ponzio 2001; Wiart 2000). Although no heterogeneity was observed $\left(I^{2}=0 \%\right)$, confidence intervals were very wide.

Two trials (two comparisons) reported that non-invasive brain stimulation resulted in no deaths (Gu 2016; Jiang 2001a) (Analysis 2.8 subgroup 2.8 .1 ).

Eight trials (eight comparisons) found that psychological therapy had no effect on adverse events compared to usual care or attention control: death (RR $0.65,95 \% \mathrm{Cl} 0.26$ to 1.66 ; 8 RCTs; 831 participants; very low-certainty evidence; Analysis 3.17 subgroup 3.17.1) (Alexopoulos 2012; Fang 2017; Gao 2017b; Lincoln 2003; Mitchell 2002; Thomas 2007; Towle 1989; Watkins 2007). We observed no heterogeneity $\left(I^{2}=0 \%\right)$ but confidence intervals were very wide.

Three trials (three comparisons) reported that a combination of non-invasive brain stimulation and pharmacological intervention resulted in no deaths compared to pharmacological intervention and sham stimulation or usual care (Du 2005; Jiang 2014b; Liu 2015)) (Analysis 5.9 subgroup 5.9.1).

\section{Adverse events: all}

Significant evidence of harm was demonstrated among adverse events, in particular, CNS effects (RR $1.55,95 \% \mathrm{Cl} 1.12$ to 2.15 ; 5 RCTs; 488 participants; very low-certainty evidence; $I^{2}=31 \%$ ) (Andersen 1994; Lipsey 1984; Murray 2002; Ponzio 2001; Wiart 2000), along with gastrointestinal effects (RR 1.62, 95\% Cl 1.19 to 2.19; 4 RCTs; 473 participants; very low-certainty evidence) (Li 2008; Murray 2002; Ponzio 2001; Wiart 2000), among those who received pharmacological interventions compared with placebo (see Analysis 1.15 subgroup 1.15 .1 and 1.15 .5 ). We observed no heterogeneity $\left(I^{2}=0 \%\right)$, but the confidence intervals were very wide. 
Two trials (two comparisons) reported that non-invasive brain stimulation resulted in no other adverse events - not listed above (e.g. dysuria, eye discomfort; Analysis 2.9) (Gu 2016; Jiang 2014a).

Four trials (four comparisons) found that psychological therapy resulted in no significant adverse events (recurrent stroke - RR 5.0, $95 \% \mathrm{Cl} 0.24$ to $103.12 ; 1 \mathrm{RCT} ; 254$ participants; vascular events - RR $0.71,95 \% \mathrm{Cl} 0.23$ to $2.19 ; 1 \mathrm{RCT} ; 254$ participants; very low-certainty evidence), nor other events - not listed above (e.g. too ill) (RR 1.02, $95 \% \mathrm{Cl} 0.15$ to $6.81 ; 2$ RCTs; 206 participants; very low-certainty evidence). See Analysis 3.18 (Mitchell 2002; Thomas 2007; Towle 1989; Watkins 2007).

Two trials (two comparisons) found that a combination of non-invasive brain stimulation and pharmacological intervention resulted in no significant adverse events (other events - not listed above, e.g. insomnia, discomfort, headache) (RR 7.0, 95\% $\mathrm{Cl} 0.38$ to 129.93; 2 RCTs; 120 participants; very low-certainty evidence). See Analysis 5.10 (Du 2005; Jiang 2014b).

\section{Adverse events: leaving the study early (including death)}

Twelve trials (13 pharmacological comparisons) reported on this outcome (Andersen 1994; Fruehwald 2003; Gao 2017a; Huang 2002; Kong 2007; Li 2008; Lipsey 1984; Murray 2002; Ponzio 2001; Robinson 2008a/Robinson 2008b; Wang 2005; Wiart 2000). Pharmacological interventions had no effect on the proportion of participants leaving the study early (including death) compared to placebo (RR 1.07, 95\% Cl 0.82 to 1.39; 13 RCTs; 1165 participants; very low-certainty evidence; Analysis 1.16 subgroup 1.16.1). Although we observed no heterogeneity $\left(I^{2}=0 \%\right)$, confidence intervals were very wide.

Seven trials (eight comparisons) revealed that psychological therapy had no effect on the proportion of participants leaving the study early (including death) compared to usual care and/ or attention control (RR $0.83,95 \% \mathrm{Cl} 0.42$ to 1.63 ; 8 RCTs; 784 participants; very low-certainty evidence; Analysis 3.19 subgroup 3.19.1) (Alexopoulos 2012; Gao 2017b; Kirkness 2017a/Kirkness 2017b; Lincoln 2003; Mitchell 2002; Towle 1989; Watkins 2007). Although we observed no heterogeneity $\left(I^{2}=0 \%\right)$, confidence intervals were very wide.

Four combination therapy trials (rTMS and pharmacological interventions) (four comparisons) reported on this outcome. A combination of rTMS and pharmacological interventions had no effect on the proportion of people leaving the study early (including death) compared to pharmacological intervention alone (RR 1.33, $95 \% \mathrm{Cl} 0.32$ to 5.58; 4 RCTs; 300 participants; very low-certainty evidence) (Du 2005; Jiang 2014b; Liu 2015; Lu 2016). See Analysis 5.11 subgroup 5.11 .1 . We observed no heterogeneity $\left(I^{2}=0 \%\right)$, but confidence intervals were very wide.

\section{DISCUSSION}

\section{Summary of main results}

In this review update, we included 49 trials (56 comparisons) involving 3342 participants that met our criteria. The large increase in the number of included trials is partially explained by expansion of the types of included interventions. This is the first time we have included other (in addition to electroconvulsive therapy (ECT)) non-invasive brain stimulation interventions and combination interventions.
Data were available for these comparisons: (1) pharmacological interventions versus placebo; (2) one of various forms of non-invasive brain stimulation versus sham stimulation or usual care; (3) one of various forms of psychological therapy versus usual care and/or attention control; (4) pharmacological intervention and various forms of psychological therapy versus pharmacological intervention and usual care and/or attention control (with two comparisons); and (5) non-invasive brain stimulation and pharmacological intervention versus pharmacological intervention and sham stimulation or usual care (with 10 comparisons).

Comparison 1. Comparing pharmacological intervention to placebo, we found very low-certainty pooled evidence suggesting benefit in treating depression to remission and reducing depressive symptom scores on mood rating scales, along with evidence of harm (more central nervous system and gastrointestinal adverse events). These results are largely unchanged from previous versions of this review. For pharmacological trials, a key requirement is that a therapeutic dose of the medication must be achieved for an adequate period of time. Guidelines from the American College of Physicians suggest that antidepressants should be continued for at least four months beyond initial recovery, and that treatment should be changed if no response has been shown by six weeks (Snow 2000). In this review, the interventions in most pharmacological trials probably were not given for an adequate length of time to show maximal or sustained response. Therefore, we are unable to comment on the long-term effects of antidepressant therapy, or to provide information on the most appropriate duration or dose of treatment; nor can we say if one group of antidepressants is more efficacious or provide stopping rules for antidepressant therapy in this group.

Comparison 2. Comparing non-invasive brain stimulation to usual care or sham stimulation, we found very low-certainty pooled evidence that repetitive transcranial magnetic stimulation (rTMS) reduces depressive symptom scores at end of treatment and after follow-up, off treatment. No reported data were related to remission. No adverse events were reported. We did not include this endpoint in previous versions of this review. The duration of treatment in these trials was short, ranging from one to four weeks. The impact of many different facets of interventions such as rTMS (including electrode placement, number of sessions, or particular frequencies on outcomes) is not within the scope of this review.

Comparison 3. Comparing psychological therapy to usual care or attention control, we found very low-certainty pooled evidence of benefit in treating depression to remission at end of treatment, but this benefit was not sustained to the end of follow-up off treatment. We did not pool data related to changes in depression symptom scores due to use of multiple measures across and within studies with no a priori primary outcome measure identified. Pooled evidence for adverse events included benefit and harm. These results are different from findings of previous versions of this review, which demonstrated no treatment effects. For psychological therapy trials, good evidence shows that efficacy is linked to delivery of adequate exposure to the intervention. This means that therapists should be trained and supervised in the therapy they are delivering, and should use a standardised, prespecified framework for therapy. To achieve this in psychological therapy trials, therapy is determined with use of a manual, and research therapists are trained and supervised in use of the 
manual. Success in brief therapy is linked to adherence to the therapeutic model, as well as to the therapists' characteristics. Future stroke psychological therapy trials should adhere to these standard psychological therapy research guidelines if there is to be any probability of demonstrating consistency and response.

Comparison 4. Comparing combined pharmacological intervention and psychological therapy to pharmacological intervention plus usual care or attention control, we found very low-certainty pooled evidence of benefit in reducing depressive symptom scores on mood rating scales. No reported data were related to remission. We did not include this endpoint in previous versions of this review.

Comparison 5. Comparing non-invasive brain stimulation and pharmacological intervention to usual care or sham stimulation and pharmacological intervention, we found very low-certainty pooled evidence of benefit in reducing depressive symptom scores on mood rating scales. No reported data were related to remission. Pooled evidence for adverse events included benefit and harm. We did not include this endpoint in previous versions of this review.

We found no trials for these comparisons: (6) pharmacological intervention and various forms of psychological therapy interventions compared with placebo and psychological therapy; (7) pharmacological intervention and non-invasive brain stimulation versus placebo plus non-invasive brain stimulation; (8) non-invasive brain stimulation and one of various forms of psychological therapy versus non-invasive brain stimulation plus usual care and/or attention control; and (9) non-invasive brain stimulation and one of various forms of psychological therapy versus sham brain stimulation or usual care plus psychological therapy.

Evidence demonstrating benefit must continue to be considered alongside several basic methodological limitations of many of these trials, including the short duration of many interventions, variation in the types of trial participants recruited and in the methods used to diagnose depression, lack of an a priori measurable endpoint, and high risk of bias in many trials. Of particular concern is the evidence of harm (more adverse events) given the small number of trials in which adverse events were systematically recorded and reported, making reliable assessment of the benefits and risks of treatments impossible.

The trials in this review included participants with depression occurring several days to more than two years following stroke. However, depression occurring in the early phase of stroke is likely to be different from that occurring several months or years after the event. Survivors in the first weeks following stroke are coping with the consequences of experiencing a potentially life-threatening event, as well as recovering from the disabling effects of the stroke itself. In the medium to long term, survivors of stroke are more likely to be adjusting to the prospects of permanent disability and changes in social and financial circumstances. It is difficult to summarise the evidence from such mixed populations, and even in doing so, whether it could be considered meaningful, especially given the high risk of relapse of depression in the first few months of recovery, which declines over time (Snow 2000).

In contrast to the wide range in the length of time between stroke onset and entry into the trial, many trials included participants with narrow demographic and clinical characteristics, in particular, they excluded people with communication problems, cognitive loss, or previous psychiatric illness. This reinforces a common criticism of depression research - that trial participants are not representative of those requiring treatment in the 'real world' (Zimmerman 2002). It would appear that this criticism is also applicable to trials of depression following stroke, where up to half of survivors may be excluded on the basis of such criteria (Turner-Stokes 2003). Given the older age of most people with stroke and the frequent presence of neurological impairments, aphasia, and comorbid medical conditions, the fact that up to half of all survivors of stroke are excluded limits the external validity (generalisability) of the results. Use of a large list of exclusions means that the results are applicable to only a small proportion of stroke survivors who have a narrow range of comorbidities and other characteristics. Such exclusions may be justifiable for trials of psychological therapy, in which participants are required to actively participate in therapy by talking, but the exclusions seem inappropriate for pharmacotherapy trials. Ideally, patients should be heterogeneous with regard to stroke diagnosis, which requires the use of standard diagnostic criteria and neuroimaging in a high proportion of cases. Given differences in the natural history and management of subarachnoid haemorrhage, it could be argued that this form of stroke should be examined separately.

Lack of a consistent method to diagnose depression at trial entry and outcomes in the included trials is a concern and a reflection of the general lack of a standard definition for a 'healthy state' among people with mood disorders (Keller 2003). Few trials have stated whether the primary goal of therapy was remission (no longer meeting the baseline criteria for depression), response (> $50 \%$ reduction in mood scores from baseline), or simply a greater reduction in mood scores (or difference in scores) in one of the randomised groups. Complete remission of symptoms is arguably the most meaningful endpoint for the patient, whereas the significance of a small reduction in mood scores on a continuous scale is generally difficult to interpret for the patient and for the treating physician. These problems with outcome assessment were further confounded by frequent use of multiple scales and selective reporting of findings between and within trials. Any one scale was used across only eight trials at most, and significantly different cut-points were used to determine depression at entry and at trial end. Given the practical difficulties and high costs of conducting psychiatric interviews in clinical trials, it seems appropriate to adopt a pragmatic approach to assess depression on the basis of a validated mood questionnaire or structured interview. It is hoped that the compulsory registration of trial protocols on publicly available databases will reduce, if not eliminate, the opportunity for selective reporting of results. It has been suggested that more than one-third of efficacy outcomes and half of harm outcomes are inadequately reported (Chan 2004). Several other methodological deficiencies in trials further limit the conclusions that can be drawn from this review. Many trials were small; less than half reported adequate concealment of the randomisation sequence, and dropout rates were high in several trials. Additionally, blinding of investigators and outcome assessors was seldom stated.

\section{Overall completeness and applicability of evidence}

The present review included 49 trials (56 comparisons) with 3342 participants. Data were available for 20 pharmacological comparisons, eight non-invasive brain stimulation comparisons, 16 psychological therapy comparisons, and 11 combination therapy trials. Overall, consistent methods used to diagnose depression 
were lacking, and we considered it inappropriate to pool outcome data for many endpoints. The accuracy of the findings of this systematic review and meta-analysis must be considered in light of the basic methodological limitations described in the Risk of bias in included studies table. Eighteen trials are considered dropouts, 21 trials are awaiting classification, and at least five ongoing trials may contribute further evidence to future updates of this review.

\section{Quality of the evidence}

We rated the certainty of evidence for all comparisons by using the five GRADE considerations (study limitations, consistency of effect, indirectness, imprecision, and publication bias; Schunemann 2011). We created a 'Summary of findings' table for each comparison. Certainty assessment was very low.

\section{Limitations in study design or execution}

For the comparison of pharmacological interventions versus placebo, we downgraded the certainty of evidence by two points for the following outcomes: meeting study criteria for depression at end of treatment, less than $50 \%$ reduction in depression scale scores, mean neurological function scores at end of treatment, and adverse events - death at end of treatment, all CNS events, and gastrointestinal events - as we rated several studies as having high or unclear risk for multiple risk of bias domains (Summary of findings for the main comparison).

For the comparison of non-invasive brain stimulation versus sham, we downgraded the certainty of evidence by two points for the following outcomes: mean depression scores at end of treatment, mean depression scores at end of follow-up, and mean neurological function scores at end of treatment, as we rated several studies as having high or unclear risk for multiple risk of bias domains (Summary of findings 2).

For the comparison of psychological therapy versus usual care and/or attention control, we downgraded the certainty of evidence by two points for the following outcomes: meeting study criteria for depression at end of treatment, average change in depression scores between baseline and end of treatment, meeting study criteria for depression at end of follow-up, and adverse events death at end of treatment and leaving the study early - as we rated several studies as having high or unclear risk for multiple risk of bias domains (Summary of findings 3).

For the comparison of pharmacological interventions and psychological therapy (combination) versus pharmacological intervention and usual care and/or attention control (single), we downgraded the certainty of evidence by two points for the following outcomes: mean depression scores at end of treatment and mean activities of daily living scores at end of treatment, as we rated two studies as having unclear risk for multiple risk of bias domains, related to allocation concealment and blinding of participants, personnel, and outcome assessors (Summary of findings 4).

For the comparison of non-invasive brain stimulation and pharmacological intervention (combination) versus pharmacological intervention with sham or usual care (single), we downgraded by two points the certainty of evidence for mean depression scores, mean activities of daily living scores at end of treatment, and leaving the study early, as we rated the study as having high risk for multiple risk of bias domains, related to blinding of participants, personnel, and outcome assessors (Summary of findings 5).

\section{Inconsistency of results}

For the comparison of pharmacological interventions versus placebo, we downgraded by two points the certainty of evidence for the following outcomes: meeting study criteria for depression, less than $50 \%$ reduction in depression scale scores, and mean neurological function scores at end of treatment, as we observed substantial heterogeneity (50\% to $89 \%)$. We also downgraded the certainty of evidence by one point for gastrointestinal events, as we observed moderate heterogeneity (30\% to 49\%) (Summary of findings for the main comparison).

For the comparison of non-invasive brain stimulation versus sham, we downgraded the certainty of evidence by two points for mean depression scores and neurological function scores at end of treatment due to considerable heterogeneity observed $(90 \%$ to $100 \%$ ) (Summary of findings 2).

For the comparison of pharmacological interventions and psychological therapy (combination) versus pharmacological intervention and usual care and/or attention control (single), we downgraded by two points the certainty of evidence for mean depression scores at end of treatment due to substantial heterogeneity (50\% to $89 \%$ ), and by two points for mean activities of daily living scores at end of treatment for considerable heterogeneity ( $90 \%$ to $100 \%$ ) observed (Summary of findings 4).

For the comparison of non-invasive brain stimulation and pharmacological intervention (combination) versus pharmacological intervention with sham or usual care (single), we downgraded by one point the certainty of evidence for mean depression scores and mean neurological function scores at end of treatment due to substantial heterogeneity (50\% to $89 \%$ ) observed. We also downgraded by two points the certainty of evidence for mean activities of daily living scores at end of treatment as considerable heterogeneity (90\% to $100 \%)$ was observed (Summary of findings 5).

\section{Indirectness of evidence}

All included trials addressed the main review questions (PICO). Thus, we did not downgrade any outcomes for indirectness of evidence (Summary of findings for the main comparison; Summary of findings 2; Summary of findings 3; Summary of findings 4; Summary of findings 5 ).

\section{Imprecision}

For the comparison of pharmacological interventions versus placebo, we downgraded the certainty of evidence by one point for the following outcomes: meeting study criteria for depression and less than $50 \%$ reduction in depression scale scores at end of treatment, as the confidence intervals were wide. We also downgraded by two points the certainty of evidence for mean neurological scores and adverse events - death, CNS events, and gastrointestinal events at end of treatment, as the confidence intervals were very wide (Summary of findings for the main comparison).

For the comparison of non-invasive brain stimulation versus sham, we downgraded the certainty of evidence by two points for the following outcomes: mean depression scores at end of treatment 
and mean depression scores at end of follow-up, as the confidence intervals were very wide. We also downgraded by one point the certainty of evidence for mean neurological function scores at end of treatment, as the confidence intervals were wide (Summary of findings 2).

For the comparison of psychological therapy versus usual care and/ or attention control, we downgraded the certainty of evidence by one point for meeting criteria for depression at end of treatment, as the confidence intervals were wide. We also downgraded by two points the certainty of evidence for the following outcomes: average change in depression scores between baseline and end of treatment, meeting study criteria for depression at end of followup, and adverse events - death at end of treatment and leaving the study early - as the confidence intervals were very wide (Summary of findings 3 ).

For the comparison of pharmacological interventions and psychological therapy (combination) versus pharmacological intervention and usual care and/or attention control (single), we downgraded the certainty of evidence by two points for mean depression scores and activities of daily living scores at end of treatment, as the confidence intervals were very wide (Summary of findings 4).
For the comparison of non-invasive brain stimulation and pharmacological intervention (combination) versus pharmacological intervention with sham or usual care (single), we downgraded the certainty of evidence by two points for the following outcomes: mean depression scores, mean activities of daily living scores, and mean neurological function scores at end of treatment and leaving the study early, as the confidence intervals were very wide (Summary of findings 5).

\section{Publication bias}

We assessed publication bias using funnel plots for the outcome meeting study criteria for depression at end of treatment for pharmaceutical interventions versus placebo; Figure 4 shows no evidence of publication bias for this outcome. We did not assess publication bias using funnel plots for the other outcomes in each comparison due to the small number of studies $(<10$ studies) contributing to the analysis. Therefore, we did not downgrade the certainty of evidence for publication bias for any outcomes per comparison (Summary of findings for the main comparison; Summary of findings 2; Summary of findings 3; Summary of findings 4; Summary of findings 5). 
Figure 4. Funnel plot of comparison: 1 Pharmacological interventions versus placebo, outcome: 1.1 Depression: meeting study criteria for depression at end of treatment.

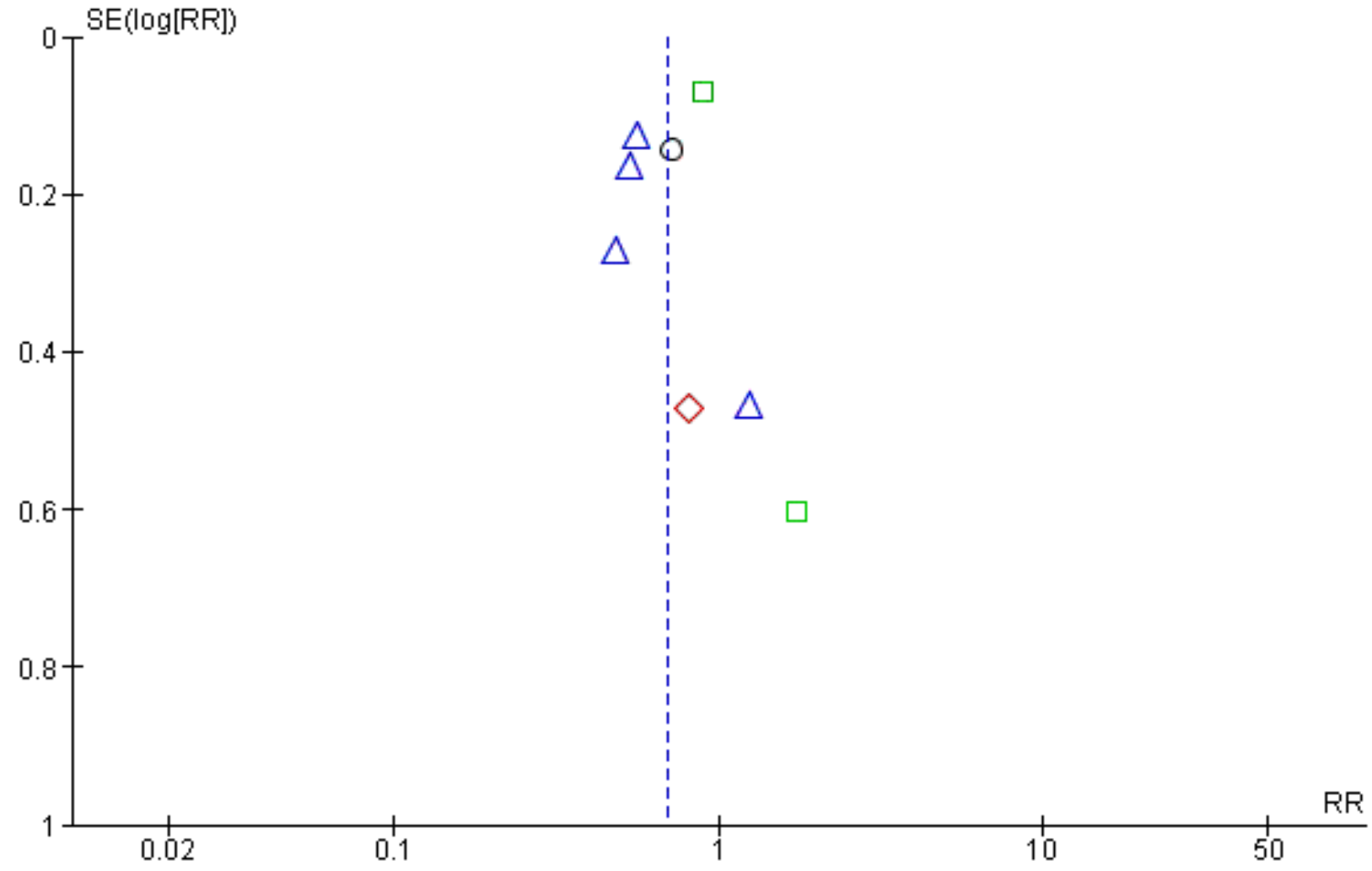

Subgroups

Clinician interview/impression (number not improved)

DSM-III

MADRS

HDRS

\section{Potential biases in the review process}

\section{Strengths and weaknesses of this review}

This review has rigorously adhered to Cochrane methods for performing systematic reviews. During the review process, we tried to avoid and minimise any biases. We undertook extensive searches of databases and additional resources. We did not apply any language restrictions during the search process. Thus, we believe that we have identified and included in this review all potentially relevant trials. We arranged for any relevant and non-relevant non-English full-text trials to be translated into English, to finalise the eligibility process. Furthermore, at least two review authors independently extracted and managed the data.

The main weaknesses of this review are the heterogeneous nature of the outcome measures and the frequent use of multiple scales between and within trials. Inadequate reporting of some trials has led us to rate some of these trials across categories as having unclear risk of bias, with an overall rating of 'very low' certainty of evidence.

\section{Agreements and disagreements with other studies or reviews}

To date, no other systematic reviews have been as comprehensive as this current review.

We found one other systematic review comparing effects of pharmacotherapy versus placebo in the stroke population (Chen 2006). Although this review appears similar, there are important differences in the inclusion criteria. We included trials of people with depression on recruitment and excluded trials with participants who were not depressed at recruitment (included in Hackett 2008a; update pending). Other reviews included trials of people with and without diagnosed depression at recruitment. This limits our ability to directly compare results. One network metaanalysis comparing pharmacotherapy to placebo in people with a diagnosis of major depressive disorder (but not stroke) also found low-quality pooled evidence of benefit of pharmacotherapy in treating depression to remission (Cipriani 2018). Many trials in that review also provided inadequate information about randomisation and allocation concealment, which restricts interpretation of their results. This indicates that limitations in study design in pharmacotherapy trials are not limited to stroke. 
One systematic review compared effects of rTMS with sham rTMS and a combination of rTMS and pharmacotherapy versus usual care or sham rTMS and pharmacotherapy in treating depression after stroke (Shen 2017). Those review authors included 22 trials (24 comparisons), of which 13 trials ( 15 comparisons) are also included in our review (Chen 2005a; Fan 2014; Jiang 2014a; Jiang 2014b; Jin 2013; Li 2013; Li 2014; Liu 2015; Lu 2016; Meng 2015; Yang 2013; Yang 2014a; Yang 2014b; Zhang 2013; Zheng 2016), and two trials (three comparisons) are awaiting classification (Liu 2010; Yan 2010a/Yan 2010b). Seven of the trials included in Shen 2017 did not meet our review criteria for the type of intervention. These trials compared rTMS and pharmacotherapy versus pharmacotherapy alone (with no sham rTMS or usual care). We did not include any additional trials in our review. This review also found lowquality pooled evidence that rTMS and a combination of rTMS and pharmacotherapy reduced depressive symptom scores at end of treatment and after follow-up. However, these findings must also be considered in light of the same limitations in study design and heterogeneity. Another systematic review compared effects of noninvasive brain stimulation (which includes rTMS and transcranial direct current stimulation (tDCS)) versus sham stimulation or usual care (Bucur 2018). Review authors included seven studies (case studies and randomised controlled trials (RCTs)), of which one trial is also included in our review (Gu 2016), and two trials are considered 'dropouts', as outcome data were not reported grouped by depressed/non-depressed participants at baseline (Jorge 2004; Valiengo 2017). Review authors did not perform a meta-analysis and only narratively described the included studies.

One systematic review reported on effects of cognitive-behavioural therapy (CBT) in treating depression after stroke. These review authors included 23 trials, two of which are included in our review (Gao 2017b; Lincoln 2003), and one is considered a 'dropout' as the outcome data (reported median and interquartile ratio (IQR)) were not suitable for pooling (Kootker 2012). The 20 trials that are not included in our review were conducted and published in China, and none were identified by our search strategy, nor were they accessible during this update. We will endeavour to locate, translate, and assess these 20 trials in time for the next update of this review.

Identification of ongoing studies and those awaiting classification indicates that this is an area of stroke research for which further evidence will evolve over the short and longer term.

\section{AUTHORS' CONCLUSIONS}

\section{Implications for practice}

Evidence from trials in people with stroke tentatively supports the use of prescription antidepressants or psychological therapy to treat depression, but this must be considered in light of evidence of an associated increase in harm. Antidepressants may produce a remission or a response in terms of lower scores on mood rating scales but may also increase adverse events. Psychological therapy does not appear to have the same associated risks. Any use of pharmacological agents in people with persistent depressive disorder after stroke would require caution, as little is known about the risks, especially of seizures, falls, delirium, and interaction with other medications.

\section{Implications for research}

We recommend that further research is needed in this area. Future trials investigating effects of pharmacological, psychological, and non-invasive brain stimulation interventions, alone and in combination, for treatment of depression in people after stroke should:

- review and refine the methods for trials of psychological endpoints in people with physical illness;

- recruit an adequate number of participants, so that variables such as time passed between stroke and recruitment, inclusion of patients with dysphasia, and subarachnoid haemorrhage (SAH) can be controlled, and modest but clinically important effects can be detected;

- recruit a representative 'real-world' sample of patients to enable results to be generalised to most stroke survivors;

- provide treatment for sufficient duration and follow-up, so that rates of relapse or maintenance of remission can be assessed;

- carefully specify and monitor psychological interventions;

- describe interventions in sufficient detail to allow their replication;

- include careful, prospective assessment and complete reporting of adverse events;

- define a priori an unambiguous, measurable primary endpoint; and

- limit the number of secondary outcomes to three or four and report results for all outcomes.

\section{ACKN OWLEDGEMENTS}

The original review was supported by a grant from the Stroke Society of Australasia in 2003, with additional financial assistance provided by the Academic Unit of Psychiatry, The University of Leeds, and the Department of Clinical Neurosciences, University of Edinburgh. We thank the Cochrane Stroke Group, particularly Brenda Thomas and Josh Cheyne, for searching the Cochrane Stroke Registers and for assisting in development and updating of search strategies. We also thank Hazel Fraser for providing assistance throughout the review process. We thank Professor Stefano Ricci for translating a study in Italian, Mansur Kutlubaev for translating studies in Russian, Simon Ladwig and Matthias Volz for translating and extracting data from a German study, and Zien Zhou and Jessica Gong for translating letters for personal communication on studies in Chinese. We also thank Dee Shneiderman, a consumer who reviewed this update. Special acknowledgement and thanks go to Dr. Judith Redfern, who led the screening, reviewing, and extracting of data for parts of this update but had to stop when she received a diagnosis of terminal cancer. Jude died in February 2018. 


\section{REFERE N CES}

\section{References to studies included in this review}

Alexopoulos 2012 \{published data only\}

* Alexopoulos GS, Wilkins VM, Marino P, Kanellopoulos D, Reding M, Sirey JA, et al. Ecosystem focused therapy in poststroke depression: a preliminary study. International Journal of Geriatric Psychiatry 2012;27:1053-60.

Kiosses DN, Alexopoulos GS, Wilkins V. Ecosystem focused therapy for treating older depressed stroke survivors. http:// www.strokecenter.org/trials/clinicalstudies/ecosystemfocused-therapy-for-treating-older-depressed-stroke-survivors (first received 22 July 2009).

NCT00944762. Ecosystem focused therapy for treating older depressed stroke survivors. http://ClinicalTrials.gov/show/ NCT00944762 (first received 23 July 2009).

\section{Andersen 1994 \{published data only\}}

Andersen G, Vestergaard K, Lauritzen L. Effective treatment of post-stroke depression with the selective serotonin reuptake inhibitor, citalopram. Journal of Neurology 1994;241 Suppl 1:S42.

* Andersen G, Vestergaard K, Lauritzen L. Effective treatment of poststroke depression with the selective serotonin reuptake inhibitor citalopram. Stroke 1994;25(6):1099-104.

Andersen G, Vestergaard K, Lauritzen L. Post-stroke depression treated with citalopram. Acta Neurologica Scandinavica 1994;89 Suppl 155:20.

Andersen G, Vestergaard K, Lauritzen L. Post-stroke depression treated with citalopram - a selective serotonin reuptake inhibitor. Canadian Journal of Neurological Sciences 1993;20(Suppl 4):S115.

Andersen G, Vestergaard K, Lauritzen L. Post-stroke depression treated with citalopram a selective serotonin reuptake inhibitor. Proceedings of the 7th Scandinavian Meeting on Cerebrovascular Disease. Jyvaskyla, Finland, 14-17 August 1993:54.

Andersen G, Vestergaard K, Lauritzen LU. Effective treatment of depression following apoplexy with citalopram. Ugeskrift for Laeger 1995;157(14):2000-3.

Flicker C, Andersen G, Bayer L. A placebo-controlled study of citalopram treatment for post-stroke depression. Proceedings of the 11th Annual Meeting of the American Association for Geriatric Psychiatry. San Diego, California, USA, 1998.

\section{Cao 2009a \{published data only\}}

Cao WW, Yu JM, Sun SY, Sun YB, Luan L, Cai XJ, et al. Group psychotherapy in treatment of post stroke depression [团体心 理治疗在脑卒中后抑郁治疗中的应用]. Chinese Mental Health Journal 2009;23(2):100-4.

\section{Cao 2009b \{published data only\}}

Cao WW, Yu JM, Sun SY, Sun YB, Luan L, Cai XJ, et al. Group psychotherapy in treatment of post stroke depression [团体心
理治疗在脑卒中后抑郁治疗中的应用]. Chinese Mental Health Journal 2009;23(2):100-4.

\section{Chen 2005a \{published data only\}}

Chen Y-P, Mei Y-W, Sun S-G, Bao M, Yu S-C. Evaluation of frequency repetitive transcranial magnetic stimulation for post-stroke depression and neurologic impairment. Zhongguo Linchuang Kangfu 2005;9:18-9.

\section{Cullen 2018 \{published data only\}}

Cullen B, Pownall J, Cummings J, Baylan S, Broomfield N, Haig C, et al. Positive PsychoTherapy in ABI Rehab (PoPsTAR): a pilot randomised controlled trial. Neuropsychological Rehabilitation 2018;28(1):17-33.

\section{Du 2005 \{published data only\}}

Du DQ, Wu YB. Living ability and cognitive function ameliorated by low frequency repetitive transcranial magnetic stimulation in patients with post-stroke depression: comparison with drug plus psychological treatment. Zhongguo Linchuang Kangfu 2005;9(16):22-3.

\section{Fan 2014 \{published data only\}}

Fan X. Duloxetine combined with repetitive transcranial magnetic stimulation in the treatment of post stroke depression effect. Chinese Journal of Practical Nervous Disease 2014;1:102-3.

\section{Fang 2017 \{published data only\}}

Fang Y, Mpofu E, Athanasou J. Reducing depressive or anxiety symptoms in post-stroke patients: pilot trial of a constructive integrative psychosocial intervention. International Journal of Health Sciences 2017;11(4):53-8.

Fruehwald 2003 \{published data only\}

Fruehwald S, Gatterbauer E, Rehak P, Baumhackl U. Early fluoxetine treatment of post-stroke depression: a three months double-blind placebo-controlled study with an open-label longterm follow up. Journal of Neurology 2003;250(3):347-51.

\section{Gao 2017a \{published data only\}}

Gao J, Lin M, Zhao J, Bi S, Ni Z, Shang X. Different interventions for post-ischaemic stroke depression in different time periods: a single-blind randomized controlled trial with stratification by time after stroke. Clinical Rehabilitation 2017;31(1):71-81.

\section{Gao 2017b \{published data only\}}

Gao J, Lin M, Zhao J, Bi S, Ni Z, Shang X. Different interventions for post-ischaemic stroke depression in different time periods: a single-blind randomized controlled trial with stratification by time after stroke. Clinical Rehabilitation 2017;31(1):71-81.

\section{Gu 2016 \{published data only\}}

Gu SY, Chang MC. The effects of $10-\mathrm{Hz}$ repetitive transcranial magnetic stimulation on depression in chronic stroke patients. Brain Stimulation 2016;10:270-4. 
Hoffmann 2015 \{published and unpublished data\}

ACTRN12609000741280. Evaluation of brief interventions for enhancing early emotional adjustment following stroke: a pilot randomised controlled trial. https://www.anzctr.org.au/Trial/ Registration/TrialReview.aspx?id=308355\&isClinicalTrial=False (first received 25 August 2009).

* Hoffmann T, Ownsworth T, Eames S, Shum D. Evaluation of brief interventions for enhancing early emotional adjustment following stroke: a pilot randomised controlled trial. Topics in Stroke Rehabilitation 2015;22(2):117-26.

Ownsworth T, Hoffman T, Stemm B, Evans E, Howlett J, Shum D. Early cognitive appraisals, benefit finding and emotional status after stroke: pre-intervention associations and preliminary intervention findings. Brain Impairment 2011;12:66.

\section{Huang 2002 \{published data only\}}

Huang, XH. The clinical correlation study and the effect of fluoxetine intervention on poststroke depression. Chinese Journal of Clinical Rehabilitation 2002;6(15):2296-7.

\section{Jiang 2001a \{published data only\}}

Jiang B, Lu W, Song X-W, Tan L-M, Hu Z-P. The effect of poststroke depression interventions on the recovery of neurological function. Modern Rehabilitation 2001;5(3):29-30.

\section{Jiang 2001b \{published data only\}}

Jiang B, Lu W, Song X-W, Tan L-M, Hu Z-P. The effect of poststroke depression interventions on the recovery of neurological function. Modern Rehabilitation 2001;5(3):29-30.

\section{Jiang 2014a \{published data only\}}

Jiang X. Effect of transcranial magnetic stimulation combined with sertraline on neurological deficits in patients with acute cerebral infarction. Chinese Journal of Practical Nervous Disease 17;21:81-3.

\section{Jiang 2014b \{published data only\}}

Jiang X. Effect of transcranial magnetic stimulation combined with sertraline on neurological deficits in patients with acute cerebral infarction. Chinese Journal of Practical Nervous Disease 2014;17(21):81-3.

\section{Jin 2013 \{published data only\}}

Jin H. Effect of repetitive transcranial magnetic stimulation on post stroke depression. Chinese Journal of Rehabilitation Medicine 2013;28(1):58-60.

\section{Kerr 2018 \{published data only\}}

Kerr D, Mackey E, Wijeratne T, McCann T. Nursing early motivational interviewing on poststroke depressive symptoms: pilot randomized controlled trial of the good mood intervention program. International Journal of Stroke 2014;19:245.

* Kerr D, McCann T, Mackey E, Wijeratne T. Effects of early motivational interviewing on post-stroke depressive symptoms: pilot randomised study of the Good Mood Intervention program. International Journal of Nursing Practice 2018;24:1-8.

\section{Kirkness 2017a \{published data only\}}

Kirkness CJ, Cain KC, Becker KJ, Tirschwell DL, Buzaitis AM, Weisman PL, et al. Randomized trial of telephone versus inperson delivery of a brief psychosocial intervention in poststroke depression. BMC Research Notes 2017;10:500.

Kirkness 2017b \{published data only\}

Kirkness CJ, Cain KC, Becker KJ, Tirschwell DL, Buzaitis AM, Weisman PL, et al. Randomized trial of telephone versus inperson delivery of a brief psychosocial intervention in poststroke depression. BMC Research Notes 2017;10:500.

\section{Kong 2007 \{published data only\}}

Kong Y, Dong WL, Liu CF. Fluoxetine for poststroke depression: a randomized placebo controlled clinical trial. Neural Regeneration Research 2007;2:162-5.

Lai 2006a \{published data only\}

Lai J, Zeng G. The effect of using paroxetine to treat post stroke depression. Journal of Guangdong Medical College 2006;24(6):585-6.

\section{Li 2008 \{published data only\}}

Li L, Wang S, Ge H, Chen J, Yue S, Yu M. The beneficial effects of the herbal medicine Free and Easy Wanderer Plus (FEWP) and fluoxetine on post-stroke depression. Journal of Alternative \& Complementary Medicine 2008;14(7):841-6.

\section{Li 2013 \{published data only\}}

Li N, Han Y. A study of efficacy mirtazapine merge rTMS to treatment of post-stroke depression. Chinese Journal of Trauma and Disability Medicine 2013;21(6):48-50.

\section{Li 2014 \{published data only\}}

Li L. Fluoxetine capsules combined with repetitive transcranial magnetic stimulation on depression after stroke treatment. Chinese Journal of Practical Nervous Disease 2014;17(20):105-6.

\section{Lincoln 2003 \{published data only\}}

Flannaghan T. Cognitive behavioural psychotherapy for the treatment of depression after stroke. Unpublished. University of Nottingham, Nottingham, 2000.

Lincoln N. Pilot evaluation of cognitive behavioural treatment of depression after stroke. National Research Register 1996.

* Lincoln NB, Flannaghan T. Cognitive behavioral psychotherapy for depression following stroke: a randomized controlled trial. Stroke 2003;34:111-5.

Thomas SA, Lincoln NB. Factors relating to depression after stroke. British Journal of Clinical Psychology 2006;45:49-61.

\section{Lipsey 1984 \{published data only\}}

Kimura M, Robinson RG, Kosier JT. Treatment of cognitive impairment after poststroke depression: a double-blind treatment trial. Stroke 2000;31:1482-6.

Kimura M, Tateno A, Robinson RG. Treatment of poststroke generalized anxiety disorder comorbid with poststroke depression: merged analysis of nortriptyline trials. American Journal of Geriatric Psychiatry 2003;11(3):320-7. 
Lipsey JR, Robinson RG. Nortriptyline for post-stroke depression. Lancet 1984;1(8380):803.

* Lipsey JR, Robinson RG, Pearlson GD, Rao K, Price TR. Nortriptyline treatment of post-stroke depression: a doubleblind study. Lancet 1984;1(8372):297-300.

Liu 2015 \{published data only\}

Liu X. The effect of repetitive transcranial magnetic stimulation on the treatment of post-stroke depression. Chinese Journal of Gerontology 2015;1:5621-2.

\section{Lu 2016 \{published data only\}}

Lu Q, Zhang H-P, Yang S-P, Zhu Z-F. Study of low frequency repetitive transcranial magnetic stimulation in the treatment of post-stroke depression. Hainan Medical Journal 2016;27(12):1963-4.

\section{Meng 2015 \{published data only\}}

Meng Y. Clinical study of transcranial magnetic therapy in the treatment of depression after cerebral infarction. Chinese Journal of Trauma and Disability Medicine 2015;23(5):141-2.

\section{Mitchell 2002 \{published data only\}}

Barer D. A brief psychosocial-behavioral intervention reduced depression after stroke more than usual care: commentary. Annals of Internal Medicine 2010; Vol. 152:JC3-10.

Becker KJ, Buzaitis A, Cain KC, Fruin M, Kohen R, Teri L, et al. Brief psychosocial/behavioral intervention with antidepressant reduces post-stroke depression significantly more than antidepressant alone. Stroke. 2008; Vol. 39:543.

Kohen R, Cain KC, Buzaitis A, Johnson V, Becker KJ, Teri L, et al. Response to psychosocial treatment in poststroke depression is associated with serotonin transporter polymorphisms. Stroke 2011;42:2068-70.

Mitchell PH, Becker KJ, Buzaitis A, Cain KC, Johnson V, Kohen R, et al. Factors associated with treatment response to combined psychosocial and antidepressant treatment of post-stroke depression (PSD). Stroke 2010;41:e235-6.

Mitchell PH, Teri L, Veith R, Buzaitis A, Tirschwell D, Becker K, et al. Living well with stroke: design and methods for a randomized controlled trial of a psychosocial behavioral intervention for poststroke depression. Journal of Stroke and Cerebrovascular Diseases 2008;17(3):109-15.

Mitchell PH, Veith R, Cain KC. Living well with stroke: psychosocial-behavioural intervention in post-stroke depression. Proceedings of the International Stroke Conference. USA, New Orleans, Louisiana: American Stroke Association, 2 February 2005:Abst. CTP39.

* Mitchell PH, Veith RC, Becker KJ, Buzaitis A, Cain KC, Fruin M, et al. Brief psychosocial-behavioral intervention with antidepressant reduces poststroke depression significantly more than usual care with antidepressant: living well with stroke: randomized, controlled trial. Stroke 2009;40:3073-8.
NCT00194454. Psychosocial/behavioral intervention in post stroke depression (PSD). http://ClinicalTrials.gov/show/ NCT00194454 (first received 19 September 2005).

Wong B. Nurse-led interventions decrease depression in stroke survivors. American Journal of Nursing 2009; Vol. 109, issue 11:19.

Murray 2002 \{published data only\}

* Murray V, Von Arbin M, Asberg M, Bartfai A, Berggren A, Landtblom A, et al. Double-blind placebo comparison of sertraline and placebo in stroke patients with depression. Unpublished 2003.

Murray V, Von Arbin M, Bartfai A, Berggren A, Landtblom A Lundmark J, et al. Double-blind comparison of sertraline and placebo in stroke patients with minor depression and less severe major depression. Journal of Clinical Psychiatry 2005;66(6):708-16.

Murray V, Von Arbin M, Varelius R, Olsson JE, Terent A, Samuelsson M, et al. Sertraline in poststroke depression: a controlled study. Stroke 2002;33(1):P292.

Ohtomo 1991 \{published data only\}

Kumar V. Post-stroke depression and treatment strategies including aniracetam. International Journal of Geriatric Psychopharmacology 1999;2:40-6.

* Ohtomo E, Hirai S, Terashi A, Hasegawa K, Tazaki Y, Araki G, et al. Clinical evaluation of aniracetam on psychiatric symptoms related to cerebrovascular disease. Journal of Clinical Experimental Medicine 1991;156:143-87.

Ponzio 2001 \{published data only\}

* An 8-week, double-blind, placebo controlled, parallel group study to assess the efficacy and tolerability of paroxetine in patients suffering from depression following stroke. http:// www.ctr.gsk.co.uk/Summary/Paroxetine/III_PAR_625.pdf issue par 625.

Ponzio F, Marini G, Riva E. The efficacy of paroxetine in some kinds of "critical" patients. European Neuropsychopharmacology 2001;11 Suppl 2:S49-S50 Abstract P.1.29.

\section{Rampello 2005 \{published data only\}}

Rampello L, Alvano A, Chiechio S, Raffaele R, Vecchio I, Malaguarnera M. An evaluation of efficacy and safety of reboxetine in elderly patients affected by "retarded" post-stroke depression: a random, placebo-controlled study. Archives of Gerontology and Geriatrics 2005;40:275-85.

\section{Reding 1986 \{published data only\}}

Reding MJ, Orto LA, Winter SW, Fortuna IM, Di Ponte P, McDowell FH. Antidepressant therapy after stroke: a doubleblind trial. Archives of Neurology 1986;43(8):763-5.

Robinson 2008a \{published data only\}

* Robinson RG, Jorge RE, Clarence-Smith K. Doubleblind randomized treatment of poststroke depression 
using nefiracetam. Journal of Neuropsychiatry and Clinical Neurosciences 2008;20(2):178-84.

Robinson RG, Jorge RE, Clarence-Smith K, Starkstein S. Double-blind treatment of apathy in patients with poststroke depression using nefiracetam. Journal of Neuropsychiatry and Clinical Neurosciences 2009;21:144-51.

Robinson 2008b \{published data only\}

* Robinson RG, Jorge RE, Clarence-Smith K. Doubleblind randomized treatment of poststroke depression using nefiracetam. Journal of Neuropsychiatry and Clinical Neurosciences 2008;20(2):178-84.

Robinson RG, Jorge RE, Clarence-Smith K, Starkstein S. Double-blind treatment of apathy in patients with poststroke depression using nefiracetam. Journal of Neuropsychiatry and Clinical Neurosciences 2009;21:144-51.

\section{Sun 2013 \{published data only\}}

Sun $\mathrm{H}$. The efficacy of repetitive transcranial magnetic stimulation in the treatment of post-stroke depression. Chinese Journal of Integrative Medicine on Cardio-Cerebrovascular Disease 2013;11(3):321-2.

\section{Thomas 2007 \{published data only\}}

N0192165295. CALM: communication and low mood. National Research Register (first received 23 September 2009).

Thomas SA, Lincoln NB, Walker MF, Macniven J, Haworth H. Communication and low mood (CALM) study: a randomised controlled trial evaluating behaviour therapy for low mood in people with aphasia after stroke. International Journal of Stroke 2011;6 Suppl 2:27-8.

* Thomas SA, Walker MF, Macniven JA, Haworth H, Lincoln NB. Communication and low mood (CALM): a randomized controlled trial of behavioural therapy for stroke patients with aphasia. Clinical Rehabilitation 2012;27(5):398-408.

\section{Towle 1989 \{published data only\}}

* Towle D, Lincoln NB, Mayfield LM. Evaluation of social work on depression after stroke. Clinical Rehabilitation 1989;3(2):89-96.

Towle D, Lincoln NB, Mayfield LM. Service provision and functional independence in depressed stroke patients and the effect of social work intervention on these. Journal of Neurology, Neurosurgery and Psychiatry 1989;52(4):519-22.

Towle D, Mayfield L, Lincoln M. Depression after stroke. Clinical Rehabilitation 1988;2:256.

\section{Wang 2004a \{published data only\}}

Wang X, Song J, Mu J. The effect of psychotherapy on depression and cognitive function of patients with cerebral stroke. Chinese Mental Health Journal 2004;18:778-81.

\section{Wang 2005 \{published data only\}}

Chen Y, Guo JJ, Zhan S, Paul NC. Treatment effects of antidepressants in patients with post-stroke depression: a meta-analysis. Annals of Pharmacotherapy 2006;40:2115-22.
* Wang ZM, Wang P, You LL. Study of effects of fluoxetine in patients with post-stroke depression, a random placebocontrolled study. Chinese Journal of Practical Nervous Diseases 2005; Vol. 8:80-1.

\section{Wang 2005a \{published data only\}}

He Y, Wang X. A clinical study of paroxetine joint psychotherapy in treating poststroke depression with anxiety. Chinese Journal of Practical Nervous Diseases 2006;9(1):34-5.

* Wang X, He Y, Xiao CL. A clinical trial of paroxetine and psychotherapy in patients with poststroke depression and anxiety. Chinese Mental Health Journal 2005;19:564-6.

\section{Watkins 2007 \{published and unpublished data\}}

Deans CF, Jack CIA. Evaluation of motivational interviewing early after acute stroke: a randomized controlled trial. Clinical Rehabilitation 2006;20:731-6.

Sutton C, Dickinson H, Leathley M, Hills K, Auton M, Lightbody E, et al. Motivational interviewing: altering outcome after stroke. 12th European Stroke Conference. Valencia, Spain, 2003 May 21-24:103.

* Watkins CL, Auton MF, Deans CF, Dickinson HA, Jack CIA, Lightbody $C E$, et al. Motivational interviewing early after acute stroke: a randomized, controlled trial. Stroke 2007;38:1004-9.

Watkins CL, Wathan JV, Leathley MJ, Auton MF, Deans CF, Dickinson $\mathrm{HA}$, et al. The 12-month effects of early motivational interviewing after acute stroke: a randomized controlled trial. Stroke 2011;42:1956-61.

\section{Wiart 2000 \{published data only\}}

Wiart L, Gassies TD, France B, Petit H, Debelleix D. A doubleblind, placebo controlled trial to study the efficacy and tolerance of fluoxetine in the treatment of early post stroke depression. Proceedings of the 152nd Annual Meeting of the American Psychiatric Association. USA, Washington: American Psychiatric Association, 15-20 May 1999.

* Wiart L, Petit H, Joseph PA, Mazaux JM, Barat M. Fluoxetine in early poststroke depression: a double-blind placebo-controlled study. Stroke 2000;31(8):1829-32.

\section{Yang 2002 \{published data only\}}

Yang J, Zhao Y, Bai S. Controlled study on antidepressant treatment of patients with post-stroke depression. Chinese Journal of Psychology 2002;16(12):871-2.

\section{Yang 2013 \{published data only\}}

Yang M. A comparative study of high frequency repetitive transcranial magnetic stimulation in the treatment of post stroke depression. Stroke Nervous Disorders 2013;20(5):303-5.

\section{Yang 2014a \{published data only\}}

Yang L, Liu Y, Liu, L, Qi, X, Shi W, Lu C, et al. The curative effect of different frequency repetitive transcranial magnetic stimulation on patients with depression after stroke. Chinese Journal of Practical Nervous Diseases 2014;17(22):18-20. 
Yang 2014b \{published data only\}

Yang L, Liu Y, Liu, L, Qi, X, Shi W, Lu C, et al. The curative effect of different frequency repetitive transcranial magnetic stimulation on patients with depression after stroke. Chinese Journal of Practical Nervous Diseases 2014;17(22):18-20.

\section{Zhang 2013 \{published data only\}}

Zhang Z, Mu J, Geng G-H, Li Q, Song J-G. Effects of repetitive transcranial magnetic stimulation on depression and cognition in the treatment of post-stroke depression. Chinese Journal of Physical Medical Rehabilitation 2013;35(3):197-200.

Zhao 2004 \{published data only\}

Zhao H-W, Zhou C-X, Su X-L, Xiao X-C, Guo Y. Effect of mental intervention on post-stroke depression and rehabilitation of neurological function. Chinese Journal of Clinical Rehabilitation 2004;8(13):2408-9.

\section{Zheng 2016 \{published data only\}}

Zheng F. Clinical observation of ultra-low frequency transcranial magnetic stimulation in the treatment of post stroke depression. Today Nurse 2016;6:116-7.

\section{References to studies excluded from this review}

\section{Aben 2014 \{published data only\}}

Aben L, Heijenbrok-Kal MH, Ponds RWHM, Busschbach JJV, Ribbers GM. Long-lasting effects of a new memory self-efficacy training for stroke patients: a randomized controlled trial. Neurorehabilitation and Neural Repair 2014;28(3):199-206.

* Aben L, Heijenbrok-Kal MH, van Loon EMP, Groet E, Ponds RWHM, Busschbach JJV, et al. Training memory selfefficacy in the chronic stage after stroke: a randomized controlled trial. Neurorehabilitation and Neural Repair 2013;27(2):110-7.

\section{Agnoli 1985 \{published data only\}}

Agnoli A, Fioravanti M, Lechner H. Efficacy of CDP-Choline in chronic cerebral vascular diseases (CCVD). In: Appia V, Kennedy EP, Nilsson BI, Galletti P editor(s). Novel Biochemical, Pharmacological and Clinical Aspects of Cytidinediphosphocholine. New York: Elsevier, 1985:305-15.

\section{Bai 2017 \{published data only\}}

Bai B, Yan Z, Hao Y, Zhang Z, Li G, Dekker J, et al. A randomised controlled multimodal intervention trial in patients with ischaemic stroke in Shandong, China: design and rationale. Lancet 2017;390(1):13.

\section{Bramanti 1989 \{published data only\}}

Bramanti P, Ricci RM, Di Bella P, De Luca GP, Sessa E, Di Leo M, et al. Neuropsychological and clinical evaluation after administration of TRH-T in cerebrovascular pathology [Valutazioni neuropsicologiche e cliniche dopo somminitrazione di TRH-T neila patologia cerebrovascolare]. Rassegna di Medicina Interna 1989;X(4):157-61.

\section{Casella 1960 \{published data only\}}

Casella C, Sokolow J. A study to determine the energizing effects of iproniazid (marsilid) on a group of hemiplegics. Archives of Physical Medicine and Rehabilitation 1960;41:381-5.

\section{Chang 2011 \{published data only\}}

Chang K, Zhang H, Xia Y, Chen C. Testing the effectiveness of knowledge and behavior therapy in patients of hemiplegic stroke. Topics in Stroke Rehabilitation 2011;18(5):525-35.

\section{Cheng 2016 \{published data only\}}

Cheng HY. The effect of a psychoeducational intervention on stroke family caregivers' outcomes and stroke survivors' utilisation of health and social services. Dissertation Abstracts International: Section B: The Sciences and Engineering 2016; Vol. 76.

\section{Choi-Kwon 2006 \{published data only\}}

Choi-Kwon S, Choi J, Kwon SU, Kang D, Kim JS. Fluoxetine is not effective in the treatment of poststroke fatigue: a doubleblind, placebo controlled study. Cerebrovascular Diseases 2007;23:103-8

Choi-Kwon S, Choi J, Kwon SU, Kang DW, Kim JS. Fluoxetine improves the quality of life in patients with post-stroke emotional disturbances. Cerebrovascular Diseases 2008;26:266-71.

* Choi-Kwon S, Han SW, Kwon SU, Kang D, Kim CS, Kim JS. Fluoxetine treatment in poststroke depression, emotional incontinence, and anger proneness: a double-blind, placebocontrolled study. Stroke 2006;37:156-61.

Choi-Kwon S, Kwon SU, Kang DW, Kim JS. Fluoxetine improves the quality of life in patients with post-stroke emotional disturbances. Stroke 2009;40:e282.

Kim JS. Post-stroke emotional disturbances. Journal of Stroke 2005;18(3):244

\section{Chollet 2011 \{published data only\}}

Chollet F, Tardy J, Albucher JF, Thalamas C, Berard E, Lamy C, et al. Fluoxetine for motor recovery after acute ischaemic stroke (FLAME): a randomised placebo-controlled trial. Lancet Neurology 2011;10:123-30.

\section{Clark 2003 \{published data only\}}

Clark MS, Rubenach S, Winsor A. A randomized controlled trial of an education and counselling intervention for families after stroke. Clinical Rehabilitation 2003;17(7):703-12.

\section{Delbari 2011 \{published data only\}}

Delbari A, Salman-Roghani R, Lokk J. Effect of methylphenidate and/or levodopa combined with physiotherapy on mood and cognition after stroke: a randomized, double-blind, placebocontrolled trial. European Neurology 2011;66:7-13.

\section{Downes 1995 \{published data only\}}

Downes B, Rooney V, Oyebode JR, Roper-Hall A, Mayer P, Main A. The effect of giving information and counselling on depression and anxiety in stroke survivors and carers (The Birmingham Stroke Counselling Project). Unpublished 1995. 


\section{Evans 1997 \{published data only\}}

Evans M, Hammond M, Wilson K, Lye M, Copeland J. Treatment of depression in the elderly: effect of physical illness on response. International Journal of Geriatric Psychiatry 1997;12:1189-94.

\section{Finkenzeller 2006 \{published data only\}}

Finkenzeller W, Zobel I, Rietz S, Schramm E, Berger M. Interpersonal psychotherapy and pharmacotherapy for poststroke depression. Feasibility and effectiveness. Der Nervenarzt 2009;80:805-12.

\section{Hadidi 2014 \{published data only\}}

Hadidi N, Buckwalter K, Lindquist R, Rangen C. Feasibility of a pilot study of problem-solving therapy for stroke survivors. Rehabilitation Nursing 2014;5:327-337.

\section{Hu 2003 \{published data only\}}

Hu Z, Hu Y, Lu Q. Impact of early rehabilitation therapy on post stroke depression. Chinese Journal of Clinical Rehabilitation 2003;3(15):1.

\section{ISRCTN88489864 \{published data only\}}

ISRCTN88489864. Influence of a single dose of fluoxetine on muscle activation patterns and functional ability in chronic stroke patients. http://www.isrctn.com/ISRCTN88489864 (first received 23 July 2005).

\section{Jiang 2004 \{published data only\}}

Jiang JB, Li GC. Effects of post-stroke depression and antidepression therapy on rehabilitation of neurological function in patients with meta method. Chinese Journal of Clinical Rehabilitation 2004;8(31):6829-31.

\section{Jorge 2004 \{published data only\}}

Jorge RE, Robinson RG, Tateno A, Narushima K, Acion L, Moser D, et al. Repetitive transcranial magnetic stimulation as treatment of poststroke depression: a preliminary study. Biological Psychiatry 2004;55:398-405.

\section{Jorge 2008 \{published data only\}}

Jorge RE, Moser DJ, Acion M, Robinson RG. Treatment of vascular depression using repetitive transcranial magnetic stimulation. Archives of General Psychiatry 2008;65(3):268-76.

\section{Kim 2010a \{published data only\}}

Kim BR, Kim D, Chun MH, Yi JH, Kwon JS. Effect of repetitive transcranial magnetic stimulation on cognition and mood in stroke patients: a double-blind, sham-controlled trial. American Journal of Physical Medicine and Rehabilitation 2010;89(5):362-8.

\section{Kim 2010b \{published data only\}}

Kim BR, Kim D, Chun MH, Yi JH, Kwon JS. Effect of repetitive transcranial magnetic stimulation on cognition and mood in stroke patients: a double-blind, sham-controlled trial. American Journal of Physical Medicine and Rehabilitation 2010;89(5):362-8.

\section{Kim 2017 \{published data only\}}

Kim JS, Lee E-J, Chang D, Park J-H, Ahn SH, Cha J-K, et al. Efficacy of early administration of escitalopram on depressive and emotional symptoms and neurological dysfunction after stroke: a multicentre, double-blind, randomised, placebocontrolled study. Lancet Psychiatry 2017;4(1):33-43.

Kim 2017a \{published data only\}

Kim K-U, Kim S-H, An T-G. The effects of repetitive transcranial magnetic stimulation (rTMS) on depression, visual perception, and activities of daily living in stroke patients. Journal of Physical Therapy Science 2017;29:1036-9.

\section{Kootker 2012 \{published data only\}}

Kootker JA, Fasotti L, Rasquin SMC, van Heugten CM, Geurts $\mathrm{ACH}$. The effectiveness of an augmented cognitive behavioural intervention for post-stroke depression with or without anxiety (PSDA): the Restore4Stroke-PSDA trial. BMC Neurology 2012;12(51):1-8.

* Kootker JA, Rasquin SMC, Lem FC, van Heughten CM, Fasotti L, Geurts ACH. Augmented cognitive behavioral therapy for poststroke depressive symptoms: a randomized controlled trial. Archives of Physical Medicine and Rehabilitation 2017;98:687-94.

Kootker JA, Rasquin SMC, Smits P, Geurts AC, van Heugten CM, Fasotti L. An augmented cognitive behavioural therapy for treating post-stroke depression: description of a treatment protocol. Clinical Rehabilitation 2015;29(9):833-43.

\section{Laska 2005 \{published data only\}}

Laska AC, van Arbin M, Kahan T, Hellblom A, Murray V. Longterm antidepressant treatment with moclobemide for aphasia in acute stroke patients: a randomised, double-blind, placebocontrolled study. Cerebrovascular Diseases 2005;19(2):125-32.

\section{Leijon 1989 \{published data only\}}

Leijon G, Boivie J. Central post-stroke pain: a controlled trial of amitriptyline and carbamazepine. Pain 1989;36(1):27-36.

\section{Lobjanidze 2010 \{published data only\}}

Lobjanidze N, Dzagnidze A, Jeiranashvili A, Kukava M, Beridze M, Khachiashvili $\mathrm{M}$, et al. Long-term effects of the use of citicoline (ceraxone) in the post-stroke cognitive-mood impairment. Cerebrovascular Diseases 2010; Vol. 5, issue 1:14.

Mauri 1988 \{published data only\}

Marui L, Arboix A, Marti-Vilalta JL. Efficacy of antidepressive treatment in affective disorders associated to ischemic vascular disease. Neurologia 1988;3 Suppl 3:10.

\section{Meara 1998 \{published data only\}}

Meara JR. A randomised double blind placebo controlled study of the treatment of post stroke depression. National Research Register.

* Meara RJ, Thalanany M, Balonwu V, Hobson P. The treatment of depression after stroke with the selective serotonin reuptake inhibitor sertraline. Cerebrovascular Diseases 1998;8 Suppl 4:90. 
Narushima 2007 \{published data only\}

Narushima K, Paradiso S, Moser D, Jorge R, Robinson R. Effect of antidepressant therapy on executive function after stroke. British Journal of Psychiatry 2007;190:260-5.

\section{Ohtomo 1985 \{published data only\}}

Ohtomo E, Kutsuzawa T, Araki G, Hirai S, Terashi A, Kuzuya F, et al. Clinical usefulness of tiapride on psychiatric symptoms caused by cerebrovascular disorders. Clinical Evaluation 1985;13:295-332.

\section{Ostwald 2014 \{published data only\}}

NCT00178529. Intervention for stroke survivors and their spousal caregivers. https://clinicaltrials.gov/ct2/show/ NCT00178529 (first received 19 July 2001).

* Ostwald S, Godwin K, Cron S, Kelley C, Hersch G, Davis S. Home-based psychoeducational and mailed information programs for stroke-caregiving dyads post-discharge: a randomized trial. Disability and Rehabilitation 2014;36(1):55-62.

Ostwald SK, Davis S, Hersch G, Kelley C, Godwin KM. Evidence based educational guidelines for stroke survivors after discharge home. Journal of Neuroscience Nursing 2008;40(3):173-91.

Ostwald SK, Wasserman J, Davis S. Medications, comorbidities, and medical complications in stroke survivors: the CAReS study. Rehabilitation Nursing 2006;31(1):10-4.

\section{Otomo 1986 \{published data only\}}

Otomo E, Tohgi H, Hirai S, Gotoh F, Hasegawa K, Tazaki Y, et al. Clinical evaluation of YM-08054 (indeloxazine) in the treatment of cerebrovascular disorder. Igaku no Ayumi 1986;136(7):535-55.

\section{Raffaele 1996 \{published data only\}}

Raffaele R, Rampello L, Vecchio I, Tornali C, Malaguanera M. Trazodone therapy of the post-stroke depression. Archives of Gerontology and Geriatrics 1996;22 Suppl 1:217-20.

\section{Rich 2016 \{published data only\}}

Rich TL, Menk J, Krach LE, Feyma T, Gillick BT. Repetitive transcranial magnetic stimulation/behavioural intervention clinical trial: long-term follow-up of outcomes in congenital hemiparesis. Journal of Child and Adolescent Psychopharmacology 2016;26(7):598-605.

\section{Robinson 2000 \{published data only\}}

Robinson RG, Schultz SK, Castillo C, Kopel T, Kosier T, Newman M, et al. Nortriptyline versus fluoxetine in the treatment of depression and in-short-term recovery after stroke: a placebo-controlled, double-blind study. American Journal of Psychiatry 2000;157(3):351-9.

\section{Robinson 2017 \{published data only\}}

Robinson RG, Jorge RE, Long J. Prevention of poststroke mortality using problem-solving therapy or escitalopram. American Journal of Geriatric Psychiatry 2017;25(5):512-9.
Rudberg 2017 \{published data only\}

Rudberg AS, Isaksson E, Lundstrom E. Efficacy of fluoxetine: a randomised controlled trial in stroke, the effects study in Sweden. European Stroke Journal 2017;2(1 Suppl 1):320-1.

\section{Sieger 2018 \{published data only\}}

Sieger FS, Poveda MYP, Moreno AP, Sanchez JAM, Romero LAL, Guzman LCR, et al. Effects of repetitive transcranial magnetic stimulation in aphasic stroke: a randomised double-blind controlled clinical trial. Neurology 2018;90(15 Suppl 1):P5.004.

\section{Sivenius 2001 \{published data only\}}

Sivenius J, Sarasoja T, Aaltonen H, Heinonen E, Kilkku O, Reinikainen $\mathrm{K}$. Selegiline treatment facilitates recovery after stroke. Journal of Neurologic Rehabilitation 2001;15(3):183-90.

\section{Su 2004a \{published data only\}}

Su XL, Xiao XC. Effect of psychotherapy on the motor functional rehabilitation in patients with post-stroke depression. Chinese Journal of Clinical Rehabilitation 2004;8(19):3720-1.

\section{Sun 2000 \{published data only\}}

Sun B, Chan JL. Effects of psychological rehabilitation on the recovery of hemiplegia patients after stroke. Modern Rehabilitation 2000;4(1):36-7.

\section{Szepfalusi 2017 \{published data only\}}

Szepfalusi N, Nemeth VL, Vekony T, Imre N, Balogh R, Holczer $A$, et al. Ten-day cognitive training program combined with transcranial direct current stimulation (tDCS) in stroke rehabilitation - a sham controlled study. European Neuropsychopharmacology 2017;27 Suppl 4:S1038-9.

* Szepfalusi N, Nemeth VL, Vekony T, Imre N, Balogh R, Holczer A, et al. The use of transcranial direct current stimulation (tDCS) combined with cognitive training in stroke rehabilitation - a sham-controlled pilot study. Cerebrovascular Diseases 2017;43 Suppl 1:61.

\section{Valiengo 2017 \{published data only\}}

NCT01525524. Treatment of major depressive disorder post stroke with transcranial direct current stimulation. https:// clinicaltrials.gov/ct2/show/NCT01525524 (first received 3 February 2012).

* Valiengo LCL, Goulart AC, de Oliveira JF, Benseñor IM, Lotufo PA, Brunoni AR. Transcranial direct current stimulation for the treatment of post-stroke depression: results from a randomised, sham-controlled, double-blinded trial. Journal of Neurology, Neurosurgery and Psychiatry 2017;88:170-5.

\section{Visser 2015 \{published data only\}}

Visser MM, Heijenbrok-Kal MH, van't Spijker A, Busschbach JJV, Ribbers GM. Poster 44: problem solving therapy during outpatient rehabilitation for stroke: short-term results of a randomized controlled trial. Stroke 2014; Vol. Suppl 1:28.

* Visser MM, Heijenbrok-Kal MH, van't Spijker A, Busschbach JJV, Ribbers GM. Problem solving therapy during outpatient stroke rehabilitation improves coping and HRQoL: a randomized controlled trial. Stroke 2015;1:1-28. 
Visser MM, Heijenbrok-Kal MH, van't Spijker A, Ribbers GM, Busschbach JJV. The effectiveness of problem solving therapy for stroke patients: study protocol for a pragmatic randomised controlled trial. BMC Neurology 2013;13(67):1-7.

Walker-Batson 1995 \{published data only\}

Walker-Batson D, Smith P, Curtis S, Unwin H, Greenlee R. Amphetamine paired with physical therapy accelerates motor recovery after stroke: further evidence. Stroke 1995;26(12):2254-9.

\section{Wang 2009 \{published data only\}}

Wang P. Influence of magnetic stimulation to treat post stroke depression on recovery of neurological function. Chinese Nursing Research 2009;23(5):1356-7.

\section{References to studies awaiting assessment}

Chen 2002a \{published data only\}

Chen W, Wang GF, Chen XH, Sheng YL, Zhu H. Effects of paroxetine on function recovery in patients with poststroke depression. Chinese Journal of Clinical Rehabilitation 2002;6(13):2014-5.

\section{Chen 2002b \{published data only\}}

Chen W, Wang GF, Chen XH, Sheng YL, Zhu H. Effects of paroxetine on function recovery in patients with poststroke depression. Chinese Journal of Clinical Rehabilitation 2002;6(13):2014-5

\section{Ding 2005 \{published data only\}}

Ding D, Zhang Z. Combination of paroxetine and psychotherapy in the treatment of post-stroke depression. Shanghai Archives of Psychiatry 2005;17(2):89-91.

\section{Evans 1985 \{published data only\}}

Evans RL, Kleinman L, Halar EM, Herzer K. Predicting outcome of group counselling with severely disabled patients. American Journal of Physical Medicine 1985;64(1):24-31.

Finkenzeller 2009 \{published data only\}

Finkenzeller W, Zobel I, Rietz S, Schramm E, Berger M. Interpersonal psychotherapy and pharmacotherapy in poststroke depression [Interpersonelle Psychotherapie und Pharmakotherapie bei Post-Stroke-Depression]. Nervenarzt 2009;80:805-12.

\section{Hanspal 2007 \{published data only\}}

Hanspal R. The effectiveness of sertraline in clinical management of depression with or without lability in braininjured. National Research Register. [N0388126828]

\section{He 2003 \{published data only\}}

$\mathrm{He}$ C. The effect of psychological intervention combined with amitriptyline on patients with depression after stroke. Chinese Journal of Clinical Rehabilitation 2003;7(5):850.

\section{He 2005 \{published data only\}}

He Y. A clinical study of paroxetine joint psychotherapy in treating poststroke depression with anxiety. Chinese Journal of Practical Nervous Diseases 2006;9(1):34-5.

* He Y. Prospective study of effects of paroxetine with mental intervention on depression and anxiety after stroke. Nervous Disease and Mental Health 2005;5(1):6-8.

Huang 2005 \{published data only\}

Huang C, Shi G, Bai C. A comparative study of venlafaxine plus cognitive therapy in the treatment of post-stroke depression. Chinese Journal of Behavioral Medical Science 2005;14(9):790-1.

Katz 1998 \{published data only\}

Katz RA, Hubbard DJ, Blaine J. The effect of group psychotherapy on post-stroke depression. Rehabilitation Psychology 1998;43(2):178.

Latow 1983 \{published data only\}

Latow J. Psychotherapy and its effect on depression, sickrole identification and rehabilitation outcome for stroke victims. Archives of Physical Medicine and Rehabilitation 1983;64(10):511-2.

\section{Lee 2005 \{published data only\}}

Lee NG, Choi IS, Kim JH, Lee SY, Han JY. The effect of repetitive transcranial magnetic stimulation on the poststroke depression. Proceedings of the Proceedings of 3rd World Congress of the International Society of Physical and Rehabilitation Medicine - ISPRM. Brazil, Sao Paulo, 10-14 April 2005:105-9.

\section{Liu 2010 \{published data only\}}

Liu J, Li X. High- frequency transcranial magnetic stimulation for alleviating post-stroke depression. Chinese Journal of Physical Medical Rehabilitation 2010;32(7):513-5.

Pearson 2005 \{published data only\}

Pearson V. Educational intervention reduces occurrence of depression in community-dwelling stroke survivors. Stroke 2005;36(2):423.

\section{Razazian 2016 \{published data only\}}

Razazian N, Esmaeili O, Almasi A. Effect of fluoxetine on motor improvement in ischemic stroke patients: a double blind clinical trial study. Zahedan Journal of Research in Medical Sciences 2016;18(7):e7549.

\section{Tang 2002 \{published data only\}}

Tang LY, Ye JH. Cognitive therapy on depression and cognitive dysfunction after stroke. Nursing Journal of Chinese People's Liberation Army 2002;19(4):6-7.

\section{Wang 2015 \{published data only\}}

Wang J, Zhang M, Xu L. The clinical observation of post-stroke depression improvement by repetitive transcranial magnetic stimulation. Chinese Journal of Rehabilitation 2015;6(30):167-70. 
Yan 2010a \{published data only\}

Yan TT, He LM, Gu ZT. A randomized, controlled study of highand low-frequency rTMS on the treatment outcome for post stroke depression [高频及低频重复 经颅磁剌激对脑卒中后抑 郁的疗效对比研究]. Qingdao Yiyao Weisheng 2010;42(2):81-5.

Yan 2010b \{published data only\}

Yan TT, He LM, Gu ZT. A randomized, controlled study of highand low-frequency rTMS on the treatment outcome for post stroke depression [高频及低频重复经硕磁刺激对脑卒中后抑 郁的疗效对比研究]. Qingdao Yiyao Weisheng 2010;42(2):81-5.

Yan 2010c \{published data only\}

Yan TT, He LM, Gu ZT. A randomized, controlled study of highand low-frequency rTMS on the treatment outcome for post stroke depression [ [高频及低频重复经乑磁剌激对脑卒中后抑 郁的疗效对比研究] ]. Qingdao Yiyao Weisheng 2010;42(2):81-5.

Yan 2010d \{published data only\}

Yan TT, He LM, Gu ZT. A randomized, controlled study of highand low-frequency rTMS on the treatment outcome for post stroke depression [高频及低频重复 经颅磁剌激对脑卒中后抑 郁的疗效对比研究]. Qingdao Yiyao Weisheng 2010;42(2):81-5.

\section{References to ongoing studies}

\section{Kirkevold 2018 \{published data only\}}

Kirkevold M, Bragstad LK, Bronken BA, Kvigne K, Martinsen R, Hjelle EG, et al. Promoting psychosocial well-being following stroke: study protocol for a randomized, controlled trial. BMC Psychology 2018;6(12):1-12.

\section{NCT03056287 \{published data only\}}

NCT03056287. Exercise and brain stimulation for post-stroke. https://clinicaltrials.gov/ct2/show/NCT03056287 (first received 17 February 2017).

\section{Tang 2017 \{published data only\}}

Tang Y, Chen A, Zhu S, Yang L, Zhou J, Pan S, et al. Repetitive transcranial magnetic stimulation for depression after basal ganglia ischaemic stroke: protocol for a multicentre randomised double-blind placebo-controlled trial. BMJ Open 2017;8:1-7.

\section{Thomas 2016 \{published data only\}}

Thomas SA, Coates E, das Nair R, Lincoln NB, Cooper C, Palmer R, et al. BEhavioural Activation therapy for Depression after Stroke (BEADS): a study protocol for a feasibility randomised controlled pilot trial of a psychological intervention for post-stroke depression. Pilot and Feasibility Studies 2016;2(45):1-12.

\section{Xu 2016 \{published data only\}}

Xu SM, Zou DZ, Shen LY, Zhou XY, Pu JC, Dong MX, et al. Efficacy and feasibility of antidepressant treatment in patients with post-stroke depression. Medicine 2016;95(45):e5349.

\section{Additional references}

\section{Anderson 1995a}

Anderson CS, Linto J, Stewart-Wynne EG. A population-based assessment of the impact and burden of caregiving for longterm stroke survivors. Stroke 1995;26:843-9.

\section{APA 1987}

American Psychiatric Association. Diagnostic and Statistical Manual of Mental Disorders: DSM-III-R. Third Edition. Washington, DC: American Psychiatric Association, 1987.

\section{APA 1994}

American Psychiatric Association. Diagnostic and Statistical Manual of Mental Disorders: DSM-IV. Fourth. Washington, DC: American Psychiatric Association, 1994.

\section{APA 2013}

American Psychiatric Association. Diagnostic and Statistical Manual of Mental Disorders: DSM-V. Fifth. Arlington, VA: American Psychiatric Publishing, 2013.

\section{Astrom 1996}

Astrom M. Generalized anxiety disorder in stroke patients: a 3year longitudinal study. Stroke 1996;27:270-5.

\section{Atkins 2004}

Atkins D, Best D, Briss PA, Eccles M, Falck-Yitter Y, Flottorp G, et al. GRADE Working Group. Grading quality of evidence and strength of recommendations. BMJ 2004;328(7454):1490.

\section{Ayerbe 2013}

Ayerbe L, Ayis S, Wolfe CDA, Rudd AG. Natural history, predictors and outcomes of depression after stroke: systematic review and meta-analysis. British Journal of Psychiatry 2013;202:14-21.

\section{Beck 1961}

Beck AT, Ward C, Mendelson M. An inventory for measuring depression. Archives of General Psychiatry 1961;4:561-71.

\section{Bucur 2018}

Bucur M, Papagno C. A systematic review of noninvasive brain stimulation for post-stroke depression. Journal of Affective Disorders 2018;238:69-78.

\section{Burvill 1995a}

Burvill PW, Johnson GA, Jamrozik KD, Anderson CS, StewartWynne EG, Chakera TM. Prevalence of depression after stroke: the Perth Community Stroke Study. British Journal of Psychiatry 1995;166:320-7.

\section{Burvill 1996}

Burvill PW, Johnson GA, Chakera TMH, Stewart-Wynne EG, Anderson CS, Jamrozik KD. The place of site of lesion in the aetiology of post-stroke depression. Cerebrovascular Diseases 1996;6:208-15.

\section{Burvill 1997}

Burvill P, Johnson G, Jamrozik KD, Anderson C. Risk factors for post-stroke depression. International Journal of Geriatric Psychiatry 1997;12:219-26. 


\section{Carson 2000}

Carson AJ, MacHale S, Allen K, Lawrie SM, Dennis M, House A, et al. Depression after stroke and lesion location: a systematic review. Lancet 2000;356:122-6.

\section{Chan 2004}

Chan A, Hrobjartsson A, Haahr MT, Gotzsche PC, Altman DG. Empirical evidence for selective reporting of outcomes in randomized trials: comparison of protocols to published articles. Journal of the American Medical Association 2004;291:2457-65.

\section{Chen 2006}

Chen Y, Guo JJ, Zhan S, Patel NC. Treatment effects of antidepressants in patients with post-stroke depression: a meta-analysis. Annals of Pharmacotherapy 2006;40(12):211522.

\section{Cipriani 2018}

Cipriani A, Furukawa TA, Salanti G, Chaimani A, Atkinson LZ, Ogawa Y, et al. Comparative efficacy and acceptability of 21 antidepressant drugs for the acute treatment of adults with major depressive disorder: a systematic review and network meta-analysis. Lancet 2018;391:1357-66.

\section{Deeks 2011}

Deeks JJ, Higgins JP, Altman DG, editor(s). Chapter 9. Analysing data and undertaking meta-analyses. In: Higgins JP, Green S, editor(s). Cochrane Handbook for Systematic Reviews of Interventions Version 5.1.0 (updated March 2011). The Cochrane Collaboration, 2011. Available from handbook.cochrane.org.

\section{Deutsch 1997}

Deutsch A, Braun S, Granger CV. The Functional Independence Measure (FIM Instrument). Journal of Rehabilitation Outcomes Measures 1997;1:67-71.

\section{Ebrahim 1987a}

Ebrahim S, Barer D, Nouri F. Affective illness after stroke. British Journal of Psychiatry 1987;151:52-6.

\section{Folstein 1975}

Folstein MF, Folstein SE, McHugh PR. 'Mini-Mental State': a practical method for grading the cognitive state of patients for the clinician. Journal of Psychiatric Research 1975;12:189-98.

\section{Fournier 2010}

Fournier JC, DeRubeis RJ, Hollon SD, Dimidjian S, Amsterdam JD, Shelton RC, et al. Antidepressant drug effect and depression severity: a patient-level meta-analysis. JAMA 2010;303(1):47-53.

\section{Gill 2000}

Gill D, Hatcher S. Antidepressants for depression in medical illness. Cochrane Database of Systematic Reviews 2001, Issue 3. [DOI: 10.1002/14651858.CD001312.pub2]

\section{Goldberg 1972}

Goldberg DP. The detection of psychiatric illness by questionnaire. Maudsley Monograph No 21. Oxford: Oxford University Press, 1972.

\section{Gompertz 1993}

Gompertz P, Pound P, Ebrahim S. The reliability of stroke outcome measurement. Clinical Rehabilitation 1993;7:290-6.

\section{GRADEproGDT 2015 [Computer program]}

Hamilton (ON): McMaster University (developed by Evidence Prime). Available at gradepro.org. GRADEproGDT. Hamilton (ON): McMaster University (developed by Evidence Prime). Available at gradepro.org, 2015.

\section{Hackett 2008a}

Hackett ML, Anderson CS, House A, Halteh C. Interventions for preventing depression after stroke. Cochrane Database of Systematic Reviews 2008, Issue 3. [DOI: 10.1002/14651858.CD003689]

\section{Hackett 2014}

Hackett M, Pickles K. Part I: frequency of depression after stroke: an updated systematic review and meta-analysis of observational studies. World Stroke Organisation 2014;9(December):1017-25.

\section{Hamilton 1960}

Hamilton M. Rating scale for depression. Journal of Neurology, Neurosurgery and Psychiatry 1960;23:56-62.

\section{Herrmann 1998}

Herrmann N, Backe SE, Lawrence J, Szekely C, Szalai JP. The Sunnybrook stroke study: a prospective study of depressive symptoms and functional outcome. Stroke 1998;29:618-24.

\section{Higgins 2011}

Higgins JP, Green S, editor(s). Cochrane Handbook for Systematic Reviews of Interventions Version 5.1.0 (updated March 2011). The Cochrane Collaboration, 2011. Available from http://handbook.cochrane.org.

\section{House 1987}

House A. Mood disorders after stroke: a review of the evidence. International Journal of Geriatric Psychiatry 1987;2:211-21.

\section{House 1989}

House A, Dennis M, Hawton K, Warlow C. Methods of identifying mood disorders in stroke patients: experience in the Oxfordshire community stroke project. Age and Aging 1989;18:371-9.

\section{House 1991}

House A, Dennis M, Mogridge L, Warlow C, Hawton K, Jones L. Mood disorders in the year after first stroke. British Journal of Psychiatry 1991;158:83-92.

\section{House 2001}

House A, Knapp P, Bamford J, Vail A. Mortality at 12 and 24 months after stroke may be associated with depressive symptoms at 1 month. Stroke 2001;32:696-701.

\section{Johnson 1991}

Johnson GA. Research into psychiatric disorder after stroke: the need for further studies. Australian \& New Zealand Journal of Psychiatry 1991;25:358-70. 


\section{Katona 1995}

Katona CLE, Watkin V. Depression in old age. Reviews in Clinical Gerontology 1995;5:427-41.

\section{Keller 2003}

Keller MB. Past, present, and future directions for defining optimal treatment outcome in depression: remission and beyond. Journal of the American Medical Association 2003;289(23):3152-60.

\section{Kirsch 2008}

Kirsch I, Deacon BJ, Huedo-Medina TB, Scoboria A, Moore TJ, Johnson BT. Initial severity and antidepressant benefits: a meta-analysis of data submitted to the Food and Drug Administration. PLoS Medicine 2008;5(2):0260-8.

\section{Lima 2001}

Lima MS, Moncrieff J. Drugs versus placebo for dysthymia. Cochrane Database of Systematic Reviews 2001, Issue 3. [DOI: 10.1002/14651858.CD001130]

\section{Lyden 2001}

Lyden PD, Lu M, Levine SR, Brott TG, Broderick J, the NINDS rtPA Stroke Study Group. A modified National Institutes of Health Stroke Scale for use in stroke clinical trials: preliminary reliability and validity. Stroke 2001;32:1310-7.

\section{Mahoney 1965}

Mahoney FI, Barthel DW. Functional evaluation: the Barthel Index. Maryland State Medical Journal 1965;14:61-5.

\section{McCusker 1998}

McCusker J, Cole M, Keller E, Bellavance F, Berard A. Effectiveness of treatments of depression in older ambulatory patients. Archives of Internal Medicine 1998;158:705-12.

\section{Mead 2012}

Mead GE, Hsieh CF, Lee R, Kutlubaev MA, Claxton A, Hankey GJ, et al. Selective serotonin reuptake inhibitors (SSRIs) for stroke recovery. Cochrane Database of Systematic Reviews 2012, Issue 11. [DOI: 10.1002/14651858.CD009286]

\section{Mittmann 1997}

Mittmann N, Herrmann N, Einarson TR, Busto UE, Lanctot KL, Liu BA, et al. The efficacy, safety and tolerability of antidepressants in late life depression: a meta-analysis. Journal of Affective Disorders 1997;46:191-217.

\section{Montgomery 1979}

Montgomery SA, Asberg M. A new depression scale designed to be sensitive to change. British Journal of Psychiatry 1979;134:382-9.

\section{Morris 1993b}

Morris PL, Robinson RG, Andrzejewski P, Samuels J, Price TR. Association of depression with 10-year poststroke mortality. American Journal of Psychiatry 1993;150:124-9.

\section{Normand 1999}

Normand ST. Meta-analysis: formulating, evaluating, combining, and reporting. Statistics in Medicine 1999;18:321-59.

\section{Parikh 1990}

Parikh RM, Robinson RG, Lipsey JR, Starkstein SE, Fedoroff JP, Price TR. The impact of poststroke depression on recovery in activities of daily living over a 2-year follow-up. Archives of Neurology 1990;47:785-9.

\section{Paul 2006}

Paul SL, Dewey HM, Sturm JW, Macdonell RAL, Thrift AG. Prevalence of depression and use of antidepressant medication at 5-years poststroke in the North East Melbourne Stroke Incidence Study. Stroke 2006;37:2854-5.

\section{Review Manager 2014 [Computer program]}

Nordic Cochrane Centre, The Cochrane Collaboration. Review Manager 5 (RevMan 5). Version 5.3. Copenhagen: Nordic Cochrane Centre, The Cochrane Collaboration, 2014.

\section{Robinson 1986}

Robinson RG, Bolla-Wilson K, Kaplan E, Lipsey JR, Price TR. Depression influences intellectual impairment in stroke patients. British Journal of Psychiatry 1986;148:541-7.

\section{Schunemann 2011}

Schunemann HJ, Oxman AD, Higgins JP, Vist GE, Glazziou P, Guyatt GH. Chapter 11. Presenting results and 'Summary of findings' tables. In: Higgins JP, Green S, editor(s). Cochrane Handbook for Systematic Reviews of Interventions Version 5.1.0 (updated March 2011). The Cochrane Collaboration. Available from handbook.cochrane.org, 2011.

\section{Sharpe 1990}

Sharpe M, Hawton K, House A, Molyneux A, Sandercock P, Bamford J, et al. Mood disorders in long-term survivors of stroke: associations with brain lesion location and volume. Psychological Medicine 1990;20:815-28.

\section{Shen 2017}

Shena XY, Liua MY, Chenga Y, Jiab C, Pana XY, Goua QY, et al. Repetitive transcranial magnetic stimulation for the treatment of poststroke depression: a systematic review and metaanalysis of randomized controlled clinical trials. Journal of Affective Disorders 2017;211:65-74.

\section{Sinyor 1986}

Sinyor D, Amato P, Kaloupek DG, Becker R, Goldenberg M, Coopersmith $\mathrm{H}$. Post-stroke depression: relationships to functional impairment, coping strategies, and rehabilitation outcome. Stroke 1986;17:1102-7.

\section{Snow 2000}

Snow V, Lascher S, Mottur-Pilson C, for the American College of Physicians-American Society of Internal Medicine. Pharmacologic treatment of acute major depression and dysthymia. Annals of Internal Medicine 2000;132(9):738-42.

\section{Stenager 1998}

Stenager EN, Madsen C, Stenager E, Boldsen J. Suicide in patients with stroke: epidemiological study. BMJ 1998;316:1206.

Pharmacological, psychological, and non-invasive brain stimulation interventions for treating depression after stroke (Review) 


\section{Turner 2012}

Turner A, Hambridge J, White J, Carter G, Clover K, Nelson L, Hackett M. Depression screening in stroke: a comparison of alternative measures with the SCID (Major Depressive Episode) as criterion standard. Stroke 2012;43:1000-5.

\section{Turner-Stokes 2003}

Turner-Stokes L. Poststroke depression: getting the full picture. Lancet 2003;361(9371):1757-8.

\section{Verhagen 2001}

Verhagen AP, de Vet HCW, de Bie RA, Boers M, van den Brandt PA. The art of quality assessment of RCTs included in systematic reviews. Journal of Clinical Epidemiology 2001;54:651-4.

\section{Wilkinson 1997}

Wilkinson P. Cognitive therapy with elderly people. Age and Ageing 1997;26:53-8.

\section{Zigmond 1983}

Zigmond AS, Snaith RP. The Hospital Anxiety and Depression Scale. Acta Psychiatrica Scandinavica 1983;67:361-70.

\section{CHARACTERISTICS OF STUDIES}

Characteristics of included studies [ordered by study ID]

\section{Zimmerman 2002}

Zimmerman M, Mattia JI, Posternak MA. Are subjects in pharmacological treatment trials of depression representative of patients in routine clinical practice?. American Journal of Psychiatry 2002;159(3):469-73.

\section{References to other published versions of this review \\ Hackett 2004}

Hackett ML, Anderson CS, House AO. Interventions for treating depression after stroke. Cochrane Database of Systematic Reviews 2004, Issue 3. [DOI: 10.1002/14651858.CD003437.pub2]

\section{Hackett 2005}

Hackett ML, Anderson CS, House AO. Management of depression after stroke: a systematic review of pharmacological therapies. Stroke 2005;36:1092-7.

\section{Hackett 2008}

Hackett ML, Anderson CS, House A, Xia J. Interventions for treating depression after stroke. Cochrane Database of Systematic Reviews 2008, Issue 4. [DOI: 10.1002/14651858.CD003437.pub3]

* Indicates the major publication for the study

Alexopoulos 2012

$\begin{array}{ll}\text { Methods } & \begin{array}{l}\text { Study design: parallel design } \\ \text { Number of arms: } 2\end{array} \\ \text { Treatment arm:ecosystem focused therapy (EFT) } \\ \text { Control arm: attention control }\end{array}$

\section{Participants}

\section{Geographical location: USA \\ Setting: inpatient}

Stroke criteria: ischaemic and haemorrhagic stroke

Method of stroke diagnosis: not reported

Time since stroke: not reported

Inclusion criteria: (1) aged 60 years or older; (2) had an ischaemic, embolic, or haemorrhagic stroke; (3) diagnosis of unipolar major depression by DSM-IV

Exclusion criteria: (1) moderately severe dementia (MMSE score < 20); (2) greater than moderate aphasia (NIHSS best language > 1); (3) expectation to be discharged to a nursing home; (4) psychotic depression (by DSM-IV); (5) suicidal intent or plan; (6) inability to speak English

Depression criteria: structured clinical interview for DSM-IV-TR and PHQ-9 cut-off score $\geq 10$

Total number randomised in this trial: 24

Number randomised to treatment group: 12 (50\% men, mean age 72 years, SD 7)

Number randomised to control group: 12 (58\% men, mean age 69 years, SD 10) 


\section{Total number included in the final analysis: 24}

Number included in treatment group for final analysis: 12 (50\% men, mean age 72 years, SD 7)

Number included in control group for final analysis: 12 (58\% men, mean age 69 years, SD 10)

Interventions

Treatment: 12 weekly 45-minute personalised sessions of EFT were offered. Treatment was designed to increase patient participation in rehabilitation and social activities, focusing on adherence, problem-solving, goal-setting, and co-ordination of care

Administered by: therapist trained in EFT using manuals; qualification of therapist not stated

Attention control: 12 weekly 45-minute sessions of Education on Stroke and Depression (ESD) Administered by: therapist trained in ESD using manuals; qualification of therapist not stated

Supervision: 3 practice cases of EFT and ESD were supervised; qualifications of the supervisor not stated

Intervention fidelity: all EFT and ESD sessions were audio-taped and rated by reviewers who were not members of the research team, using specially devised EFT and ESD fidelity scales ( 5 grades: $1=$ poor, 5 = excellent). Mean EFT scores ranged from 4.0 to 4.4; mean ESD scores ranged from 4.6 to 4.9, indicating good intervention fidelity for both arms

Duration: 12 weeks

Follow-up: none

\begin{tabular}{ll}
\hline Outcomes & Primary outcomes \\
- Depressive symptoms measured using the HDRS \\
Secondary outcomes \\
- Remission of depression $($ HDRS $<10)$ \\
- Disability measured using the WHODAS-II
\end{tabular}

Notes

Author contact: emailed study authors to ask how missing data were handled and to ask for information on sample size calculation 19 November 2018

\section{Risk of bias}

\begin{tabular}{|c|c|c|}
\hline Bias & Authors' judgement & Support for judgement \\
\hline $\begin{array}{l}\text { Random sequence genera- } \\
\text { tion (selection bias) }\end{array}$ & Low risk & $\begin{array}{l}\text { Quote: "the subjects were randomly assigned to EFT or ESD using random } \\
\text { numbers" (p. 1055) }\end{array}$ \\
\hline $\begin{array}{l}\text { Allocation concealment } \\
\text { (selection bias) }\end{array}$ & Unclear risk & Comment: method of allocation concealment not reported \\
\hline $\begin{array}{l}\text { Blinding of participants } \\
\text { and personnel (perfor- } \\
\text { mance bias) }\end{array}$ & High risk & $\begin{array}{l}\text { Quote: "four therapists were trained and offered both EFT and ESD..." (p. } \\
\text { 1056) }\end{array}$ \\
\hline All outcomes & & $\begin{array}{l}\text { Comment: due to the nature of the trial, it was not possible to mask partici- } \\
\text { pants, therapists, or researchers to the treatment allocation }\end{array}$ \\
\hline $\begin{array}{l}\text { Blinding of outcome as- } \\
\text { sessment (detection bias) } \\
\text { All outcomes }\end{array}$ & High risk & $\begin{array}{l}\text { Quote: ".....the raters could not be blinded to the treatment condition, al- } \\
\text { though they were unaware of the study hypotheses" (p. 1058) }\end{array}$ \\
\hline
\end{tabular}


Alexopoulos 2012 (Continued)

Incomplete outcome data Unclear risk Comment: in the intervention arm, 2 died, 1 LTF was reported; in the control (attrition bias) arm, 1 discontinued treatment. Analysis includes all patients (ITT), but how All outcomes missing data were handled was not reported

Selective reporting (re- Unclear risk porting bias)
Comment: all pre-specified outcomes were reported. No trial protocol or registry record available to compare with the publication

Other bias Low risk

Comment: no statistically significant differences in demographic characteristics, age of depression between EFT- and ESD-treated participants

\section{Andersen 1994}

Study design: parallel design
Mumber of arms: 2
Treatment arm: citalopram (SSRI)
Control arm: matched placebo
Geographical location: Denmark
Setting: mixed outpatient and inpatient
Stroke criteria: ischaemic stroke and primary intracerebral haemorrhage
Method of stroke diagnosis: diagnosis via clinical signs and CT (100\%)
Time since stroke: 2 to 52 weeks (average time 12 weeks)
Inclusion criteria: (1) had stroke 2 to 52 weeks before randomisation
Exclusion criteria: (1) patients with subarachnoid haemorrhage or Binswanger's disease; (2) with pre-
vious degenerative or expansive neurological disease (such as multiple sclerosis, amyotrophic lateral
sclerosis, tumour, and hydrocephalus); (3) with history of psychiatric illness (except depression more
than 1 year earlier); (4) decreased consciousness; (5) dementia; (6) aphasia to such a degree that they
could not explain themselves or gave conflicting verbal and non-verbal signals

Depression criteria: HDRS score > 12 (score transformed to appropriate DSM-III-R criteria)

Total number randomised in this trial: 66

Number randomised to treatment group: 33 (36\% men, mean age 68 years, SD 4) Number randomised to control group: 33 ( $66 \%$ men, mean age 66 years, SD 9)

Total number included in the final analysis: 66

Number included in treatment group for final analysis: 33 (36\% men, mean age 68 years, SD 4)

Number included in control group for final analysis: 33 (66\% men, mean age 66 years, SD 9)

Treatment: citalopram (SSRI), $10 \mathrm{mg}$ in participants > 66 years, $20 \mathrm{mg}$ in participants $<67$ years, daily; dose doubled if no response to treatment within 3 weeks

Control: matched placebo

Duration: 6 weeks; treatment continued only for responders at 6 weeks (these data not included in review)

Follow-up: none 
Andersen 1994 (Continued)

- Depression measured using the HDRS

- Proportion no longer meeting entry criteria (HDRS score < 13)

- Depression measured using the Melancholia Scale

\section{Secondary outcomes}

- Disability measured using the BI

- Social functioning measured using the Social Activities Index

- Cognitive functioning measured using the MMSE

\section{Notes}

\section{Risk of bias}

\begin{tabular}{lll}
\hline Bias & Authors' judgement & Support for judgement \\
\hline $\begin{array}{l}\text { Random sequence genera- } \\
\text { tion (selection bias) }\end{array}$ & Unclear risk & $\begin{array}{l}\text { Quote: "to ensure approximately equal numbers of patients in the treatment } \\
\text { groups, randomization was carried out in groups of 4, with } 2 \text { assigned to citalo- } \\
\text { pram" } \\
\text { Comment: method of sequence generation not reported }\end{array}$ \\
\end{tabular}

\begin{tabular}{|c|c|c|}
\hline $\begin{array}{l}\text { Allocation concealment } \\
\text { (selection bias) }\end{array}$ & High risk & $\begin{array}{l}\text { Comment: opaque envelopes with codes concealed until end of the study } \\
\text { were used. After study authors were contacted for more information, this de- } \\
\text { tail was provided }\end{array}$ \\
\hline
\end{tabular}

\begin{tabular}{|c|c|c|}
\hline $\begin{array}{l}\text { Blinding of participants } \\
\text { and personnel (perfor- } \\
\text { mance bias) } \\
\text { All outcomes }\end{array}$ & Low risk & $\begin{array}{l}\text { Quote: "the trial was designed as a randomized, double-blind, placebo-con- } \\
\text { trolled study" (p. 1100) } \\
\text { Comment: who was blinded was not reported }\end{array}$ \\
\hline $\begin{array}{l}\text { Blinding of outcome as- } \\
\text { sessment (detection bias) } \\
\text { All outcomes }\end{array}$ & Low risk & $\begin{array}{l}\text { Quote: "the trial was designed as a randomized, double-blind, placebo-con- } \\
\text { trolled study" (p. 1100) } \\
\text { Comment: who was blinded was not reported }\end{array}$ \\
\hline $\begin{array}{l}\text { Incomplete outcome data } \\
\text { (attrition bias) } \\
\text { All outcomes }\end{array}$ & Low risk & $\begin{array}{l}\text { Comment: ITT analysis (all participants including dropout were included). See } \\
\text { Table } 2 \text { (p. 1101) for last observation for dropout carried forward }\end{array}$ \\
\hline $\begin{array}{l}\text { Selective reporting (re- } \\
\text { porting bias) }\end{array}$ & Unclear risk & $\begin{array}{l}\text { Comment: all pre-specified outcomes reported; no trial protocol or registry } \\
\text { record available to compare with the publication }\end{array}$ \\
\hline Other bias & Low risk & $\begin{array}{l}\text { Comment: there were no differences in baseline demographic characteristics } \\
\text { between groups }\end{array}$ \\
\hline
\end{tabular}

\section{Cao 2009a}

\begin{tabular}{ll}
\hline Methods & Study design: parallel design \\
& Number of arms: 2 \\
& Treatment arm: fluoxetine $($ SSRI $)+$ psychotherapy + usual care \\
& Control arm: fluoxetine (SSRI) + usual care \\
\hline Participants & $\begin{array}{l}\text { Geographical location: China } \\
\text { Setting: inpatient }\end{array}$ \\
\hline
\end{tabular}


Stroke criteria: cerebral haemorrhage and infarct

Method of stroke diagnosis: not reported

Time since stroke: not reported

Inclusion criteria: (1) 24-item HDRS score > 20; (2) can sign informed consent; (3) voluntary participation; (4) strong desire to change themselves; (5) willingness to communicate with others; (6) completion of 12 therapy sessions (treatment arm only)

Exclusion criteria: (1) history of psychiatric illness; (2) severe cognitive impairment; (3) verbal communication barrier; (4) severe illness (e.g. myocardial infarction)

Depression criteria: Chinese version of 24-item HDRS score $>20$

Total number randomised in this trial: 144 ( $48 \%$ of total group men; mean age of total group 60 years, SD 9)

Number randomised to treatment group: 72 (as above)

Number randomised to control group: 72

Total number included in the final analysis: 144 (48\% of total group men; mean age of total group 60 years, SD 9)

Number included in treatment group for final analysis: 72 (as above)

Number included in control group for final analysis: 72 (as above)

Interventions

Treatment: fluoxetine (SSRI) $20 \mathrm{mg} / \mathrm{d}+$ group psychotherapy with 4 phases: an introductory session to build group security and trust

Administered by: each group has 1 leader and 1 assistant. 2 neurologists qualified with group psychotherapy (national counsellors, grade 2) serve as leaders, and 3 nurses with professional training serve as assistants

Supervision: not reported

Intervention fidelity: not reported

Control: fluoxetine (SSRI) $20 \mathrm{mg} / \mathrm{d}$

Duration of psychotherapy: 30 to 40 minutes, once/week for 12 weeks

Duration of fluoxetine: first depression 4 to 6 months, then taper and discontinue; recurrent depression: extended additional 3 to 6 months; depression episodes $\geq 3$ times: more prolonged period

Follow-up: none

Outcomes Primary outcomes

- Depression measured using 24-item HDRS

Secondary outcomes

- Disability measured using BI

Notes

\section{Risk of bias}


Cao 2009a (Continued)

Random sequence genera- Unclear risk Comment: method of sequence generation not reported tion (selection bias)

Allocation concealment Unclear risk Comment: method of allocation concealment not reported
(selection bias)

Blinding of participants Unclear risk Comment: blinding of participants and personnel not reported
and personnel (perfor-
mance bias)
All outcomes

\begin{tabular}{lll}
\hline $\begin{array}{l}\text { Blinding of outcome as- } \\
\text { sessment (detection bias) } \\
\text { All outcomes }\end{array}$ & Unclear risk & Comment: blinding of outcome assessment not reported \\
\hline $\begin{array}{l}\text { Incomplete outcome data } \\
\text { (attrition bias) }\end{array}$ & High risk & $\begin{array}{l}\text { Comment: ITT; no missing data reported, but randomised participants who } \\
\text { did not complete the } 12 \text { sessions appear to have been excluded; dropouts/ } \\
\text { All outcomes }\end{array}$
\end{tabular}

\begin{tabular}{lll}
\hline $\begin{array}{l}\text { Selective reporting (re- } \\
\text { porting bias) }\end{array}$ & Unclear risk & $\begin{array}{l}\text { Comment: all pre-specified outcomes reported; no trial protocol or registry } \\
\text { record to compare with the publication }\end{array}$ \\
\hline Other bias & Low risk & $\begin{array}{l}\text { Comment: no differences in baseline 24-item HDRS and BI. Baseline demo- } \\
\text { graphic information not reported }\end{array}$ \\
\hline
\end{tabular}

Cao 2009b

\begin{tabular}{l} 
Study design: parallel design \\
Number of arms: 2 \\
Treatment arm: psychotherapy + usual care \\
Control arm: usual care \\
\hline
\end{tabular}

Participants

\author{
Geographical location: China \\ Setting: inpatient
}

Stroke criteria: cerebral haemorrhage and infarct

Method of stroke diagnosis: not reported

Time since stroke: not reported

Inclusion criteria: (1) 24-item HDRS score > 20; (2) can sign informed consent; (3) voluntary participation; (4) strong desire to change themselves; (5) willingness to communicate with others; (6) completion of 12 therapy sessions (treatment arm only)

Exclusion criteria: (1) history of psychiatric illness; (2) severe cognitive impairment; (3) verbal communication barrier; (4) severe illness (e.g. myocardial infarction)

Depression criteria: Chinese version of 24-item HDRS $>20$

Total number randomised in this trial: 144 ( $48 \%$ of total group men; mean age of total group 60 years, SD 9)

Number randomised to treatment group: 72 (as above)

Number randomised to control group: 72 (as above) 
Cao 2009b (Continued)

Total number included in the final analysis: 144 (48\% of total group men; mean age of total group 60 years, SD 9)

Number included in treatment group for final analysis: 72 (as above)

Number included in control group for final analysis: 72 (as above)

Interventions

Treatment: group psychotherapy with 4 phases: an introductory session to build group security and trust

Administered by: each group has 1 leader and 1 assistant. 2 neurologists qualified with group psychotherapy (national counsellors, grade 2) serve as leaders, and 3 nurses with professional training serve as assistants

Supervision: not reported

Intervention fidelity: not reported

Control: usual care

Duration of psychotherapy: 30 to 40 minutes, once/week for 12 weeks

Follow-up: none

- Depression measured using 24-item HDRS

\section{Secondary outcomes}

- Disability measured using BI

Notes

\section{Risk of bias}

\begin{tabular}{lll}
\hline Bias & Authors' judgement & Support for judgement \\
\hline $\begin{array}{l}\text { Random sequence genera- } \\
\text { tion (selection bias) }\end{array}$ & Unclear risk & Comment: method of sequence generation not reported \\
\hline $\begin{array}{l}\text { Allocation concealment } \\
\text { (selection bias) }\end{array}$ & Unclear risk & Comment: method of allocation concealment not reported \\
\hline
\end{tabular}

Blinding of participants Unclear risk Comment: blinding of participants and personnel not reported
and personnel (performance bias)

All outcomes

\begin{tabular}{lll}
\hline $\begin{array}{l}\text { Blinding of outcome as- } \\
\text { sessment (detection bias) } \\
\text { All outcomes }\end{array}$ & Unclear risk & Comment: blinding of outcome assessment not reported \\
\hline $\begin{array}{l}\text { Incomplete outcome data } \\
\begin{array}{l}\text { (attrition bias) } \\
\text { All outcomes }\end{array}\end{array}$ & High risk & $\begin{array}{l}\text { Comment: ITT; no missing data reported but randomised participants who did } \\
\text { not complete the } 12 \text { sessions appear to have been excluded; dropouts/cross- } \\
\text { overs not reported }\end{array}$ \\
\hline
\end{tabular}

Selective reporting (re- Unclear risk porting bias)

Comment: all pre-specified outcomes reported; no trial protocol or registry record to compare with the publication 
Cao 2009b (Continued)

$\begin{array}{ll}\text { Other bias } \quad \text { Low risk } & \begin{array}{l}\text { Comment: no differences in baseline 24-item HDRS and BI; baseline demo- } \\ \text { graphic information not reported }\end{array}\end{array}$
graphic information not reported

Chen 2005a

\begin{tabular}{l} 
Study design: parallel design \\
Number of arms: 2 \\
Treatment arm: rTMS + cerebrovascular disease routine care \\
Control arm: cerebrovascular disease routine care \\
\hline
\end{tabular}

Participants

Geographical location: China

Setting: mixed outpatient and inpatient

Stroke criteria: ischaemic stroke

Method of stroke diagnosis: diagnosis consistent with diagnostic criteria for stroke formulated by the Fourth National Symposium on Cerebrovascular Disease in 1995; confirmation by brain CT or MRI

Time since stroke: 2 months

Inclusion criteria: (1) disease course of stroke on average > 2 months; (2) patients and family gave informed consent

Exclusion criteria: (1) history of psychiatric illness; (2) obvious comprehension impairment; (3) obvious aphasia; (4) severe physical illness; (5) epilepsy

Depression criteria: depression was diagnosed by clinical interview according to the CCMD-2-R; 17 item HDRS score $>17$

Total number randomised in this trial: 32

Number randomised to treatment group: 16 (62\% men, mean age 61 years, SD 4.9; modified SSS 18.3, SD 4.8)

Number randomised to control group: 16 (56\% men, mean age 61.2 years, SD 4.7; modified SSS 17.5, SD 4.4)

Total number included in final analysis: 32

Number included in treatment group for final analysis: 16 (62\% men, mean age 61 years, SD 4.9; modified SSS 18.3, SD 4.8)

Number included in control group for final analysis: 16 (56\% men, mean age 61.2 years, SD 4.7; modified SSS 17.5 SD, 4.4) quency $0.5 \mathrm{~Hz}, 1$ sequence included 30 stimulations in each side of the pre-frontal lobe; plus cerebrovascular disease routine care

Control: cerebrovascular disease routine care

Treatment duration: 1 sequence a day for 7 successive days

Administration: unclear

Follow-up: none 
Chen 2005a (Continued)

- Depression measured using 17-item HDRS

- Impairments measured using modified SSS

\title{
Secondary outcomes
}

- Adverse events

Notes

\section{Risk of bias}

\begin{tabular}{lll}
\hline Bias & Authors' judgement & Support for judgement \\
\hline $\begin{array}{l}\text { Random sequence genera- } \\
\text { tion (selection bias) }\end{array}$ & High risk & Comment: randomisation performed by drawing lots, which is prone to bias \\
\hline $\begin{array}{l}\text { Allocation concealment } \\
\text { (selection bias) }\end{array}$ & Unclear risk & Comment: method of allocation concealment not reported \\
\hline $\begin{array}{l}\text { Blinding of participants } \\
\text { and personnel (perfor- } \\
\text { mance bias) } \\
\text { All outcomes }\end{array}$ & High risk & $\begin{array}{l}\text { Comment: participants and personnel not blinded to group allocation. Study } \\
\text { used a prospective, randomised open-blinded endpoint (PROBE) design }\end{array}$ \\
\hline
\end{tabular}

Blinding of outcome as- Low risk Comment: outcome assessors blinded to group allocation
sessment (detection bias)

All outcomes

\begin{tabular}{lll}
\hline $\begin{array}{l}\text { Incomplete outcome data } \\
\text { (attrition bias) } \\
\text { All outcomes }\end{array}$ & Low risk & Comment: ITT analysis reported; no missing data \\
\hline $\begin{array}{l}\text { Selective reporting (re- } \\
\text { porting bias) }\end{array}$ & Unclear risk & $\begin{array}{l}\text { Comment: all pre-specified outcomes reported; no trial protocol or registry } \\
\text { record to compare with the publication }\end{array}$ \\
\hline Other bias & Low risk & $\begin{array}{l}\text { Comment: no differences in baseline demographic characteristics between } \\
\text { groups }\end{array}$ \\
\hline
\end{tabular}

Cullen 2018

Study design: parallel design
Number of arms: 2
Treatment arm: brief positive psychotherapy + usual care
Control arm: usual care

Participants

\author{
Geographical location: Scotland
}

Setting: outpatient

Stroke criteria: cerebrovascular infarct and haemorrhagic stroke

Method of stroke diagnosis: confirmed by local clinician based on clinical and/or radiological evidence

Time since stroke: 3 to 36 months 
Inclusion criteria: (1) adults aged 18 or over; (2) diagnosis of acquired, non-progressive brain injury; (3) between 3 and 12 months post injury at time of recruitment; (4) presence of emotional distress (score in moderate or above range on at least 1 subscale of the DASS-21; (5) medically stable; (6) able to consent

Exclusion criteria: (1) significant communication impairment; (2) diagnosis of mild traumatic brain injury; (3) comorbid developmental learning disability or degenerative neurological condition

Depression criteria: presence of emotional distress (score in moderate or above range on at least 1 subscale of the DASS-21)

\section{Total number randomised in this trial (stroke participants only): 24}

Number included in treatment group: 12 (67\% men; mean age 55 years, SD 10)

Number included in control group: 12 (67\% men; mean age 60 years, SD 9)

Total number included in final analysis (stroke participants only): 24

Number included in treatment group for final analysis: 12 (67\% men; mean age 55 years, SD 10)

Number included in control group for final analysis: 12 (67\% men; mean age 60 years, SD 9)

Interventions

Treatment: participants in intervention arm received a brief positive psychotherapy intervention delivered over 8 weeks, in addition to accessing usual care within the clinical service. Study intervention followed a manualised programme designed by the research team and based on aspects of Rashid and Seligman's (2013) programme, incorporating psychoeducation about ABI and positive psychology (week 1), a range of therapeutic exercises, and homework focused on using signature character strengths and reflecting on positive events (weeks 2 to 7 inclusive, with mid-point review at week 4), and final review and plan for maintenance (week 8)

Administered by: not reported

Supervision: not reported

Intervention fidelity: not reported

Control: participants in control arm received usual care within the clinical service; the content of usual care was not standardised: input varied between services and participants, but all participants could access clinical psychology input if required

Duration: 8 weeks

Follow-up: 20 weeks

Outcomes Primary outcomes

- Depression measured using DASS-21 Depression

- Anxiety measured using DASS-21 Anxiety

- Stress measured using DASS-21 Stress

- Depression measured using AHI

\section{Secondary outcomes}

- Overall function measured using Mayo-Portland Adaptability Inventory-4 (MPAI-4) total (participant)

- Overall function measured using MPAI-4 total (informant)

- Caregiver Strain measured using Modified-Caregiver Strain Index AHI, and DASS-21 Anxiety for stroke patients only 09/11/2018) 
Cullen 2018 (Continued)

Risk of bias

\begin{tabular}{lll}
\hline Bias & Authors' judgement & Support for judgement \\
\hline $\begin{array}{ll}\text { Random sequence genera- } \\
\text { tion (selection bias) }\end{array}$ & Low risk & $\begin{array}{l}\text { Quote: "stratified randomisation with blocking was used to allocate partici- } \\
\text { pants to two groups of equal size, stratified by service setting (stroke versus } \\
\text { CTCBI). Because service setting was a proxy for injury type (stroke versus non- } \\
\text { stroke) and for the nature of usual care that would be available to participants, } \\
\text { either of which could have influenced outcomes, including this as a stratifica- } \\
\text { tion factor ensured these aspects would be balanced across the intervention } \\
\text { and control groups" (p. 24) }\end{array}$
\end{tabular}

Comment: computer-generated numbers were used based on correspondence with author

$\begin{array}{ll}\begin{array}{l}\text { Allocation concealment } \\ \text { (selection bias) }\end{array} & \text { Low risk } \\ & \begin{array}{l}\text { Quote: "the allocation system was managed by the Robertson Centre for Bio- } \\ \text { statistics and was accessed via an automated telephone service after the base- } \\ \text { line assessment had been completed" (p. 24) }\end{array}\end{array}$

Blinding of participants Unclear risk and personnel (perfor-

Quote: "screening, baseline assessments, allocation and interventions were mance bias)

All outcomes carried out by one RA (who was blinded to randomisation block length), and the interim and follow-up measures were administered by a second RA, each of whom was blind to the other's findings. The second RA was blind to participant allocation; a standard script was used to prevent unblinding during follow-up telephone calls, and postal materials included clear instructions to participants not to reveal treatment allocation information" (p. 24)

Comment: due to the nature of the intervention, it is unlikely that participants were blinded to the group allocation

\begin{tabular}{|c|c|c|}
\hline $\begin{array}{l}\text { Blinding of outcome as- } \\
\text { sessment (detection bias) } \\
\text { All outcomes }\end{array}$ & Low risk & $\begin{array}{l}\text { Quote: "a blinded assessor administered the DASS- } 21 \text { and the AHI at } 5,9 \text { and } \\
20 \text { weeks post-baseline. Of } 27 \text { participants randomised (median age } 57 ; 63 \% \\
\text { men; } 82 \% \text { ischaemic stroke survivors; median } 5.7 \text { months post-injury), } 14 \text { were } \\
\text { assigned to positive psychotherapy, of whom } 8 \text { completed treatment" (p. } 31 \text { ) }\end{array}$ \\
\hline
\end{tabular}

Incomplete outcome data High risk

Comment: per protocol analysis reported only; $11 / 27$ participants not includ(attrition bias) ed in the analysis

All outcomes

\begin{tabular}{|c|c|c|}
\hline $\begin{array}{l}\text { Selective reporting (re- } \\
\text { porting bias) }\end{array}$ & Unclear risk & $\begin{array}{l}\text { Comment: all pre-specified outcomes reported; no trial protocol or registry } \\
\text { record available to compare with the publication }\end{array}$ \\
\hline
\end{tabular}

\begin{tabular}{ll}
\hline Other bias $\quad$ Low risk & $\begin{array}{l}\text { Comment: no differences in baseline demographic characteristics between } \\
\text { groups }\end{array}$
\end{tabular}

\section{Du 2005}

Methods Study design: parallel design

Number of arms: 2

Treatment arm: rTMS + fluoxetine (SSRI)

Control arm: fluoxetine (SSRI)

$\begin{array}{ll}\text { Participants } & \text { Geographical location: China } \\ \text { Setting: inpatient }\end{array}$


Du 2005 (Continued)

Stroke criteria: stroke, types not stated

Method of stroke diagnosis: diagnosis consistent with diagnostic criteria for stroke formulated by the Fourth National Symposium on Cerebrovascular Disease in 1995; confirmation by brain CT or MRI

Time since stroke: not reported

Inclusion criteria: (1) 17-item HDRS score $\geq 8$ points; (2) can sign informed consent

Exclusion criteria: (1) previous depression or psychiatric illness history; (2) aphasia; (3) severe cardiac, pulmonary, hepatic, and renal impairment

Total number randomised in this trial: 60

Number randomised to treatment group: 30 (53\% men; age range 59 to 82 years)

Number randomised to control group: 30 (53\% men; age range 56 to 83 years)

Total number included in final analysis: 60

Number included in treatment group for final analysis: 30

Number included in control group for final analysis: 30

Interventions

Treatment: low-frequency rTMS + 20 mg fluoxetine (SSRI) daily. Patients' bilateral frontal lobes were stimulated with $60 \%$ of maximal stimulus intensity, 30 times for each side. Frequency was $0.5 \mathrm{~Hz}, 1 \mathrm{se}-$ quence every day continuous for 5 days as a course, with an interval of 2 days between courses

Control: $20 \mathrm{mg}$ fluoxetine (SSRI) daily

Treatment duration: 4 weeks

Follow-up: none

\begin{tabular}{ll}
\hline Outcomes & Primary outcomes \\
- Depression measured using 17-item HDRS \\
- Cognitive functioning measured using MMSE \\
- Disability measured using BI
\end{tabular}

Notes

Risk of bias

Bias Authors' judgement Support for judgement

Random sequence genera- High risk Comment: drawing lots used to generate randomisation sequence; this tion (selection bias) method of sequence generation is prone to bias

\begin{tabular}{lll}
\hline $\begin{array}{l}\text { Allocation concealment } \\
\text { (selection bias) }\end{array}$ & Unclear risk & Comment: method of allocation concealment not reported \\
\hline $\begin{array}{l}\text { Blinding of participants } \\
\text { and personnel (perfor- }\end{array}$ & High risk & Comment: participants and personnel not blinded to group allocation \\
mance bias) & & \\
All outcomes & & \\
\hline
\end{tabular}

$\begin{array}{ll}\text { Blinding of outcome as- } & \text { High risk } \quad \text { Comment: outcome assessors not blinded to group allocation } \\ \text { sessment (detection bias) }\end{array}$

All outcomes 
Du 2005 (Continued)

Incomplete outcome data Low risk Comment: ITT; no missing data reported
(attrition bias)

All outcomes

\begin{tabular}{|c|c|c|}
\hline $\begin{array}{l}\text { Selective reporting (re- } \\
\text { porting bias) }\end{array}$ & Unclear risk & $\begin{array}{l}\text { Comment: all pre-specified outcomes reported; no trial protocol or registry } \\
\text { record available to compare with the publication }\end{array}$ \\
\hline
\end{tabular}

\begin{tabular}{ll}
\hline Other bias $\quad$ Low risk & $\begin{array}{l}\text { Comment: no differences in baseline demographic characteristics between } \\
\text { groups }\end{array}$ \\
\hline
\end{tabular}

\section{Fan 2014}

\begin{tabular}{ll}
\hline Study design: parallel design \\
Number of arms: 2 \\
Experimental arm: rTMS + duloxetine (SNRI) + stroke usual care \\
Control arm: duloxetine (SNRI) + stroke usual care
\end{tabular}

Setting: unclear

Stroke criteria: not reported

Method of stroke diagnosis: not reported

Time since stroke: not reported

Inclusion criteria: (1) meeting diagnostic criteria of the CCMD-2-R for depression; (2) 17-item HDRS score $\geq 8$; (3) stable condition; (4) could tolerate rTMS; (5) patient or family member can sign informed consent; (6) age 18 to 80 years

Exclusion criteria: (1) with previous depression, psychiatric illness history; (2) without 1-week washout period of previous antidepressants; (3) consciousness disturbance or severe cognitive impairment; (4) with epilepsy or severe cardiac, pulmonary, hepatic, or renal disease; (5) critical conditions or unstable acute stage of stroke

Depression criteria: must meet diagnostic criteria of the CCMD-2-R for depression and the 17-item HDRS score $\geq 8$

Total number randomised in this trial: 90

Number randomised to treatment group: 45 (42\% men, mean age 61.43, SD 8.74)

Number randomised to control group: 45 (51\% men, mean age 64.78, SD 7.23)

Total number included in final analysis: 90

Number included in treatment group for final analysis: 45 (42\% men, mean age 61.43, SD 8.74 )

Number included in treatment group for final analysis: 45 (51\% men, mean age 64.78, SD 7.23)

Interventions

Treatment: rTMS (frequency: $1 \mathrm{~Hz}$, intensity: 100\% motor threshold, 30 times for a series, 10 series for each treatment; location: bilateral dorsolateral pre-frontal) + duloxetine (SNRI) $60 \mathrm{mg} / \mathrm{d}+$ stroke usual care (routine medication and rehabilitation)

Control: duloxetine (SNRI) + stroke usual care

Duration: 4 weeks 
Fan 2014 (Continued)

Follow-up: none

\begin{tabular}{ll}
\hline Outcomes & Primary outcomes \\
- Depression measured using 17-item HDRS \\
- Disability measured using MBI
\end{tabular}

Notes

\section{Risk of bias}

\begin{tabular}{lll}
\hline Bias & Authors' judgement & Support for judgement \\
\hline $\begin{array}{l}\text { Random sequence genera- } \\
\text { tion (selection bias) }\end{array}$ & Unclear risk & Comment: method of sequence generation not reported \\
\hline
\end{tabular}

Allocation concealment $\quad$ Unclear risk $\quad$ Comment: method of allocation concealment not reported
(selection bias)

Blinding of participants Unclear risk Comment: double-blind stated but who was blinded not reported
and personnel (perfor-
mance bias)
All outcomes

\begin{tabular}{ll}
\hline Blinding of outcome as- & Unclear risk $\quad$ Comment: double-blind stated but who was blinded not reported \\
sessment (detection bias)
\end{tabular}

All outcomes

\begin{tabular}{lll}
\hline $\begin{array}{l}\text { Incomplete outcome data } \\
\text { (attrition bias) } \\
\text { All outcomes }\end{array}$ & Low risk & Comment: ITT analysis; no missing data reported \\
\hline $\begin{array}{l}\text { Selective reporting (re- } \\
\text { porting bias) }\end{array}$ & Unclear risk & $\begin{array}{l}\text { Comment: all pre-specified outcomes reported; no trial protocol available to } \\
\text { compare with the publication }\end{array}$ \\
\hline Other bias & Low risk & Comment: no differences in baseline demographics between groups
\end{tabular}

Fang 2017

\begin{tabular}{ll} 
Study design: parallel design \\
Number of arms: 2 \\
Experimental arm: constructive integrative psychosocial intervention (CIPI) \\
Control arm: standard care \\
\hline
\end{tabular}

Participants

Geographical location: Singapore

Setting: inpatient

Stroke criteria: ischaemic and haemorrhagic stroke

Method of stroke diagnosis: clinically diagnosed new stroke

Time since stroke: 1 week 
Fang 2017 (Continued)

Inclusion criteria: (1) had satisfactory mental status MMSE > 23; (2) had clinically diagnosed new stroke within a week; (3) only patients who spoke English or Mandarin

Exclusion criteria: (1) other non-stroke-related neurological conditions such as brain tumour or traumatic brain injury; (2) patients discharged to a nursing home

Depression criteria: HADS score $\geq 8$

Total number randomised in this trial: 42

Number randomised to treatment group: 23 (\% men, age not recorded in the study)

Number randomised to control group: 19 (\% men, age not recorded in the study)

Total number included in final analysis: 19

Number included in treatment group for final analysis: 13 (\% men, age not recorded in the study)

Number included in control group for final analysis: 6 (\% men, age not recorded in the study)

Treatment: CIPI result in a positive construction of experience of illness by patients and significant others. This addresses their cognitions related to living with stroke and related behavioural response to the stroke experience. Key qualities include evidence-supported components of psychosocial-behavioural intervention life review and education

Administered by: not reported

Supervision: not reported

Intervention fidelity: not reported

Control: standard care

Duration: 6 months

Follow-up: none

Outcomes

\section{Primary outcome}

- Depression measured using HADS at 1, 3, and 6 months

\section{Secondary outcome}

- Cognitive functioning measured using MMSE at 1,3, and 6 months

\section{Notes}

\section{Risk of bias}

Bias Authors' judgement Support for judgement

Random sequence genera- Low risk tion (selection bias)
Quote: "it used a randomized control group in an acute stroke unit with pretest-posttest...."

Comment: based on study authors' responses; random number tables used

\begin{tabular}{lll}
\hline $\begin{array}{l}\text { Allocation concealment } \\
\text { (selection bias) }\end{array}$ & High risk & $\begin{array}{l}\text { Comment: based on study authors' responses; sealed envelopes used to con- } \\
\text { ceal allocation. This method of allocation concealment can be tampered with }\end{array}$ \\
\hline $\begin{array}{l}\text { Blinding of participants } \\
\begin{array}{l}\text { and personnel (perfor- } \\
\text { mance bias) }\end{array}\end{array}$ & High risk & $\begin{array}{l}\text { Comment: due to the nature of the trial, it was not possible to mask partici- } \\
\text { pants, clinicians, and researchers to treatment allocation }\end{array}$ \\
All outcomes & \\
\hline
\end{tabular}

Pharmacological, psychological, and non-invasive brain stimulation interventions for treating depression after stroke (Review) 
Fang 2017 (Continued)

Blinding of outcome as- Low risk Comment: based on study authors' responses: outcome assessors blinded sessment (detection bias)

All outcomes

\begin{tabular}{lll}
\hline $\begin{array}{l}\text { Incomplete outcome data } \\
\text { (attrition bias) } \\
\text { All outcomes }\end{array}$ & High risk & $\begin{array}{l}\text { Comment: per protocol analysis reported; 3/23 in treatment group not includ- } \\
\text { ed in the analysis }\end{array}$ \\
\hline $\begin{array}{l}\text { Selective reporting (re- } \\
\text { porting bias) }\end{array}$ & Unclear risk & $\begin{array}{l}\text { Comment: all pre-specified outcomes reported; no trial protocol available to } \\
\text { compare with the publication }\end{array}$ \\
\hline Other bias & Unclear risk & Comment: baseline demographic information not reported \\
\hline
\end{tabular}

Fruehwald 2003

\begin{tabular}{ll}
\hline Methods & Study design: parallel design \\
Number of arms: 2 \\
Treatment arm: fluoxetine (SSRI) \\
Control arm: matched placebo
\end{tabular}

Participants Geographical location: Austria

Setting: inpatients

Stroke criteria: ischaemic stroke and primary intracerebral haemorrhage

Method of stroke diagnosis: diagnosis via clinical signs and CT (100\%)

Time since stroke: 11 days

Inclusion criteria: (1) stroke on average 11 days before randomisation

Exclusion criteria: (1) MMSE < 20, more than mild communication deficit; (2) disease of the CNS and previous degenerative or expansive neurological disorder

Depression criteria: psychiatric interview and HDRS score $>15$

Total number randomised in this trial: 54

Number randomised to treatment group: 28 ( $46 \%$ men, mean age 65 years, SD 14)

Number randomised to control group: 26 ( $71 \%$ men, mean age 64 years, SD 14)

Total number included in final analysis: 40

Number included in treatment group for final analysis: 22 (\% men and mean age not reported)

Number included in control group for final analysis: 18 (\% men and mean age not reported)

Interventions

Treatment: fluoxetine (SSRI) $20 \mathrm{mg}$ daily; dose escalation at 4 weeks if HDRS score $>13$

Control: matched placebo

Duration: 12 weeks. Open-label treatment was continued for a further 15 months for all (these data not included in the review)

Follow-up: 18 months

\begin{tabular}{ll}
\hline Outcomes & Primary outcomes \\
- Depression measured using HDRS, BDI, and CGI Scale-1 & - Proportion of responders (HDRS $<13$ )
\end{tabular}

Pharmacological, psychological, and non-invasive brain stimulation interventions for treating depression after stroke (Review) 
Fruehwald 2003 (Continued)

\section{Secondary outcomes}

- Stroke impairment measured using SSS

- Adverse events

Notes

Risk of bias

\begin{tabular}{|c|c|c|}
\hline Bias & Authors' judgement & Support for judgement \\
\hline $\begin{array}{l}\text { Random sequence genera- } \\
\text { tion (selection bias) }\end{array}$ & Low risk & $\begin{array}{l}\text { Quote: "the randomization code list was generated by a computer program in } \\
\text { a random permuted block design for each centre" (p. 348) }\end{array}$ \\
\hline $\begin{array}{l}\text { Allocation concealment } \\
\text { (selection bias) }\end{array}$ & Low risk & $\begin{array}{l}\text { Quote: "all patients were randomly assigned to either fluoxetine or placebo } \\
\text { treatment by the drug company independently of the research teams and the } \\
\text { study centres" (p. 348) }\end{array}$ \\
\hline $\begin{array}{l}\text { Blinding of participants } \\
\text { and personnel (perfor- } \\
\text { mance bias) } \\
\text { All outcomes }\end{array}$ & Low risk & $\begin{array}{l}\text { Quote: "... neither patients nor relatives, clinical examiners nor nursing staff } \\
\text { were aware of the drug treatment being given" (p. 348) }\end{array}$ \\
\hline $\begin{array}{l}\text { Blinding of outcome as- } \\
\text { sessment (detection bias) } \\
\text { All outcomes }\end{array}$ & Low risk & $\begin{array}{l}\text { Quote: "... neither patients nor relatives, clinical examiners nor nursing staff } \\
\text { were aware of the drug treatment being given" (p. 348) }\end{array}$ \\
\hline $\begin{array}{l}\text { Incomplete outcome data } \\
\text { (attrition bias) } \\
\text { All outcomes }\end{array}$ & High risk & $\begin{array}{l}\text { Comment: per protocol analysis reported only; } 4 / 54(7.4 \%) \text { not included in } \\
\text { analyses }\end{array}$ \\
\hline $\begin{array}{l}\text { Selective reporting (re- } \\
\text { porting bias) }\end{array}$ & Unclear risk & $\begin{array}{l}\text { Comment: all pre-specified outcomes reported. No trial protocol or registry } \\
\text { record available to compare with the publication }\end{array}$ \\
\hline Other bias & Low risk & $\begin{array}{l}\text { Comment: non-significant trends towards more women and right-sided lesion } \\
\text { strokes in treatment group }\end{array}$ \\
\hline
\end{tabular}

Gao 2017a

Methods

Study design: parallel design

Number of arms: 2

Treatment arm: citalopram (SSRI) + 'attention control' psychological intervention (group B)

Control arm: placebo + 'attention control' psychological intervention (group A)

\section{Participants}

Geographical location: China

Setting: outpatient

Stroke criteria: ischaemic stroke

Method of stroke diagnosis: occurrence of an ischaemic stroke that met the standards of WHO diagnostic criteria. Radiological MRI confirmation of an anatomical infarct observed on diffusion-weighted acute MRI

Time since stroke: not reported 
Inclusion criteria: (1) first-ever acute ischaemic stroke; (2) no history of depression; (3) no antidepressant treatments received before our interventions; (4) over 18 years of age

Exclusion criteria: (1) presence of pre-stroke disease leading to pre-stroke disability; Barthel Index < 10

Depression criteria: 20 -item BDI scores $>10$

Total number randomised in this trial: 136

Number randomised to treatment group: 91 (50\% men, mean age 66 years, SD 7)

Number randomised to control group: $45^{\star \star}(53 \%$ men, mean age 67 years, SD 10)

Total number included in final analysis: 128

Number included in treatment group for final analysis: 85 (\% men and mean age were not reported)

Number included in control group for final analysis: $43^{\star *}$ (\% men and mean age were not reported)

Treatment: patients received active citalopram tablets (SSRI) and participated in similar placebo psychological discussions as group A

Control: patients received placebo tablets and participated in a placebo psychological intervention, 1-hour discussions with non-psychological clinical doctors twice a week for 3 months; discussions focused on inquiries about stroke recovery and changes in daily life

Administered by: non-psychological clinical doctors

Supervision: not reported

Duration: 3 months

Follow-up: none

\begin{tabular}{ll}
\hline Outcomes & Primary outcomes \\
- Depression measured using HDRS \\
- Depression measured using Melancholia Scale
\end{tabular}

\section{Secondary outcomes}

- Disability measured using BI

$\begin{array}{ll}\text { Notes } & \text { Author contact: emailed study authors to request AE tables with numbers for all groups } 23 \text { Octoer } \\ 2018\end{array}$

\section{Risk of bias}

\begin{tabular}{lll}
\hline Bias & Authors' judgement & Support for judgement \\
\hline $\begin{array}{l}\text { Random sequence genera- } \\
\text { tion (selection bias) }\end{array}$ & Low risk & $\begin{array}{l}\text { Quote: "randomization into one of three intervention groups was undertaken } \\
\text { by an independent researcher using computer-generated random number se- } \\
\text { quences" (p. 73) }\end{array}$
\end{tabular}

\begin{tabular}{|c|c|c|}
\hline $\begin{array}{l}\text { Allocation concealment } \\
\text { (selection bias) }\end{array}$ & High risk & $\begin{array}{l}\text { Quote: "... were prepared in advance and placed in consecutively numbered, } \\
\text { sealed, opaque envelopes" (p. 73) }\end{array}$ \\
\hline
\end{tabular}

Comment: sealed, opaque envelopes can be tampered with

\begin{tabular}{|c|c|c|}
\hline $\begin{array}{l}\text { Blinding of participants } \\
\text { and personnel (perfor- } \\
\text { mance bias) }\end{array}$ & High risk & $\begin{array}{l}\text { Quote: "when patients were enrolled, they were told to participate in drug } \\
\text { therapy, talk with doctors, and engage in rehabilitation at the same time. No } \\
\text { breaches in blinding were detected during the trial" (p. 74) }\end{array}$ \\
\hline
\end{tabular}


Gao 2017a (Continued) All outcomes "the study therapists were asked not to divulge any treatment information to their patients" (p. 75)

Comment: therapists delivering the intervention were not blinded to group allocation
Blinding of outcome as- High risk sessment (detection bias)

All outcomes
Quote: "the study therapists acted as clinical evaluators..." (p. 74)

\begin{tabular}{lll}
\hline $\begin{array}{l}\text { Incomplete outcome data } \\
\text { (attrition bias) } \\
\text { All outcomes }\end{array}$ & High risk & $\begin{array}{l}\text { Comment: per protocol analysis reported only; 5/91 in control, 6/91 in treat- } \\
\text { ment not included in the analysis }\end{array}$ \\
\hline $\begin{array}{l}\text { Selective reporting (re- } \\
\text { porting bias) }\end{array}$ & Unclear risk & $\begin{array}{l}\text { Comment: all pre-specified outcomes reported; no trial protocol or registry } \\
\text { record available to compare with the publication }\end{array}$ \\
\hline Other bias & Low risk & $\begin{array}{l}\text { Comment: no differences in baseline demographic characteristics between } \\
\text { groups }\end{array}$ \\
\hline
\end{tabular}

\section{Gao 2017b}

\begin{tabular}{l} 
Study design: parallel design \\
Number of arms: 2 \\
Treatment arm: 'active' psychological intervention + placebo (group C) \\
Control arm: 'attention control' psychological intervention + placebo (group A) \\
\hline
\end{tabular}

Setting: outpatient

Stroke criteria: ischaemic stroke

Method of stroke diagnosis: occurrence of an ischaemic stroke that met the standards of WHO diagnostic criteria. Radiological MRI confirmation of an anatomical infarct observed on diffusion-weighted acute MRI

Time since stroke: not reported

Inclusion criteria: (1) first-ever acute ischaemic stroke; (2) no history of depression; (3) no antidepressant treatments received before our interventions; (4) over 18 years of age

Exclusion criteria: (1) presence of pre-stroke disease leading to pre-stroke disability; Barthel Index < 10

Depression criteria: 20 -item BDI scores $>10$

Total number randomised in this trial: 138

Number randomised to treatment group: 92 (52\% men, mean age 65 years, SD 8)

Number randomised to control group: $46^{\star \star}$ (53\% men, mean age years 67, SD 10)

Total number included in final analysis: 130

Number included in treatment group for final analysis: 87 (\% men and mean age not reported)

Number included in control group for final analysis: $43^{\star \star}(\%$ men and mean age not reported) 
Gao 2017b (Continued)

Interventions
Treatment: patients received placebo tablets and had an 'active' psychological intervention: professional cognitive-behavioural therapy with psychologists who were trained by a professional cognitive therapist for 1 week. The manual-based treatment included cognitive and behavioural courses that consisted of education, activities, graded task assignments, and identifying and modifying useless beliefs and thoughts. Interventional measures were altered to meet individual demands

Administered by: psychologist trained in professional cognitive therapy

Supervision: not reported

Control: patients received placebo tablets and participated in a placebo psychological intervention, 1-hour discussions with non-psychological clinical doctors twice a week for 3 months; discussions focused on inquiries about stroke recovery and changes in daily life

Administered by: non-psychological clinical doctors

Supervision: not reported

Intervention fidelity: not reported

Duration: 3 months

Follow-up: none

\begin{tabular}{ll}
\hline Outcomes & Primary outcomes \\
- Depression measured using HDRS \\
- Depression measured using Melancholia Scale
\end{tabular}

Secondary outcomes

- Disability measured using BI

Notes

Author contact: emailed study authors to request AE tables with numbers for all groups 23 October 2018

\section{Risk of bias}

\begin{tabular}{lll}
\hline Bias & Authors' judgement & Support for judgement \\
\hline $\begin{array}{l}\text { Random sequence genera- } \\
\text { tion (selection bias) }\end{array}$ & Low risk & $\begin{array}{l}\text { Quote: "randomization into one of three intervention groups was undertaken } \\
\text { by an independent researcher using computer-generated random number se- } \\
\text { quences" (p. 73) }\end{array}$ \\
& & ald
\end{tabular}

Allocation concealment High risk (selection bias)

Quote: "....were prepared in advance and placed in consecutively numbered, sealed, opaque envelopes" (p. 73)

Comment: sealed, opaque envelopes can be tampered with

Blinding of participants High risk and personnel (performance bias)

All outcomes
Quote: "when patients were enrolled, they were told to participate in drug therapy, talk with doctors, and engage in rehabilitation at the same time. No breaches in blinding were detected during the trial" (p. 74)

"the study therapists were asked not to divulge any treatment information to their patients" (p. 75)

Comment: therapists delivering the intervention not blinded to group allocation
Blinding of outcome as- High risk sessment (detection bias)
Quote: "the study therapists acted as clinical evaluators ..." (p. 74) 
Gao 2017b (Continued)

All outcomes

$\begin{array}{ll}\begin{array}{l}\text { Incomplete outcome data } \\ \text { (attrition bias) }\end{array} & \text { High risk } \\ \text { ment not included in the analysis }\end{array}$

All outcomes

ment not included in the analysis

\begin{tabular}{lll}
\hline $\begin{array}{l}\text { Selective reporting (re- } \\
\text { porting bias) }\end{array}$ & Unclear risk & $\begin{array}{l}\text { Comment: all pre-specified outcomes reported; no trial protocol or registry } \\
\text { record available to compare with the publication }\end{array}$ \\
\hline Other bias & Low risk & $\begin{array}{l}\text { Comment: no differences in baseline demographic characteristics between } \\
\text { groups }\end{array}$ \\
\hline
\end{tabular}

Gu 2016

Study design: parallel design
Number of arms: 2
Treatment arm: rTMS
Control arm: sham rTMS

Participants

Geographical location: South Korea

Setting: unclear

Number of participants: 24

Stroke criteria: infarct and haemorrhage

Method of stroke diagnosis: not reported

Time since stroke: $>6$ months

Inclusion criteria: (1) absence of depression or medication history of antidepressants before stroke onset; (2) absence of severe cognitive dysfunction or aphasia; (3) absence of serious medical complications such as pneumonia or cardiac problems; (4) admitted > 6 months after stroke onset; (5) aged between 21 and 80 years only

Exclusion criteria: (1) history of depression before stroke onset; (2) medication history of antidepressants before stroke onset; (3) serious medical complications such as pneumonia or cardiac problems

Depression criteria: BDI scores $>12$ and 17 -item HDRS scores $>6$

Total number randomised in this trial: 24

Number randomised to treatment group: 12 (50\% men, mean age 58 years, SD 9)

Number randomised to control group: 12 (42\% men, mean age 58 years, SD 8)

Total number included in final analysis: 24

Number included in treatment group for final analysis: 12 (50\% men, mean age 58 years, SD 9)

Number included in control group for final analysis: 12 (42\% men, mean age 58 years, SD 8)

Interventions

Treatment: Magstim Super Rapid Magnetic Stimulator (The Magstim Company, Wales, UK) with 70$\mathrm{mm}$, air-cooled coil in the shape of a figure of 8 . The coil was held with the handle posterior and oriented sagittally. rTMS was performed over the left F3 on the scalp according to the 10/20 electroencephalography system (i.e. the DLPFC). For patients in the rTMS group, rTMS was delivered over the DLPFC at $10 \mathrm{~Hz}$, at an intensity of $110 \%$ of the motor threshold, duration of 5 seconds, and total of 20 
Gu 2016 (Continued)

trains separated by 1-minute pauses (total of 1000 pulses). Each patient underwent 10 consecutive sessions (Monday to Friday, 5 times per week for 2 weeks)

Control: sham stimulation was delivered using the same protocol, except that the angle of the coil was at 90 perpendicular to the skull rather than tangential to it. Thus, the magnetic field could not penetrate the brain, although patients could hear the sound that was produced

Administered by: psychiatrist

Duration: 2 weeks

Follow-up: 4 weeks

Primary outcomes
- Depression measured using BDI and 17-item HDRS
Secondary outcomes
- Motor function measured using Upper limb Motoricity Index (MI-UE), lower limb MI-LE, Modified
Brunnstrom Classification (MBC), and Functional Ambulatory Category (FAC)

Notes

Author contact: emailed study authors for method of randomisation, details of blinding of patients, method of stroke diagnosis, number of patients screened/eligible, and sample size calculations 24 October 2018

\section{Risk of bias}

\begin{tabular}{lll}
\hline Bias & Authors' judgement & Support for judgement \\
\hline $\begin{array}{l}\text { Random sequence genera- } \\
\text { tion (selection bias) }\end{array}$ & Unclear risk & $\begin{array}{l}\text { Quote: "all patients were randomly assigned to two groups, the rTMS and } \\
\text { sham groups..."(p. 271) } \\
\text { Comment: } \text { method of sequence generation not reported }\end{array}$ \\
\hline $\begin{array}{l}\text { Allocation concealment } \\
\text { (selection bias) }\end{array}$ & Unclear risk & Comment: method of allocation concealment not reported \\
\hline $\begin{array}{l}\text { Blinding of participants } \\
\text { and personnel (perfor- } \\
\text { mance bias) }\end{array}$ & Unclear risk & $\begin{array}{l}\text { Quote: "a psychiatrist who was blinded to the study protocol performed rTMS } \\
\text { using a Magstim Super Rapid Magnetic Stimulator" (p. 271) }\end{array}$ \\
\hline $\begin{array}{l}\text { Blinding of outcome as- } \\
\text { sessment (detection bias) } \\
\begin{array}{l}\text { All outcomes } \\
\text { Comment: double-blind stated but not reported whether participants were } \\
\text { blinded }\end{array}\end{array}$ & Low risk & $\begin{array}{l}\text { Quote: "the experimenters who applied the rTMS or sham stimulations were } \\
\text { different from the experimenters who assessed the degree of depression and } \\
\text { motor function. The experimenters who assessed depression and motor func- } \\
\text { tion were blinded to the group assignment" (p. 271) }\end{array}$
\end{tabular}

Incomplete outcome data Low risk

Comment: ITT analysis reported; all participants included in the analysis

(attrition bias)

All outcomes

\begin{tabular}{|c|c|c|}
\hline $\begin{array}{l}\text { Selective reporting (re- } \\
\text { porting bias) }\end{array}$ & Unclear risk & $\begin{array}{l}\text { Comment: all pre-specified outcomes reported; no trial protocol or registry } \\
\text { record available to compare with the publication }\end{array}$ \\
\hline
\end{tabular}

porting bias)

Low risk

Comment: no differences in baseline BDI scores and demographic characteristics between groups 
Hoffmann 2015

\begin{tabular}{ll}
\hline Methods & Study design: parallel design \\
& Number of arms: 2 \\
Experimental arm: cognitive-behavioural therapy \\
Control arm: usual care \\
\hline
\end{tabular}

Participants

Geographical location: Australia

Setting: inpatient

Stroke criteria: unclear

Method of stroke diagnosis: diagnosis of stroke confirmed by chart review

Time since stroke: not reported

Inclusion criteria: (1) > 18 years old; (2) adequate cognitive capacity to provide informed consent; (3) adequate English and expressive and receptive communication skills

Exclusion criteria: (1) neurodegenerative disorder (e.g. dementia); (2) living > 50 km away from hospital

Depression criteria: depression score not an entry criteria. For unpublished analysis, HADS $\geq 8$ used for depression criteria

Total number randomised in this trial: 22

Number randomised to treatment group: 12 (75\% men; mean age 60.8, SD 11.7)

Number randomised to control group: 10 (60\% men; mean age 57.0, SD 14.2)

Total number included in final analysis: 17

Number included in treatment group for final analysis: 12 (75\% men; mean age 60.8, SD 11.7)

Number included in control group for final analysis: 5 (60\% men; mean age 57.0, SD 14.2) with first 2 sessions in hospital, then 6 delivered at home

Administered by: clinical psychologist

Supervision: psychologist

Intervention fidelity: $9 / 11$ patients received 8 sessions; $7 / 11$ received sessions in the intended location

Control: usual care

Duration: 8 weeks

Follow-up: 3 months

- Depression measured using HADS and MADRS

- Anxiety measured using HADS

\section{Secondary outcomes}

- Disability measured using MBI

- Self-efficacy measured using Stroke Self Efficacy Questionnaire 
Hoffmann 2015 (Continued)

- Functional capacity measured using Nottingham EADL

- Knowledge of stroke measured using Stroke Knowledge Questionnaire

- Quality of life measured using SAQoL

Notes

This trial had 3 arms (self-management therapy, cognitive-behavioural therapy, and usual care), but only data from cognitive-behavioural therapy compared with usual care ( $\mathrm{n}=17$ participants) are presented here

\section{Risk of bias}

\begin{tabular}{|c|c|c|}
\hline Bias & Authors' judgement & Support for judgement \\
\hline $\begin{array}{l}\text { Random sequence genera- } \\
\text { tion (selection bias) }\end{array}$ & Low risk & $\begin{array}{l}\text { Quote: "... randomly allocated using a predetermined computer generated } \\
\text { randomisation sequence ..." (p. 118) }\end{array}$ \\
\hline $\begin{array}{l}\text { Allocation concealment } \\
\text { (selection bias) }\end{array}$ & High risk & $\begin{array}{l}\text { Comment: sealed opaque envelopes reported; this method of allocation con- } \\
\text { cealment can be tampered with }\end{array}$ \\
\hline $\begin{array}{l}\text { Blinding of participants } \\
\text { and personnel (perfor- } \\
\text { mance bias) } \\
\text { All outcomes }\end{array}$ & High risk & $\begin{array}{l}\text { Comment: due to the nature of the trial, it was not possible to mask partici- } \\
\text { pants, personnel delivering the intervention, and researchers to treatment al- } \\
\text { location }\end{array}$ \\
\hline
\end{tabular}

\begin{tabular}{|c|c|c|}
\hline $\begin{array}{l}\text { Blinding of outcome as- } \\
\text { sessment (detection bias) } \\
\text { All outcomes }\end{array}$ & Low risk & $\begin{array}{l}\text { Quote: "outcomes were assessed in a face-to-face interview conducted by a } \\
\text { research assistant (a registered psychologist) who was blind to group alloca- } \\
\text { tion" (p. 118) }\end{array}$ \\
\hline
\end{tabular}

\begin{tabular}{|c|c|c|}
\hline $\begin{array}{l}\text { Incomplete outcome data } \\
\text { (attrition bias) } \\
\text { All outcomes }\end{array}$ & Low risk & $\begin{array}{l}\text { Quote: "analysis was completed using ... and on an intention to treat basis and } \\
\text { missing data were addressed using the last observation carried forward proce- } \\
\text { dure" (p. 120) }\end{array}$ \\
\hline
\end{tabular}

All outcomes dure" (p. 120)

Comment: ITT analysis reported. From whole data set, including depressed and non-depressed, 1 intervention and 1 control withdrew post randomisation

\begin{tabular}{|c|c|c|}
\hline $\begin{array}{l}\text { Selective reporting (re- } \\
\text { porting bias) }\end{array}$ & Unclear risk & $\begin{array}{l}\text { Comment: all pre-specified outcomes reported; no trial protocol available to } \\
\text { compare with the publication }\end{array}$ \\
\hline
\end{tabular}

\begin{tabular}{ll}
\hline Other bias $\quad$ Unclear risk $\quad$ Comment: baseline demographic information not reported \\
\hline
\end{tabular}

Huang 2002

\begin{tabular}{ll}
\hline Sethods & Numb design: parallel design \\
Number of arms: 2 \\
Experimental arm: fluoxetine (SSRI) \\
Control arm: matched placebo \\
\hline Geographical location: China \\
Setting: inpatient \\
Stroke criteria: first-ever ischaemic or haemorrhagic stroke \\
Method of stroke diagnosis: diagnosis is consistent with the diagnostic criteria for acute stroke formu- \\
lated by the Chinese Medical Association with 1 single and unilateral lesion confirmed by brain CT or \\
MRI
\end{tabular}


Time since stroke: unclear

Inclusion criteria: none reported

Exclusion criteria: (1) history of psychiatric illness; (2) severe heart disease; (3) previous organic brain disease; (4) severe liver or kidney disease; (5) history of drug allergy

Depression criteria: psychiatric interview to confirm diagnosis meets depression diagnostic criteria of the CCMD-2-R

Total number randomised in this trial: 80 (overall percentage of men 45\%; 80 patients were a depressive subgroup of 168 patients whose mean age was 62.2 years, SD 8.1)

Number randomised to treatment group: 40 ( $\%$ men and mean age in treatment group not reported)

Number randomised to control group: 40 (\% men and mean age in control group not reported; total group as above)

Total number included in final analysis: 80 (overall percentage of men $45 \% ; 80$ patients were a depressive subgroup of 168 patients whose mean age was 62.2 years, SD 8.1)

Number included in treatment group for final analysis: 40 (\% men and mean age in treatment group not reported)

Number included in control group for final analysis: 40 (\% men and mean age in control group not reported; total group as above)

Interventions

Treatment: fluoxetine (SSRI) $20 \mathrm{mg} / \mathrm{d}$ in the morning

Control: matched placebo

Duration: 4 weeks

Follow-up: none

\begin{tabular}{ll}
\hline Outcomes & Primary outcomes \\
- Depression measured using CCMD-2-R and 17-iter & Secondary outcomes \\
- Neurological impairment measured using CSS \\
- Adverse events
\end{tabular}

Notes

\section{Risk of bias}

Bias Authors' judgement Support for judgement

Random sequence genera- Unclear risk Comment: method of sequence generation not reported tion (selection bias)

Allocation concealment Unclear risk Comment: method of allocation concealment not reported
(selection bias)

Blinding of participants Unclear risk Comment: blinding of participants and personnel not reported
and personnel (perfor-
mance bias)
All outcomes

\begin{tabular}{l}
\hline $\begin{array}{l}\text { Blinding of outcome as- } \\
\text { sessment (detection bias) }\end{array}$ \\
\hline Pharmacological, psychological, and non-invasive brain stimulation interventions for treating depression after stroke (Review) \\
Copyright @ 2020 The Cochrane Collaboration. Published by John Wiley \& Sons, Ltd.
\end{tabular}


Huang 2002 (Continued)

All outcomes

Incomplete outcome data Low risk Comment: ITT analysis reported; no missing data reported
(attrition bias)

(attrition bias)

All outcomes

\begin{tabular}{lll}
\hline $\begin{array}{l}\text { Selective reporting (re- } \\
\text { porting bias) }\end{array}$ & Unclear risk & $\begin{array}{l}\text { Comment: all pre-specified outcomes reported; no trial protocol available to } \\
\text { compare with the publication }\end{array}$ \\
\hline Other bias & Low risk & Comment: no differences in baseline HAMD and CSS scores between groups \\
\hline
\end{tabular}

Jiang 2001a

\begin{tabular}{ll}
\hline Methods & $\begin{array}{l}\text { Study design: parallel design } \\
\text { Number of arms: } 2\end{array}$ \\
Experimental arm: amitriptyline (TCA) \\
Control arm: placebo (not matched) \\
Geographical location: China \\
Setting: inpatient \\
Stroke criteria: unclear \\
Method of stroke diagnosis: diagnosis via CT or MRI (100\%) \\
Time since stroke: 0 to 7 days \\
Inclusion criteria: $(1)$ Chinese Stroke Scale score > 8; (2) can independently complete HDRS, aged < 80 \\
years; (3) no severe negative life events in past year; (4) first stroke; (5) no previous psychosis; (6) no an- \\
tidepressant medication
\end{tabular}

Exclusion criteria: (1) with history of psychosis; (2) on antidepressant medication

Depression criteria: $\mathrm{HDRS}>8$

Total number randomised in this trial: 45

Number randomised to treatment group: 30 (57\% men, mean age 62 years, SD 14)

Number randomised to control group: $15^{\star \star}(60 \%$ men, mean age 63 years, SD 15)

Total number included in final analysis: 45

Number included in treatment group for final analysis: 30 (57\% men, mean age 62 years, SD 14)

Number included in control group for final analysis: $15^{\star \star}(60 \%$ men, mean age 63 years, SD 15)

\begin{tabular}{ll}
\hline Interventions & Treatment: amitriptyline (TCA) 50 mg increasing by $25 \mathrm{mg}$ per day to $200 \mathrm{mg}$ daily \\
Control: placebo (not matched) 2 tablets per day \\
Duration: 6 months \\
Follow-up: none
\end{tabular}

Outcomes

Primary outcomes

- Depression measured using HDRS

Secondary outcomes

- Impairment measured using CSS 
Jiang 2001a (Continued)

- Adverse events

Notes

\section{Risk of bias}

\begin{tabular}{|c|c|c|}
\hline Bias & Authors' judgement & Support for judgement \\
\hline $\begin{array}{l}\text { Random sequence genera- } \\
\text { tion (selection bias) }\end{array}$ & Unclear risk & Comment: method of sequence generation not reported \\
\hline $\begin{array}{l}\text { Allocation concealment } \\
\text { (selection bias) }\end{array}$ & High risk & $\begin{array}{l}\text { Comment: } 3 \text {-armed trial. Placebo frequency matched to Deanxit (intervention } \\
\text { in third arm) - not to amitriptyline }\end{array}$ \\
\hline $\begin{array}{l}\text { Blinding of participants } \\
\text { and personnel (perfor- } \\
\text { mance bias) } \\
\text { All outcomes }\end{array}$ & High risk & Comment: participants blinded but personnel not blinded \\
\hline $\begin{array}{l}\text { Blinding of outcome as- } \\
\text { sessment (detection bias) } \\
\text { All outcomes }\end{array}$ & Unclear risk & Comment: blinding of outcome assessment not reported \\
\hline $\begin{array}{l}\text { Incomplete outcome data } \\
\text { (attrition bias) } \\
\text { All outcomes }\end{array}$ & Low risk & $\begin{array}{l}\text { Comment: ITT analysis reported (complete follow-up of all randomised partic- } \\
\text { ipants) }\end{array}$ \\
\hline $\begin{array}{l}\text { Selective reporting (re- } \\
\text { porting bias) }\end{array}$ & Unclear risk & $\begin{array}{l}\text { Comment: all pre-specified outcomes reported; no trial protocol available to } \\
\text { compare with the publication }\end{array}$ \\
\hline Other bias & High risk & $\begin{array}{l}\text { Comment: intervention group was younger and had higher HDRS score and } \\
\text { lower CSS score }\end{array}$ \\
\hline
\end{tabular}

Jiang 2001b

\begin{tabular}{ll}
\hline Methods & $\begin{array}{l}\text { Study design: parallel design } \\
\text { Number of arms: } 2\end{array}$ \\
Experimental arm: Deanxit \\
Control arm: placebo (not matched) \\
Geographical location: China \\
Setting: inpatient \\
Stroke criteria: unclear \\
Method of stroke diagnosis: diagnosis via CT or MRI (100\%) \\
Time since stroke: 0 to 7 days \\
Inclusion criteria: (1) CSS score > 8; (2) can independently complete HDRS, aged < 80 years; $(3)$ no se- \\
vere negative life events in past year; (4) first stroke; (5) no previous psychosis; $(6)$ no antidepressant \\
medication
\end{tabular}

Exclusion criteria: (1) with history of psychosis; (2) on antidepressant medication

Depression criteria: $\mathrm{HDRS}>8$ 


\section{Total number randomised in this trial: 45}

Number randomised to treatment group: 30 ( $58 \%$ men, mean age 62 years, SD 14)

Number randomised to control group: $15^{\star \star}(60 \%$ men, mean age 63 years, SD 15)

Total number included in final analysis: 45

Number included in treatment group for final analysis: 30 (58\% men, mean age 62 years, SD 14)

Number included in control group for final analysis: $15^{\star \star}(60 \%$ men, mean age 63 years, SD 15)

\begin{tabular}{ll}
\hline Interventions & Treatment: Deanxit 2 tablets daily \\
Control: placebo (not matched but frequency matched) \\
Duration: 6 months \\
Follow-up: none
\end{tabular}

Outcomes

Primary outcomes

- Depression measured using HDRS

Secondary outcomes

- Impairment measured using CSS

- Adverse events

Notes

\section{Risk of bias}

\begin{tabular}{lll}
\hline Bias & Authors' judgement & Support for judgement \\
\hline $\begin{array}{l}\text { Random sequence genera- } \\
\text { tion (selection bias) }\end{array}$ & Unclear risk & Comment: method of sequence generation not reported \\
\hline $\begin{array}{l}\text { Allocation concealment } \\
\text { (selection bias) }\end{array}$ & High risk & $\begin{array}{l}\text { Comment: 3-armed trial. Placebo frequency matched to Deanxit (intervention } \\
\text { in third arm) - not to amitriptyline }\end{array}$ \\
\hline $\begin{array}{l}\text { Blinding of participants } \\
\text { and personnel (perfor- } \\
\text { mance bias) }\end{array}$ & High risk & Comment: participants blinded but personnel not blinded \\
All outcomes & & \\
\hline
\end{tabular}

\begin{tabular}{ll}
\hline Blinding of outcome as- & Unclear risk
\end{tabular} Comment: blinding of outcome assessment not reported

All outcomes

\begin{tabular}{lll}
\hline $\begin{array}{l}\text { Incomplete outcome data } \\
\text { (attrition bias) } \\
\text { All outcomes }\end{array}$ & Low risk & $\begin{array}{l}\text { Comment: ITT analysis reported (complete follow-up of all randomised partic- } \\
\text { ipants) }\end{array}$ \\
\hline $\begin{array}{l}\text { Selective reporting (re- } \\
\text { porting bias) }\end{array}$ & Unclear risk & $\begin{array}{l}\text { Comment: all pre-specified outcomes reported; no trial protocol available to } \\
\text { compare with the publication }\end{array}$ \\
\hline
\end{tabular}

Other bias

High risk

Comment: intervention group was younger and had higher HDRS score and lower CSS score 
Jiang 2014a

Methods

Study design: parallel design

Number of arms: 2

Experimental arm: TMS + acute stroke usual care

Control arm: acute stroke usual care

Participants

Geographical location: China

Setting: inpatient

Stroke criteria: Internal carotid artery territory infarct

Method of stroke diagnosis: clinical diagnosis plus confirmation by imaging that a relevant lesion needed to be visible

Time since stroke: 3 to 10 days

Inclusion criteria: (1) first-ever stroke; (2) age 30 to 70 years; (3) NIHSS at admission 8 to 20 points; (4) GCS scale score > 8; (5) education level: at least high school, able to complete questionnaires; (6) no communication barriers, able to communicate with medical staff; (7) can sign informed consent

Exclusion criteria: (1) comorbid severe organ failure; (2) history of epilepsy or consciousness disturbance; (3) contraindication for transcranial magnetic stimulation such as pacemaker implanted, severe cardiac dysrhythmia; (4) worsened clinical condition, new infarct, or haemorrhagic transformation

Depression criteria: not reported

Total number randomised in this trial: 100

Number randomised to treatment group: 50 (\% men and mean age not reported)

Number randomised to control group: 50 (\% men and mean age not reported)

Total number included in final analysis: 100

Number included in treatment group for final analysis: 50 (\% men and mean age not reported)

Number included in control group for final analysis: 50 (\% men and mean age not reported)

Control: acute stroke usual care

Duration: 12 weeks

Follow-up: 3 months

Outcomes Primary outcomes

- Depression measured using HDRS

- Impairment measured using NIHSS

- Activities of daily living measured using ADL

\section{Notes}

\section{Risk of bias}

Bias Authors' judgement Support for judgement


Jiang 2014a (Continued)

Random sequence genera- Low risk Comment: random numbers table used for sequence generation tion (selection bias)

Allocation concealment $\quad$ Unclear risk $\quad$ Comment: method of allocation concealment not reported
(selection bias)

\section{Blinding of participants Unclear risk} and personnel (performance bias)

All outcomes

\begin{tabular}{lll}
\hline $\begin{array}{l}\text { Blinding of outcome as- } \\
\text { sessment (detection bias) } \\
\text { All outcomes }\end{array}$ & Low risk & Comment: outcome assessors blinded \\
\hline $\begin{array}{l}\text { Incomplete outcome data } \\
\begin{array}{l}\text { (attrition bias) } \\
\text { All outcomes }\end{array}\end{array}$ & High risk & $\begin{array}{l}\text { Comment: per protocol analysis reported only; 1 participant dropped out and } \\
\text { was not included in the analysis }\end{array}$ \\
\hline $\begin{array}{l}\text { Selective reporting (re- } \\
\text { porting bias) }\end{array}$ & Unclear risk & $\begin{array}{l}\text { Comment: all pre-specified outcomes reported; no trial protocol available to } \\
\text { compare with the publication }\end{array}$ \\
\hline Other bias & Low risk & Comment: no differences in baseline demographics between groups \\
\hline
\end{tabular}

\begin{tabular}{ll}
\hline Study design: parallel design \\
Number of arms: 2 \\
Experimental arm: TMS + sertraline (SSRI) + acute stroke usual care \\
Control arm: sertraline (SSRI) + acute stroke usual care \\
\hline
\end{tabular}

\section{Geographical location: China}

Setting: inpatient

Stroke criteria: internal carotid artery territory infarct

Method of stroke diagnosis: clinical diagnosis plus confirmation by imaging that a relevant lesion needed to be visible

Time since stroke: 3 to 10 days

Inclusion criteria: (1) first-ever stroke; (2) age 30 to 70 years; (3) NIHSS at admission 8 to 20 points; (4) GCS scale score > 8; (5) education level: at least high school, able to complete questionnaires; (6) no communication barriers, able to communicate with medical staff; (7) can sign informed consent

Exclusion criteria: (1) comorbid severe organ failure; (2) history of epilepsy or consciousness disturbance; (3) contraindication for transcranial magnetic stimulation such as pacemaker implanted, severe cardiac dysrhythmia; (4) worsening clinical condition, new infarct, or haemorrhagic transformation

Depression criteria: not reported

Total number randomised in this trial: 100

Number randomised to treatment group: 50 (\% men and mean age not reported)

Number randomised to control group: 50 (\% men and mean age not reported) 
Jiang 2014b (Continued)

Total number included in final analysis: 99

Number included in treatment group for final analysis: 50

Number included in control group for final analysis: 49
Treatment: TMS + sertraline (SSRI) $50 \mathrm{mg} / \mathrm{d}$ + acute stroke usual care; frequency: start 3 to 10 days after stroke onset, 2 times a day, 20 minutes each time, for successive 14 days, location: motor cortex on the healthy side

Control: sertraline (SSRI) $50 \mathrm{mg} / \mathrm{d}+$ acute stroke usual care

Duration: 12 weeks

Follow-up: 3 months

\section{Primary outcomes}

- Depression measured using HDRS

- Impairment measured using NIHSS

- Activities of daily living measured using ADL

\section{Notes}

\section{Risk of bias}

\begin{tabular}{|c|c|c|}
\hline Bias & Authors' judgement & Support for judgement \\
\hline $\begin{array}{l}\text { Random sequence genera- } \\
\text { tion (selection bias) }\end{array}$ & Low risk & Comment: random numbers table used for sequence generation \\
\hline $\begin{array}{l}\text { Allocation concealment } \\
\text { (selection bias) }\end{array}$ & Unclear risk & Comment: method of allocation concealment not reported \\
\hline $\begin{array}{l}\text { Blinding of participants } \\
\text { and personnel (perfor- } \\
\text { mance bias) } \\
\text { All outcomes }\end{array}$ & Unclear risk & $\begin{array}{l}\text { Comment: information about blinding of participants and personnel not re- } \\
\text { ported }\end{array}$ \\
\hline $\begin{array}{l}\text { Blinding of outcome as- } \\
\text { sessment (detection bias) } \\
\text { All outcomes }\end{array}$ & Low risk & Comment: outcome assessors blinded \\
\hline $\begin{array}{l}\text { Incomplete outcome data } \\
\text { (attrition bias) } \\
\text { All outcomes }\end{array}$ & High risk & $\begin{array}{l}\text { Comment: per protocol analysis reported only; } 1 \text { participant dropped out and } \\
\text { was not included in the analysis }\end{array}$ \\
\hline $\begin{array}{l}\text { Selective reporting (re- } \\
\text { porting bias) }\end{array}$ & Unclear risk & $\begin{array}{l}\text { Comment: all pre-specified outcomes reported; no trial protocol available to } \\
\text { compare with the publication }\end{array}$ \\
\hline Other bias & Low risk & Comment: no differences in baseline demographics between groups \\
\hline
\end{tabular}

Jin 2013

Methods Study design: parallel design

Number of arms: 2 
Jin 2013 (Continued)

Experimental arm: rTMS + sertraline (SSRI) + usual care

Control arm: sertraline (SSRI) + usual care

Participants

Geographical location: China

Setting: inpatient

Stroke criteria: ischaemic stroke

Method of stroke diagnosis: clinical diagnosis plus confirmation by imaging that a relevant lesion needed to be visible

Inclusion criteria: (1) without cerebral haemorrhage; (2) cerebral infarct history; (3) without epilepsy history; (4) EEG showing no epileptiform discharge; (5) without head injury or intracranial infection history; (6) without intracranial metal or other foreign body

Exclusion criteria: not reported

Depression criteria: 17 -item HDRS score $\geq 17$

Total number randomised in this trial: 60

Number randomised to treatment group: 30 ( $63 \%$ men; mean age 56.0, SD 9.8)

Number randomised to control group: 30 (51\% men; mean age 54.0, SD 10.2)

Total number included in final analysis: 60

Number included in treatment group for final analysis: 30

Number included in control group for final analysis: 30 motor threshold, with each stimulation lasting 4 seconds with an interval of 56 seconds, total 20 minutes each treatment, 1 treatment per day, 5 treatments per week, location: left DLPFC

Control: sertraline (SSRI) $100 \mathrm{mg} / \mathrm{d}+$ usual care

Duration: 4 weeks

Follow-up: none

\begin{tabular}{ll}
\hline Outcomes & Primary outcomes \\
& - Depression measured using 17-item HDRS \\
- Impairment measured using NIHSS
\end{tabular}

Notes

Risk of bias

\begin{tabular}{lll}
\hline Bias & Authors' judgement & Support for judgement \\
\hline $\begin{array}{l}\text { Random sequence genera- } \\
\text { tion (selection bias) }\end{array}$ & Unclear risk & Comment: method of sequence generation not reported \\
\hline $\begin{array}{l}\text { Allocation concealment } \\
\text { (selection bias) }\end{array}$ & Unclear risk & Comment: method of allocation concealment not reported \\
\hline $\begin{array}{l}\text { Blinding of participants } \\
\text { and personnel (perfor- } \\
\text { mance bias) }\end{array}$ & Unclear risk & $\begin{array}{l}\text { Comment: information about blinding of participants and personnel not re- } \\
\text { ported }\end{array}$ \\
\hline
\end{tabular}

Pharmacological, psychological, and non-invasive brain stimulation interventions for treating depression after stroke (Review) 
Jin 2013 (Continued)

All outcomes

Blinding of outcome as-
sessment (detection bias) $\quad$ Unclear risk Comment: information about blinding of outcome assessors not reported
sessment (detection bias)

All outcomes

Incomplete outcome data Low risk Comment: ITT analysis reported; no missing data
(attrition bias)

All outcomes

\begin{tabular}{|c|c|c|}
\hline $\begin{array}{l}\text { Selective reporting (re- } \\
\text { porting bias) }\end{array}$ & Unclear risk & $\begin{array}{l}\text { Comment: all pre-specified outcomes reported; no trial protocol available to } \\
\text { compare with the publication }\end{array}$ \\
\hline
\end{tabular}

porting bias) compare with the publication

\begin{tabular}{ll}
\hline Other bias Low risk Comment: no differences in baseline demographics between groups \\
\hline
\end{tabular}

Kerr 2018

\begin{tabular}{ll}
\hline Methods & Study design: parallel design \\
Number of arms: 2 \\
Experimental arm: individual motivational interviewing \\
Control arm: usual care
\end{tabular}

Participants

Geographical location: Australia

Setting: inpatient

Stroke criteria: cerebral infarction/intracerebral haemorrhage

Method of stroke diagnosis: medical diagnosis confirmed by neurologist in the medical notes

Time since stroke: not reported

Inclusion criteria: (1) acute presentation after acute stroke (cerebral infarction/intracerebral haemorrhage; (2) cognitively alert

Exclusion criteria: (1) subarachnoid haemorrhage; (2) mental health conditions, including depressive symptoms requiring professional support within 1 month; (3) severe communication problems (e.g. significant dysphasia or aphasia); (4) myocardial infarction; (5) concurrent neurological disease/trauma

Depression criteria: none

Total number randomised in this trial (stroke participants only): 10

Number randomised to treatment group: 4 (25\% men, mean age 57 years, SD 20.8)

Number randomised to control group: 6 (50\% men, mean age 65.8 years, SD 12.9)

Total number included in final analysis (stroke participants only): 9

Number randomised to treatment group: 4

Number included in control group: 5

Interventions

Treatment: the over-arching principle of the intervention was to support the stroke survivor in adjusting to life after stroke. The purpose of Session 1 was to set the agenda and encourage the patient to talk about adjustment to stroke. In Session 2, the patient was encouraged to identify realistic goals for recovery and barriers to achieving goals. In Session 3, the goals were to identify any ambivalence that the patient had about achieving goals; to support the patient's optimism and self-efficacy, and to as- 
Kerr 2018 (Continued)

sist in identification of solutions to problems. Participants were encouraged to summarise their goals and commitment and to clarify any information from the first 2 sessions. Sessions were scheduled for 30 minutes

Administered by: trained facilitators

Supervision: not stated

Intervention fidelity: not reported

Control: usual care

Duration: not reported

Follow-up: 1 month and 3 months

Primary outcomes
- Feasibility (application, recruitment, and retention)
Primary clinical outcomes
- Depression measured using HADS and PHQ-9
- Anxiety measured using HADS
- Quality of life measured using quality of life Index

Secondary outcomes

- Disability measured using MBI

Notes

Risk of bias

\begin{tabular}{lll}
\hline Bias & Authors' judgement & Support for judgement \\
\hline $\begin{array}{l}\text { Random sequence genera- } \\
\text { tion (selection bias) }\end{array}$ & Low risk & $\begin{array}{l}\text { Quote: "a computer-generated block randomisation list equally divided all } \\
\text { numbers between } 1 \text { and } 60 \text { into either treatment or control groups" (p. 3) }\end{array}$ \\
\hline
\end{tabular}

Allocation concealment Low risk (selection bias)

Quote: "allocation to the intervention or control arms was concealed from participants until after recruitment and baseline data collection. Envelopes were prepared by the Principal Investigator and stored in a locked cupboard in the ward. The envelopes were numbered sequentially, indicating the order in which participants were enrolled into the study (e.g. the first participant received the envelope labelled "Number 1 ", the second participant received the envelope "Number 2 ", etc.). A note in the envelope indicated the allocation (to intervention or control group), concealed by coloured paper to protect the identity of the allocation group. The project manager opened the randomisation envelopes after baseline data collection" (p. 3)

\begin{tabular}{|c|c|c|}
\hline $\begin{array}{l}\text { Blinding of participants } \\
\text { and personnel (perfor- } \\
\text { mance bias) } \\
\text { All outcomes }\end{array}$ & High risk & $\begin{array}{l}\text { Quote: "allocation to the intervention or control arms was concealed from } \\
\text { participants until after recruitment and baseline data collection" (p. 3) "Al- } \\
\text { though intentionally blinded, the research assistant may have become aware } \\
\text { of the allocation in conversation with the participant" (p. 5) }\end{array}$ \\
\hline
\end{tabular}

\begin{tabular}{lll}
\hline Blinding of outcome as- & High risk & Quote: "the research assistant, a nurse with significant research experience, \\
sessment (detection bias) & was employed to collect data at the 2 follow-up time points. Although inten- \\
All outcomes & tionally blinded, the research assistant may have become aware of the alloca- \\
& tion in conversation with the participant" (p. 5)
\end{tabular}

Incomplete outcome data Unclear risk (attrition bias)
Comment: per protocol analysis reported only; $10 / 48$ participants not included in the analysis

Pharmacological, psychological, and non-invasive brain stimulation interventions for treating depression after stroke (Review) 
Kerr 2018 (Continued)

All outcomes

Selective reporting (re- High risk Comment: Barthel Index not reported in the publication
porting bias)

\begin{tabular}{ll}
\hline Other bias $\quad$ Low risk & $\begin{array}{l}\text { Comment: no differences in baseline demographic characteristics between } \\
\text { groups }\end{array}$
\end{tabular}

Kirkness 2017a

\begin{tabular}{ll}
\hline Study design: parallel design \\
Number of arms: 2 \\
Experimental arm: brief psychosocial-behavioural intervention (in-person) \\
Control arm: usual care
\end{tabular}

Participants

\section{Geographical location: USA}

Setting: outpatient

Stroke criteria: ischaemic or haemorrhagic stroke

Method of stroke diagnosis: ischaemic or haemorrhagic stroke (verified by CT or MRI)

Time since stroke: 4 months

Inclusion criteria: (1) those with ischaemic or haemorrhagic stroke; (2) GDS score > 11; (3) within 4 months of stroke onset

Exclusion criteria: (1) GDS score < 11; (2) not within 4 months of stroke onset

Depression criteria: GDS score $<11$

Total number randomised in this trial: 49

Number randomised to treatment group: 35 (48.6\% men, mean age 58.5 years, SD not reported)

Number randomised to control group: $14^{\star \star}$ (50\% men, mean age 60.7 years, SD not reported)

Total number included in final analysis: 44

Number included in treatment group for final analysis: 31

Number included in control group for final analysis: $13^{* *}$

Treatment: brief in-person psychosocial-behavioural intervention (had 1 in-person orientation session with the psychosocial nurse practitioner therapist, either at home or at our study offices. Participant received participant manuals, discussed goals and expectations of each session, and learned how to fill out homework sections

Administered by: psychosocial nurse practitioner therapist

Supervised by: not reported

Treatment fidelity: not reported

Control: usual care (participants reported on their progress at follow-up visits in their homes from research nurses at 8 weeks, 21 weeks, and 12 months following entry to the study).

Duration: 8 weeks 
Kirkness 2017a (Continued)

Follow-up: 10 months

\begin{tabular}{|c|c|}
\hline Outcomes & $\begin{array}{l}\text { Primary outcomes } \\
\text { - Response (per cent reduction in HDRS) } \\
\text { - Remission (HDRS score }<10 \text { ) at } 8 \text { weeks and } 12 \text { months post treatment }\end{array}$ \\
\hline Notes & $\begin{array}{l}\text { Emailed study authors to request mean and SD for HDRS, BI, and NIHSS score at } 8 \text { weeks and } 12 \\
\text { months post treatment for all } 3 \text { groups } 23 \text { October } 2018 \text { (reply received - mean SD and remission for } \\
\text { HDRS and BI for all treatment groups sent by study author 06/11/2018) }\end{array}$ \\
\hline
\end{tabular}

\section{Risk of bias}

\begin{tabular}{lll}
\hline Bias & Authors' judgement & Support for judgement \\
\hline $\begin{array}{ll}\text { Random sequence genera- } \\
\text { tion (selection bias) }\end{array}$ & Low risk & $\begin{array}{l}\text { Quote: "the algorithm was based on an imbalance score which measured, for } \\
\text { a given set of random assignments, how far out of balance the study would be } \\
\text { within strata for each factor and then summed over factors. When a new sub- } \\
\text { ject was available for randomization, we computed what the imbalance score } \\
\text { would be if this subject were assigned to usual care, or to telephone interven- } \\
\text { tion, or to in-person intervention. Then randomization was done to allocate } \\
\text { two intervention participants to each control with each new assignment hav- } \\
\text { ing a higher probability of less imbalance. The schema did not require equal } \\
\text { numbers in each arm" (p. 4) }\end{array}$
\end{tabular}

\begin{tabular}{|c|c|c|}
\hline $\begin{array}{l}\text { Allocation concealment } \\
\text { (selection bias) }\end{array}$ & Unclear risk & $\begin{array}{l}\text { Quote: "the study statistician generated the algorithm, which was secure- } \\
\text { ly stored and accessible only by the statistician and research nurse supervi- } \\
\text { sor" (p. 5) }\end{array}$ \\
\hline
\end{tabular}

Comment: method of allocation concealment not reported

\begin{tabular}{|c|c|c|}
\hline $\begin{array}{l}\text { Blinding of participants } \\
\text { and personnel (perfor- } \\
\text { mance bias) } \\
\text { All outcomes }\end{array}$ & High risk & $\begin{array}{l}\text { Quote: "participants were asked not to reveal their study arm to the outcome } \\
\text { assessors" (p. 5) } \\
\text { Comment: blinding of personnel not reported }\end{array}$ \\
\hline $\begin{array}{l}\text { Blinding of outcome as- } \\
\text { sessment (detection bias) } \\
\text { All outcomes }\end{array}$ & Low risk & $\begin{array}{l}\text { Quote: "... masking outcome assessors to the participant's randomization sta- } \\
\text { tus. Participants were asked not to reveal their study arm to the outcome as- } \\
\text { sessors. We did not detect any breaches in masking" (p. 5) }\end{array}$ \\
\hline $\begin{array}{l}\text { Incomplete outcome data } \\
\text { (attrition bias) } \\
\text { All outcomes }\end{array}$ & High risk & $\begin{array}{l}\text { Comment: per protocol analysis reported only. } 9 \text { participants not included in } \\
\text { the analysis }\end{array}$ \\
\hline $\begin{array}{l}\text { Selective reporting (re- } \\
\text { porting bias) }\end{array}$ & Unclear risk & $\begin{array}{l}\text { Comment: all pre-specified outcomes reported; no trial protocol to compare } \\
\text { with the publication }\end{array}$ \\
\hline Other bias & Low risk & $\begin{array}{l}\text { Comment: no differences in baseline demographic characteristics between } \\
\text { groups }\end{array}$ \\
\hline
\end{tabular}

Kirkness 2017b

Study design: parallel design
Number of arms: 2
Experimental arm: brief psychosocial-behavioural intervention (telephone)


Kirkness 2017b (Continued)

Control arm: usual care

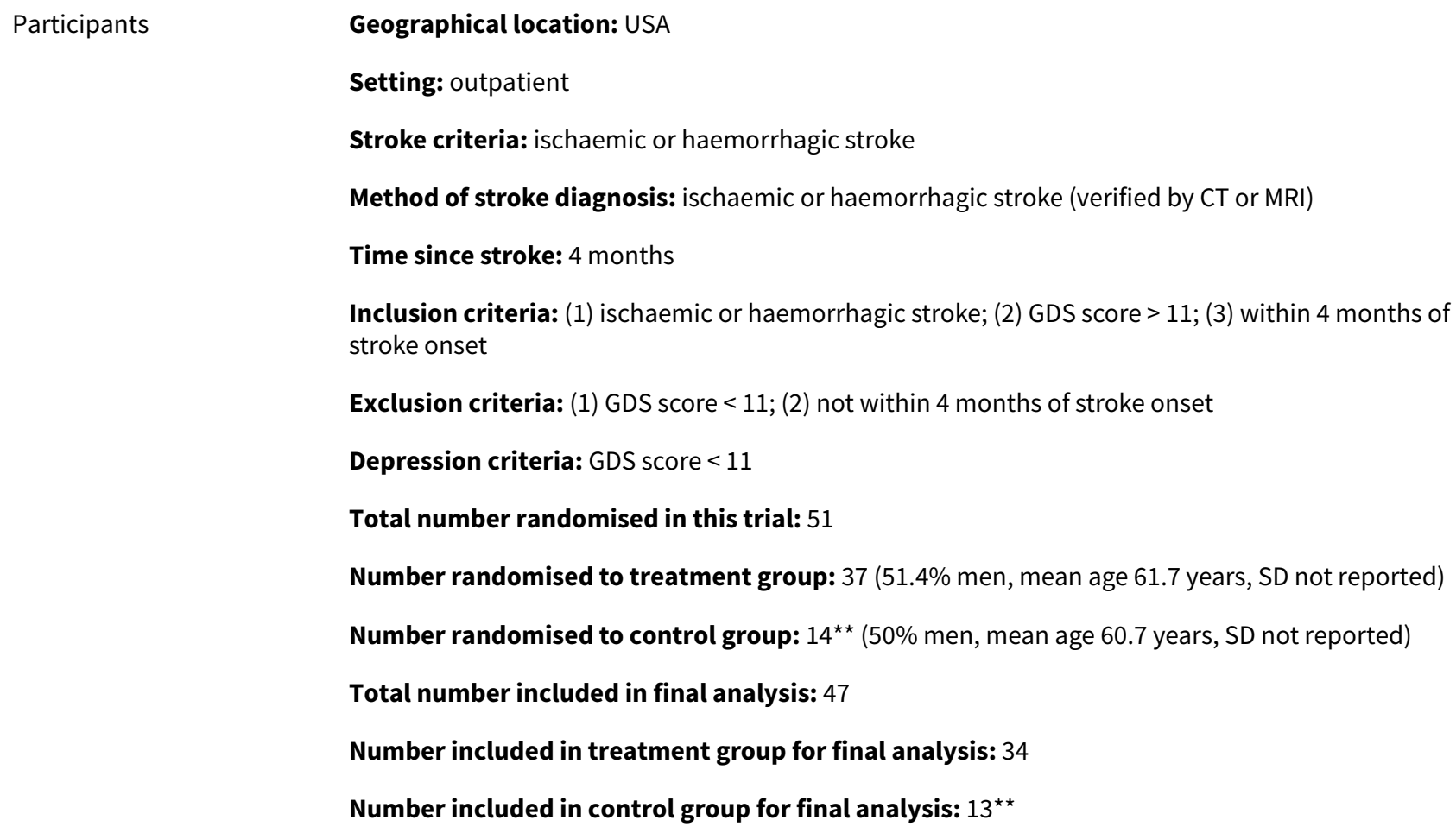
sion with psychosocial nurse practitioner therapist, either at home or at our study offices. Participants received participant manuals, discussed goals and expectations of each session, and learned how to fill out homework sections

Administered by: psychosocial nurse practitioner therapist

Supervised by: not reported

Treatment fidelity: not reported

Control: usual care (participants reported on their progress at follow-up visits in their homes from research nurses at 8 weeks, 21 weeks, and 12 months following entry to the study)

Duration: 8 weeks

Follow-up: 10 months

\begin{tabular}{ll} 
Outcomes & Primary outcomes \\
& - Response (per cent reduction in HDRS) \\
& Remission (HDRS score < 10) at 8 weeks and 12 months post treatment \\
\hline Notes & $\begin{array}{l}\text { Emailed study authors to request mean and SD for HDRS, BI, and NIHSS score at } 8 \text { weeks and } 12 \\
\text { months post treatment for all } 3 \text { groups } 23 / 10 / 2018 \text { (reply received - mean SD and remission for HDRS } \\
\text { and BI for all treatment groups sent by trial author } 06 / 11 / 2018)\end{array}$
\end{tabular}

\section{Risk of bias}

\begin{tabular}{lll}
\hline Bias & Authors' judgement & Support for judgement \\
\hline $\begin{array}{l}\text { Random sequence genera- } \\
\text { tion (selection bias) }\end{array}$ & Low risk & $\begin{array}{l}\text { Quote: "the algorithm was based on an imbalance score which measured, for } \\
\text { a given set of random assignments, how far out of balance the study would be }\end{array}$
\end{tabular}

Pharmacological, psychological, and non-invasive brain stimulation interventions for treating depression after stroke (Review) 
within strata for each factor and then summed over factors. When a new subject was available for randomization, we computed what the imbalance score would be if this subject were assigned to usual care, or to telephone intervention, or to in-person intervention. Then randomization was done to allocate two intervention participants to each control with each new assignment having a higher probability of less imbalance. The schema did not require equal numbers in each arm" (p. 4)

\begin{tabular}{ll}
\hline $\begin{array}{l}\text { Allocation concealment } \\
\text { (selection bias) }\end{array}$ & Unclear risk \\
& $\begin{array}{l}\text { Quote: "the study statistician generated the algorithm, which was secure- } \\
\text { ly stored and accessible only by the statistician and research nurse supervi- } \\
\text { sor" (p. 5) }\end{array}$
\end{tabular}

Comment: method of allocation concealment not reported

\begin{tabular}{|c|c|c|}
\hline $\begin{array}{l}\text { Blinding of participants } \\
\text { and personnel (perfor- } \\
\text { mance bias) } \\
\text { All outcomes }\end{array}$ & High risk & $\begin{array}{l}\text { Quote: "participants were asked not to reveal their study arm to the outcome } \\
\text { assessors" (p. 5) } \\
\text { Comment: blinding of personnel not reported }\end{array}$ \\
\hline $\begin{array}{l}\text { Blinding of outcome as- } \\
\text { sessment (detection bias) } \\
\text { All outcomes }\end{array}$ & Low risk & $\begin{array}{l}\text { Quote: "....masking outcome assessors to the participant's randomization sta- } \\
\text { tus. Participants were asked not to reveal their study arm to the outcome as- } \\
\text { sessors. We did not detect any breaches in masking" (p. 5) }\end{array}$ \\
\hline $\begin{array}{l}\text { Incomplete outcome data } \\
\text { (attrition bias) } \\
\text { All outcomes }\end{array}$ & High risk & $\begin{array}{l}\text { Comment: per protocol analysis reported only; } 9 \text { participants not included in } \\
\text { the analysis }\end{array}$ \\
\hline $\begin{array}{l}\text { Selective reporting (re- } \\
\text { porting bias) }\end{array}$ & Unclear risk & $\begin{array}{l}\text { Comment: all pre-specified outcomes reported; no trial protocol to compare } \\
\text { with the publication }\end{array}$ \\
\hline Other bias & Low risk & $\begin{array}{l}\text { Comment: no differences in baseline demographic characteristics between } \\
\text { groups }\end{array}$ \\
\hline
\end{tabular}

Kong 2007

Study design: parallel design
Number of arms: 2
Experimental arm: fluoxetine $(\mathrm{SSRI}) 20 \mathrm{mg} / \mathrm{d}$
Control arm: placebo

Participants

\author{
Geographical location: China \\ Setting: inpatient
}

Stroke criteria: ischaemic stroke

Method of stroke diagnosis: diagnosis met the diagnostic criteria of various cerebrovascular diseases formulated at the 4th National Cerebrovascular Disease Conference and confirmed as stroke by skull CT or MRI

Time since stroke: $<7$ days

Inclusion criteria: (1) all patients were < 7 days from their first-ever stroke; (2) able to understand and carry out verbal instructions

Exclusion criteria: (1) diagnosis of major depression at evaluation or at any earlier period during the index episode; (2) active suicidal ideation; (3) bipolar disorder, schizophrenia, or other psychotic disor- 
Kong 2007 (Continued)

der; (4) currently taking antidepressants; (5) MMSE score $\leq 23$; (6) medical contraindication to fluoxetine; (7) history of allergy to fluoxetine; (8) history of substance abuse; (9) obvious liver and renal function deficit

Depression criteria: 24 -item HDRS score $\geq 8$ and $\leq 20$

Total number randomised in this trial: 90

Number randomised to treatment group: 48 (60\% men; mean age 64 years, SD 7; $62 \%$ ischaemic; NIHSS 14.6, SD 5.8)

Number randomised to control group: 42 (58\% men; mean age 62 years, SD 7; 58\% ischaemic; NIHSS 14.3, SD 6.1)

Total number included in final analysis: 73

Number included in treatment group for final analysis: 37

Number included in control group for final analysis: 36

$\begin{array}{ll}\text { Interventions } & \text { Treatment: fluoxetine (SSRI) } 20 \mathrm{mg} / \mathrm{d} \text {; no further details given } \\ \text { Control: placebo (vitamin C). Dose not specified but capsules described as identical to treatment cap- } \\ \text { sules } \\ \text { Duration: } 8 \text { weeks } \\ \text { Follow-up: none }\end{array}$

Outcomes

Primary outcomes

- Depression measured using 24-item HDRS

\section{Secondary outcomes}

- Functional capacity measured using BI

- Impairment measured using NIHSS

\section{Notes}

\section{Risk of bias}

\begin{tabular}{lll}
\hline Bias & Authors' judgement & Support for judgement \\
\hline $\begin{array}{l}\text { Random sequence genera- } \\
\text { tion (selection bias) }\end{array}$ & Low risk & $\begin{array}{l}\text { Quote: "treatment allocation was based on a computer-generated list of treat- } \\
\text { ment numbers" (p. 163) }\end{array}$ \\
\hline
\end{tabular}

\begin{tabular}{|c|c|c|}
\hline $\begin{array}{l}\text { Allocation concealment } \\
\text { (selection bias) }\end{array}$ & Low risk & $\begin{array}{l}\text { Quote: "....were given as a single morning dose in identical capsules in coded } \\
\text { boxes" (p. 163) }\end{array}$ \\
\hline $\begin{array}{l}\text { Blinding of participants } \\
\text { and personnel (perfor- } \\
\text { mance bias) }\end{array}$ & Low risk & $\begin{array}{l}\text { Quote: "the patient, relatives and the researchers were not aware of the drug } \\
\text { being given" (p. 163) } \\
\text { Comment: blinding of those who delivered the intervention not reported }\end{array}$ \\
\hline All outcomes & & Comment: blinding of those who delivered the intervention not reported \\
\hline $\begin{array}{l}\text { Blinding of outcome as- } \\
\text { sessment (detection bias) } \\
\text { All outcomes }\end{array}$ & Unclear risk & Comment: blinding of outcome assessors not reported \\
\hline $\begin{array}{l}\text { Incomplete outcome data } \\
\text { (attrition bias) } \\
\text { All outcomes }\end{array}$ & High risk & $\begin{array}{l}\text { Quote: "seventy-three of the } 90 \text { randomized patients accomplished the trial. } \\
\text { In the treatment group, } 11 \text { patients dropped out, including insufficient clinical } \\
\text { response }(n=4) \text {, somatic side effects }(n=2) \text {, intervening medical illness }(n=1) \text {, } \\
\text { hypomania }(n=3) \text {, and other reasons }(n=2) \text {. In the placebo group, } 6 \text { patients }\end{array}$ \\
\hline
\end{tabular}


Kong 2007 (Continued)

existed, including insufficient clinical response $(n=2)$, somatic side effects $(n=$

$1)$ and other reasons $(n=3) "$

Comment: per protocol analysis reported only

\begin{tabular}{lll}
\hline $\begin{array}{l}\text { Selective reporting (re- } \\
\text { porting bias) }\end{array}$ & Unclear risk & $\begin{array}{l}\text { Comment: all pre-specified outcomes reported; no trial protocol available to } \\
\text { compare with the publication }\end{array}$ \\
\hline Other bias & Low risk & $\begin{array}{l}\text { Comment: } \text { no differences in baseline demographic characteristics between } \\
\text { groups }\end{array}$ \\
\hline
\end{tabular}

Lai 2006a

Methods Study design: parallel design

Number of arms: 2

Experimental arm: paroxetine (SSRI)

Control arm: placebo

Participants Geographical location: China

Setting: inpatient

Stroke criteria: acute stroke

Method of stroke diagnosis: diagnosis via CT

Time since stroke: unclear

Inclusion criteria: not reported

Exclusion criteria: not reported

Depression criteria: HDRS score $>6$

Total number randomised in this trial: 80

Number included in treatment group: 40 ( $54 \%$ men in total, mean age 60 years, SD 14)

Number included in control group: 40 (54\% men in total, mean age 60 years, SD 14)

Total number included in final analysis: 80

Number included in treatment group for final analysis: 40

Number included in control group for final analysis: 40

$\begin{array}{ll}\text { Interventions } & \text { Treatment: paroxetine (SSRI) } 20 \mathrm{mg} / \mathrm{d} \\ \text { Control: placebo } \\ \text { Duration: } 2 \text { months } \\ \text { Follow-up: not reported }\end{array}$

Outcomes Primary outcomes

- Depression measured using HDRS and ZDS

- Impairment measured using SSS

\section{Secondary outcomes}

- Death 
Lai 2006a (Continued)

- Adverse events

Notes

\section{Risk of bias}

\begin{tabular}{|c|c|c|}
\hline Bias & Authors' judgement & Support for judgement \\
\hline $\begin{array}{l}\text { Random sequence genera- } \\
\text { tion (selection bias) }\end{array}$ & Unclear risk & Comment: method of sequence generation not reported \\
\hline $\begin{array}{l}\text { Allocation concealment } \\
\text { (selection bias) }\end{array}$ & Unclear risk & Comment: method of allocation concealment not reported \\
\hline $\begin{array}{l}\text { Blinding of participants } \\
\text { and personnel (perfor- } \\
\text { mance bias) } \\
\text { All outcomes }\end{array}$ & Unclear risk & Comment: blinding of participants and personnel not reported \\
\hline $\begin{array}{l}\text { Blinding of outcome as- } \\
\text { sessment (detection bias) } \\
\text { All outcomes }\end{array}$ & Unclear risk & Comment: blinding of outcome assessment not reported \\
\hline $\begin{array}{l}\text { Incomplete outcome data } \\
\text { (attrition bias) } \\
\text { All outcomes }\end{array}$ & Low risk & $\begin{array}{l}\text { Comment: ITT analysis reported (complete follow-up of all randomised partic- } \\
\text { ipants) }\end{array}$ \\
\hline $\begin{array}{l}\text { Selective reporting (re- } \\
\text { porting bias) }\end{array}$ & Unclear risk & $\begin{array}{l}\text { Comment: all pre-specified outcomes reported; no trial protocol available to } \\
\text { compare with the publication }\end{array}$ \\
\hline Other bias & Unclear risk & Comment: baseline demographic information not reported \\
\hline
\end{tabular}

Li 2008

\begin{tabular}{ll} 
Methods & Study design: parallel design \\
Number of arms: 2 \\
Experimental arm: fluoxetine (SSRI) \\
Control arm: matched placebo \\
\hline
\end{tabular}

\section{Participants}

\section{Geographical location: China.}

Setting: unclear

Stroke criteria: ischaemic or haemorrhagic stroke

Method of stroke diagnosis: each patient evaluated for inclusion by a neuro-psychiatrist. Presence of recent $<6$ weeks ischaemic or haemorrhagic stroke documented by CT or MRI before the study

\section{Time since stroke: 4.78 days}

Inclusion criteria: (1) lack of treatment with antidepressants during the 2 weeks before this study; (2) only single ischaemic and haemorrhagic stroke

Exclusion criteria: (1) cognitive impairment (MMSE < 23); (2) severe aphasia; (3) history of alcoholism, abnormal thyroid, or epilepsy

Depression criteria: HDRS score $>20$ 


\section{Total number randomised in this trial: 90}

Number randomised to treatment group: 60 (47\% men; mean age 68.5 years, SD 4.1; mean time since stroke 4.83 weeks, SD 0.57)

Number randomised to control group: 30 (57\% men; mean age 67.8 years, SD 3.9; mean time since stroke 4.82, SD 0.67)

Total number included in final analysis: 86

Number included in treatment group for final analysis: 58

Number included in control group for final analysis: 28

Treatment: fluoxetine (SSRI) 20 to $40 \mathrm{mg}$ depending on tolerability together with placebo to make up 6 tablets

Control: matched placebo (composition not specified) 18 grams in 6 tablets twice daily

Duration: 8 weeks

Follow-up: none

Outcomes Primary outcomes

- Depression measured using HDRS (mean HDRS score at end of trial)

- Percentage of responders (measure of clinical response defined as $>50 \%$ reduction in HDRS score compared with baseline score)

\section{Secondary outcomes}

- Depression measured using HDRS (at 4 weeks)

\section{Notes}

\section{Risk of bias}

\begin{tabular}{lll}
\hline Bias & Authors' judgement & Support for judgement \\
\hline $\begin{array}{l}\text { Random sequence genera- } \\
\text { tion (selection bias) }\end{array}$ & Low risk & Quote: "a computer-generated randomisation was carried out..." (p. 843) \\
\hline $\begin{array}{l}\text { Allocation concealment } \\
\text { (selection bias) }\end{array}$ & Low risk & $\begin{array}{l}\text { Quote: "both placebo and herbal tablets were prepared to be identical to the } \\
\text { fluoxetine..." (p. 842) }\end{array}$ \\
\hline
\end{tabular}

Blinding of participants High risk and personnel (performance bias)

All outcomes
Blinding of outcome as- Low risk sessment (detection bias) All outcomes
Quote: "neither the examiners involved nor the patients were aware of the type of the administered medications" (p. 842)

Comment: physician initiated and moderated treatment dose based on patient's tolerability and response. It is likely that the physician was not blinded

Quote: "neither the examiners involved nor the patients were aware of the type of the administered medications" (p. 842)

\begin{tabular}{|c|c|c|}
\hline $\begin{array}{l}\text { Incomplete outcome data } \\
\text { (attrition bias) } \\
\text { All outcomes }\end{array}$ & High risk & $\begin{array}{l}\text { Comment: } 2 / 60 \text { patients in the fluoxetine group withdrew from the study due } \\
\text { to recurrent stroke; } 2 / 30 \text { withdrew due to increased depressive symptoms } \\
\text { within } 4 \text { weeks of the start of the trial. Per protocol analysis reported only }\end{array}$ \\
\hline $\begin{array}{l}\text { Selective reporting (re- } \\
\text { porting bias) }\end{array}$ & Unclear risk & $\begin{array}{l}\text { Comment: all pre-specified outcomes reported; no trial protocol available to } \\
\text { compare with the publication }\end{array}$ \\
\hline
\end{tabular}


Li 2008 (Continued) $\begin{array}{ll}\text { Other bias } \quad \text { Low risk } & \begin{array}{l}\text { Comment: no differences in baseline demographic characteristics between } \\ \text { groups }\end{array}\end{array}$

\section{Number of arms: 2}

Experimental arm: rTMS + mirtazapine + stroke usual care

Control arm: mirtazapine + stroke usual care

\section{Participants}

Geographical location: China

Setting: inpatient

Stroke criteria: not reported

Method of stroke diagnosis: clinical diagnosis plus confirmation by imaging that a relevant lesion needed to be visible

Inclusion criteria: (1) aged over 65 years; (2) patient or guardian can sign informed consent; (3) meeting diagnostic criteria of the CCMD-3 for depression

Exclusion criteria: (1) comorbid with aphasia, comprehension, or expression impairment, or severe mental retardation; (2) with severe cardiac, hepatic, or renal disease, or with epilepsy; (3) intracranial metal implant, possible history of allergy to mirtazapine

Depression criteria: meeting diagnostic criteria of the CCMD-3 for depression and 17-item HDRS score $\geq 17$

Total number randomised in this trial: 60

Number included in treatment group: 30 (56\% men; mean age 64.8, SD 5.4)

Number included in control group: 30 (53\% men; mean age 65.2, SD 4.8)

Total number included in final analysis: 60

Number included in treatment group for final analysis: 30

Number included in control group for final analysis: 30

Treatment: rTMS + mirtazapine (starting from $15 \mathrm{mg} / \mathrm{d}$ at night, if tolerable, increase to $30 \mathrm{mg} / \mathrm{d}$ in 2 to 3 days) + stroke usual care (medications + rehabilitation). Frequency: $1 \mathrm{~Hz}$, intensity: $90 \%$ motor threshold, each treatment lasting for 20 minutes, 5 treatments a week, location: right DLPFC

Control: mirtazapine + stroke usual care

Duration: 4 weeks

Follow-up: none

\section{Outcomes}

\section{Primary outcomes}

- Depression measured using HDRS

\section{Secondary outcomes}

- Impairment measured using NIHSS 
Li 2013 (Continued)

Notes

\section{Risk of bias}

\begin{tabular}{lll}
\hline Bias & Authors' judgement & Support for judgement \\
\hline $\begin{array}{l}\text { Random sequence genera- } \\
\text { tion (selection bias) }\end{array}$ & Unclear risk & Comment: method of sequence generation not reported \\
\hline
\end{tabular}

Allocation concealment Unclear risk Comment: method of allocation concealment not reported
(selection bias)

\begin{tabular}{|c|c|c|}
\hline $\begin{array}{l}\text { Blinding of participants } \\
\text { and personnel (perfor- } \\
\text { mance bias) } \\
\text { All outcomes }\end{array}$ & Unclear risk & $\begin{array}{l}\text { Comment: information about blinding of participants and personnel not re- } \\
\text { ported }\end{array}$ \\
\hline
\end{tabular}

\begin{tabular}{ll}
\hline Blinding of outcome as- & Unclear risk
\end{tabular}

All outcomes

Incomplete outcome data Low risk Comment: ITT analysis reported; no missing data
(attrition bias)
All outcomes

\begin{tabular}{lll}
\hline $\begin{array}{l}\text { Selective reporting (re- } \\
\text { porting bias) }\end{array}$ & Unclear risk & $\begin{array}{l}\text { Comment: all pre-specified outcomes reported; no trial protocol available to } \\
\text { compare with the publication }\end{array}$ \\
\hline Other bias & Low risk & $\begin{array}{l}\text { Comment: } \text { no significant differences in baseline demographics between } \\
\text { groups }\end{array}$ \\
\hline
\end{tabular}

\begin{tabular}{ll}
\hline Study design: parallel design \\
Number of arms: 2 \\
Experimental arm: rTMS + fluoxetine (SSRI) + stroke usual care \\
Control arm: fluoxetine (SSRI) + stroke usual care \\
Geographical location: China \\
Setting: inpatient \\
Stroke criteria: ischaemic and haemorrhagic stroke \\
Method of stroke diagnosis: clinical diagnosis plus confirmation by imaging that a relevant lesion \\
needed to be visible \\
Inclusion criteria: (1) meeting diagnostic criteria of the CCMD-3 for depression; (2) 17-item HDRS score \\
$\geq 18$ \\
Exclusion criteria: not reported \\
Depression criteria: meeting diagnostic criteria of the CCMD-3 for depression and for 17-item HDRS \\
score $\geq 18$ \\
Total number randomised in this trial: 93
\end{tabular}


Number randomised to treatment group: 47 (49\% men; mean age 57.6, SD 6.8)

Number randomised to control group: 46 ( $52 \%$ men; mean age 56.5, SD 6.7)

Total number included in final analysis: 93

Number included in treatment group for final analysis: 47

Number included in control group for final analysis: 46

\begin{tabular}{ll}
\hline Interventions & Treatment: rTMS + fluoxetine (SSRI) $20 \mathrm{mg} / \mathrm{d}+$ stroke usual care (medications + rehabilitation) \\
Frequency: $10 \mathrm{~Hz}$, intensity: $80 \%$ motor threshold, with each series lasting 4 seconds with an interval of \\
56 seconds, successive 20 series per day, 5 treatments a week, location: left DLPFC \\
Control: fluoxetine (SSRI) + stroke usual care \\
Duration: 4 weeks \\
Follow-up: none \\
Primary outcomes \\
- Depression measured using HDRS \\
- Disability measured using MBI
\end{tabular}

Notes

\section{Risk of bias}

\begin{tabular}{lll}
\hline Bias & Authors' judgement & Support for judgement \\
\hline $\begin{array}{l}\text { Random sequence genera- } \\
\text { tion (selection bias) }\end{array}$ & Low risk & Comment: random number table used for sequence generation \\
\hline
\end{tabular}

\begin{tabular}{lll}
\hline $\begin{array}{l}\text { Allocation concealment } \\
\text { (selection bias) }\end{array}$ & Unclear risk & Comment: method of allocation concealment not reported \\
\hline $\begin{array}{l}\text { Blinding of participants } \\
\text { and personnel (perfor- } \\
\text { mance bias) }\end{array}$ & Unclear risk & $\begin{array}{l}\text { Comment: information about blinding of participants and personnel not re- } \\
\text { ported }\end{array}$ \\
All outcomes &
\end{tabular}

\begin{tabular}{|c|c|c|}
\hline $\begin{array}{l}\text { Blinding of outcome as- } \\
\text { sessment (detection bias) } \\
\text { All outcomes }\end{array}$ & Unclear risk & Comment: information about blinding of outcome assessors not reported \\
\hline
\end{tabular}

\begin{tabular}{lll}
\hline $\begin{array}{l}\text { Incomplete outcome data } \\
\text { (attrition bias) } \\
\text { All outcomes }\end{array}$ & Low risk & Comment: ITT analysis reported; no missing data \\
\hline $\begin{array}{l}\text { Selective reporting (re- } \\
\text { porting bias) }\end{array}$ & Unclear risk & $\begin{array}{l}\text { Comment: all pre-specified outcomes reported; no trial protocol available to } \\
\text { compare with the publication }\end{array}$ \\
\hline Other bias & Low risk & Comment: no differences in baseline demographics between groups \\
\hline
\end{tabular}


Lincoln 2003

Methods

Study design: parallel design

Number of arms: 3

Experimental arm: cognitive-behavioural therapy

Control arm 1: attention control

Control arm 2: usual care

\section{Participants}

\section{Geographical location: UK}

Setting: outpatient

Stroke criteria: all subtypes

Method of stroke diagnosis: diagnosis via clinical signs and symptoms and CT

Time since stroke: 1 to 6 months

Inclusion criteria: not reported

Exclusion criteria: (1) blindness; (2) deafness; (3) participant did not speak English; (4) dementia documented in medical records; (5) treated for depression in previous 5 years; (6) lived outside specified locality; (7) participant could not complete questionnaire unaided

Depression criteria: psychiatric interview (SCAN), BDI score $>10$, WDI score $>18$

Total number randomised in this trial: 123

Number randomised to treatment group: 39 (51\% men, mean age 67 years, SD 13)

Number randomised to attention control and usual care group^: 84 (51\% men, mean age 66 years, SD 14)

Total number included in final analysis: 111

Number included in treatment group for final analysis: 34

Number included in control group for final analysis: 77

Interventions

Treatment: cognitive-behavioural therapy (techniques included education, graded task assignment, activity scheduling, and identification and modification of unhelpful thoughts and beliefs. Interventions were tailored to meet the individual's needs. Frequency and duration of sessions were $10 \times 1$ hour sessions over 13 weeks

Administered by: trained therapist

Supervision: therapist received training and clinical supervision by experienced cognitive therapist

Intervention fidelity: not reported

Attention control: no formal therapeutic intervention; conversation focused on day-to-day occurrences and discussion regarding physical effects of stroke and life changes ( $10 \times 1$ hour visits over 13 weeks)

Control: usual care (no contact)

Duration: 13 weeks

Follow-up: 3 months

Outcomes Primary outcomes

- Depression measured using BDI, WDI, GHQ 28

- Activities of daily living measured using EADL scale

- Leaving the study early 
Lincoln 2003 (Continued)

$$
\text { - Death }
$$

\section{Notes}

\section{Risk of bias}

\begin{tabular}{lll}
\hline Bias & Authors' judgement & Support for judgement \\
\hline $\begin{array}{ll}\text { Random sequence genera- } \\
\text { tion (selection bias) }\end{array}$ & Low risk & $\begin{array}{l}\text { Quote: "a computer generated random number sequence was prepared in ad- } \\
\text { vance and sealed in opaque, consecutively numbered envelopes by an inde- } \\
\text { pendent researcher" (p. 112) }\end{array}$ \\
& & \\
\hline
\end{tabular}

Allocation concealment High risk Quote: "....prepared in advance and sealed in opaque, consecutively num-
(selection bias) bered envelopes by an independent researcher" (p. 112)

Comment: this method of allocation concealment can be tampered with

\begin{tabular}{lll}
\hline $\begin{array}{l}\text { Blinding of participants } \\
\text { and personnel (perfor- }\end{array}$ & High risk & Comment: due to the nature of the intervention, it was not possible to mask \\
participants, CBT therapists, or researchers to treatment allocation
\end{tabular}
mance bias)

All outcomes

\begin{tabular}{ll}
\hline Blinding of outcome as- & High risk \\
sessment (detection bias)
\end{tabular}
gist, who was blind to the group allocation, 3 and 6 months after randomization. The primary outcome measures were the BDI and WDI, which were sent for patients to complete prior to a visit" (p. 112)

\begin{tabular}{|c|c|c|}
\hline $\begin{array}{l}\text { Incomplete outcome data } \\
\text { (attrition bias) }\end{array}$ & High risk & $\begin{array}{l}\text { Comment: per protocol analysis reported only; } 5 / 121(4.1 \%) \text { not included in } \\
\text { analyses }\end{array}$ \\
\hline
\end{tabular}

All outcomes

Comment: per protocol analysis reported only; 5/121 (4.1\%) not included in

Selective reporting (re- Unclear risk porting bias)

Comment: all pre-specified outcomes were reported; no trial protocol available to compare with the publication

Other bias High risk

Comment: significantly more participants in the treatment group with an ICD-10 diagnosis of depression

\section{Lipsey 1984}

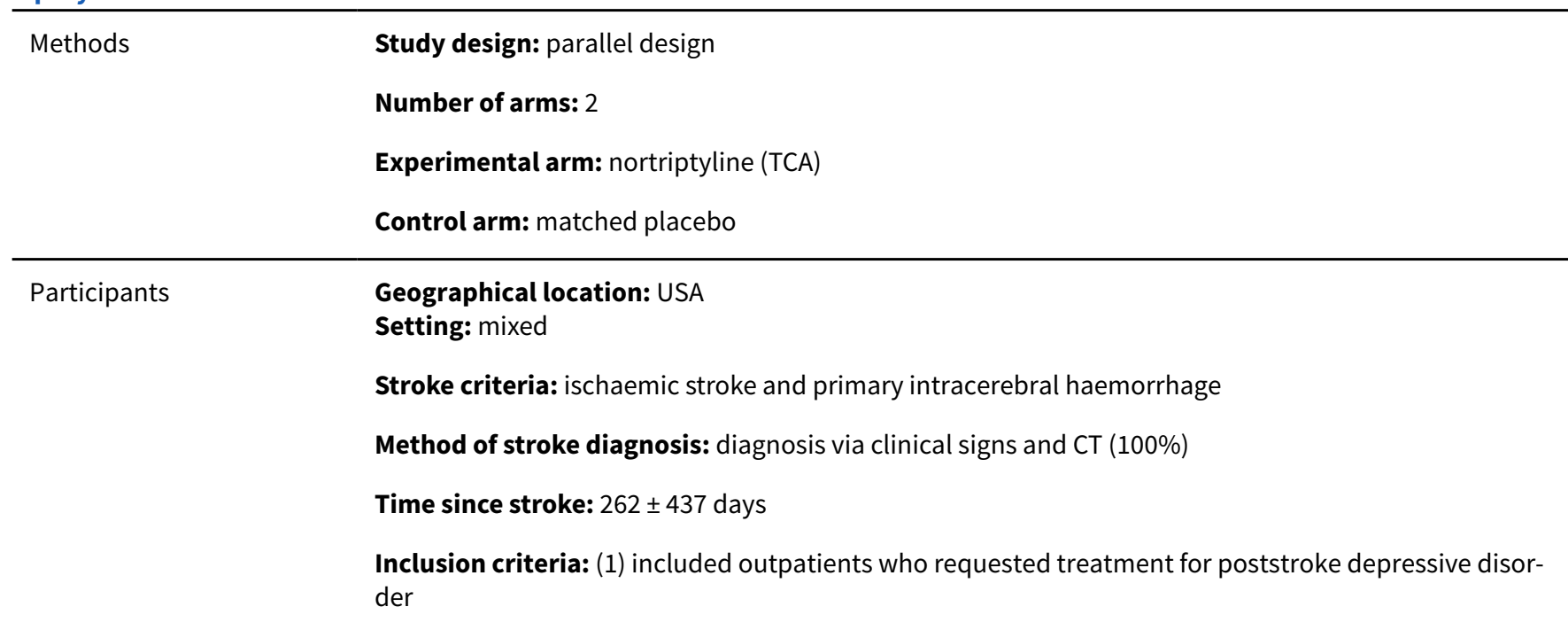


Exclusion criteria: (1) current treatment for depression; (2) severe comprehension deficit; (3) medical contraindication to nortriptyline

Depression criteria: psychiatric interview (PSE, DSM-III)

Total number randomised in this trial: 39

Number randomised to treatment group: 17

Number randomised to control group: 22

Total number included in final analysis: 34

Number included in treatment group for final analysis: 14 (64\% men, mean age 62 years, SD 9)

Number included in control group for final analysis: 20 (65\% men, mean age 60 years, SD 12)

Interventions

Treatment: nortriptyline (TCA) 20 to $100 \mathrm{mg}$ daily; 2 treatment regimens combined; dose escalation over treatment period to $100 \mathrm{mg}$

Control: matched placebo

Duration: 4 to 6 weeks

Follow-up: not reported

$\begin{array}{ll}\text { Putcomes } & \text { Primary outcomes } \\ \text { - Depression (proportion no longer meeting entry criteria (DSM-III), measured using HDRS and ZDS)^ } \dagger \\ \text { Secondary outcomes } \\ \text { - Death } \\ \text { - Adverse events }\end{array}$

Notes

\section{Risk of bias}

\begin{tabular}{lll}
\hline Bias & Authors' judgement & Support for judgement \\
\hline $\begin{array}{l}\text { Random sequence genera- } \\
\text { tion (selection bias) }\end{array}$ & Low risk & $\begin{array}{l}\text { Quote: "all patients were randomly assigned to nortriptyline or placebo treat- } \\
\text { ment by means of a random number table" (p. 297) }\end{array}$ \\
\hline $\begin{array}{l}\text { Allocation concealment } \\
\text { (selection bias) }\end{array}$ & Low risk & Quote: "nortriptyline and placebo were supplied in identical capsules" (p. 297) \\
\hline
\end{tabular}

Blinding of participants Low risk Quote: "patients and their families, clinical examiners and nursing staff were
and personnel (perfor- $\quad$ unaware of the drug treatment being given" (p. 297)

mance bias)

All outcomes

\section{Blinding of outcome as- Low risk} sessment (detection bias) All outcomes
Quote: "patients and their families, clinical examiners and nursing staff were unaware of the drug treatment being given" (p. 297)

Incomplete outcome data High risk

Comment: per protocol analysis reported; 5/39 (13\%) not included in analyses

(attrition bias)

All outcomes

Selective reporting (re- Unclear risk porting bias)

Comment: all pre-specified outcomes reported; no trial protocol available to compare with the publication 
Lipsey 1984 (Continued) $\begin{array}{ll}\text { Other bias } \quad \text { Low risk } & \begin{array}{l}\text { Comment: no differences in baseline demographic characteristics between } \\ \text { groups }\end{array}\end{array}$

Liu 2015

Study design: parallel design
Number of arms: 2
Experimental arm: rTMS + citalopram (SSRI) + short-term benzodiazepines (BZDs) if needed for insom-
nia
Control arm: citalopram (SSRI) + short-term BZDs if needed for insomnia

Participants

Geographical location: China

Setting: mixed

Stroke criteria: ischaemic stroke

Method of stroke diagnosis: clinical diagnosis plus confirmation by imaging that a relevant lesion needed to be visible

Inclusion criteria: (1) can sign informed consent; (2) 17-item HDRS score $\geq 17$

Exclusion criteria: (1) drug dependence history in recent 6 months; (2) bleeding tendency, severe hepatic or renal impairment, or other physical illness; (3) epilepsy history, head injury with consciousness loss history, history of cranial operation, metal implant or electronic devices in the body

Depression criteria: 17 -item HDRS score $\geq 17$

Total number randomised in this trial: 60

Number included in treatment group: 30 (56\% men; mean age 64.2, SD 3.1)

Number included in control group: 30 (53\% men; mean age 65.1, SD 3.5)

Total number included in final analysis: 60

Number included in treatment group for final analysis: 30

Number included in control group for final analysis: 30

Interventions

Treatment: rTMS + citalopram (SSRI), starting from $10 \mathrm{mg} / \mathrm{d}$ in the morning, may titrate up to $20 \mathrm{mg} /$ $\mathrm{d}$ according to the patient's condition + short-term BZDs (only for difficulty in falling asleep; combined duration: less than 1 week). Frequency: $10 \mathrm{~Hz}$, intensity: $80 \%$ resting motor threshold, 1 stimulation lasts 5 seconds and stops for 20 seconds, total treatment time: 20 minutes, 1 treatment per day, 5 treatments a week, total 4 weeks, location: left DLPFC

Control: citalopram (SSRI) + short-term BZDs

Duration: 4 weeks

Follow-up: none

Outcomes Primary outcome

- Depression measured using 17-item HDRS

\section{Secondary outcome}


Liu 2015 (Continued)

- Impairment measured using NIHSS

Notes

\section{Risk of bias}

\begin{tabular}{|c|c|c|}
\hline Bias & Authors' judgement & Support for judgement \\
\hline $\begin{array}{l}\text { Random sequence genera- } \\
\text { tion (selection bias) }\end{array}$ & Unclear risk & Comment: method of sequence generation not reported \\
\hline $\begin{array}{l}\text { Allocation concealment } \\
\text { (selection bias) }\end{array}$ & Unclear risk & Comment: method of allocation concealment not reported \\
\hline $\begin{array}{l}\text { Blinding of participants } \\
\text { and personnel (perfor- } \\
\text { mance bias) } \\
\text { All outcomes }\end{array}$ & Unclear risk & $\begin{array}{l}\text { Comment: information about blinding of participants and personnel not re- } \\
\text { ported }\end{array}$ \\
\hline $\begin{array}{l}\text { Blinding of outcome as- } \\
\text { sessment (detection bias) } \\
\text { All outcomes }\end{array}$ & Unclear risk & Comment: information about blinding of outcome assessors not reported \\
\hline $\begin{array}{l}\text { Incomplete outcome data } \\
\text { (attrition bias) } \\
\text { All outcomes }\end{array}$ & Low risk & Comment: ITT analysis reported; no missing data \\
\hline $\begin{array}{l}\text { Selective reporting (re- } \\
\text { porting bias) }\end{array}$ & Unclear risk & $\begin{array}{l}\text { Comment: all pre-specified outcomes reported; no trial protocol available to } \\
\text { compare with the publication }\end{array}$ \\
\hline Other bias & Low risk & Comment: no differences in baseline demographics between groups \\
\hline
\end{tabular}

Lu 2016

\begin{tabular}{ll}
\hline Study design: parallel design \\
Number of arms: 2 \\
Experimental arm: rTMS + duloxetine (SNRI) + ischaemic stroke routine care \\
Control arm: duloxetine (SNRI) + ischaemic stroke routine care \\
\hline
\end{tabular}

Participants

Geographical location: China

Setting: inpatient

Stroke criteria: ischaemic stroke

Method of stroke diagnosis: clinical diagnosis plus confirmation by imaging that a relevant lesion needed to be visible

Inclusion criteria: (1) clear consciousness; (2) 24-item HDRS score $\geq 20$; (3) meeting diagnostic criteria of ICD-10 for depression

Exclusion criteria: (1) cognitive impairment; (2) no language impairment; (3) severe cardiac or pulmonary disease, hepatic or renal impairment; (4) bleeding tendency

Depression criteria: meeting diagnostic criteria of ICD-10 for depression and 24-item HDRS score $\geq 20$ 
Total number randomised in this trial: 80

Number randomised to treatment group: 40 (57.5\% men; mean age 65.3 , SD 8.8)

Number randomised to control group: 40 (52.5\% men; mean age 63.8, SD 8.4)

Total number included in final analysis: 73

Number included in treatment group for final analysis: 36

Number included in control group for final analysis: 37 tensity: $110 \%$ resting motor threshold, 1 treatment lasts 5 minutes, 5 treatments a week, location: left temporoparietal area

Control: duloxetine (SNRI) + ischaemic stroke routine care

Duration: 4 weeks

Follow-up: none

\begin{tabular}{ll}
\hline Outcomes & Primary outcomes \\
- Depression measured using MADRS \\
- Depression measured using 24-item HDRS \\
- Dependence measured using SDS
\end{tabular}

Notes

\section{Risk of bias}

\begin{tabular}{|c|c|c|}
\hline Bias & Authors' judgement & Support for judgement \\
\hline $\begin{array}{l}\text { Random sequence genera- } \\
\text { tion (selection bias) }\end{array}$ & Low risk & Comment: random numbers table used for sequence generation \\
\hline $\begin{array}{l}\text { Allocation concealment } \\
\text { (selection bias) }\end{array}$ & Unclear risk & Comment: method of allocation concealment not reported \\
\hline $\begin{array}{l}\text { Blinding of participants } \\
\text { and personnel (perfor- } \\
\text { mance bias) } \\
\text { All outcomes }\end{array}$ & Unclear risk & $\begin{array}{l}\text { Comment: information about blinding of participants and personnel not re- } \\
\text { ported }\end{array}$ \\
\hline $\begin{array}{l}\text { Blinding of outcome as- } \\
\text { sessment (detection bias) } \\
\text { All outcomes }\end{array}$ & Unclear risk & Comment: information about blinding of outcome assessors not reported \\
\hline $\begin{array}{l}\text { Incomplete outcome data } \\
\text { (attrition bias) } \\
\text { All outcomes }\end{array}$ & High risk & $\begin{array}{l}\text { Comment: per protocol analysis reported only; } 7 / 80 \text { not included in the analy- } \\
\text { sis }\end{array}$ \\
\hline $\begin{array}{l}\text { Selective reporting (re- } \\
\text { porting bias) }\end{array}$ & Unclear risk & $\begin{array}{l}\text { Comment: all pre-specified outcomes reported; no trial protocol available to } \\
\text { compare with the publication }\end{array}$ \\
\hline Other bias & Low risk & Comment: no differences in baseline demographics between groups \\
\hline
\end{tabular}


Meng 2015

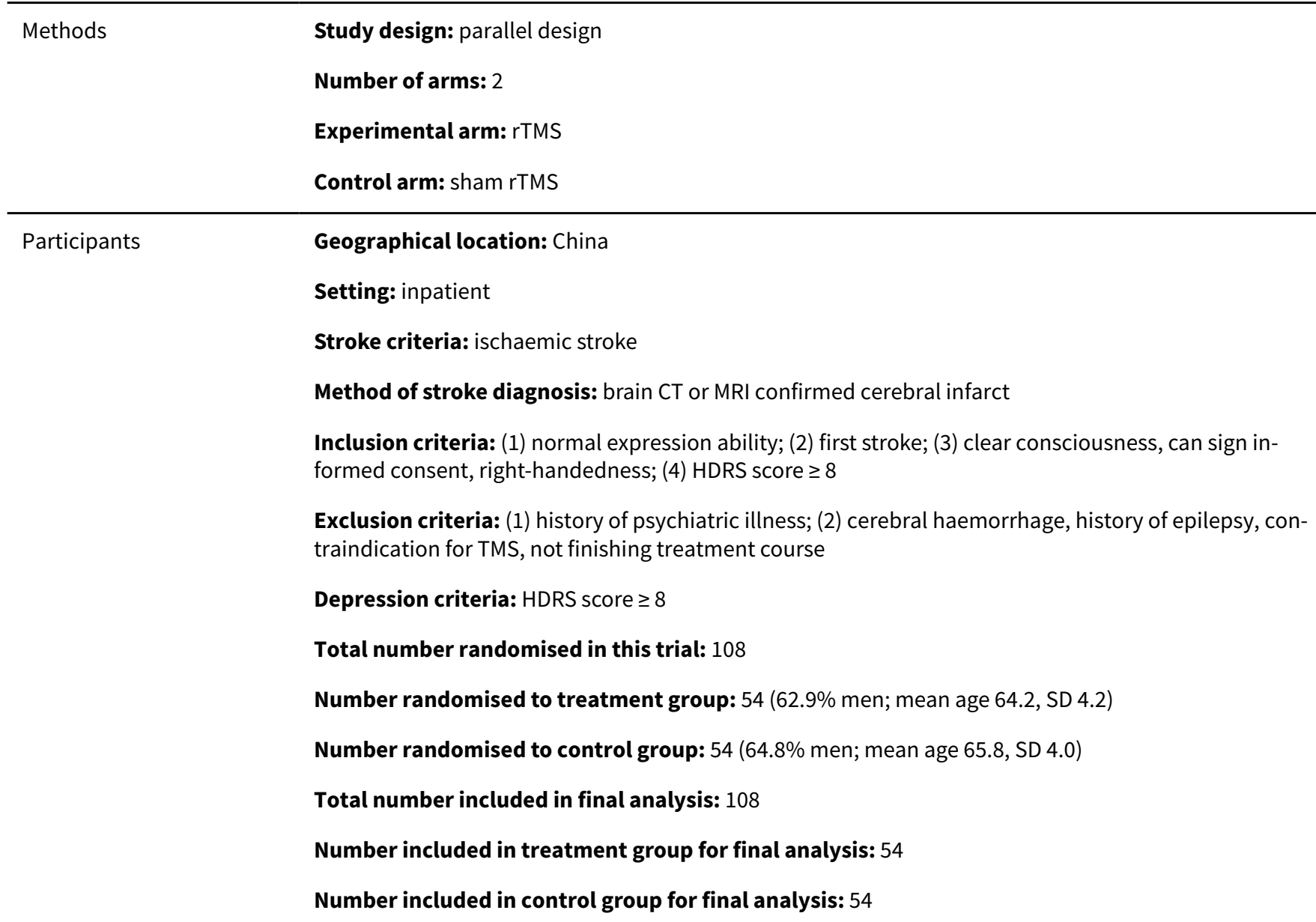

Treatment: rTMS + usual care (which includes antidepressants if already on them, no change of antidepressant dosage or medication during treatment). Frequency: $10 \mathrm{~Hz}$, intensity: $80 \%$ motor threshold, 1 stimulation lasts 4.9 seconds and stops for 20 seconds, 86 cycles a day, total 1960 impulses a day, location: left DLPFC

Control: sham rTMS, keeping coils at 90-degree angles with the scalp + usual care (which includes antidepressants if already on them, no change in antidepressant dosage or medication during treatment)

Duration: 2 weeks

Follow-up: 4 weeks

\begin{tabular}{|c|c|c|}
\hline Outcomes & $\begin{array}{l}\text { Primary outcomes } \\
\text { - Depression measu } \\
\text { - Disability measure } \\
\text { - Impairment meas }\end{array}$ & $\begin{array}{l}\text { d using HDRS } \\
\text { using BI } \\
\text { d using CSS }\end{array}$ \\
\hline \multicolumn{3}{|l|}{ Notes } \\
\hline \multicolumn{3}{|l|}{ Risk of bias } \\
\hline Bias & Authors' judgement & Support for judgement \\
\hline $\begin{array}{l}\text { Random sequence genera- } \\
\text { tion (selection bias) }\end{array}$ & Low risk & Comment: random numbers table used for sequence generation \\
\hline
\end{tabular}


Meng 2015 (Continued)

Allocation concealment Unclear risk Comment: method of allocation concealment not reported
(selection bias)
(selection bias)

Blinding of participants Unclear risk

Comment: information about blinding of participants and personnel not reand personnel (perforported mance bias)

All outcomes

Blinding of outcome as- $\quad$ Unclear risk $\quad$ Comment: information about blinding of outcome assessors not reported
sessment (detection bias)
sessment (detection bias)

All outcomes

Incomplete outcome data Low risk Comment: ITT analysis reported; no missing data
(attrition bias)

All outcomes

\begin{tabular}{ll}
\hline $\begin{array}{l}\text { Selective reporting (re- } \\
\text { porting bias) }\end{array}$ & Unclear risk
\end{tabular}$\quad \begin{aligned} & \text { Comment: all pre-specified outcomes reported; no trial protocol available to } \\
& \text { compare with the publication }\end{aligned}$
porting bias) compare with the publication

Other bias Unclear risk comment: significant differences in age between groups

\section{Mitchell 2002}

\begin{tabular}{|c|c|}
\hline Methods & $\begin{array}{l}\text { Study design: parallel design } \\
\text { Number of arms: } 2 \\
\text { Experimental arm: cognitive-behavioural therapy plus problem-solving } \\
\text { Control arm: written information from the Stroke Association including information about depression }\end{array}$ \\
\hline \multirow{10}{*}{ Participants } & $\begin{array}{l}\text { Stroke criteria: ischaemic stroke } \\
\text { Method of stroke diagnosis: verified by CT or MRI }\end{array}$ \\
\hline & Time since stroke: within 4 months \\
\hline & Inclusion criteria: (1) stroke within 4 months; (2) 21 years of age and older \\
\hline & $\begin{array}{l}\text { Exclusion criteria: }(1) \text { subarachnoid or intracranial haemorrhagic stroke; (2) global aphasia; (3) re- } \\
\text { duced level of consciousness }(G C S<15)\end{array}$ \\
\hline & $\begin{array}{l}\text { Depression criteria: diagnosis of depression validated by the Diagnostic Interview and Structured } \\
\text { Hamilton among those who scored }>10 \text { on the GDS }\end{array}$ \\
\hline & Total number randomised in this trial: 101 \\
\hline & Number randomised to treatment group: 48 (60\% men, mean age 57 years, age range 25 to 88 years) \\
\hline & Number randomised to control group: 53 (60\% men, mean age 57 years, age range 29 to 88 years) \\
\hline & Total number included in final analysis: 92 \\
\hline & Number included in control group for final analysis: 48 \\
\hline
\end{tabular}


Mitchell 2002 (Continued)

Interventions
Treatment: cognitive-behavioural therapy plus problem-solving. Sessions were focused on the individual; however, a participant could opt to have a family member or an informal caregiver join these sessions. The brief psychosocial-behavioural intervention was adapted from the "Seattle Protocols" shown to reduce disability associated with depression in Alzheimer disease. All participants received written information from the Stroke Association including information about depression. Participants could receive antidepressant medication at the discretion of their usual care provider. Frequency and duration: 9 sessions over 8 weeks

Administered by: therapists

Supervision: all therapists met monthly with the clinical psychologist who developed the intervention

Intervention fidelity: sessions were audio-taped, and session content was compared to the content specified for each visit

Control: all participants received written information from the Stroke Association including information about depression. Participants could receive antidepressant medication at the discretion of their usual care provider

Duration: 8 weeks

Follow-up: 12 months

\begin{tabular}{ll}
\hline Outcomes & Primary outcomes \\
- Depression measured using HDRS \\
- Adverse event data systematically collected included worsening of depression, suicidal ideation, and \\
suicide attempts
\end{tabular}

Notes

\section{Risk of bias}

\begin{tabular}{lll}
\hline Bias & Authors' judgement & Support for judgement \\
\hline $\begin{array}{l}\text { Random sequence genera- } \\
\text { tion (selection bias) }\end{array}$ & Low risk & $\begin{array}{l}\text { Quote: "randomization status was generated by a computerized adaptive ran- } \\
\text { domisation procedure..." (p. 3075) }\end{array}$ \\
\hline $\begin{array}{l}\text { Allocation concealment } \\
\text { (selection bias) }\end{array}$ & Unclear risk & Comment: method of allocation concealment not reported \\
\hline
\end{tabular}

Blinding of participants High risk and personnel (performance bias)

All outcomes
Comment: due to the nature of the trial, it was not possible to mask participants, clinicians, and researchers to treatment allocation

\begin{tabular}{|c|c|c|}
\hline $\begin{array}{l}\text { Blinding of outcome as- } \\
\text { sessment (detection bias) } \\
\text { All outcomes }\end{array}$ & Low risk & $\begin{array}{l}\text { Quote: "all outcome assessors were masked to the participant's randomiza- } \\
\text { tion status at each data collection point. We did not detect any breaches in } \\
\text { masking" (p. 3075) }\end{array}$ \\
\hline
\end{tabular}

All outcomes masking" (p. 3075)

Incomplete outcome data High risk (attrition bias)

All outcomes
Comment: 9/101 participants were not included in the analysis (per protocol analysis reported only)

\begin{tabular}{|c|c|c|}
\hline $\begin{array}{l}\text { Selective reporting (re- } \\
\text { porting bias) }\end{array}$ & High risk & $\begin{array}{l}\text { Comment: caregiving burden and benefit (Sense of Competence Scale) out- } \\
\text { come in the protocol not reported in the publication }\end{array}$ \\
\hline
\end{tabular}

Other bias Low risk

Comment: no differences in baseline demographic characteristics between groups 
Murray 2002

\begin{tabular}{ll}
\hline Study design: parallel design \\
Number of arms: 2 \\
Experimental arm: sertraline (SSRI) \\
Control arm: matched placebo \\
Geographical location: Sweden \\
Setting: mixed \\
Stroke criteria: all subtypes \\
Method of stroke diagnosis: diagnosis via clinical signs and CT (100\%) \\
Time since stroke: 12 months \\
Inclusion criteria: (1) > 17 years of age; (2) stroke within previous 12 months \\
Exclusion criteria: (1) under 18 years of age; (2) severely impaired communication; (3) apparent diffi- \\
culties in adhering to study protocol; (4) acute myocardial infarction; (5) psychiatric illness other than \\
depression; (6) significant risk of suicide; (7) antidepressants during the month before randomisation; \\
(8) current use of psychotropic medication or opiate analgesic drugs; (9) < 20\% reduction in MADRS \\
score at 6 weeks
\end{tabular}

Depression criteria: psychiatric interview (DSM-IV, major and minor) and MADRS > 9

Total number randomised in this trial: 123

Number randomised to treatment group: 62 ( $52 \%$ men, mean age 71 years, SD 10)

Number randomised to control group: 61 ( $44 \%$ men, mean age 71 years, SD 10)

Total number included in final analysis: 123

Number included in treatment group for final analysis: 62

Number included in control group for final analysis: 61

\begin{tabular}{ll} 
Interventions & $\begin{array}{l}\text { Treatment: sertraline (SSRI) } 50 \mathrm{mg} \text { daily; possible dose escalation to } 100 \mathrm{mg} \text { after } 4 \text { weeks } \\
\text { Control: matched placebo } \\
\text { Duration: } 26 \text { weeks } \\
\text { Follow-up: not reported }\end{array}$ \\
\hline Primary outcomes \\
- Depression measured using MADRS (change in scores from baseline to end of treatment on MADRS) \\
Secondary outcomes \\
- Death \\
- Adverse events
\end{tabular}

Notes

\section{Risk of bias}

\begin{tabular}{lll}
\hline Bias & Authors' judgement & Support for judgement \\
\hline $\begin{array}{l}\text { Random sequence genera- } \\
\text { tion (selection bias) }\end{array}$ & Low risk & $\begin{array}{l}\text { Quote: "a centralised randomization procedure was applied. The Central } \\
\text { Pharmacy in Stockholm kept the randomization list" (p. 709) }\end{array}$ \\
\hline
\end{tabular}


Murray 2002 (Continued)

$\begin{array}{ll}\begin{array}{l}\text { Allocation concealment } \\ \text { (selection bias) }\end{array} \quad \text { Low risk } & \begin{array}{l}\text { Quote: "each centre pharmacy received a consecutive series of presealed } \\ \text { treatment packages" (p. 709) }\end{array}\end{array}$

Blinding of participants Low risk and personnel (performance bias)

All outcomes

\section{Blinding of outcome as- Low risk} sessment (detection bias)

All outcomes

Quote: "patients received double-blind identical capsules of either sertraline $50 \mathrm{mg}$ or placebo, once a day as a starting dose" (p. 709)
Comment: double-blind placebo-controlled trial, which suggests that outcome assessors were blinded
Incomplete outcome data High risk (attrition bias)

All outcomes

Quote: "efficacy analyses were based on the intention to treat (ITT), last observation carried forward population..." (p. 710)

"... response and remission rates were calculated for those patients who completed the study" (p. 710)

Comment: continuous outcomes analysed by ITT; dichotomous outcomes analysed per protocol (data reported for 38/62, 61\% intervention participants; $31 / 61,51 \%$ control participants)

\begin{tabular}{lll}
\hline $\begin{array}{l}\text { Selective reporting (re- } \\
\text { porting bias) }\end{array}$ & Unclear risk & $\begin{array}{l}\text { Comment: all pre-specified outcomes reported; no trial protocol available to } \\
\text { compare with the publication }\end{array}$ \\
\hline Other bias & Unclear risk & $\begin{array}{l}\text { Comment: significant trend towards more left hemisphere lesion strokes in } \\
\text { treatment group }\end{array}$ \\
\hline
\end{tabular}

Ohtomo 1991

\begin{tabular}{ll}
\hline Methods & Study design: parallel design \\
Number of arms: 2 \\
Experimental arm: Aniracetam (nootropic agent) \\
Control arm: matched placebo
\end{tabular}

Participants

\author{
Geographical location: Japan \\ Setting: unclear
}

Stroke criteria: ischaemic stroke

Method of stroke diagnosis: not reported

Time since stroke: not reported

Inclusion criteria: not reported

Exclusion criteria: not reported

Depression criteria: based on physician's impression, no scale used for evaluation

Total number randomised in this trial: 285

Number randomised to treatment group: 150 (details unclear) Number randomised to control group: 135 (details unclear)

Total number included in final analysis: 206 
Ohtomo 1991 (Continued)

Number included in treatment group for final analysis: unclear

Number included in control group for final analysis: unclear

\begin{tabular}{ll}
\hline Interventions & $\begin{array}{l}\text { Treatment: Aniracetam (nootropic agent) } 600 \text { mg twice daily } \\
\text { Control: matched placebo } \\
\text { Duration: } 12 \text { weeks }\end{array}$ \\
& Follow-up: not reported \\
\hline Outcomes & Primary outcomes \\
- Depression measured by physician assessment of change in depression from baseline to end of treat- \\
ment \\
- Anxiety measured by physician assessment of change
\end{tabular}

Notes

\section{Risk of bias}

\begin{tabular}{|c|c|c|}
\hline Bias & Authors' judgement & Support for judgement \\
\hline $\begin{array}{l}\text { Random sequence genera- } \\
\text { tion (selection bias) }\end{array}$ & High risk & Comment: generation sequence controlled by Professor Furukawa \\
\hline $\begin{array}{l}\text { Allocation concealment } \\
\text { (selection bias) }\end{array}$ & Unclear risk & Comment: method of allocation concealment not reported \\
\hline $\begin{array}{l}\text { Blinding of participants } \\
\text { and personnel (perfor- } \\
\text { mance bias) } \\
\text { All outcomes }\end{array}$ & Low risk & Comment: double-blind reported and matched placebo used \\
\hline $\begin{array}{l}\text { Blinding of outcome as- } \\
\text { sessment (detection bias) } \\
\text { All outcomes }\end{array}$ & Low risk & $\begin{array}{l}\text { Comment: double-blind reported, so likely that outcome assessment was } \\
\text { blinded }\end{array}$ \\
\hline $\begin{array}{l}\text { Incomplete outcome data } \\
\text { (attrition bias) } \\
\text { All outcomes }\end{array}$ & High risk & $\begin{array}{l}\text { Comment: per protocol analyses reported only; } 79 / 285 \text { (27.3\%) missing from } \\
\text { depression analyses }\end{array}$ \\
\hline $\begin{array}{l}\text { Selective reporting (re- } \\
\text { porting bias) }\end{array}$ & Unclear risk & $\begin{array}{l}\text { Comment: all pre-specified outcomes reported. No trial protocol available to } \\
\text { compare with the publication }\end{array}$ \\
\hline Other bias & Unclear risk & $\begin{array}{l}\text { Comment: differences in baseline demographics between groups not report- } \\
\text { ed }\end{array}$ \\
\hline
\end{tabular}

Ponzio 2001

Methods Study design: parallel design

Number of arms: 2

Experimental arm: paroxetine (SSRI)

Control arm: matched placebo

Participants Geographical location: Italy


Ponzio 2001 (Continued)

\author{
Setting: outpatient \\ Stroke criteria: unclear \\ Method of stroke diagnosis: not reported \\ Time since stroke: not reported \\ Inclusion criteria: (1) 18 to 85 years of age; (2) MMSE score $>23$
}

Exclusion criteria: (1) concurrent predominant psychiatric disorders; (2) receiving psychotropic pharmacotherapy; (3) with substance abuse/dependence; (4) participation in other clinical trials; (5) suicide risk; (6) concomitant medication intolerance to paroxetine

Depression criteria: MADRS $>18$

Total number randomised in this trial: 229

Number randomised to treatment group: 112 (54\% men, mean age 64 years, SD 11)

Number randomised to control group: 117 (55\% men, mean age 66 years, SD 11)

Total number included in final analysis: 229

Number included in treatment group for final analysis: 112

Number included in control group for final analysis: 117

\begin{tabular}{ll}
\hline Interventions & $\begin{array}{l}\text { Treatment: paroxetine (SSRI) } 20 \text { to } 40 \text { mg daily } \\
\text { Control: } \text { matched placebo } \\
\text { Duration: } 8 \text { weeks }\end{array}$ \\
Follow-up: not reported \\
\hline Primary outcomes \\
- Depression (change in scores from baseline to end of treatment) measured using MADRS and CGI \\
Secondary outcomes \\
- Proportion scoring < 7 on MADRS and responders on CGI \\
- Disability (change in scores from baseline to end of treatment) measured using BI \\
- Functional capacity (change in scores from baseline to end of treatment) measured using Rankin scale
\end{tabular}

Notes

\title{
Risk of bias
}

\begin{tabular}{lll}
\hline Bias & Authors' judgement & Support for judgement \\
\hline $\begin{array}{l}\text { Random sequence genera- } \\
\text { tion (selection bias) }\end{array}$ & Unclear risk & Quote: "subjects randomised to paroxetine..." (p. 1) \\
& & Comment: method of sequence generation not reported \\
\hline
\end{tabular}

Allocation concealment Unclear risk $\quad$ Comment: method of allocation concealment not reported
(selection bias)

\begin{tabular}{|c|c|c|}
\hline $\begin{array}{l}\text { Blinding of participants } \\
\text { and personnel (perfor- } \\
\text { mance bias) }\end{array}$ & Unclear risk & $\begin{array}{l}\text { Quote: "blinding of study medication was maintained by referring to } \\
\text { dosage..." (p. 1) }\end{array}$ \\
\hline All outcomes & & $\begin{array}{l}\text { Comment: in study design, it states that this study is a 'double-blind, place- } \\
\text { bo-controlled' trial, but in treatment, this is a 'single-blind placebo' trial }\end{array}$ \\
\hline
\end{tabular}


Ponzio 2001 (Continued)

Blinding of outcome as- Unclear risk Comment: in study design, it states that this is a 'double-blind, placebo consessment (detection bias)

trolled' trial, but in treatment, this is a 'single-blind placebo' trial

All outcomes

$\begin{aligned} & \text { Incomplete outcome data } \\ & \begin{array}{l}\text { (attrition bias) } \\ \text { Allow risk }\end{array}\end{aligned} \quad \begin{aligned} & \text { Quote: "the primary analysis (post stroke depression) population was the in- } \\ & \text { tention-to-treat (ITT) population...." (p. 1) }\end{aligned}$

All outcomes

tention-to-treat (ITT) population...." (p. 1)

\begin{tabular}{|c|c|c|}
\hline $\begin{array}{l}\text { Selective reporting (re- } \\
\text { porting bias) }\end{array}$ & Unclear risk & $\begin{array}{l}\text { Comment: all pre-specified outcomes reported; no trial protocol available to } \\
\text { compare with the publication }\end{array}$ \\
\hline
\end{tabular}

\begin{tabular}{ll}
\hline Other bias Low risk & $\begin{array}{l}\text { Comment: no differences in baseline demographic characteristics between } \\
\text { groups }\end{array}$ \\
\hline
\end{tabular}

Rampello 2005

\begin{tabular}{ll}
\hline Study design: parallel design \\
Number of arms: 2 \\
Experimental arm: reboxetine (NRI) \\
Control arm: matched placebo \\
\hline
\end{tabular}

\title{
Participants
}

\author{
Geographical location: Italy \\ Setting: outpatient \\ Stroke criteria: single ischaemic or haemorrhagic stroke
}

Method of stroke diagnosis: diagnosis via CT and MRI

Time since stroke: 2 weeks

Inclusion criteria: (1) presence of major or minor depression; (2) presence of retarded depression; (3) lack of treatment with antidepressants 2 weeks before randomisation; (4) absence of treatment with neuroleptic drugs during 3 months before enrolment; (5) can sign informed consent

Exclusion criteria: (1) previous degenerative or expansive neurological disease; (2) tumour, multiple sclerosis, amyotrophic sclerosis, hydrocephalus, SAH, Binswanger's disease; (3) history of psychiatric illness (other than depression); (4) severe aphasia; (5) severe cognitive deficit; (6) chronic alcoholism

Depression criteria: psychiatric interview, HDRS $>20, \mathrm{BDI}>15$

Total number randomised in this trial: 31

Number randomised to treatment group: 16 (44\% men, mean age 78 years, SD 4)

Number randomised to control group: 15 (46\% men, mean age 77 years, SD 4)

Total number included in final analysis: 31

Number included in treatment group for final analysis: 16

Number included in control group for final analysis: 15

Interventions

Treatment: reboxetine (NRI) 4 mg twice daily

Control: matched placebo

Duration: 16 weeks

Follow-up: not reported

Outcomes Primary outcomes

Pharmacological, psychological, and non-invasive brain stimulation interventions for treating depression after stroke (Review) 
Rampello 2005 (Continued)

\title{
- Depression measured using HDRS and BDI
}

\section{Secondary outcomes}

- Adverse events

Notes

\section{Risk of bias}

\begin{tabular}{lll}
\hline Bias & Authors' judgement & Support for judgement \\
\hline $\begin{array}{l}\text { Random sequence genera- } \\
\text { tion (selection bias) }\end{array}$ & Low risk & $\begin{array}{l}\text { Quote: "a computer-generated randomization was carried out by a physician } \\
\text { who was not involved in the evaluation of patients..." (p. 277) }\end{array}$ \\
\hline
\end{tabular}

Allocation concealment Low risk

(selection bias)

Quote: "the generator of randomization assigned a code number (0) to patients who were treated with reboxetine, and a different code (1) was given to patients treated with placebo. Code 0 was stuck on totally white boxes, without any marks, sealed, containing the tablets of..." (p. 278)

Blinding of participants High risk
and personnel (perfor-
mance bias)
All outcomes

Quote: "the generator of randomization handed over, for each patient, the box marked with the code and containing the tablets that should be taken" (p. 279)

All outcomes

Comment: participants were blinded but the personnel who delivered the intervention knew the treatment allocation

\begin{tabular}{|c|c|c|}
\hline $\begin{array}{l}\text { Blinding of outcome as- } \\
\text { sessment (detection bias) }\end{array}$ & Low risk & $\begin{array}{l}\text { Quote: "the other physician was in charge of the follow-up visits and of the } \\
\text { evaluation of the outcome measures" (p. 279) }\end{array}$ \\
\hline
\end{tabular}

Incomplete outcome data Low risk

(attrition bias)

Comment: follow-up of all participants was complete; ITT analysis reported

All outcomes

\begin{tabular}{|c|c|c|}
\hline $\begin{array}{l}\text { Selective reporting (re- } \\
\text { porting bias) }\end{array}$ & Unclear risk & $\begin{array}{l}\text { Comment: all pre-specified outcomes reported; no trial protocol to compare } \\
\text { with the publication }\end{array}$ \\
\hline
\end{tabular}

Other bias Low risk

Reding 1986

$\begin{array}{ll}\text { Methods } & \text { Study design: parallel design } \\ & \text { Number of arms: } 2 \\ & \text { Experimental arm: trazodone- } \mathrm{HCl}(\mathrm{TCA}) \\ \text { Control arm: matched placebo }\end{array}$

Participants

\author{
Geographical location: USA \\ Setting: inpatients \\ Stroke criteria: all subtypes
}

Method of stroke diagnosis: diagnosis via clinical signs and CT (\% not reported)

Time since stroke: 45 to 48 days

Inclusion criteria: not reported 
Reding 1986 (Continued)

Exclusion criteria: (1) myocardial infarction within previous month; (2) on antiarrhythmic medication

Depression criteria: psychiatric interview (DSM-III, major and minor)

Total number randomised in this trial: 17

Number randomised to treatment group: 11 (66\% men, mean age 68 years, SE 2) Number randomised to control group: 6 ( $73 \%$ men, mean age 68 years, SE 3)

Total number included in final analysis: 17

Number included in treatment group for final analysis: 11

Number included in control group for final analysis: 6

Treatment: trazodone- $\mathrm{HCl}$ (TCA) $50 \mathrm{mg}$ daily; dose escalation every 3 days to target dose of $200 \mathrm{mg}$

Control: matched placebo

Duration: $32 \pm 6$ days (treatment group) and $24 \pm 4$ days (control group)

Follow-up: not reported

Primary outcomes
Outcomes $\quad$ Depression measured using clinical diagnosis of depression and ZDS
Secondary outcomes
- Disability measured using BI

Notes

\section{Risk of bias}

\begin{tabular}{lll}
\hline Bias & Authors' judgement & Support for judgement \\
\hline $\begin{array}{l}\text { Random sequence genera- } \\
\text { tion (selection bias) }\end{array}$ & Low risk & $\begin{array}{l}\text { Quote: "patients were assigned to either treatment or placebo groups accord- } \\
\text { ing to a table of random numbers" (p. 763) }\end{array}$ \\
\hline $\begin{array}{l}\text { Allocation concealment } \\
\text { (selection bias) }\end{array}$ & Low risk & $\begin{array}{l}\text { Quote: "...or placebo in an identical capsule was administered orally..." (p. } \\
763)\end{array}$ \\
\hline $\begin{array}{l}\text { Blinding of participants } \\
\text { and personnel (perfor- } \\
\text { mance bias) } \\
\text { All outcomes }\end{array}$ & Low risk & $\begin{array}{l}\text { Quote: "...or placebo in an identical capsule was administered orally..." (p. } \\
763)\end{array}$ \\
\hline
\end{tabular}

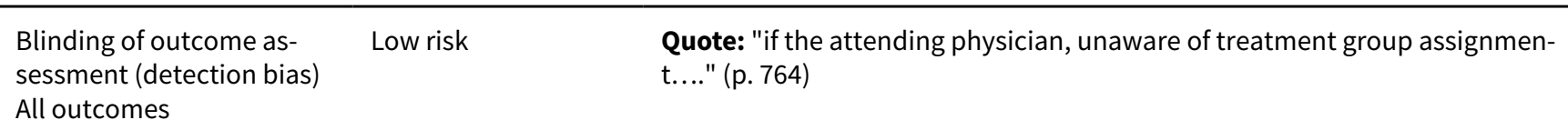

\begin{tabular}{lll}
\hline $\begin{array}{l}\text { Incomplete outcome data } \\
\text { (attrition bias) } \\
\text { All outcomes }\end{array}$ & Low risk & $\begin{array}{l}\text { Comment: follow-up of all participants was complete; ITT analysis reported in } \\
\text { table }\end{array}$ \\
\hline $\begin{array}{l}\text { Selective reporting (re- } \\
\text { porting bias) }\end{array}$ & Unclear risk & $\begin{array}{l}\text { Comment: all pre-specified outcomes reported; no trial protocol to compare } \\
\text { with the publication }\end{array}$ \\
\hline Other bias & Unclear risk & Comment: baseline demographic information not reported \\
\hline
\end{tabular}




\begin{tabular}{ll}
\hline Methods & Study design: parallel design \\
& Number of arms: 2 \\
Experimental arm: nefiracetam (nootropic agent) \\
Control arm: matched placebo
\end{tabular}

Participants

Geographical location: USA

Setting: unclear

Stroke criteria: ischaemic and primary intracerebral haemorrhage

Method of stroke diagnosis: unclear

Time since stroke: 10 days to 3 months

Inclusion criteria: not reported

Exclusion criteria: (1) other psychiatric or neurological disease (e.g. Alzheimer's disease, Parkinson's disease); (2) depression or suicidal plans requiring psychiatric hospitalisation; (3) on psychotropic medication (excluding benzodiazepines or insomnia medication); (4) comprehension deficit precluding verbal interview; (5) life-threatening illness; (6) previous subarachnoid haemorrhage

Depression criteria: psychiatric interview to confirm DSM-IV diagnosis of "depression due to stroke with major depressive-like episode" plus HDRS score $\geq 18$

Total number randomised in this trial: 76

Number randomised to treatment group: 48 (40\% men; mean age 68.1, SD 11.9)

Number randomised to control group: $28^{\star \star}$ (54\% men; mean age 66.8, SD 13.0)

Total number included in final analysis: 66

Number included in treatment group for final analysis: 41

Number included in control group for final analysis: $25^{\star *}$

Interventions

Treatment: nefiracetam (nootropic agent) $900 \mathrm{mg}, 3 \times 150 \mathrm{mg}$ capsule twice/d

Control: matching placebo $3 \times 150 \mathrm{mg}$ capsule twice/d

Duration: 12 weeks

Follow-up: not reported

\section{Outcomes}

\section{Primary outcomes}

- Depression measured using HDRS

- Depression measured using BDI

\section{Secondary outcomes}

- Apathy measured using Apathy Scale

- Leaving the trial early

- Adverse events

\section{Notes}


Robinson 2008a (Continued)

\begin{tabular}{lll} 
Bias & Authors' judgement & Support for judgement \\
\hline $\begin{array}{l}\text { Random sequence genera- } \\
\text { tion (selection bias) }\end{array}$ & Low risk & $\begin{array}{l}\text { Comment: based on the study author's responses, sequence generation was } \\
\text { attained with computer-generated numbers }\end{array}$ \\
\hline
\end{tabular}

\begin{tabular}{|c|c|c|}
\hline $\begin{array}{l}\text { Allocation concealment } \\
\text { (selection bias) }\end{array}$ & Low risk & $\begin{array}{l}\text { Quote: "nefiracetam or placebo was administered double-blind in three iden- } \\
\text { tical } 150 \text { mg capsules..." (p. 179) }\end{array}$ \\
\hline
\end{tabular}

\begin{tabular}{|c|c|c|}
\hline $\begin{array}{l}\text { Blinding of participants } \\
\text { and personnel (perfor- }\end{array}$ & Low risk & $\begin{array}{l}\text { Comment: study author states that this study was double-blinded but does } \\
\text { not state who was blinded }\end{array}$ \\
\hline
\end{tabular}
not state who was blinded

ce bias)

All outcomes

Blinding of outcome as- Low risk sessment (detection bias) All outcomes
Comment: study author states that this study was double-blinded but does not state who was blinded
Incomplete outcome data High risk
(attrition bias)

All outcomes

Selective reporting (re- High risk porting bias)
Quote: "...missing data points were estimated using LOCF..." (p. 146)

"attrition related bias cannot be ruled out" (p. 149)

Comment: the number of dropouts reported and the number analysed are inconsistent within and between publications

Comment: study author reports that a number of measures were assessed but does not provide details of these measures in the publication

Other bias Unclear risk Comment: baseline demographic information was not reported

Robinson 2008b

$\begin{array}{ll}\text { Methods } & \text { Study design: parallel design } \\ \text { Number of arms: } 2 \\ \text { Experimental arm: nefiracetam (nootropic agent) } \\ \text { Control arm: matched placebo }\end{array}$

Participants Geographical location: USA

Setting: unclear

Stroke criteria: ischaemic and primary intracerebral haemorrhage

Method of stroke diagnosis: unclear

Time since stroke: 10 days to 3 months

Inclusion criteria: not reported

Exclusion criteria: (1) other psychiatric or neurological disease (e.g. Alzheimer's disease, Parkinson's disease); (2) depression or suicidal plans requiring psychiatric hospitalisation; (3) on psychotropic medication (excluding benzodiazepines or insomnia medication); (4) comprehension deficit precluding verbal interview; (5) life-threatening illness; (6) previous subarachnoid haemorrhage

Depression criteria: psychiatric interview to confirm DSM-IV diagnosis of "depression due to stroke with major depressive-like episode" plus HDRS score $\geq 18$

Total number randomised in this trial: 83 
Robinson 2008b (Continued)

Number included in treatment group: 55 (40\% men; mean age 64.7, SD 11.9)

Number included in control group: $28^{\star \star}$ (54\% men; mean age 66.8 , SD 13.0)

Total number included in final analysis: 72

Number included in treatment group for final analysis: 47

Number included in control group for final analysis: $25^{\star *}$

\begin{tabular}{ll}
\hline Interventions & Treatment: nefiracetam $600 \mathrm{mg}, 3 \times 150 \mathrm{mg}$ \\
& Control: matching placebo $3 \times 150 \mathrm{mg}$ cap \\
& Duration: 12 weeks \\
& Follow-up: not reported \\
\hline Outcomes & Primary outcomes \\
- Depression measured using HDRS \\
- Depression measured using BDI \\
Secondary outcomes \\
- Apathy measured using Apathy Scale \\
- Leaving the trial early \\
- Adverse events
\end{tabular}

Notes

Risk of bias

\begin{tabular}{lll}
\hline Bias & Authors' judgement & Support for judgement \\
\hline $\begin{array}{l}\text { Random sequence genera- } \\
\text { tion (selection bias) }\end{array}$ & Low risk & $\begin{array}{l}\text { Comment: based on the study author's responses, sequence generation was } \\
\text { attained with computer-generated numbers }\end{array}$ \\
\hline $\begin{array}{l}\text { Allocation concealment } \\
\text { (selection bias) }\end{array}$ & Low risk & $\begin{array}{l}\text { Quote: "nefiracetam or placebo was administered double-blind in three iden- } \\
\text { tical } 150 \text { mg capsules..." (p. 179) }\end{array}$ \\
\hline
\end{tabular}

Blinding of participants Low risk and personnel (perfor-

mance bias)

All outcomes
Comment: study author states that this study was double-blinded but does not state who was blinded

\begin{tabular}{|c|c|c|}
\hline $\begin{array}{l}\text { Blinding of outcome as- } \\
\text { sessment (detection bias) } \\
\text { All outcomes }\end{array}$ & Low risk & $\begin{array}{l}\text { Comment: study author states that this study was double-blinded but does } \\
\text { not state who was blinded }\end{array}$ \\
\hline \multirow{2}{*}{$\begin{array}{l}\text { Incomplete outcome data } \\
\text { (attrition bias) } \\
\text { All outcomes }\end{array}$} & High risk & $\begin{array}{l}\text { Quote: "...missing data points were estimated using LOCF..." (p. 146) } \\
\text { "attrition related bias cannot be ruled out" (p. 149) }\end{array}$ \\
\hline & & $\begin{array}{l}\text { Comment: the number of dropouts reported and the number analysed are in- } \\
\text { consistent within and between publications }\end{array}$ \\
\hline $\begin{array}{l}\text { Selective reporting (re- } \\
\text { porting bias) }\end{array}$ & High risk & $\begin{array}{l}\text { Comment: study author reports that a number of measures were assessed but } \\
\text { does not provide details of these measures in the publication }\end{array}$ \\
\hline Other bias & Unclear risk & Comment: baseline demographic information was not reported \\
\hline
\end{tabular}


Number of arms: 2

Experimental arm: rTMS + Deanxit (flupentixol and melitracen)

Control arm: Deanxit (flupentixol and melitracen)

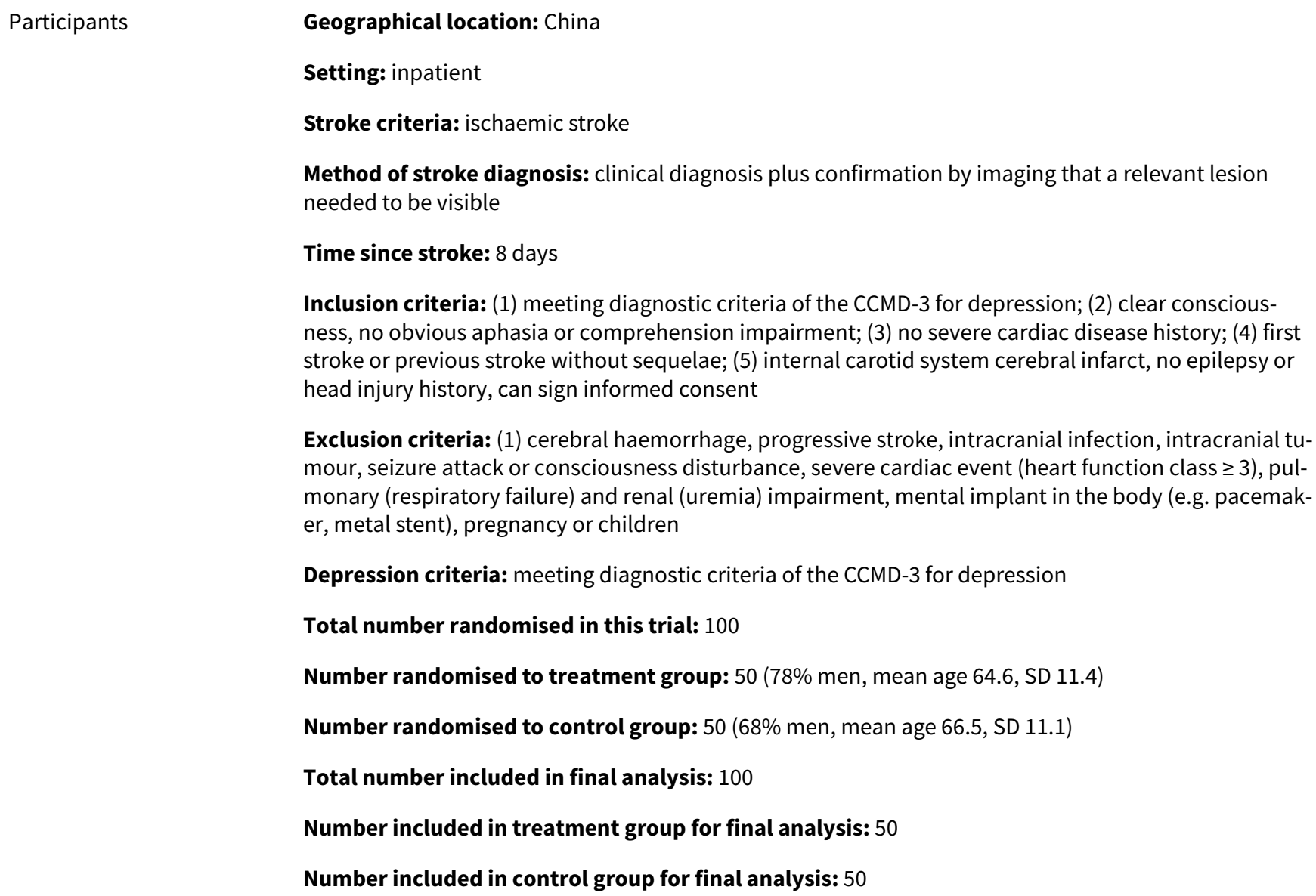

Exclusion criteria: (1) cerebral haemorrhage, progressive stroke, intracranial infection, intracranial tumour, seizure attack or consciousness disturbance, severe cardiac event (heart function class $\geq 3$ ), pulmonary (respiratory failure) and renal (uremia) impairment, mental implant in the body (e.g. pacemaker, metal stent), pregnancy or children

Depression criteria: meeting diagnostic criteria of the CCMD-3 for depression

Total number randomised in this trial: 100

Number randomised to treatment group: 50 (78\% men, mean age 64.6, SD 11.4)

Number randomised to control group: 50 (68\% men, mean age 66.5, SD 11.1)

Total number included in final analysis: 100

Number included in treatment group for final analysis: 50

Number included in control group for final analysis: 50

Control: Deanxit (flupentixol and melitracen)

Duration: 2 weeks

Follow-up: none

\section{Outcomes}

\section{Primary outcomes}

- Depression measured using HDRS

- Depression measured using SDS

\section{Notes}

\section{Risk of bias}


Sun 2013 (Continued)

\begin{tabular}{lll} 
Bias & Authors' judgement & Support for judgement \\
\hline $\begin{array}{l}\text { Random sequence genera- } \\
\text { tion (selection bias) }\end{array}$ & Unclear risk & Comment: method of sequence generation not reported \\
\hline $\begin{array}{l}\text { Allocation concealment } \\
\text { (selection bias) }\end{array}$ & Unclear risk & Comment: method of allocation concealment not reported \\
\hline $\begin{array}{l}\text { Blinding of participants } \\
\text { and personnel (perfor- } \\
\text { mance bias) }\end{array}$ & Unclear risk & $\begin{array}{l}\text { Comment: information about blinding of participants and personnel not re- } \\
\text { All outcomes }\end{array}$ \\
\hline
\end{tabular}

\begin{tabular}{ll}
\hline Blinding of outcome as- & Unclear risk $\quad$ Comment: information about blinding of outcome assessors not reported \\
sessment (detection bias) &
\end{tabular}

All outcomes

Incomplete outcome data Low risk Comment: ITT analysis reported; no missing data
(attrition bias)
All outcomes

\begin{tabular}{lll}
\hline $\begin{array}{l}\text { Selective reporting (re- } \\
\text { porting bias) }\end{array}$ & Unclear risk & $\begin{array}{l}\text { Comment: all pre-specified outcomes reported; no trial protocol available to } \\
\text { compare with the publication }\end{array}$ \\
\hline Other bias & Low risk & Comment: no differences in baseline demographics between groups \\
\hline
\end{tabular}

Thomas 2007

Methods Study design: parallel design

\section{Number of arms: 2}

Experimental arm: behavioural psychotherapy

Control arm: usual care

Participants Geographical location: UK

Setting: mixed

Stroke criteria: unclear

Method of stroke diagnosis: not reported

Time since stroke: 8.85 days

Inclusion criteria: (1) presence of aphasia confirmed by a speech and language therapist (hospital or community participants) or using the Sheffield Screening Test for Acquired Language Disorders (voluntary sector participants)

Exclusion criteria: (1) receiving treatment for depression pre-stroke (at the time of stroke), (2) with dementia, (3) blind or deaf; (4) unable to speak English before stroke

Depression criteria: using the 'sad' item of the VAMS and the 10-item hospital version of the SAD-Q, completed by a nurse, relative, or carer. Those identified as having low mood on the 'sad' item of the VAMS (cut-off $>50$ ) or the SAD-Q (cut-off $>6$ )

Total number randomised in this trial: 105

Number randomised to treatment group: 51 (57\% men, mean age 68.5 years, SD 13.1) 
Total number included in final analysis: 89

Number included in treatment group for final analysis: 43

Number included in control group for final analysis: 46

Treatment 1: behavioural psychotherapy up to 20 sessions of treatment over 3 months, with each session lasting approximately 1 hour. The manual had been developed from studies of cognitive-behavioural therapy for depression after stroke and with older adults, and from guidelines on conducting cognitive-behavioural therapy with people with aphasia. The intensity of therapy was left to the discretion of the assistant psychologist. The intervention was tailored to the individual's needs, and communication resources such as pictures, photographs, and letter charts were used

Administered by: assistant psychologist

Supervision: therapy was delivered by an assistant psychologist supervised weekly by a clinical psychologist. All assistant psychologists attended a joint monthly supervision meeting with a consultant clinical neuropsychologist. Assistant psychologists received training in supported communication from speech and language therapists and were provided with a therapy manual

Intervention fidelity: delivery of therapy was monitored by observation of therapy sessions by the chief investigator. The content of therapy was documented using record forms completed by the assistant psychologist after each session

Control: usual care

Duration: 3 months

Follow-up: 3 months

Outcomes Primary outcomes

- Depression measured using the 21-item hospital version of the SAD-Q - an observational measure of mood completed by a relative or primary carer

- Depression measured using the 'sad' item of VAMS

\section{Secondary outcomes}

- Self-esteem measured using Visual Analogue Self-Esteem Scale

- Activities of daily measured using Nottingham Leisure Questionnaire

- Caregiver strain measured using CSI

- Patient and carer satisfaction with care measured using 100-mm VAS

Notes

\section{Risk of bias}

\begin{tabular}{lll}
\hline Bias & Authors' judgement & Support for judgement \\
\hline $\begin{array}{l}\text { Random sequence genera- } \\
\text { tion (selection bias) }\end{array}$ & Low risk & $\begin{array}{l}\text { Quote: "participants were randomly allocated to one of two groups.....using a } \\
\text { computer generated pseudo-random list..." (p. 400) }\end{array}$ \\
\hline $\begin{array}{l}\text { Allocation concealment } \\
\text { (selection bias) }\end{array}$ & Low risk & $\begin{array}{l}\text { Quote: "the assistant psychologist providing treatment accessed the alloca- } \\
\text { tion by logging into a secure computer server, thus ensuring concealment of } \\
\text { allocation" (p. 400) }\end{array}$ \\
\hline
\end{tabular}

\begin{tabular}{lll}
\hline $\begin{array}{l}\text { Blinding of participants } \\
\text { and personnel (perfor- }\end{array}$ & High risk & $\begin{array}{l}\text { Comment: due to the nature of the trial, not possible to mask participants, } \\
\text { personnel, and researchers to treatment allocation }\end{array}$
\end{tabular}
mance bias)

All outcomes 
Thomas 2007 (Continued)

Blinding of outcome as- Unclear risk Comment: primary endpoint self-assessed by relative or carer who was aware sessment (detection bias)

All outcomes of treatment allocation. Secondary endpoints assessed using a blinded assessor

Incomplete outcome data High risk (attrition bias)

All outcomes

Quote: "outcomes were analysed by intention to treat" (p. 401)

"...missing data using the last observation carried forward on the assumption of no change..." (p. 402)

Comment: only per protocol analysis reported

\begin{tabular}{lll}
\hline $\begin{array}{l}\text { Selective reporting (re- } \\
\text { porting bias) }\end{array}$ & High risk & $\begin{array}{l}\text { Comment: one secondary outcome measure (Extended Activities of Daily Liv- } \\
\text { ing Scale) not reported in the publication }\end{array}$ \\
\hline Other bias & Low risk & $\begin{array}{l}\text { Comment: no differences in baseline demographic characteristics between } \\
\text { groups }\end{array}$
\end{tabular}

Towle 1989

\begin{tabular}{ll}
\hline Study design: parallel design \\
Number of arms: 2 \\
Experimental arm: pragmatic approach (counselling) \\
Control arm: custom-designed information booklet \\
\hline
\end{tabular}

Participants

Geographical location: UK

Setting: outpatients

Stroke criteria: all subtypes

Method of stroke diagnosis: diagnosis via clinical signs

Time since stroke: 6 to 7 months

Inclusion criteria: (1) able to complete questionnaires unaided

Exclusion criteria: (1) stroke < 1 year before randomisation; (2) residence in hospital or residential care

Depression criteria: WDI score $>17$ or GHQ-28 score $>9$

Total number randomised in this trial: 44

Number randomised to treatment group: 21 (43\% men, mean age 70 years, SD 9)

Number randomised to control group: 23 (30\% men, mean age 69 years, SD 7)

Total number included in final analysis: 43

Number included in treatment group for final analysis: 21

Number included in control group for final analysis: 22

Treatment: pragmatic approach dealing with problems identified by social worker and patients; included counselling the patient and caregiver, giving opportunity to reflect upon their situation and express their feelings (duration: 2 to 11 visits over 16 weeks, mean visits $6.8 \pm 2.8$; however, length and content of visits varied)

Administered by: not reported

Supervision: not reported 
Towle 1989 (Continued)

Intervention fidelity: unclear; no report of formal evaluation of the quality or content of therapy pro-

vided

Control: custom-designed information booklet (covered areas believed to be of use and interest to stroke survivors and their families, such as details on housing and financial benefits; aids to daily living; addresses of stroke clubs and self-help groups; telephone number of local social services department),

1 visit, no ongoing visits

Administered by: social worker

Duration: 16 weeks

Follow-up: not reported

Outcomes Primary outcomes

- Depression (change in scores from baseline to end of treatment) measured using WDI and GHQ-28

\section{Notes}

\section{Risk of bias}

\begin{tabular}{lll}
\hline Bias & Authors' judgement & Support for judgement \\
\hline $\begin{array}{l}\text { Random sequence genera- } \\
\text { tion (selection bias) }\end{array}$ & Low risk & $\begin{array}{l}\text { Quote: "the order of the envelopes had been decided before the study using } \\
\text { random number tables" (p. 520) }\end{array}$ \\
\hline $\begin{array}{l}\text { Allocation concealment } \\
\text { (selection bias) }\end{array}$ & High risk & $\begin{array}{l}\text { Quote: "the patients were then allocated randomly to one of two groups using } \\
\text { sealed envelopes each containing a slip of paper stating either "treatment" or } \\
\text { "no treatment"' (p. 520) }\end{array}$ \\
\hline
\end{tabular}

Blinding of participants High risk and personnel (performance bias)

All outcomes

Quote: "the patients were then allocated randomly to one of two groups using sealed envelopes each containing a slip of paper stating either "treatment" or "no treatment"" (p. 520)

Comment: due to the nature of the trial, it was not possible to mask participants or social worker to treatment allocation

$\begin{array}{lll}\begin{array}{l}\text { Blinding of outcome as- } \\ \text { sessment (detection bias) }\end{array} & \text { Unclear risk } & \begin{array}{l}\text { Quote: "each patient was visited } 8 \text { weeks and } 16 \text { weeks later by the indepen- } \\ \text { dent assessor who repeated the pre-intervention questionnaires" }\end{array}\end{array}$

All outcomes

Comment: it is unclear whether the independent assessor was blinded to treatment allocation

\begin{tabular}{lll}
\hline $\begin{array}{l}\text { Incomplete outcome data } \\
\text { (attrition bias) } \\
\text { All outcomes }\end{array}$ & High risk & $\begin{array}{l}\text { Comment: } 1 / 44 \text { participants was excluded from the analysis; only per protocol } \\
\text { analysis reported }\end{array}$ \\
\hline $\begin{array}{l}\text { Selective reporting (re- } \\
\text { porting bias) }\end{array}$ & Unclear risk & $\begin{array}{l}\text { Comment: all pre-specified outcomes reported; no trial protocol available to } \\
\text { compare with the publication }\end{array}$
\end{tabular}

\begin{tabular}{ll}
\hline Other bias Low risk & $\begin{array}{l}\text { Comment: no differences in baseline demographic characteristics between } \\
\text { groups }\end{array}$ \\
\hline
\end{tabular}

Wang 2004a

Study design: parallel design
Number of arms: 2
Experimental arm: psychological therapy


Wang 2004a (Continued)

Control arm: usual care

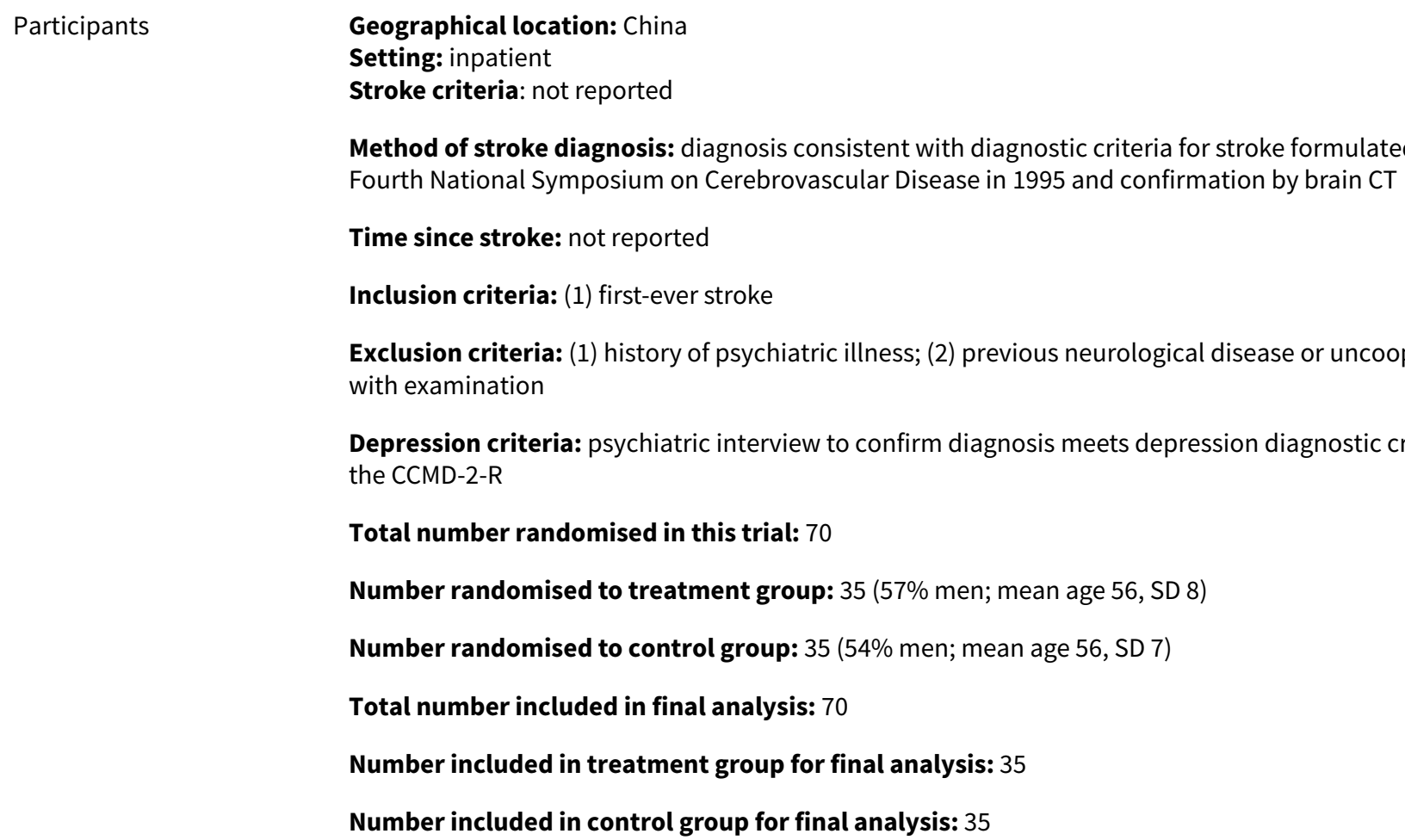
Fourth National Symposium on Cerebrovascular Disease in 1995 and confirmation by brain CT

Time since stroke: not reported

Inclusion criteria: (1) first-ever stroke with examination the CCMD-2-R

Total number randomised in this trial: 70

Number randomised to treatment group: 35 (57\% men; mean age 56, SD 8)

Number randomised to control group: 35 (54\% men; mean age 56, SD 7)

Total number included in final analysis: 70

Number included in treatment group for final analysis: 35

Number included in control group for final analysis: 35

Method of stroke diagnosis: diagnosis consistent with diagnostic criteria for stroke formulated by the

Exclusion criteria: (1) history of psychiatric illness; (2) previous neurological disease or uncooperative

Depression criteria: psychiatric interview to confirm diagnosis meets depression diagnostic criteria of

Administered by: not reported

Supervision: not reported

Intervention fidelity: not reported

Control: usual care

Duration: 5 weeks

Follow-up: none

\begin{tabular}{ll}
\hline Outcomes & Primary outcomes \\
& - Depression measured using ZDS \\
- Cognition measured by P300
\end{tabular}

Notes

\section{Risk of bias}

\begin{tabular}{lll}
\hline Bias & Authors' judgement & Support for judgement \\
\hline $\begin{array}{l}\text { Random sequence genera- } \\
\text { tion (selection bias) }\end{array}$ & Unclear risk & Comment: method of sequence generation not reported \\
\hline $\begin{array}{l}\text { Allocation concealment } \\
\text { (selection bias) }\end{array}$ & Unclear risk & Comment: method of allocation concealment not reported \\
\hline
\end{tabular}


Wang 2004a (Continued)

Blinding of participants Unclear risk Comment: blinding of participants and personnel not reported and personnel (performance bias)

All outcomes

\begin{tabular}{ll}
\hline Blinding of outcome as- & Unclear risk
\end{tabular} Comment: blinding of outcome assessment not reported

All outcomes

Incomplete outcome data Low risk Comment: ITT analysis reported; no missing data
(attrition bias)

All outcomes

\begin{tabular}{|c|c|c|}
\hline $\begin{array}{l}\text { Selective reporting (re- } \\
\text { porting bias) }\end{array}$ & Unclear risk & $\begin{array}{l}\text { Comment: all pre-specified outcomes reported; no trial protocol available to } \\
\text { compare with the publication }\end{array}$ \\
\hline
\end{tabular}

Other bias Low risk $\quad \begin{aligned} & \text { Comment: no differences in baseline demographic characteristics between } \\ & \text { groups }\end{aligned}$
groups

Wang 2005

Study design: parallel design
Number of arms: 2
Experimental arm: fluoxetine (SSRI)
Control arm: matched placebo

Participants

Geographical location: China

Setting: inpatient

Stroke criteria: all stroke

Method of stroke diagnosis: diagnosis consistent with Diagnostic Criteria for Cerebrovascular Disease formulated by the Fourth National Conference of Chinese Medical Association in 1995

Inclusion criteria: not reported

Exclusion criteria: (1) history of psychiatric illness; (2) dementia; (3) aphasia; (4) disturbance of consciousness

Depression criteria: HDRS scores $>17$

Total number randomised in this trial: 108

Number randomised to treatment group: 54 (57\% men, mean age 58.9 years for total sample)

Number randomised to control group: 54 (57\% men, mean age 58.9 years for total sample)

Total number included in final analysis: 108

Number included in treatment group for final analysis: 54

Number included in control group for final analysis: 54

\footnotetext{
Interventions

Treatment: fluoxetine (SSRI) 20 to $40 \mathrm{mg} / \mathrm{d}$. If reduction in HDRS scores $\leq 5$ points after 2 weeks of treatment, increase dosage to $40 \mathrm{mg} / \mathrm{d}$
} 
Wang 2005 (Continued)

Control: matched placebo

Duration: 4 weeks

Follow-up: none

Outcomes Primary outcomes

- Depression measured using HDRS (remission: no depression symptoms and HDRS < 7; improved depression symptoms: reduction of HDRS scores by $\geq 5$; ineffective: severely depressed mood and reduction in HDRS scores $<4$ )

\section{Secondry outcomes}

- Neurological Impairment measured using CSS

- Leaving the trial early

Notes

\section{Risk of bias}

\begin{tabular}{lll}
\hline Bias & Authors' judgement & Support for judgement \\
\hline $\begin{array}{l}\text { Random sequence genera- } \\
\text { tion (selection bias) }\end{array}$ & Unclear risk & Comment: method of sequence generation not reported \\
\hline
\end{tabular}

tion (selection bias)

\begin{tabular}{l}
$\begin{array}{l}\text { Allocation concealment } \\
\text { (selection bias) }\end{array}$ Unclear risk Comment: method of allocation concealment not reported \\
\hline
\end{tabular}

Blinding of participants Unclear risk Comment: single-blind reported but who was blinded not reported
and personnel (perfor-
mance bias)
All outcomes

\begin{tabular}{ll}
\hline Blinding of outcome as- & Unclear risk
\end{tabular} Comment: blinding of outcome assessment not reported

All outcomes

\begin{tabular}{|c|c|c|}
\hline $\begin{array}{l}\text { Incomplete outcome data } \\
\text { (attrition bias) } \\
\text { All outcomes }\end{array}$ & High risk & $\begin{array}{l}\text { Comment: ITT (last observation carried forward) for dichotomous endpoints; } \\
\text { unclear for continuous endpoints }\end{array}$ \\
\hline
\end{tabular}

\begin{tabular}{lll}
\hline $\begin{array}{l}\text { Selective reporting (re- } \\
\text { porting bias) }\end{array}$ & Unclear risk & $\begin{array}{l}\text { Comment: all pre-specified outcomes reported; no trial protocol available to } \\
\text { compare with the publication }\end{array}$ \\
\hline Other bias & Unclear risk & Comment: difference in baseline demographic characteristics not reported \\
\hline
\end{tabular}

Wang 2005a

\begin{tabular}{ll}
\hline Methods & Study design: parallel design \\
& Number of arms: 2 \\
& Experimental arm: combined psychotherapy + paroxetine (SSRI) \\
& Control arm: paroxetine (SSRI) \\
\hline Participants & Geographical location: China
\end{tabular}


Wang 2005a (Continued)

\section{Setting: inpatient}

Stroke criteria: ischaemic and haemorrhagic stroke; haemorrhagic subtypes not specified

Method of stroke diagnosis: diagnosis consistent with diagnostic criteria for cerebrovascular disease formulated by the National Symposium on Cerebrovascular Disease of Chinese Medical Association in 1995 and confirmation by brain CT or MRI

Time since stroke: 21.85 days

Inclusion criteria: (1) first-ever stroke

Exclusion criteria: (1) history of psychiatric illness, depressive phase of bipolar disorders; (2) antidepressants and antipsychotics in the previous 3 months; (3) severe cognitive impairment, aphasia; (4) severe cardiac impairment, hepatic or renal impairment; (5) coma; (6) too severe clinical condition to receive interview; (7) allergy to paroxetine

Depression criteria: meeting both organic depression and organic anxiety diagnostic criteria of the CCMD-3

Total number randomised in this trial: 54

Number included in treatment group: 27 (52\% men; mean age 64.0, SD 5.3)

Number included in control group: 27 (52\% men; mean age 62.4, SD 6.1)

Total number included in final analysis: 54

Number included in treatment group for final analysis: 27

Number included in control group for final analysis: 27

Interventions

Treatment: combined psychotherapy, 1 session/week variable length 30 to 60 minutes administered by a psychotherapist + SSRI (paroxetine) $20 \mathrm{mg} / \mathrm{d}$ in the morning. Psychotherapy was described as having a supportive focus

Administered by: not reported

Supervision: not reported

Intervention fidelity: not reported

Control: paroxetine (SSRI) $20 \mathrm{mg} / \mathrm{d}$ in the morning

Duration: 6 weeks

Follow-up: none

Outcomes

Primary outcomes

- Depression measured using HDRS

Secondary outcomes

- Anxiety measured using HARS

- Disability measured using BI

- Impairment measure using SSS 
Wang 2005a (Continued)

Random sequence genera- Unclear risk Comment: method of sequence generation not reported tion (selection bias)

Allocation concealment $\quad$ Unclear risk
(selection bias)

Blinding of participants Unclear risk Comment: blinding of participants and personnel not reported

and personnel (perfor-

mance bias)

All outcomes

\begin{tabular}{lll}
\hline $\begin{array}{l}\text { Blinding of outcome as- } \\
\text { sessment (detection bias) } \\
\text { All outcomes }\end{array}$ & Unclear risk & Comment: blinding of outcome assessment not reported \\
\hline $\begin{array}{l}\text { Incomplete outcome data } \\
\text { (attrition bias) }\end{array}$ & Unclear risk & $\begin{array}{l}\text { Comment: } 2 / 54 \text { treatment and } 0 / 54 \text { control dropped out. ITT for categorical } \\
\text { outcome variable: clinical efficacy of participants with missing data regarded } \\
\text { all outcomes } \\
\text { ported }\end{array}$
\end{tabular}

\begin{tabular}{lll}
\hline $\begin{array}{l}\text { Selective reporting (re- } \\
\text { porting bias) }\end{array}$ & Unclear risk & $\begin{array}{l}\text { Comment: all pre-specified outcomes reported; no trial protocol available to } \\
\text { compare with the publication }\end{array}$ \\
\hline Other bias & Low risk & $\begin{array}{l}\text { Comment: no differences in baseline demographic characteristics between } \\
\text { groups }\end{array}$ \\
\hline
\end{tabular}

Watkins 2007

Methods Study design: parallel design

Number of arms: 2

Experimental arm: motivational interviewing

Control arm: usual care

Participants

Geographical location: UK

Setting: inpatient

Stroke criteria: all subtypes

Method of stroke diagnosis: diagnosis via clinical signs and CT (100\%)

Time since stroke: 5 to 28 days

Inclusion criteria: (1) over 18 years of age

Exclusion criteria: (1) severe cognitive and communication problems; (2) moving out of the area after discharge; (3) already receiving psychiatric or clinical psychology intervention

Depression criteria: GHQ score $>4$

Total number randomised in this trial: 254

Number randomised to treatment group: 127 (52\% men, mean age 68 years, SD 12)

Number randomised to control group: 127 (53\% men, mean age 68 years, SD 12)

Total number included in final analysis: 254

Number included in treatment group for final analysis: 127 
Watkins 2007 (Continued)

\section{Number included in control group for final analysis: 127}

Interventions Treatment: motivational interviewing, up to 4 sessions, 1 per week, with same therapist

Administered by: therapists

Supervision: therapists received 4 days of training in motivational interviewing by a specialist followed by up to 10 practice sessions until competent and confident of the technique. Therapists were supervised by a clinical psychologist through team meetings and 1-to-1 clinical supervision sessions on a monthly basis with additional informal support throughout the study

Intervention fidelity: therapy sessions were audio recorded. The quality of the application of motivational interviewing was assessed by analysing a purposive sample of 60 sessions from different patients. A clinical psychologist reviewed the content of 20 therapist utterances around the midpoint of each session using a structured evaluation tool, "Motivational Interviewing Skill Code (version 2)". Utterances rated motivational interviewing-consistent included open questions, reflections, advise with permission, affirm, emphasise control, reflect, re-frame, and support. Utterances rated motivational interviewing-inconsistent included advise without permission, confront, direct, raise concern without permission, and warn. The percentage of motivational interviewing-consistent utterances was determined (total MI-consistent/(total MI-consistent plus MI-inconsistent)). Unclear if or how this information was fed back to therapists

Control: usual care

Delivered by: nurses and non-clinical psychologists

Duration: 4 weeks

Follow-up: none

$\begin{array}{ll}\text { Putcomes } & \text { Primary outcomes } \\ \text { - Depression (proportion no longer meeting study criteria for depression, change in scores from base- } \\ \text { line to end of treatment) measured using GHQ-28 } \\ \text { - Disability measured using BI } \\ \text { - Stroke Impairment measured using Stroke Expectations Questionnaire }\end{array}$

Notes Additional unpublished data provided by study authors

\section{Risk of bias}

\section{Bias \\ Authors' judgement Support for judgement}

Random sequence genera- Low risk tion (selection bias)
Quote: "a research nurse randomized patients (1:1 ratio) to either usual care (control) or MI (intervention) using minimization over sex, age (65 and 65 years), baseline function in activities of daily living (ADL; Barthel: 18 to 20; 11 to $17 ; 0$ to 10$)$, and location (acute stroke unit)"

\section{Allocation concealment High risk} (selection bias)
Quote: "the same nurse then assigned intervention group patients to 1 of 4 therapists using an opaque sealed envelope in a pseudorandomized blocked design" (p. 1957)

\section{Blinding of participants High risk} and personnel (performance bias)

All outcomes
Quote: "the same nurse then assigned intervention group patients to 1 of 4 therapists using an opaque sealed envelope in a pseudorandomized blocked design" (p. 1957)

Comment: due to the nature of the intervention, it was not possible to mask participants, nurses, and researchers to treatment allocation
High risk

Blinding of outcome assessment (detection bias)

All outcomes
Quote: "surviving patients were sent a questionnaire. Patients not returning questionnaires within 2 weeks were telephoned by a second research nurse, blind to group allocation, and given the option of declining, having a further 
Watkins 2007 (Continued)

questionnaire posted, completing the questionnaire over the telephone, or receiving a home visit to assist" (p. 1957)

\begin{tabular}{lll}
\hline $\begin{array}{l}\text { Incomplete outcome data } \\
\text { (attrition bias) } \\
\text { All outcomes }\end{array}$ & Low risk & $\begin{array}{l}\text { Quote: "where data were missing, imputations were performed as described } \\
\text { previously" (p. 1958) }\end{array}$ \\
\hline $\begin{array}{l}\text { Selective reporting (re- } \\
\text { porting bias) }\end{array}$ & Unclear risk & $\begin{array}{l}\text { Comment: ITT analysis reported } \\
\text { compare with the publication }\end{array}$ \\
\hline $\begin{array}{l}\text { Other bias } \\
\text { Cow risk }\end{array}$ & $\begin{array}{l}\text { Comment: } \text { no differences in baseline demographic characteristics between } \\
\text { groups }\end{array}$ \\
\hline
\end{tabular}

Wiart 2000

\begin{tabular}{ll}
\hline Methods & Study design: parallel design \\
& Number of arms: 2 \\
& Experimental arm: fluoxetine (SSRI) \\
Control arm: matched placebo
\end{tabular}

Participants

Geographical location: France

Setting: not reported

Stroke criteria: ischaemic stroke and primary intracerebral haemorrhage

Method of stroke diagnosis: diagnosis via clinical signs and CT (100\%)

Time since stroke: 48 days

Inclusion criteria: (1) all antidepressant or neuroleptic drugs stopped 10 days before enrolment

Exclusion criteria: (1) severe psychiatric problems that required hospitalisation; (2) severe cognitive impairment; (3) chronic alcoholism; (4) chronic associated handicapping pathology; (5) contraindication to fluoxetine

Depression criteria: psychiatric interview (ICD-10 criteria) and MADRS score $>19$

Total number randomised in this trial: 31

Number randomised to treatment group: 16 (56\% men, mean age 66 years, SD 7)

Number randomised to control group: 15 (40\% men, mean age 69 years, SD 12)

Total number included in final analysis: 31

Number included in treatment group for final analysis: 16

Number included in control group for final analysis: 15

\begin{tabular}{ll}
\hline Interventions & $\begin{array}{l}\text { Treatment: fluoxetine (SSRI) } 20 \text { mg daily } \\
\text { Control: matched placebo } \\
\text { Duration: } 45 \text { days }\end{array}$ \\
Follow-up: none
\end{tabular}

Outcomes

Primary outcomes 
Wiart 2000 (Continued)

- Depression (change in scores from baseline to end of treatment, 50\% reduction in score) measured using MADRS

\section{Secondary outcomes}

- Functional capacity measured using FIM

- Cognitive function measured using MMSE

- Motor function measured using Motoricity Index

- Leaving the study early

- Adverse events

- Death

Notes

\section{Risk of bias}

\begin{tabular}{lll}
\hline Bias & Authors' judgement & Support for judgement \\
\hline $\begin{array}{l}\text { Random sequence genera- } \\
\text { tion (selection bias) }\end{array}$ & Unclear risk & Comment: method of sequence generation not reported \\
\hline $\begin{array}{l}\text { Allocation concealment } \\
\text { (selection bias) }\end{array}$ & Low risk & $\begin{array}{l}\text { Quote: "treatment lasted up to } 45 \text { days (end point) and was given in the form } \\
\text { of identical white capsules containing } 20 \text { mg of either fluoxetine or placebo, } \\
\text { delivered in boxes coded by the central pharmacy of the University Hospital } \\
\text { complex of Bordeaux" (p. 1829) }\end{array}$ \\
\hline $\begin{array}{l}\text { Blinding of participants } \\
\text { and personnel (perfor- } \\
\text { mance bias) }\end{array}$ & Low risk & $\begin{array}{l}\text { Quote: "treatment lasted up to 45 days (end point) and was given in the form } \\
\text { of identical white capsules containing 20 mg of either fluoxetine or placebo, } \\
\text { delivered in boxes coded by the central pharmacy of the University Hospital } \\
\text { complex of Bordeaux" (p. 1829) }\end{array}$
\end{tabular}

\begin{tabular}{|c|c|c|}
\hline $\begin{array}{l}\text { Blinding of outcome as- } \\
\text { sessment (detection bias) } \\
\text { All outcomes }\end{array}$ & Low risk & Comment: double-blind reported but who was blinded not reported \\
\hline $\begin{array}{l}\text { Incomplete outcome data } \\
\text { (attrition bias) } \\
\text { All outcomes }\end{array}$ & Low risk & $\begin{array}{l}\text { Quote: "an intent-to-treat statistical analysis was conducted in which the last } \\
\text { visit recorded was used as an end point" (p. 1830) } \\
\text { Comment: missing data were handled using last observation carried forward } \\
\text { method }\end{array}$ \\
\hline $\begin{array}{l}\text { Selective reporting (re- } \\
\text { porting bias) }\end{array}$ & Unclear risk & $\begin{array}{l}\text { Comment: all pre-specified outcomes reported; no trial protocol available to } \\
\text { compare with the publication }\end{array}$ \\
\hline Other bias & Low risk & $\begin{array}{l}\text { Comment: no differences in baseline demographic characteristics between } \\
\text { groups }\end{array}$ \\
\hline
\end{tabular}

Yang 2002

\begin{tabular}{ll}
\hline Methods & Study design: parallel design \\
& Number of arms: 2
\end{tabular}

Number of arms: 2

Experimental arm: paroxetine (SSRI)

Control arm: matched placebo

Participants Geographical location: China

Pharmacological, psychological, and non-invasive brain stimulation interventions for treating depression after stroke (Review) 
Yang 2002 (Continued)

Setting: outpatient

Stroke criteria: ischaemic and haemorrhagic stroke

Method of stroke diagnosis: not reported

Inclusion criteria: not reported

Exclusion criteria: not reported

Depression criteria: HDRS score $>7$

Total number randomised in this trial: 121

Number included in treatment group: 64 (63\% men, mean age 64 years, SD 3)

Number included in control group: 57 ( $56 \%$ men, mean age 63 years, SD 5)

Total number included in final analysis: 110

Number included in treatment group for final analysis: unclear

Number included in control group for final analysis: unclear

\begin{tabular}{ll}
\hline Interventions & Treatment: paroxetine (SSRI) $20 \mathrm{mg}$ daily \\
Control: matched placebo \\
Duration: 4 months \\
Follow-up: none
\end{tabular}

Outcomes

Primary outcomes

- Depression (50\% reduction in scores from baseline to end of treatment) measured using HDRS

Notes

\section{Risk of bias}

\begin{tabular}{lll}
\hline Bias & Authors' judgement & Support for judgement \\
\hline $\begin{array}{l}\text { Random sequence genera- } \\
\text { tion (selection bias) }\end{array}$ & Unclear risk & Comment: method of sequence generation not reported \\
\hline $\begin{array}{l}\text { Allocation concealment } \\
\text { (selection bias) }\end{array}$ & Unclear risk & Comment: method of allocation concealment not reported \\
\hline
\end{tabular}

Blinding of participants Unclear risk Comment: blinding of participants and personnel not reported
and personnel (perfor-

mance bias)

All outcomes

\begin{tabular}{lll}
\hline $\begin{array}{l}\text { Blinding of outcome as- } \\
\text { sessment (detection bias) } \\
\text { All outcomes }\end{array}$ & Unclear risk & Comment: blinding of outcome assessment not reported \\
\hline $\begin{array}{l}\text { Incomplete outcome data } \\
\text { (attrition bias) } \\
\text { All outcomes }\end{array}$ & High risk & $\begin{array}{l}\text { Comment: per protocol analysis reported only; } 11 / 121(9 \%) \text { excluded from } \\
\text { analysis }\end{array}$ \\
\hline $\begin{array}{l}\text { Selective reporting (re- } \\
\text { porting bias) }\end{array}$ & Unclear risk & $\begin{array}{l}\text { Comment: all pre-specified outcomes reported; no trial protocol available to } \\
\text { compare with the publication }\end{array}$ \\
\hline
\end{tabular}


Yang 2013

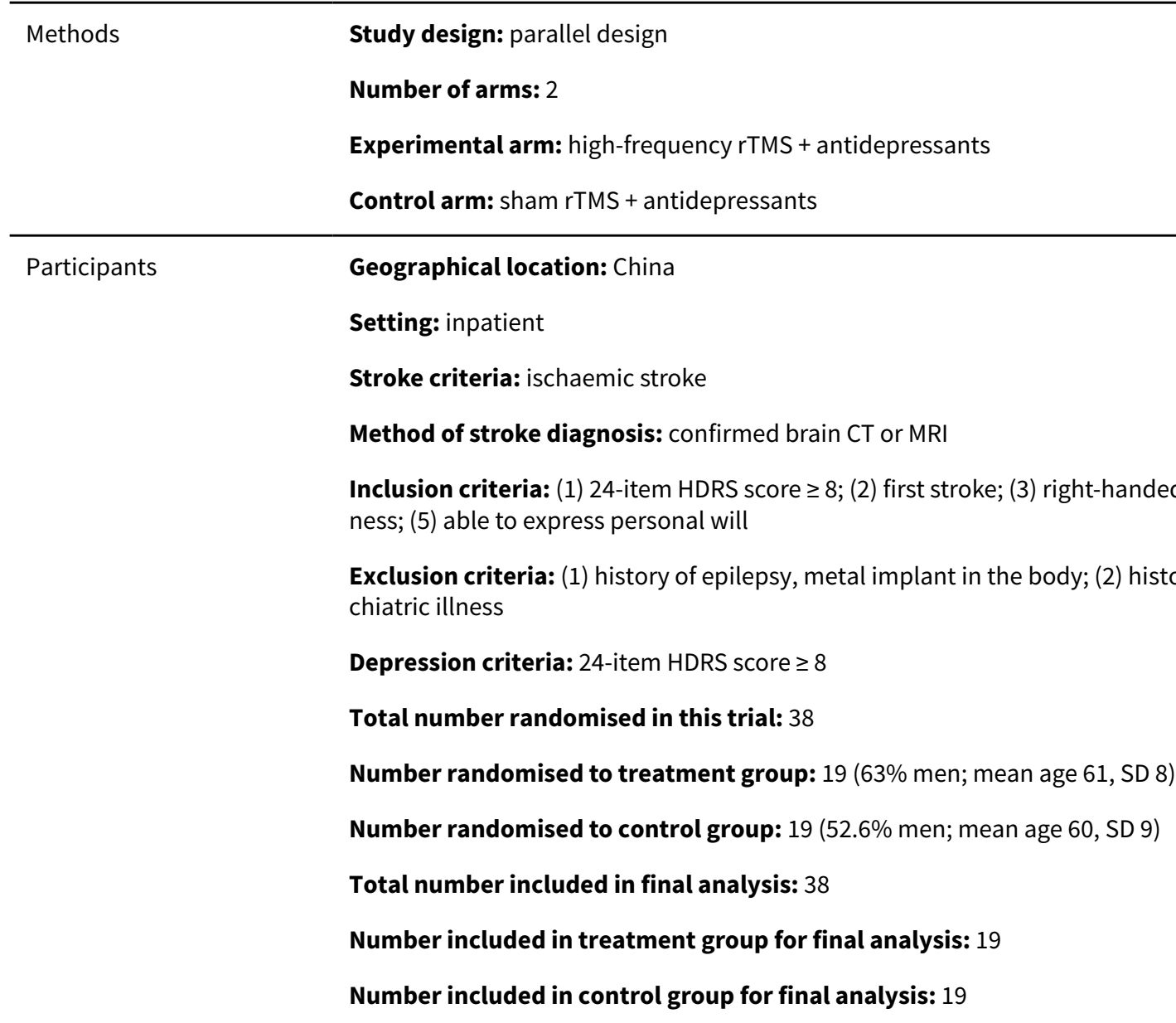

Interventions

Treatment: high-frequency rTMS + antidepressants. Frequency: $10 \mathrm{~Hz}$, intensity: $80 \%$ motor threshold, 1 stimulation lasts 4.9 seconds and stops for 20 seconds, total impulse number: 1960/d, 16 minutes per day, for 10 working days, location: left DLPFC

Control: sham rTMS + antidepressants. Keeping the coils at 90-degree angles with the scalp

Duration: 2 weeks

Follow-up: 4 weeks

Outcomes Primary outcomes

- Depression measured using HDRS

Notes

\section{Risk of bias}

\begin{tabular}{lll}
\hline Bias & Authors' judgement & Support for judgement \\
\hline $\begin{array}{l}\text { Random sequence genera- } \\
\text { tion (selection bias) }\end{array}$ & Unclear risk & Comment: method of sequence generation not reported \\
\hline $\begin{array}{l}\text { Allocation concealment } \\
\text { (selection bias) }\end{array}$ & Unclear risk & Comment: method of allocation concealment not reported \\
\hline
\end{tabular}


Yang 2013 (Continued)

Blinding of participants Unclear risk Comment: information about blinding of participants and personnel not reand personnel (perforported mance bias)

All outcomes

Blinding of outcome as-
sessment (detection bias) $\quad$ Unclear risk Comment: information about blinding of outcome assessors not reported

All outcomes

Incomplete outcome data Low risk Comment: ITT analysis reported; no missing data
(attrition bias)

All outcomes

\begin{tabular}{|c|c|c|}
\hline $\begin{array}{l}\text { Selective reporting (re- } \\
\text { porting bias) }\end{array}$ & Unclear risk & $\begin{array}{l}\text { Comment: all pre-specified outcomes reported; no trial protocol available to } \\
\text { compare with the publication }\end{array}$ \\
\hline
\end{tabular}

Other bias Low risk Comment: no differences in baseline demographics between groups

Yang 2014a

\begin{tabular}{ll}
\hline Study design: parallel design \\
Number of arms: 2 \\
Experimental arm: high-frequency rTMS \\
Control arm: sham rTMS \\
\hline
\end{tabular}

Participants

\author{
Geographical location: China \\ Setting: mixed \\ Stroke criteria: not reported
}

Method of stroke diagnosis: clinical diagnosis plus confirmation by imaging that a relevant lesion needed to be visible

Inclusion criteria: (1) meeting diagnostic criteria of the CCMD-3 for depression; (2) 24-item HDRS score $\geq 8$; (3) first stroke; (4) clear consciousness; (5) able to express personal will and to sign informed consent

Exclusion criteria: (1) history or family history of psychiatric illness; (2) unable to co-operate with the examination due to obvious aphasia or severe cognitive dysfunction; (3) history of epilepsy, metal implant in the body

Depression criteria: meeting diagnostic criteria of the CCMD-3 for depression and 24-item HDRS score $\geq 8$

Total number randomised in this trial: 56

Number randomised to treatment group: 37 (75.6\% men; mean age 56.6, SD 13.6)

Number randomised to control group: $19^{\star \star}$ ( $73 \%$ men; mean age 53.3 , SD 14.6)

Total number included in final analysis: 55

Number included in treatment group for final analysis: 37

Number included in control group for final analysis: $19^{\star *}$ 
Yang 2014a (Continued) Interventions
Treatment: high-frequency rTMS. Frequency: $10 \mathrm{~Hz}$, intensity: $90 \%$ motor threshold, 1 stimulation lasts 5 seconds and stops for 35 seconds, total impulse number: 1500, location: left DLPFC

Control: sham rTMS. With coils kept at 90-degree angles with the scalp and with coils contacting the scalp, participants could hear the click sounds

Duration: 2 weeks

Follow-up: 4 weeks

Outcomes Primary outcomes

- Depression measured using HDRS

Notes

\section{Risk of bias}

\begin{tabular}{lll}
\hline Bias & Authors' judgement & Support for judgement \\
\hline $\begin{array}{l}\text { Random sequence genera- } \\
\text { tion (selection bias) }\end{array}$ & Unclear risk & Comment: method of sequence generation not reported
\end{tabular}

\begin{tabular}{lll}
\hline $\begin{array}{l}\text { Allocation concealment } \\
\text { (selection bias) }\end{array}$ & Unclear risk & Comment: method of allocation concealment not reported \\
\hline $\begin{array}{l}\text { Blinding of participants } \\
\text { and personnel (perfor- } \\
\text { mance bias) } \\
\begin{array}{l}\text { All outcomes } \\
\text { Blinding of outcome as- }\end{array}\end{array}$ & Unclear risk & $\begin{array}{l}\text { Comment: information about blinding of participants and personnel not re- } \\
\text { ported }\end{array}$ \\
$\begin{array}{l}\text { All outcomes } \\
\text { Incomplete outcome data } \\
\text { (attrition bias) } \\
\text { All outcomes }\end{array}$ & Low risk & Comment: information about blinding of outcome assessors not reported \\
\hline $\begin{array}{l}\text { Selective reporting (re- } \\
\text { porting bias) }\end{array}$ & Unclear risk & Comment: ITT analysis reported; no missing data \\
\hline \begin{tabular}{l} 
Other bias \\
\hline
\end{tabular} & Low risk & $\begin{array}{l}\text { Comment: all pre-specified outcomes reported; no trial protocol available to } \\
\text { compare with the publication }\end{array}$ \\
\hline
\end{tabular}

Yang 2014b

Study design: parallel design
Number of arms: 2
Experimental arm: low-frequency rTMS
Control arm: sham rTMS

Participants

Geographical location: China

Setting: mixed 
Yang 2014b (Continued)

Stroke criteria: not reported

Method of stroke diagnosis: clinical diagnosis plus confirmation by imaging that a relevant lesion needed to be visible

Inclusion criteria: (1) meeting diagnostic criteria of the CCMD-3 for depression; (2) 24-item HDRS score $\geq 8$; (3) first stroke; (4) clear consciousness; (5) able to express personal will and to sign informed consent

Exclusion criteria: (1) history or family history of psychiatric illness; (2) unable to co-operate with the examination due to obvious aphasia or severe cognitive dysfunction; (3) history of epilepsy, metal implant in the body

Depression criteria: meeting diagnostic criteria of the CCMD-3 for depression and 24-item HDRS score $\geq 8$

Total number randomised in this trial: 55

Number randomised to treatment group: 37 (81\% men; mean age 52.3, SD 11)

Number randomised to control group: $18^{\star \star}$ ( $73 \%$ men; mean age 53.3, SD 14.6$)$

Total number included in final analysis: 55

Number included in treatment group for final analysis: 37

Number included in control group for final analysis: $18^{\star \star}$

Interventions

Treatment: low-frequency rTMS. Frequency: $1 \mathrm{~Hz}$, intensity: 90\% motor threshold, 1 stimulation lasts 10 seconds and stops for 2 seconds, total impulse number: 1000 , location: left DLPFC

Control: sham rTMS. With coils kept at 90-degree angles with the scalp and with coils contacting the scalp, participants could hear the click sounds

Duration: 2 weeks

Follow-up: 4 weeks

Outcomes Primary outcomes

- Depression measured using HDRS

\section{Notes}

\section{Risk of bias}

\begin{tabular}{|c|c|c|}
\hline Bias & Authors' judgement & Support for judgement \\
\hline $\begin{array}{l}\text { Random sequence genera- } \\
\text { tion (selection bias) }\end{array}$ & Unclear risk & Comment: method of sequence generation not reported \\
\hline $\begin{array}{l}\text { Allocation concealment } \\
\text { (selection bias) }\end{array}$ & Unclear risk & Comment: method of allocation concealment not reported \\
\hline $\begin{array}{l}\text { Blinding of participants } \\
\text { and personnel (perfor- } \\
\text { mance bias) } \\
\text { All outcomes }\end{array}$ & Unclear risk & $\begin{array}{l}\text { Comment: information about blinding of participants and personnel not re- } \\
\text { ported }\end{array}$ \\
\hline $\begin{array}{l}\text { Blinding of outcome as- } \\
\text { sessment (detection bias) } \\
\text { All outcomes }\end{array}$ & Unclear risk & Comment: information about blinding of outcome assessors not reported \\
\hline
\end{tabular}


Yang 2014b (Continued)

Incomplete outcome data Low risk Comment: ITT analysis reported; no missing data
(attrition bias)

All outcomes

\begin{tabular}{|c|c|c|}
\hline $\begin{array}{l}\text { Selective reporting (re- } \\
\text { porting bias) }\end{array}$ & Unclear risk & $\begin{array}{l}\text { Comment: all pre-specified outcomes reported; no trial protocol available to } \\
\text { compare with the publication }\end{array}$ \\
\hline
\end{tabular}

Other bias Low risk Comment: no differences in baseline demographics between groups

Zhang 2013

Study design: parallel design
Number of arms: 2
Experimental arm: rTMS + fluoxetine + stroke medications
Control arm: fluoxetine + stroke medications

Participants

Geographical location: China

Setting: inpatient

Stroke criteria: ischaemic and haemorrhagic stroke

Method of stroke diagnosis: complying with diagnostic criteria for cerebral infarction and cerebral haemorrhage formulated by the Fourth National Conference on Cerebrovascular Diseases

Inclusion criteria: (1) meeting diagnostic criteria of the CCMD-3 for depression; (2) 17-item HDRS score $\geq 17$; (3) no history of psychiatric illness and history of drug abuse or alcohol; (4) not taking any antipsychotic drugs 2 weeks before enrolment; (5) relatively stable clinical condition, able to clearly express feelings, no communication obstacle; (6) age 40 to 70 years, Han ethnic group, co-operative during treatment, able to complete all exams and to sign informed consent, educational level: junior high school or above

Exclusion criteria: not reported

Depression criteria: meeting diagnostic criteria of the CCMD-3 for depression and 17-item HDRS score $\geq 17$

Total number randomised in this trial: 82

Number randomised to treatment group: 41 (56\% men; mean age 56.9, SD 5.8)

Number randomised to control group: 41 (53.6\% men; mean age 57.7, SD 6.6)

Total number included in final analysis: 82

Number included in treatment group for final analysis: 41

Number included in control group for final analysis: 41

Treatment: rTMS + fluoxetine $(20 \mathrm{mg} / \mathrm{d})$ + stroke medications. Frequency: $10 \mathrm{~Hz}$, intensity: $90 \%$ motor threshold, 1 stimulation lasts 4 seconds in 1 series, 20 series a day, 3 times a week, location: left DLPFC

Control: fluoxetine + stroke medications

Duration: 8 weeks

Follow-up: none 
Zhang 2013 (Continued)

Outcomes

Primary outcome

- Depression measured using HDRS

Notes

\title{
Risk of bias
}

\begin{tabular}{|c|c|c|}
\hline Bias & Authors' judgement & Support for judgement \\
\hline $\begin{array}{l}\text { Random sequence genera- } \\
\text { tion (selection bias) }\end{array}$ & Low risk & Comment: random number table used for sequence generation \\
\hline $\begin{array}{l}\text { Allocation concealment } \\
\text { (selection bias) }\end{array}$ & Unclear risk & Comment: method of allocation concealment not reported \\
\hline $\begin{array}{l}\text { Blinding of participants } \\
\text { and personnel (perfor- } \\
\text { mance bias) } \\
\text { All outcomes }\end{array}$ & Unclear risk & $\begin{array}{l}\text { Comment: information about blinding of participants and personnel not re- } \\
\text { ported }\end{array}$ \\
\hline $\begin{array}{l}\text { Blinding of outcome as- } \\
\text { sessment (detection bias) } \\
\text { All outcomes }\end{array}$ & Unclear risk & Comment: information about blinding of outcome assessors not reported \\
\hline $\begin{array}{l}\text { Incomplete outcome data } \\
\text { (attrition bias) } \\
\text { All outcomes }\end{array}$ & Low risk & Comment: ITT analysis reported; no missing data \\
\hline $\begin{array}{l}\text { Selective reporting (re- } \\
\text { porting bias) }\end{array}$ & Unclear risk & $\begin{array}{l}\text { Comment: all pre-specified outcomes reported; no trial protocol available to } \\
\text { compare with the publication }\end{array}$ \\
\hline Other bias & Low risk & Comment: no differences in baseline demographics between groups \\
\hline
\end{tabular}

\section{Zhao 2004}

Method

Study design: parallel design

Number of arms: 2

Experimental arm: psychoeducation

Control arm: usual care

\section{Participants}

\author{
Geographical location: China \\ Setting: inpatient
}

Stroke criteria: not reported

Method of stroke diagnosis: diagnosis via CT or MRI (100\%)

Time since stroke: not reported

Inclusion criteria: (1) cognitively competent; (2) no acute medical problems

Exclusion criteria: (1) serious mental problems; (2) low intelligence; (3) other serious neurological condition; (4) heart failure; (5) other acute disease 
Zhao 2004 (Continued)

$$
\text { Depression criteria: HDRS score }>17
$$

Total number randomised in this trial: 70

Number randomised to treatment group: 35 ( $57 \%$ men, mean age 65 years, SD 13)

Number randomised to control group: 35 (51\% men, mean age 61 years, SD 14)

Total number included in final analysis: 70

Number included in treatment group for final analysis: 35

Number included in control group for final analysis: 35

Interventions

Treatment: psychoeducation, daily, less than 30 minutes

Administered by: special personnel who received 2 weeks training before the trial started

Supervision: not reported

Intervention fidelity: unclear; no formal evaluation of the quality or content of therapy provided

Control: usual care

Duration: 4 weeks

Follow-up: none

Outcomes Primary outcomes

- Depression (reduction in scores from baseline to end of treatment) measured using HDRS

Notes

\section{Risk of bias}

\begin{tabular}{lll}
\hline Bias & Authors' judgement & Support for judgement \\
\hline $\begin{array}{l}\text { Random sequence genera- } \\
\text { tion (selection bias) }\end{array}$ & Unclear risk & Comment: method of sequence generation not reported \\
\hline $\begin{array}{l}\text { Allocation concealment } \\
\text { (selection bias) }\end{array}$ & Unclear risk & Comment: method of allocation concealment not stated \\
\hline $\begin{array}{l}\text { Blinding of participants } \\
\text { and personnel (perfor- } \\
\text { mance bias) }\end{array}$ & High risk & Comment: single-blind reported; participants not blinded \\
All outcomes & &
\end{tabular}

$\begin{array}{lll}\text { Blinding of outcome as- } & \text { Low risk } \quad \text { Comment: outcome assessment blinded } \\ \text { sessment (detection bias) }\end{array}$

All outcomes

Incomplete outcome data Low risk

(attrition bias)

All outcomes
Selective reporting (re- Unclear risk porting bias)
Comment: ITT analysis reported (complete follow-up of all randomised participants)

Other bias

Low risk

Comment: all pre-specified outcomes reported; no trial protocol available to compare with the publication

Comment: no differences in baseline demographic characteristics between groups 
Zheng 2016

Methods

Study design: parallel design

Number of arms: 2

Experimental arm: intra-low frequency (ILF)-TMS + cerebrovascular disease routine care + early rehabilitation

Control arm: cerebrovascular disease routine care + early rehabilitation

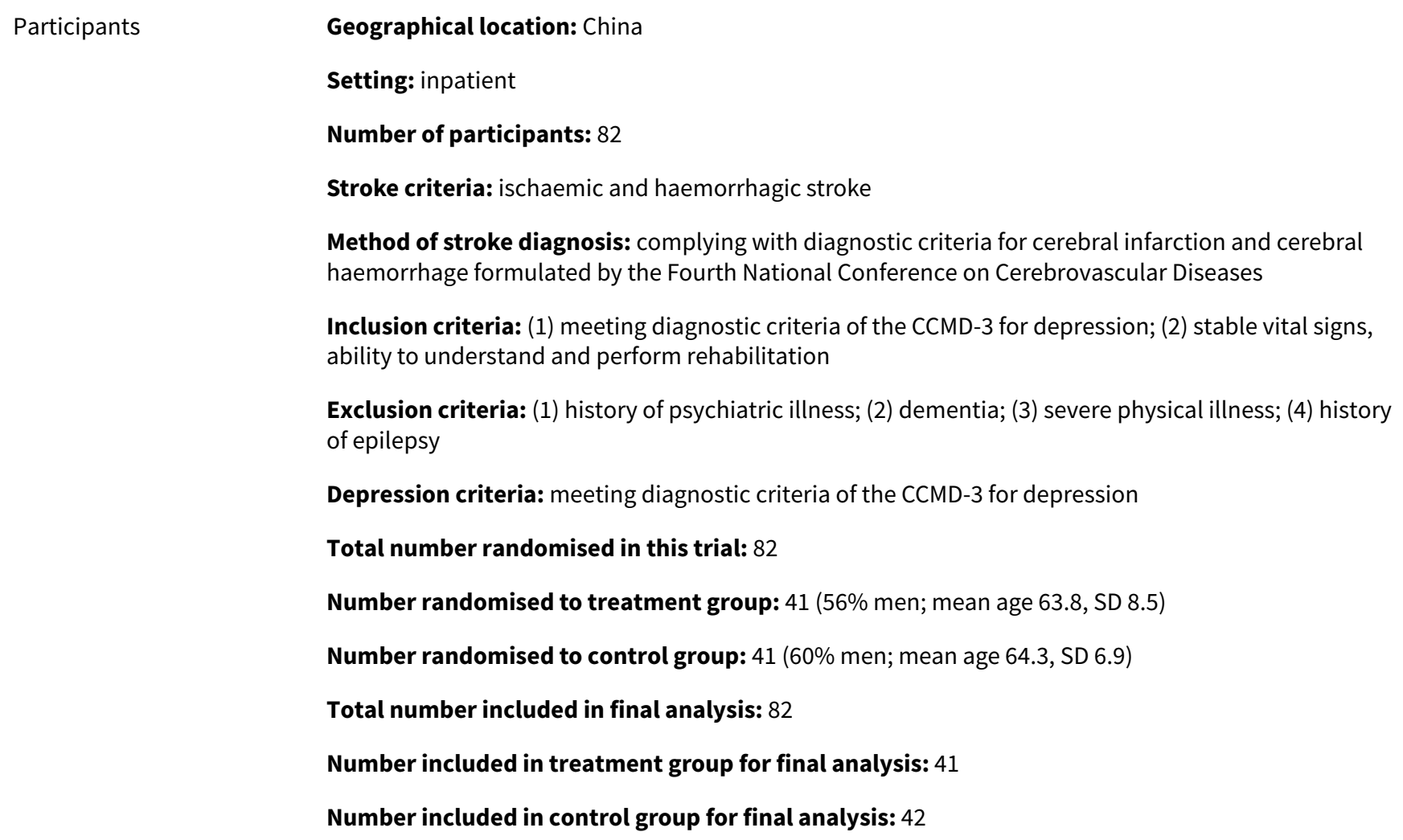

- Impairment measured using SSS 
Zheng 2016 (Continued)

Random sequence genera- Unclear risk Comment: method of sequence generation not reported tion (selection bias)

Allocation concealment $\quad$ Unclear risk
(selection bias)

$\begin{array}{ll}\begin{array}{l}\text { Blinding of participants } \\ \text { and personnel (perfor- }\end{array} & \text { Unclear risk }\end{array}$

mance bias)

All outcomes

Blinding of outcome as-
sessment (detection bias) $\quad$ Unclear risk Comment: information about blinding of outcome assessors not reported

All outcomes

Incomplete outcome data Low risk Comment: ITT analysis reported; no missing data

(attrition bias)

All outcomes

\begin{tabular}{|c|c|c|}
\hline $\begin{array}{l}\text { Selective reporting (re- } \\
\text { porting bias) }\end{array}$ & Unclear risk & $\begin{array}{l}\text { Comment: all pre-specified outcomes reported; no trial protocol available to } \\
\text { compare with the publication }\end{array}$ \\
\hline
\end{tabular}

Other bias Low risk Comment: no differences in baseline demographics between groups

** Results for control group halved.

$\wedge$ Results for attention control and control group pooled.

$A B I$ : acquired brain injury.

$A D L$ : activities of daily living.

$\mathrm{AE}$ : adverse event.

AHI: Authentic Happiness Inventory.

BDI: Beck Depression Inventory.

BI: Barthel Index.

BZDs: benzodiazepines.

CBT: cognitive-behavioural therapy.

CCMD-2-R: Chinese Classification of Mental Disorders, Second Edition, Revised.

CCMD-3: Chinese Classification of Mental Disorders, Third Edition.

CGI: Clinical Global Impression Scale.

CIPI: constructive integrative psychosocial intervention.

CNS: central nervous system.

CSI: Caregiver Strain Index.

CSS: Chinese Stroke Scale.

CT: computed tomography.

DASS-21: Depression Anxiety Stress Scales - 21 items.

DLPFC: dorsolateral pre-frontal cortex.

DSM: Diagnostic and Statistical Manual.

EADL: extended activities of daily living.

EFT: ecosystem focused therapy.

ESD: Education on Stroke and Depression.

FAC: Functional Ambulatory Category.

FIM: Functional Independence Measure.

GCS: Glasgow Coma Scale.

GDS: Geriatric Depression Scale.

GHQ-28: 28-item General Health Questionnaire.

HADS: Hospital Anxiety Depression Scale.

HARS: Hamilton Anxiety Rating Scale.

HDRS-24: 24-item Hamilton Depression Rating Scale.

HDRS-17: 17-item Hamilton Depression Rating Scale.

HRQoL: health-related quality of life. 
Hz: Hertz.

ICD: International Classification of Diseases.

ILF: intra-low frequency.

ITT: intention to treat.

LTF: loss to follow-up.

LOCF: last observation carried forward.

MADRS: Montgomery Asberg Depression Rating Scale.

MBC: Modified Brunnstrom Classification.

MBI: Modified Barthel Index.

MI: motivational interviewing.

MMSE: Mini Mental State Examination.

MPAI-4: Mayo-Portland Adaptability Inventory-4.

MRI: magnetic resonance imaging.

NIHSS: National Institute of Health Stroke Scale.

NRI: norepinephrine reuptake inhibitor.

P300: the P300 is a wave that represents a positive deflection in the human event-related potential. It is most commonly elicited when a patient detects an occasional "target" stimulus in a regular train of standard stimuli.

PHQ-9: 9-item Patient Health Questionnaire.

PSE: Present State Examination.

QoL: quality of life.

rTMS: repetitive transcranial magnetic stimulation.

SAD-Q: Stroke Aphasia Depression Questionnaire.

SAH: subarachnoid haemorrhage.

SAQoL: Stroke Aphasia Quality of Life Scale.

SCAN: Schedules for Clinical Assessment in Neuropsychiatry.

SD: standard deviation.

SDS: Severity of Dependence Scale.

SE: standard error.

SNRI: selective norepinephrine reuptake inhibitor.

SSRI: selective serotonin reuptake inhibitor.

SSS: Scandinavian Stroke Scale.

TCA: tricyclic antidepressant.

TMS: transcranial magnetic stimulation.

VAMS: Visual Analogue Mood Scale.

VAS: visual analogue scale (100 mm).

WDI: Wakefield Depression Inventory.

WHO: World Health Organization.

WHODAS- II: World Health Organization Disability Assessment Schedule.

ZDS: Zung Depression Scale.

Characteristics of excluded studies [ordered by study ID]

\begin{tabular}{ll}
\hline Study & Reason for exclusion \\
\hline Aben 2014 & Depression not the primary outcome of this study \\
\hline Agnoli 1985 & Inability to isolate stroke patients \\
\hline Bai 2017 & Depression not the primary outcome of this study \\
\hline Bramanti 1989 & Data not available for depressed participants only \\
\hline Casella 1960 & Depression not the primary outcome of this study \\
\hline Chang 2011 & Data not available for depressed participants only \\
\hline Cheng 2016 & Depression not the primary outcome of this study \\
\hline Choi-Kwon 2006 & Data not available for depressed participants only \\
\hline \hline
\end{tabular}

Pharmacological, psychological, and non-invasive brain stimulation interventions for treating depression after stroke (Review) 


\begin{tabular}{|c|c|}
\hline Study & Reason for exclusion \\
\hline Chollet 2011 & Depression not the primary outcome of this study \\
\hline Clark 2003 & Data not available for depressed participants only \\
\hline Delbari 2011 & Data not available for depressed participants only \\
\hline Downes 1995 & Data not available for depressed participants only \\
\hline Evans 1997 & $\begin{array}{l}\text { Participants were acute geriatric medical inpatients with depression. We were unable to isolate any } \\
\text { chronic stroke patients. No acute stroke patients were included in the sample }\end{array}$ \\
\hline Finkenzeller 2006 & Depression assessments not available at a consistent time point \\
\hline Hadidi 2014 & Data not available for depressed participants only \\
\hline Hu 2003 & Depression not the primary outcome of this study \\
\hline ISRCTN88489864 & Depression not the primary outcome of this study \\
\hline Jiang 2004 & Depression not the primary outcome of this study \\
\hline Jorge 2004 & Data not available for depressed participants only \\
\hline Jorge 2008 & Data not available for depressed participants only \\
\hline Kim 2010a & Data not available for depressed participants only \\
\hline Kim 2010b & Data not available for depressed participants only \\
\hline Kim 2017 & Data not available for depressed participants only \\
\hline Kim 2017a & Data not available for depressed participants only \\
\hline Kootker 2012 & Data not available in the format suitable for this review \\
\hline Laska 2005 & Depression not an outcome of this study \\
\hline Leijon 1989 & Depression not an outcome of this study \\
\hline Lobjanidze 2010 & Depression not the primary outcome of this study \\
\hline Mauri 1988 & Data not available in a format suitable for this review \\
\hline Meara 1998 & Data not available for depressed participants only \\
\hline Narushima 2007 & Depression not the primary outcome of this study \\
\hline Ohtomo 1985 & Data not available for depressed participants only \\
\hline Ostwald 2014 & Data not available for depressed participants only \\
\hline Otomo 1986 & Participants not depressed at entry into the study \\
\hline Raffaele 1996 & Data not available for depressed participants only \\
\hline
\end{tabular}




\begin{tabular}{|c|c|}
\hline Study & Reason for exclusion \\
\hline Rich 2016 & Depression not the primary outcome of this study \\
\hline Robinson 2000 & Data not available for depressed participants only \\
\hline Robinson 2017 & Depression not the primary outcome of this study \\
\hline Rudberg 2017 & Depression not the primary outcome of this study \\
\hline Sieger 2018 & Depression not the primary outcome of this study \\
\hline Sivenius 2001 & Depression not the primary outcome of this study \\
\hline Su $2004 a$ & Depression not the primary outcome of this study \\
\hline Sun 2000 & Data not available for depressed participants only \\
\hline Szepfalusi 2017 & Depression not the primary outcome of this study \\
\hline Valiengo 2017 & Data not available for depressed participants only \\
\hline Visser 2015 & Depression not the primary outcome of this study \\
\hline Walker-Batson 1995 & Depression not the primary outcome of this study \\
\hline Wang 2009 & Depression not the primary outcome of this study \\
\hline
\end{tabular}

Characteristics of studies awaiting assessment [ordered by study ID]

Chen 2002a

\begin{tabular}{ll}
\hline Study design: parallel design \\
Number of arms: 2 \\
Experimental arm: paroxetine (SSRI) \\
Control arm: placebo \\
\hline Geographical location: China \\
Setting: unclear \\
Number of participants: 36 \\
Stroke criteria: unclear \\
Method of stroke diagnosis: not reported \\
Inclusion criteria: not reported \\
Exclusion criteria: (1) cognitive impairment (MMSE <24); (2) depression deterioration (HDRS $>24) ;$ \\
(3) suicidal mood; (4) drug intolerability \\
Depression criteria: unclear \\
Total number randomised in this trial: 36 \\
Number randomised to treatment group: 24 \\
Number randomised to control group: $12^{\star \star}$
\end{tabular}


Total number included in final analysis: 34

Number included in treatment group for final analysis: 24

Number included in control group for final analysis: $10^{\star *}$

\begin{tabular}{ll}
\hline Interventions & Treatment: paroxetine (SSRI) $200 \mathrm{mg}$ once daily \\
& Control: placebo (guvitamine) $10 \mathrm{mg} 3 \times$ daily \\
& Duration: 8 weeks \\
& Follow-up: none \\
\hline Outcomes & Primary outcomes \\
& Secondary outcomes \\
& - Disability measured using BI \\
& Impairment measured using CSS \\
\hline Notes & Unable to obtain information on the primary outcome: whether depression or functional recovery \\
\hline
\end{tabular}

Chen 2002b

\begin{tabular}{|c|c|}
\hline Methods & $\begin{array}{l}\text { Study design: parallel design } \\
\text { Number of arms: } 2 \\
\text { Experimental arm: doxepin } \\
\text { Control arm: placebo }\end{array}$ \\
\hline Participants & $\begin{array}{l}\text { Geographical location: China } \\
\text { Setting: unclear } \\
\text { Number of participants: } 36 \\
\text { Stroke criteria: unclear } \\
\text { Method of stroke diagnosis: not reported } \\
\text { Inclusion criteria: not reported } \\
\text { Exclusion criteria: (1) cognitive impairment (MMSE <24); (2) depression deterioration (HDRS > 24); } \\
\text { (3) suicidal mood; (4) drug intolerability } \\
\text { Depression criteria: unclear } \\
\text { Total numbers randomised in this trial: } 36 \\
\text { Numbers randomised to treatment group: } 24 \\
\text { Numbers randomised to control group: } 12^{\star \star} \\
\text { Total numbers included in final analysis: } 26 \\
\text { Numbers included in treatment group for final analysis: } 16 \\
\text { Numbers included in control group for final analysis: } 10^{\star \star}\end{array}$ \\
\hline Interventions & Treatment: doxepin 25 mg $3 \times$ daily \\
\hline
\end{tabular}


Chen 2002b (Continued)

Control: placebo (guvitamine) $10 \mathrm{mg} 3 \times$ daily

Duration: 8 weeks

Follow-up: none

Outcomes

Primary outcome

- Depression measured using HDRS

\section{Secondary outcomes}

- Disability measured using BI

- Impairment measured using CSS

\section{Number of arms: 2}

Treatment arm: paroxetine (SSRI) + psychotherapy + education

Control arm: paroxetine (SSRI)

\section{Participants}

\section{Geographical location: China}

Setting: outpatient

Stroke criteria: ischaemic and haemorrhagic stroke

Method of stroke diagnosis: clinical diagnosis with imaging consistent with stroke using Oxford Community Stroke Project classification and structural brain CT classification (by anatomical location)

Time since stroke: 2 to 6 months

Inclusion criteria: (1) meeting depression diagnostic criteria of the CCMD-3 and 17-item HDRS score $>17$ )

Exclusion criteria: (1) bipolar disorders; (2) drug dependence or abuse

Depression criteria: psychiatric interview; meeting depression diagnostic criteria of the CCMD-3; 17-item HDRS score > 17; HARS score > 7; clinical impression

Total number randomised in this trial: 68

Number randomised to treatment group: 34 (56\% men; mean age 61.3 years, SD 9.3)

Number randomised to control group: 34 (47\% men; mean age 60.5 years, SD 10.4)

Total number included in final analysis: 68

Number included in treatment group for final analysis: 34 (56\% men; mean age 61.3 years, SD 9.3)

Number included in control group for final analysis: 34 (47\% men; mean age 60.5 years, SD 10.4) 
Ding 2005 (Continued)

pression; behavioural therapy targeted at attitudes in practice and education. Psychotherapy was delivered in 40 to 60 -minute sessions, 2 to 3 sessions a week

Administered by: a professional physician; training in psychotherapy unclear

Supervision of therapists: not reported

Intervention fidelity: not reported

Control: paroxetine (SSRI, variable dose, started from $10 \mathrm{mg} / \mathrm{d}$, titrated up to 20 to $30 \mathrm{mg} / \mathrm{d}$ )

Duration: 8 weeks

Follow-up: 4 months

Outcomes Primary outcomes

- Depression measured using HDRS

Secondary outcomes

- Anxiety measured using HARS

- Activities of daily living measured using BI

- Symptoms measured using Treatment Emergent Symptom Scale

Notes

Unable to obtain information to determine if the psychotherapy component of the intervention meets the review criteria for psychotherapy

Evans 1985

Methods

Study design: parallel design

Number of arms: 2

Experimental arm: telephone counselling

Control arm: usual care

Participants

Geographical location: USA

Setting: outpatient

Stroke criteria: unclear (also includes people with spinal cord injury, CNS disease, and 'other')

Method of stroke diagnosis: not reported

Inclusion criteria: (1) patients discharged from rehabilitation centre; (2) housebound; (3) able to hear; (4) ordinary speech; (5) sufficient cognitive ability to engage in meaningful conversation

Exclusion criteria: not reported

Depression criteria: score taken from the Life Satisfaction Index (LSI); unclear how scored

Total number randomised in this trial: 38

Number randomised to treatment group: 19 (95\% men, mean age 54.8 years, SD 11.9 years); 4 with stroke

Number randomised to control group: 19 (95\% men, mean age 54.8 years, SD 10.2 years); 5 with stroke

Total number included in final analysis: unclear 
Number included in treatment group for final analysis: unclear

Number included in control group for final analysis: unclear

Interventions

Treatment: 8-weekly hour-long counselling sessions by phone with groups of 4 patients. Formulation of behaviorally specific goals encouraged and developed with each patient, and discussion directed at finding ways to meet those goals

Administered by: an experienced counsellor

Supervision: not reported

Control: usual care (no contact)

Duration: not reported

Follow-up: not reported

\section{Outcomes}

\section{Primary outcome}

- Depression - unclear what measure was used

\section{Notes}

Unable to obtain any more information on this trial or series of trials despite multiple attempts since 2003

Finkenzeller 2009

\begin{tabular}{ll}
\hline Study design: parallel design \\
Number of arms: 2 \\
Experimental arm: sertraline (SSRI) + psychological therapy \\
Control arm: sertraline (SSRI)
\end{tabular}

Participants

Geographical location: Germany

Setting: inpatient

Stroke criteria: all subtypes

Method of stroke diagnosis: unclear

Time since stroke: $<3$ months

Inclusion criteria: (1) onset of stroke no longer than 3 months

Exclusion criteria: (1) previous or current psychiatric disorder like substance abuse, borderline or antisocial personality disorder, or other prominent Axis I disorder; (2) with previous depressive disorder, only if participants were still treated with antidepressive medication for this matter; (3) stronger cognitive impairment (e.g. dementia, aphasia, delirium) (no defined criteria or cut-off)

Depression criteria: HADS > 7 on the subscale Depression, HDRS score $>13$

Total number randomised in this trial: 21

Number randomised to treatment group: 9 (39\% men, mean age 64.7, SD 11.1)

Number randomised to control group: 12 (50\% men, mean age 71.7, SD 7.1)

Total number included in final analysis: 21 
Administered by: not reported

Supervision: not reported

Intervention fidelity: not reported

Control: sertraline (SSRI)

Duration: 4 to 8 weeks

Follow-up: none

\begin{tabular}{ll}
\hline Outcomes & Primary outcomes \\
& - Depression (response $>50 \%$ reduction in initial score) measured using HDRS \\
& Depression (remission) measured using HDRS $(<8)$ \\
\hline Notes & $\begin{array}{l}\text { Unable to obtain information to determine if the psychotherapy component of the intervention } \\
\text { meets the review criteria for psychotherapy }\end{array}$
\end{tabular}

Hanspal 2007

\section{Methods}

Study design: parallel design

Number of arms: 2

Experimental arm: sertraline (SSRI)

Control arm: placebo

Geographical location: UK
Setting: unclear
Stroke criteria: unclear (also includes people with non-vascular events such as trauma, hypoxia,
or encephalitis)
Method of stroke diagnosis: not reported
Inclusion criteria: not reported
Exclusion criteria: not reported
Depression criteria: unclear
Total number randomised in this trial: unclear
Number randomised to treatment group: unclear
Number randomised to control group: unclear
Total number included in final analysis: unclear
Number included in treatment group for final analysis: unclear
Number included in control group for final analysis: unclear


Hanspal 2007 (Continued)

Interventions
Treatment: sertraline (SSRI)

Control: placebo

Duration: not reported

Follow-up: not reported

\section{Outcomes}

\section{Primary outcome}

- Depression: unclear what measure was used

\section{Number of arms: 2}

Treatment arm: amitriptyline (TCA) + psychological intervention + routine drugs for cerebrovascular disease

Control arm: amitriptyline (TCA) + routine drugs for cerebrovascular disease

\section{Participants}

\section{Geographical location: China}

Setting: unclear

Stroke criteria: cerebral infarction and haemorrhage

Method of stroke diagnosis: stroke diagnosed according to the standards of National Fourth Cerebral Vascular Disease Meeting of Chinese Medical Association in 1995

Inclusion criteria: (1) score > 8 in the CCMD-2-R

Exclusion criteria: (1) history of mental disorder; (2) patients with coma, anepia, intelligence disorder; (3) patients with severe disease of heart, liver, and lung

Depression criteria: score $>8$ in the CCMD-2-R

Total number randomised in this trial: 67

Number randomised to treatment group: 35 (54.3\% men, mean 64 years, SD 9)

Number randomised to control group: 32 (percentage of men and mean age not reported for this group)

Total number included in final analysis: unclear

Number included in treatment group for final analysis: unclear

Number included in control group for final analysis: unclear

Treatment: amitriptyline (TCA + psychological intervention + routine drugs for cerebrovascular disease). Psychological intervention included (1) treatment of cognitive behaviour; (2) supportive psychological treatment; (3) education about hypertension, coronary heart disease, and diabetes; (4) education about psychological hygiene

Administered by: not reported

Supervision: not reported 
He 2003 (Continued)

\title{
Intervention fidelity: not reported
}

Control: amitriptyline (TCA) + routine drugs for cerebrovascular disease

Duration: 6 weeks

Follow-up: none

\begin{tabular}{ll}
\hline Outcomes & Primary outcomes \\
& - Depression measured using HDRS \\
& - Activities of daily living (unclear what measure was used) \\
\hline Notes & Unable to obtain information on the intervention of this trial \\
\hline
\end{tabular}

He 2005

Methods Study design: parallel design

Number of arms: 2

Experimental arm: paroxetine (SSRI)

Control arm: psychotherapy + paroxetine (SSRI)

\section{Participants}

\author{
Geographical location: China \\ Setting: inpatient \\ Stroke criteria: ischaemic stroke and cerebral haemorrhage
}

Method of stroke diagnosis: first-ever stroke with a diagnosis consistent with diagnostic criteria for cerebral infarct formulated by the Fourth National Conference on Cerebrovascular Disease and confirmation by brain CT or MRI

Time since stroke: not reported

Inclusion criteria: (1) first-ever stroke; (2) meeting organic depressive disorder/organic anxiety disorder diagnostic criteria of ICD-10; (3) 17-item HDRS score $\geq 17$; HARS score $\geq 14$

Exclusion criteria: (1) history of psychiatric illness; (2) taking antidepressants and neuroleptics in the previous 3 months; (3) aphasia; (4) severe cognitive impairment; (5) allergy to paroxetine; (6) suicidal behaviour; (7) in a coma

Depression criteria: meeting organic depressive disorder/organic anxiety disorder diagnostic criteria of ICD-10 and 17-item HDRS score $\geq 17$; HARS score $\geq 14$

\section{Total number randomised in this trial: 54}

Number randomised to treatment group: 27 (52\% men; mean age 64, SD 5.3)

Number randomised to control group: 27 (52\% men; mean age 62.4, SD 6.1)

Total number included in final analysis: 54

Number included in treatment group for final analysis: 27

Number included in control group for final analysis: 27 
He 2005 (Continued)

\section{Intervention fidelity: not reported}

Control: paroxetine (SSRI) $20 \mathrm{mg} / \mathrm{d}$

Duration: 6 weeks

Follow-up: none

\begin{tabular}{ll}
\hline Primary outcomes \\
- Depression measured using HDRS \\
- Anxiety measured by HARS \\
Secondary outcomes \\
- Symptoms measured using Treatment Emergent Symptom Scale \\
- Disability measured using BI \\
- Impairment measured using SSS \\
Unable to obtain information to determine if the psychotherapy component of the intervention \\
meets the review criteria for psychotherapy
\end{tabular}

Huang 2005

Study design: parallel design
Number of arms: 2
Experimental arm: venlafaxine (SNRI) + cognitive therapy
Control arm: venlafaxine (SNRI)

Participants

\section{Geographical location: China \\ Setting: mixed \\ Stroke criteria: ischaemic stroke only}

Method of stroke diagnosis: first-ever stroke with diagnosis consistent with diagnostic criteria for cerebral infarct formulated by the Fourth National Conference on Cerebrovascular Disease and confirmation by brain CT or MRI

Time since stroke: not reported

Inclusion criteria: (1) first-ever stroke; (2) depression developed in the acute stage of cerebral infarct; (3) HDRS score $\geq 18$

Exclusion criteria: (1) history of psychiatric illness; (2) dementia; (3) aphasia; (4) consciousness disturbance; (5) apraxia; (6) other organic disease; (7) systematic disease; (8) depression developed in the acute stage of cerebral infarct

Depression criteria: HDRS score $\geq 18$; depression developed in the acute stage of cerebral infarct

Total number randomised in this trial: 82

Number randomised to treatment group: 41 (\% men not reported, mean age 62.2 years, SD 8.3 )

Number randomised to control group: 41 (\% men not reported, mean age 61.8 years, SD 8.7)

Total number included in final analysis: 80

Number included in treatment group for final analysis: 40 (63\% men, mean age not reported)

Number included in control group for final analysis: 40 (61\% men, mean age not reported) 
Huang 2005 (Continued)

Interventions
Treatment: venlafaxine (SNRI) $121.56 \mathrm{mg} / \mathrm{d}+$ combined cognitive therapy (more than 1 hour every session, 1 session/week initially, 1 session fortnightly 1 month later, and 1 to 2 sessions/month 2 months later)

Administered by: not reported

Supervision: not reported

Intervention fidelity: not reported

Control: venlafaxine (SNRI) $121.56 \mathrm{mg} / \mathrm{d}$

Duration: 3 months

Follow-up: none

\section{Primary outcomes}

- Depression measured using HDRS

\section{Secondary outcomes}

- Symptoms measured using Treatment Emergent Symptom Scale meets the review criteria for psychotherapy

\section{Katz 1998}

Methods

Study design: unclear

Number of arms: 4

Experimental arm 1: group psychotherapy

Experimental arm 2: behavioral therapy

Experimental arm 3: combined antidepressant and individual psychotherapy plus group psychotherapy

Control arm: unclear

Participants

Geographical location: unclear

Setting: unclear

Stroke criteria: unclear

Method of stroke diagnosis: unclear

Inclusion criteria: not reported

Exclusion criteria: not reported

Depression criteria: unclear

Total number randomised in this trial: unclear

Number randomised to treatment group: unclear

Number randomised to control group: unclear 
Katz 1998 (Continued)

Total number included in final analysis: unclear

Number included in treatment group for final analysis: unclear

Number included in control group for final analysis: unclear

Interventions

Treatment 1: group psychotherapy

Treatment 2: behavioural therapy

Treatment 3: combined antidepressant and individual psychotherapy plus group psychotherapy

Control: unclear

Duration: not reported

Follow-up: not reported

Outcomes Primary outcome

- Depression - unclear what measure was used

Notes

Unable to obtain any more information on this trial or series of trials despite multiple attempts since 2002

Latow 1983

Methods

Study design: unclear

Number of arms: unclear

Experimental arm: psychotherapy

Control arm: unclear

Participants

Geographical location: unclear

Setting: unclear

Number of participants: unclear

Stroke criteria: unclear

Method of stroke diagnosis: not reported

Inclusion criteria: not reported

Exclusion criteria: not reported

Depression criteria: unclear

Total number randomised in this trial: unclear

Number randomised to treatment group: unclear

Number randomised to control group: unclear

Total number included in final analysis: unclear

Number included in treatment group for final analysis: unclear

Number included in control group for final analysis: unclear 
Latow 1983 (Continued)

Interventions

Treatment: psychotherapy

Control: unclear

Duration: unclear

Follow-up: unclear

\section{Outcomes}

\section{Primary outcome}

- Depression - unclear what measure was used

Notes Unable to obtain a copy of this article, which also may be a book

Lee 2005

Methods

Study design: parallel design

Number of arms: 2

Experimental arm: rTMS

Control arm: sham stimulation

Participants

Geographical location: Republic of Korea

Setting: not reported

Stroke criteria: ischaemic stroke

Method of stroke diagnosis: radiological diagnosis of location of infarct is given, but it is unclear whether this was used to make the diagnosis

Inclusion criteria: (1) patients who did not respond to conventional antidepressant medication (paroxetine $20 \mathrm{mg} / \mathrm{d}$ ); (2) Rancho Los Amogos cognitive function scale more than VIla

Exclusion criteria: (1) history of psychiatric illness; (2) aphasia; (3) arrhythmia; (4) left pre-frontal cortical lesion; (5) seizure or internal metallic device

Depression criteria: $\mathrm{BDI}>17$

Total number randomised in this trial: 20

Number randomised to treatment group: 10 (70\% men, mean age 67.8, SD 2.3)

Number randomised to control group: 10 (60\% men, mean age 66.3, SD 3.0)

Total number included in final analysis: unclear

Number included in treatment group for final analysis: unclear

Number included in control group for final analysis: unclear

Administered by: not reported

Control: sham stimulation

Frequency: 10 trains separated by 60 seconds

Duration: for 10 days during a 2-week period 
Lee 2005 (Continued)

\section{Follow-up: none}

Outcomes

\section{Primary outcomes}

- Depression measured using HDRS

- Depression measured using BDI

\section{Secondary outcomes}

- Cognitive function measured using MMSE

Notes Unable to obtain any more information on this trial despite multiple attempts since 2008

Liu 2010

Methods

Study design: parallel design

Number of arms: 2

Experimental arm: rTMS + routine care + physical factors treatment + acupuncture + psychotherapy

Control arm: sham rTMS + routine care + physical factors treatment + acupuncture + psychotherapy

Participants

Geographical location: China

Setting: inpatient

Stroke criteria: ischaemic stroke

Method of stroke diagnosis: clinical diagnosis plus confirmation by imaging that a relevant lesion needed to be visible

Inclusion criteria: (1) no dementia; (2) no aphasia; (3) clear consciousness; (4) age < 75 years

Exclusion criteria: (1) cerebral haemorrhage; (2) history of epilepsy; (3) metal implant in the body; (4) other serious physical illness; (5) history of psychiatric illness or family history

Depression criteria: meeting diagnostic criteria of ICD-10 for depression and 24-item HDRS score > 20

Total number randomised in this trial: 60

Number randomised to treatment group: 30 (36\% men; mean age 59, SD 9)

Number randomised to control group: 30 (30\% men; mean age 58, SD 11)

Total number included in final analysis: 60

Number included in treatment group for final analysis: 30

Number included in control group for final analysis: 30

Interventions

Treatment: rTMS + routine care (medications (sertraline or citalopram), physical factors treatment (musical therapy, high-voltage static current therapy), Chinese medicine (acupuncture), and psychotherapy (patient-centred therapy, cognitive therapy, behaviour therapy)). Frequency: 10 to 15 $\mathrm{Hz}$, intensity: $90 \%$ motor threshold, 1 stimulation lasting 1 second and stop for 10 seconds, total 1200 stimulations per day, for 10 days, location: left DLPFC

Control: sham rTMS + routine care (medications (sertraline or citalopram), physical factors treatment (musical therapy, high-voltage static current therapy), Chinese medicine (acupuncture), and 
Liu 2010 (Continued)

psychotherapy (patient-centred therapy, cognitive therapy, behaviour therapy)). Keeping the coils at 90-degree angle with the scalp, keeping the coils at a distance of $8 \mathrm{~cm}$ from treatment area

Duration: 10 days

Follow-up: 40 days

\section{Outcomes}

\section{Primary outcome}

- Depression measured using HDRS

\begin{tabular}{|c|c|}
\hline Methods & $\begin{array}{l}\text { Study design: parallel design } \\
\text { Number of arms: } 2 \\
\text { Experimental arm: nurse-led education intervention } \\
\text { Control arm: unclear }\end{array}$ \\
\hline Participants & $\begin{array}{l}\text { Geographical location: unclear } \\
\text { Setting: outpatient } \\
\text { Number of participants: } 41 \\
\text { Stroke criteria: unclear } \\
\text { Method of stroke diagnosis: not reported } \\
\text { Inclusion criteria: not reported } \\
\text { Exclusion criteria: not reported } \\
\text { Depression criteria: unclear } \\
\text { Total number randomised in this trial: } 41 \\
\text { Number randomised to treatment group: } 20 \\
\text { Number randomised to control group: } 21 \\
\text { Total number included in final analysis: unclear } \\
\text { Number included in treatment group for final analysis: unclear } \\
\text { Number included in control group for final analysis: unclear }\end{array}$ \\
\hline Interventions & $\begin{array}{l}\text { Treatment: Orem's self-care model of nursing, Knowles' principles of adult learning, nurse-led ed- } \\
\text { ucational intervention } \\
\text { Control: unclear } \\
\text { Duration: } 16 \text { hours } \\
\text { Follow-up: not reported }\end{array}$ \\
\hline Outcomes & Primary outcome \\
\hline
\end{tabular}




\begin{tabular}{ll}
\hline Study design: parallel design \\
Number of arms: 2 \\
Experimental arm: fluoxetine (SSRI) \\
Control arm: placebo
\end{tabular}

Geographical location: Iran
Setting: inpatient
Stroke criteria: acute ischaemic stroke
Method of stroke diagnosis: documented with CT scan
Time since stroke: not reported
Inclusion criteria: (1) acute ischaemic stroke (documented with CT scan) that leads monoparesis,
hemiparesis, or hemiplegia; (2) not in a comatose state and stable
Exclusion criteria: (1) death due to any cause during assessment; (2) pregnancy; (3) poor compli-
ance of drugs and physiotherapy; (4) miscarriage returning of patient for further exams and assess-
ments; (5) any drug complication during assessment (prospected or not); (6) any metabolic disease
(liver, renal, cardiac impairment, and hyperthyroidism); (7) ischaemic stroke in the territory of an-
terior cerebral artery (ACA) or posterior cerebral artery (PCA), using any interfering drugs with flu-
oxetine (such as cyproheptadine, selegiline)

Depression criteria: none

Total number randomised in this trial: 172

Number randomised to treatment group: 86 (50.6\% men; mean age 63.2, SD 11.4)

Number randomised to control group: 86 (41.3\% men; mean age 64.6, SD 11.9)

Total number included in final analysis: 150

Number included in treatment group for final analysis: 75

Number included in control group for final analysis: 75

\begin{tabular}{ll}
\hline Interventions & Treatment: fluoxetine (SSRI) $20 \mathrm{mg} / \mathrm{C}$ \\
& Control: placebo \\
& Duration: 45 days \\
& Follow-up: 90 days \\
\hline Outcomes & Primary outcomes \\
& - Motor impairment \\
& - Depression measured using ZDS \\
& Disability measured using BI
\end{tabular}


Razazian 2016 (Continued)

\title{
Number of arms: 2
}

Experimental arm: paroxetine (SSRI) + cognitive therapy (frequency unknown)

Control arm: paroxetine (SSRI)

Participants

\author{
Geographical location: China \\ Setting: inpatient \\ Stroke criteria: unclear
}

Method of stroke diagnosis: diagnosis consistent with diagnostic criteria for stroke formulated by the Second National Symposium on Cerebrovascular Disease and confirmation by brain CT or MRI

Time since stroke: 2 weeks ago

Inclusion criteria: (1) no history of aphasia or agnosia; (2) clear consciousness; (3) stroke onset at least 2 weeks ago

Exclusion criteria: (1) history of psychiatric illness; (2) organic or reactive depression; (3) comorbid with other severe psychiatric symptoms, or family history

Depression criteria: psychiatric interview to confirm diagnosis meets diagnostic criteria of CCMD-2-R; ZDS score $\geq 50$

Total number randomised in this trial: 41

Number randomised to treatment group: 20 (60\% men; mean age 57.5, SD 5.2)

Number randomised to control group: 21 (57\% men; mean age 56.3, SD 5.7)

Total number included in final analysis: 41

Number included in treatment group for final analysis: 20

Number included in control group for final analysis: 21 unknown). Cognitive therapy entailed guiding patients to apply cognitive remediation for negative thoughts; recognise situations causing depression; re-establish healthy ideas and attitudes; establish family co-operation

Administered by: not reported

Supervision: not reported

Intervention fidelity: not reported

Control: paroxetine (SSRI) $20 \mathrm{mg} / \mathrm{d}$ in the morning

Duration: 1 month

Follow-up: none 
Tang 2002 (Continued)

\section{Secondary outcomes}

- Cognitive functioning measured using MMSE

- Evaluation of clinical status, stratifying clinical status as recovered (disappearance of symptoms, insight recovery, social function recovery), obviously improved (most symptoms disappear, insight partial recovery), improved (only slightly improved), not efficacious (no any improvement and even worse)

Notes

Unable to obtain information to determine if the psychotherapy component of the intervention meets the review criteria for psychotherapy

Wang 2015

Methods

Study design: parallel design

Number of arms: 2

Experimental arm: rTMS + conventional drugs, rehabilitation training, and psychological counselling therapy

Control arm: conventional drugs, rehabilitation training, and psychological counselling therapy

Participants

Geographical location: China

Setting: inpatient

Stroke criteria: not reported

Method of stroke diagnosis: clinical criteria only

Inclusion criteria: (1) meeting diagnostic criteria of ICD for organic depression; (2) 17-item HDRS score $\geq 8$; (3) over 65 years of age

Exclusion criteria: not reported

Depression criteria: meeting diagnostic criteria of ICD for organic depression and 17-item HDRS score $\geq 8$

Total number randomised in this trial: 150

Number randomised to treatment group: 75 (56\% men; mean age 56.7, SD 7.2)

Number randomised to control group: 75 (53\% men; mean age 57.9, SD 6.8)

Total number included in final analysis: 150

Number included in treatment group for final analysis: 75

Number included in control group for final analysis: 75

Interventions

Treatment: rTMS + conventional drugs, rehabilitation training, and psychological counselling therapy. Frequency: $10 \mathrm{~Hz}$, intensity: $60 \%$ motor threshold, 1 stimulation lasts 4 seconds and stops for 56 seconds, 30 stimulations for 1 series, 5 series a week, for successive 12 weeks, location: left DLF$\mathrm{PC}$

Control: conventional drugs, rehabilitation training, and psychological counselling therapy

Duration: 12 weeks

Follow-up: none 
- Depression measured using HDRS

- Disability measured using BI

Notes

Unable to obtain information to determine if the psychotherapy component of the intervention meets the review criteria for psychotherapy

Yan 2010a

Study design: parallel design
Number of arms: 2
Experimental arm: high-frequency rTMS + routine care + flupentixol and melitracen $10.5 \mathrm{mg} /$
tablet, 1 tablet twice a day + psychotherapy
Control arm: sham rTMS + routine care + flupentixol and melitracen $10.5 \mathrm{mg} /$ tablet, 1 tablet twice
a day + psychotherapy

\section{Participants}

\section{Geographical location: China \\ Setting: inpatient \\ Stroke criteria: not reported}

Method of stroke diagnosis: diagnosis consistent with diagnostic criteria for stroke formulated by the Fourth National Symposium on Cerebrovascular Disease in 1995 and confirmation by brain CT or MRI stated

Time since stroke: $<6$ months

Inclusion criteria: (1) right-handedness; (2) disease course < 6 months; (3) can sign informed consent

Exclusion criteria: (1) history of psychiatric illness or family history; (2) aphasia; (3) severe dementia; (4) severe physical illness, consciousness disturbance, or deafness, which influences the expression of depressed mood; (4) psychoactive or non-addiction-producing substance-induced depression; (5) various reasons to refuse trial or difficulty in finishing trial

Depression criteria: depression diagnosed according to CCMD-3

Total number randomised in this trial: 20

Number randomised to treatment group: 10 (50\% men; mean age 68.65, SD 7.62)

Number randomised to control group: $10^{\star \star}$ ( $55 \%$ men; mean age 68.70 , SD 8.94)

Total number included in final analysis: 20

Number included in treatment group for final analysis: 10

Number included in control group for final analysis: $10^{\star \star}$

Treatment: high-frequency rTMS + routine care + flupentixol and melitracen $10.5 \mathrm{mg} /$ tablet, 1 tablet twice a day + psychotherapy. High rTMS frequency: $10 \mathrm{~Hz}$; intensity: $110 \%$ motor threshold; location: left DLPFC; 1 sequence included continuous stimulations for 30 minutes, frequency of treatment: 1 sequence a day during 09:00 to 10:00

Control: sham rTMS + routine care + flupentixol and melitracen $10.5 \mathrm{mg} /$ tablet, 1 twice a day + psychotherapy. Sham rTMS $0 \mathrm{~Hz}$; intensity: 0; location: left or right DLPFC; 1 sequence included continuous stimulations for 30 minutes, frequency of treatment: 1 sequence a day during 09:00 to 10:00

Duration: 7 days 
Yan 2010a (Continued)

\title{
Follow-up: none
}

$\begin{array}{ll}\text { Outcomes } & \text { Primary outcomes } \\ \text { - Depression measured using HDRS } \\ \text { Secondary outcomes } \\ \text { - Adverse events } \\ \text { - Leaving the trial early } \\ \text { - Death }\end{array}$

Notes

Unable to obtain information to determine if the psychotherapy component of the intervention meets the review criteria for psychotherapy

Number of arms: 2

Experimental arm: low-frequency rTMS + routine care + flupentixol and melitracen $10.5 \mathrm{mg} /$ tablet, 1 tablet twice a day + psychotherapy

Control arm: sham rTMS + routine care + flupentixol and melitracen $10.5 \mathrm{mg} /$ tablet, 1 tablet twice a day + psychotherapy

\section{Participants}

\author{
Geographical location: China \\ Setting: inpatient \\ Stroke criteria: not reported
}

Method of stroke diagnosis: diagnosis consistent with diagnostic criteria for stroke formulated by the Fourth National Symposium on Cerebrovascular Disease in 1995 and confirmation by brain CT or MRI stated

Time since stroke: $<6$ months

Inclusion criteria: (1) right-handedness; (2) disease course < 6 months; (3) can sign informed consent

Exclusion criteria: (1) history of psychiatric illness or family history; (2) aphasia; (3) severe dementia; (4) severe physical illness, consciousness disturbance, or deafness, which influences the expression of depressed mood; (4) psychoactive or non-addiction-producing substance-induced depression; (5) various reasons to refuse trial or difficulty in finishing trial

Depression criteria: depression diagnosed according to the CCMD-3

Total number randomised in this trial: 20

Number randomised to treatment group: 10 (55\% men; mean age $69.65 \pm 5.81$ )

Number randomised to control group: $10^{\star \star}$ (55\% men; mean age $\left.68.70 \pm 8.94\right)$

Total number included in final analysis: 20

Number included in treatment group for final analysis: 10

Number included in control group for final analysis: $10^{\star \star *}$ 
Yan 2010b (Continued)

Interventions
Treatment: low-frequency rTMS + routine care + flupentixol and melitracen $10.5 \mathrm{mg} /$ tablet, 1 tablet twice a day + psychotherapy. Low rTMS frequency: $1 \mathrm{~Hz}$; intensity: $110 \%$ motor threshold; location: left DLPFC; 1 sequence included continuous stimulations for 30 minutes; frequency of treatment: 1 sequence a day during 09:00 to 10:00

Control: sham rTMS + routine care + flupentixol and melitracen $10.5 \mathrm{mg} /$ tablet, 1 tablet twice a day + psychotherapy. Sham rTMS $0 \mathrm{~Hz}$; intensity: 0; location: left or right DLPFC; 1 sequence included continuous stimulations for 30 minutes; frequency of treatment: 1 sequence a day during 09:00 to 10:00

Duration: 7 days

Follow-up: none

- Depression measured using HDRS

- Impairment measured using NIHSS

\section{Secondary outcomes}

- Adverse events

- Leaving the trial early

- Death

Notes Unable to obtain information to determine if the psychotherapy component of the intervention meets the review criteria for psychotherapy

Yan 2010c

Methods

Study design: parallel design

Number of arms: 2

Experimental arm: high-frequency rTMS + routine care + flupentixol and melitracen $10.5 \mathrm{mg}$ / tablet, 1 tablet twice a day + psychotherapy

Control arm: routine care + flupentixol and melitracen $10.5 \mathrm{mg} /$ tablet, 1 tablet twice a day + psychotherapy

\section{Participants}

\section{Geographical location: China \\ Setting: inpatient \\ Stroke criteria: not reported}

Method of stroke diagnosis: diagnosis consistent with diagnostic criteria for stroke formulated by the Fourth National Symposium on Cerebrovascular Disease in 1995 and confirmation by brain CT or MRI stated

Time since stroke: $<6$ months

Inclusion criteria: (1) right-handedness; (2) disease course < 6 months; (3) can sign informed consent

Exclusion criteria: (1) history of psychiatric illness or family history; (2) aphasia; (3) severe dementia; (4) severe physical illnesses, consciousness disturbance, or deafness, which influences the expression of depressed mood; (4) psychoactive or non-addiction-producing substance-induced depression; (5) various reasons to refuse trial or difficulty in finishing trial

Depression criteria: depression diagnosed according to the CCMD-3 
Yan 2010c (Continued)

Total number randomised in this trial: 20

Number randomised to treatment group: 10 (50\% men; mean age 68.65, SD 7.62)

Number randomised to control group: $10^{\star \star}$ (60\% men; mean age 67.25 , SD 9.15)

Total number included in final analysis: 20

Number included in treatment group for final analysis: 10

Number included in control group for final analysis: $10^{\star \star *}$

Interventions
Treatment: high-frequency rTMS + routine care + flupentixol and melitracen $10.5 \mathrm{mg} /$ tablet, 1 tablet twice a day + psychotherapy. High rTMS frequency: $10 \mathrm{~Hz}$; intensity: $110 \%$ motor threshold; location: left DLPFC; 1 sequence included continuous stimulations for 30 minutes; frequency of treatment: 1 sequence a day during 09:00 to 10:00

Control: routine care + flupentixol and melitracen $10.5 \mathrm{mg} /$ tablet, 1 tablet twice a day + psychotherapy

Duration: 7 days

Follow-up: none

\begin{tabular}{ll}
\hline Outcomes & Primary outcomes \\
- & Depression measured using HDRS \\
Secondary outcomes \\
- Adverse events \\
- Leaving the trial early \\
- Death \\
\hline Unable to obtain information to determine if the psychotherapy component of the intervention \\
meets the review criteria for psychotherapy \\
\hline
\end{tabular}

Yan 2010d

Methods Study design: parallel design

Number of arms: 2

Experimental arm: low-frequency rTMS + routine care + flupentixol and melitracen $10.5 \mathrm{mg} /$ tablet, 1 tablet twice a day + psychotherapy

Control arm: routine care + flupentixol and melitracen $10.5 \mathrm{mg} /$ tablet, 1 tablet twice a day + psychotherapy

Participants

\section{Geographical location: China \\ Setting: inpatient \\ Stroke criteria: not reported}

Method of stroke diagnosis: diagnosis consistent with diagnostic criteria for stroke formulated by the Fourth National Symposium on Cerebrovascular Disease in 1995 and confirmation by brain CT or MRI stated

Time since stroke: $<6$ months

Inclusion criteria: (1) right-handedness; (2) disease course < 6 months; (3) can sign informed consent 
Yan 2010d (Continued)

Exclusion criteria: (1) history of psychiatric illness or family history; (2) aphasia; (3) severe dementia; (4) severe physical illness, consciousness disturbance, or deafness, which influences the expression of depressed mood; (4) psychoactive or non-addiction-producing substance-induced depression; (5) various reasons to refuse trial or difficulty in finishing trial

Depression criteria: depression diagnosed according to the CCMD-3

Total numbers randomised in this trial: 20

Numbers randomised to treatment group: 10 (55\% men; mean age 69.65, SD 5.81)

Numbers randomised to control group: $10^{\star \star}$ (60\% men; mean age 67.25 , SD 9.15)

Total numbers included in final analysis: 20

Numbers included in treatment group for final analysis: 10

Numbers included in control group for final analysis: $10^{\star *}$

Interventions

Treatment: low-frequency rTMS + routine care + flupentixol and melitracen $10.5 \mathrm{mg} /$ tablet, 1 tablet twice a day + psychotherapy. Low rTMS frequency: $1 \mathrm{~Hz}$; intensity: $110 \%$ motor threshold; location: left DLPFC; 1 sequence included continuous stimulations for 30 minutes; frequency of treatment: 1 sequence a day during 09:00 to 10:00

Control: routine care + flupentixol and melitracen $10.5 \mathrm{mg} /$ tablet, 1 tablet twice a day + psychotherapy

Duration: 7 days

Follow-up: none

\begin{tabular}{ll}
\hline Outcomes & Primary outcomes \\
- & Depression measured using HDRS \\
- Impairment measured using NIHSS
\end{tabular}

\section{Secondary outcomes}

- Adverse events

- Leaving the trial early

- Death

Notes

Unable to obtain information to determine if the psychotherapy component of the intervention meets the review criteria for psychotherapy

** Results for control group halved.

ACA: anterior cerebral artery.

BDI: Beck Depression Inventory.

BI: Barthel Index.

CCMD-2-R: Chinese Classification of Mental Disorders, Second Edition, Revised.

CCMD-3: Chinese Classification of Mental Disorders, Third Edition.

CNS: central nervous system.

CSS: Chinese Stroke Scale.

$\mathrm{CT}$ : computed tomography.

DLPFC: dorsolateral pre-frontal cortex.

HADS: Hospital Anxiety Depression Scale.

HARS: Hamilton Anxiety Rating Scale.

HDRS-17: 17-item Hamilton Depression Rating Scale.

HDRS-24: 24-item Hamilton Depression Rating Scale.

$\mathrm{Hz}$ : hertz.

ICD: International Classification of Diseases.

LSI: Life Satisfaction Index.

Pharmacological, psychological, and non-invasive brain stimulation interventions for treating depression after stroke (Review) 
MMSE: Mini Mental State Examination.

MRI: magnetic resonance imaging.

NIHSS: National Institute of Health Stroke Scale.

PCA: posterior cerebral artery.

rTMS: repetitive transcranial magnetic stimulation.

SD: standard deviation.

SNRI: selective norepinephrine reuptake inhibitor.

SSRI: selective serotonin reuptake inhibitor.

SSS: Scandinavian Stroke Scale.

TCA: tricyclic antidepressant.

ZDS: Zung Depression Scale.

Characteristics of ongoing studies [ordered by study ID]

\section{Kirkevold 2018}

Trial name or title

Promoting psychosocial well-being following stroke: study protocol for a randomised, controlled trial

\begin{tabular}{ll}
\hline Methods & Study design: parallel design \\
Number of arms: 2 \\
Experimental arm: dialogue-based intervention
\end{tabular}

Control arm: usual care

Participants

Geographical location: Norway

Setting: mixed

Stroke criteria: unclear

Method of stroke diagnosis: not reported

Inclusion criteria: (1) adults over 18 years of age; (2) acute stroke within the last month before inclusion; (3) medically stable; (4) sufficient cognitive functioning to participate (assessed by physician/stroke team); (5) interested in participating; (6) able to understand and speak Norwegian; (7) able to give informed consent

Exclusion criteria: (1) serious somatic or psychiatric disease, as these are assumed to impact ability to participate in the intervention; (2) severe dementia; (3) significant impressive aphasia or severe expressive aphasia

Depression criteria: no criteria for depression at entry

Interventions

Treatment: dialogue-based intervention to promote psychosocial well-being. Intervention consists of 8 one to one and a half hour dialogue-based sessions between the stroke survivor and a specially trained health professional (RN or OT). Each meeting has a guiding topical outline, which addresses significant issues described in the research literature (e.g. bodily changes, emotional challenges, personal relations, daily life issues, meaningful activities, existential issues, important values)

Administered by: trained health professional (RN or OT)

Supervision: not reported

Control: usual care

Duration: 6 months

Follow-up: 2 weeks

\section{Outcomes}

\section{Primary outcome}


Kirkevold 2018 (Continued)

- Depression measured using GHQ-28

\section{Secondary outcomes}

- Coherence measured using SOC-13

- Health-related quality of life measured using SAQoL-39

\begin{tabular}{ll}
\hline Starting date & December 2014 \\
\hline Contact information & $\begin{array}{l}\text { Dr. Marit Kirkevold, Institute of Health and Society and Research Center for Rehabilitation and Re- } \\
\text { habilitation services and models (CHARM), University of Oslo, PO Box 1130, Blindern, 0318 Oslo, } \\
\text { Norway } \\
\text { Email: marit.kirkevold@medisin.uio.no }\end{array}$ \\
\hline
\end{tabular}

Notes

\section{NCT03056287}

\section{Trial name or title}

Methods

\section{Exercise and brain stimulation for post-stroke}

Study design: parallel design

Number of arms: 2

Experimental arm: rTMS

Control arm: Sham rTMS

\section{Participants}

\section{Geographical location: USA}

Setting: unclear

Stroke criteria: unclear

Method of stroke diagnosis: not reported

Inclusion criteria: (1) major depressive disorder (PHQ-9 > 10); (2) no antidepressant medications or clinically able to discontinue medications

Exclusion criteria: (1) unable to ambulate at least 150 feet before stroke, or experienced intermittent claudication while walking; (2) history of congestive heart failure, unstable cardiac arrhythmias, hypertrophic cardiomyopathy, severe aortic stenosis, angina or dyspnoea at rest or during ADLs; (3) history of oxygen dependence; (4) pre-existing neurological disorders, dementia, or previous stroke; (5) history of major head trauma; (6) legal blindness or severe visual impairment; (7) history of psychosis or other Axis I disorder that is primary; (8) life expectancy < 1 year; (9) severe arthritis or other problem that limits passive range of motion; (10) history of DVT or pulmonary embolism within 6 months; (11) uncontrolled diabetes with recent weight loss, diabetic coma, or frequent insulin reactions; (12) severe hypertension with systolic $>200 \mathrm{mmHg}$ and diastolic $>110$ $\mathrm{mmHg}$ at rest; (13) suicide attempt in the last 2 years or at suicidal risk as assessed by SCID interview; (14) previous or current enrolment in a clinical trial to enhance motor recovery; (15) currently exercising $\geq 2$ times per week ( $\geq 20$ minutes); (16) presence of non-MRI compatible implants, pregnancy, or severe claustrophobia

Depression criteria: PHQ-9 > 10 and diagnosed according to DSM-IV

\section{Treatment: rTMS}

Control: sham rTMS 
NCT03056287 (Continued)

Duration: 8 weeks

Follow-up: 8 weeks

\begin{tabular}{ll}
\hline Outcomes & Primary outcome \\
& - Depression measured using HDRS \\
& Secondary outcome \\
& Walking speed \\
\hline Starting date & 1 January 2016 \\
\hline Contact information & Dr. Chris Gregory, Medical University of South Carolina, Charleston, South Carolina, United States \\
& 29425 \\
& Email: gregoryc@musc.edu \\
\hline Notes & \\
\hline
\end{tabular}

\section{Tang 2017}

Trial name or title

Repetitive transcranial magnetic stimulation for depression after basal ganglia ischaemic stroke: protocol for a multicentre randomised double-blind placebo-controlled trial

\section{Methods}

\section{Study design: parallel design}

\section{Number of arms: 2}

Experimental arm: active rTMS

Control arm: sham rTMS

\section{Participants}

\section{Geographical location: China}

Setting: inpatient

Stroke criteria: ischaemic stroke

Method of stroke diagnosis: clinical and MRI or CT findings of basal ganglia ischaemic stroke Inclusion criteria: (1) first-time ischaemic stroke; (2) recent stroke (within 3 weeks to 3 months)

Exclusion criteria: (1) prior history of depressive disorders or major trauma within 1 year, severe depression, or any other severe mental disorder; (2) current or prior antidepressant use for any reason; (3) aphasia or severe cognitive impairment, severe hearing impairment, or severe language comprehension deficit due to other causes; (4) other cerebral disease such as Parkinson's disease, encephalitis, dementia, multiple sclerosis, head injury, severe systemic disease, or ongoing neoplasia; (5) ongoing postoperative recovery

Depression criteria: DSM-IV diagnosis of depression due to stroke (ICD-10-CM code 293.83 (F06.32)) 
Tang 2017 (Continued)

Follow-up: not reported

Outcomes

Primary outcome

- Depression measured using 24-item HDRS

Secondary outcomes

- Impairment measured using NIHSS

- Activities of Daily Living measured using ADLS

- Cognitive functioning measured using MoCA

- Aphasia measured using Aphasia Battery in Chinese, Social Support Revalued Scale

Starting date 20 November 2017

Contact information Dr. Lianxu Zhao

Email: zhaolianxu@smu.edu.cn

Notes

Thomas 2016

Trial name or title

BEhavioural Activation therapy for Depression after Stroke (BEADS): a study protocol for a feasibility randomised controlled pilot trial of a psychological intervention for post-stroke depression

Methods

Study design: parallel design

Number of arms: 2

Experimental arm: behavioural activation therapy

Control arm: usual care

Participants

Geographical location: UK

Setting: mixed

Number of participants: unclear

Stroke criteria: ischaemic or haemorrhagic stroke

Method of stroke diagnosis: not reported

Inclusion criteria: (1) minimum of 3 months and maximum of 5 years post stroke; (2) 18 years of age or older; (3) living in community settings (including nursing homes)

Exclusion criteria: (1) receiving medical or psychological treatment for depression at the time at which they had their stroke (based on self-report by patient/carer) and/or currently receiving psychological intervention; (2) diagnosis of dementia before the stroke (based on self-report by patient/carer); (3) communication difficulties that would impact their capacity to take part in the intervention; (4) visual or hearing impairment that would impact capacity to take part in the intervention (based on the therapist's discretion at baseline assessment); (5) unable to communicate in English before the stroke or without mental capacity to consent to take part in the trial

Depression criteria: $\mathrm{PHQ}-9$ score $\geq 10$. For participants with communication difficulties or severe cognitive difficulties who are unable to complete the PHQ-9, a score of at least 50/100 on VAMS Sad item 
Thomas 2016 (Continued)

Interventions
Treatment: behavioural activation (BA) therapy is a structured and individualised treatment that aims to increase people's level of activity, particularly the frequency of pleasant or enjoyable events, to improve mood. Maximum of 15 sessions of BA over 4 months, with an expected average of 10 sessions. Therapy sessions were face-to-face on an individual basis, at participants' residences, and lasted about 1 hour. A BA treatment manual was developed

Administered by: assistant psychologist

Supervision: not reported

Intervention fidelity: not reported

Control: usual care

Duration: 4 months

Follow-up: 6 months

Outcomes Primary outcome

- Depression measured using PHQ-9, SAD-Q Hospital version (observer-rated depression)

\section{Secondary outcomes}

- Activities of daily living measured using Nottingham Leisure Questionnaire

- Functional outcome measured using Nottingham EADL

- Health-related quality of life measured using EQ5D

Starting date 12 December 2014

Contact information

Dr. Shirley Thomas; Division of Rehabilitation and Ageing, School of Medicine, B Floor Medical School, Queens Medical Centre, University of Nottingham, Nottingham NG7 2UH, UK

Email: shirley.thomas@nottingham.ac.uk

Notes

Author contact:emailed study authors to check if they can share findings (reply received: the funder has advised the author not to share findings until published 25 October 2018)

Xu 2016

Trial name or title Efficacy and feasibility of antidepressant treatment in patients with post-stroke depression

Methods

Study design: parallel design

Number of arms: 2

Experimental arm: rTMS

Control arm: sham rTMS

Participants

Geographical location: China

Setting: unclear

Number of participants: unclear

Stroke criteria: ischaemic stroke

Method of stroke diagnosis: ischaemic brain region or infarction confirmed by CT or MRI 
Xu 2016 (Continued)

Inclusion criteria: (1) 2 weeks to 3 months after acute ischaemic stroke

Exclusion criteria: (1) all kinds of serious mental disorders other than depressive disorder; confirmed cases of various types of depression, or history of major mental trauma within 1 year; (2) verbal communication failure (aphasia, severe cognitive impairment, severe hearing loss, etc.); (3) other systemic diseases that have a serious impact on abilities of daily living; (4) brain disease other than stroke (such as Parkinson's disease, encephalitis, multiple sclerosis, brain trauma, etc.); (5) nuclear magnetic resonance or transcranial magnetic stimulation contraindications

Depression criteria: diagnostic criteria of depression disorder caused by other somatic disease accorded with American Diagnostic and Statistical Manual of Mental Disorders (DSM-V, ICD-10-CM 293.83 (F06.32))

\begin{tabular}{|c|c|}
\hline Interventions & $\begin{array}{l}\text { Treatment: rTMS } \\
\text { Control: sham rTMS } \\
\text { Duration: not reported } \\
\text { Follow-up: not reported }\end{array}$ \\
\hline Outcomes & $\begin{array}{l}\text { Primary outcome } \\
\text { - Depression measured using HDRS } \\
\text { Secondary outcomes } \\
\text { - Dependence measured using Social Support Revalued Scale } \\
\text { - Disability and impairments measured using Medical Coping Modes Questionnaire }\end{array}$ \\
\hline Starting date & 1 January 2016 \\
\hline Contact information & $\begin{array}{l}\text { Dr. Suiyi Xu } \\
\text { Email: suiyixu@sina.com }\end{array}$ \\
\hline Notes & $\begin{array}{l}\text { Author contact: emailed study authors to check if there are any published results for the trial } 3 \text { De- } \\
\text { cember 2018; no reply received }\end{array}$ \\
\hline
\end{tabular}

ADLs: activities of daily living.

ADLS: Activities of Daily Living Scale.

BA: behavioural activation.

$\mathrm{CT}$ : computed tomography.

DSM-IV: Diagnostic and Statistical Manual of Mental Disorders, Fourth Edition.

DSM-v: Diagnostic and Statistical Manual of Mental Disorders, Fifth Edition.

DVT: deep vein thrombosis.

EADL: Extended Activities of Daily Living.

EQ5D: EuroQoL 5-dimensions.

GHQ-28: 28-item General Health Questionnaire.

HDRS-24: 24-item Hamilton Depression Rating Scale.

ICD: International Classification for Diseases.

MoCA: Montreal Cognitive Assessment.

MRI: magnetic resonance imaging.

NIHSS: National Institutes of Health Stroke Scale.

OT: occupational therapist.

PHQ-9: 9-item Patient Health Questionnaire.

RN: registered nurse.

rTMS: repetitive transcranial magnetic stimulation.

SAD-Q: Stroke Aphasia Depression Questionnaire - hospital version.

SAQoL-39: Stroke Aphasia Quality of Life Scale.

SCID: severe combined immunodeficiency. 
SOC-13: Sense of Coherence.

VAMS: Visual Analog Mood Scale.

DATA AND ANALYSES

Comparison 1. Pharmacological interventions versus placebo

\begin{tabular}{|c|c|c|c|c|}
\hline Outcome or subgroup title & No. of studies & $\begin{array}{l}\text { No. of partici- } \\
\text { pants }\end{array}$ & Statistical method & Effect size \\
\hline $\begin{array}{l}1 \text { Depression: meeting study criteria } \\
\text { for depression at end of treatment }\end{array}$ & 8 & 1025 & $\begin{array}{l}\text { Risk Ratio (M-H, Random, } \\
95 \% \mathrm{Cl})\end{array}$ & $0.70[0.55,0.88]$ \\
\hline $\begin{array}{l}\text { 1.1 Clinician interview/impression } \\
\text { (number not improved) }\end{array}$ & 1 & 285 & $\begin{array}{l}\text { Risk Ratio (M-H, Random, } \\
95 \% \mathrm{Cl})\end{array}$ & $0.72[0.54,0.95]$ \\
\hline $1.2 \mathrm{DSM}-\mathrm{III}$ & 1 & 39 & $\begin{array}{l}\text { Risk Ratio (M-H, Random, } \\
95 \% \mathrm{Cl})\end{array}$ & $0.81[0.32,2.03]$ \\
\hline 1.3 MADRS & 2 & 352 & $\begin{array}{l}\text { Risk Ratio (M-H, Random, } \\
95 \% \mathrm{Cl})\end{array}$ & $0.98[0.59,1.60]$ \\
\hline 1.4 HDRS & 4 & 349 & $\begin{array}{l}\text { Risk Ratio (M-H, Random, } \\
95 \% \mathrm{Cl})\end{array}$ & $0.56[0.46,0.68]$ \\
\hline $\begin{array}{l}2 \text { Depression: }<50 \% \text { reduction in scale } \\
\text { scores at end of treatment }\end{array}$ & 6 & 511 & $\begin{array}{l}\text { Risk Ratio (M-H, Random, } \\
95 \% \mathrm{Cl})\end{array}$ & $0.48[0.32,0.70]$ \\
\hline 2.1 HDRS & 4 & 357 & $\begin{array}{l}\text { Risk Ratio (M-H, Random, } \\
95 \% \mathrm{Cl} \text { ) }\end{array}$ & $0.40[0.25,0.61]$ \\
\hline 2.2 MADRS & 2 & 154 & $\begin{array}{l}\text { Risk Ratio (M-H, Random, } \\
95 \% \mathrm{Cl} \text { ) }\end{array}$ & $0.77[0.59,1.01]$ \\
\hline $\begin{array}{l}3 \text { Depression: average change in scores } \\
\text { between baseline and end of treat- } \\
\text { ment }\end{array}$ & 5 & & $\begin{array}{l}\text { Std. Mean Difference (IV, Ran- } \\
\text { dom, } 95 \% \mathrm{CI} \text { ) }\end{array}$ & $\begin{array}{l}\text { Totals not select- } \\
\text { ed }\end{array}$ \\
\hline 3.1 BDI (high score = more depressed) & 1 & & $\begin{array}{l}\text { Std. Mean Difference (IV, Ran- } \\
\text { dom, } 95 \% \mathrm{CI} \text { ) }\end{array}$ & $0.0[0.0,0.0]$ \\
\hline $\begin{array}{l}3.2 \text { CGI (low score }=\text { improvement } / \text { high } \\
\text { score = deterioration) }\end{array}$ & 1 & & $\begin{array}{l}\text { Std. Mean Difference (IV, Ran- } \\
\text { dom, } 95 \% \mathrm{CI} \text { ) }\end{array}$ & $0.0[0.0,0.0]$ \\
\hline $\begin{array}{l}\text { 3.3 HDRS (high score = more de- } \\
\text { pressed) }\end{array}$ & 2 & & $\begin{array}{l}\text { Std. Mean Difference (IV, Ran- } \\
\text { dom, } 95 \% \mathrm{CI} \text { ) }\end{array}$ & $0.0[0.0,0.0]$ \\
\hline $\begin{array}{l}\text { 3.4 MADRS (high score = more de- } \\
\text { pressed) }\end{array}$ & 3 & & $\begin{array}{l}\text { Std. Mean Difference (IV, Ran- } \\
\text { dom, } 95 \% \mathrm{CI} \text { ) }\end{array}$ & $0.0[0.0,0.0]$ \\
\hline $\begin{array}{l}4 \text { Depression: mean scores at end of } \\
\text { treatment }\end{array}$ & 15 & & $\begin{array}{l}\text { Std. Mean Difference (IV, Ran- } \\
\text { dom, } 95 \% \mathrm{CI} \text { ) }\end{array}$ & $\begin{array}{l}\text { Totals not select- } \\
\text { ed }\end{array}$ \\
\hline 4.1 BDI (high score = more depressed) & 4 & & $\begin{array}{l}\text { Std. Mean Difference (IV, Ran- } \\
\text { dom, } 95 \% \mathrm{Cl} \text { ) }\end{array}$ & $0.0[0.0,0.0]$ \\
\hline
\end{tabular}




\begin{tabular}{|c|c|c|c|c|}
\hline Outcome or subgroup title & No. of studies & $\begin{array}{l}\text { No. of partici- } \\
\text { pants }\end{array}$ & Statistical method & Effect size \\
\hline $\begin{array}{l}\text { 4.2 CGI (low score = improvement } / \text { high } \\
\text { score = deterioration) }\end{array}$ & 1 & & $\begin{array}{l}\text { Std. Mean Difference (IV, Ran- } \\
\text { dom, } 95 \% \mathrm{Cl} \text { ) }\end{array}$ & $0.0[0.0,0.0]$ \\
\hline $\begin{array}{l}4.3 \text { HDRS (high score = more de- } \\
\text { pressed) }\end{array}$ & 13 & & $\begin{array}{l}\text { Std. Mean Difference (IV, Ran- } \\
\text { dom, } 95 \% \mathrm{Cl} \text { ) }\end{array}$ & $0.0[0.0,0.0]$ \\
\hline $\begin{array}{l}\text { 4.4 MADRS (high score = more de- } \\
\text { pressed) }\end{array}$ & 2 & & $\begin{array}{l}\text { Std. Mean Difference (IV, Ran- } \\
\text { dom, } 95 \% \mathrm{Cl} \text { ) }\end{array}$ & $0.0[0.0,0.0]$ \\
\hline $\begin{array}{l}4.5 \text { Melancholia scale (high score }= \\
\text { more depressed) }\end{array}$ & 1 & & $\begin{array}{l}\text { Std. Mean Difference (IV, Ran- } \\
\text { dom, } 95 \% \mathrm{Cl} \text { ) }\end{array}$ & $0.0[0.0,0.0]$ \\
\hline $\begin{array}{l}\text { 4.6 Zung Depression Scale (high score } \\
=\text { more depressed) }\end{array}$ & 1 & & $\begin{array}{l}\text { Std. Mean Difference (IV, Ran- } \\
\text { dom, } 95 \% \mathrm{CI} \text { ) }\end{array}$ & $0.0[0.0,0.0]$ \\
\hline $\begin{array}{l}5 \text { Anxiety: meeting study criteria for } \\
\text { anxiety at end of treatment }\end{array}$ & 1 & & $\begin{array}{l}\text { Odds Ratio (M-H, Fixed, 95\% } \\
\mathrm{Cl})\end{array}$ & $\begin{array}{l}\text { Totals not select- } \\
\text { ed }\end{array}$ \\
\hline 5.1 Clinician interview/impression & 1 & & $\begin{array}{l}\text { Odds Ratio (M-H, Fixed, 95\% } \\
\mathrm{Cl})\end{array}$ & $0.0[0.0,0.0]$ \\
\hline $\begin{array}{l}6 \text { Cognitive function: average change } \\
\text { in scores between baseline and end of } \\
\text { treatment }\end{array}$ & 1 & & $\begin{array}{l}\text { Mean Difference (IV, Fixed, } \\
95 \% \mathrm{CI} \text { ) }\end{array}$ & $\begin{array}{l}\text { Totals not select- } \\
\text { ed }\end{array}$ \\
\hline $\begin{array}{l}6.1 \mathrm{MMSE} \text { (low score = cognitive im- } \\
\text { pairment) }\end{array}$ & 1 & & $\begin{array}{l}\text { Mean Difference (IV, Fixed, } \\
95 \% \mathrm{Cl} \text { ) }\end{array}$ & $0.0[0.0,0.0]$ \\
\hline $\begin{array}{l}7 \text { Cognitive function: mean scores at } \\
\text { end of treatment }\end{array}$ & 1 & & $\begin{array}{l}\text { Mean Difference (IV, Fixed, } \\
95 \% \mathrm{CI} \text { ) }\end{array}$ & $\begin{array}{l}\text { Totals not select- } \\
\text { ed }\end{array}$ \\
\hline $\begin{array}{l}7.1 \mathrm{MMSE} \text { (low score = cognitive im- } \\
\text { pairment) }\end{array}$ & 1 & & $\begin{array}{l}\text { Mean Difference (IV, Fixed, } \\
95 \% \mathrm{Cl} \text { ) }\end{array}$ & $0.0[0.0,0.0]$ \\
\hline $\begin{array}{l}8 \text { Activities of daily living: average } \\
\text { change in scores between baseline and } \\
\text { end of treatment }\end{array}$ & 2 & 256 & $\begin{array}{l}\text { Mean Difference (IV, Random, } \\
95 \% \mathrm{CI} \text { ) }\end{array}$ & $-8.0[-24.18,8.18]$ \\
\hline $\begin{array}{l}\text { 8.1 Barthel Index (high score = more } \\
\text { dependent) }\end{array}$ & 2 & 256 & $\begin{array}{l}\text { Mean Difference (IV, Random, } \\
95 \% \mathrm{CI} \text { ) }\end{array}$ & $-8.0[-24.18,8.18]$ \\
\hline $\begin{array}{l}9 \text { Activities of daily living: mean scores } \\
\text { at end of treatment }\end{array}$ & 3 & 316 & $\begin{array}{l}\text { Mean Difference (IV, Random, } \\
95 \% \mathrm{CI})\end{array}$ & $3.14[-0.97,7.26]$ \\
\hline $\begin{array}{l}\text { 9.1 Barthel Index (high score = more } \\
\text { dependent) }\end{array}$ & 3 & 316 & $\begin{array}{l}\text { Mean Difference (IV, Random, } \\
95 \% \mathrm{CI} \text { ) }\end{array}$ & $3.14[-0.97,7.26]$ \\
\hline $\begin{array}{l}10 \text { Disability: average change in scores } \\
\text { between baseline and end of treat- } \\
\text { ment }\end{array}$ & 3 & & $\begin{array}{l}\text { Std. Mean Difference (IV, Ran- } \\
\text { dom, } 95 \% \mathrm{Cl} \text { ) }\end{array}$ & $\begin{array}{l}\text { Totals not select- } \\
\text { ed }\end{array}$ \\
\hline $\begin{array}{l}\text { 10.1 Functional Independence Mea- } \\
\text { sure (low score = dependence) }\end{array}$ & 1 & & $\begin{array}{l}\text { Std. Mean Difference (IV, Ran- } \\
\text { dom, } 95 \% \mathrm{Cl} \text { ) }\end{array}$ & $0.0[0.0,0.0]$ \\
\hline
\end{tabular}




\begin{tabular}{|c|c|c|c|c|}
\hline Outcome or subgroup title & No. of studies & $\begin{array}{l}\text { No. of partici- } \\
\text { pants }\end{array}$ & Statistical method & Effect size \\
\hline $\begin{array}{l}10.2 \text { Motoricity Index (low score }=\text { more } \\
\text { motor impairment) }\end{array}$ & 1 & & $\begin{array}{l}\text { Std. Mean Difference (IV, Ran- } \\
\text { dom, } 95 \% \mathrm{Cl} \text { ) }\end{array}$ & $0.0[0.0,0.0]$ \\
\hline $\begin{array}{l}\text { 10.3 Scandinavian Stroke Scale (low } \\
\text { score = more neurological deficit) }\end{array}$ & 1 & & $\begin{array}{l}\text { Std. Mean Difference (IV, Ran- } \\
\text { dom, } 95 \% \mathrm{Cl} \text { ) }\end{array}$ & $0.0[0.0,0.0]$ \\
\hline $\begin{array}{l}\text { 10.4 Rankin Scale (high score = more } \\
\text { disability) }\end{array}$ & 1 & & $\begin{array}{l}\text { Std. Mean Difference (IV, Ran- } \\
\text { dom, } 95 \% \mathrm{Cl} \text { ) }\end{array}$ & $0.0[0.0,0.0]$ \\
\hline $\begin{array}{l}11 \text { Disability: mean scores at end of } \\
\text { treatment }\end{array}$ & 3 & & $\begin{array}{l}\text { Mean Difference (IV, Fixed, } \\
95 \% \mathrm{CI})\end{array}$ & $\begin{array}{l}\text { Totals not select- } \\
\text { ed }\end{array}$ \\
\hline $\begin{array}{l}\text { 11.1 Functional Independence Mea- } \\
\text { sure (low score = dependence) }\end{array}$ & 2 & & $\begin{array}{l}\text { Mean Difference (IV, Fixed, } \\
95 \% \mathrm{CI})\end{array}$ & $0.0[0.0,0.0]$ \\
\hline $\begin{array}{l}11.2 \text { Motoricity Index (low score }=\text { more } \\
\text { motor impairment) }\end{array}$ & 1 & & $\begin{array}{l}\text { Mean Difference (IV, Fixed, } \\
95 \% \mathrm{Cl} \text { ) }\end{array}$ & $0.0[0.0,0.0]$ \\
\hline $\begin{array}{l}11.3 \text { Scandinavian Stroke Scale (low } \\
\text { score = more neurological deficit) }\end{array}$ & 1 & & $\begin{array}{l}\text { Mean Difference (IV, Fixed, } \\
95 \% \mathrm{Cl} \text { ) }\end{array}$ & $0.0[0.0,0.0]$ \\
\hline $\begin{array}{l}12 \text { Neurological function: average } \\
\text { change in scores between baseline and } \\
\text { end of treatment }\end{array}$ & 1 & & $\begin{array}{l}\text { Mean Difference (IV, Fixed, } \\
95 \% \mathrm{CI} \text { ) }\end{array}$ & $\begin{array}{l}\text { Totals not select- } \\
\text { ed }\end{array}$ \\
\hline $\begin{array}{l}\text { 12.1 Chinese Stroke Scale (high score = } \\
\text { more impairment) }\end{array}$ & 1 & & $\begin{array}{l}\text { Mean Difference (IV, Fixed, } \\
95 \% \mathrm{Cl} \text { ) }\end{array}$ & $0.0[0.0,0.0]$ \\
\hline $\begin{array}{l}13 \text { Neurological function: mean scores } \\
\text { at end of treatment }\end{array}$ & 4 & 304 & $\begin{array}{l}\text { Std. Mean Difference (IV, Ran- } \\
\text { dom, } 95 \% \mathrm{Cl} \text { ) }\end{array}$ & $\begin{array}{l}-0.95[-1.44 \\
-0.45]\end{array}$ \\
\hline $\begin{array}{l}\text { 13.1 Chinese Stroke Scale (high score = } \\
\text { more impairment) }\end{array}$ & 3 & 231 & $\begin{array}{l}\text { Std. Mean Difference (IV, Ran- } \\
\text { dom, } 95 \% \mathrm{Cl} \text { ) }\end{array}$ & $\begin{array}{l}-1.15[-1.59 \\
-0.72]\end{array}$ \\
\hline $\begin{array}{l}13.2 \text { National Institutes of Health } \\
\text { Stroke Scale (high score = more impair- } \\
\text { ment }\end{array}$ & 1 & 73 & $\begin{array}{l}\text { Std. Mean Difference (IV, Ran- } \\
\text { dom, } 95 \% \mathrm{CI} \text { ) }\end{array}$ & $-0.40[-0.87,0.06]$ \\
\hline 14 Adverse events: death & 9 & 848 & $\begin{array}{l}\text { Risk Ratio (M-H, Random, } \\
95 \% \mathrm{Cl} \text { ) }\end{array}$ & $0.64[0.20,2.07]$ \\
\hline 14.1 At end of treatment & 9 & 848 & $\begin{array}{l}\text { Risk Ratio (M-H, Random, } \\
95 \% \mathrm{Cl})\end{array}$ & $0.64[0.20,2.07]$ \\
\hline 15 Adverse events: all & 10 & & $\begin{array}{l}\text { Risk Ratio (M-H, Fixed, 95\% } \\
\mathrm{Cl})\end{array}$ & Subtotals only \\
\hline $\begin{array}{l}\text { 15.1 Central nervous system events } \\
\text { (e.g. confusion, sedation, tremor) }\end{array}$ & 5 & 488 & $\begin{array}{l}\text { Risk Ratio (M-H, Fixed, 95\% } \\
\mathrm{Cl})\end{array}$ & $1.55[1.12,2.15]$ \\
\hline $\begin{array}{l}15.2 \text { Psychiatric events (e.g. anxiety, in- } \\
\text { creased depression) }\end{array}$ & 3 & 183 & $\begin{array}{l}\text { Risk Ratio (M-H, Fixed, 95\% } \\
\mathrm{Cl})\end{array}$ & $0.28[0.05,1.70]$ \\
\hline 15.3 Recurrent stroke & 3 & 195 & $\begin{array}{l}\text { Risk Ratio (M-H, Fixed, 95\% } \\
\mathrm{Cl})\end{array}$ & $1.51[0.29,7.76]$ \\
\hline
\end{tabular}




\begin{tabular}{|c|c|c|c|c|}
\hline Outcome or subgroup title & No. of studies & $\begin{array}{l}\text { No. of partici- } \\
\text { pants }\end{array}$ & Statistical method & Effect size \\
\hline $\begin{array}{l}\text { 15.4 Vascular events - not stroke (e.g. } \\
\text { dizziness, palpitation) }\end{array}$ & 7 & 587 & $\begin{array}{l}\text { Risk Ratio (M-H, Fixed, 95\% } \\
\mathrm{Cl})\end{array}$ & $1.44[0.94,2.22]$ \\
\hline $\begin{array}{l}15.5 \text { Gastrointestinal effects (e.g. con- } \\
\text { stipation, diarrhoea) }\end{array}$ & 4 & 473 & $\begin{array}{l}\text { Risk Ratio (M-H, Fixed, 95\% } \\
\mathrm{Cl})\end{array}$ & $1.62[1.19,2.19]$ \\
\hline $\begin{array}{l}\text { 15.6 Other events - not listed above } \\
\text { (e.g. dysuria, eye discomfort) }\end{array}$ & 7 & 638 & $\begin{array}{l}\text { Risk Ratio (M-H, Fixed, 95\% } \\
\mathrm{Cl} \text { ) }\end{array}$ & $1.32[1.00,1.75]$ \\
\hline $\begin{array}{l}15.7 \text { Protocol violation (e.g. refused } \\
\text { treatment, withdrew consent) }\end{array}$ & 5 & 334 & $\begin{array}{l}\text { Risk Ratio (M-H, Fixed, 95\% } \\
\mathrm{Cl})\end{array}$ & $1.10[0.45,2.68]$ \\
\hline $\begin{array}{l}16 \text { Adverse events: leaving the study } \\
\text { early (including death) }\end{array}$ & 13 & & $\begin{array}{l}\text { Risk Ratio (M-H, Random, } \\
95 \% \mathrm{Cl} \text { ) }\end{array}$ & Subtotals only \\
\hline 16.1 All dropouts and withdrawals & 13 & 1165 & $\begin{array}{l}\text { Risk Ratio (M-H, Random, } \\
95 \% \mathrm{Cl} \text { ) }\end{array}$ & $1.07[0.82,1.39]$ \\
\hline
\end{tabular}

Analysis 1.1. Comparison 1 Pharmacological interventions versus placebo, Outcome 1 Depression: meeting study criteria for depression at end of treatment.

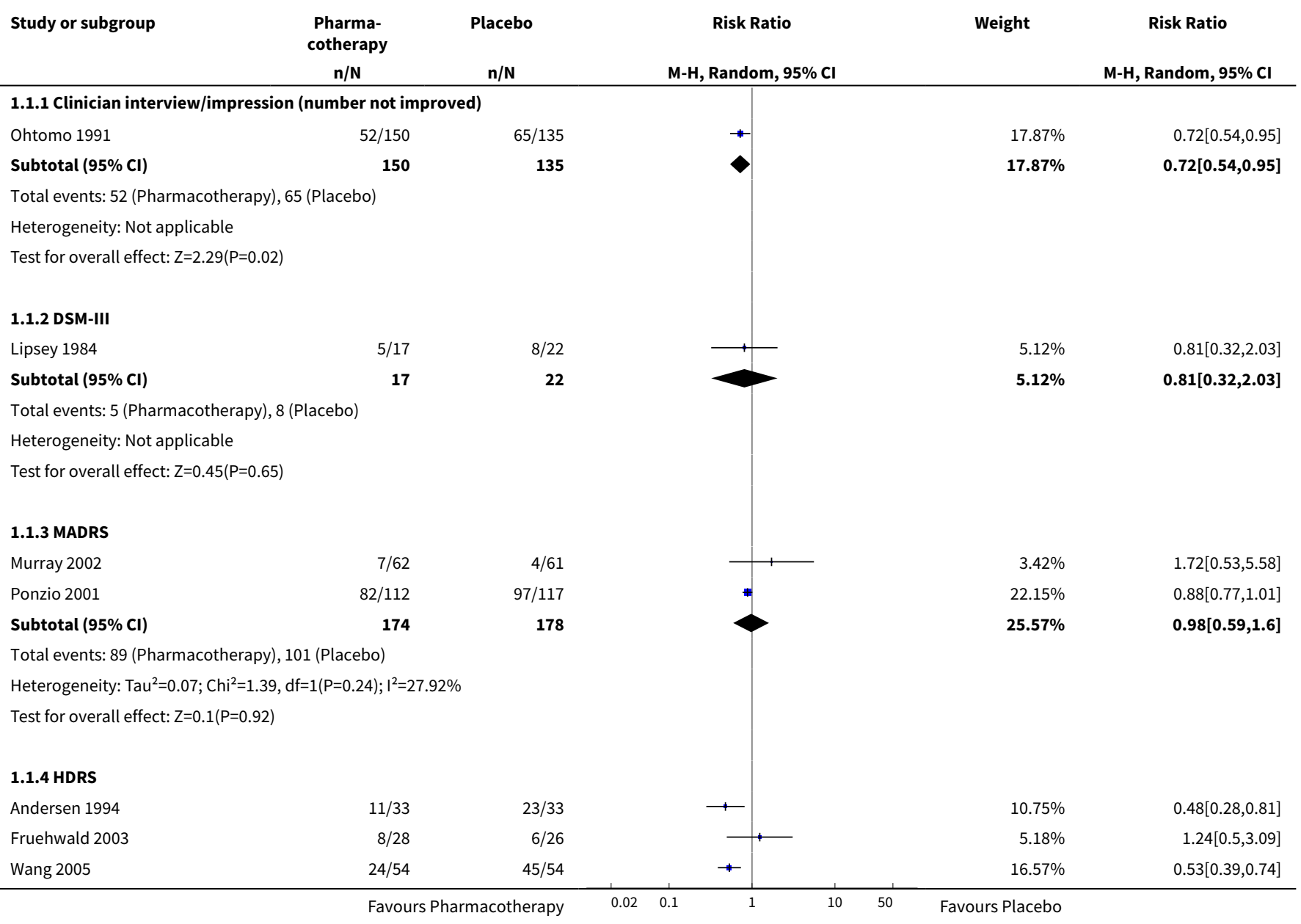




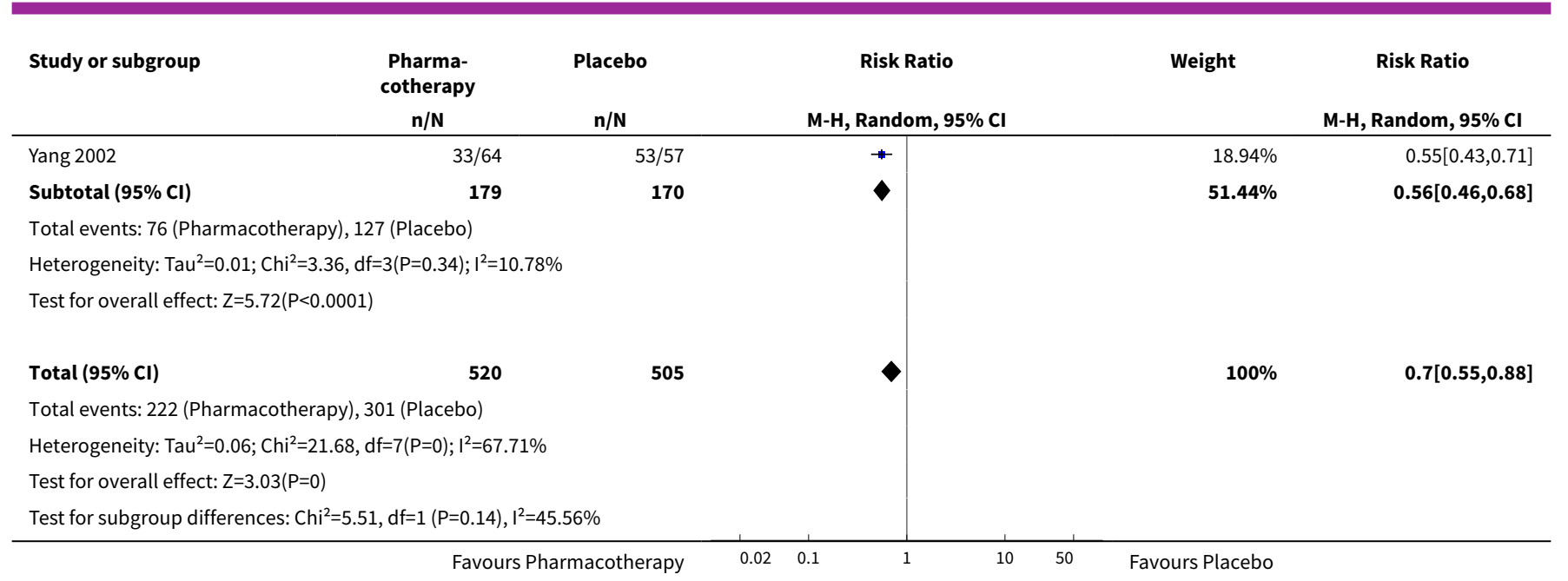

Analysis 1.2. Comparison 1 Pharmacological interventions versus placebo, Outcome 2 Depression: $<50 \%$ reduction in scale scores at end of treatment.

\begin{tabular}{ccccc} 
Study or subgroup & $\begin{array}{c}\text { Pharma- } \\
\text { cotherapy } \\
\mathrm{n} / \mathrm{N}\end{array}$ & Placebo & Risk Ratio & Weight \\
& $\mathrm{n} / \mathrm{N}$ & $\mathrm{M}-\mathrm{H}, \mathrm{Random}, 95 \% \mathrm{Cl}$ & $\mathrm{M}-\mathrm{H}, \mathrm{Random}, 95 \% \mathrm{Cl}$ \\
\hline
\end{tabular}

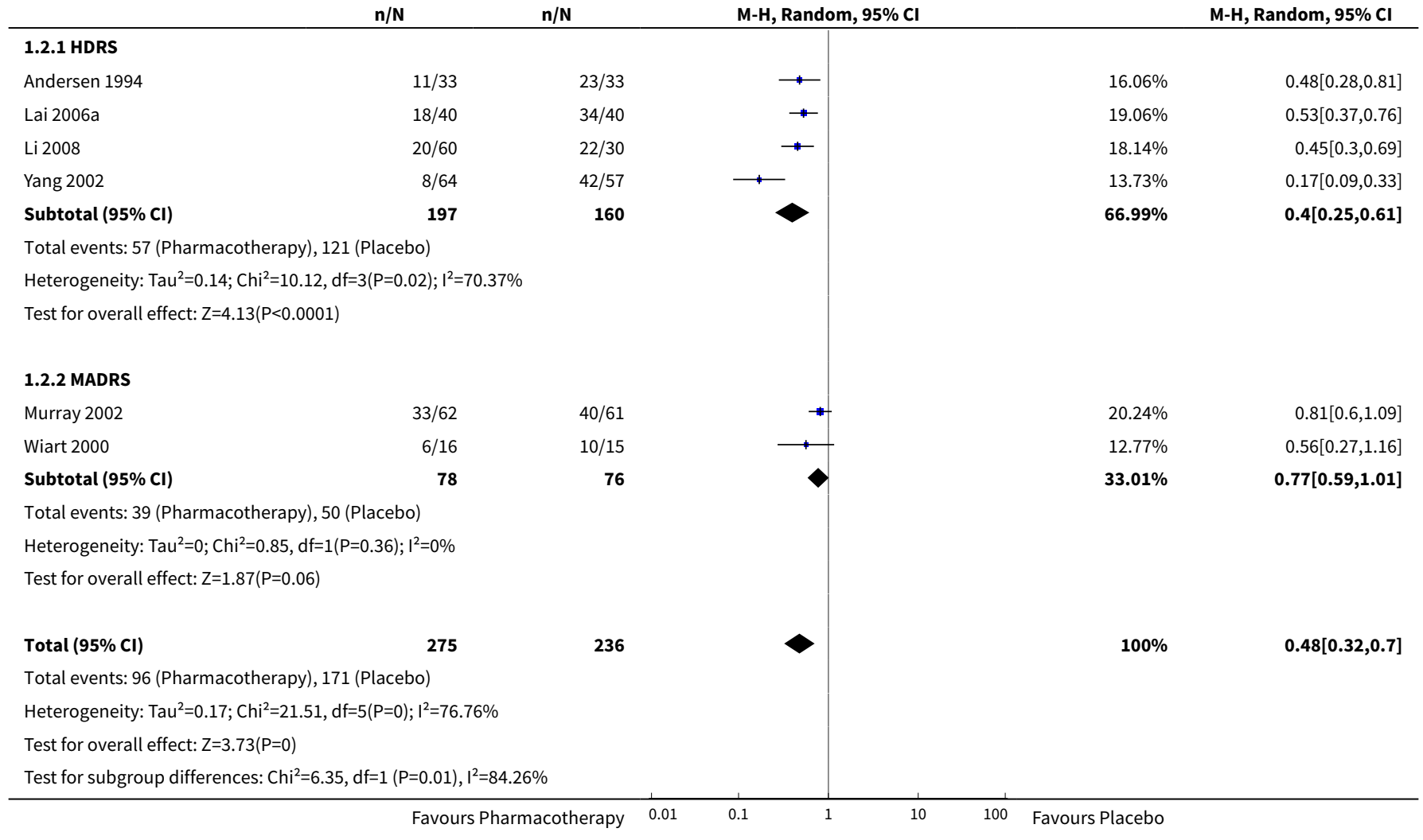


Analysis 1.3. Comparison 1 Pharmacological interventions versus placebo, Outcome 3 Depression: average change in scores between baseline and end of treatment.

\begin{tabular}{|c|c|c|c|c|c|c|}
\hline \multirow[t]{2}{*}{ Study or subgroup } & \multicolumn{2}{|c|}{ Pharmacotherapy } & \multicolumn{2}{|c|}{ Placebo } & \multirow{2}{*}{$\begin{array}{c}\text { Std. Mean Difference } \\
\text { Random, } 95 \% \mathrm{Cl}\end{array}$} & \multirow{2}{*}{$\begin{array}{c}\text { Std. Mean Difference } \\
\text { Random, } 95 \% \mathrm{Cl}\end{array}$} \\
\hline & $\mathbf{N}$ & Mean(SD) & $\mathbf{N}$ & Mean(SD) & & \\
\hline \multicolumn{7}{|c|}{ 1.3.1 BDI (high score = more depressed) } \\
\hline Fruehwald 2003 & 28 & $-6.1(5.6)$ & 26 & $-4.1(6.5)$ & + & $-0.33[-0.86,0.21]$ \\
\hline \multicolumn{7}{|c|}{ 1.3.2 CGI (low score = improvement/high score = deterioration) } \\
\hline Fruehwald 2003 & 28 & $-2.7(1.6)$ & 26 & $-2.1(1.7)$ & + & $-0.36[-0.9,0.18]$ \\
\hline \multicolumn{7}{|c|}{ 1.3.3 HDRS (high score = more depressed) } \\
\hline Fruehwald 2003 & 28 & $-23.3(12)$ & 26 & $-19.1(15.1)$ & + & $-0.3[-0.84,0.23]$ \\
\hline Jiang 2001a & 30 & $-20.1(6.8)$ & 15 & $-11.8(7.5)$ & 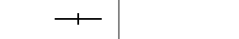 & $-1.15[-1.82,-0.49]$ \\
\hline \multicolumn{7}{|c|}{ 1.3.4 MADRS (high score = more depressed) } \\
\hline Murray 2002 & 62 & $-8.5(8.9)$ & 61 & $-7.6(9.3)$ & + & $-0.1[-0.45,0.26]$ \\
\hline Wiart 2000 & 16 & $-16.6(8.1)$ & 15 & $-8.4(7.8)$ & $千$ & $-1[-1.76,-0.25]$ \\
\hline
\end{tabular}

\section{Analysis 1.4. Comparison 1 Pharmacological interventions versus} placebo, Outcome 4 Depression: mean scores at end of treatment.

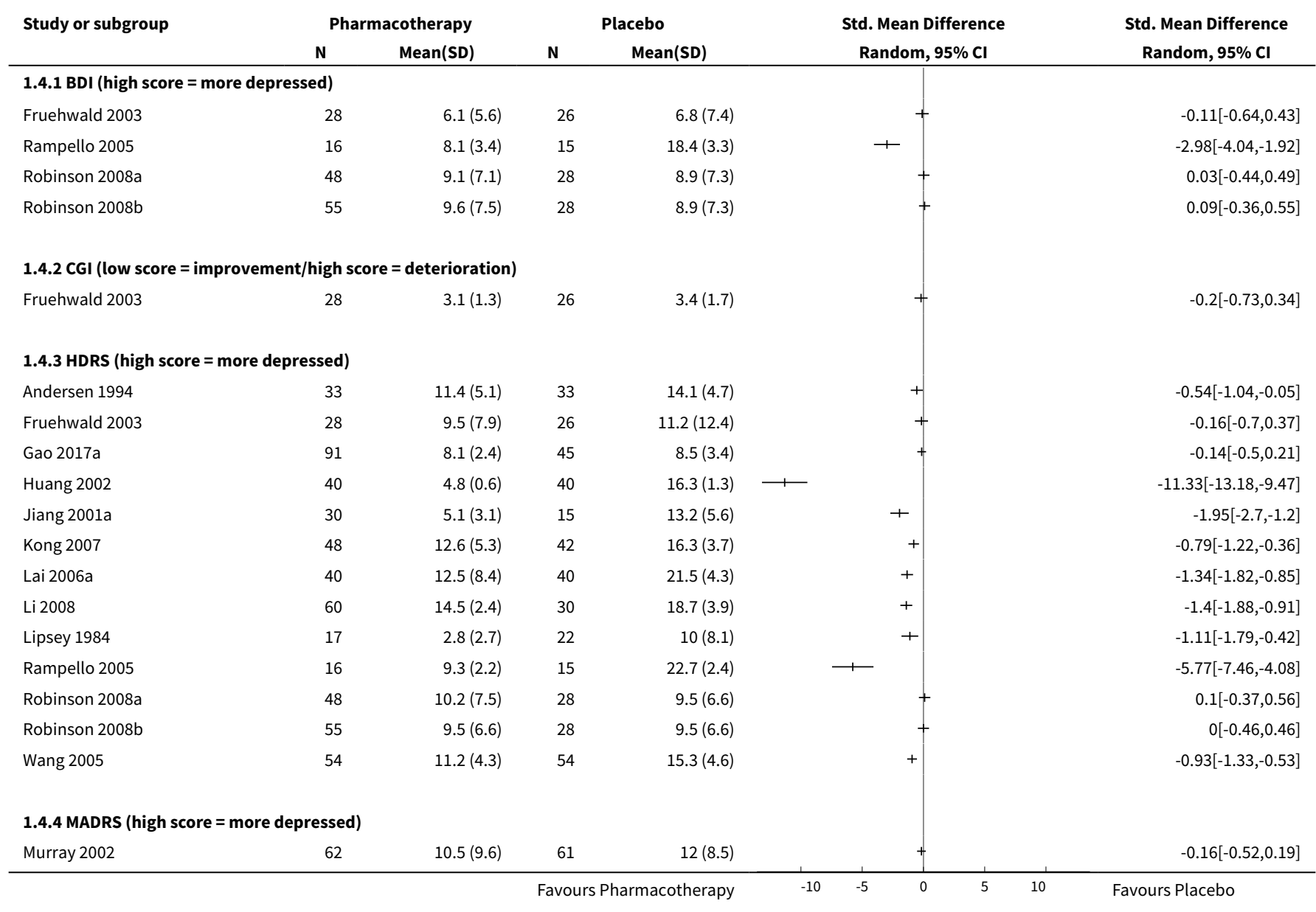




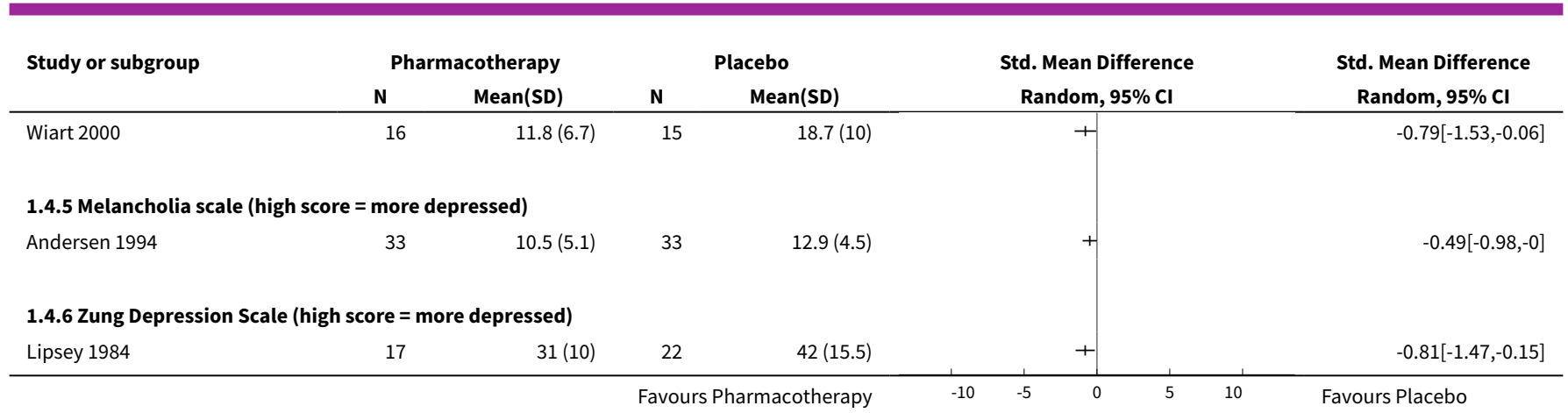

Analysis 1.5. Comparison 1 Pharmacological interventions versus placebo, Outcome 5 Anxiety: meeting study criteria for anxiety at end of treatment.

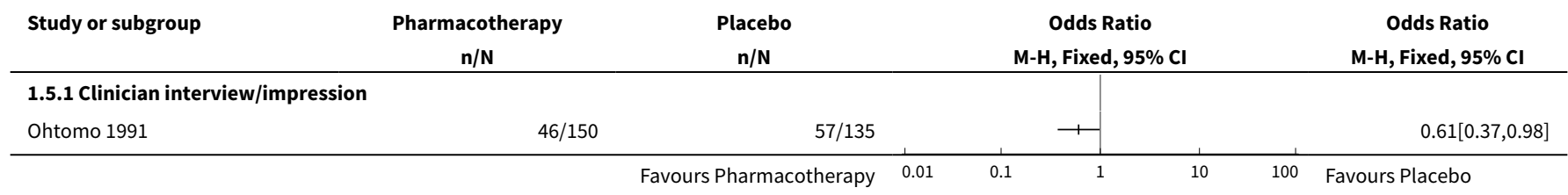

Analysis 1.6. Comparison 1 Pharmacological interventions versus placebo, Outcome 6 Cognitive function: average change in scores between baseline and end of treatment.

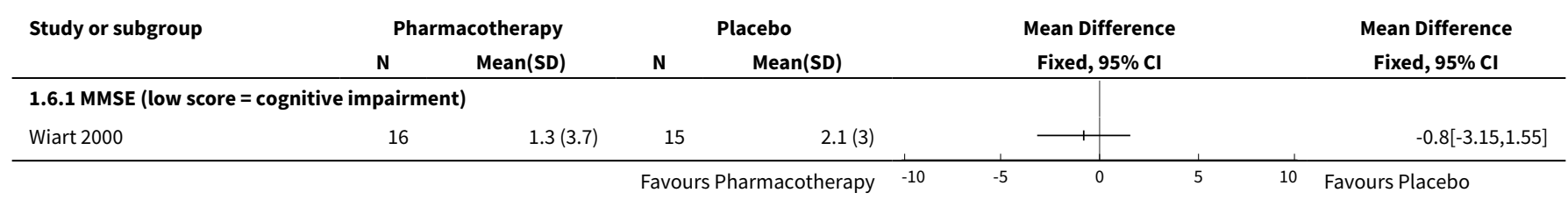

Analysis 1.7. Comparison 1 Pharmacological interventions versus placebo, Outcome 7 Cognitive function: mean scores at end of treatment.

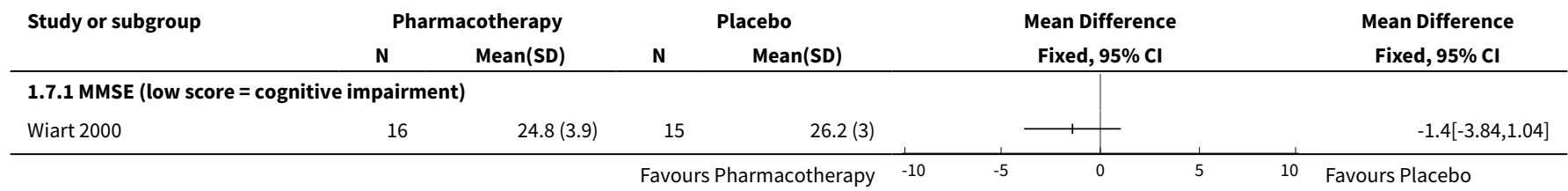

Analysis 1.8. Comparison 1 Pharmacological interventions versus placebo, Outcome 8 Activities of daily living: average change in scores between baseline and end of treatment.

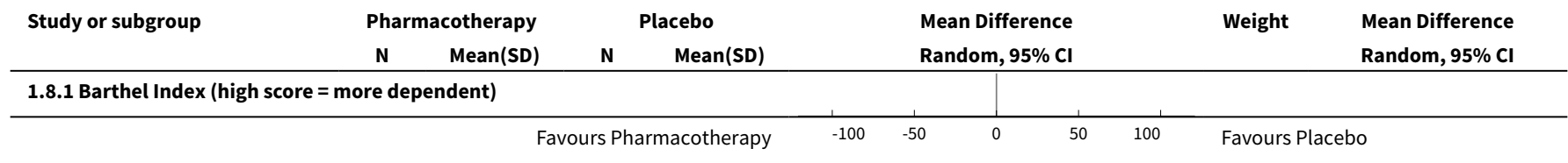




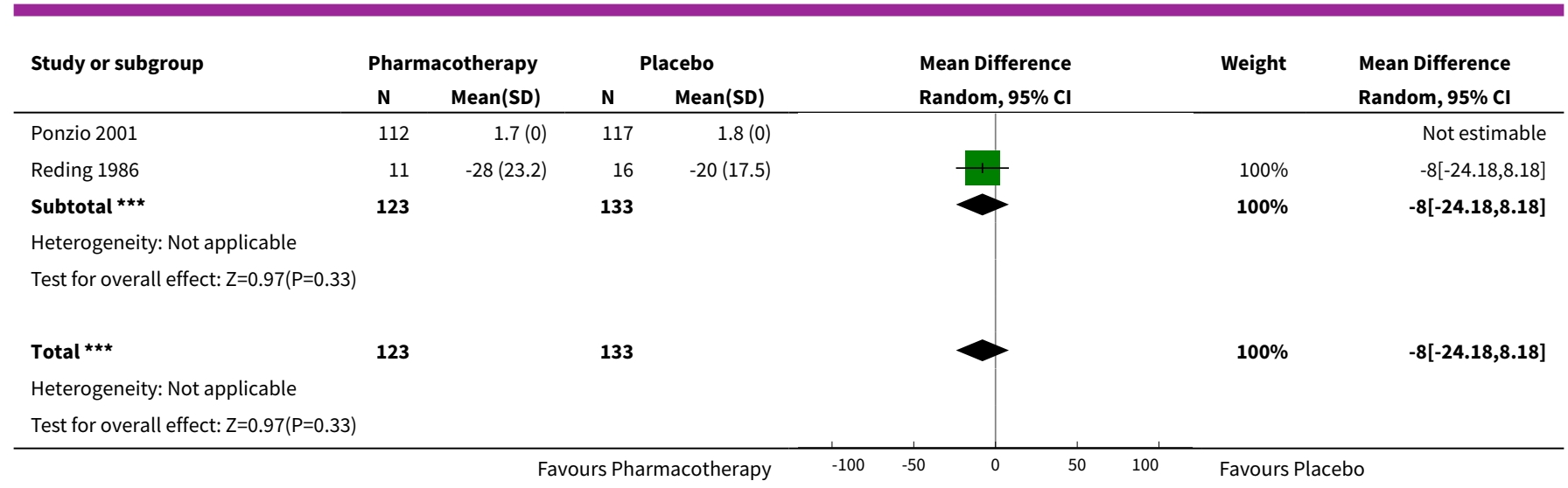

Analysis 1.9. Comparison 1 Pharmacological interventions versus placebo, Outcome 9 Activities of daily living: mean scores at end of treatment.

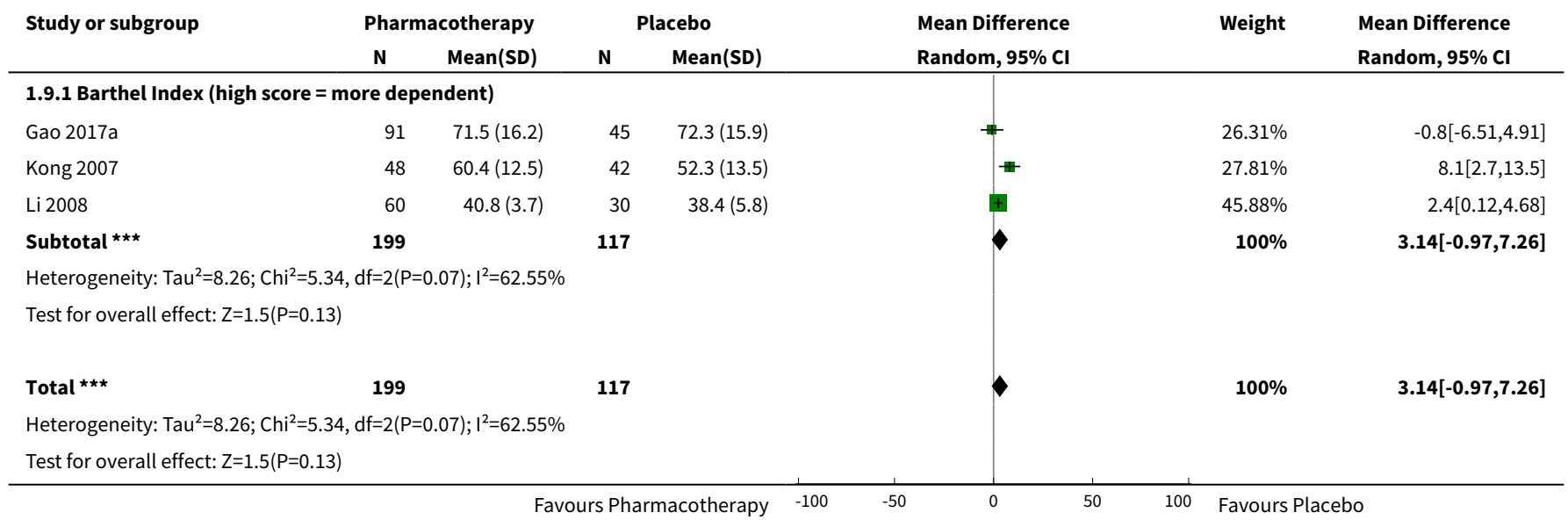

Analysis 1.10. Comparison 1 Pharmacological interventions versus placebo, Outcome 10 Disability: average change in scores between baseline and end of treatment.

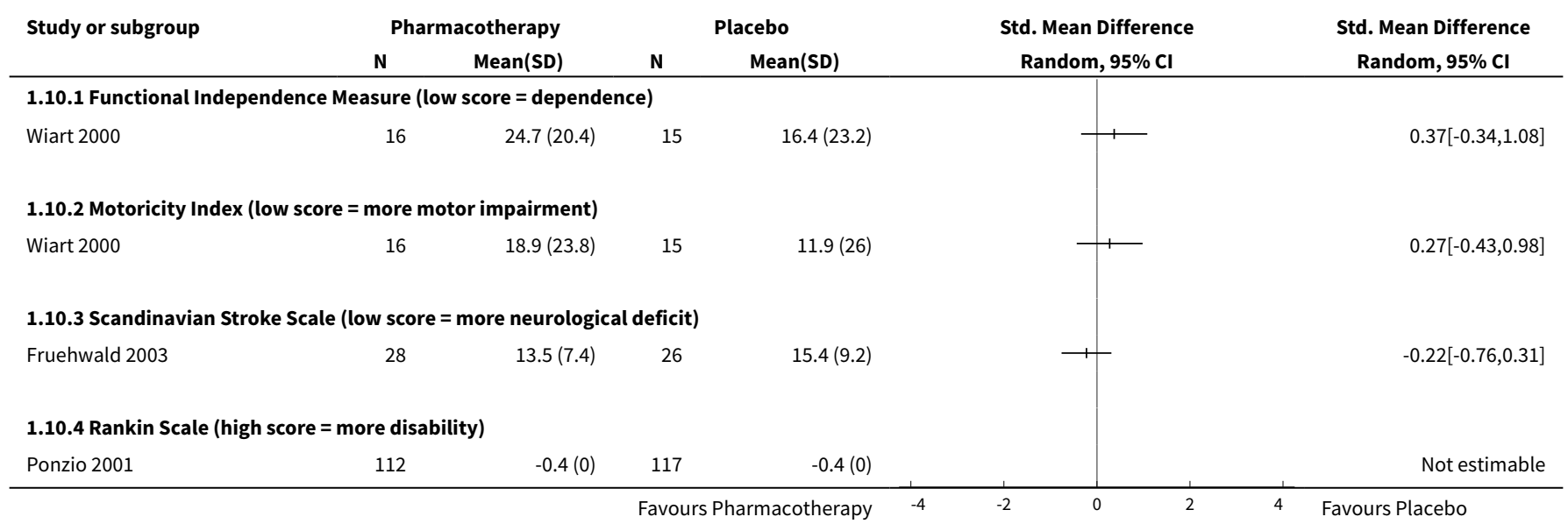




\section{Analysis 1.11. Comparison 1 Pharmacological interventions versus} placebo, Outcome 11 Disability: mean scores at end of treatment.

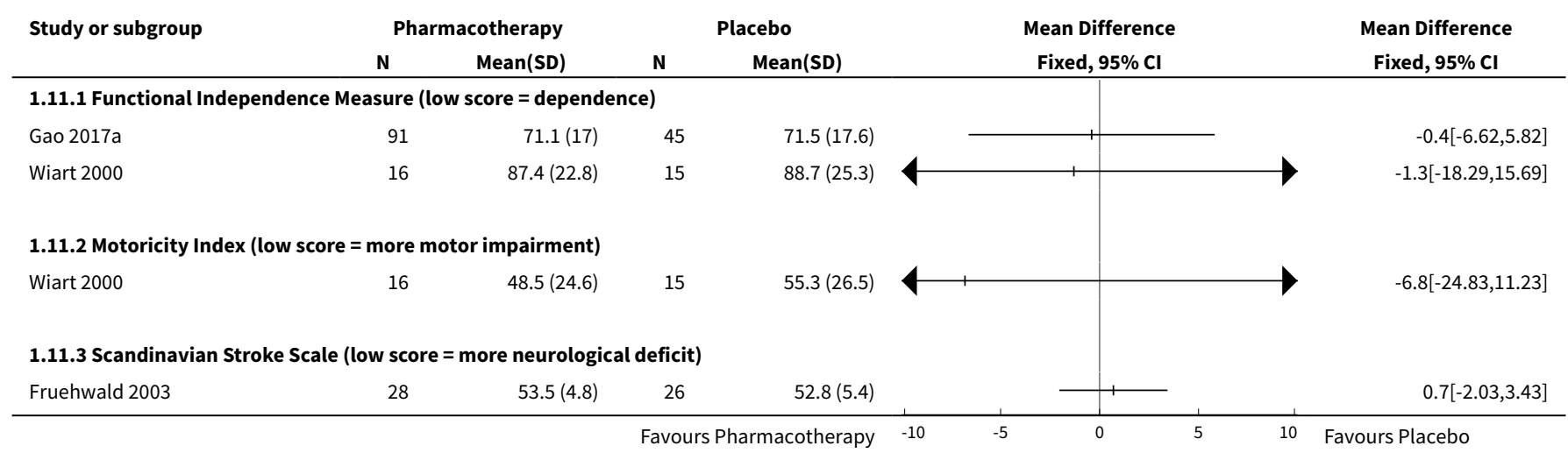

Analysis 1.12. Comparison 1 Pharmacological interventions versus placebo, Outcome 12 Neurological function: average change in scores between baseline and end of treatment.

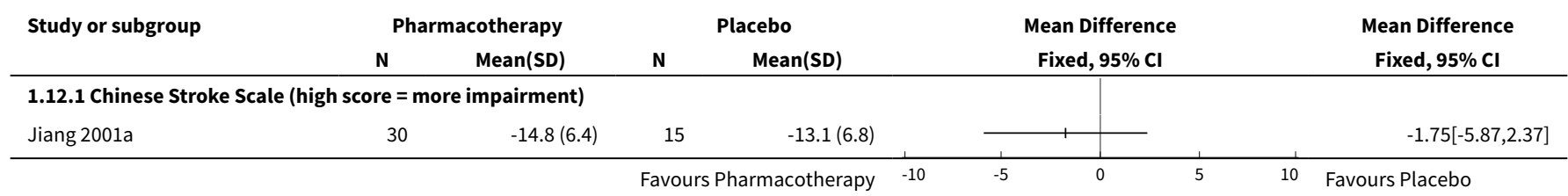

Analysis 1.13. Comparison 1 Pharmacological interventions versus placebo, Outcome 13 Neurological function: mean scores at end of treatment.

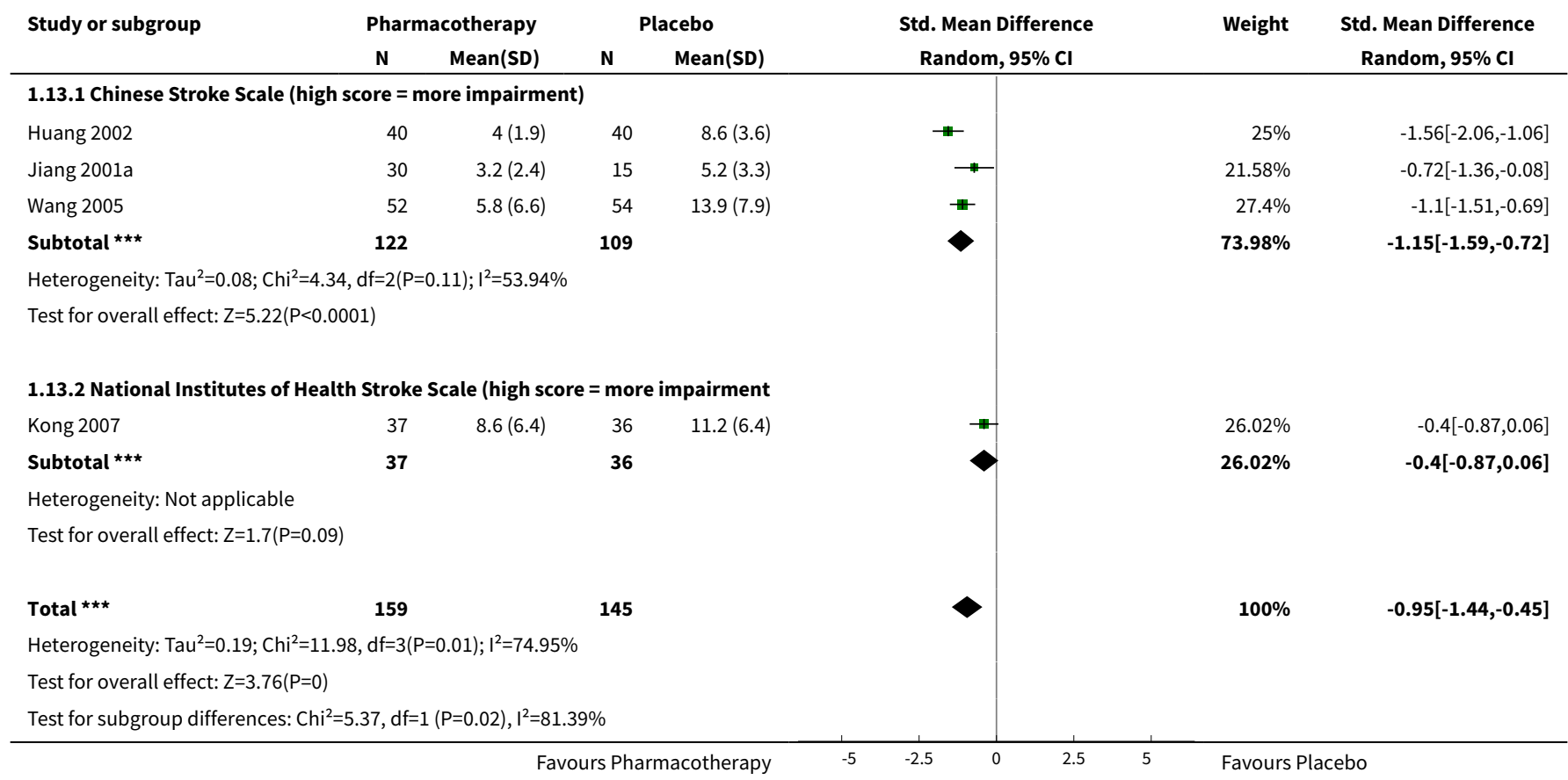


Analysis 1.14. Comparison 1 Pharmacological interventions versus placebo, Outcome 14 Adverse events: death.

\begin{tabular}{|c|c|c|c|c|c|}
\hline Study or subgroup & $\begin{array}{c}\text { Pharma- } \\
\text { cotherapy } \\
n / N\end{array}$ & $\begin{array}{l}\text { Placebo } \\
\mathrm{n} / \mathrm{N} \\
\end{array}$ & $\begin{array}{c}\text { Risk Ratio } \\
\text { M-H, Random, } 95 \% \mathrm{CI}\end{array}$ & Weight & $\begin{array}{c}\text { Risk Ratio } \\
\text { M-H, Random, } 95 \% \mathrm{Cl}\end{array}$ \\
\hline \multicolumn{6}{|c|}{ 1.14.1 At end of treatment } \\
\hline Andersen 1994 & $2 / 33$ & $2 / 33$ & $\longrightarrow$ & $37.81 \%$ & $1[0.15,6.68]$ \\
\hline Fruehwald 2003 & $1 / 28$ & $0 / 26$ & & $13.69 \%$ & $2.79[0.12,65.66]$ \\
\hline Gao 2017a & $1 / 91$ & $1 / 45$ & $\rightarrow$ & $18.06 \%$ & $0.49[0.03,7.73]$ \\
\hline Huang 2002 & $0 / 40$ & $0 / 40$ & & & Not estimable \\
\hline Li 2008 & $0 / 60$ & $0 / 30$ & & & Not estimable \\
\hline Lipsey 1984 & $0 / 17$ & $2 / 22$ & & $15.44 \%$ & $0.26[0.01,5]$ \\
\hline Murray 2002 & $0 / 62$ & $2 / 61$ & 4 & $15 \%$ & $0.2[0.01,4.02]$ \\
\hline Ponzio 2001 & $0 / 112$ & $0 / 117$ & & & Not estimable \\
\hline Wiart 2000 & $0 / 16$ & $0 / 15$ & & & Not estimable \\
\hline Subtotal $(95 \% \mathrm{Cl})$ & 459 & 389 & & $100 \%$ & $0.64[0.2,2.07]$ \\
\hline \multicolumn{6}{|c|}{ Total events: 4 (Pharmacotherapy), 7 (Placebo) } \\
\hline \multicolumn{6}{|c|}{ Heterogeneity: $\operatorname{Tau}^{2}=0 ; \mathrm{Chi}^{2}=2.05, \mathrm{df}=4(\mathrm{P}=0.73) ; I^{2}=0 \%$} \\
\hline \multicolumn{6}{|c|}{ Test for overall effect: $Z=0.74(P=0.46)$} \\
\hline Total $(95 \% \mathrm{Cl})$ & 459 & 389 & & $100 \%$ & $0.64[0.2,2.07]$ \\
\hline \multicolumn{6}{|c|}{ Total events: 4 (Pharmacotherapy), 7 (Placebo) } \\
\hline \multicolumn{6}{|c|}{ Heterogeneity: $\operatorname{Tau}^{2}=0 ; \mathrm{Chi}^{2}=2.05, \mathrm{df}=4(\mathrm{P}=0.73) ; 1^{2}=0 \%$} \\
\hline Test for overall effect & & & & & \\
\hline
\end{tabular}

Analysis 1.15. Comparison 1 Pharmacological interventions versus placebo, Outcome 15 Adverse events: all.

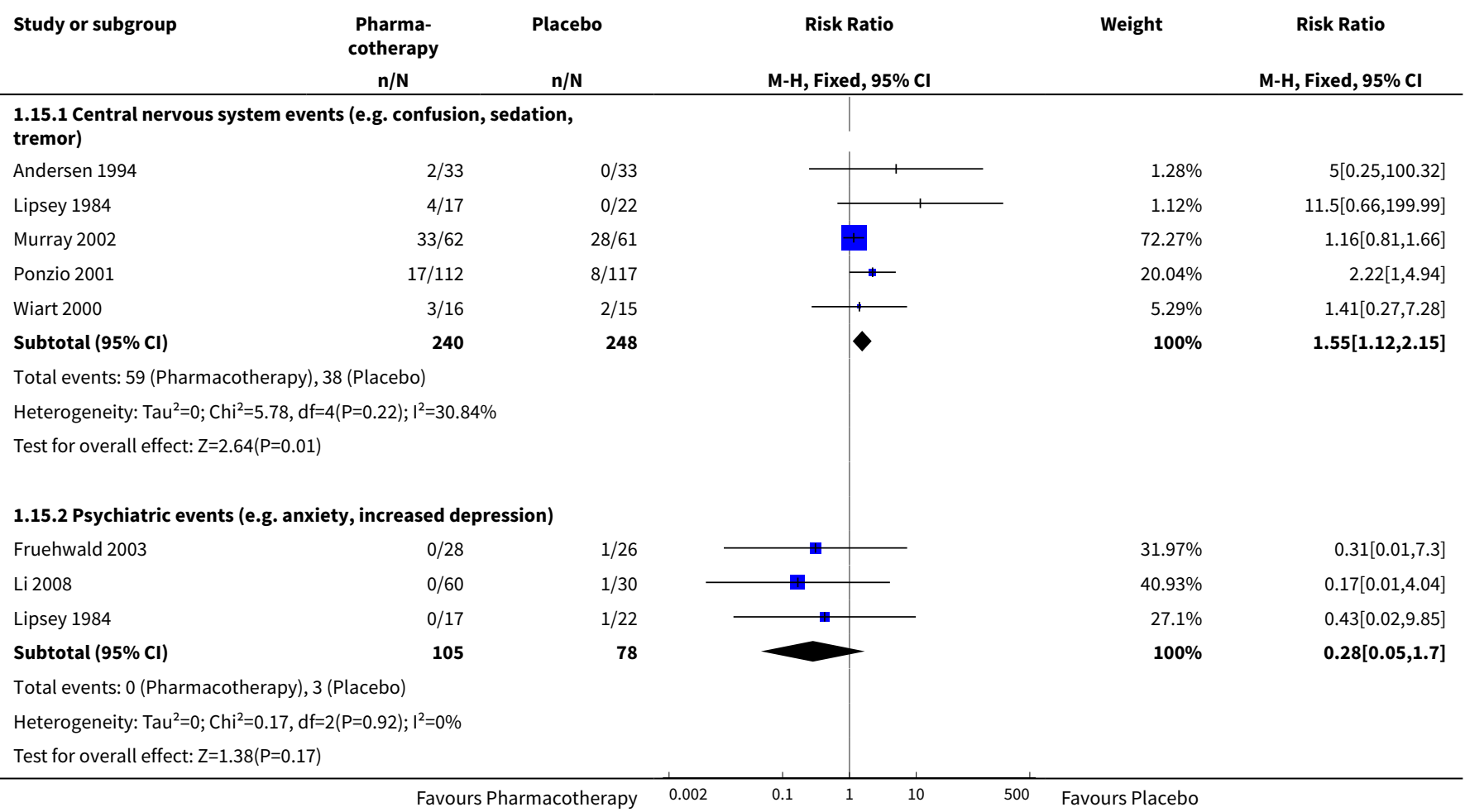




\begin{tabular}{lcccc} 
Study or subgroup & $\begin{array}{c}\text { Pharma- } \\
\text { cotherapy } \\
\mathrm{n} / \mathrm{N}\end{array}$ & Placebo & Risk Ratio & Reight \\
& $\mathrm{n} / \mathrm{N}$ & $\mathrm{M}-\mathrm{H}$, Fixed, $95 \% \mathrm{Cl}$ & $\mathrm{M}-\mathrm{H}, \mathrm{Fixed}, 95 \% \mathrm{Cl}$ \\
\hline
\end{tabular}

\subsubsection{Recurrent stroke}

Andersen 1994

Li 2008

Lipsey 1984

Subtotal $(95 \% \mathrm{CI})$

110

Total events: 3 (Pharmacotherapy), 1 (Placebo)

Heterogeneity: $\mathrm{Tau}^{2}=0 ; \mathrm{Chi}^{2}=0.92, \mathrm{df}=2(\mathrm{P}=0.63) ; \mathrm{I}^{2}=0 \%$

Test for overall effect: $\mathrm{Z}=0.49(\mathrm{P}=0.62)$

1.15.4 Vascular events - not stroke (e.g. dizziness, palpitation)

Andersen 1994

Fruehwald 2003

Jiang 2001a

$1 / 33$

$1 / 28$

$7 / 30$

Lipsey 1984

$2 / 17$

Murray 2002

$22 / 62$

Ponzio 2001

$9 / 112$

Wiart 2000

$0 / 16$

Subtotal $(95 \% \mathrm{Cl})$

298

Total events: 42 (Pharmacotherapy), 27 (Placebo)

Heterogeneity: $\mathrm{Tau}^{2}=0 ; \mathrm{Chi}^{2}=3.29, \mathrm{df}=6(\mathrm{P}=0.77) ; \mathrm{I}^{2}=0 \%$

Test for overall effect: $Z=1.68(P=0.09)$

\subsubsection{Gastrointestinal effects (e.g. constipation, diarrhoea)}

Li 2008

$6 / 60$

Murray 2002

$44 / 62$

$17 / 112$

Ponzio 2001

$1 / 16$

Wiart 2000

250

Subtotal $(95 \% \mathrm{CI})$

Total events: 68 (Pharmacotherapy), 40 (Placebo)

Heterogeneity: $\mathrm{Tau}^{2}=0 ; \mathrm{Chi}^{2}=2.86, \mathrm{df}=3(\mathrm{P}=0.41) ; \mathrm{I}^{2}=0 \%$

Test for overall effect: $Z=3.09(P=0)$

1.15.6 Other events - not listed above (e.g. dysuria, eye discomfort)

$\begin{array}{lrr}\text { Andersen 1994 } & 1 / 33 & 0 / 33 \\ \text { Fruehwald 2003 } & 0 / 28 & 1 / 26 \\ \text { Jiang 2001a } & 2 / 30 & 0 / 15 \\ \text { Li 2008 } & 4 / 60 & 1 / 30 \\ \text { Murray 2002 } & 37 / 62 & 26 / 61 \\ \text { Ponzio 2001 } & 29 / 112 & 26 / 117 \\ \text { Wiart 2000 } & 1 / 16 & 0 / 15 \\ \text { Subtotal (95\% Cl) } & \mathbf{3 4 1} & \mathbf{2 9 7}\end{array}$

Total events: 74 (Pharmacotherapy), 54 (Placebo)

Heterogeneity: $\mathrm{Tau}^{2}=0 ; \mathrm{Chi}^{2}=2.02, \mathrm{df}=6(\mathrm{P}=0.92) ; \mathrm{I}^{2}=0 \%$

Test for overall effect: $Z=1.93(P=0.05)$

1.15.7 Protocol violation (e.g. refused treatment, withdrew consent)

$\begin{array}{lll}\text { Andersen } 1994 & 1 / 33 & 0 / 33 \\ \text { Kong } 2007 & 4 / 48 & 4 / 42 \\ \text { Lipsey } 1984 & 0 / 17 & 3 / 22 \\ \text { Wang } 2005 & 2 / 54 & 0 / 54\end{array}$

$2 / 30$

$27 / 61$

$8 / 117$

$3 / 15$

223
$0 / 33$

$0 / 30$

$1 / 22$

85 


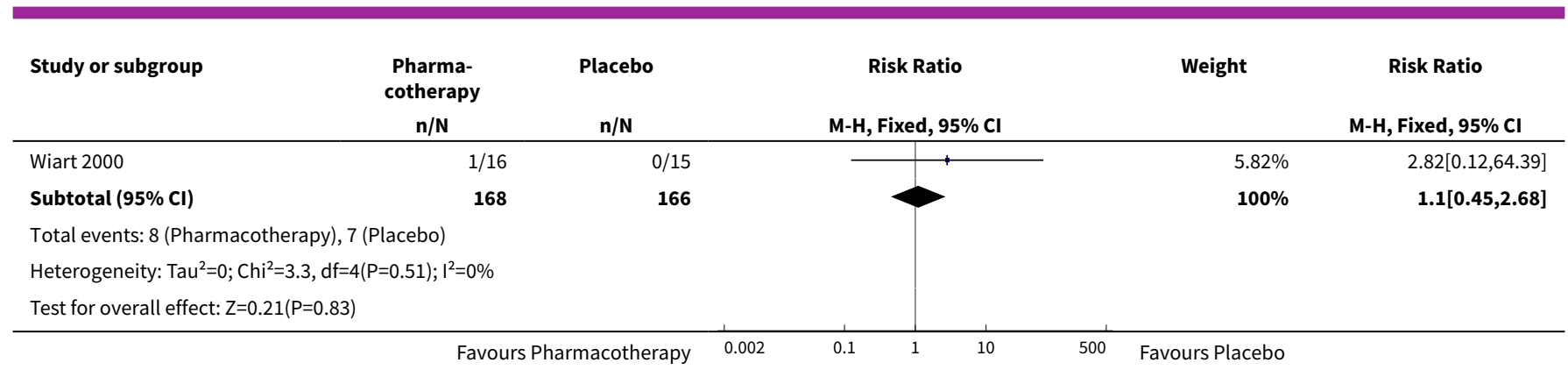

Analysis 1.16. Comparison 1 Pharmacological interventions versus placebo, Outcome 16 Adverse events: leaving the study early (including death).

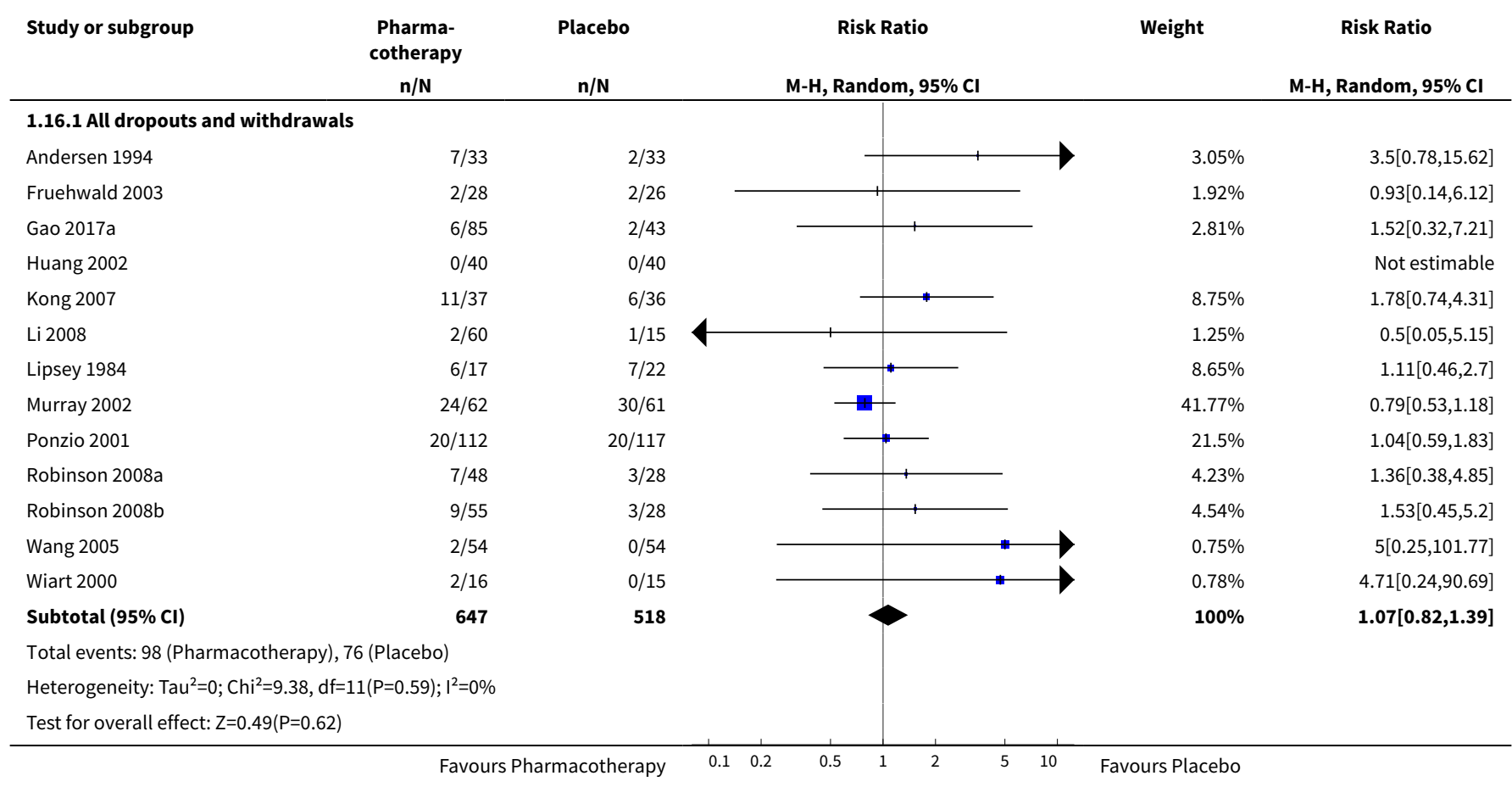

\section{Comparison 2. Non-invasive brain stimulation versus sham non-invasive brain stimulation and/or usual care}

\begin{tabular}{lllll}
\hline Outcome or subgroup title & No. of studies & $\begin{array}{l}\text { No. of partici- } \\
\text { pants }\end{array}$ & Statistical method & Effect size \\
\hline $\begin{array}{l}\text { 1 Depression: meeting study criteria } \\
\text { for depression at end of treatment }\end{array}$ & 0 & 0 & $\begin{array}{l}\text { Risk Ratio (M-H, Random, } \\
95 \% \mathrm{Cl})\end{array}$ & $0.0[0.0,0.0]$ \\
\hline $\begin{array}{l}\text { 2 Depression: }<50 \% \text { reduction in scale } \\
\text { scores at end of treatment }\end{array}$ & 1 & Risk Ratio (M-H, Random, & $\begin{array}{l}\text { Totals not select- } \\
\text { ed }\end{array}$ \\
\hline $\begin{array}{l}2.1 \text { HDRS } \\
\end{array}$ & 1 & & $\begin{array}{l}\text { Risk Ratio (M-H, Random, } \\
95 \% \mathrm{Cl})\end{array}$ & $0.0[0.0,0.0]$ \\
\hline
\end{tabular}




\begin{tabular}{|c|c|c|c|c|}
\hline Outcome or subgroup title & No. of studies & $\begin{array}{l}\text { No. of partici- } \\
\text { pants }\end{array}$ & Statistical method & Effect size \\
\hline $\begin{array}{l}3 \text { Depression: mean scores at end of } \\
\text { treatment }\end{array}$ & 8 & 495 & $\begin{array}{l}\text { Mean Difference (IV, Random, } \\
95 \% \mathrm{CI})\end{array}$ & $\begin{array}{l}-6.63[-9.71 \\
-3.55]\end{array}$ \\
\hline $\begin{array}{l}\text { 3.1 HDRS (high score = more de- } \\
\text { pressed) }\end{array}$ & 8 & 495 & $\begin{array}{l}\text { Mean Difference (IV, Random, } \\
95 \% \mathrm{CI} \text { ) }\end{array}$ & $\begin{array}{l}-6.63[-9.71 \\
-3.55]\end{array}$ \\
\hline $\begin{array}{l}4 \text { Depression: mean scores at end of } \\
\text { follow-up }\end{array}$ & 3 & 170 & $\begin{array}{l}\text { Mean Difference (IV, Random, } \\
95 \% \mathrm{CI})\end{array}$ & $\begin{array}{l}-2.60[-3.33 \\
-1.87]\end{array}$ \\
\hline $\begin{array}{l}\text { 4.1 HDRS (high score = more de- } \\
\text { pressed) }\end{array}$ & 3 & 170 & $\begin{array}{l}\text { Mean Difference (IV, Random, } \\
95 \% \mathrm{CI})\end{array}$ & $\begin{array}{l}-2.60[-3.33 \\
-1.87]\end{array}$ \\
\hline $\begin{array}{l}5 \text { Activities of daily living: mean scores } \\
\text { at end of treatment }\end{array}$ & 2 & 208 & $\begin{array}{l}\text { Std. Mean Difference (IV, Ran- } \\
\text { dom, } 95 \% \mathrm{Cl} \text { ) }\end{array}$ & $1.84[-1.40,5.08]$ \\
\hline $\begin{array}{l}\text { 5.1 Barthel Index (high score = more } \\
\text { dependent) }\end{array}$ & 1 & 108 & $\begin{array}{l}\text { Std. Mean Difference (IV, Ran- } \\
\text { dom, } 95 \% \mathrm{CI} \text { ) }\end{array}$ & $0.20[-0.18,0.57]$ \\
\hline $\begin{array}{l}\text { 5.2 ADL (high score = more impair- } \\
\text { ment) }\end{array}$ & 1 & 100 & $\begin{array}{l}\text { Std. Mean Difference (IV, Ran- } \\
\text { dom, } 95 \% \mathrm{Cl} \text { ) }\end{array}$ & $3.50[2.87,4.13]$ \\
\hline $\begin{array}{l}6 \text { Neurological function: average } \\
\text { change in scores between baseline and } \\
\text { end of treatment }\end{array}$ & 1 & & $\begin{array}{l}\text { Mean Difference (IV, Fixed, } \\
95 \% \mathrm{CI})\end{array}$ & $\begin{array}{l}\text { Totals not select- } \\
\text { ed }\end{array}$ \\
\hline $\begin{array}{l}\text { 6.1 Chinese Stroke Scale (high score = } \\
\text { more impairment) }\end{array}$ & 1 & & $\begin{array}{l}\text { Mean Difference (IV, Fixed, } \\
95 \% \mathrm{CI})\end{array}$ & $0.0[0.0,0.0]$ \\
\hline $\begin{array}{l}7 \text { Neurological function: mean scores } \\
\text { at end of treatment }\end{array}$ & 3 & 290 & $\begin{array}{l}\text { Std. Mean Difference (IV, Ran- } \\
\text { dom, } 95 \% \mathrm{Cl} \text { ) }\end{array}$ & $\begin{array}{l}-2.21[-3.32 \\
-1.09]\end{array}$ \\
\hline $\begin{array}{l}\text { 7.1 Chinese Stroke Scale (high score = } \\
\text { more impairment) }\end{array}$ & 2 & 190 & $\begin{array}{l}\text { Std. Mean Difference (IV, Ran- } \\
\text { dom, } 95 \% \mathrm{Cl} \text { ) }\end{array}$ & $\begin{array}{l}-1.79[-2.94 \\
-0.64]\end{array}$ \\
\hline $\begin{array}{l}7.2 \text { National Institutes of Health Stroke } \\
\text { Scale (high score = more impairment) }\end{array}$ & 1 & 100 & $\begin{array}{l}\text { Std. Mean Difference (IV, Ran- } \\
\text { dom, } 95 \% \mathrm{Cl} \text { ) }\end{array}$ & $\begin{array}{l}-3.04[-3.63 \\
-2.46]\end{array}$ \\
\hline 8 Adverse events: death & 2 & 124 & $\begin{array}{l}\text { Risk Ratio (M-H, Random, } \\
95 \% \mathrm{Cl})\end{array}$ & $0.0[0.0,0.0]$ \\
\hline 8.1 At end of treatment & 2 & 124 & $\begin{array}{l}\text { Risk Ratio (M-H, Random, } \\
95 \% \mathrm{Cl})\end{array}$ & $0.0[0.0,0.0]$ \\
\hline 9 Adverse events: all & 2 & 496 & $\begin{array}{l}\text { Risk Ratio (M-H, Random, } \\
95 \% \mathrm{Cl})\end{array}$ & $0.0[0.0,0.0]$ \\
\hline $\begin{array}{l}\text { 9.1 Central nervous system events (e.g. } \\
\text { confusion, sedation, tremor) }\end{array}$ & 2 & 124 & $\begin{array}{l}\text { Risk Ratio (M-H, Random, } \\
95 \% \mathrm{Cl})\end{array}$ & $0.0[0.0,0.0]$ \\
\hline $\begin{array}{l}9.2 \text { Gastrointestinal effects (e.g. consti- } \\
\text { pation, diarrhoea) }\end{array}$ & 2 & 124 & $\begin{array}{l}\text { Risk Ratio (M-H, Random, } \\
95 \% \mathrm{Cl})\end{array}$ & $0.0[0.0,0.0]$ \\
\hline 9.3 Recurrent stroke & 2 & 124 & $\begin{array}{l}\text { Risk Ratio (M-H, Random, } \\
95 \% \mathrm{Cl})\end{array}$ & $0.0[0.0,0.0]$ \\
\hline
\end{tabular}




\begin{tabular}{lllll}
\hline Outcome or subgroup title & No. of studies & $\begin{array}{l}\text { No. of partici- } \\
\text { pants }\end{array}$ & Statistical method & Effect size \\
\hline $\begin{array}{l}\text { 9.4 Other events - not listed above (e.g. } \\
\text { dysuria, eye discomfort) }\end{array}$ & 2 & 124 & $\begin{array}{l}\text { Risk Ratio (M-H, Random, } \\
95 \% \mathrm{Cl})\end{array}$ & $0.0[0.0,0.0]$ \\
\hline $\begin{array}{l}10 \text { Adverse events: leaving the study } \\
\text { early (including death) }\end{array}$ & 2 & 124 & $\begin{array}{l}\text { Risk Ratio (M-H, Random, } \\
95 \% \mathrm{Cl})\end{array}$ & $0.0[0.0,0.0]$ \\
\hline 10.1 All dropouts and withdrawals & 2 & 124 & $\begin{array}{l}\text { Risk Ratio (M-H, Random, } \\
95 \% \text { Cl) }\end{array}$ & $0.0[0.0,0.0]$ \\
\hline
\end{tabular}

Analysis 2.2. Comparison 2 Non-invasive brain stimulation versus sham non-invasive brain stimulation and/or usual care, Outcome 2 Depression: $<50 \%$ reduction in scale scores at end of treatment.

\begin{tabular}{|c|c|c|c|c|c|c|c|}
\hline $\begin{array}{l}\text { Study or subgroup } \\
2.2 .1 \text { HDRS }\end{array}$ & $\begin{array}{c}\text { Non-invasive stimulation } \\
n / N\end{array}$ & $\begin{array}{c}\text { Sham or usual care } \\
n / N\end{array}$ & \multicolumn{3}{|c|}{$\begin{array}{c}\text { Risk Ratio } \\
\text { M-H, Random, } 95 \% \text { CI }\end{array}$} & & $\begin{array}{c}\text { Risk Ratio } \\
\text { M-H, Random, } 95 \% \text { Cl } \\
\end{array}$ \\
\hline Zheng 2016 & $39 / 41$ & $29 / 41$ & & & + & & $1.34[1.09,1.66]$ \\
\hline
\end{tabular}

Analysis 2.3. Comparison 2 Non-invasive brain stimulation versus sham non-invasive brain stimulation and/or usual care, Outcome 3 Depression: mean scores at end of treatment.

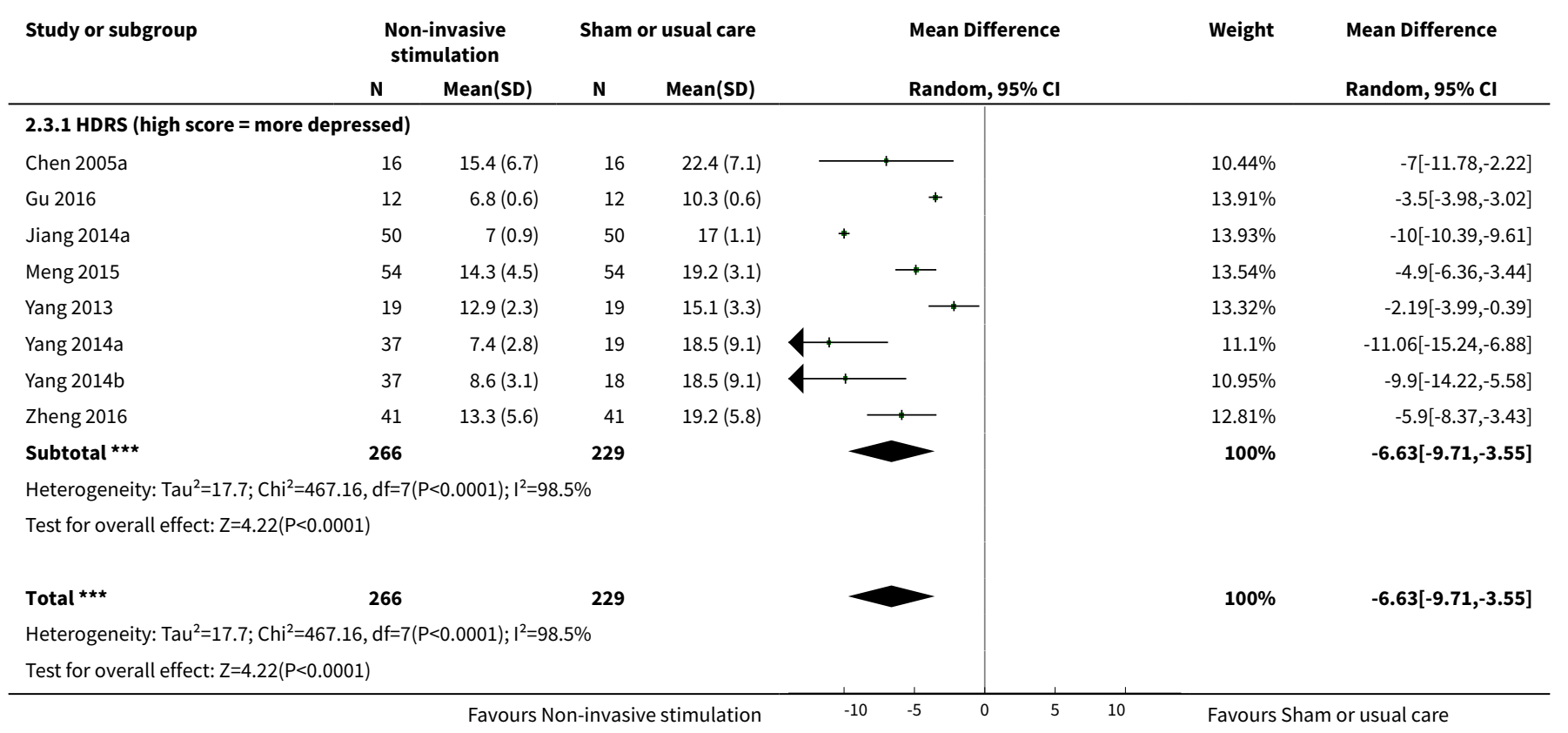


Analysis 2.4. Comparison 2 Non-invasive brain stimulation versus sham non-invasive brain stimulation and/or usual care, Outcome 4 Depression: mean scores at end of follow-up.

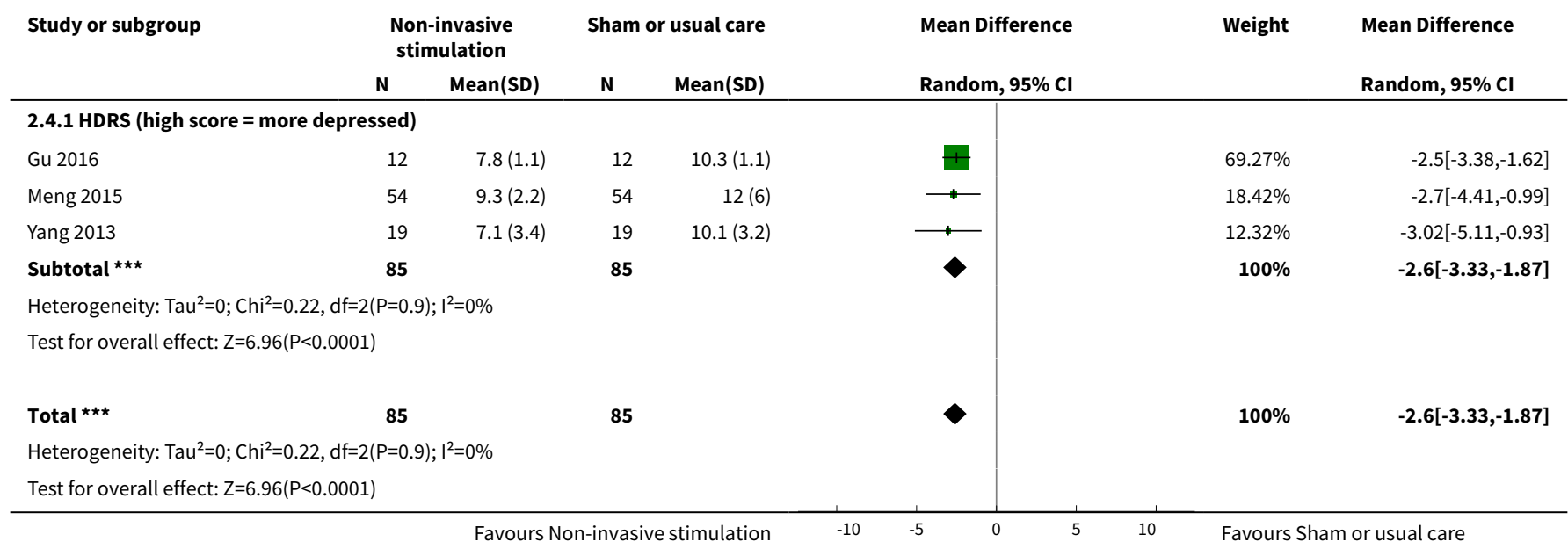

Analysis 2.5. Comparison 2 Non-invasive brain stimulation versus sham non-invasive brain stimulation and/or usual care, Outcome 5 Activities of daily living: mean scores at end of treatment.

\begin{tabular}{|c|c|c|c|c|c|c|c|}
\hline \multirow[t]{2}{*}{ Study or subgroup } & \multicolumn{2}{|c|}{$\begin{array}{l}\text { Non-invasive } \\
\text { stimulation }\end{array}$} & \multicolumn{2}{|c|}{ Sham or usual care } & \multirow{2}{*}{$\begin{array}{l}\text { Std. Mean Difference } \\
\text { Random, } 95 \% \mathrm{Cl}\end{array}$} & \multirow[t]{2}{*}{ Weight } & \multirow{2}{*}{$\begin{array}{l}\text { Std. Mean Difference } \\
\text { Random, } 95 \% \mathrm{Cl}\end{array}$} \\
\hline & $\mathbf{N}$ & Mean(SD) & $\mathbf{N}$ & Mean(SD) & & & \\
\hline \multicolumn{8}{|c|}{ 2.5.1 Barthel Index (high score = more dependent) } \\
\hline Meng 2015 & 54 & $76.8(20.4)$ & 54 & $72.9(19.3)$ & \# & $50.3 \%$ & $0.2[-0.18,0.57]$ \\
\hline 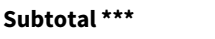 & 54 & & 54 & & & $50.3 \%$ & $0.2[-0.18,0.57]$ \\
\hline \multicolumn{8}{|c|}{ Heterogeneity: Not applicable } \\
\hline \multicolumn{8}{|c|}{ 2.5.2 ADL (high score = more impairment) } \\
\hline Jiang 2014a & 50 & $78(5.1)$ & 50 & $60(5.1)$ & \# & $49.7 \%$ & $3.5[2.87,4.13]$ \\
\hline Subtotal ${ }^{\star \star \star}$ & 50 & & 50 & & & $49.7 \%$ & $3.5[2.87,4.13]$ \\
\hline \multicolumn{8}{|c|}{ Heterogeneity: Not applicable } \\
\hline \multicolumn{8}{|c|}{ Test for overall effect: $Z=10.87(P<0.0001)$} \\
\hline 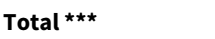 & 104 & & 104 & & & $100 \%$ & $1.84[-1.4,5.08]$ \\
\hline \multicolumn{8}{|c|}{ Heterogeneity: $\mathrm{Tau}^{2}=5.4 ; \mathrm{Chi}^{2}=77.54, \mathrm{df}=1(\mathrm{P}<0.0001) ; \mathrm{I}^{2}=98.71 \%$} \\
\hline \multicolumn{8}{|c|}{ Test for overall effect: $\mathrm{Z}=1.11(\mathrm{P}=0.27)$} \\
\hline Test for subgroup dif & $.54, \mathrm{df}=$ & $(P<0.0001), I^{2}$ & $98.71 \%$ & & & & \\
\hline
\end{tabular}

Analysis 2.6. Comparison 2 Non-invasive brain stimulation versus sham non-invasive brain stimulation and/or usual care, Outcome 6 Neurological function: average change in scores between baseline and end of treatment.

\begin{tabular}{|c|c|c|c|c|c|c|c|c|}
\hline \multirow[t]{2}{*}{ Study or subgroup } & \multicolumn{2}{|c|}{ Non-invasive stimulation } & \multicolumn{2}{|c|}{ Sham or usual care } & \multirow{2}{*}{\multicolumn{2}{|c|}{$\begin{array}{c}\text { Mean Difference } \\
\text { Fixed, } 95 \% \mathrm{Cl}\end{array}$}} & & \multirow{2}{*}{$\begin{array}{c}\text { Mean Difference } \\
\text { Fixed, } 95 \% \mathrm{Cl}\end{array}$} \\
\hline & $\mathbf{N}$ & Mean(SD) & $\mathbf{N}$ & $\operatorname{Mean}(S D)$ & & & & \\
\hline \multicolumn{9}{|c|}{ 2.6.1 Chinese Stroke Scale (high score = more impairment) } \\
\hline \multirow[t]{2}{*}{ Meng 2015} & 54 & $9.3(2.8)$ & 54 & $12.2(1.8)$ & + & & & $-2.9[-3.79,-2.01]$ \\
\hline & & & No & ive stimulation & -5 & 0 & 10 & $\begin{array}{l}\text { Favours Sham or usual } \\
\text { care }\end{array}$ \\
\hline
\end{tabular}


Analysis 2.7. Comparison 2 Non-invasive brain stimulation versus sham non-invasive brain stimulation and/or usual care, Outcome 7 Neurological function: mean scores at end of treatment.

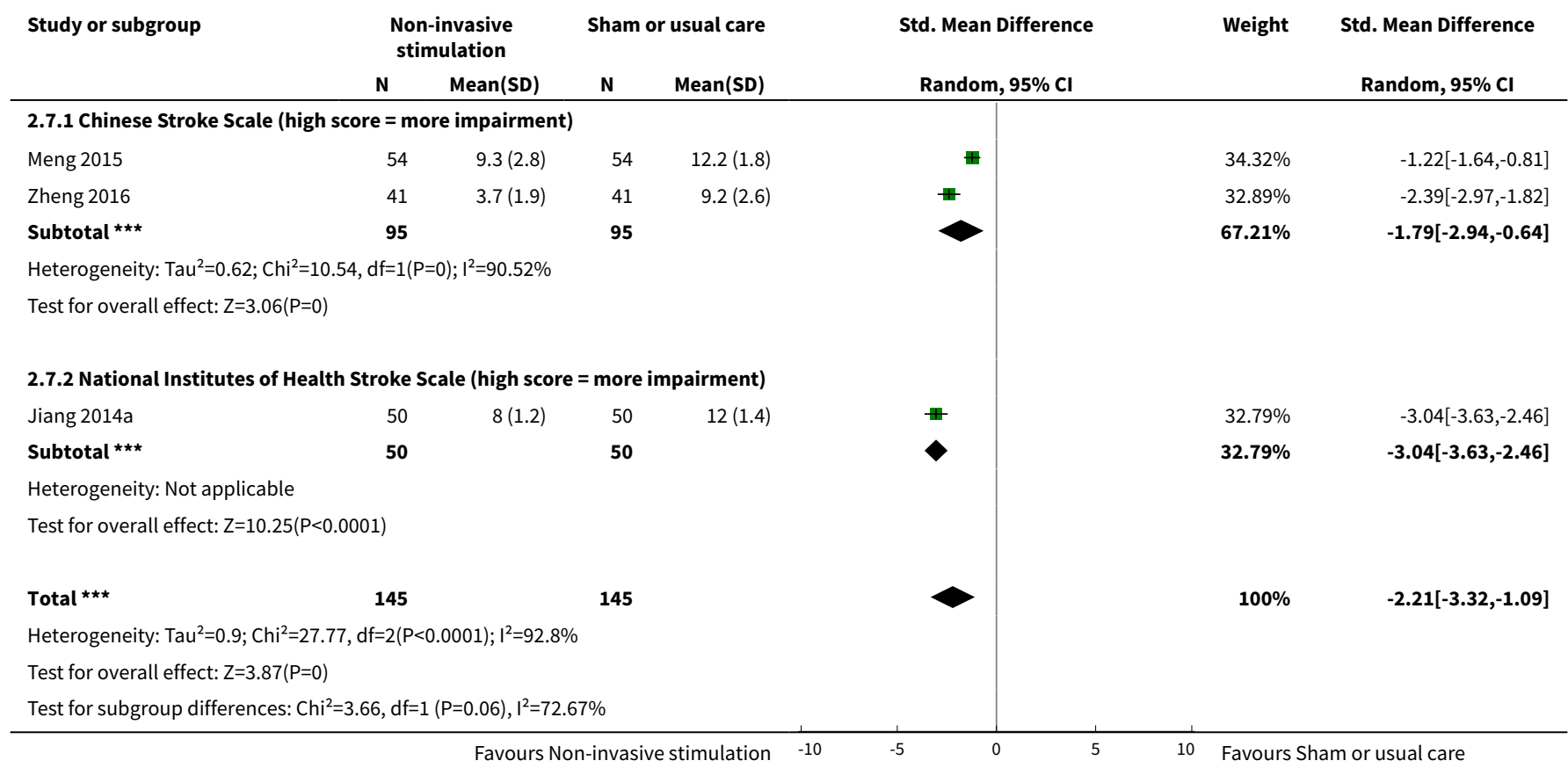

Analysis 2.8. Comparison 2 Non-invasive brain stimulation versus sham noninvasive brain stimulation and/or usual care, Outcome 8 Adverse events: death.

\begin{tabular}{ccccc} 
Study or subgroup & $\begin{array}{c}\text { Non-invasive } \\
\text { stimulation } \\
n / N\end{array}$ & $\begin{array}{c}\text { Sham or } \\
\text { usual care } \\
n / N\end{array}$ & Risk Ratio & Risk Ratio \\
\hline
\end{tabular}

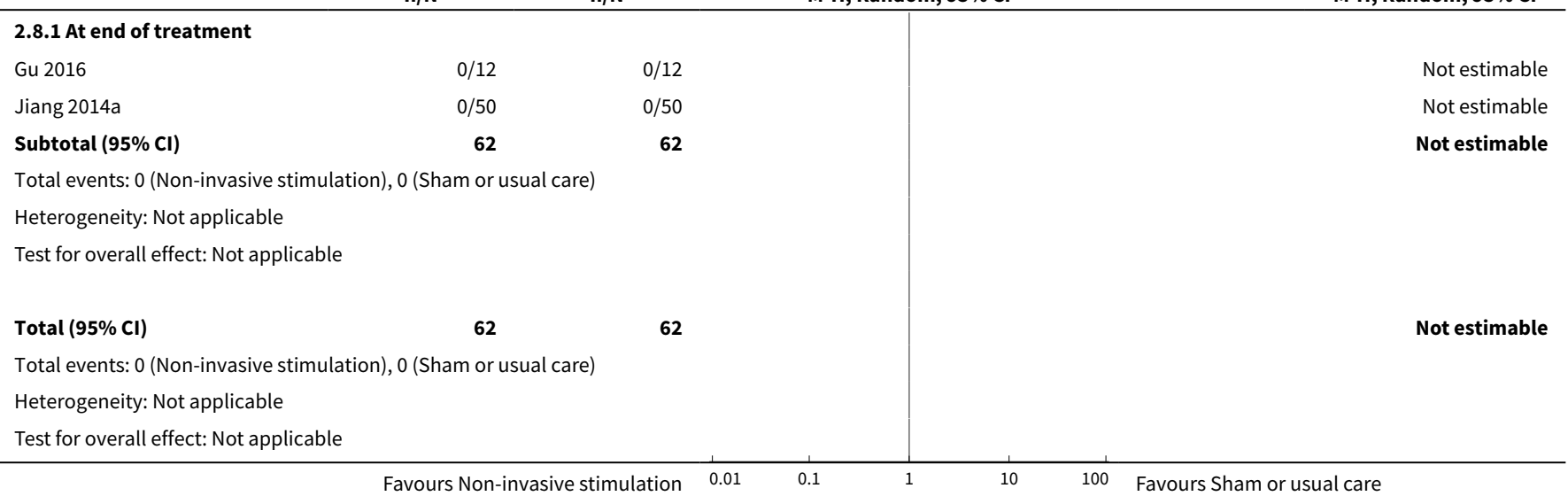


Analysis 2.9. Comparison 2 Non-invasive brain stimulation versus sham noninvasive brain stimulation and/or usual care, Outcome 9 Adverse events: all.

\begin{tabular}{ccccc} 
Study or subgroup & $\begin{array}{c}\text { Non-invasive } \\
\text { stimulation } \\
\mathrm{n} / \mathrm{N}\end{array}$ & $\begin{array}{c}\text { Sham or } \\
\text { usual care } \\
\mathrm{n} / \mathrm{N}\end{array}$ & Risk Ratio & Wisk Ratio \\
\hline
\end{tabular}

$\begin{array}{lcr}\text { 2.9.1 Central nervous system events (e.g. confusion, sedation, tremor) } \\ \text { Gu } 2016 & 0 / 12 & 0 / 12 \\ \text { Jiang 2014a } & 0 / 50 & 0 / 50 \\ \text { Subtotal }(\mathbf{9 5 \%} \mathbf{C l}) & \mathbf{6 2} & \mathbf{6 2}\end{array}$

Total events: 0 (Non-invasive stimulation), 0 (Sham or usual care)

Heterogeneity: Not applicable

Test for overall effect: Not applicable

2.9.2 Gastrointestinal effects (e.g. constipation, diarrhoea)

Jiang 2014a $\quad 0 / 50$

Subtotal $(95 \% \mathrm{Cl})$

Total events: 0 (Non-invasive stimulation), 0 (Sham or usual care)

Test for overall effect: Not applicable

\subsubsection{Recurrent stroke}

$\begin{array}{lrr}\text { Gu } 2016 & 0 / 12 & 0 / 12 \\ \text { Jiang 2014a } & 0 / 50 & 0 / 50 \\ \text { Subtotal (95\% CI) } & \mathbf{6 2} & \mathbf{6 2}\end{array}$

Total events: 0 (Non-invasive stimulation), 0 (Sham or usual care)

Test for overall effect: Not applicable

2.9.4 Other events - not listed above (e.g. dysuria, eye discomfort)

Gu 2016

Total events: 0 (Non-invasive stimulation), 0 (Sham or usual care)

Heterogeneity: Not applicable

Test for overall effect: Not applicable

Total events: 0 (Non-invasive stimulation), 0 (Sham or usual care)

Heterogeneity: Not applicable

Test for overall effect: Not applicable

Test for subgroup differences: Not applicable

$\begin{array}{lllllll}\text { Favours Non-invasive stimulation } & 0.01 & 0.1 & 1 & 10 & 100 & \text { Favours Sham or usual care }\end{array}$

Analysis 2.10. Comparison 2 Non-invasive brain stimulation versus sham non-invasive brain stimulation and/or usual care, Outcome 10 Adverse events: leaving the study early (including death).

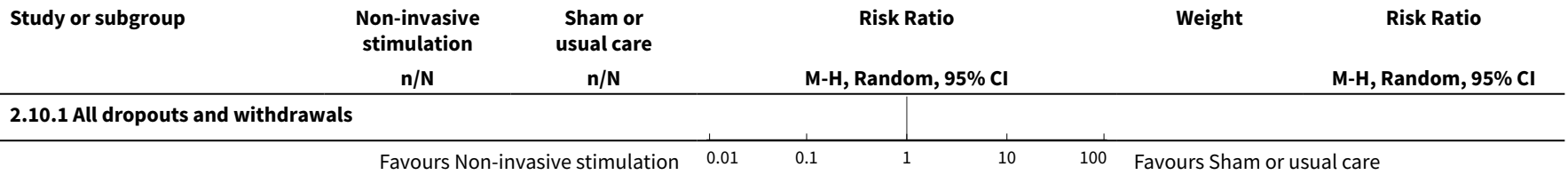




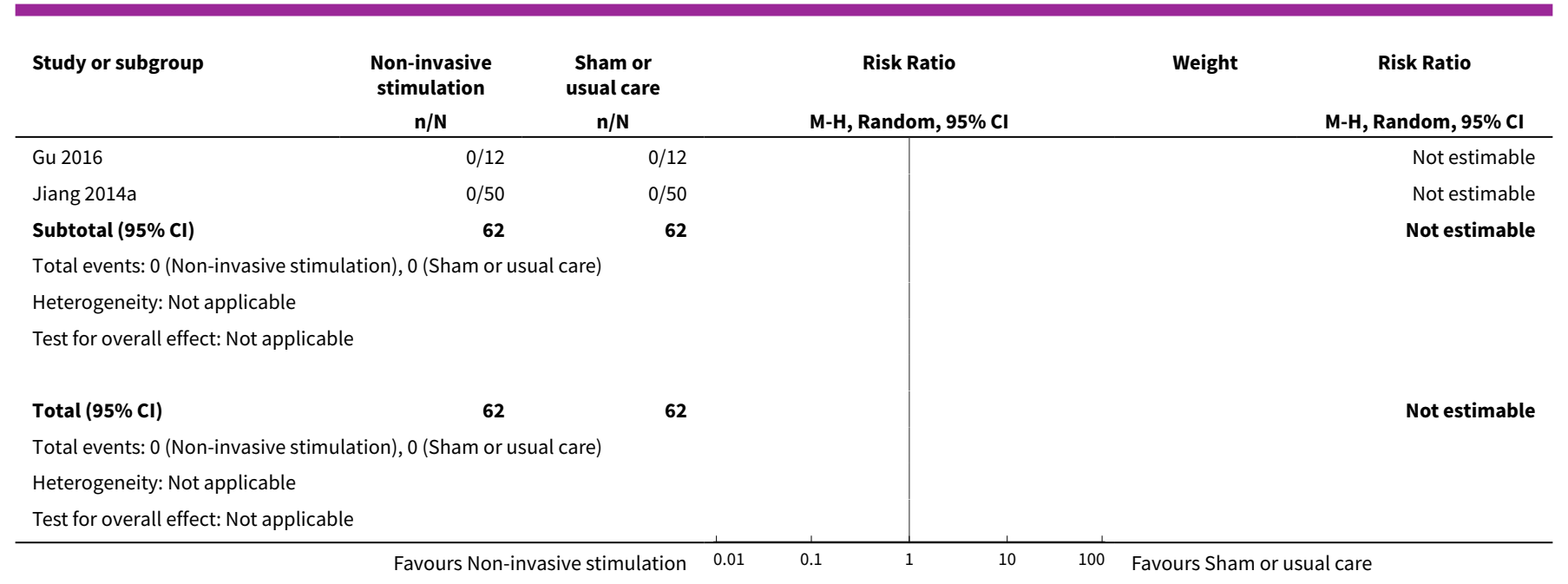

\section{Comparison 3. Psychological therapy versus usual care and/or attention control}

\begin{tabular}{|c|c|c|c|c|}
\hline Outcome or subgroup title & No. of studies & $\begin{array}{l}\text { No. of partici- } \\
\text { pants }\end{array}$ & Statistical method & Effect size \\
\hline $\begin{array}{l}1 \text { Depression: meeting study criteria } \\
\text { for depression at end of treatment }\end{array}$ & 6 & 521 & $\begin{array}{l}\text { Risk Ratio (M-H, Random, } \\
95 \% \mathrm{Cl})\end{array}$ & $0.77[0.62,0.95]$ \\
\hline $\begin{array}{l}1.1 \mathrm{GHQ}-28 \text { (high score = greater psy- } \\
\text { chological distress) }\end{array}$ & 1 & 254 & $\begin{array}{l}\text { Risk Ratio (M-H, Random, } \\
95 \% \mathrm{Cl})\end{array}$ & $0.89[0.76,1.05]$ \\
\hline $\begin{array}{l}1.2 \text { HDRS (high score = more de- } \\
\text { pressed) }\end{array}$ & 4 & 225 & $\begin{array}{l}\text { Risk Ratio (M-H, Random, } \\
95 \% \mathrm{Cl})\end{array}$ & $0.69[0.54,0.88]$ \\
\hline $\begin{array}{l}1.3 \text { HADS (high score = more de- } \\
\text { pressed) }\end{array}$ & 1 & 42 & $\begin{array}{l}\text { Risk Ratio (M-H, Random, } \\
95 \% \mathrm{Cl})\end{array}$ & $1.65[0.16,16.85]$ \\
\hline $\begin{array}{l}2 \text { Depression: }<50 \% \text { reduction in scale } \\
\text { scores at end of treatment }\end{array}$ & 0 & 0 & $\begin{array}{l}\text { Risk Ratio (M-H, Random, } \\
95 \% \mathrm{Cl})\end{array}$ & $0.0[0.0,0.0]$ \\
\hline $\begin{array}{l}3 \text { Depression: average change in scores } \\
\text { between baseline and end of treat- } \\
\text { ment }\end{array}$ & 3 & 189 & $\begin{array}{l}\text { Mean Difference (IV, Random, } \\
95 \% \mathrm{CI} \text { ) }\end{array}$ & $\begin{array}{l}-6.20[-8.24 \\
-4.16]\end{array}$ \\
\hline $\begin{array}{l}\text { 3.1 HDRS (high score = more de- } \\
\text { pressed) }\end{array}$ & 3 & 189 & $\begin{array}{l}\text { Mean Difference (IV, Random, } \\
95 \% \mathrm{CI} \text { ) }\end{array}$ & $\begin{array}{l}-6.20[-8.24 \\
-4.16]\end{array}$ \\
\hline $\begin{array}{l}4 \text { Depression: mean scores at end of } \\
\text { treatment }\end{array}$ & 10 & & $\begin{array}{l}\text { Std. Mean Difference (IV, Ran- } \\
\text { dom, } 95 \% \mathrm{CI} \text { ) }\end{array}$ & $\begin{array}{l}\text { Totals not select- } \\
\text { ed }\end{array}$ \\
\hline 4.1 BDI (high score = more depressed) & 1 & & $\begin{array}{l}\text { Std. Mean Difference (IV, Ran- } \\
\text { dom, } 95 \% \mathrm{Cl} \text { ) }\end{array}$ & $0.0[0.0,0.0]$ \\
\hline 4.2 WDI (high score $=$ more depressed) & 1 & & $\begin{array}{l}\text { Std. Mean Difference (IV, Ran- } \\
\text { dom, } 95 \% \mathrm{CI} \text { ) }\end{array}$ & $0.0[0.0,0.0]$ \\
\hline $\begin{array}{l}4.3 \text { HDRS (high score = more de- } \\
\text { pressed) }\end{array}$ & 5 & & $\begin{array}{l}\text { Std. Mean Difference (IV, Ran- } \\
\text { dom, } 95 \% \mathrm{Cl} \text { ) }\end{array}$ & $0.0[0.0,0.0]$ \\
\hline
\end{tabular}




\begin{tabular}{|c|c|c|c|c|}
\hline Outcome or subgroup title & No. of studies & $\begin{array}{l}\text { No. of partici- } \\
\text { pants }\end{array}$ & Statistical method & Effect size \\
\hline $\begin{array}{l}\text { 4.4 SAD-Q 21-item (high score = more } \\
\text { depressed) }\end{array}$ & 1 & & $\begin{array}{l}\text { Std. Mean Difference (IV, Ran- } \\
\text { dom, } 95 \% \mathrm{Cl} \text { ) }\end{array}$ & $0.0[0.0,0.0]$ \\
\hline $\begin{array}{l}\text { 4.5 Zung SDS (high score = more de- } \\
\text { pressed) }\end{array}$ & 1 & & $\begin{array}{l}\text { Std. Mean Difference (IV, Ran- } \\
\text { dom, } 95 \% \mathrm{Cl} \text { ) }\end{array}$ & $0.0[0.0,0.0]$ \\
\hline $\begin{array}{l}\text { 4.6 MADRS (high score= more de- } \\
\text { pressed }\end{array}$ & 1 & & $\begin{array}{l}\text { Std. Mean Difference (IV, Ran- } \\
\text { dom, } 95 \% \mathrm{Cl} \text { ) }\end{array}$ & $0.0[0.0,0.0]$ \\
\hline $\begin{array}{l}\text { 4.7 HADS (high score = more de- } \\
\text { pressed) }\end{array}$ & 1 & & $\begin{array}{l}\text { Std. Mean Difference (IV, Ran- } \\
\text { dom, } 95 \% \mathrm{CI} \text { ) }\end{array}$ & $0.0[0.0,0.0]$ \\
\hline $\begin{array}{l}4.8 \text { Visual Analog Mood Scale (high } \\
\text { score = more depressed) }\end{array}$ & 1 & & $\begin{array}{l}\text { Std. Mean Difference (IV, Ran- } \\
\text { dom, } 95 \% \mathrm{Cl} \text { ) }\end{array}$ & $0.0[0.0,0.0]$ \\
\hline 4.9 AHI (high score = more depressed & 1 & & $\begin{array}{l}\text { Std. Mean Difference (IV, Ran- } \\
\text { dom, } 95 \% \mathrm{Cl} \text { ) }\end{array}$ & $0.0[0.0,0.0]$ \\
\hline $\begin{array}{l}4.10 \text { DASS- } 21 \text { (high score = more de- } \\
\text { pressed) }\end{array}$ & 1 & & $\begin{array}{l}\text { Std. Mean Difference (IV, Ran- } \\
\text { dom, } 95 \% \mathrm{CI} \text { ) }\end{array}$ & $0.0[0.0,0.0]$ \\
\hline $\begin{array}{l}5 \text { Depression: meeting study criteria } \\
\text { for depression at end of follow-up }\end{array}$ & 3 & 201 & $\begin{array}{l}\text { Risk Ratio (M-H, Random, } \\
95 \% \mathrm{Cl})\end{array}$ & $0.85[0.59,1.21]$ \\
\hline $\begin{array}{l}5.1 \text { HDRS (high score = more de- } \\
\text { pressed) }\end{array}$ & 3 & 201 & $\begin{array}{l}\text { Risk Ratio (M-H, Random, } \\
95 \% \mathrm{Cl})\end{array}$ & $0.85[0.59,1.21]$ \\
\hline $\begin{array}{l}6 \text { Depression: average change in scores } \\
\text { between baseline and end of follow-up }\end{array}$ & 1 & & $\begin{array}{l}\text { Mean Difference (IV, Fixed, } \\
95 \% \mathrm{Cl} \text { ) }\end{array}$ & $\begin{array}{l}\text { Totals not select- } \\
\text { ed }\end{array}$ \\
\hline $\begin{array}{l}6.1 \text { HDRS (high score = more de- } \\
\text { pressed) }\end{array}$ & 1 & & $\begin{array}{l}\text { Mean Difference (IV, Fixed, } \\
95 \% \mathrm{CI} \text { ) }\end{array}$ & $0.0[0.0,0.0]$ \\
\hline $\begin{array}{l}7 \text { Depression: mean scores at end of } \\
\text { follow-up }\end{array}$ & 5 & & $\begin{array}{l}\text { Std. Mean Difference (IV, Ran- } \\
\text { dom, } 95 \% \mathrm{Cl} \text { ) }\end{array}$ & $\begin{array}{l}\text { Totals not select- } \\
\text { ed }\end{array}$ \\
\hline $7.1 \mathrm{BDI}$ (high score = more depressed) & 1 & & $\begin{array}{l}\text { Std. Mean Difference (IV, Ran- } \\
\text { dom, } 95 \% \mathrm{Cl} \text { ) }\end{array}$ & $0.0[0.0,0.0]$ \\
\hline 7.2 WDI (high score = more depressed) & 1 & & $\begin{array}{l}\text { Std. Mean Difference (IV, Ran- } \\
\text { dom, } 95 \% \mathrm{Cl} \text { ) }\end{array}$ & $0.0[0.0,0.0]$ \\
\hline $\begin{array}{l}\text { 7.3 SAD-Q 21-item (high score = more } \\
\text { depressed) }\end{array}$ & 1 & & $\begin{array}{l}\text { Std. Mean Difference (IV, Ran- } \\
\text { dom, } 95 \% \mathrm{Cl} \text { ) }\end{array}$ & $0.0[0.0,0.0]$ \\
\hline $\begin{array}{l}\text { 7.4 HDRS (high score = more de- } \\
\text { pressed) }\end{array}$ & 1 & & $\begin{array}{l}\text { Std. Mean Difference (IV, Ran- } \\
\text { dom, } 95 \% \mathrm{Cl} \text { ) }\end{array}$ & $0.0[0.0,0.0]$ \\
\hline $\begin{array}{l}7.5 \text { HADS (high score = more de- } \\
\text { pressed) }\end{array}$ & 2 & & $\begin{array}{l}\text { Std. Mean Difference (IV, Ran- } \\
\text { dom, } 95 \% \mathrm{Cl} \text { ) }\end{array}$ & $0.0[0.0,0.0]$ \\
\hline $\begin{array}{l}7.6 \text { MADRS (high score = more de- } \\
\text { pressed) }\end{array}$ & 1 & & $\begin{array}{l}\text { Std. Mean Difference (IV, Ran- } \\
\text { dom, } 95 \% \mathrm{Cl} \text { ) }\end{array}$ & $0.0[0.0,0.0]$ \\
\hline
\end{tabular}




\begin{tabular}{|c|c|c|c|c|}
\hline Outcome or subgroup title & No. of studies & $\begin{array}{l}\text { No. of partici- } \\
\text { pants }\end{array}$ & Statistical method & Effect size \\
\hline $\begin{array}{l}7.7 \text { VAMS (high score = more de- } \\
\text { pressed) }\end{array}$ & 1 & & $\begin{array}{l}\text { Std. Mean Difference (IV, Ran- } \\
\text { dom, } 95 \% \mathrm{Cl} \text { ) }\end{array}$ & $0.0[0.0,0.0]$ \\
\hline $\begin{array}{l}\text { 7.8 PHQ-9 (high score = more de- } \\
\text { pressed) }\end{array}$ & 1 & & $\begin{array}{l}\text { Std. Mean Difference (IV, Ran- } \\
\text { dom, } 95 \% \mathrm{Cl} \text { ) }\end{array}$ & $0.0[0.0,0.0]$ \\
\hline $\begin{array}{l}8 \text { Psychological distress: average } \\
\text { change in scores between baseline and } \\
\text { end of treatment }\end{array}$ & 2 & 377 & $\begin{array}{l}\text { Mean Difference (IV, Random, } \\
95 \% \mathrm{CI})\end{array}$ & $-0.21[-1.89,1.48]$ \\
\hline $\begin{array}{l}8.1 \mathrm{GHQ}-28 \text { (high score = greater psy- } \\
\text { chological distress) }\end{array}$ & 2 & 377 & $\begin{array}{l}\text { Mean Difference (IV, Random, } \\
95 \% \mathrm{CI} \text { ) }\end{array}$ & $-0.21[-1.89,1.48]$ \\
\hline $\begin{array}{l}9 \text { Psychological distress: mean scores } \\
\text { at end of treatment }\end{array}$ & 2 & 377 & $\begin{array}{l}\text { Mean Difference (IV, Random, } \\
95 \% \mathrm{CI} \text { ) }\end{array}$ & $-0.43[-2.17,1.31]$ \\
\hline $\begin{array}{l}\text { 9.1 GHQ-28 (high score = greater psy- } \\
\text { chological distress) }\end{array}$ & 2 & 377 & $\begin{array}{l}\text { Mean Difference (IV, Random, } \\
95 \% \mathrm{CI} \text { ) }\end{array}$ & $-0.43[-2.17,1.31]$ \\
\hline $\begin{array}{l}10 \text { Anxiety: meeting study criteria for } \\
\text { anxiety at end of treatment }\end{array}$ & 1 & & $\begin{array}{l}\text { Risk Ratio (M-H, Fixed, 95\% } \\
\mathrm{Cl} \text { ) }\end{array}$ & $\begin{array}{l}\text { Totals not select- } \\
\text { ed }\end{array}$ \\
\hline $\begin{array}{l}\text { 10.1 HADS Anxiety (high score = more } \\
\text { anxious) }\end{array}$ & 1 & & $\begin{array}{l}\text { Risk Ratio (M-H, Fixed, 95\% } \\
\mathrm{Cl} \text { ) }\end{array}$ & $0.0[0.0,0.0]$ \\
\hline $\begin{array}{l}11 \text { Anxiety: mean scores at end of } \\
\text { treatment }\end{array}$ & 2 & & $\begin{array}{l}\text { Std. Mean Difference (IV, Ran- } \\
\text { dom, } 95 \% \mathrm{Cl} \text { ) }\end{array}$ & $\begin{array}{l}\text { Totals not select- } \\
\text { ed }\end{array}$ \\
\hline $\begin{array}{l}11.1 \text { HADS Anxiety (high score = more } \\
\text { anxious) }\end{array}$ & 2 & & $\begin{array}{l}\text { Std. Mean Difference (IV, Ran- } \\
\text { dom, } 95 \% \mathrm{Cl} \text { ) }\end{array}$ & $0.0[0.0,0.0]$ \\
\hline $\begin{array}{l}\text { 11.2 State Trait Anxiety Inventory- Trait } \\
\text { (high score = more anxious) }\end{array}$ & 1 & & $\begin{array}{l}\text { Std. Mean Difference (IV, Ran- } \\
\text { dom, } 95 \% \mathrm{Cl} \text { ) }\end{array}$ & $0.0[0.0,0.0]$ \\
\hline $\begin{array}{l}\text { 11.3 State Trait Anxiety Inventory- } \\
\text { State (high score = more anxious) }\end{array}$ & 1 & & $\begin{array}{l}\text { Std. Mean Difference (IV, Ran- } \\
\text { dom, } 95 \% \mathrm{CI} \text { ) }\end{array}$ & $0.0[0.0,0.0]$ \\
\hline $\begin{array}{l}12 \text { Anxiety: mean scores at end of fol- } \\
\text { low-up }\end{array}$ & 1 & & $\begin{array}{l}\text { Std. Mean Difference (IV, Ran- } \\
\text { dom, } 95 \% \mathrm{Cl} \text { ) }\end{array}$ & $\begin{array}{l}\text { Totals not select- } \\
\text { ed }\end{array}$ \\
\hline $\begin{array}{l}\text { 12.1 State Trait Anxiety Inventory - Trait } \\
\text { (high score = more anxious) }\end{array}$ & 1 & & $\begin{array}{l}\text { Std. Mean Difference (IV, Ran- } \\
\text { dom, } 95 \% \mathrm{Cl} \text { ) }\end{array}$ & $0.0[0.0,0.0]$ \\
\hline $\begin{array}{l}\text { 12.2 State Trait Anxiety Inventory - } \\
\text { State (high score = more anxious) }\end{array}$ & 1 & & $\begin{array}{l}\text { Std. Mean Difference (IV, Ran- } \\
\text { dom, } 95 \% \mathrm{CI} \text { ) }\end{array}$ & $0.0[0.0,0.0]$ \\
\hline $\begin{array}{l}13 \text { Activities of daily living: average } \\
\text { change in scores from baseline to end } \\
\text { of treatment }\end{array}$ & 2 & 377 & $\begin{array}{l}\text { Std. Mean Difference (IV, Ran- } \\
\text { dom, } 95 \% \mathrm{Cl} \text { ) }\end{array}$ & $-0.03[-0.24,0.18]$ \\
\hline $\begin{array}{l}\text { 13.1 Nottingham EADL (high score = } \\
\text { more independent) }\end{array}$ & 1 & 123 & $\begin{array}{l}\text { Std. Mean Difference (IV, Ran- } \\
\text { dom, } 95 \% \mathrm{Cl} \text { ) }\end{array}$ & $-0.10[-0.48,0.28]$ \\
\hline $\begin{array}{l}\text { 13.2 Barthel Index (high score }=\text { more } \\
\text { dependent) }\end{array}$ & 1 & 254 & $\begin{array}{l}\text { Std. Mean Difference (IV, Ran- } \\
\text { dom, } 95 \% \mathrm{Cl} \text { ) }\end{array}$ & $0.0[-0.25,0.25]$ \\
\hline
\end{tabular}




\begin{tabular}{|c|c|c|c|c|}
\hline Outcome or subgroup title & No. of studies & $\begin{array}{l}\text { No. of partici- } \\
\text { pants }\end{array}$ & Statistical method & Effect size \\
\hline $\begin{array}{l}14 \text { Activities of daily living: mean } \\
\text { scores at end of treatment }\end{array}$ & 8 & & $\begin{array}{l}\text { Std. Mean Difference (IV, Ran- } \\
\text { dom, } 95 \% \mathrm{CI} \text { ) }\end{array}$ & $\begin{array}{l}\text { Totals not select- } \\
\text { ed }\end{array}$ \\
\hline $\begin{array}{l}\text { 14.1 Barthel Index (high score = more } \\
\text { dependent) }\end{array}$ & 8 & & $\begin{array}{l}\text { Std. Mean Difference (IV, Ran- } \\
\text { dom, } 95 \% \mathrm{Cl} \text { ) }\end{array}$ & $0.0[0.0,0.0]$ \\
\hline $\begin{array}{l}\text { 14.2 Nottingham EADL (high score = } \\
\text { more independent) }\end{array}$ & 1 & & $\begin{array}{l}\text { Std. Mean Difference (IV, Ran- } \\
\text { dom, } 95 \% \mathrm{CI} \text { ) }\end{array}$ & $0.0[0.0,0.0]$ \\
\hline $\begin{array}{l}15 \text { Activities of daily living: mean } \\
\text { scores at end of follow-up }\end{array}$ & 1 & & $\begin{array}{l}\text { Mean Difference (IV, Fixed, } \\
95 \% \mathrm{Cl})\end{array}$ & $\begin{array}{l}\text { Totals not select- } \\
\text { ed }\end{array}$ \\
\hline $\begin{array}{l}\text { 15.1 Modified Barthel Index (high score } \\
=\text { more dependent) }\end{array}$ & 1 & & $\begin{array}{l}\text { Mean Difference (IV, Fixed, } \\
95 \% \mathrm{Cl} \text { ) }\end{array}$ & $0.0[0.0,0.0]$ \\
\hline $\begin{array}{l}16 \text { Disability: mean scores at end of } \\
\text { treatment }\end{array}$ & 2 & 162 & $\begin{array}{l}\text { Std. Mean Difference (IV, Ran- } \\
\text { dom, } 95 \% \mathrm{Cl} \text { ) }\end{array}$ & $-0.16[-0.48,0.17]$ \\
\hline 16.1 WHODAS-II total & 1 & 24 & $\begin{array}{l}\text { Std. Mean Difference (IV, Ran- } \\
\text { dom, } 95 \% \mathrm{CI} \text { ) }\end{array}$ & $-0.51[-1.33,0.30]$ \\
\hline 16.2 FIM Motor & 1 & 138 & $\begin{array}{l}\text { Std. Mean Difference (IV, Ran- } \\
\text { dom, } 95 \% \mathrm{CI} \text { ) }\end{array}$ & $-0.09[-0.44,0.27]$ \\
\hline 17 Adverse events: death & 8 & & $\begin{array}{l}\text { Risk Ratio (M-H, Random, } \\
95 \% \mathrm{Cl})\end{array}$ & Subtotals only \\
\hline 17.1 At end of treatment & 8 & 831 & $\begin{array}{l}\text { Risk Ratio (M-H, Random, } \\
95 \% \mathrm{Cl} \text { ) }\end{array}$ & $0.65[0.26,1.66]$ \\
\hline 18 Adverse events: all & 4 & & $\begin{array}{l}\text { Risk Ratio (M-H, Random, } \\
95 \% \mathrm{Cl} \text { ) }\end{array}$ & Subtotals only \\
\hline 18.1 Recurrent stroke & 1 & 254 & $\begin{array}{l}\text { Risk Ratio (M-H, Random, } \\
95 \% \mathrm{Cl} \text { ) }\end{array}$ & $5.0[0.24,103.12]$ \\
\hline $\begin{array}{l}18.2 \text { Vascular events - not stroke (e.g. } \\
\text { transient ischaemic attack) }\end{array}$ & 1 & 254 & $\begin{array}{l}\text { Risk Ratio (M-H, Random, } \\
95 \% \mathrm{Cl})\end{array}$ & $0.71[0.23,2.19]$ \\
\hline $\begin{array}{l}\text { 18.3 Other events - not listed above } \\
\text { (e.g. too ill) }\end{array}$ & 2 & 206 & $\begin{array}{l}\text { Risk Ratio (M-H, Random, } \\
95 \% \mathrm{Cl} \text { ) }\end{array}$ & $1.02[0.15,6.81]$ \\
\hline $\begin{array}{l}\text { 18.4 Protocol violation (e.g. refused } \\
\text { treatment, withdrew consent) }\end{array}$ & 3 & 250 & $\begin{array}{l}\text { Risk Ratio (M-H, Random, } \\
95 \% \mathrm{Cl} \text { ) }\end{array}$ & $1.08[0.21,5.50]$ \\
\hline $\begin{array}{l}19 \text { Adverse events: leaving the study } \\
\text { early (including death) }\end{array}$ & 8 & & $\begin{array}{l}\text { Risk Ratio (M-H, Random, } \\
95 \% \mathrm{Cl})\end{array}$ & Subtotals only \\
\hline 19.1 All dropouts and withdrawals & 8 & 784 & $\begin{array}{l}\text { Risk Ratio (M-H, Random, } \\
95 \% \mathrm{Cl} \text { ) }\end{array}$ & $0.83[0.42,1.63]$ \\
\hline
\end{tabular}


Analysis 3.1. Comparison 3 Psychological therapy versus usual care and/or attention control, Outcome 1 Depression: meeting study criteria for depression at end of treatment.

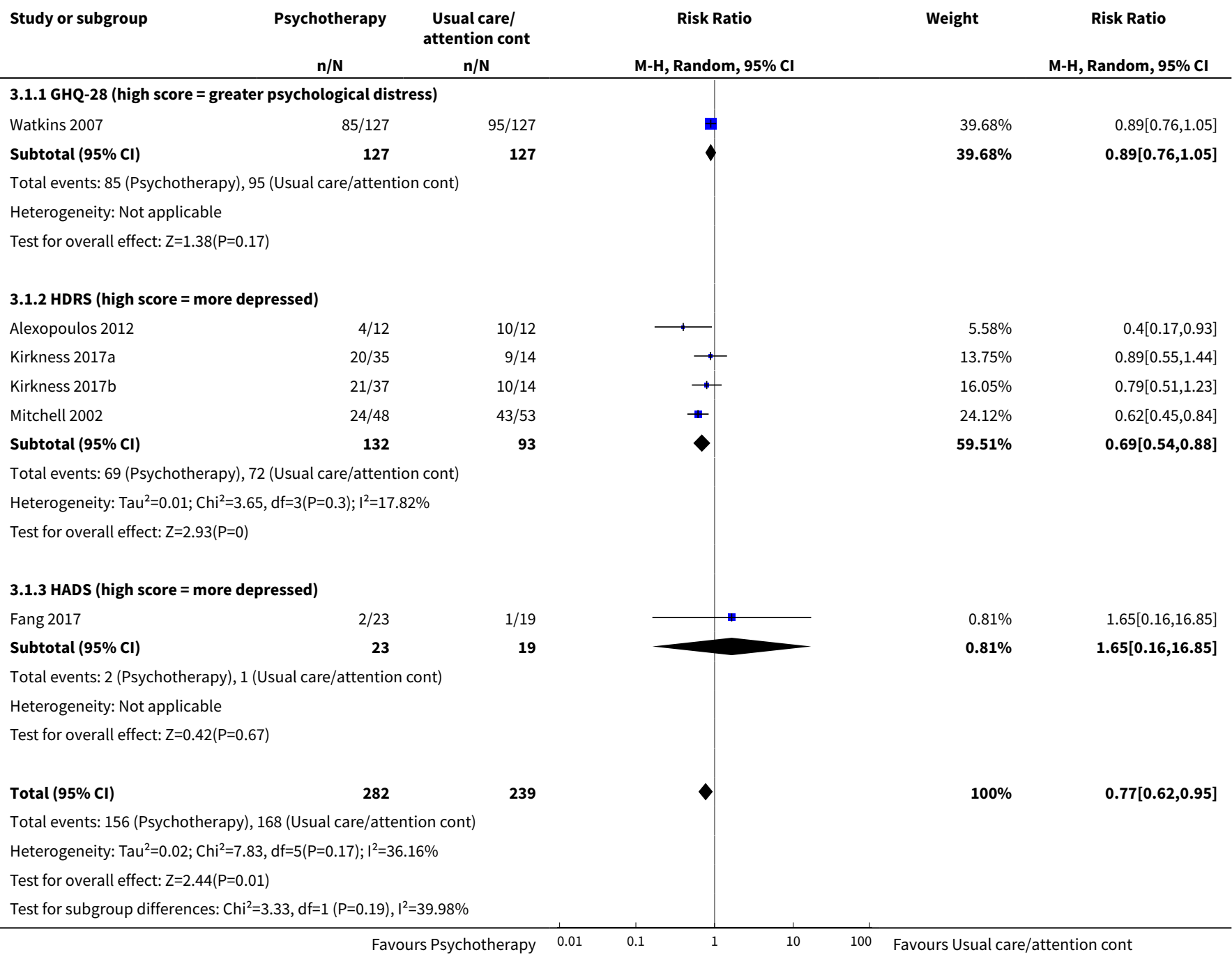

\section{Analysis 3.3. Comparison 3 Psychological therapy versus usual care and/or attention control, Outcome 3 Depression: average change in scores between baseline and end of treatment.}

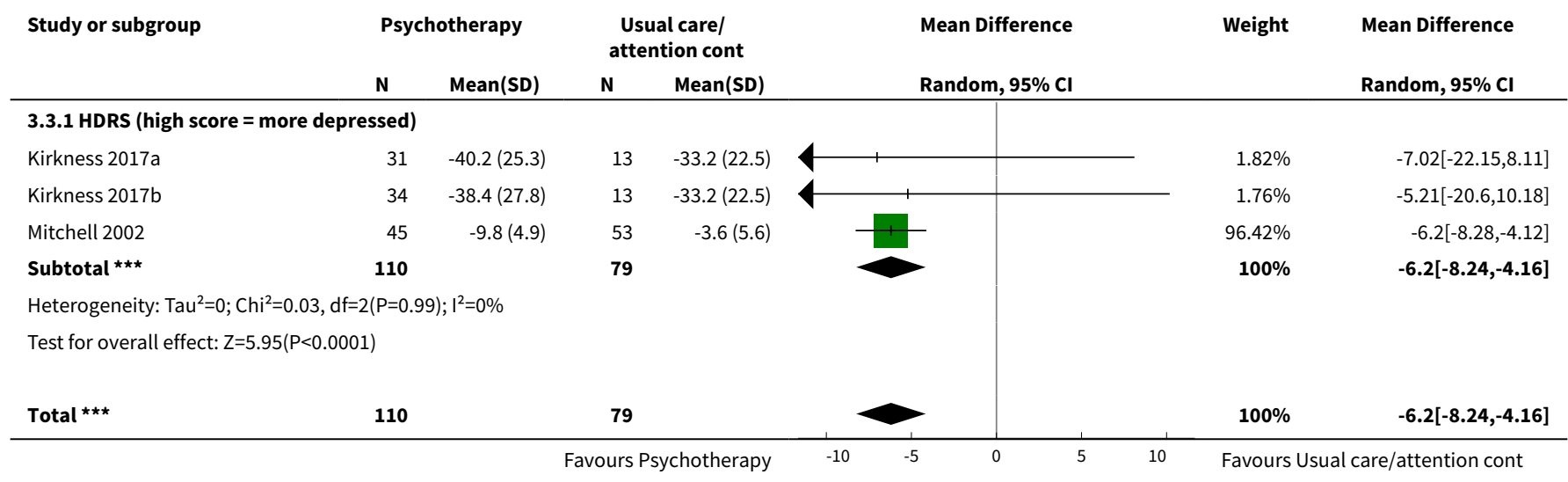




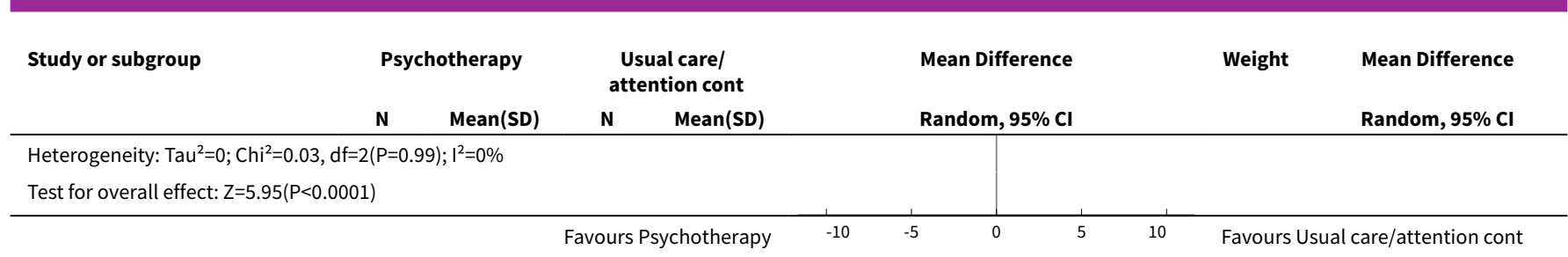

Analysis 3.4. Comparison 3 Psychological therapy versus usual care and/ or attention control, Outcome 4 Depression: mean scores at end of treatment.

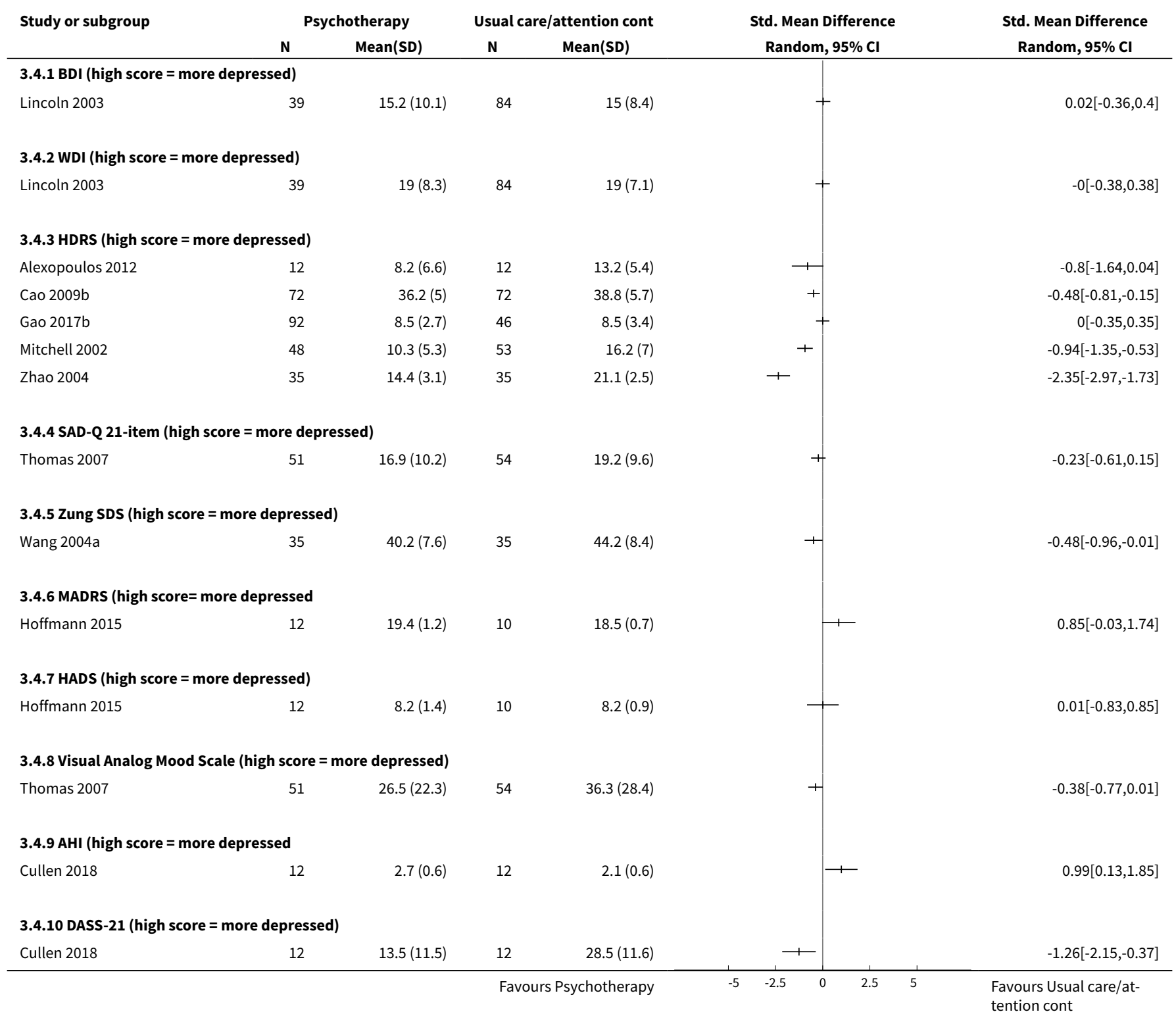


Analysis 3.5. Comparison 3 Psychological therapy versus usual care and/or attention control, Outcome 5 Depression: meeting study criteria for depression at end of follow-up.

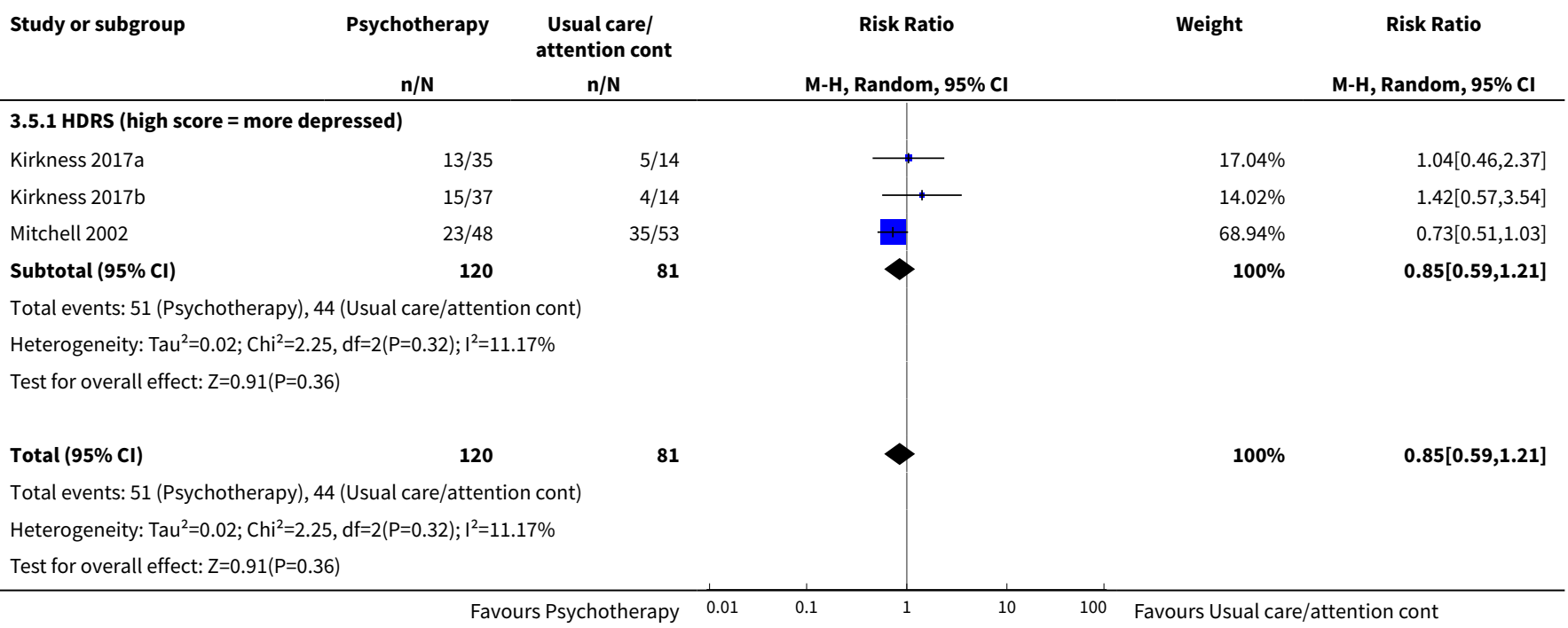

\section{Analysis 3.6. Comparison 3 Psychological therapy versus usual care and/or attention control, Outcome 6 Depression: average change in scores between baseline and end of follow-up.}

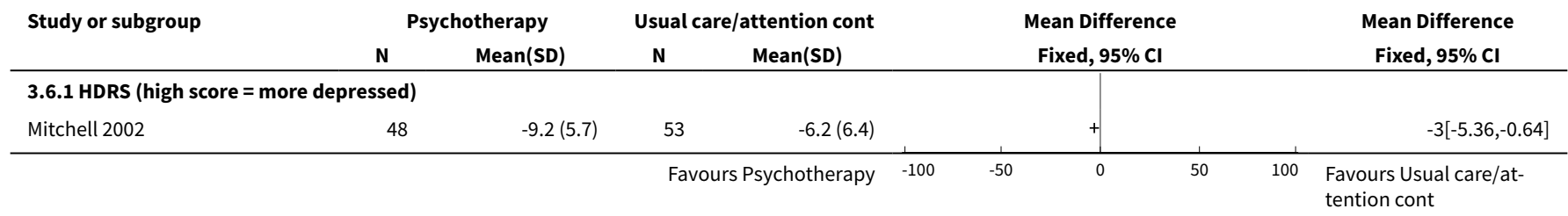

Analysis 3.7. Comparison 3 Psychological therapy versus usual care and/ or attention control, Outcome 7 Depression: mean scores at end of follow-up.

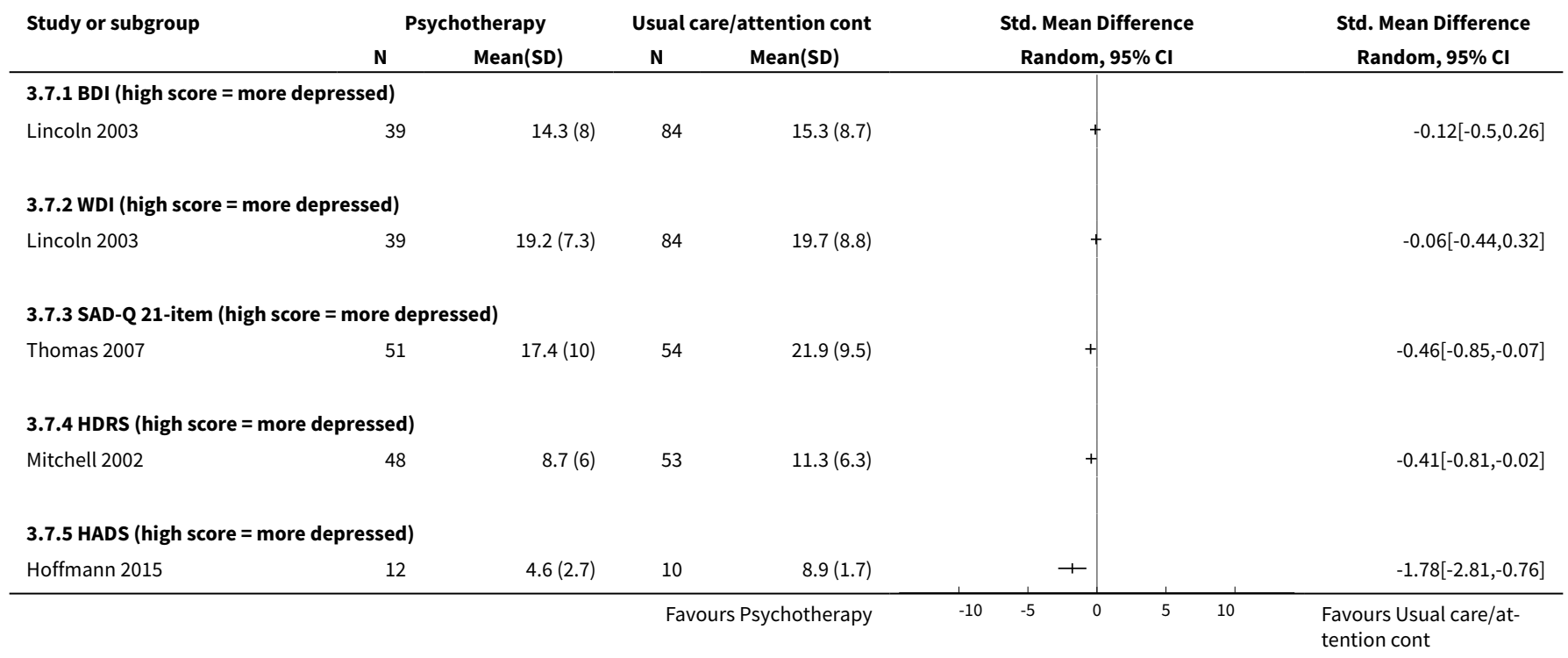




\begin{tabular}{|c|c|c|c|c|c|c|c|}
\hline \multirow[t]{2}{*}{ Study or subgroup } & \multicolumn{2}{|c|}{ Psychotherapy } & \multicolumn{2}{|c|}{ Usual care/attention cont } & \multirow{2}{*}{\multicolumn{2}{|c|}{$\begin{array}{c}\text { Std. Mean Difference } \\
\text { Random, } 95 \% \mathrm{Cl}\end{array}$}} & \multirow{2}{*}{$\begin{array}{c}\text { Std. Mean Difference } \\
\text { Random, } 95 \% \mathrm{Cl}\end{array}$} \\
\hline & $\mathbf{N}$ & Mean(SD) & $\mathbf{N}$ & Mean(SD) & & & \\
\hline Kerr 2018 & 4 & $4.5(3.9)$ & 6 & $7(2.7)$ & & 1 & $-0.7[-2.03,0.62]$ \\
\hline \multicolumn{8}{|c|}{ 3.7.6 MADRS (high score = more depressed) } \\
\hline Hoffmann 2015 & 12 & $19.8(1.8)$ & 10 & $18.7(1.1)$ & & + & $0.73[-0.14,1.6]$ \\
\hline \multicolumn{8}{|c|}{ 3.7.7 VAMS (high score $=$ more depressed) } \\
\hline Thomas 2007 & 51 & $25.5(21.5)$ & 54 & $32.1(29.3)$ & & + & $-0.25[-0.64,0.13]$ \\
\hline \multicolumn{8}{|c|}{ 3.7.8 PHQ-9 (high score = more depressed) } \\
\hline \multirow[t]{2}{*}{ Kerr 2018} & 4 & $1.7(2.9)$ & 6 & $6(7.4)$ & & 1 & $-0.64[-1.95,0.68]$ \\
\hline & & & & sychotherapy & -10 & -5 & $\begin{array}{l}\text { Favours Usual care/at- } \\
\text { tention cont }\end{array}$ \\
\hline
\end{tabular}

Analysis 3.8. Comparison 3 Psychological therapy versus usual care and/or attention control, Outcome 8 Psychological distress: average change in scores between baseline and end of treatment.

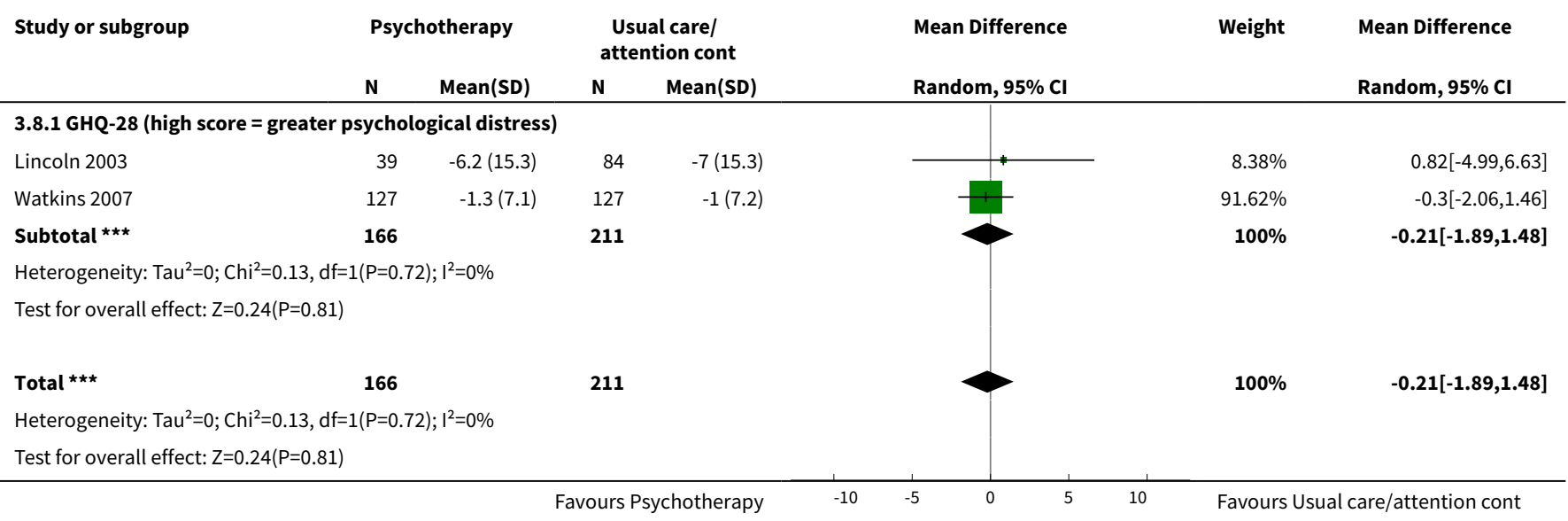

\section{Analysis 3.9. Comparison 3 Psychological therapy versus usual care and/or attention} control, Outcome 9 Psychological distress: mean scores at end of treatment.

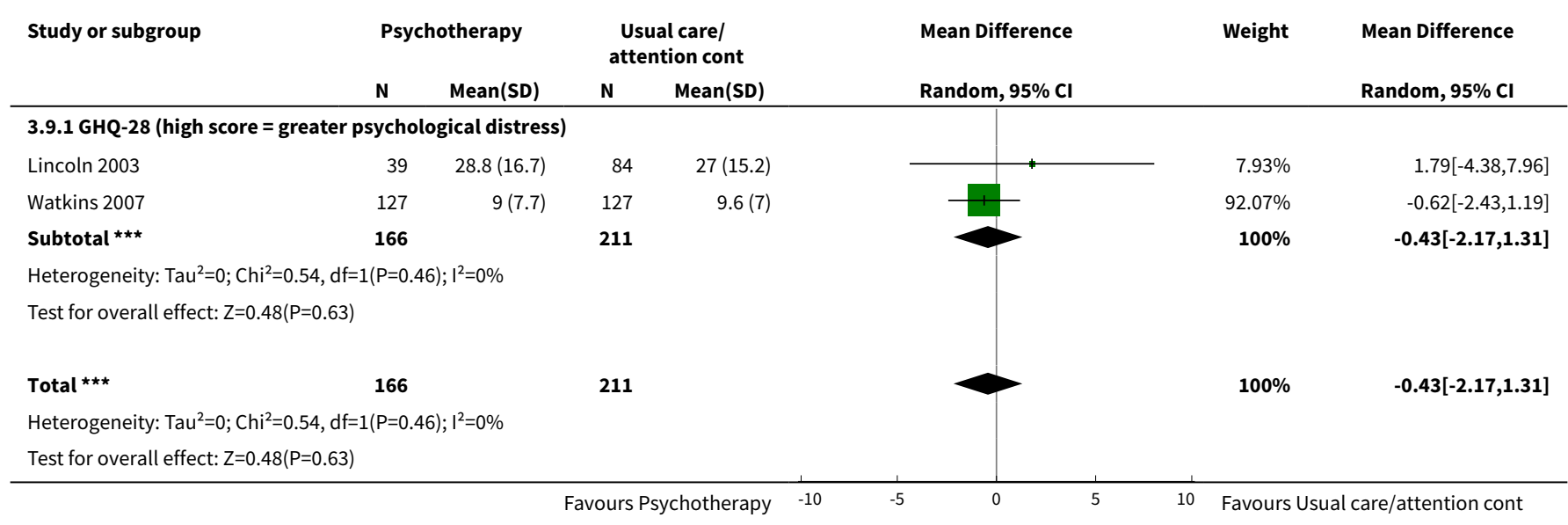


Analysis 3.10. Comparison 3 Psychological therapy versus usual care and/or attention control, Outcome 10 Anxiety: meeting study criteria for anxiety at end of treatment.

\begin{tabular}{|c|c|c|c|c|c|c|c|}
\hline Study or subgroup & $\begin{array}{c}\text { Psychotherapy } \\
\mathrm{n} / \mathrm{N} \\
\end{array}$ & $\begin{array}{l}\text { Usual care/attention cont } \\
\qquad \mathrm{n} / \mathrm{N}\end{array}$ & & \multicolumn{2}{|c|}{$\begin{array}{c}\text { Risk Ratio } \\
\text { M-H, Fixed, 95\% Cl }\end{array}$} & & \multirow[t]{2}{*}{$\begin{array}{c}\text { Risk Ratio } \\
\text { M-H, Fixed, 95\% Cl } \\
\end{array}$} \\
\hline 3.10.1 HADS Anxiet & anxious) & & & & & & \\
\hline Fang 2017 & $4 / 23$ & $2 / 19$ & & & 1 & & $1.65[0.34,8.06]$ \\
\hline
\end{tabular}

Analysis 3.11. Comparison 3 Psychological therapy versus usual care and/ or attention control, Outcome 11 Anxiety: mean scores at end of treatment.

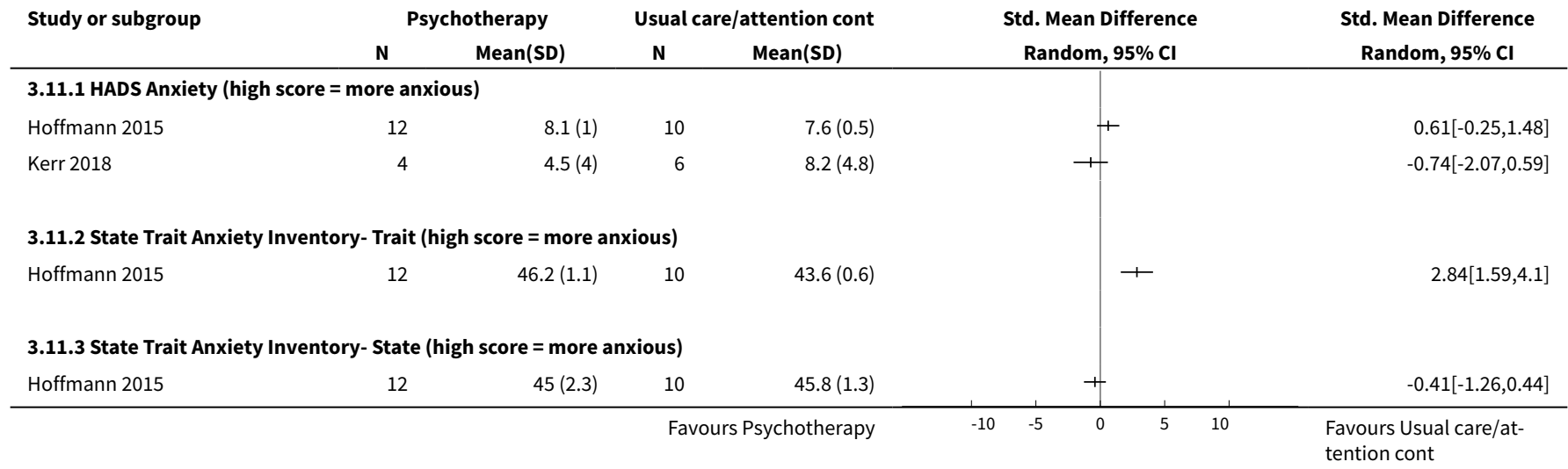

Analysis 3.12. Comparison 3 Psychological therapy versus usual care and/ or attention control, Outcome 12 Anxiety: mean scores at end of follow-up.

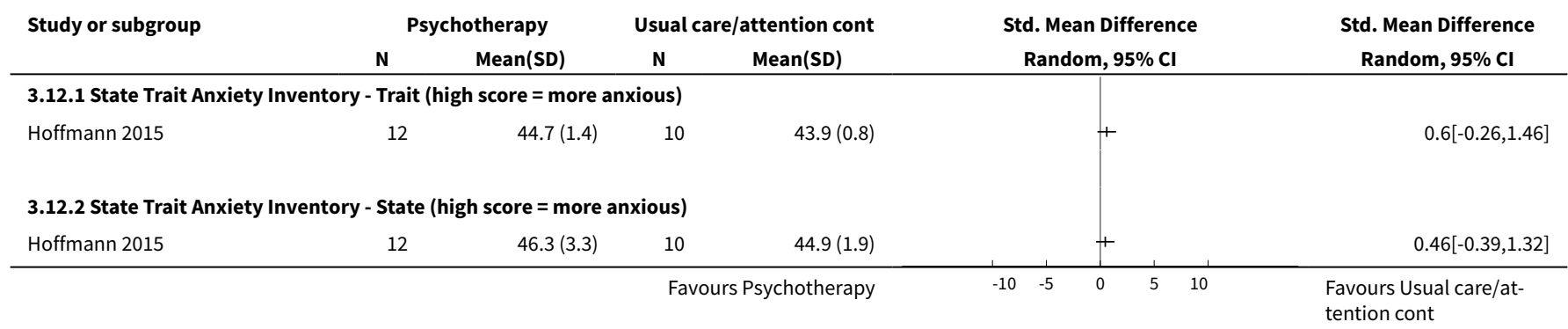

Analysis 3.13. Comparison 3 Psychological therapy versus usual care and/or attention control, Outcome 13 Activities of daily living: average change in scores from baseline to end of treatment.

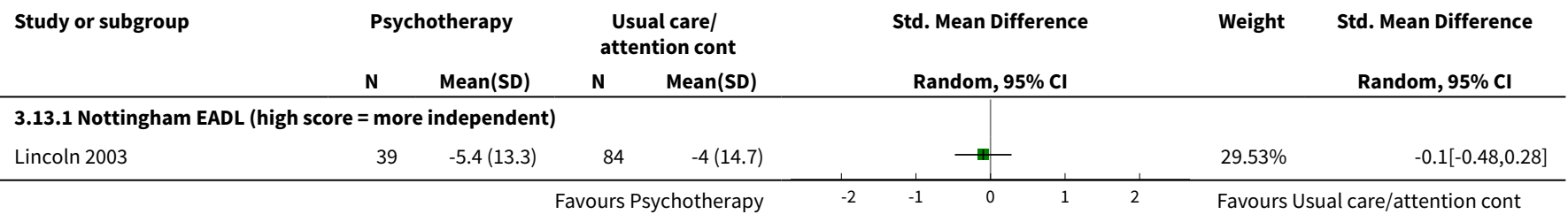




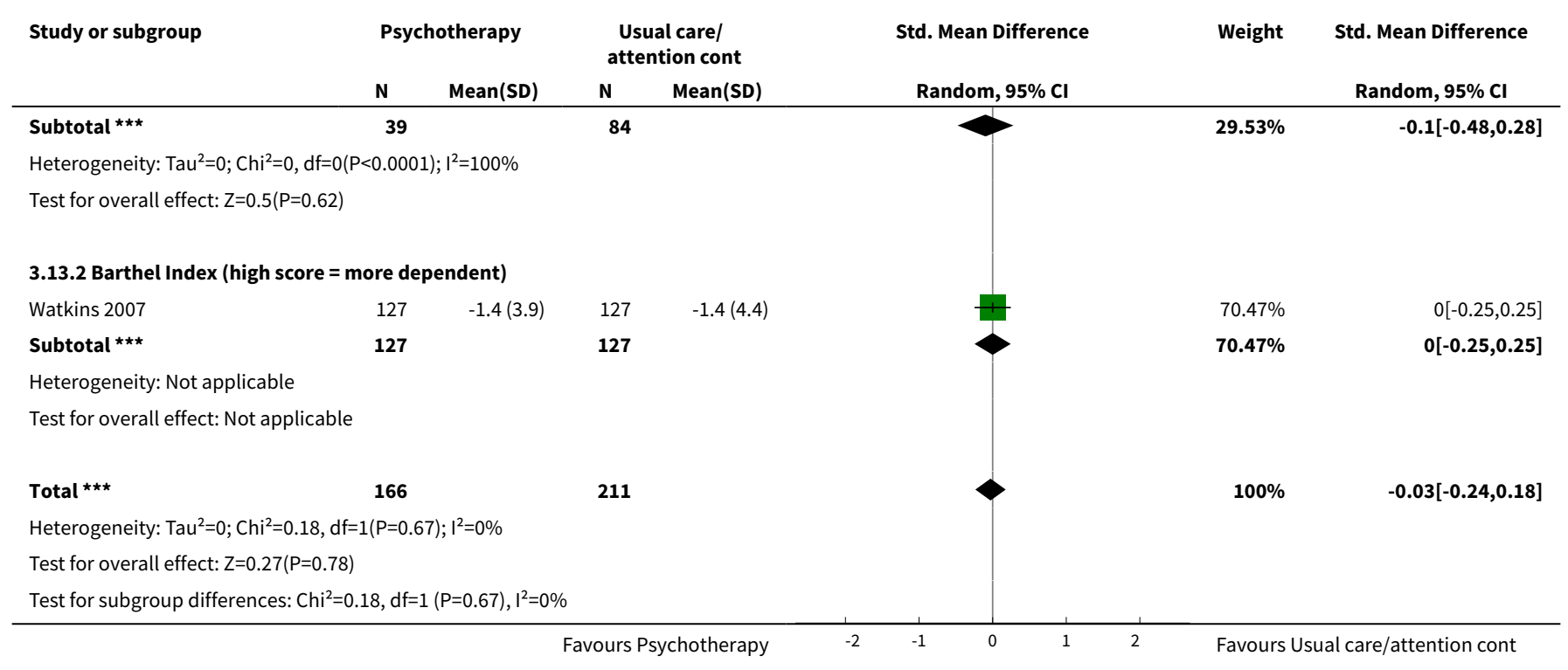

Analysis 3.14. Comparison 3 Psychological therapy versus usual care and/or attention control, Outcome 14 Activities of daily living: mean scores at end of treatment.

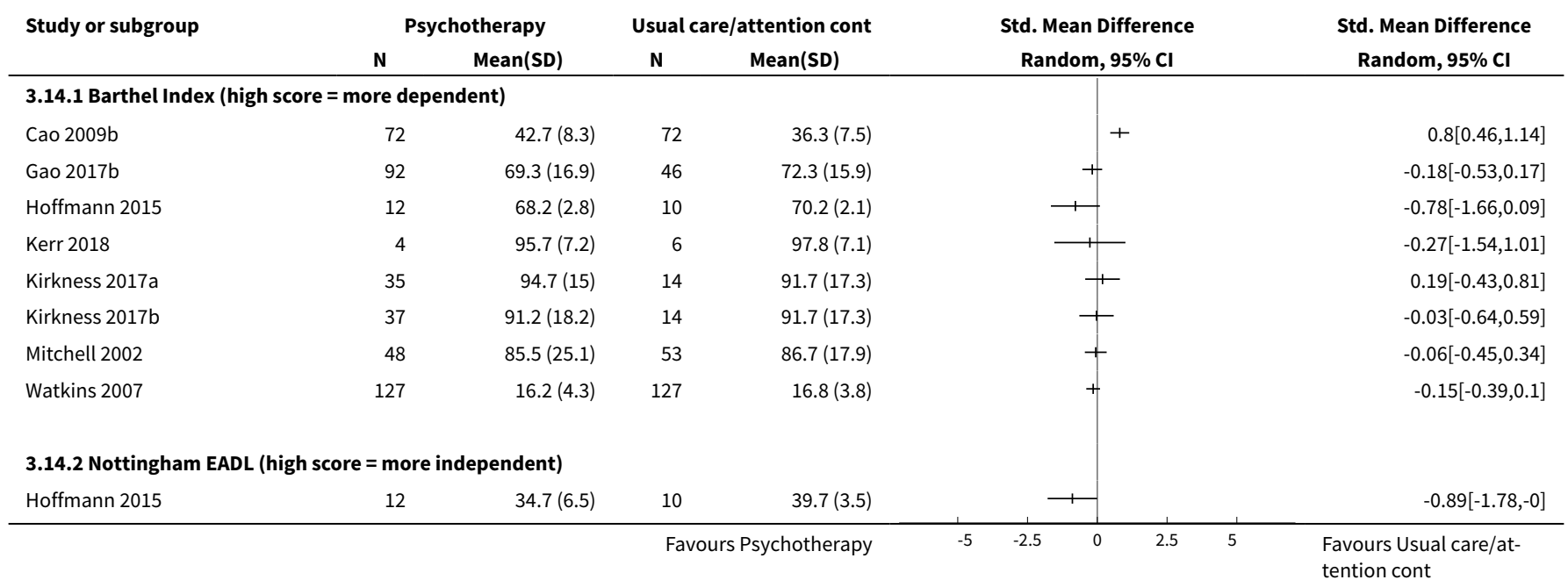

Analysis 3.15. Comparison 3 Psychological therapy versus usual care and/or attention control, Outcome 15 Activities of daily living: mean scores at end of follow-up.

\begin{tabular}{|c|c|c|c|c|c|c|c|c|c|}
\hline \multirow[t]{2}{*}{ Study or subgroup } & \multicolumn{2}{|c|}{ Psychotherapy } & \multicolumn{2}{|c|}{ Usual care/attention cont } & \multirow{2}{*}{\multicolumn{3}{|c|}{$\begin{array}{c}\text { Mean Difference } \\
\text { Fixed, } 95 \% \mathrm{Cl}\end{array}$}} & & \multirow{2}{*}{$\begin{array}{c}\text { Mean Difference } \\
\text { Fixed, } 95 \% \mathrm{Cl}\end{array}$} \\
\hline & $\mathbf{N}$ & Mean(SD) & $\mathbf{N}$ & Mean(SD) & & & & & \\
\hline Hoffmann 2015 & 2 & $76.3(5.6)$ & 5 & $76.4(4.3)$ & & & + & & $-0.12[-8.73,8.49]$ \\
\hline
\end{tabular}


Analysis 3.16. Comparison 3 Psychological therapy versus usual care and/ or attention control, Outcome 16 Disability: mean scores at end of treatment.

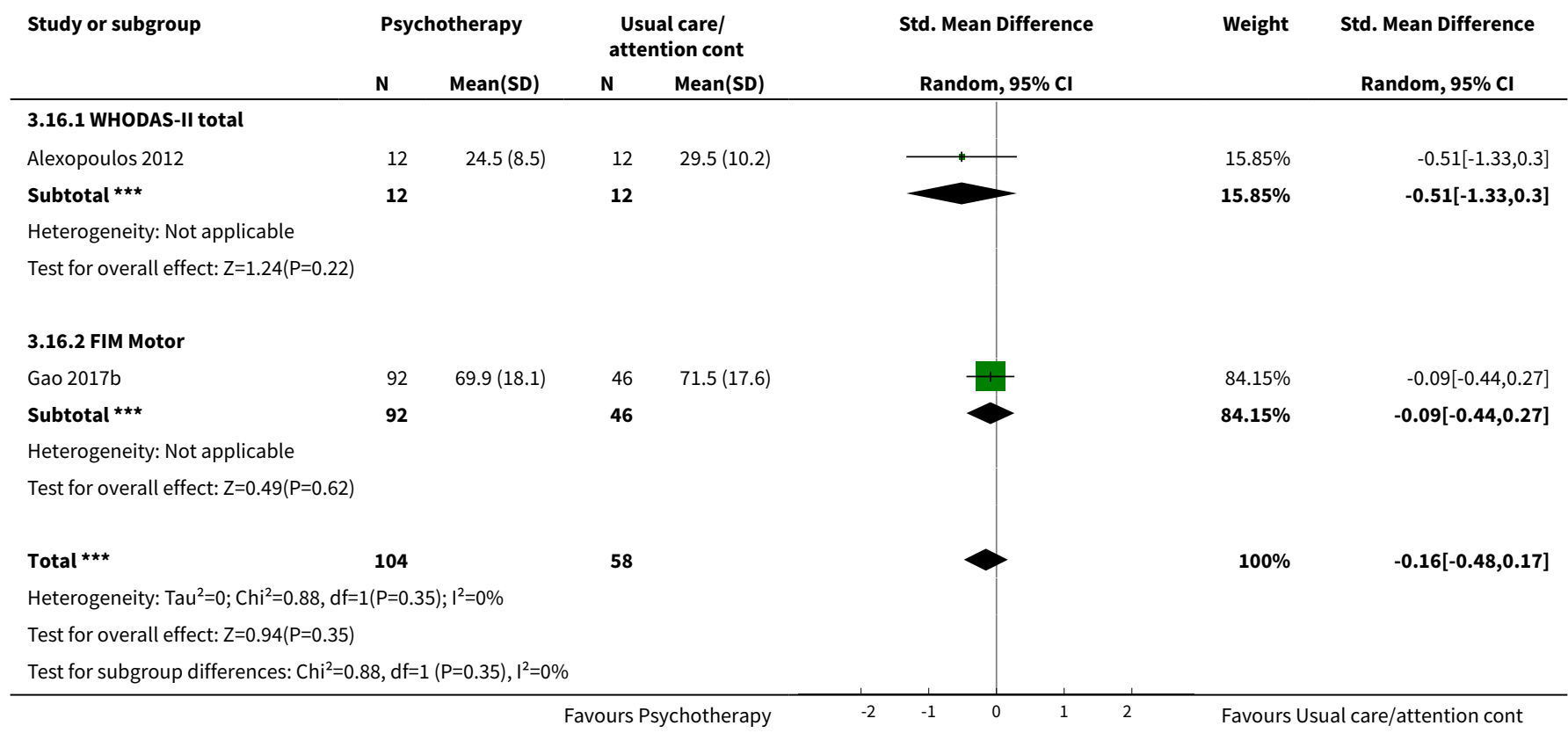

Analysis 3.17. Comparison 3 Psychological therapy versus usual care and/or attention control, Outcome 17 Adverse events: death.

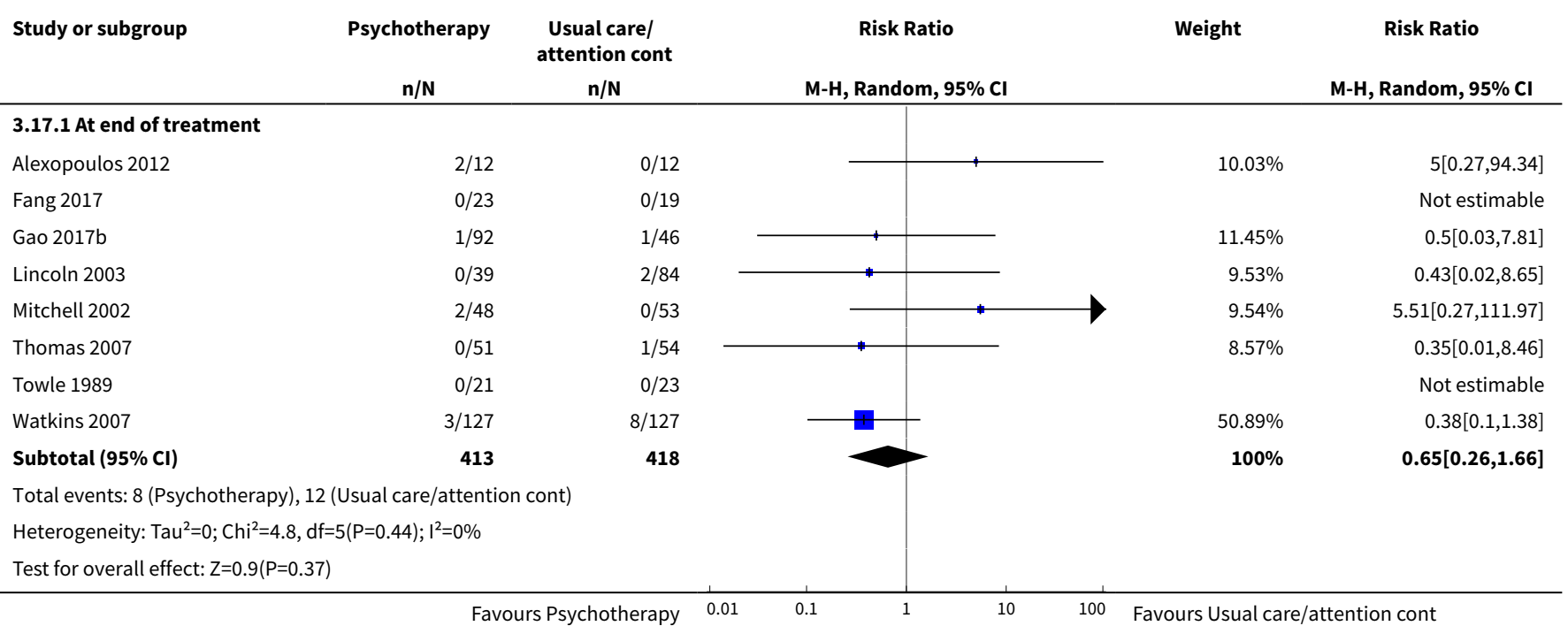


Analysis 3.18. Comparison 3 Psychological therapy versus usual care and/or attention control, Outcome 18 Adverse events: all.

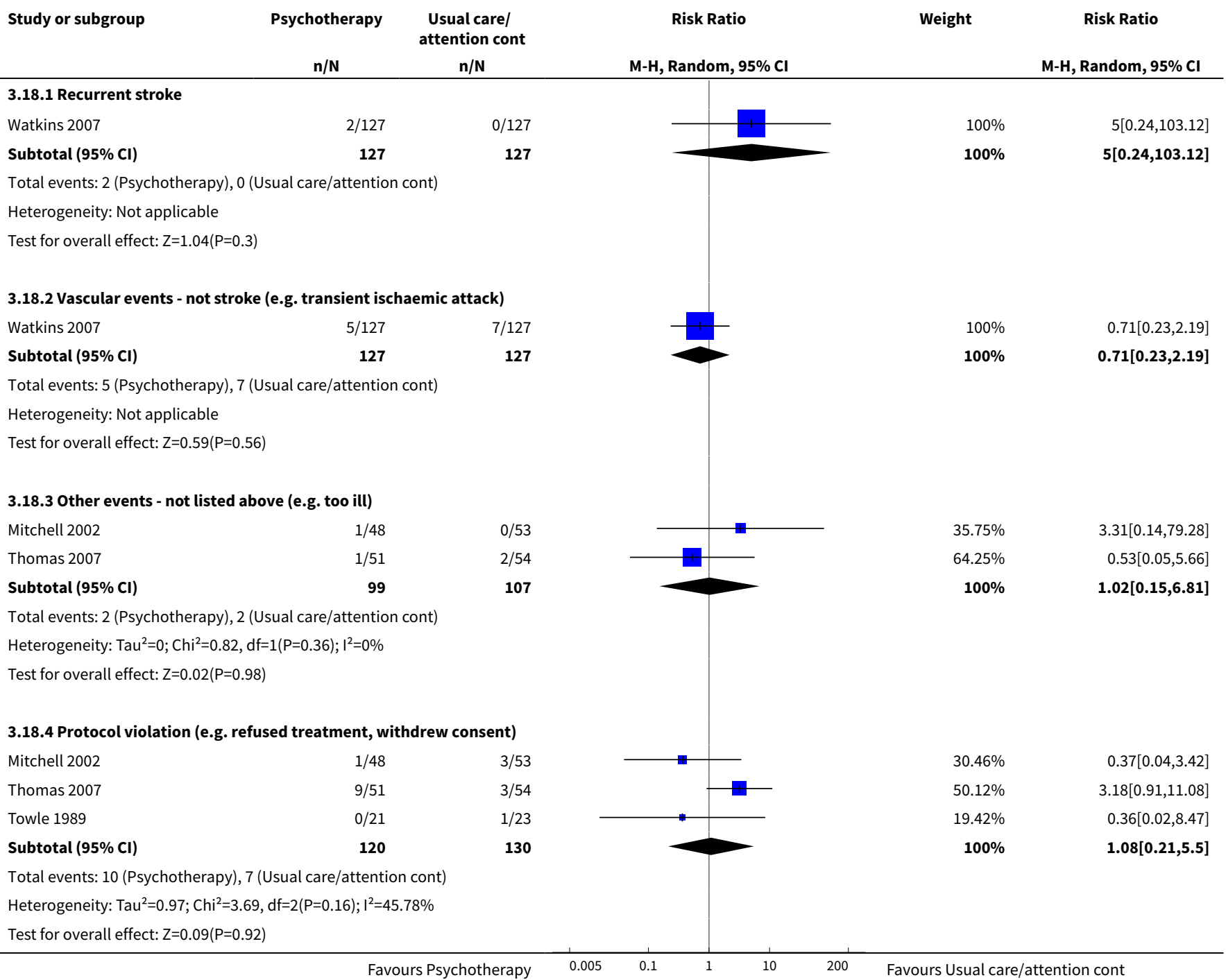

Analysis 3.19. Comparison 3 Psychological therapy versus usual care and/or attention control, Outcome 19 Adverse events: leaving the study early (including death).

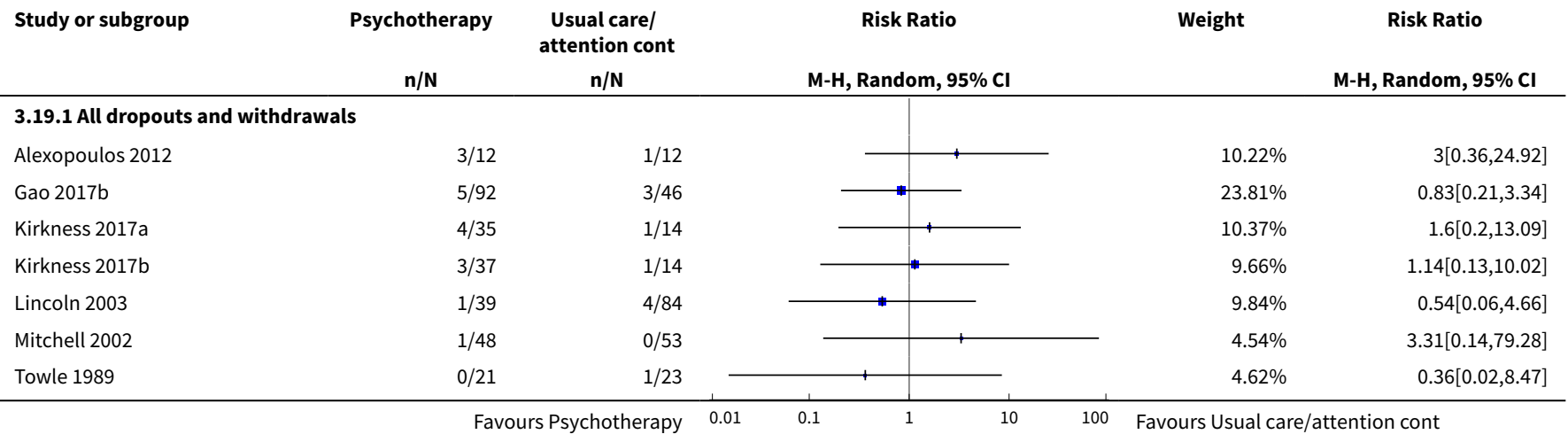




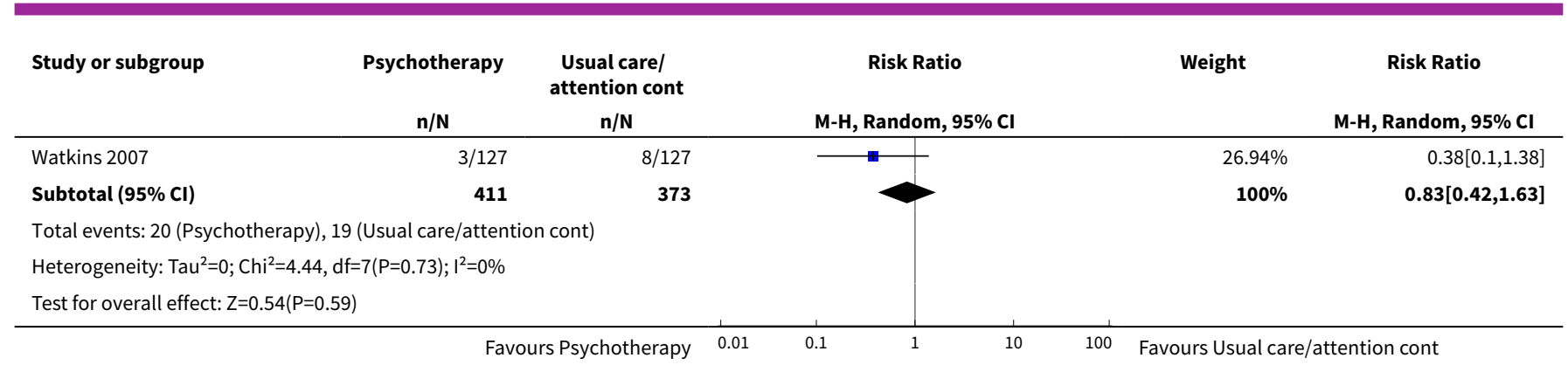

\section{Comparison 4. Pharmacological intervention and psychotherapy (combination) versus a pharmacological intervention and usual care or attention control (single)}

\begin{tabular}{|c|c|c|c|c|}
\hline Outcome or subgroup title & No. of studies & $\begin{array}{l}\text { No. of partici- } \\
\text { pants }\end{array}$ & Statistical method & Effect size \\
\hline $\begin{array}{l}1 \text { Depression: meeting study criteria for } \\
\text { depression at end of treatment }\end{array}$ & 0 & 0 & $\begin{array}{l}\text { Risk Ratio (M-H, Random, } \\
95 \% \mathrm{Cl})\end{array}$ & $0.0[0.0,0.0]$ \\
\hline $\begin{array}{l}2 \text { Depression: }<50 \% \text { reduction in scale } \\
\text { scores at end of treatment }\end{array}$ & 0 & 0 & $\begin{array}{l}\text { Risk Ratio (M-H, Random, } \\
95 \% \mathrm{Cl})\end{array}$ & $0.0[0.0,0.0]$ \\
\hline $\begin{array}{l}3 \text { Depression: mean scores at end of } \\
\text { treatment }\end{array}$ & 2 & 198 & $\begin{array}{l}\text { Mean Difference (IV, Fixed, } \\
95 \% \mathrm{CI})\end{array}$ & $\begin{array}{l}-1.53[-2.10 \\
-0.96]\end{array}$ \\
\hline 3.1 HDRS (high score = more depressed) & 2 & 198 & $\begin{array}{l}\text { Mean Difference (IV, Fixed, } \\
95 \% \mathrm{CI})\end{array}$ & $\begin{array}{l}-1.53[-2.10 \\
-0.96]\end{array}$ \\
\hline $\begin{array}{l}4 \text { Anxiety: mean scores at end of treat- } \\
\text { ment }\end{array}$ & 1 & & $\begin{array}{l}\text { Mean Difference (IV, Fixed, } \\
95 \% \mathrm{CI} \text { ) }\end{array}$ & $\begin{array}{l}\text { Totals not select- } \\
\text { ed }\end{array}$ \\
\hline 4.1 HAMA (high score = more anxious) & 1 & & $\begin{array}{l}\text { Mean Difference (IV, Fixed, } \\
95 \% \mathrm{CI})\end{array}$ & $0.0[0.0,0.0]$ \\
\hline $\begin{array}{l}5 \text { Activities of daily living: mean scores } \\
\text { at end of treatment }\end{array}$ & 2 & 198 & $\begin{array}{l}\text { Mean Difference (IV, Ran- } \\
\text { dom, } 95 \% \mathrm{CI} \text { ) }\end{array}$ & $\begin{array}{l}11.83[0.27 \\
23.40]\end{array}$ \\
\hline $\begin{array}{l}5.1 \text { Barthel Index (high score = more de- } \\
\text { pendent) }\end{array}$ & 2 & 198 & $\begin{array}{l}\text { Mean Difference (IV, Ran- } \\
\text { dom, } 95 \% \mathrm{CI} \text { ) }\end{array}$ & $\begin{array}{l}11.83[0.27 \\
23.40]\end{array}$ \\
\hline $\begin{array}{l}6 \text { Neurological function: mean scores at } \\
\text { end of treatment }\end{array}$ & 1 & & $\begin{array}{l}\text { Mean Difference (IV, Fixed, } \\
95 \% \mathrm{CI})\end{array}$ & $\begin{array}{l}\text { Totals not select- } \\
\text { ed }\end{array}$ \\
\hline $\begin{array}{l}\text { 6.1 Scandinavian Stroke Scale (low score } \\
=\text { more neurological deficit) }\end{array}$ & 1 & & $\begin{array}{l}\text { Mean Difference (IV, Fixed, } \\
95 \% \mathrm{CI})\end{array}$ & $0.0[0.0,0.0]$ \\
\hline 7 Adverse events: death & 1 & & $\begin{array}{l}\text { Risk Ratio (M-H, Fixed, 95\% } \\
\mathrm{Cl})\end{array}$ & $\begin{array}{l}\text { Totals not select- } \\
\text { ed }\end{array}$ \\
\hline 7.1 At end of treatment & 1 & & $\begin{array}{l}\text { Risk Ratio (M-H, Fixed, 95\% } \\
\mathrm{Cl} \text { ) }\end{array}$ & $0.0[0.0,0.0]$ \\
\hline 8 Adverse events: all & 1 & & $\begin{array}{l}\text { Risk Ratio (M-H, Fixed, 95\% } \\
\text { Cl) }\end{array}$ & $\begin{array}{l}\text { Totals not select- } \\
\text { ed }\end{array}$ \\
\hline
\end{tabular}




\begin{tabular}{lllll}
\hline Outcome or subgroup title & No. of studies & $\begin{array}{l}\text { No. of partici- } \\
\text { pants }\end{array}$ & Statistical method & Effect size \\
\hline $\begin{array}{l}\text { 8.1 Gastrointestinal effects (e.g. consti- } \\
\text { pation, diarrhoea) }\end{array}$ & 1 & & $\begin{array}{l}\text { Risk Ratio (M-H, Fixed, 95\% } \\
\mathrm{Cl})\end{array}$ & $0.0[0.0,0.0]$ \\
\hline $\begin{array}{l}\text { 9 Adverse events: leaving the study early } \\
\text { (including death) }\end{array}$ & 1 & $\begin{array}{l}\text { Risk Ratio (M-H, Fixed, 95\% } \\
\text { Cl) }\end{array}$ & $\begin{array}{l}\text { Totals not select- } \\
\text { ed }\end{array}$ \\
\hline 9.1 All dropouts and withdrawals & 1 & $\begin{array}{l}\text { Risk Ratio (M-H, Fixed, 95\% } \\
\text { Cl) }\end{array}$ & $0.0[0.0,0.0]$ \\
\hline
\end{tabular}

Analysis 4.3. Comparison 4 Pharmacological intervention and psychotherapy (combination) versus a pharmacological intervention and usual care or attention control (single), Outcome 3 Depression: mean scores at end of treatment.

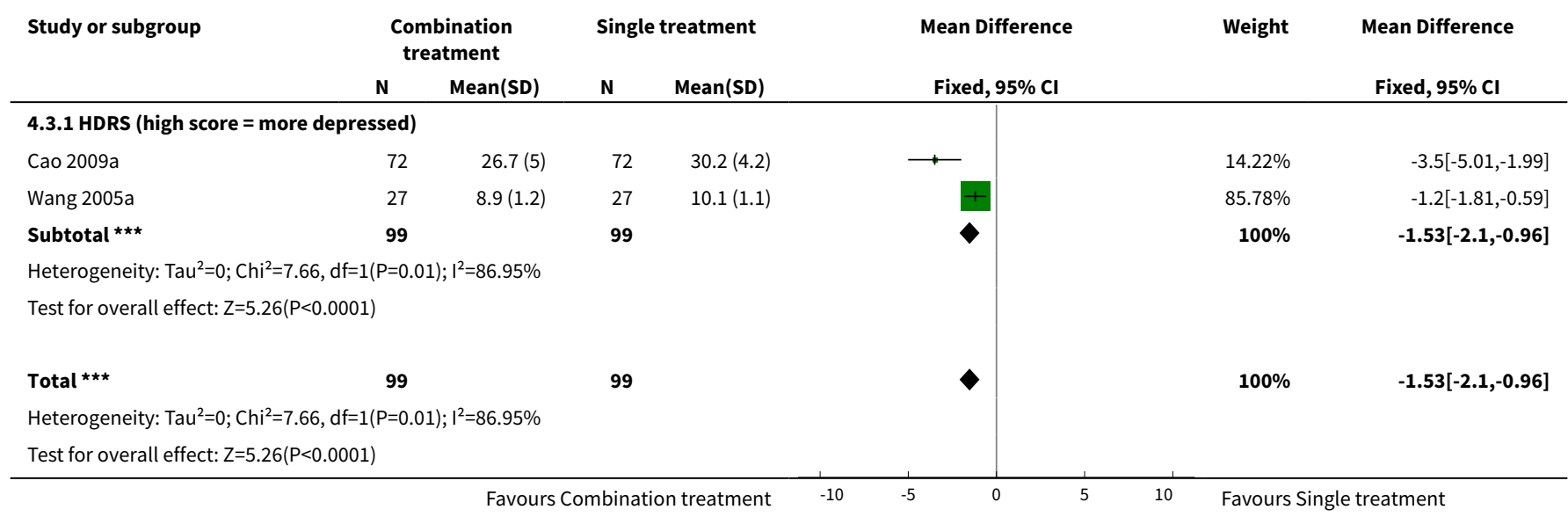

Analysis 4.4. Comparison 4 Pharmacological intervention and psychotherapy

(combination) versus a pharmacological intervention and usual care or attention control (single), Outcome 4 Anxiety: mean scores at end of treatment.

\begin{tabular}{|c|c|c|c|c|c|c|c|}
\hline \multirow[t]{2}{*}{ Study or subgroup } & \multicolumn{2}{|c|}{ Combination treatment } & \multicolumn{2}{|c|}{ Single treatment } & \multirow{2}{*}{\multicolumn{2}{|c|}{$\begin{array}{c}\text { Mean Difference } \\
\text { Fixed, } 95 \% \mathrm{Cl}\end{array}$}} & \multirow{2}{*}{$\begin{array}{c}\text { Mean Difference } \\
\text { Fixed, } 95 \% \mathrm{Cl}\end{array}$} \\
\hline & $\mathbf{N}$ & Mean(SD) & $\mathbf{N}$ & Mean(SD) & & & \\
\hline \multicolumn{8}{|c|}{ 4.4.1 HAMA (high score = more anxious) } \\
\hline Wang 2005a & 27 & $3.8(1.8)$ & 27 & $5.4(1.7)$ & + & & $-1.6[-2.53,-0.67]$ \\
\hline
\end{tabular}


Analysis 4.5. Comparison 4 Pharmacological intervention and psychotherapy

(combination) versus a pharmacological intervention and usual care or attention control (single), Outcome 5 Activities of daily living: mean scores at end of treatment.

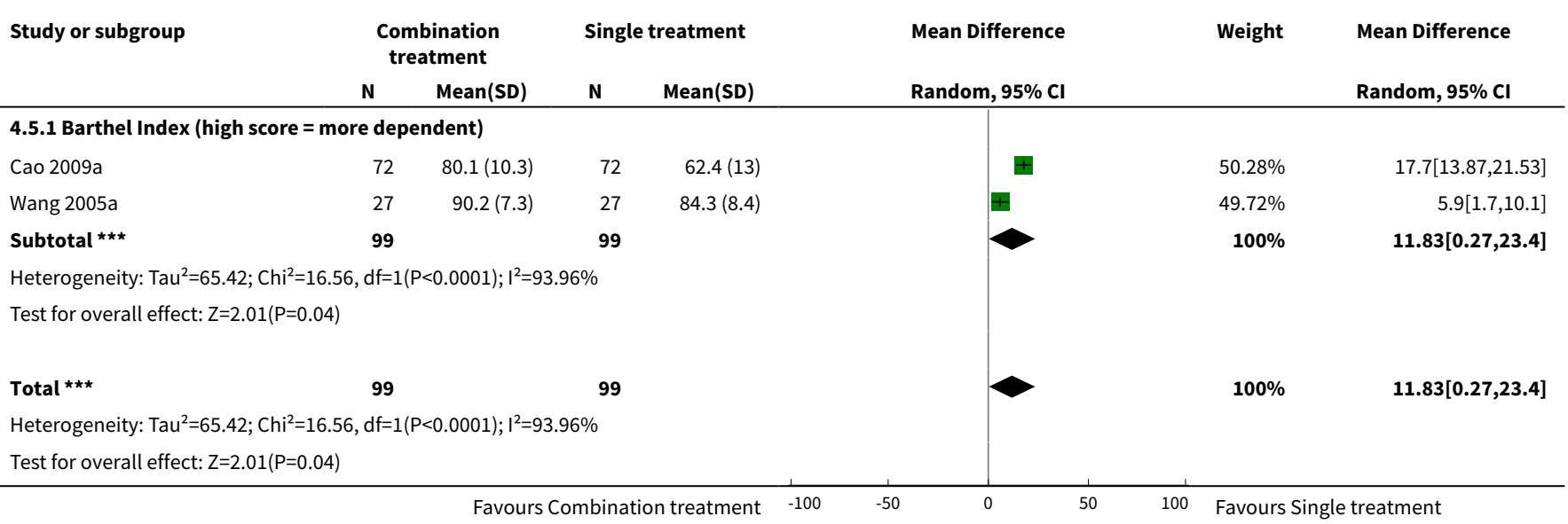

Analysis 4.6. Comparison 4 Pharmacological intervention and psychotherapy (combination) versus a pharmacological intervention and usual care or attention control (single), Outcome 6 Neurological function: mean scores at end of treatment.

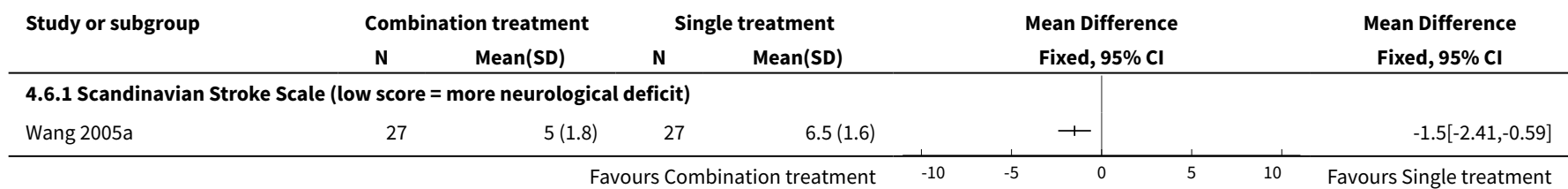

Analysis 4.7. Comparison 4 Pharmacological intervention and psychotherapy (combination) versus a pharmacological intervention and usual care or attention control (single), Outcome 7 Adverse events: death.

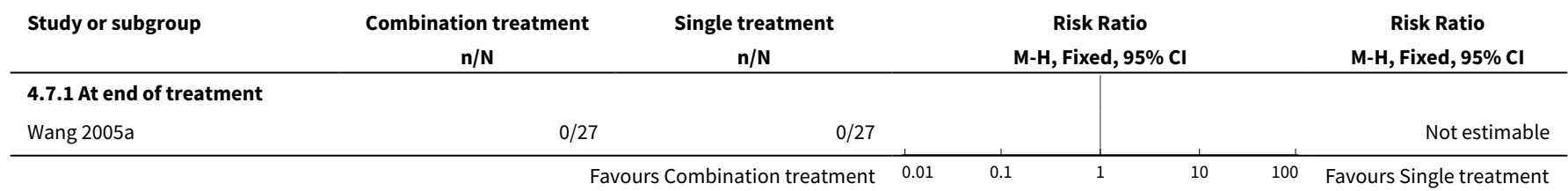

Analysis 4.8. Comparison 4 Pharmacological intervention and psychotherapy (combination) versus a pharmacological intervention and usual care or attention control (single), Outcome 8 Adverse events: all.

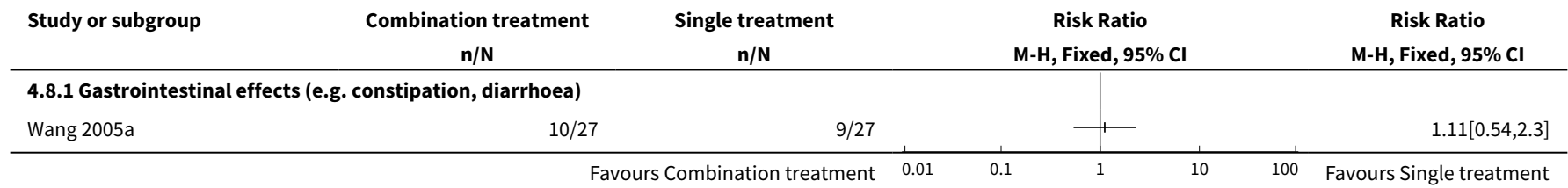


Analysis 4.9. Comparison 4 Pharmacological intervention and psychotherapy (combination) versus a pharmacological intervention and usual care or attention control (single), Outcome 9 Adverse events: leaving the study early (including death).

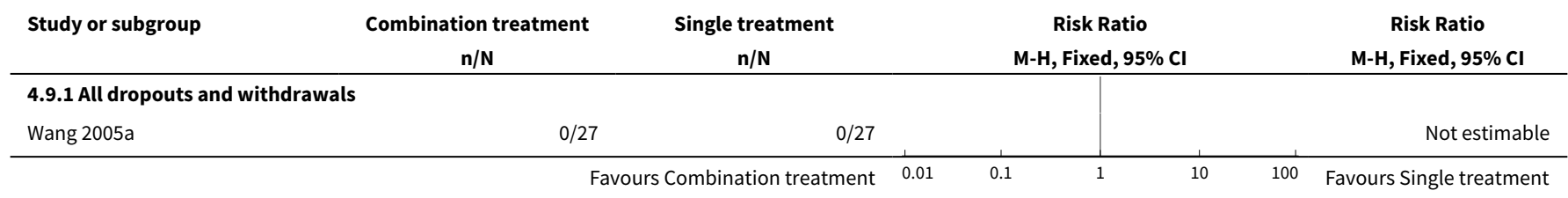

Comparison 5. Non-invasive brain stimulation and a pharmacological intervention (combination) versus a pharmacological intervention and sham stimulation or usual care (single)

\begin{tabular}{|c|c|c|c|c|}
\hline Outcome or subgroup title & No. of studies & $\begin{array}{l}\text { No. of partici- } \\
\text { pants }\end{array}$ & Statistical method & Effect size \\
\hline $\begin{array}{l}1 \text { Depression: meeting the criteria } \\
\text { for depression at end of treatment }\end{array}$ & 1 & & Risk Ratio (M-H, Fixed, 95\% CI) & $\begin{array}{l}\text { Totals not select- } \\
\text { ed }\end{array}$ \\
\hline $\begin{array}{l}1.1 \text { HDRS (high score = more de- } \\
\text { pressed) }\end{array}$ & 1 & & Risk Ratio (M-H, Fixed, 95\% Cl) & $0.0[0.0,0.0]$ \\
\hline $\begin{array}{l}2 \text { Depression: }<50 \% \text { reduction in } \\
\text { scale scores at end of treatment }\end{array}$ & 1 & & Risk Ratio (M-H, Fixed, 95\% Cl) & $\begin{array}{l}\text { Totals not select- } \\
\text { ed }\end{array}$ \\
\hline $2.1 \mathrm{HDRS}$ & 1 & & Risk Ratio (M-H, Fixed, 95\% Cl) & $0.0[0.0,0.0]$ \\
\hline $\begin{array}{l}3 \text { Depression: mean scores at end of } \\
\text { treatment }\end{array}$ & 9 & 685 & $\begin{array}{l}\text { Mean Difference (IV, Random, } \\
95 \% \mathrm{Cl} \text { ) }\end{array}$ & $-4.09[-5.61,-2.57]$ \\
\hline $\begin{array}{l}\text { 3.1 HDRS (high score = more de- } \\
\text { pressed) }\end{array}$ & 9 & 685 & $\begin{array}{l}\text { Mean Difference (IV, Random, } \\
95 \% \mathrm{CI} \text { ) }\end{array}$ & $-4.09[-5.61,-2.57]$ \\
\hline $\begin{array}{l}4 \text { Depression: mean scores at end of } \\
\text { follow-up }\end{array}$ & 1 & & $\begin{array}{l}\text { Mean Difference (IV, Fixed, 95\% } \\
\mathrm{Cl})\end{array}$ & $\begin{array}{l}\text { Totals not select- } \\
\text { ed }\end{array}$ \\
\hline $\begin{array}{l}4.1 \text { HDRS (high score = more de- } \\
\text { pressed) }\end{array}$ & 1 & & $\begin{array}{l}\text { Mean Difference (IV, Fixed, 95\% } \\
\mathrm{Cl} \text { ) }\end{array}$ & $0.0[0.0,0.0]$ \\
\hline $\begin{array}{l}5 \text { Cognitive function: mean scores at } \\
\text { end of treatment }\end{array}$ & 1 & & $\begin{array}{l}\text { Mean Difference (IV, Fixed, 95\% } \\
\mathrm{Cl} \text { ) }\end{array}$ & $\begin{array}{l}\text { Totals not select- } \\
\text { ed }\end{array}$ \\
\hline $\begin{array}{l}5.1 \mathrm{MMSE} \text { (low score = cognitive im- } \\
\text { pairment) }\end{array}$ & 1 & & $\begin{array}{l}\text { Mean Difference (IV, Fixed, 95\% } \\
\mathrm{CI} \text { ) }\end{array}$ & $0.0[0.0,0.0]$ \\
\hline $\begin{array}{l}6 \text { Activities of daily living: mean } \\
\text { scores at end of treatment }\end{array}$ & 5 & 403 & $\begin{array}{l}\text { Std. Mean Difference (IV, Ran- } \\
\text { dom, } 95 \% \mathrm{CI} \text { ) }\end{array}$ & $2.03[1.21,2.85]$ \\
\hline $\begin{array}{l}\text { 6.1 Barthel Index (high score }=\text { more } \\
\text { dependent) }\end{array}$ & 3 & 243 & $\begin{array}{l}\text { Std. Mean Difference (IV, Ran- } \\
\text { dom, } 95 \% \mathrm{CI} \text { ) }\end{array}$ & $2.49[1.78,3.19]$ \\
\hline $\begin{array}{l}\text { 6.2 ADL (high score = more impair- } \\
\text { ment) }\end{array}$ & 2 & 160 & $\begin{array}{l}\text { Std. Mean Difference (IV, Ran- } \\
\text { dom, } 95 \% \mathrm{CI} \text { ) }\end{array}$ & $1.33[-0.28,2.94]$ \\
\hline
\end{tabular}




\begin{tabular}{|c|c|c|c|c|}
\hline Outcome or subgroup title & No. of studies & $\begin{array}{l}\text { No. of partici- } \\
\text { pants }\end{array}$ & Statistical method & Effect size \\
\hline $\begin{array}{l}7 \text { Disability: mean scores at end of } \\
\text { treatment }\end{array}$ & 2 & 180 & $\begin{array}{l}\text { Mean Difference (IV, Random, } \\
95 \% \mathrm{CI})\end{array}$ & $\begin{array}{l}-10.02[-20.14 \\
0.11]\end{array}$ \\
\hline 7.1 SDS (high score = more disability & 2 & 180 & $\begin{array}{l}\text { Mean Difference (IV, Random, } \\
95 \% \mathrm{CI})\end{array}$ & $\begin{array}{l}-10.02[-20.14 \\
0.11]\end{array}$ \\
\hline $\begin{array}{l}8 \text { Neurological function: mean } \\
\text { scores at end of treatment }\end{array}$ & 4 & 280 & $\begin{array}{l}\text { Mean Difference (IV, Random, } \\
95 \% \mathrm{CI})\end{array}$ & $-2.78[-4.13,-1.44]$ \\
\hline $\begin{array}{l}8.1 \mathrm{NIHSS} \text { (high score = more impair- } \\
\text { ment) }\end{array}$ & 4 & 280 & $\begin{array}{l}\text { Mean Difference (IV, Random, } \\
95 \% \mathrm{CI} \text { ) }\end{array}$ & $-2.78[-4.13,-1.44]$ \\
\hline 9 Adverse events: death & 3 & 220 & $\begin{array}{l}\text { Risk Ratio (M-H, Random, 95\% } \\
\mathrm{Cl} \text { ) }\end{array}$ & $0.0[0.0,0.0]$ \\
\hline 9.1 At end of treatment & 3 & 220 & $\begin{array}{l}\text { Risk Ratio (M-H, Random, 95\% } \\
\mathrm{Cl} \text { ) }\end{array}$ & $0.0[0.0,0.0]$ \\
\hline 10 Adverse events: all & 2 & 120 & $\begin{array}{l}\text { Risk Ratio (M-H, Random, 95\% } \\
\mathrm{Cl} \text { ) }\end{array}$ & $7.0[0.38,129.93]$ \\
\hline $\begin{array}{l}10.1 \text { Other events - not listed } \\
\text { above (e.g. insomnia, discomfort, } \\
\text { headaches) }\end{array}$ & 2 & 120 & $\begin{array}{l}\text { Risk Ratio (M-H, Random, 95\% } \\
\mathrm{Cl} \text { ) }\end{array}$ & $7.0[0.38,129.93]$ \\
\hline $\begin{array}{l}11 \text { Adverse events: leaving the study } \\
\text { early (including death) }\end{array}$ & 4 & 300 & $\begin{array}{l}\text { Risk Ratio (M-H, Random, 95\% } \\
\mathrm{Cl} \text { ) }\end{array}$ & $1.33[0.32,5.58]$ \\
\hline 11.1 All dropouts and withdrawals & 4 & 300 & $\begin{array}{l}\text { Risk Ratio (M-H, Random, 95\% } \\
\mathrm{Cl} \text { ) }\end{array}$ & $1.33[0.32,5.58]$ \\
\hline
\end{tabular}

\section{Analysis 5.1. Comparison 5 Non-invasive brain stimulation and a pharmacological intervention (combination) versus a pharmacological intervention and sham stimulation or usual care (single), Outcome 1 Depression: meeting the criteria for depression at end of treatment.}

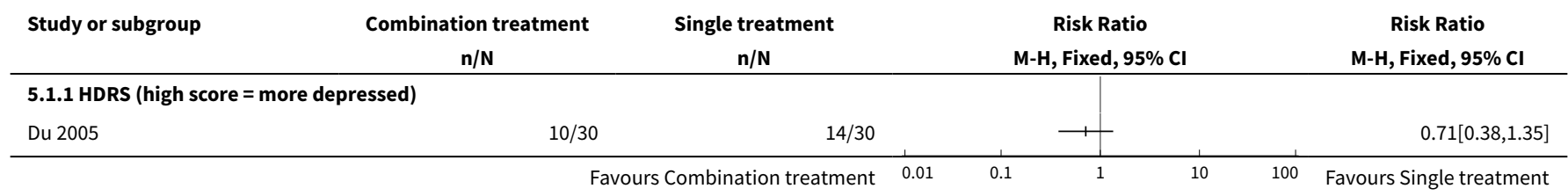


Analysis 5.2. Comparison 5 Non-invasive brain stimulation and a pharmacological intervention (combination) versus a pharmacological intervention and sham stimulation or usual care (single), Outcome 2 Depression: $<50 \%$ reduction in scale scores at end of treatment.

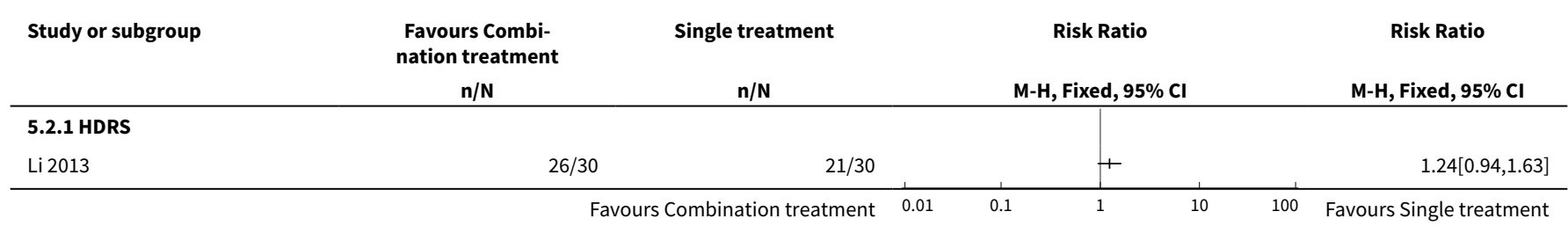

Analysis 5.3. Comparison 5 Non-invasive brain stimulation and a pharmacological intervention (combination) versus a pharmacological intervention and sham stimulation or usual care (single), Outcome 3 Depression: mean scores at end of treatment.

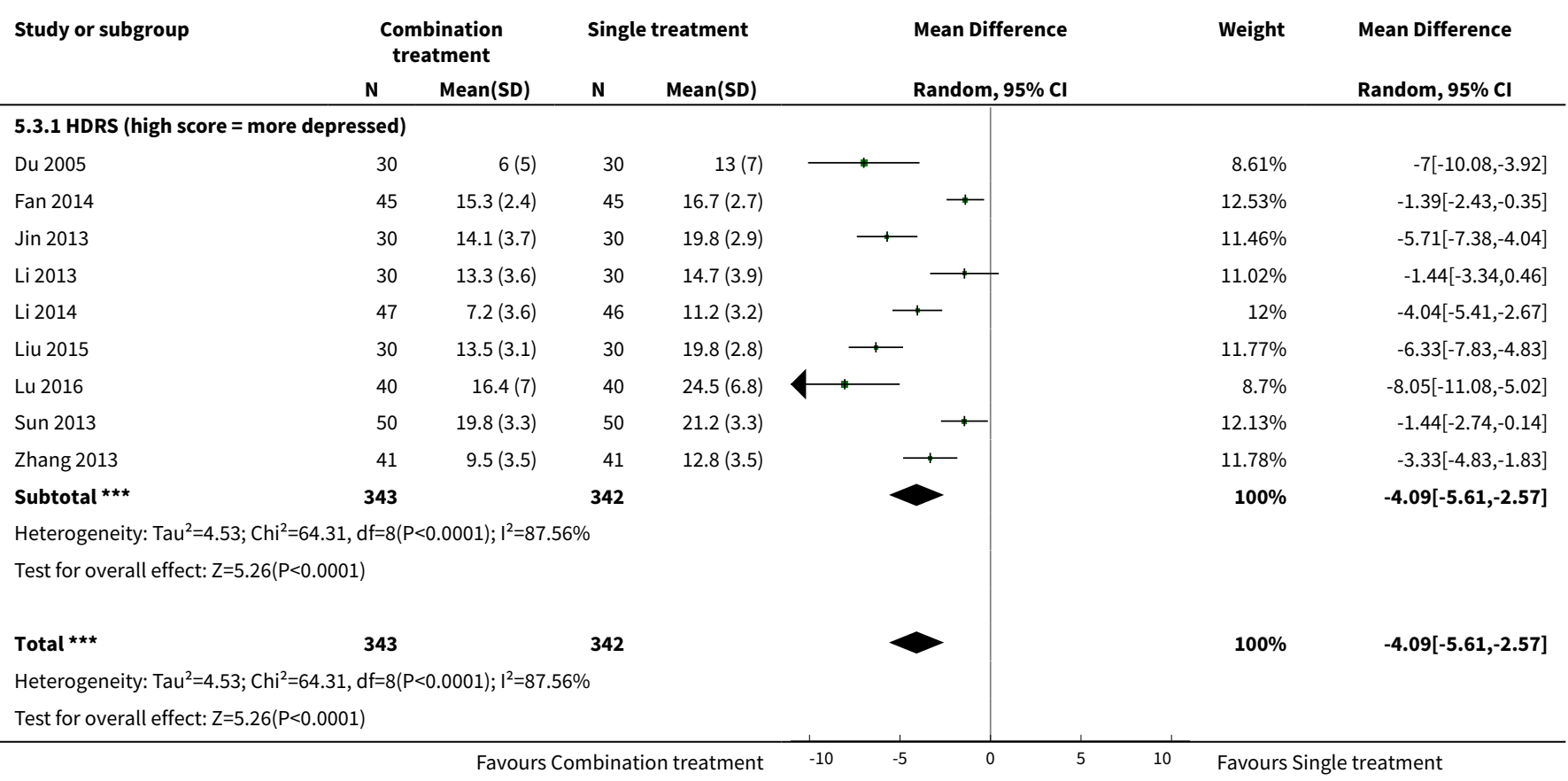

Analysis 5.4. Comparison 5 Non-invasive brain stimulation and a pharmacological intervention (combination) versus a pharmacological intervention and sham stimulation or usual care (single), Outcome 4 Depression: mean scores at end of follow-up.

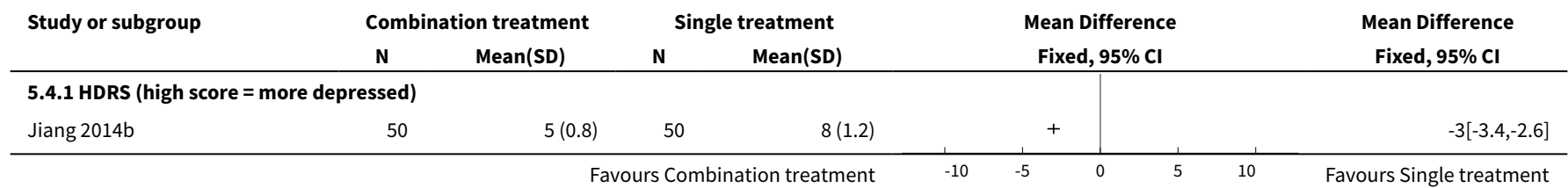


Analysis 5.5. Comparison 5 Non-invasive brain stimulation and a pharmacological intervention (combination) versus a pharmacological intervention and sham stimulation or usual care (single), Outcome 5 Cognitive function: mean scores at end of treatment.

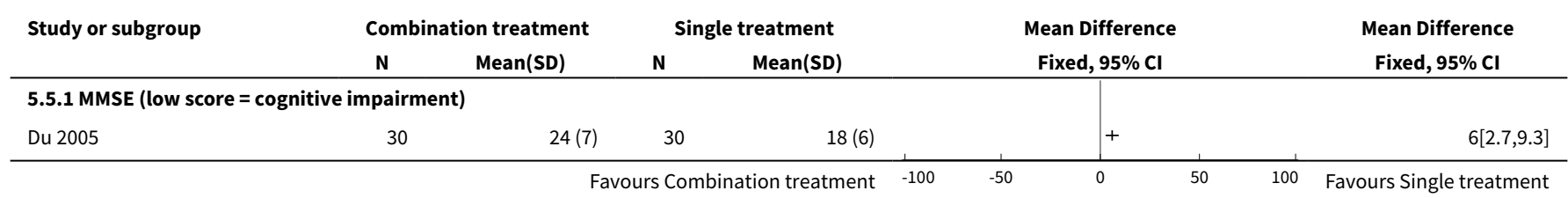

Analysis 5.6. Comparison 5 Non-invasive brain stimulation and a pharmacological intervention (combination) versus a pharmacological intervention and sham stimulation or usual care (single), Outcome 6 Activities of daily living: mean scores at end of treatment.

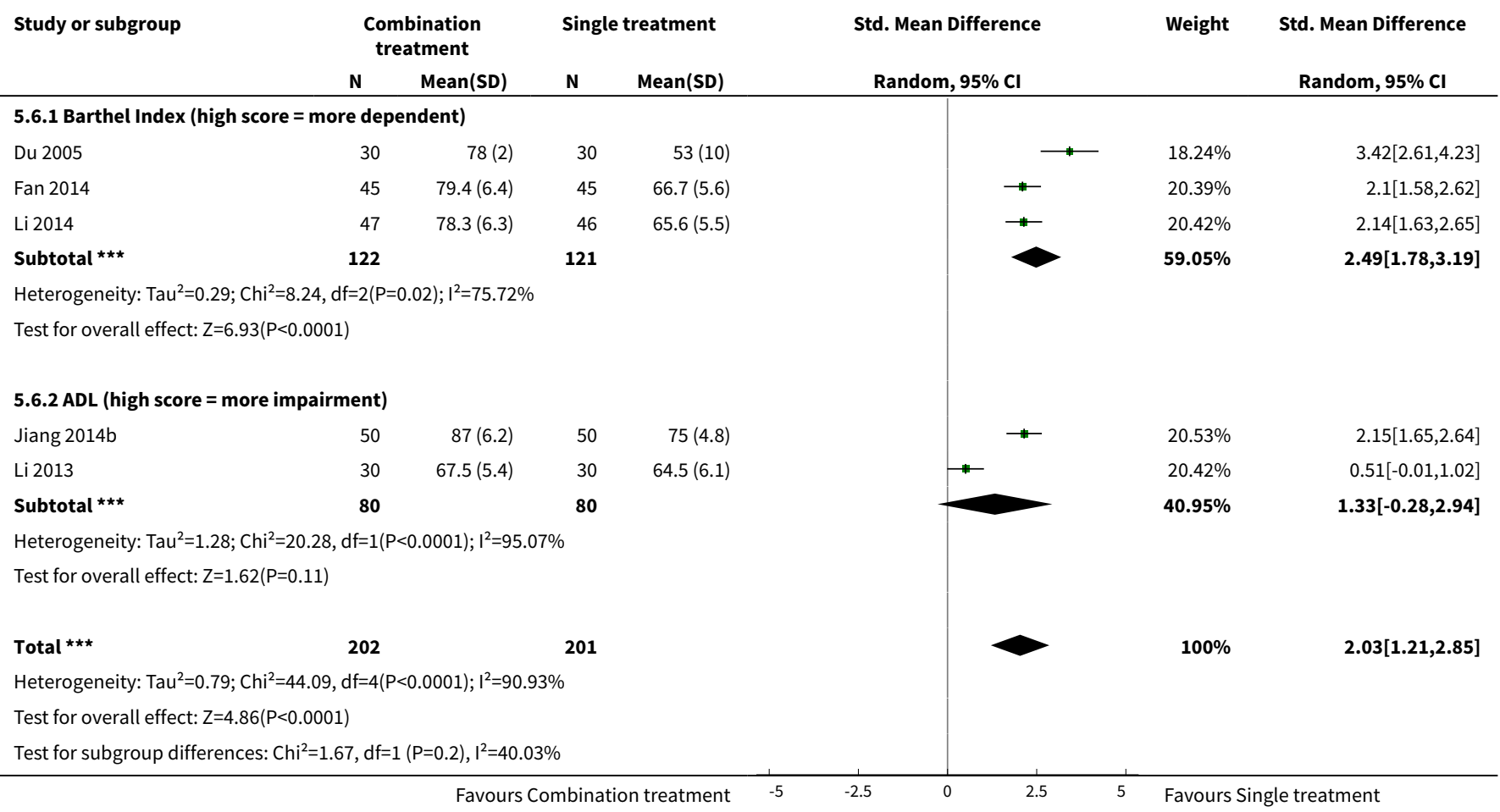

Analysis 5.7. Comparison 5 Non-invasive brain stimulation and a pharmacological intervention (combination) versus a pharmacological intervention and sham stimulation or usual care (single), Outcome 7 Disability: mean scores at end of treatment.

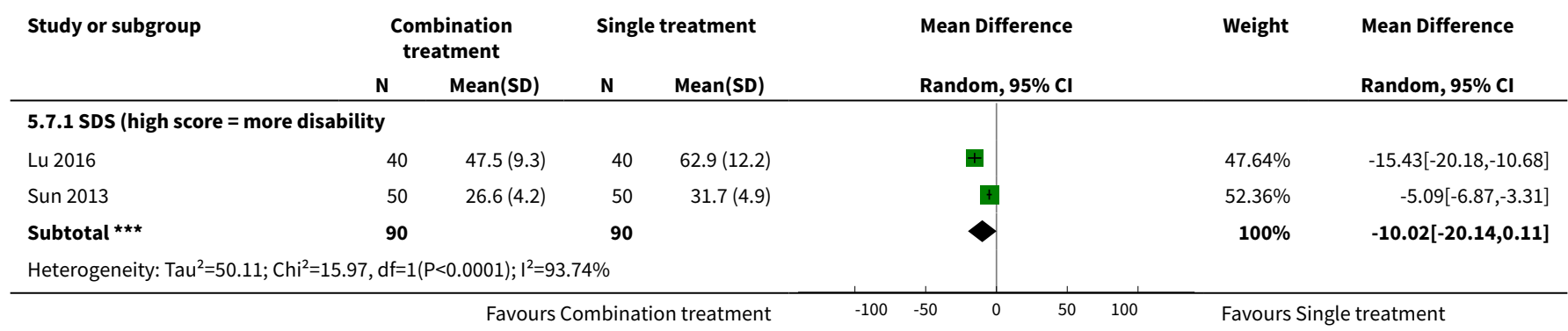




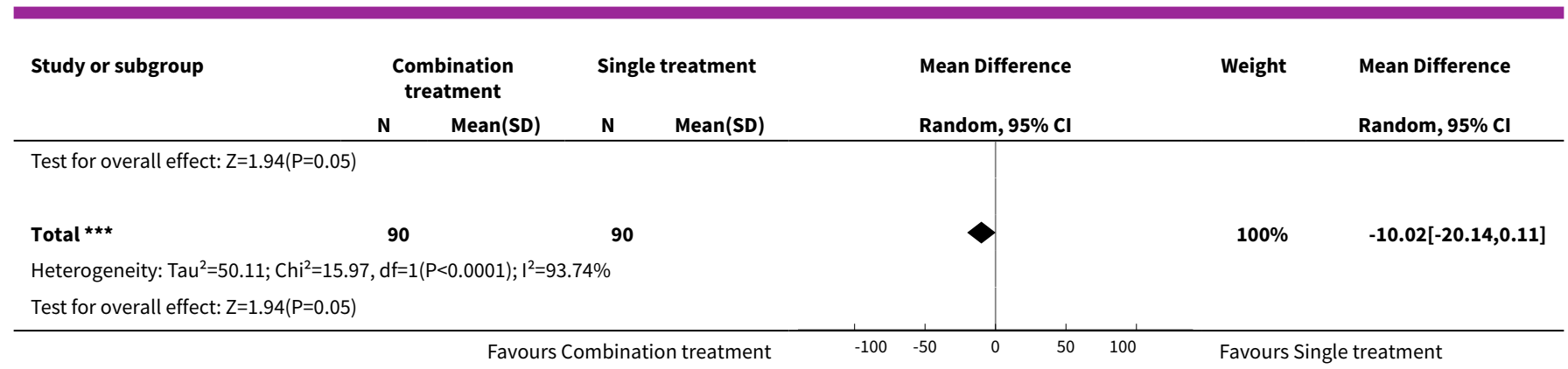

Analysis 5.8. Comparison 5 Non-invasive brain stimulation and a pharmacological intervention (combination) versus a pharmacological intervention and sham stimulation or usual care (single), Outcome 8 Neurological function: mean scores at end of treatment.

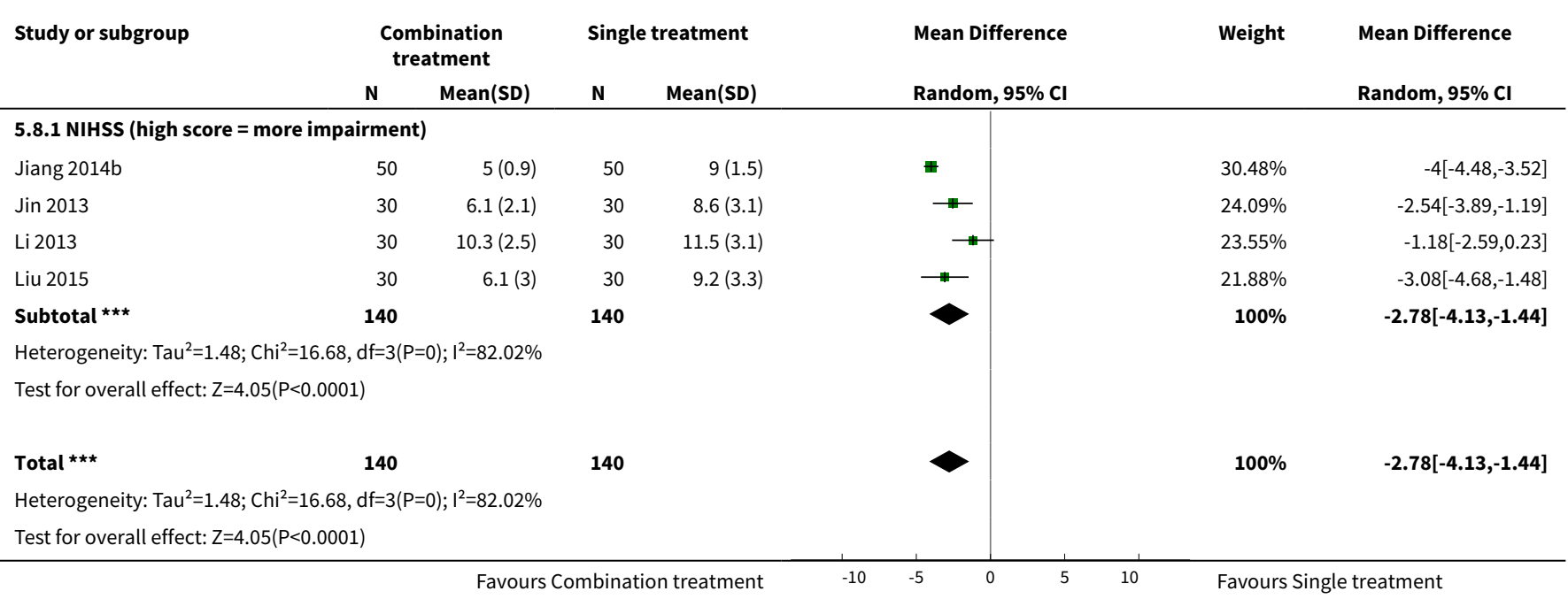

Analysis 5.9. Comparison 5 Non-invasive brain stimulation and a pharmacological intervention (combination) versus a pharmacological intervention and sham stimulation or usual care (single), Outcome 9 Adverse events: death.

\begin{tabular}{|c|c|c|c|c|c|c|}
\hline Study or subgroup & $\begin{array}{c}\text { Combination } \\
\text { treatment } \\
n / N \\
\end{array}$ & $\begin{array}{c}\text { Single } \\
\text { treatment } \\
\mathrm{n} / \mathrm{N}\end{array}$ & & $\begin{array}{c}\text { Risk Ratio } \\
\text { M-H, Random, } 95 \% \mathrm{CI}\end{array}$ & Weight & \multirow[t]{2}{*}{$\begin{array}{c}\text { Risk Ratio } \\
\text { M-H, Random, } 95 \% \mathrm{CI}\end{array}$} \\
\hline \multicolumn{6}{|c|}{ 5.9.1 At end of treatment } & \\
\hline Du 2005 & $0 / 30$ & $0 / 30$ & & & & Not estimable \\
\hline Jiang 2014b & $0 / 50$ & $0 / 50$ & & & & Not estimable \\
\hline Liu 2015 & $0 / 30$ & $0 / 30$ & & & & Not estimable \\
\hline Subtotal $(95 \% \mathrm{Cl})$ & 110 & 110 & & & & Not estimable \\
\hline \multicolumn{7}{|c|}{ Total events: 0 (Combination treatment), 0 (Single treatment) } \\
\hline \multicolumn{7}{|c|}{ Heterogeneity: Not applicable } \\
\hline \multicolumn{7}{|c|}{ Test for overall effect: Not applicable } \\
\hline Total $(95 \% \mathrm{Cl})$ & 110 & 110 & & & & Not estimable \\
\hline \multicolumn{7}{|c|}{ Total events: 0 (Combination treatment), 0 (Single treatment) } \\
\hline
\end{tabular}




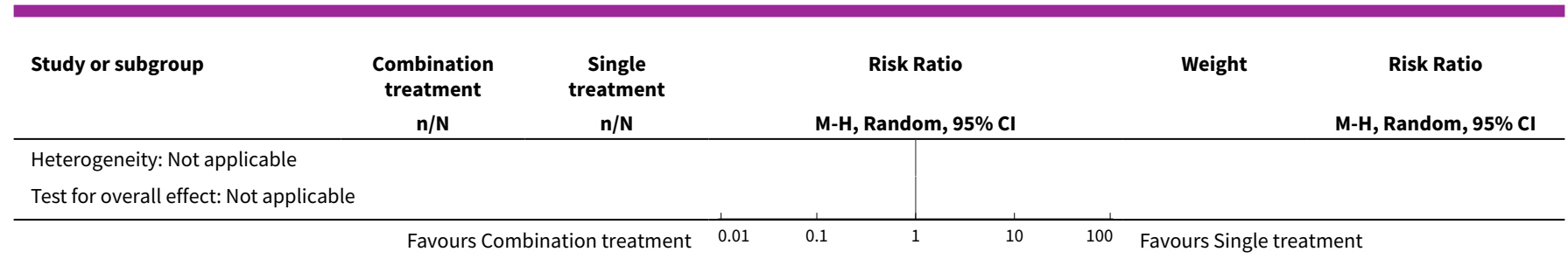

Analysis 5.10. Comparison 5 Non-invasive brain stimulation and a pharmacological intervention (combination) versus a pharmacological intervention and sham stimulation or usual care (single), Outcome 10 Adverse events: all.

\begin{tabular}{|c|c|c|c|c|c|}
\hline Study or subgroup & $\begin{array}{c}\text { Combination } \\
\text { treatment } \\
n / N \\
\end{array}$ & $\begin{array}{c}\text { Single } \\
\text { treatment } \\
\mathrm{n} / \mathrm{N}\end{array}$ & M-H, Random, $95 \% \mathrm{Cl}$ & Weight & $\begin{array}{c}\text { Risk Ratio } \\
\text { M-H, Random, } 95 \% \mathrm{CI}\end{array}$ \\
\hline \multicolumn{3}{|c|}{$\begin{array}{l}5.10 .1 \text { Other events - not listed above (e.g. insomnia, discomfort, } \\
\text { headaches) }\end{array}$} & & & \\
\hline Du 2005 & $0 / 30$ & $0 / 30$ & & & Not estimable \\
\hline Liu 2015 & $3 / 30$ & $0 / 30$ & & $100 \%$ & $7[0.38,129.93]$ \\
\hline Subtotal $(95 \% \mathrm{Cl})$ & 60 & 60 & & $100 \%$ & $7[0.38,129.93]$ \\
\hline \multicolumn{6}{|c|}{ Total events: 3 (Combination treatment), 0 (Single treatment) } \\
\hline \multicolumn{6}{|c|}{ Heterogeneity: Not applicable } \\
\hline \multicolumn{6}{|c|}{ Test for overall effect: $Z=1.31(P=0.19)$} \\
\hline Total $(95 \% \mathrm{Cl})$ & 60 & 60 & & $100 \%$ & $7[0.38,129.93]$ \\
\hline \multicolumn{6}{|c|}{ Total events: 3 (Combination treatment), 0 (Single treatment) } \\
\hline \multicolumn{6}{|c|}{ Heterogeneity: Not applicable } \\
\hline \multicolumn{6}{|c|}{ Test for overall effect: $Z=1.31(P=0.19)$} \\
\hline
\end{tabular}

Analysis 5.11. Comparison 5 Non-invasive brain stimulation and a pharmacological intervention (combination) versus a pharmacological intervention and sham stimulation or usual care (single), Outcome 11 Adverse events: leaving the study early (including death).

\begin{tabular}{ccccc} 
Study or subgroup & $\begin{array}{c}\text { Combination } \\
\text { treatment } \\
\mathrm{n} / \mathrm{N}\end{array}$ & $\begin{array}{c}\text { Single } \\
\text { treatment } \\
\mathrm{n} / \mathrm{N}\end{array}$ & Risk Ratio & Weight \\
& & $\mathrm{M}-\mathrm{H}$, Random, 95\% Cl & $\mathrm{M}-\mathrm{H}, \mathrm{Random}, 95 \% \mathrm{Cl}$ \\
\hline
\end{tabular}

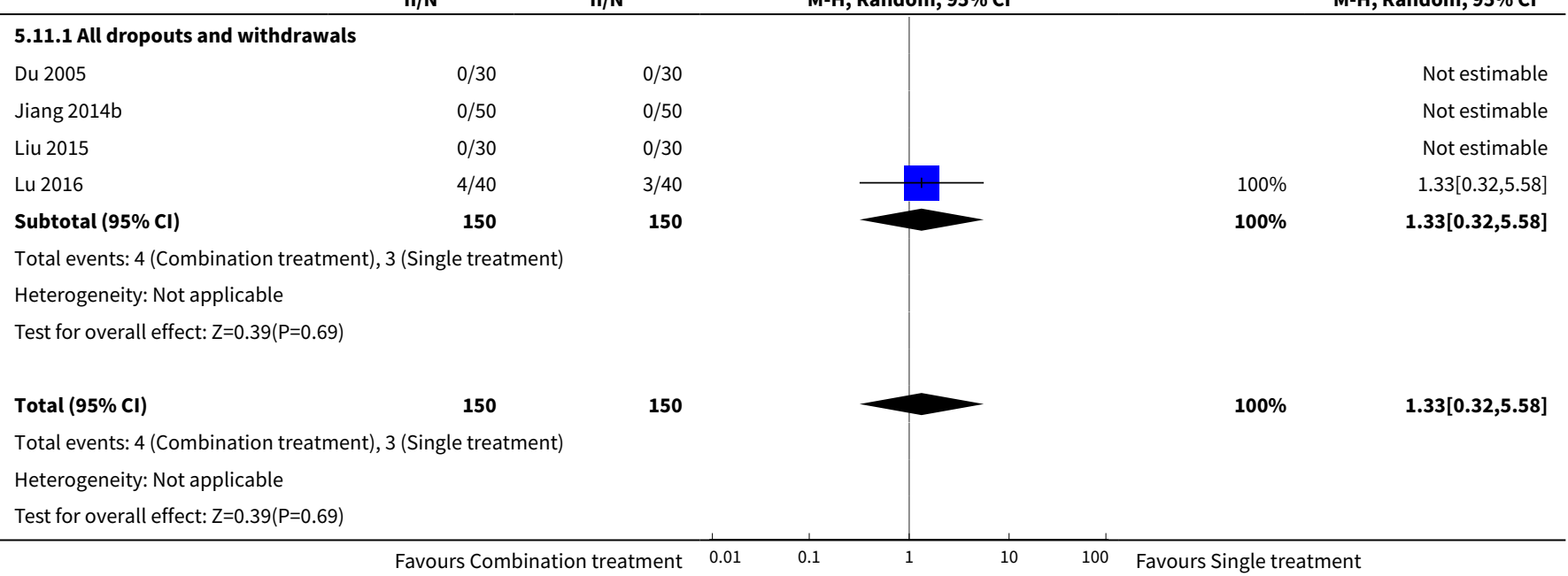


ADDITIONAL TABLES

Table 1. Characteristics of 'dropout' studies

\begin{tabular}{|c|c|c|c|c|c|}
\hline Study ID & Methods & Participants & Interventions & Outcomes & Notes \\
\hline Bramanti 1989 & $\begin{array}{l}\text { Study design: } \\
\text { parallel de- } \\
\text { sign } \\
\text { Number of } \\
\text { arms: } 2 \\
\text { Experimen- } \\
\text { tal arm: pro- } \\
\text { tirelin tartrate } \\
\text { (TRH-T) } \\
\text { Control arm: } \\
\text { placebo }\end{array}$ & $\begin{array}{l}\text { Geographical location: Italy } \\
\text { Setting: unclear } \\
\text { Number of participants: } 30 \\
\text { Stroke criteria: acute stroke } \\
\text { Method of stroke diagnosis: not re- } \\
\text { ported } \\
\text { Inclusion criteria: not reported } \\
\text { Exclusion criteria: not reported } \\
\text { Depression criteria: not reported } \\
\text { Total number included in this tri- } \\
\text { al: unclear (63\% men, mean age } \\
72.2, \text { SD not reported for the overall } \\
\text { cohort) } \\
\text { Number included in treatment } \\
\text { group: unclear } \\
\text { Number included in control } \\
\text { group: unclear }\end{array}$ & $\begin{array}{l}\text { Treatment: protirelin } \\
\text { tartrate (TRH-T) } 2 \mathrm{mg} / \\
\mathrm{d} \\
\text { Control: placebo } \\
\text { Duration: } 2 \text { weeks } \\
\text { Follow-up: none }\end{array}$ & $\begin{array}{l}\text { Depression } \\
\text { measured } \\
\text { using HDRS }\end{array}$ & $\begin{array}{l}\text { Results not } \\
\text { available in } \\
\text { format suit- } \\
\text { able for this } \\
\text { review }\end{array}$ \\
\hline Chang 2011 & $\begin{array}{l}\text { Study design: } \\
\text { parallel de- } \\
\text { sign } \\
\text { Number of } \\
\text { arms: } 2 \\
\text { Experimental } \\
\text { arm: rational } \\
\text { emotive be- } \\
\text { haviour ther- } \\
\text { apy (REBT) + } \\
\text { usual care } \\
\text { Control arm: } \\
\text { usual care }\end{array}$ & $\begin{array}{l}\text { Geographical location: China } \\
\text { Setting: inpatient } \\
\text { Number of participants: } 16 \\
\text { Stroke criteria: ischaemic strokes } \\
\text { Method of stroke diagnosis: diag- } \\
\text { nosis confirmed by imaging } \\
\text { Inclusion criteria: not reported } \\
\text { Exclusion criteria: (1) history of } \\
\text { mental illness; (2) cognitive impair- } \\
\text { ment; (3) severe aphasia; (4) > } 2 \\
\text { weeks post stroke } \\
\text { Depression criteria: Chinese ver- } \\
\text { sion of HDRS score } \geq 35 \\
\text { Total number included in this tri- } \\
\text { al: } 16 \text { (\% men and age unknown) } \\
\text { Number included in treatment } \\
\text { group: } 8 \\
\text { Number included in control }\end{array}$ & $\begin{array}{l}\text { Treatment: REBT + } \\
\text { usual care. REBT coun- } \\
\text { selling therapy (1 to } 2 \\
\text { hour sessions/week) } \\
\text { consisting of a knowl- } \\
\text { edge component (ed- } \\
\text { ucation about health } \\
\text { psychology and recov- } \\
\text { ery from hemiplegic } \\
\text { stroke) and a behav- } \\
\text { ioural training compo- } \\
\text { nent (belief changes, } \\
\text { forgiveness training, } \\
\text { anger management) } \\
\text { Administered by: a } \\
\text { trained psychology } \\
\text { graduate (regular care } \\
\text { administered by hos- } \\
\text { pital nurses) } \\
\text { Supervision: unclear } \\
\text { Intervention fidelity: } \\
\text { not reported }\end{array}$ & 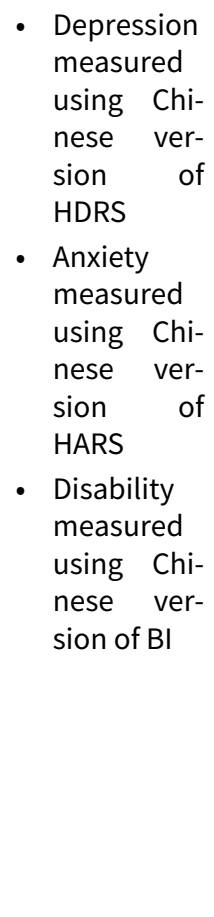 & $\begin{array}{l}\text { Unable to iso- } \\
\text { late outcome } \\
\text { data for those } \\
\text { with depres- } \\
\text { sion at ran- } \\
\text { domisation }\end{array}$ \\
\hline
\end{tabular}

Control: usual care 
Table 1. Characteristics of 'dropout' studies (Continued)

Duration: 1 month

\begin{tabular}{|c|c|c|c|c|c|}
\hline $\begin{array}{l}\text { Choi-Kwon } \\
2006\end{array}$ & $\begin{array}{l}\text { Study design: } \\
\text { parallel de- } \\
\text { sign } \\
\text { Number of } \\
\text { arms: } 2 \\
\text { Experimental } \\
\text { arm: fluoxe- } \\
\text { tine (SSRI) } \\
\text { Control arm: } \\
\text { matched } \\
\text { placebo }\end{array}$ & $\begin{array}{l}\text { Geographical location: South Ko- } \\
\text { rea } \\
\text { Setting: outpatients } \\
\text { Number of participants: } 152 \\
\text { Stroke criteria: ischaemic stroke } \\
\text { Method of stroke diagnosis: diag- } \\
\text { nosis via CT and MRI scans; inter- } \\
\text { view performed on average of } 14 \\
\text { months after stroke } \\
\text { Inclusion criteria: not reported } \\
\text { Exclusion criteria: (1) did not un- } \\
\text { dergo imaging (CT/MRI) studies; ( } 2 \text { ) } \\
\text { SAH; (3) had TIA without progres- } \\
\text { sion to stroke; (4) severe communi- } \\
\text { cation problems (aphasia, demen- } \\
\text { tia, or dysarthria); (5) scored < } 23 \text { on } \\
\text { MMSE; (6) history of depression or } \\
\text { psychiatric illness before onset of } \\
\text { stroke; ( } 7 \text { ) already treated with psy- } \\
\text { chiatric regimens; (8) lived alone } \\
\text { Depression criteria: psychiatric in- } \\
\text { terview, BDI score > 13 } \\
\text { Total number included in this tri- } \\
\text { al: } 152 \\
\text { Number included in treatment } \\
\text { group: } 76 \text { ( } 75 \% \text { men, mean age } 58 \\
\text { years, SD 9) } \\
\text { Number included in control } \\
\text { group: } 76 \text { ( } 79 \% \text { men, mean age } 58 \\
\text { years, SD 9) }\end{array}$ & $\begin{array}{l}\text { Treatment: fluoxetine } \\
\text { (SSRI) } 20 \text { mg daily } \\
\text { Control: matched } \\
\text { placebo } \\
\text { Duration: } 3 \text { months }\end{array}$ & $\begin{array}{l}\text { - Depression } \\
\text { measured } \\
\text { using BDI } \\
\text { - Leaving the } \\
\text { study early } \\
\text { - Adverse } \\
\text { events }\end{array}$ & $\begin{array}{l}\text { Unable to iso- } \\
\text { late outcome } \\
\text { data for those } \\
\text { with depres- } \\
\text { sion at ran- } \\
\text { domisation }\end{array}$ \\
\hline Delbari 2011 & $\begin{array}{l}\text { Study design: } \\
\text { parallel de- } \\
\text { sign } \\
\text { Number of } \\
\text { arms: } 4 \\
\text { Experimen- } \\
\text { tal arm A: } \\
\text { methylphenidate } \\
+ \text { placebo } \\
\text { Experimen- } \\
\text { tal arm B: } \\
\text { levodopa + } \\
\text { placebo } \\
\text { Experimen- } \\
\text { tal arm C: } \\
\text { methylphenidate } \\
+ \text { levodopa } \\
\text { Control arm: } \\
2 \times 10 \text { mg }\end{array}$ & $\begin{array}{l}\text { Geographical location: Iran } \\
\text { Setting: inpatient } \\
\text { Number of participants: } 78 \\
\text { Stroke criteria: ischaemic stroke } \\
\text { Method of stroke diagnosis: not re- } \\
\text { ported } \\
\text { Inclusion criteria: (1) only patients } \\
\text { with limb (arm or leg) paresis } \\
\text { Exclusion criteria: (1) unable to re- } \\
\text { spond or directly consent; (2) co- } \\
\text { morbidities requiring strict blood } \\
\text { pressure control and put at risk by } \\
\text { the potential of hypertension from } \\
\text { MPH therapy (history of haemor- } \\
\text { rhagic stroke, recent myocardial in- } \\
\text { farction within 4-week period, de- } \\
\text { compensated cardiac insufficien- } \\
\text { cy, tachycardia, uncontrolled hy- }\end{array}$ & $\begin{array}{l}\text { Treatment A: } 2 \times 10 \\
\text { mg methylphenidate }+ \\
125 \text { mg placebo (con- } \\
\text { tent unknown) } \\
\text { Treatment B: } 1 \times 12.5 \\
\text { mg levodopa }+2 \times 10 \\
\text { mg placebo } \\
\text { Treatment C: } 2 \times 10 \\
\text { mg methylphenidate + } \\
1 \times 125 \text { mg levodopa } \\
\text { Control: } 2 \times 10 \text { mg } \\
\text { placebo }+1 \times 125 \text { mg } \\
\text { placebo } \\
\text { Duration: } 5 \text { days a } \\
\text { week for a total of } 15 \\
\text { sessions }\end{array}$ & $\begin{array}{l}\text { Depression } \\
\text { measured } \\
\text { using GDS } \\
\text { - Cognitive } \\
\text { function } \\
\text { measured } \\
\text { using } \\
\text { MMSE }\end{array}$ & $\begin{array}{l}\text { Unable to iso- } \\
\text { late outcome } \\
\text { data for those } \\
\text { with depres- } \\
\text { sion at ran- } \\
\text { domisation }\end{array}$ \\
\hline
\end{tabular}


Table 1. Characteristics of 'dropout' studies (Continued) placebo $+1 \times \quad$ pertension, unstable metabolic $125 \mathrm{mg}$ place- disease, glaucoma); (3) potential bo

for adverse outcomes from stimulant effects of MPH, including seizure and agitation major cognitive deficits preventing adequate study participation; (4) currently taking alpha-adrenergic agonists, antagonists, neuroleptics, benzodiazepines, MAO inhibitors, or antidepressants; (5) known hypersensitivity to MPH or LD

\section{Depression criteria: GDS $<7.8$}

Total number included in this trial: 78

Number included in Treatment A:

19 (47\% men, mean age 64.05 , SD

$10.8)$

Number included in Treatment B: 20 (70\% men, mean age 66.3, SD 9.5)

Number included in Treatment C: 19 (58\% men, 60.2, SD 9.1)

Number included in control group: 20 (70\% men, mean age 65.3, SD 9.6)

\begin{tabular}{|c|c|c|c|c|c|}
\hline Downes 1995 & $\begin{array}{l}\text { Study design: } \\
\text { parallel de- } \\
\text { sign } \\
\text { Number of } \\
\text { arms: } 3 \\
\text { Experimen- } \\
\text { tal arm 1: in- } \\
\text { formation + } \\
\text { counselling } \\
\text { Experimental } \\
\text { arm 2: infor- } \\
\text { mation pack } \\
\text { Control arm: } \\
\text { standard care }\end{array}$ & $\begin{array}{l}\text { Geographical location: UK } \\
\text { Setting: outpatient } \\
\text { Number of participants: } 62 \\
\text { Stroke criteria: not reported } \\
\text { Method of stroke diagnosis: not re- } \\
\text { ported } \\
\text { Inclusion criteria: (1) lived at } \\
\text { home; (2) had an informal carer; } \\
\text { (3) stroke increase in mRS; (4) post- } \\
\text { stroke mRS score of } 2 \text { to } 5 \\
\text { Exclusion criteria: (1) not living at } \\
\text { home; (2) not having an informal } \\
\text { carer; (3) having no increase in dis- } \\
\text { ability or change in lifestyle/depen- } \\
\text { dency } \\
\text { Total number included in this tri- } \\
\text { al: } 62 \\
\text { Number included in treatment 1: } \\
22 \text { (50\% men, age not reported) } \\
\text { Number included in treatment 2: } \\
22 \text { (55\% men, age not reported) } \\
\text { Number included in control } \\
\text { group: } 18 \text { (44\% men, age not re- } \\
\text { ported) }\end{array}$ & $\begin{array}{l}\text { Treatment 1: infor- } \\
\text { mation plus coun- } \\
\text { selling. Egan's prob- } \\
\text { lem-solving approach, } \\
\text { individual is helped } \\
\text { to explore concerns, } \\
\text { clarify problems, set } \\
\text { goals, and take appro- } \\
\text { priate action. Protocol } \\
\text { discussed first and for- } \\
\text { mulated into a coun- } \\
\text { sellor/client contract. } \\
\text { Information pack con- } \\
\text { taining information } \\
\text { on physical, cognitive, } \\
\text { behavioural, and emo- } \\
\text { tional effects of stroke, } \\
\text { carer well-being, and } \\
\text { local services } \\
\text { Treatment 2: infor- } \\
\text { mation only: informa- } \\
\text { tion pack containing } \\
\text { information on physi- } \\
\text { cal, cognitive, behav- } \\
\text { ioural, and emotional } \\
\text { effects of stroke, carer } \\
\text { well-being, and local } \\
\text { services } \\
\text { Control: standard } \\
\text { care, no visit(s) or in- }\end{array}$ & $\begin{array}{l}\text { - Depression } \\
\text { measured } \\
\text { using } \\
\text { HADS- } \\
\text { Depression } \\
\text { - Anxiety } \\
\text { measured } \\
\text { using } \\
\text { HADS- } \\
\text { Anxiety }\end{array}$ & $\begin{array}{l}\text { Unable to iso- } \\
\text { late outcome } \\
\text { data for those } \\
\text { with depres- } \\
\text { sion at ran- } \\
\text { domisation }\end{array}$ \\
\hline
\end{tabular}


Table 1. Characteristics of 'dropout' studies (Continued)

formation pack pro-

vided

Duration: informa-

tion session consist-

ed of 1 visit and pro-

vision of the informa-

tion pack. Counselling

consisted of up to 8

counselling sessions

over 4 to 6 months

Delivered by: nurse

counsellor

\begin{tabular}{|c|c|c|c|c|c|}
\hline Hadidi 2014 & $\begin{array}{l}\text { Study design: } \\
\text { parallel de- } \\
\text { sign } \\
\text { Number of } \\
\text { arms: } 2 \\
\text { Experimen- } \\
\text { tal arm: prob- } \\
\text { lem-solving } \\
\text { therapy (PST) } \\
\text { Control arm: } \\
\text { weekly tele- } \\
\text { phone calls }\end{array}$ & $\begin{array}{l}\text { Geographical location: USA } \\
\text { Setting: inpatient } \\
\text { Number of participants: } \\
\text { Stroke criteria: first-time diagnosis } \\
\text { of ischaemic stroke < } 48 \text { hours } \\
\text { Method of stroke diagnosis: not re- } \\
\text { ported } \\
\text { Inclusion criteria: (1) Mini-Cog } \\
\text { score of } 3 ; \geq 50 \text { years of age; ( } 2 \text { ) able } \\
\text { to read and write in English } \\
\text { Exclusion criteria: (1) previous his- } \\
\text { tory of mental health problems; (2) } \\
\text { diagnosis of severe aphasia as iden- } \\
\text { tified by a speech pathologist; (3) } \\
\text { haemorrhagic stroke or transient is- } \\
\text { chaemic attack; (4) medical instabil- } \\
\text { ity requiring transfer to critical care } \\
\text { Depression criteria: CES-D score } \\
\text { measured at baseline but patients } \\
\text { recruited regardless of their CES-D } \\
\text { score. If CES-D score > } 10 \text {, or suicidal } \\
\text { ideation, the primary physician was } \\
\text { notified } \\
\text { Total number included in this tri- } \\
\text { al: } 22 \\
\text { Number included in treatment } \\
\text { group: } 11 \text { ( } 18 \% \text { men, mean age } 73 \text { ) } \\
\text { Number included in control } \\
\text { group: } 11 \text { ( } 45 \% \text { men, mean age } 69 \text { ) }\end{array}$ & $\begin{array}{l}\text { Treatment: 1-on-1 } \\
\text { problem-solving ther- } \\
\text { apy sessions lasting } \\
1 \text { to } 2 \text { hours. Therapy } \\
\text { entails providing pa- } \\
\text { tient information on } \\
\text { impact and guidance } \\
\text { to enable the patient } \\
\text { to identify and define } \\
\text { the problem; brain- } \\
\text { storm all potential } \\
\text { solutions; select the } \\
\text { most appropriate and } \\
\text { feasible solution; cre- } \\
\text { ate and implement a } \\
\text { SMART (Specific, Mea- } \\
\text { sureable, Achievable, } \\
\text { Realistic, and Timely) } \\
\text { goal; evaluate and re- } \\
\text { view progress in fol- } \\
\text { low-up sessions } \\
\text { Administered by: a } \\
\text { doctoral nursing stu- } \\
\text { dent who received PST } \\
\text { training through a 13- } \\
\text { module online pro- } \\
\text { gram adapted from } \\
\text { standard 3-day in-per- } \\
\text { son training } \\
\text { Supervision: principal } \\
\text { Investigator who had } \\
\text { undergone in-person } \\
\text { PST training } \\
\text { Intervention fidelity: } \\
\text { not reported } \\
\text { Control: weekly tele- } \\
\text { phone calls to assess } \\
\text { CES-D and FIM scores } \\
\text { Duration: once per } \\
\text { week for } 10 \text { weeks }\end{array}$ & $\begin{array}{l}\text { Depression } \\
\text { measured } \\
\text { using CES- } \\
\text { D } \\
\text { - Impair- } \\
\text { ment mea- } \\
\text { sured using } \\
\text { FIM } \\
\text { - Leaving the } \\
\text { trial early }\end{array}$ & $\begin{array}{l}\text { Unable to iso- } \\
\text { late outcome } \\
\text { data for those } \\
\text { with depres- } \\
\text { sion at ran- } \\
\text { domisation }\end{array}$ \\
\hline
\end{tabular}


Table 1. Characteristics of 'dropout' studies (Continued)

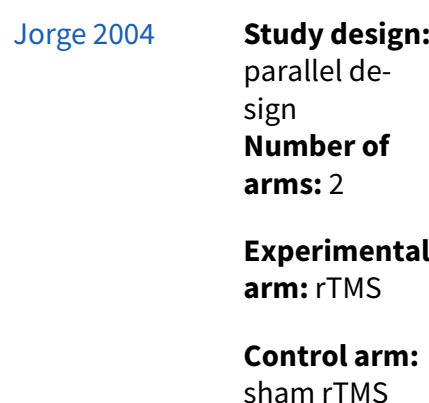
confirmed by imaging

\section{Geographical location: USA Setting: outpatient Number of participants: 20}

Stroke criteria: ischaemic stroke

Method of stroke diagnosis: clinical diagnosis of ischaemic stroke

\section{Inclusion criteria: not reported}

Exclusion criteria: (1) haemorrhagic stroke; (2) clinical evidence of dementia MMSE scores < 23; (3) aphasia with severe language comprehension deficits; (4) alcohol or drug abuse during past 12 months; (5) severe systemic disease or ongoing neoplasia; (6) neurodegenerative disorders such as Parkinson's disease or Alzheimer's disease; (7) contraindications to rTMS including prior occurrence of induced seizures; major head trauma; or history of idiopathic epilepsy; presence of metal in the skull, cranial cavity, or brain parenchyma; cardiac pacemaker, implanted defibrillator, or intracardiac lines

Depression criteria: psychiatric diagnosis (i.e. depression due to stroke with major depressive-like episode or research criteria for minor depression) was made using symptoms elicited by a version of the Present State Examination modified to identify DSM-IV symptoms of depression and anxiety disorder; evidence that depression was unresponsive to at least 2 treatments with antidepressants given in adequate doses; clear clinical indication of a significant change in the course or severity of depressive disorder after stroke

\section{Total number included in this tri-} al: 20

Number included in treatment group: 10 (60\% men; mean age 63.1, SD 8.1)

Number included in control group: 10 (50\% men; mean age 66.5, SD 12.2)
Treatment: rTMS delivered over the left pre-frontal cortex at frequency of $10 \mathrm{~Hz}$, intensity of $110 \%$ of the motor threshold, duration of 5 seconds, and total of 20 trains separated by 60 -second pauses. Cumulative rTMS exposure for the $10-\mathrm{Hz}$ stimuli was 5 seconds $\times 20$ per session $\times 10$ sessions $\times 1000$ seconds of cumulative exposure or a total of 10,000 magnetic pulses

Control: sham stimulation: similar stimulation parameters to the rTMS stated but with the coil angled off the head, to produce a $67 \%$ to $73 \%$ reduction in the magnetic field

Administered by: investigators at the ECT facility in the Department of Psychiatry

Duration: 2 weeks
- Depression clinical response (reduction in HDRS total score $\geq 50 \%$ and patient no longer meeting DSM-IV criteria for depression diagnosis)

- Remission of depression (reduction in HDRS total score $\geq 50 \%$ with final HDRS score $<8)$

- Depression measured using 17item HDRS

- Cognitive function measured using MMSE

- Adverse events
Unable to isolate outcome data for those with depression at randomisation

\begin{tabular}{llllll}
\hline Jorge 2008 & Study design: & Geographical location: USA & Treatment A: $10 \mathrm{rT}-$ & - Depression Unable to ob- \\
& parallel de- & MS sessions in the left & - unclear tain informa- \\
& sign & Setting: mixed & DLPFC at frequency & what mea- tion about \\
\hline
\end{tabular}

Pharmacological, psychological, and non-invasive brain stimulation interventions for treating depression after stroke (Review) 
Table 1. Characteristics of 'dropout' studies (Continued) Number of Number of participants: unclear arms: 4

Experimental

Stroke criteria: not an entry critearm A: 10 rT-

MS sessions

Experimental

Arm B: 15 rT-

MS sessions

Control arm

A: 10 sham rTMS

Control arm

B: 15 sham rTMS

ria. Includes patients with clinical diagnosis of vascular depression

Inclusion criteria: not reported

Exclusion criteria: (1) presence of severe heart or respiratory failure or renal or hepatic failure, or occurrence of ongoing neoplastic process; (2) neurodegenerative disorders such as idiopathic Parkinson's disease or probable tia Rating Scale score 0.5); (3) deAlzheimer's disease and clinical evidence of dementia (Clinical Demenpressed patients who were actively suicidal, who presented with prominent psychotic features, or with comorbid alcohol or other drug abuse that was active within 2 years before the study; (4) prior occurrence of induced seizures, major head trauma, and history of epilepsy; (5) metal in the skull, cranial cavity, or brain parenchyma; cardiac pacemaker, implanted defibrillator, or medication pump

Depression criteria: diagnosis of major depression during current depressive episode

Total number included in this trial: number of stroke patients unclear

Number included in treatment group: number of stroke patients unclear

Number included in control group: number of stroke patients unclear of $10 \mathrm{~Hz}$ and intensity of $110 \%$ of the motor threshold during a 6 second period, with a total of 20 trains separated by 1-minute pauses. Treatment was administered during a 10-day period for a TCD of 12,000 pulses (i.e. TCD-12K group)

Treatment B: 15 rT-

MS sessions in the left pre-frontal cortex at frequency of $10 \mathrm{~Hz}$ and intensity of $110 \%$ of the motor threshold during a 6-second period, with a total of 20 trains separated by 1-minute pauses. Treatment was administered during a 10-day period with 2 sessions per day for 5 days to achieve a TCD of 18,000 pulses (i.e. TCD-18K group)

Control A: 10 sham stimulation sessions with matched pulses but performed with a specially designed coil that looks exactly like the standard stimulating coil but produces scalp sensation without actual cortical stimulation

Control B: 15 sham stimulation sessions

Duration: 10 days sure was whether any used participants in this study have a diagnosis of stroke and whether some participants who received treatment $A$ are the same as those reported in Jorge 2004

\begin{tabular}{|c|c|c|c|c|c|}
\hline Kim 2017 & $\begin{array}{l}\text { Study design: } \\
\text { parallel de- } \\
\text { sign } \\
\text { Number of } \\
\text { arms: } 2 \\
\text { Experimental } \\
\text { arm: escitalo- } \\
\text { pram } \\
\text { Control arm: } \\
\text { placebo }\end{array}$ & $\begin{array}{l}\text { Geographical location: South Ko- } \\
\text { rea } \\
\text { Setting: unclear } \\
\text { Number of participants: } 478 \\
\text { Stroke criteria: ischaemic stroke or } \\
\text { intracerebral haemorrhage } \\
\text { Method of diagnosis: diagnosis } \\
\text { confirmed by MRI or CT } \\
\text { Inclusion criteria: (1) acute is- } \\
\text { chaemic stroke or intracerebral }\end{array}$ & $\begin{array}{l}\text { Treatment: escitalo- } \\
\text { pram (5 mg daily as a } \\
\text { starting dose, dose in- } \\
\text { creased to } 10 \mathrm{mg} \text { daily } \\
\text { from the second week } \\
\text { and then every other } \\
\text { day for } 1 \text { week) } \\
\text { Control: placebo } \\
\text { Duration: } 12 \text { weeks } \\
\text { Follow-up: } 6 \text { months }\end{array}$ & $\begin{array}{l}\text { - Depression } \\
\text { measured } \\
\text { using } \\
\text { MADRS } \\
\text { - Emotional } \\
\text { inconti- } \\
\text { nence mea- } \\
\text { sured using } \\
\text { Kim's crite- } \\
\text { ria } \\
\text { - Anger } \\
\text { proneness } \\
\text { measured } \\
\text { using Spiel- }\end{array}$ & $\begin{array}{l}\text { Unable to iso- } \\
\text { late outcome } \\
\text { data for those } \\
\text { with depres- } \\
\text { sion at ran- } \\
\text { domisation }\end{array}$ \\
\hline
\end{tabular}


Table 1. Characteristics of 'dropout' studies (Continued) haemorrhage within previous 21 days

Exclusion criteria: (1) history of diagnosed depression or other psychiatric diseases before index stroke; (2) severe dementia, defined as requiring assistance from others to maintain activities of daily living because of cognitive dysfunction (stages 5 to 7 of the Global Deterioration Scale); (3) aphasia resulting in communication difficulties regardless of reasons; (4) exhibiting strong suicidal thoughts (combined MADRS score $>8$ on ninth and tenth questions); (5) seizures; (6) history of other brain disease or head trauma within 30 days before screening; (7) abnormal blood tests such as abnormal liver function test or renal insufficiency; (8) pregnant or lactating

\section{Depression criteria: none}

Total number included in this trial: 478

\section{Number included in treatment} group: 241 (57\% men, mean age 63.6, SD 12.6)

Number included in control group: 237 (65\% men, mean age 63.5, SD 12.0)

\begin{tabular}{|c|c|c|c|c|c|}
\hline Kim 2017a & $\begin{array}{l}\text { Study design: } \\
\text { parallel de- } \\
\text { sign } \\
\text { Number of } \\
\text { arms: } \\
2 \\
\text { Experimen- } \\
\text { tal arm: rTMS } \\
\text { Control arm: } \\
\text { sham rTMS }\end{array}$ & $\begin{array}{l}\text { Georgraphical location: South Ko- } \\
\text { rea } \\
\text { Setting: inpatient } \\
\text { Number of participants: } 44 \\
\text { Stroke criteria: right hemisphere } \\
\text { ischaemic or haemorrhagic stroke } \\
\text { Method of stroke diagnosis: un- } \\
\text { clear } \\
\text { Inclusion criteria: (1) diagnosis } \\
\text { of right hemisphere ischaemic or } \\
\text { haemorrhagic stroke } \\
\text { Exclusion criteria: (1) severe cog- } \\
\text { nitive impairment that made it diffi- } \\
\text { cult to understand instructions; (2) } \\
\text { seizures; (3) severe head trauma; (4) } \\
\text { metal skull implant; (5) pacemaker } \\
\text { Depression criteria: none } \\
\text { Total number included in this tri- } \\
\text { al: } 44\end{array}$ & $\begin{array}{l}\text { Treatment: rTMS. rT- } \\
\text { MS stimulus was tar- } \\
\text { geted at P3, over the } \\
\text { left parieto-occipital } \\
\text { cortex, and at P4, over } \\
\text { the right parieto-oc- } \\
\text { cipital cortex. To set } \\
\text { the motor threshold } \\
\text { before stimulation, } \\
\text { a cotton cap with a } \\
\text { grid ( } 1 \times 1 \mathrm{~cm}^{2} \text { ) was } \\
\text { fixed to the scalp from } \\
\text { the nasion to the in- } \\
\text { ion, a magnetic stim- } \\
\text { ulus was applied to } \\
\text { the cranium, and mo- } \\
\text { tor-evoked poten- } \\
\text { tials were measured. } \\
\text { Low-frequency rTMS } \\
\text { stimulation was ap- } \\
\text { plied to P3 on the left, } \\
\text { healthy side, using a } \\
\text { 1-Hz stimulus at } 90 \% \\
\text { motor threshold, } 4 \\
\text { times, for } 5 \text { minutes at }\end{array}$ & $\begin{array}{l}\text { - Depression } \\
\text { measured } \\
\text { using BD } \\
\text { - Activities of } \\
\text { daily liv- } \\
\text { ing mea- } \\
\text { sured using } \\
\text { FIM }\end{array}$ & $\begin{array}{l}\text { Unable to iso- } \\
\text { late outcome } \\
\text { data for those } \\
\text { with depres- } \\
\text { sion at ran- } \\
\text { domisation }\end{array}$ \\
\hline
\end{tabular}

Pharmacological, psychological, and non-invasive brain stimulation interventions for treating depression after stroke (Review) 208
berg Train
Anger Scale
- Impair-
ment mea-
sured using
NIHSS
- Disability
measured
using $\mathrm{mRS}$
and $\mathrm{BI}$ 
Table 1. Characteristics of 'dropout' studies (Continued) Number included in treatment group: 22 ( $82 \%$ men, mean age 52.6, SD 10.6)

Number included in control group: 22 (59\% men, mean age 64.3, SD 11.5) a time, separated by 1 minute intervals. Highfrequency rTMS was applied to P4 on the right, affected side, using a $5-\mathrm{Hz}$ stimulus at $90 \%$ motor threshold, 20 times, for 5 seconds at a time, separated by 55 -second intervals

Control: sham rTMS. Mock stimulus used the same protocol as low-frequency rTMS, except that the coil was not placed against the skull, and the stimulus was applied in the vertical direction Duration: 12 weeks

Follow-up: 8 weeks

\section{Kootker 2012}

\section{Study design: parallel de- sign \\ Number of arms: 2}

Experimental arm: tailored cognitive-behavioural therapy (CBT)

Control arm: computer cognitive training (CCT)
Geographical location: The Netherlands

Setting: outpatient

Number of participants: 61

Stroke criteria: all subtypes

Method of stroke diagnosis: clinically confirmed stroke

Inclusion criteria: (1) sustained any type of clinically confirmed stroke at least 3 months earlier; (2) only mild cognitive impairment (MMSE score); (3) scoring positively on communication-related items of NIHSS; (4) master Dutch language

Exclusion criteria: (1) pre-stroke major depression requiring psychiatric care; (2) poststroke major depression requiring a start with medication; (3) pre-morbid disability as reflected in a BI score $<19$ (out of 20); (4) severe comorbidity that might affect mood (e.g. cancer)

Depression criteria: HADS score $>7$

Total number included in this trial: 61

Number included in treatment group: 31 (61.3\% men, mean age 61, SD not reported)
Treatment: tailored cognitive-behavioural therapy. Each session consisted of $2 \times$ 20 to 25 -minute blocks divided by a 10 to 15 minute break. Therefore, each session lasted approximately 1 hour. Goals for attaining daily life activities were primarily set together by the patient and the therapist using pictures from the Activity Card Sort. Concurrently with psychological sessions, the CBT intervention was augmented with 3 sessions of occupational therapy or movement therapy. During these sessions, an occupational or movement therapist helped patients in establishing and attaining goals aimed at meaningful activities and social participation. These goals were attuned to the content of the psychological sessions
- Depression measured using HADSDepression review

- Anxiety measured using HADSAnxiety

- Quality of life measured using EQ5D
Results not available in format suitable for this 
Table 1. Characteristics of 'dropout' studies (Continued) Number included in control group: 30 (63.3\%, mean age $61, \mathrm{SD}$ not reported)
Administered by: certified healthcare psychologist (therapist)

Supervision: not reported

Intervention fidelity: not reported

Control: computer cognitive training. A desktop was set up with headphones and a keyboard with coloured patches attached to 2 keys. Patients could select any (or a combination) of 4 specific cognitive domains for training (i.e. attention, memory, executive functioning, visual attention). As patients improved, the Cogniplus Program adjusted the level of difficulty for each training task accordingly. In this way, each patient trained at his/her individual level and pace

Administered by: selfadministered, but cognitive trainers or psychological assistants were present to assist participants during training

Duration: 4 months

Follow-up: 12 months

\begin{tabular}{|c|c|c|c|c|c|}
\hline Mauri 1988 & $\begin{array}{l}\text { Study design: } \\
\text { parallel de- } \\
\text { sign } \\
\text { Number of } \\
\text { arms: } 2 \\
\text { Experimental } \\
\text { arm: } \\
\text { mianserin } \\
\text { Control arm: } \\
\text { placebo }\end{array}$ & $\begin{array}{l}\text { Geographical location: Spain } \\
\text { Setting: unclear } \\
\text { Number of participants: unclear } \\
\text { Stroke criteria: ischaemic stroke } \\
\text { Method of diagnosis: unclear } \\
\text { Inclusion criteria: not reported } \\
\text { Exclusion criteria: not reported } \\
\text { Depression criteria: GDS (15 item) } \\
\text { score > } 4 \\
\text { Total number included in this tri- } \\
\text { al: unclear }\end{array}$ & $\begin{array}{l}\text { Treatment: mianserin } \\
\text { Control: placebo } \\
\text { Duration: } 6 \text { weeks }\end{array}$ & $\begin{array}{l}\text { Depression } \\
\text { - unclear } \\
\text { what mea- } \\
\text { sure was } \\
\text { used }\end{array}$ & $\begin{array}{l}\text { Results not } \\
\text { available in } \\
\text { format suit- } \\
\text { able for this } \\
\text { review }\end{array}$ \\
\hline
\end{tabular}

Pharmacological, psychological, and non-invasive brain stimulation interventions for treating depression after stroke (Review) 
Table 1. Characteristics of 'dropout' studies (Continued)

Number included in treatment

group: unclear

Number included in control

group: unclear

\begin{tabular}{|c|c|c|c|c|c|}
\hline Meara 1998 & $\begin{array}{l}\text { Study design: } \\
\text { parallel de- } \\
\text { sign } \\
\text { Number of } \\
\text { arms: } 2 \\
\text { Experimental } \\
\text { arm: sertra- } \\
\text { line } \\
\text { Control arm: } \\
\text { placebo }\end{array}$ & $\begin{array}{l}\text { Geographical location: UK } \\
\text { Setting: inpatient } \\
\text { Number of participants: unclear } \\
\text { Stroke criteria: ischaemic stroke } \\
\text { Method of stroke diagnosis: un- } \\
\text { clear } \\
\text { Inclusion criteria: not reported } \\
\text { Exclusion criteria: (1) moderate to } \\
\text { severe dementia; (2) severe apha- } \\
\text { sia, communication difficulties; (3) } \\
\text { poorly controlled epilepsy } \\
\text { Depression criteria: GDS (15 item) } \\
\text { score > } 4 \\
\text { Total number included in this tri- } \\
\text { al: unclear } \\
\text { Number included in treatment: } \\
\text { unclear } \\
\text { Number included in control } \\
\text { group: unclear }\end{array}$ & $\begin{array}{l}\text { Treatment: sertraline, } \\
50 \text { mg daily. Dose es- } \\
\text { calation to } 100 \text { mg for } \\
\text { non-responders at } 2 \\
\text { weeks } \\
\text { Control: matched } \\
\text { placebo } \\
\text { Duration: } 6 \text { weeks }\end{array}$ & $\begin{array}{l}\text { Depression } \\
\text { measured } \\
\text { using GDS }\end{array}$ & $\begin{array}{l}\text { Results not } \\
\text { available in } \\
\text { format suit- } \\
\text { able for this } \\
\text { review }\end{array}$ \\
\hline Ohtomo 1985 & $\begin{array}{l}\text { Study design: } \\
\text { parallel de- } \\
\text { sign } \\
\text { Number of } \\
\text { arms: } 2 \\
\text { Experimental } \\
\text { arm: tiapride } \\
\text { Control arm: } \\
\text { placebo }\end{array}$ & $\begin{array}{l}\text { Geographical location: Japan } \\
\text { Setting: unclear } \\
\text { Number of participants: } 188 \\
\text { Stroke criteria: all subtypes } \\
\text { Method of stroke diagnosis: diag- } \\
\text { nosis via clinical signs and CT } \\
\text { Inclusion criteria: (1) > } 40 \text { years of } \\
\text { age, high blood pressure (> 160/90 } \\
\text { mmHg), and hypertensive changes } \\
\text { on fundoscopy changes; (2) stable } \\
\text { neuroleptic, minor tranquilliser, an- } \\
\text { tidepressant, brain metabolic acti- } \\
\text { vators, cerebro-vasodilators washed } \\
\text { out for } 3 \text { to } 7 \text { days before randomi- } \\
\text { sation } \\
\text { Exclusion criteria: (1) severe apha- } \\
\text { sia; (2) severe dementia; (3) drug } \\
\text { dependence; (4) inadequate condi- } \\
\text { tions for the study } \\
\text { Depression criteria: not reported } \\
\text { Total number included in this tri- } \\
\text { al- } 288\end{array}$ & $\begin{array}{l}\text { Treatment: tiapride, } \\
75 \text { mg daily for } 1 \text { week, } \\
\text { dose escalation to } 150 \\
\text { to } 225 \text { mg daily for } 5 \\
\text { weeks according to } \\
\text { clinical response } \\
\text { Control: } \text { matched } \\
\text { placebo } \\
\text { Duration: } 6 \text { weeks }\end{array}$ & $\begin{array}{l}\text { - Depression } \\
\text { - unclear } \\
\text { what mea- } \\
\text { sure was } \\
\text { used }\end{array}$ & $\begin{array}{l}\text { Unable to iso- } \\
\text { late outcome } \\
\text { data for those } \\
\text { with depres- } \\
\text { sion at ran- } \\
\text { domisation }\end{array}$ \\
\hline
\end{tabular}


Table 1. Characteristics of 'dropout' studies (Continued)

Number included in treatment

group: 141 (54\% men, mean age not

reported)

Number included in control

group: 147 (61\% men, mean age not reported)

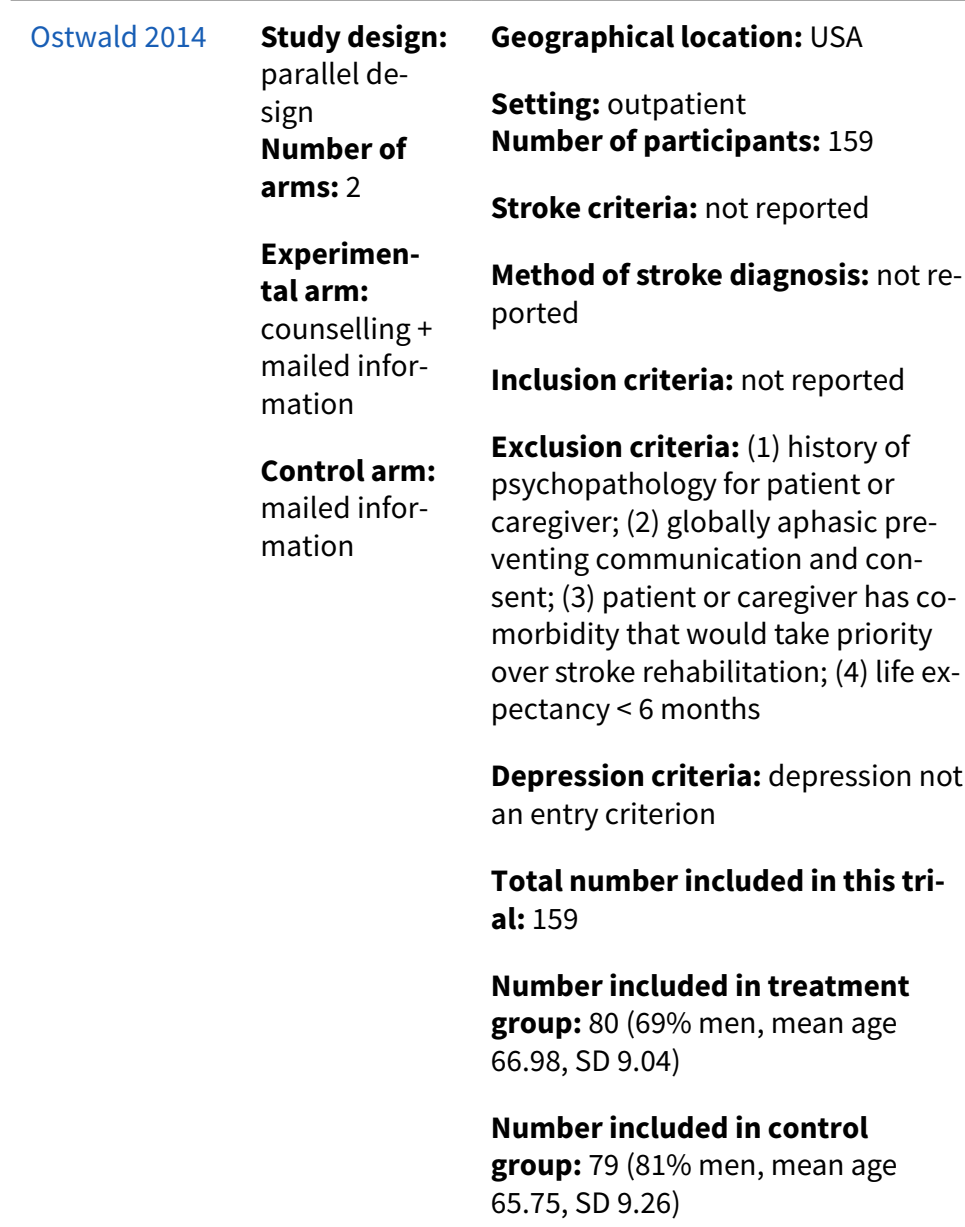

Treatment: home vis its from a multi-disciplinary therapy team to provide education, support, skill training, counselling, and linkages to social and community resources + mailed information. Average dose 36.7 hours

Administered by: ad vanced practice nurses, occupational and physical therapists

Supervision: not reported

Intervention fidelity: not reported

Control: mailed information

Duration: 6 months
- Depression measured using GDS

- Disability measured using FIM

- Quality of life measured using SF-36
Unable to isolate outcome data for those with depression at randomisation

\begin{tabular}{|c|c|c|c|c|c|}
\hline Raffaele 1996 & $\begin{array}{l}\text { Study design: } \\
\text { parallel de- } \\
\text { sign } \\
\text { Number of } \\
\text { arms: } 2 \\
\text { Experimen- } \\
\text { tal arm: tra- } \\
\text { zodone } \\
\text { Control arm: } \\
\text { placebo }\end{array}$ & $\begin{array}{l}\text { Geographical location: Italy } \\
\text { Setting: outpatient } \\
\text { Number of participants: } 22 \\
\text { Stroke criteria: unclear } \\
\text { Method of stroke diagnosis: not re- } \\
\text { ported } \\
\text { Inclusion criteria: not reported } \\
\text { Exclusion criteria: not reported } \\
\text { Depression criteria: ZDS } \\
\text { Total number included in this tri- } \\
\text { al: } 22\end{array}$ & $\begin{array}{l}\text { Treatment: trazodone } \\
300 \mathrm{mg} / \mathrm{d} \\
\text { Control: placebo } \\
\text { Duration: } 30 \text { to } 45 \\
\text { days } \\
\text { Follow-up: unclear }\end{array}$ & 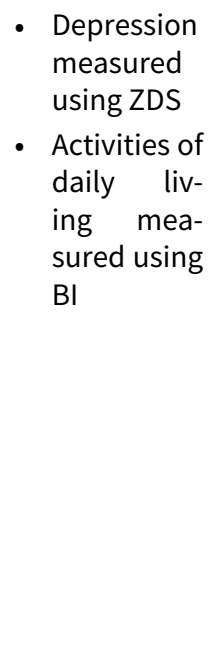 & $\begin{array}{l}\text { Unable to iso- } \\
\text { late outcome } \\
\text { data for those } \\
\text { with depres- } \\
\text { sion at ran- } \\
\text { domisation }\end{array}$ \\
\hline
\end{tabular}


Table 1. Characteristics of 'dropout' studies (Continued) Number included in treatment group: $11(45.4 \%$ men, mean age 69.5, SD 2.3)

Number included in control group: $11(72.7 \%$ men, mean age 70.4, SD 3.0)

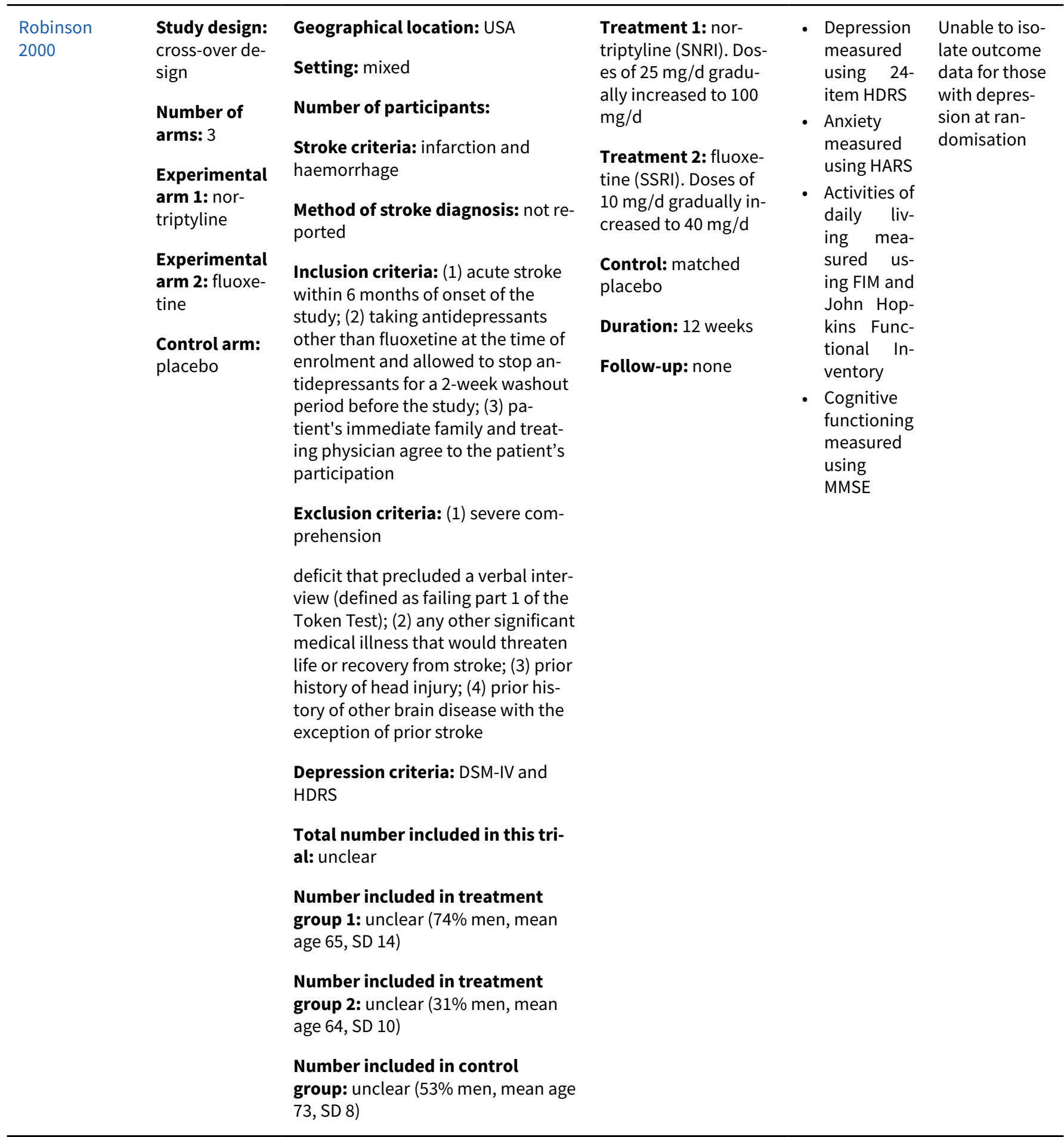


Table 1. Characteristics of 'dropout' studies (Continued)

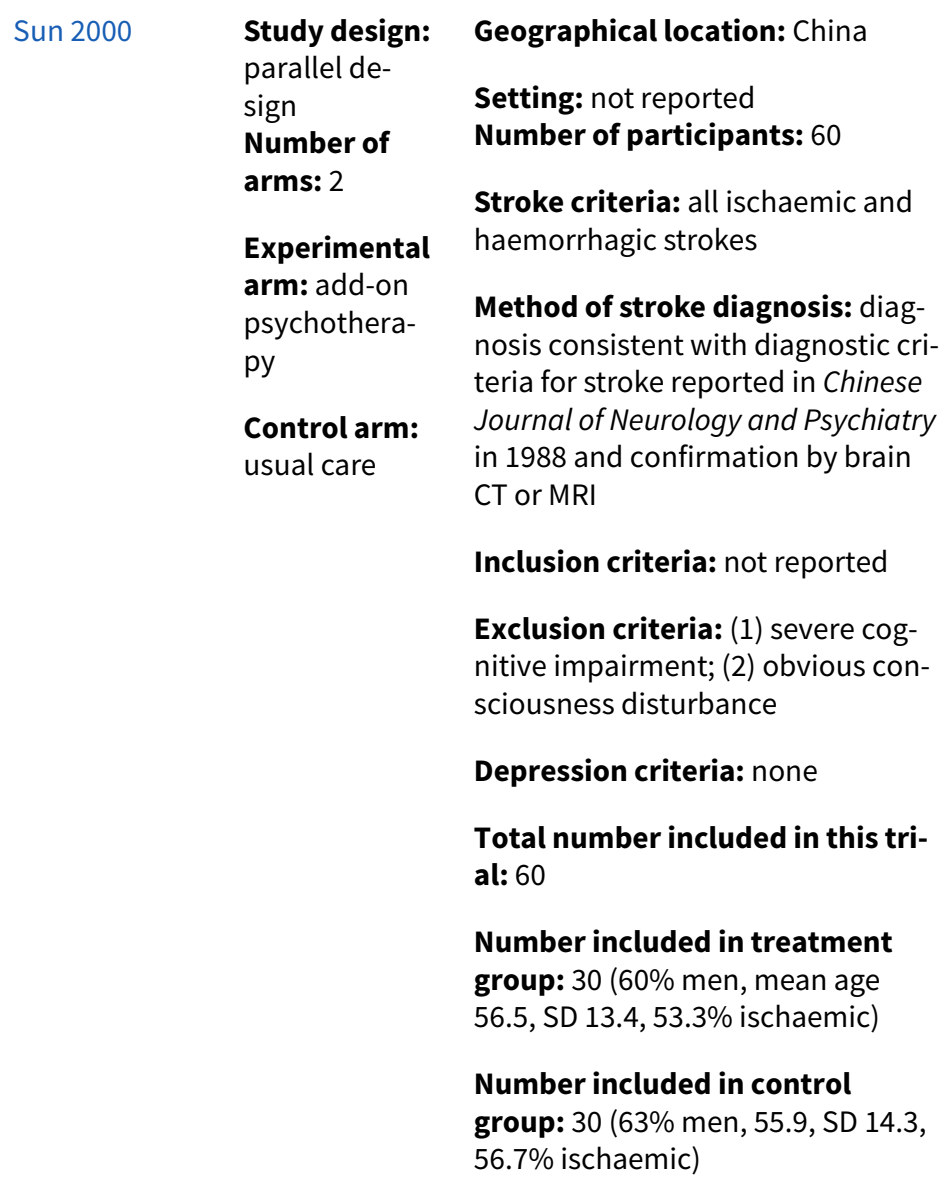

Treatment: add-on psychotherapy entailing understanding the patient's reaction to sudden illness and letting the patient talk about concerns in mind, to give sympathy, care, and support; inducing correct understanding of the illness by the patient, helping him/her to analyse current problems and building confidence to overcome the disease; promoting the family's help and co-operation; giving praise, encouragement, or small prizes for patient improvement

Administered by: not reported

Supervision: not reported

Intervention fidelity: not reported

Control: usual care

\section{- Depression Unable to iso- - unclear late outcome what mea- data for those sure was with depres- used sion at ran- domisation}

\begin{tabular}{ll}
\hline Valiengo 2017 & $\begin{array}{l}\text { Study design: } \\
\text { parallel de- } \\
\text { sign }\end{array}$ \\
& $\begin{array}{l}\text { Number of } \\
\text { arms: } 2\end{array}$ \\
& Experimental \\
arm: tDCS & \\
& $\begin{array}{l}\text { Control arm: } \\
\text { sham tDCS }\end{array}$
\end{tabular}

Geographical location: Brazil

Setting: outpatient

Number of participants: 48

Stroke criteria: ischaemic and PICH stroke

Method of stroke diagnosis: diagnosis was confirmed with brain CT or MRI

Inclusion criteria: (1) aged 30 to 60 years; (2) first stroke only; (3) time since stroke $<5$ years

Exclusion criteria: (1) on antidepressants, antipsychotics, benzodiazepines, or diazepam; (2) dementia and epilepsy, life-threatening condition, suicide risk (score $\leq 2$ on third item HDRS)

Depression criteria: depression diagnosed by a trained psychiatrist

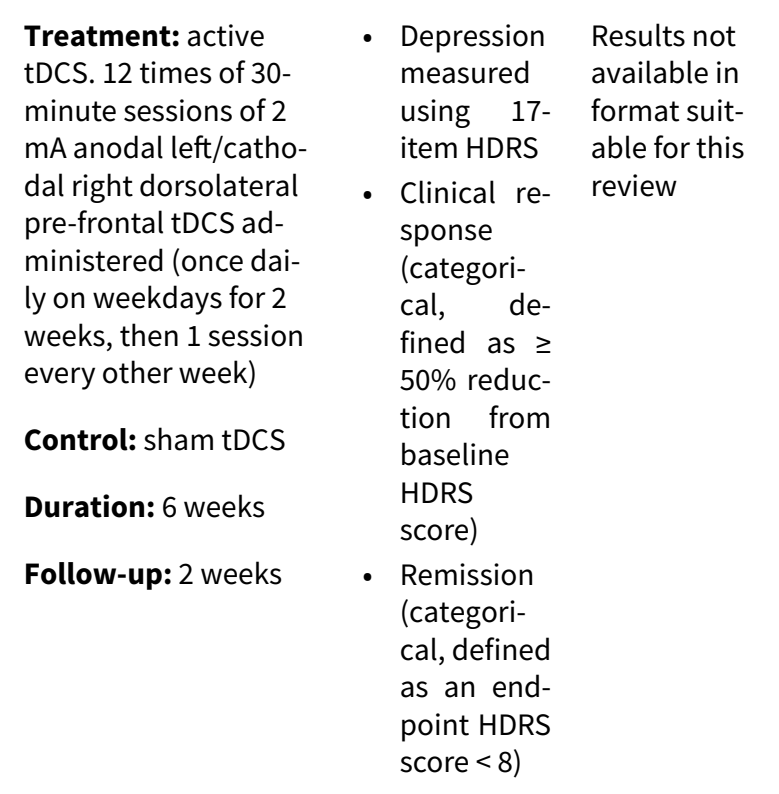


Table 1. Characteristics of 'dropout' studies (Continued)

with the MINI for DSM-IV psychiatric

disorders

Total number included in this tri-

al: 48

Number included in treatment

group: 24 ( $50 \%$ men, mean age

62.2, SD not reported)

Number included in control

group: 24 ( $50 \%$ men, mean age

61.3, SD not reported)

BDI: Beck Depression Inventory.

BI: Barthel Index.

CES-D: Centre for Epidemiologic Studies Depression Scale.

$\mathrm{CT}$ : computed tomography.

DLPFC: dorsolateral pre-frontal cortex.

DSM- IV: Diagnostic and Statistical Manual of Mental Disorders, Fourth Edition.

ECT: electroconvulsive therapy.

EQ5D: EuroQoL 5-dimension.

FIM: Functional Independence Measure.

GDS: Geriatric Depression Scale.

HADS: Hospital Anxiety and Depression Scale.

HARS: Hamilton Anxiety Rating Scale.

HDRS: Hamilton Depression Rating Scale.

$\mathrm{Hz}$ : hertz.

LD: levodopa.

MADRS: Montgomery Asberg Depression Rating Scale.

MAO: monoamine oxidase.

MINI: Mini-International Neuropsychiatry Interview.

MMSE: Mini Mental State Examination.

MPH: methylphenidate.

MRI: magnetic resonance imaging.

mRS: modified Rankin Scale.

NIHSS: National Institute of Health Stroke Scale.

$\mathrm{PICH}$ : primary intracerebral haemorrhage.

PHQ-9: 9-item Patient Health Questionnaire.

rTMS: repetitive transcranial magnetic stimulation.

SAH: subarachnoid haemorrhage.

SD: standard deviation.

SF-36: Short-Form 36.

SNRI: selective nortriptyline reuptake inhibitor.

SSRI: selective serotonin reuptake inhibitor.

TIA: transient ischaemic attack.

tDCS: transcranial direct current stimulation.

ZDS: Zung Depression Scale.

\section{AP PE N DICES}

\section{Appendix 1. Search review - 2008}

\section{Electronic searches}

Cochrane Stroke Trial Register - searched October 2007; Cochrane Anxiety and Neurosis Trial Register - searched February 2008.

The remaining databases were searched May 2006.

- Cochrane Central Register of Controlled Trials (CENTRAL)

Pharmacological, psychological, and non-invasive brain stimulation interventions for treating depression after stroke (Review) 
- MEDLINE

- Embase

- CINAHL

- Psycinfo

- Applied Science and Technology Plus

- Arts and Humanities Index

- Biological Abstracts

- BIOSIS Previews

- General Science Plus

- Science Citation Index

- Social Sciences Citation Index

- ISI Web of Science

- Dissertations and Theses

The following search strategy with a combination of controlled vocabulary and free-text terms for MEDLINE and CINAHL (Ovid), and modified to suit the other databases.

1 exp cerebrovascular disorders/

2 (stroke\$ or poststroke\$ or cva\$).tw.

3 (cerebrovascular\$ or cerebral vascular).tw.

4 (cerebral or cerebellar or brain $\$$ or vertebrobasilar).tw.

5 (infarct\$ or isch?emi\$ or thrombo $\$$ or emboli\$ or apoplexy).tw.

6 (cerebral or intracerebral or intracranial or brain\$).tw.

7 (haemorrhage or hemorrhage or bleed\$).tw.

84 and 5

96 and 7

101 or 2 or 3 or 8 or 9

11 Depression/

12 Depression, involutional/ or Depressive disorder/ or Dysthymic disorder/

13 (depress\$ or dysthymi\$).tw.

1411 or 12 or 13

1510 and 14

16 randomized controlled trial.pt.

17 randomized controlled trials/

18 controlled clinical trial.pt.

19 controlled clinical trials/

20 random allocation/

21 double-blind method/

22 single-blind method/

23 clinical trial.pt.

24 exp clinical trials/

25 (clin\$ adj25 trial\$).tw.

26 ((singl\$ or doubl\$ or tripl\$ or trebl\$) adj25 (blind\$ or mask\$)).tw.

27 placebos/

28 placebo\$.tw.

29 random\$.tw.

30 research design/

31 clinical trial phase ii.pt.

32 clinical trial phase iii.pt.

33 clinical trial phase iv.pt.

34 meta analysis.pt.

35 multicenter study.pt.

36 intervention studies/

37 cross-over studies/

38 meta-analysis/

39 control\$.tw.

40 alternate treatment.tw.

41 "comparative study"/

42 exp evaluation studies/

43 Follow-up studies/

Pharmacological, psychological, and non-invasive brain stimulation interventions for treating depression after stroke (Review) 
44 Prospective studies/

45 prospective.tw.

46 (versus or sham or intervention group or comparative stud\$).tw.

47 or $/ 16-46$

4815 and 47

49 limit 48 to human

\section{Additional searches}

The following conference abstracts and proceedings were searched.

- European Stroke Conferences (2000 to 2007)

- Stroke Society of Australasia Annual Scientific Meetings (1999 to 2007)

Online clinical trials and research registries were also searched August 2007.

- www.strokecenter.org/trials

- www.ClinicalTrials.gov

- www.Clinicalstudyresults.org

- www.anzctr.org.au

\section{Reference lists}

Reference lists of relevant studies were searched to identify studies not already included.

\section{Personal communication}

Professional bodies, authors of included studies, and pharmaceutical companies were contacted for information on published and unpublished information.

\section{Appendix 2. Search review 2018 - CENTRAL}

Search strategy for CENTRAL, August 2018

\begin{tabular}{|c|c|}
\hline \# & Query \\
\hline$\# 1$ & $\begin{array}{l}\text { [mh }{ }^{\wedge} " \text { cerebrovascular disorders"] or [mh "basal ganglia cerebrovascular disease"] or [mh "brain } \\
\text { ischemia"] or [mh "carotid artery diseases"] or [mh "intracranial arterial diseases"] or [mh "in- } \\
\text { tracranial embolism and thrombosis"] or [mh "intracranial hemorrhages"] or [mh ^stroke] or [mh } \\
\text { "brain infarction"] or [mh ^"stroke, lacunar"] or [mh ^^"vasospasm, intracranial"] or [mh ^"vertebral } \\
\text { artery dissection"] }\end{array}$ \\
\hline$\# 2$ & 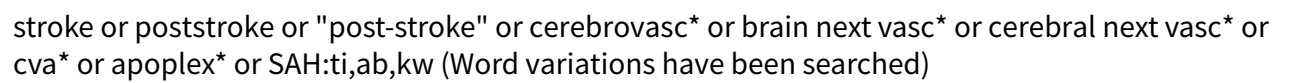 \\
\hline \#3 & 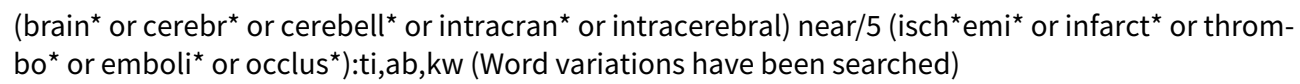 \\
\hline \#4 & $\begin{array}{l}\text { (brain* } \text { or cerebr }^{\star} \text { or cerebell* or intracerebral or intracranial or subarachnoid) near } / 5 \text { (haemor- } \\
\text { rhage }^{\star} \text { or hemorrhage }{ }^{\star} \text { or haematoma* or hematoma* or bleed*):ti,ab,kw (Word variations have } \\
\text { been searched) }\end{array}$ \\
\hline \#5 & [mh hemiplegia] or [mh paresis] \\
\hline \#6 & hemipleg* or hemipar ${ }^{\star}$ or paresis or paretic:ti,ab,kw (Word variations have been searched) \\
\hline \#7 & $\# 1$ or \#2 or \#3 or \#4 or \#5 or \#6 \\
\hline
\end{tabular}


[mh^"depressive disorder"] or [mh ^"depressive disorder, major"] or [mh ^"depressive disorder, treatment-resistant"] or [mh^"dysthymic disorder"] or [mh^^depression] or [mh "antidepressive agents"]

\begin{tabular}{ll}
\hline$\# 9$ & $\begin{array}{l}\text { depress }{ }^{*} \text { or dysthymi*or dysphor }{ }^{\star} \text { or antidepress }{ }^{\star} \text { or anti-depress }{ }^{\star}: \text { ti,ab,kw (Word variations have } \\
\text { been searched) }\end{array}$ \\
\hline$\# 10$ & $\# 8$ or \#9 \\
\hline$\# 11$ & $\# 7$ and \#10 \\
\hline
\end{tabular}

\section{Appendix 3. Search review 2018 - MEDLINE}

\section{Search strategy for MEDLINE, August 2018}

1. cerebrovascular disorders/ or exp basal ganglia cerebrovascular disease/ or exp brain ischemia/ or exp carotid artery diseases/ or exp intracranial arterial diseases/ or exp "intracranial embolism and thrombosis"/ or exp intracranial hemorrhages/ or stroke/ or exp brain infarction/ or stroke, lacunar/ or vasospasm, intracranial/ or vertebral artery dissection/

2. (stroke or poststroke or post-stroke or cerebrovasc $\$$ or brain vasc $\$$ or cerebral vasc $\$$ or cva $\$$ or apoplex\$ or SAH).tw.

3. ((brain\$ or cerebr\$ or cerebell\$ or intracran\$ or intracerebral) adj5 (isch?emi\$ or infarct\$ or thrombo $\$$ or emboli\$ or occlus\$)).tw.

4. ((brain $\$$ or cerebr\$ or cerebell\$ or intracerebral or intracranial or subarachnoid) adj5 (haemorrhage\$ or hemorrhage\$ or haematoma

\$ or hematoma\$ or bleed\$)).tw.

5. hemiplegia/ or exp paresis/

6. (hemipleg\$ or hemipar\$ or paresis or paretic).tw.

7. 1 or 2 or 3 or 4 or 5 or 6

8. depressive disorder/ or depressive disorder, major/ or depressive disorder, treatment-resistant/ or dysthymic disorder/ or Depression/ or exp Antidepressive Agents/

9. (depress\$ or dysthymi or dysphor\$ or antidepress\$ or anti-depress\$).tw.

10. 8 or 9

11. Randomized Controlled Trials as Topic/

12. random allocation/

13. Controlled Clinical Trials as Topic/

14. control groups/

15. clinical trials as topic/ or clinical trials, phase i as topic/ or clinical trials, phase ii as topic/ or clinical trials, phase iii as topic/ or clinical trials, phase iv as topic/

16. double-blind method/

17. single-blind method/

18. Placebos/

19. placebo effect/

20. cross-over studies/

21. Therapies, Investigational/

22. Drug Evaluation/

23. Research Design/

24. randomized controlled trial.pt.

25. controlled clinical trial.pt.

26. (clinical trial or clinical trial phase i or clinical trial phase ii or clinical trial phase iii or clinical trial phase iv).pt.

27. (random\$ or RCT or RCTs).tw.

28. (controlled adj5 (trial\$ or stud\$)).tw.

29. (clinical\$ adj5 trial\$).tw.

30. ((control or treatment or experiment\$ or intervention) adj5 (group\$ or subject\$ or patient\$)).tw.

31. (quasi-random $\$$ or quasi random $\$$ or pseudo-random $\$$ or pseudo random $\$$ ).tw.

32. ((control or experiment $\$$ or conservative) adj5 (treatment or therapy or procedure or manage\$)).tw.

33. ((singl\$ or doubl\$ or tripl\$ or trebl\$) adj5 (blind\$ or mask\$)).tw.

34. (cross-over or cross over or crossover).tw.

35. (placebo $\$$ or sham).tw.

36. trial.ti.

37. (assign\$ or allocat\$).tw.

38. or/11-37

39. 7 and 10 and 38 
40. exp animals/ not humans.sh.

41. 39 not 40

\section{Appendix 4. Search review 2018 - Embase}

\section{Search strategy for Embase, August 2018}

1. cerebrovascular disease/ or basal ganglion hemorrhage/ or exp brain hematoma/ or exp brain hemorrhage/ or exp brain infarction/ or exp brain ischemia/ or exp carotid artery disease/ or cerebral artery disease/ or exp cerebrovascular accident/ or exp intracranial aneurysm/ or exp occlusive cerebrovascular disease/

2. stroke patient/ or stroke unit/

3. (stroke or poststroke or post-stroke or cerebrovasc $\$$ or brain vasc $\$$ or cerebral vasc\$ or cva\$ or apoplex\$ or SAH).tw.

4. ((brain $\$$ or cerebr\$ or cerebell\$ or intracran\$ or intracerebral) adj5 (isch?emi\$ or infarct\$ or thrombo $\$$ or emboli\$ or occlus\$)).tw.

5. ((brain\$ or cerebr\$ or cerebell\$ or intracerebral or intracranial or subarachnoid) adj5 (haemorrhage\$ or hemorrhage\$ or haematoma

\$ or hematoma\$ or bleed\$)).tw.

6. hemiparesis/ or hemiplegia/

7. (hemipleg\$ or hemipar\$ or paresis or paretic).tw.

8.1 or 2 or 3 or 4 or 5 or 6 or 7

9. depression/ or agitated depression/ or atypical depression/ or dysphoria/ or dysthymia/ or endogenous depression/ or involutional depression/ or late life depression/ or major depression/ or masked depression/ or reactive depression/ or recurrent brief depression/ or treatment resistant depression/

10. exp antidepressant agent/

11. (depress\$ or dysthymi\$ or dysphor $\$$ or antidepress $\$$ or anti-depress\$).tw.

12. 9 or 10 or 11

13. Randomized Controlled Trial/ or "randomized controlled trial (topic)"/

14. Randomization/

15. Controlled clinical trial/ or "controlled clinical trial (topic)"/

16. control group/ or controlled study/

17. clinical trial/ or "clinical trial (topic)"/ or phase 1 clinical trial/ or phase 2 clinical trial/ or phase $3 \mathrm{clinical}$ trial/ or phase $4 \mathrm{clinical}$ trial/

18. Crossover Procedure/

19. Double Blind Procedure/

20. Single Blind Procedure/ or triple blind procedure/

21. placebo/ or placebo effect/

22. (random\$ or RCT or RCTs).tw.

23. (controlled adj5 (trial\$ or stud\$)).tw.

24. (clinical\$ adj5 trial\$).tw.

25. ((control or treatment or experiment\$ or intervention) adj5 (group\$ or subject\$ or patient\$)).tw.

26. (quasi-random $\$$ or quasi random $\$$ or pseudo-random $\$$ or pseudo random $\$$ ).tw.

27. ((control or experiment\$ or conservative) adj5 (treatment or therapy or procedure or manage\$)).tw.

28. ((singl\$ or doubl\$ or tripl\$ or trebl\$) adj5 (blind\$ or mask\$)).tw.

29. (cross-over or cross over or crossover).tw.

30. (placebo\$ or sham).tw.

31. trial.ti.

32. (assign\$ or allocat\$).tw.

33. or/13-32

34. 8 and 12 and 33

35. (exp animals/ or exp invertebrate/ or animal experiment/ or animal model/ or animal tissue/ or animal cell/ or nonhuman/) not (human/ or normal human/ or human cell/)

36. 34 not 35

\section{Appendix 5. Search review 2018 - PsycINFO}

\section{Search strategy for PsycINFO, August 2018}

1. cerebrovascular disorders/ or cerebral hemorrhage/ or exp cerebral ischemia/ or cerebral small vessel disease/ or cerebrovascular accidents/ or subarachnoid hemorrhage/

2. (stroke or poststroke or post-stroke or cerebrovasc $\$$ or brain vasc $\$$ or cerebral vasc $\$$ or cva\$ or apoplex\$ or SAH).tw.

3. ((brain $\$$ or cerebr\$ or cerebell\$ or intracran\$ or intracerebral) adj5 (isch?emi\$ or infarct $\$$ or thrombo $\$$ or emboli\$ or occlus\$)).tw.

4. ((brain $\$$ or cerebr $\$$ or cerebell\$ or intracerebral or intracranial or subarachnoid) adj5 (haemorrhage $\$$ or hemorrhage $\$$ or haematoma

\$ or hematoma\$ or bleed\$)).tw.

5. hemiparesis/ or hemiplegia/

6. (hemipleg\$ or hemipar\$ or paresis or paretic).tw.

7. 1 or 2 or 3 or 4 or 5 or 6 
8. major depression/ or dysthymic disorder/ or endogenous depression/ or reactive depression/ or recurrent depression/ or treatment resistant depression/ or atypical depression/ or "depression (emotion)"/

9. exp antidepressant drugs/

10. (depress $\$$ or dysthymi $\$$ or dysphor $\$$ or antidepress $\$$ or anti-depress $\$$ ).tw.

11.8 or 9 or 10

12. clinical trials/ or treatment effectiveness evaluation/ or placebo/

13. treatment outcome clinical trial.md.

14. (random\$ or RCT or RCTs).tw.

15. (controlled adj5 (trial\$ or stud\$)).tw.

16. (clinical\$ adj5 trial\$).tw.

17. ((control or treatment or experiment\$ or intervention) adj5 (group\$ or subject\$ or patient\$)).tw.

18. (quasi-random $\$$ or quasi random $\$$ or pseudo-random $\$$ or pseudo random\$).tw.

19. ((control or experiment $\$$ or conservative) adj5 (treatment or therapy or procedure or manage $\$)$ ).tw.

20. ((singl\$ or doubl\$ or tripl\$ or trebl\$) adj5 (blind\$ or mask\$)).tw.

21. (cross-over or cross over or crossover).tw.

22. (placebo\$ or sham).tw.

23. trial.ti.

24. (assign\$ or allocat\$).tw.

25. or/12-24

26. 7 and 11 and 25

\section{Appendix 6. Search review 2018 - CINAHL}

\section{Search strategy for CINAHL, August 2018}

\begin{tabular}{ll}
\hline$\#$ & Query \\
\hline S1 & (MH "Cerebrovascular Disorders") OR (MH "Basal Ganglia Cerebrovascular Disease+") OR (MH \\
& "Carotid Artery Diseases+") OR (MH "Cerebral Ischemia+") OR (MH "Cerebral Vasospasm") OR (MH \\
& "Intracranial Arterial Diseases+") OR (MH "Intracranial Embolism and Thrombosis") OR (MH "In- \\
& tracranial Hemorrhage+") OR (MH "Stroke") OR (MH "Vertebral Artery Dissections") \\
\hline
\end{tabular}

S2 (MH "Stroke Patients") OR (MH "Stroke Units")
apoplex or SAH ) or AB ( stroke or poststroke or post-stroke or cerebrovasc* or brain vasc* or cerebral vasc or cva or apoplex or SAH )

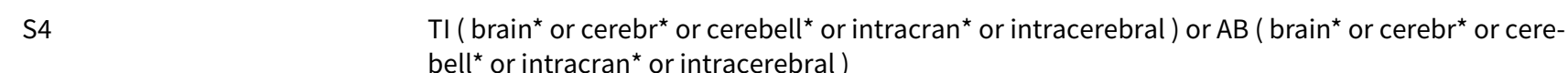
bell* or intracran* or intracerebral)

S5 TI (ischemi ${ }^{\star}$ or ischaemi ${ }^{\star}$ or infarct ${ }^{\star}$ or thrombo ${ }^{\star}$ or emboli* or occlus ${ }^{\star}$ ) or AB (ischemi ${ }^{\star}$ or ischae-
$\mathrm{mi}^{\star}$ or infarct* or thrombo* or emboli* or occlus ${ }^{\star}$ )

\begin{tabular}{ll}
\hline S6 & S4 and S5 \\
\hline S7 & $\begin{array}{l}\text { TI ( brain* or cerebr } \\
\left.\text { cerebr or cerebell* or intracerebral or intracranial or subarachnoid }) \text { or } \text { or }^{\star} \text { intracerebral or intracranial or subarachnoid }\right)\end{array}$
\end{tabular}

SI ( haemorrhage* or hemorrhage* or haematoma* or hematoma* or bleed ${ }^{\star}$ ) or AB ( haemor-
rhage* or hemorrhage* or haematoma* or hematoma* or bleed*)

\begin{tabular}{ll}
\hline S9 & S7 and S8 \\
\hline S10 & (MH "Hemiplegia") \\
\hline S11 & $\begin{array}{l}\text { TI ( hemipleg* or hemipar* or paresis or paretic) or AB ( hemipleg* or hemipar or paresis or paret- } \\
\text { ic) }\end{array}$ \\
\hline
\end{tabular}




\begin{tabular}{|c|c|}
\hline S13 & (MH "Depression") OR (MH "Depression, Reactive") OR (MH "Dysthymic Disorder") \\
\hline S14 & (MH "Antidepressive Agents+") \\
\hline S15 & 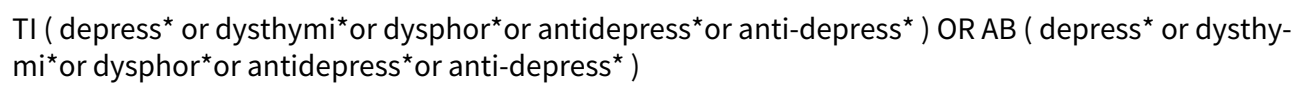 \\
\hline S16 & S13 OR S14 OR S15 \\
\hline S17 & (MH "Randomized Controlled Trials") or (MH "Random Assignment") or (MH "Random Sample+") \\
\hline S18 & (MH "Clinical Trials") or (MH "Intervention Trials") or (MH "Therapeutic Trials") \\
\hline S19 & (MH "Double-Blind Studies") or (MH "Single-Blind Studies") or (MH "Triple-Blind Studies") \\
\hline S20 & (MH "Control (Research)") or (MH "Control Group") or (MH "Placebos") or (MH "Placebo Effect") \\
\hline S21 & (MH "Crossover Design") OR (MH "Quasi-Experimental Studies") \\
\hline $\mathrm{S} 22$ & PT (clinical trial or randomized controlled trial) \\
\hline S23 & $\mathrm{TI}$ (random* or RCT or RCTs) or AB (random* or RCT or RCTs) \\
\hline S24 & TI (controlled N5 (trial ${ }^{\star}$ or stud $\left.\left.{ }^{\star}\right)\right)$ or AB (controlled N5 (trial ${ }^{\star}$ or stud $\left.\left.{ }^{\star}\right)\right)$ \\
\hline S25 & $\mathrm{TI}\left(\right.$ clinical $^{\star} \mathrm{N} 5$ trial $\left.^{\star}\right)$ or AB (clinical* N5 trial $^{\star}$ ) \\
\hline S26 & $\begin{array}{l}\left.\left.\text { TI ((control or treatment or experiment }{ }^{\star} \text { or intervention) N5 (group* or subject* or patient }{ }^{\star}\right)\right) \text { or AB } \\
\left.\left.\text { ((control or treatment or experiment }{ }^{\star} \text { or intervention) N5 (group* } \text { or subject }^{\star} \text { or patient }^{\star}\right)\right)\end{array}$ \\
\hline S27 & $\begin{array}{l}\mathrm{TI}((\text { control or experiment* or conservative) N5 (treatment or therapy or procedure or manage* })) \text { or } \\
\mathrm{AB}\left(\left(\text { control or experiment }{ }^{\star} \text { or conservative) N5 (treatment or therapy or procedure or manage }\right)\right)\end{array}$ \\
\hline S28 & 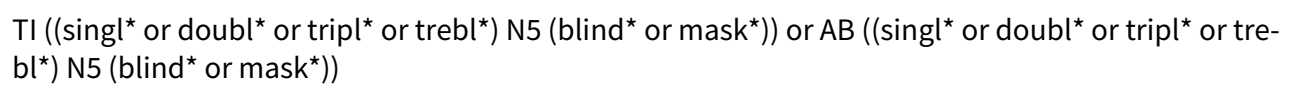 \\
\hline S29 & $\mathrm{TI}$ (cross-over or cross over or crossover) or AB (cross-over or cross over or crossover) \\
\hline S30 & $\mathrm{TI}$ (placebo* or sham) or AB (placebo* or sham) \\
\hline S31 & TI trial \\
\hline S32 & $\mathrm{TI}$ (assign ${ }^{\star}$ or allocat ${ }^{\star}$ ) or AB (assign ${ }^{\star}$ or allocat ${ }^{\star}$ ) \\
\hline S33 & $\mathrm{TI}$ controls or $\mathrm{AB}$ controls \\
\hline S34 & $\begin{array}{l}\mathrm{TI} \text { (quasi-random* or quasi random* or pseudo-random* or pseudo random*) or AB (quasi-ran- } \\
\text { dom }^{\star} \text { or quasi random* or pseudo-random* or pseudo random }{ }^{\star} \text { ) }\end{array}$ \\
\hline S35 & $\begin{array}{l}\text { S17 OR S18 OR S19 OR S20 OR S21 OR S22 OR S23 OR S24 OR S25 OR S26 OR S27 OR S } 28 \text { OR S29 OR } \\
\text { S30 OR S31 OR S32 OR S33 OR S34 S36.S12 AND S16 AND S35 }\end{array}$ \\
\hline S36 & S6 AND S16 AND S35 \\
\hline
\end{tabular}




\section{Appendix 7. Search review 2018 - Web of Science}

\section{Search strategy for Web of Science, August 2018}

The following indexes Science Citation Index Expanded (SCI-EXPANDED), Social Sciences Citation Index (SSCI), and Arts \& Humanities Citation Index (A\&HCl) within Web of Science were searched from January 2002 to August 2018.

\begin{tabular}{|c|c|}
\hline \# & Query \\
\hline \#1 & $\begin{array}{l}\text { TS }=\left(\text { stroke or poststroke or post-stroke or cerebrovasc }^{\star} \text { or brain vasc }^{\star} \text { or cerebral vasc } \text { or cva* or }^{*}\right. \\
\text { apoplex }{ }^{\star} \text { or SAH) }\end{array}$ \\
\hline \#2 & $\begin{array}{l}\text { TS }=\left(\left(\text { brain }^{\star} \text { or cerebr* or cerebell* or intracran }{ }^{\star} \text { or intracerebral) NEAR/5 (isch*emi* } \text { or infarct* or }^{\star}\right.\right. \\
\left.\left.\text { thrombo }{ }^{\star} \text { or emboli* or occlus }\right)\right)\end{array}$ \\
\hline \#3 & $\begin{array}{l}\text { TS }=\left(\left(\text { brain }^{\star} \text { or cerebr* or cerebell }{ }^{\star} \text { or intracerebral or intracranial or subarachnoid) NEAR/5 (haem- }\right.\right. \\
\left.\left.\text { orrhage }^{\star} \text { or hemorrhage* or haematoma* or hematoma* or bleed }{ }^{\star}\right)\right)\end{array}$ \\
\hline \#4 & TS=(hemipleg ${ }^{\star}$ or hemipar ${ }^{\star}$ or paresis or paretic or hemineglect or hemi-neglect) \\
\hline \#5 & $\mathrm{TS}=(($ unilateral or spatial or hemi*spatial or visual) NEAR/5 neglect) \\
\hline \#6 & \#5 OR \#4 OR \#3 OR \#2 OR \#1 \\
\hline \#7 & TS $=\left(\right.$ depress $^{\star}$ or dysthymi*or dysphor ${ }^{\star}$ or antidepress ${ }^{\star}$ or anti-depress $\left.{ }^{\star}\right)$ \\
\hline \#8 & $\mathrm{TS}=\left(\right.$ random ${ }^{*}$ or RCT or RCTs) \\
\hline \#9 & $\mathrm{TS}=\left(\right.$ controlled NEAR/5 $\left(\right.$ trial $^{\star}$ or stud $\left.\left.{ }^{\star}\right)\right)$ \\
\hline \#10 & TS $=\left(\right.$ clinical $^{\star}$ NEAR $/ 5$ trial $\left.^{\star}\right)$ \\
\hline \#11 & $\mathrm{TS}=\left(\left(\right.\right.$ control or treatment or experiment ${ }^{\star}$ or intervention) NEAR/5 (group or subject $^{\star}$ or patient $\left.\left.{ }^{\star}\right)\right)$ \\
\hline \#12 & TS=(quasi-random* or quasi random* or pseudo-random* or pseudo random*) \\
\hline \#13 & $\begin{array}{l}\text { TS }=\left(\left(\text { control or experiment }{ }^{\star} \text { or conservative) NEAR/5 (treatment or therapy or procedure or man- }\right.\right. \\
\left.\left.\text { age }^{\star}\right)\right)\end{array}$ \\
\hline \#14 & $\mathrm{TS}=\left(\left(\right.\right.$ singl ${ }^{\star}$ or doubl ${ }^{\star}$ or tripl ${ }^{\star}$ or trebl $\left.l^{\star}\right)$ NEAR/5 $\left(\right.$ blind $^{\star}$ or mask $\left.\left.^{\star}\right)\right)$ \\
\hline \#15 & $\mathrm{TS}=($ cross-over or cross over or crossover) \\
\hline \#16 & $\mathrm{TS}=($ placebo* or sham) \\
\hline \#17 & $\mathrm{Tl}=$ trial \\
\hline \#18 & TS $=\left(\right.$ assign $^{\star}$ or allocat $\left.{ }^{\star}\right)$ \\
\hline \#19 & $\mathrm{TS}=$ controls \\
\hline \#20 & \#8 OR \#9 OR \#10 OR \#11 OR \#12 OR \#13 OR \#14 OR \#15 OR \#16 OR \#17 OR \#18 or \#19 \\
\hline \#21 & \#6 AND \#7 AND \#20 \\
\hline
\end{tabular}




\section{Appendix 8. Search review 2018 - other sources}

\section{Additional searches}

The following conference abstracts and proceedings were searched.

1. European Stroke Conference (2011- 2018)

2. Stroke Society of Australasia Annual Scientific Meetings (2011- 2017)

3. World Stroke Congress (2000-2016)

4. Asia Pacific Stroke Conference (2011-2017)

Online clinical trials and research registers were also searched August 2018.

- www.ClinicalTrials.gov (https://clinicaltrials.gov/)

(depression OR low mood) AND (Brain Infarction OR Intracranial Hemorrhages OR Carotid Artery Diseases OR Brain Ischemia OR Cerebral Hemorrhage OR Cerebrovascular Disorders OR Stroke)

- WHO International Clinical Trials Registry Platform (https://www.who.int/ictrp/search/en/)

Condition: stroke AND depression OR low mood

Recruitment status is: ALL

Phases are: ALL

Hide synonyms

- 9-52 DEPRESSIVE DISORDERS, BEREAVEMENT, DEPRESSED, DEPRESSED - SYMPTOM, DEPRESSED MOOD, DEPRESSED MOOD (FINDING), DEPRESSED MOOD (PHYSICAL FINDING), DEPRESSED STATE, DEPRESSIVE DIS, DEPRESSIVE DISORDER, DEPRESSIVE DISORDER (DISORDER), DEPRESSIVE DISORDER [DISEASE/FINDING], DEPRESSIVE DISORDER NOS, DEPRESSIVE DISORDER, NOS, DEPRESSIVE DISORDERS, DEPRESSIVE DISORDERS NOS, DEPRESSIVE ILLNESS, DEPRESSIVE NEUROSES, DEPRESSIVE NEUROSIS, DEPRESSIVE STATE, DEPRESSIVE STATE NOS, DEPRESSIVE; DISORDER, DEPRESSIVE; NEUROSIS, DEPRESSIVE; STATE, DISORDER, DEPRESSIVE, DISORDER; DEPRESSIVE, DISORDERS, DEPRESSIVE, DYSTHYMIC DISORDER, FEELING BLUE, FEELING DOWN, FEELING;DOWN, LOW MOOD, MELANCHOLY, MISERABLE, MOOD DEPRESSED, MOOD DISORDER OF DEPRESSED TYPE, MOOD DISORDER OF DEPRESSED TYPE (DISORDER), MOROSE MOOD, NEUROSES, DEPRESSIVE, NEUROSIS, DEPRESSIVE, NEUROSIS; DEPRESSIVE, PUSH DOWN OR DEPRESS, STATE; DEPRESSIVE, depression - DEPRESSED, DEPRESSED MOOD, DEPRESSED MOOD (FINDING), DEPRESSED MOOD (PHYSICAL FINDING), FEELING BLUE, FEELING DOWN, FEELING;DOWN, MELANCHOLY, MOOD DEPRESSED, MOOD DEPRESSION, MOOD DEPRESSIONS, MOROSE MOOD, low mood - ACCIDENT CEREBROVASCULAR, ACCIDENT; CEREBRAL, ACCIDENT; CEREBROVASCULAR, APOPLEXY, APOPLEXY, CEREBROVASCULAR, APOPLEXY; CEREBRAL, BRAIN ATTACK, BRAIN VASCULAR ACCIDENT, BRAIN VASCULAR ACCIDENTS, CEREBRAL VASCULAR ACCIDENT, CEREBRAL VASCULAR EVENTS, CEREBRAL; ACCIDENT, CEREBRAL; APOPLEXY, CEREBROVASCULAR ACCIDENT, CEREBROVASCULAR ACCIDENT (DISORDER), CEREBROVASCULAR ACCIDENT NOS, CEREBROVASCULAR ACCIDENT, NOS, CEREBROVASCULAR ACCIDENTS, CEREBROVASCULAR APOPLEXY, CEREBROVASCULAR; ACCIDENT, CVA, CVA (CEREBRAL VASCULAR ACCIDENT), CVA (CEREBROVASCULAR ACCIDENT), CVA NOS, CVAS (CEREBROVASCULAR ACCIDENT), NEURO: CEREBROVASCULAR ACCIDENT, VASCULAR ACCIDENT, BRAIN, VASCULAR ACCIDENTS, BRAIN, stroke

\section{WHAT'S NEW}

\begin{tabular}{lll}
\hline Date & Event & Description \\
\hline 13 August 2018 & New search has been performed & $\begin{array}{l}\text { New interventions are included: combination psychological and } \\
\text { pharmacological interventions vs a single intervention, and non- } \\
\text { invasive brain stimulation interventions }\end{array}$
\end{tabular}

Thirty-three new trials (39 comparisons) with 2753 participants are included in the review. A total of 49 trials (56 comparisons) with 3342 participants are included in the review. Data were available for 20 pharmacological comparisons, 8 non-invasive brain stimulation comparisons, 16 psychological therapy comparisons, and 12 combination therapy trials

Covidence was used to collate and screen identified titles and abstracts

$\mathrm{MH}$ extracted additional data from previously included trials 


\begin{tabular}{lll}
\hline Date & Event & Description \\
\hline & Searches for the review were completed to 13 August 2018 & New citation required and conclusions \\
\hline have changed & Nugust 2018 & \begin{tabular}{l} 
Newcluded. New authors are included \\
\hline
\end{tabular}
\end{tabular}

\section{HIS T O R Y}

Protocol first published: Issue 1, 2002

Review first published: Issue 3, 2004

\begin{tabular}{lll}
\hline Date & Event & Description \\
\hline 28 March 2008 & Amended & Review was converted to new review format \\
\hline 14 March 2008 & New search has been performed & Searches for the review were completed to February 2008 \\
& $\begin{array}{l}\text { Seven new trials have been added: } 6 \text { pharmacological interven- } \\
\text { tions, making a total of 13, and 2 psychological interventions, } \\
\text { making a total of } 4 \text { comparisons. A total of } 16 \text { trials with } 1655 \\
\text { participants are now included }\end{array}$
\end{tabular}

Eight trials require more information before they can be assessed for inclusion in the review (down from 14 in the previous version). Nine trials appear to meet the review inclusion criteria, but information is not available in a format suitable for pooling. Three studies are ongoing (up from 0 in the previous version)

14 March $2008 \quad$ New citation required and conclusions have changed
This version of the review found a small but significant effect of pharmacotherapy (not psychotherapy) on treating depression and reducing depressive symptoms in stroke patients

There has been a change in authorship

\section{CONTRIBUTIONSOF AUTHORS}

SA: contributed to writing the review, completed title screening and inclusion/exclusion review, extracted data, performed meta-analyses and GRADE assessment.

KC: completed title screening and inclusion/exclusion review and data extraction.

$\mathrm{CFH}$ : assisted with obtaining, translating, and extracting data from Chinese studies for the current updated review. $\mathrm{HL}$ : completed title screening and cross-checked data extraction.

$\mathrm{AH}$ : conceived the idea for the review; contributed to development, writing, and editing of the protocol; and undertook the work necessary to complete the 2004 and 2008 reviews.

$\mathrm{MH}$ : contributed to development, writing, and editing of the protocol; undertook the work necessary to complete the 2004 and 2008 reviews; and oversaw each version of the review updates.

All review authors read and edited this update.

\section{DECLARATIONS OF INTEREST}

SA: none known.

KC: none known.

C-FH: none known.

$\mathrm{HL}$ : none known.

$\mathrm{AH}$ : none known.

$\mathrm{MH}$ : none known. 


\section{SOURCES OF SUPPORT}

\section{Internal sources}

- The George Institute for International Health, Australia.

\section{External sources}

- Stroke Society of Australasia, Overseas Study Scholarship, Australia.

- The Academic Unit of Psychiatry, The University of Leeds, UK.

- The Department of Clinical Neurosciences, The University of Edinburgh, UK.

- The Clinical Trials Research Unit, The University of Auckland, New Zealand.

\section{DIFFERENCES BETWEEN PROTOCOL AND REVIEW}

In this update, the review was expanded to include other non-invasive brain stimulation interventions such as (1) transcranial magnetic stimulation or repetitive transcranial magnetic stimulation (TMS or rTMS, where a magnetic 'coil' is placed near the head of the person receiving treatment without making physical contact); (2) transcranial direct current stimulation (tDCS, where a constant, low current is delivered directly to the brain area of interest via small electrodes); (3) cranial electrotherapy stimulation (CES, where a small, pulsed electrical current is applied across a patient's head); and (4) magnetic seizure therapy (MST), a type of convulsive therapy that involves replacing the electrical stimulation used in ECT with a rapidly alternating strong magnetic stimulation; and (5) combinations of all included interventions compared with a single intervention plus a respective control.

\section{INDEX TERMS}

\section{Medical Subject Headings (MeSH)}

Antidepressive Agents [ ${ }^{\star}$ therapeutic use]; Depressive Disorder [etiology] [ ${ }^{*}$ therapy]; Electric Stimulation Therapy [ ${ }^{\star}$ methods]; Psychotherapy [*methods]; Quality of Life; Randomized Controlled Trials as Topic; Stroke [ ${ }^{*}$ psychology]

\section{MeSH check words}

Humans 